\title{
CLOSURE REPORT FOR CORRECTIVE ACTION UNIT 92: AREA 6 DECONTAMINATION POND NEVADA TEST SITE, NEVADA
}

Controlled Copy No.:

Revision: 0

April 1999

Prepared for the

U.S. Department of Energy

Nevada Operations Office

Work Performed Under Contract No. DE-AC08-96NV11718 
Available to the public from:

U.S. Department of Commerce

National Technical Information Service

5285 Port Royal Road

Springfield, VA 22161-0002

(703) 487-4650

Available electronically at http://www.doe gov/bridge. Available to U.S. Department of Energy and its contractors in paper from:

U.S. Department of Energy

Office of Scientific and Technical Information

P.O. Box 62

Oak Ridge, TN 37831-0062

(423) 576-8401

Reference herein to any specific commercial product, process, or service by trade name, trademark, manufacturer, or otherwise, does not necessarily constitute or imply its endorsement, recommendation, or favoring by the U.S. Government or any agency thereof or its contractors or subcontractors. 


\section{CLOSURE REPORT FOR CORRECTIVE ACTION UNIT 92: \\ AREA 6 DECONTAMINATION POND NEVADA TEST SITE, NEVADA}

Controlled Copy No.:

Revision: 0

April 1999

Prepared for the

U. S. Department of Energy

Nevada Operations Office

Work Performed Under Contract No. DE-AC08-96NV11718 
THIS PAGE INTENTIONALLY LEFT BLANK 


\section{CLOSURE REPORT FOR CORRECTIVE ACTION UNIT 92: AREA 6 DECONTAMINATION POND NEVADA TEST SITE, NEVADA}

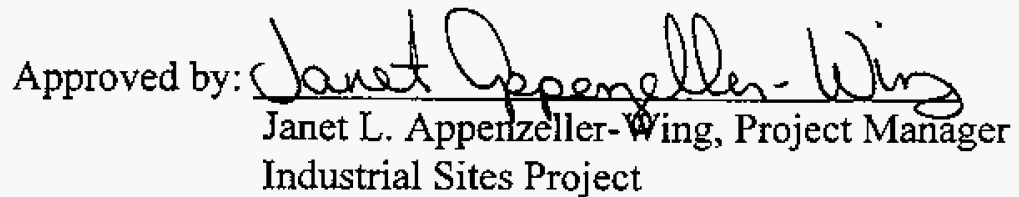

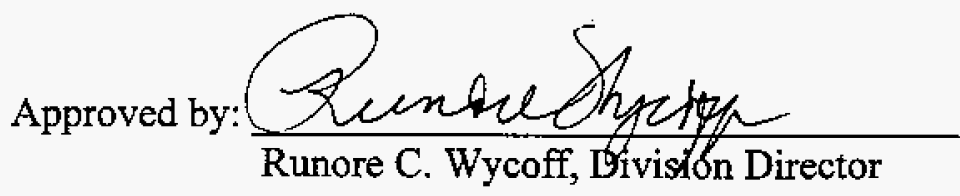
Nevada Environmental Division
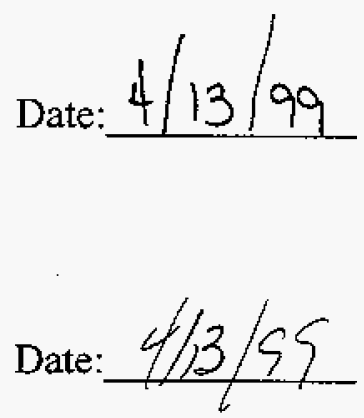
THIS PAGE INTENTIONALLY LEFT BLANK 
ACRONYMS AND ABBREVIATIONS $\ldots \ldots \ldots \ldots \ldots \ldots \ldots \ldots \ldots \ldots \ldots \ldots \ldots$ vii

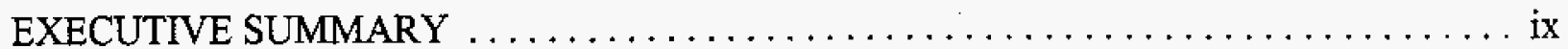

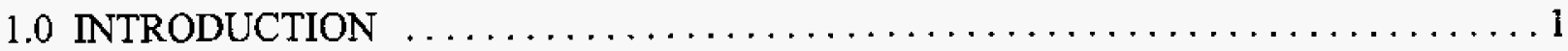

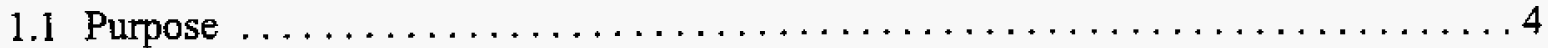

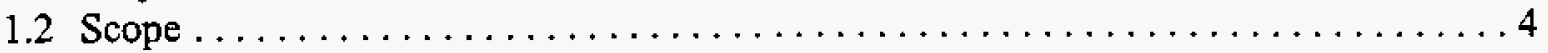

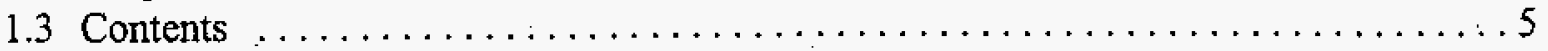

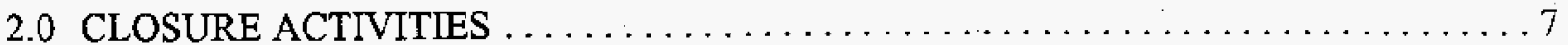

2.1 Description of Corrective Action Activities . . . . . . . . . . . . . . . . . . 7

2.1.1 Decontamination Pad Oil/Water Separator - CAS 06-04-01 Corrective Action . 7

2.1.1.1 Excavation and Pipe Removal $\ldots \ldots \ldots \ldots \ldots \ldots \ldots \ldots \ldots$

2.1.1.2 Soil Sampling $\ldots \ldots \ldots \ldots \ldots \ldots \ldots \ldots \ldots \ldots \ldots \ldots \ldots \ldots$

2.1.1.3 Grouting and Backfilling $\ldots \ldots \ldots \ldots \ldots \ldots \ldots \ldots \ldots \ldots \ldots \ldots \ldots \ldots$

2.1.1.4 Waste Disposal .............................. 10

2.1.2 Decontamination Pond - CAS 06-05-02 Corrective Action $\ldots \ldots \ldots \ldots \ldots, 10$

2.1.2.1 Berm Removal and Placement $\ldots \ldots \ldots \ldots \ldots \ldots \ldots \ldots \ldots \ldots \ldots$

2.1.2.2 Shaping Fill Placement $\ldots \ldots \ldots \ldots \ldots \ldots \ldots \ldots \ldots \ldots \ldots \ldots \ldots \ldots \ldots$

2.1.2.3 Bedding/GCL/Buffer Layer Placement $\ldots \ldots \ldots \ldots \ldots \ldots \ldots \ldots .11$

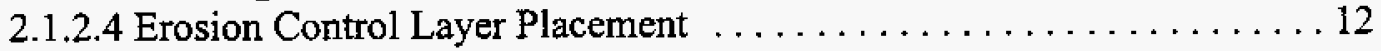

2.1.3 Certification of Closure . . . . . . . . . . . . . . . . . . . . . . . 12

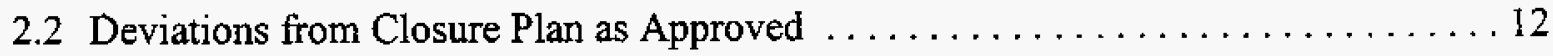

2.3 Corrective Action Schedule as Completed $\ldots \ldots \ldots \ldots \ldots \ldots \ldots \ldots \ldots \ldots \ldots \ldots \ldots$

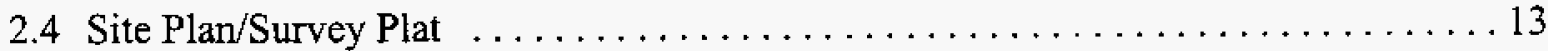

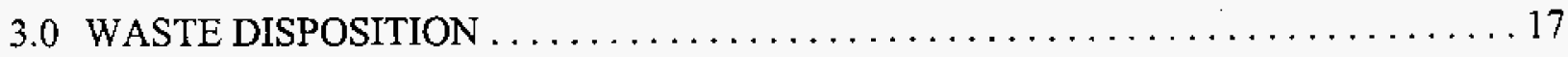

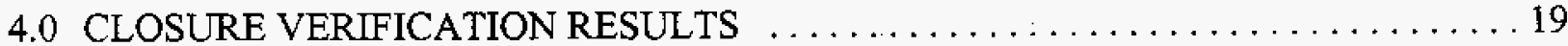

4.1 Decontamination Pad Oil/Water Separator - CAS 06-04-01 Verification Results . . . . 19

4.2 Decontamination Pond - CAS 06-05-02 Verification Results . . . . . . . . . . 27

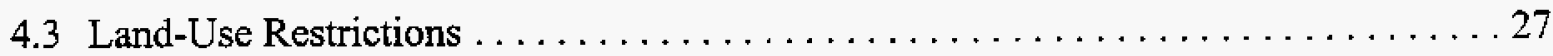

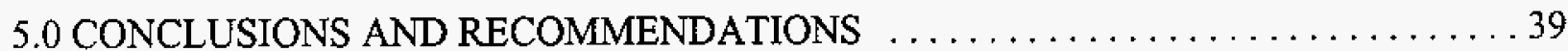

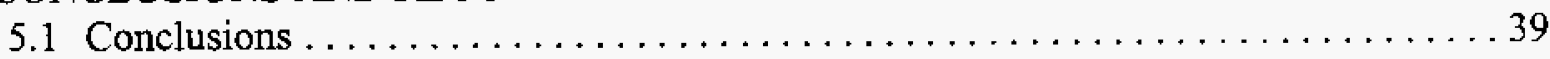

5.1.1 Decontamination Pad Oil/Water Separator - CAS 06-04-01 . . . . . . . . . 39

5.1.2 Decontamination Pond - CAS 06-05-02 .................... 39

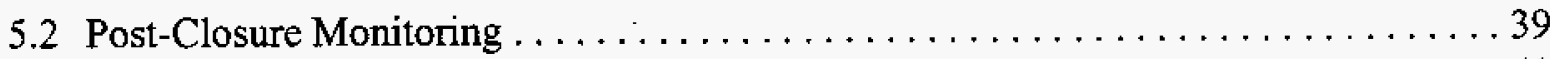

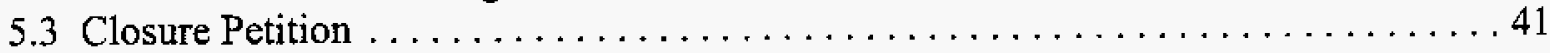

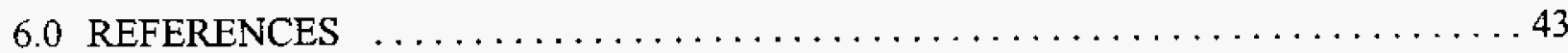


APPENDIX A - PROJECT PHOTOGRAPHS

APPENDIX B - SAMPLE ANALYTICAL RESULTS

APPENDIX C - CLOSURE CERTIFICATION

APPENDIX D - DESIGN CHANGE NOTICE

APPENDIX E - AS-BUILT DRAWINGS FOR THE DECONTAMINATION POND COVER

APPENDIX F - APPROVAL MATRIX AND CHECKLIST

APPENDIX G - COMMENT RESPONSE DOCUMENTATION

DISTRIBUTION LIST

\section{FIGURES}

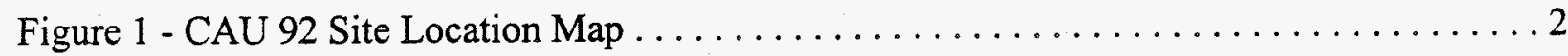

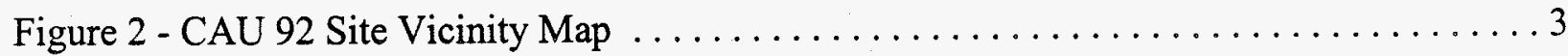

Figure 3 - Oil/Water Separator and Pipeline Verification Sampling Locations $\ldots \ldots \ldots \ldots .9$

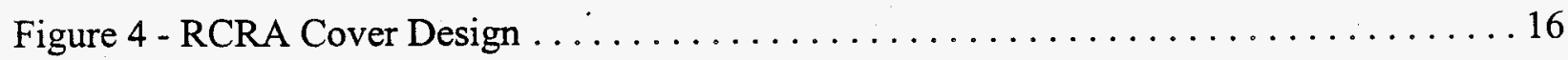

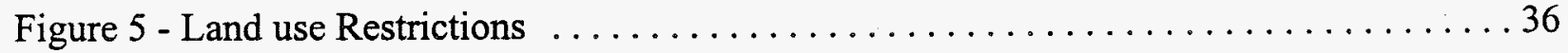

Figure 6 - Locations for the RCRA Cover Nuclear Density Tests $\ldots \ldots \ldots \ldots \ldots \ldots \ldots$

\section{TABLES}

Table 1 - CAU 92 Closure Schedule as Completed ............................ 14

Table 2 - Decontamination Oil/Water Separator and Pipeline Verification Sample Results $\ldots 20$

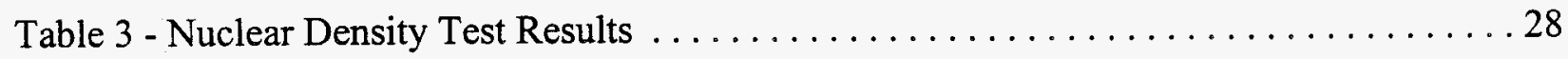




\begin{tabular}{ll} 
ASTM & American Standard Testing Methods \\
BN & Bechtel Nevada \\
CAP & Corrective Action Plan \\
CAS & Corrective Action Site \\
CAU & Corrective Action Unit \\
CFR & Code of Federal Regulations \\
cm & centimeter \\
CMS & Corrective Measures Study \\
COC & Constituent of Concern \\
CR & Closure Report \\
CQA & Construction Quality Assurance \\
DOE & U.S. Department of Energy, Nevada Operations Office \\
EPA & U.S. Environmental Protection Agency \\
FFACO & Federal Facility Agreement and Consent Order \\
ft & feet \\
GCL & Geosythetic Clay Liner \\
IDW & Investigation Derived Waste \\
m & meter \\
NAC & milligrams per kilograms \\
mill & mevada Administrative Code \\
\hline
\end{tabular}




\section{ACRONYMS AND ABBREVIATIONS (CONTINUED)}

NDEP Nevada Division of Environmental Protection

PCB Polychlorinated biphenyls

$\mathrm{pCi} / \mathrm{g} \quad$ picocuries per gram

RCRA Resource Conservation and Recovery Act

SVOC Semivolatile Organic Compounds

TCLP Toxicity Characteristic Leaching Potential

TPH Total Petroleum Hydrocarbons

ug/kg micrograms per kilogram

VOC Volatile Organic Compounds 
The Area 6 Decontamination Pond, Corrective Action Unit (CAU) 92, was closed in accordance with the Resource Conservation and Recovery Act (RCRA) Part B operational permit (Nevada Division of Environmental Protection [NDEP], 1995) and the Federal Facility Agreement and Consent Order (NDEP et al, 1996). The Decontamination Pond CAU consists of two Corrective Action Sites (CASs): the Decontamination Pond (CAS 06-05-02) and the Decontamination Pad Oil/Water Separator (separator \#3) (CAS 06-04-01). The two CASs were connected by approximately 180 meters $(\mathrm{m})$ ( 600 feet [ft]) of clay pipe. Historically the Decontamination Pond was used for the disposal of partially treated liquid effluent discharged from the Decontamination Facility (Building 6-05) and the Industrial Laundry (Building 6-07) (U:S. Department of Energy [DOE], 1996a).

Heavy equipment and critical tolerance equipment used in weapons testing were processed in Building 6-605 to remove radioactive contamination. The equipment was taken into the decontamination bay and treated using degreasers, caustic or acid solutions, and detergents, and then spray washed with pressurized hot water (DOE, 1996a). The laundry process used standard industrial water softeners and laundry detergents to decontaminate personnel protective equipment (DOE, 1996a).

The Decontamination Pond was constructed and became operational in 1979. Releases of RCRA-regulated hazardous waste or hazardous waste constituents have not been discharged to the Decontamination Pond since 1988 (DOE, 1996a). The pipe connecting the Decontamination Pond and Decontamination Facility and Laundry were cut and sealed at the Decontamination Pad Oil/Water Separator in 1992.

The Decontamination Pond characterization activities detected the following RCRA-regulated hazardous waste constituents: chlorobenzene, 1,1,1-trichloroethane, and lead. Xylene isomers as petroleum hydrocarbons were also identified (DOE, 1997a). In addition, the following radionuclides were detected: cobalt-60, cesium-137, plutonium-238, and tritium (DOE, 1997a).

Closure of CAU 92 was completed using a NDEP-approved Closure Plan, Revision 2, dated November 1998 (DOE, 1998a). The closure plan was based on the recommendations presented in a Corrective Measures Study (CMS) (DOE, 1997b). The CMS was developed from the analytical results of three separate characterization studies (DOE, 1997a). The Area 6 Decontamination Pond, CAU 92 was closed in the following manner:

- Decontamination Pad Oil/Water Separator, CAS 06-04-01 was closed in place by sealing the separator with expanding cement. The excavation exposing the separator was backfilled with clean fill material.

- The clay pipe from $6.1 \mathrm{~m}(20 \mathrm{ft})$ north of the Decontamination Facility fence line to the CAS 06-04-01 was closed in place by sealing the pipe with cement grout. 
- The clay pipe from $6.1 \mathrm{~m}(20 \mathrm{ft})$ north of the Decontamination Facility fence line to the Decontamination Pond was removed and placed on the base of the Decontamination Pond prior to construction of the cover. After the pipe and impacted soil were excavated, verification soil samples were collected. Analytical data from one verification sample (DECON-P3) indicated that Americium-241 was slightiy above the minimal detectable activity. The soil sumounding this verification sample location was over excavated and the soil disposed in the base of the Decontamination Pond. One additional verification sample was collected to confirm that all impacted soil had been excavated.

- Impacted soils, clay pipe, and characterization investigation waste was removed and placed on the base of the decontamination pond prior to the start of the RCRA cover closure activities.

- The Decontamination Pond, CAS 06-05-02 was closed in place by installing a RCRA cover. Following cover construction, a fence was constructed around the cover to prevent accidental damage to the cover.

Closure activities were certified by an independent registered professional. Post-closure monitoring at the site will consist of quarterly inspections of the RCRA cover. Inspections will also be conducted if:

- Precipitation occurs in excess of 1.28 centimeters (cm) $(0.50$ inches [in]) in a 24 hour period, or

- Earthquake occurs with a magnitude exceeding 4.5 on the Richter scale within 100 kilometers (62 miles). 


\subsection{INTRODUCTION}

This closure report (CR) provides documentation for the closure of Corrective Action Unit (CAU) 92 identified in the Federal Facility Agreement and Consent Order (FFACO) (NDEP, 1996). The site is located at the Nevada Test Site Area 6, adjacent to the Decontamination Facility in the southem portion of Yucca Flat (Figure 1). The CAU consists of two Corrective Action Sites (CAS) 06-05-02 and CAS 06-04-01 and the clay pipe which connects the two CASs. The locations of the various parts of the urit are found in Figure 2.

The Decontamination Pond was used for the disposal of partially treated liquid effluent discharged from Buildings 6-605 (Decontamination Facility) and 6-607 (Industrial Laundry). Effluent from Buildings 6-605 and 6-607 was piped into the Decontamination Pad Oil/Water Separator which is the boundary between the Decontamination Facility (CAU 244) and the Decontamination Pond (CAU 92). Waste was piped from the separator northeast to the Decontamination Pond, a distance of approximately 180 meters $(m)$ (600 feet [ft]) (L.S. Department of Energy [DOE], 1996a).

Radioactively contaminated site worker clothing (i.e., coveralls, gloves, and boot covers) are processed in the laundry in Building 6-607. The laundry process used standard industrial water softeners and laundry detergents to decontaminate the personnel protective equipment. The waste water from this operation discharged into the Oil/Water Separator (Sump No. 3) which is the first component of the Decontamination Pond CAU (DOE, 1996a).

Heavy equipment and critical tolerance equipment used in weapons testing were processed in Building 6-605 to remove radioactive contamination. The equipment was taken into the decontamination bay and treated using degreasers, caustic or acid solutions, and detergents and then spray washed with pressurized hot water. The wash water was collected in floor drains, and the water was piped through a sand filter/separator. This partialiy treated water also fed into the Oil/Water Separator (Sump No. 3), which is the first component of the Decontamination Pond CAC. The Decontamination Facility and associated pipe upgradient of Sump 3, will be addressed after the Decontamination Facility is closed (DOE, 1996a).

The Decontamination Pond was constructed and became operational in 1979. It is a square unit, approximately $60 \mathrm{~m}(200 \mathrm{ft})$ long on each interior side with perimeter berms $1.5 \mathrm{~m}(5 \mathrm{ft})$ above surrounding grade. A discharge pipe from the Decontamination Pad oil/water separator enters the pond on the southwest side approximately $0.3 \mathrm{~m}(1 \mathrm{ft})$ above the base of the pond. The pond is unlined and is surrounded by an earth berm on all four sides with a two-to-one grade (DOE, 1996a).

Limited documentation on the volume of waste water entering the Decontamination Pond is available. During a typical year approximately 14,000 cubic meters ( 3.6 million gallons) of effluent was discharged into the Decontamination Pond (DOE, 1996a). The volume of water discharged into the Decontamination Pond was dependent on the amount of nuclear testing conducted each year. Three previous characterization activities were done at the Area 6 


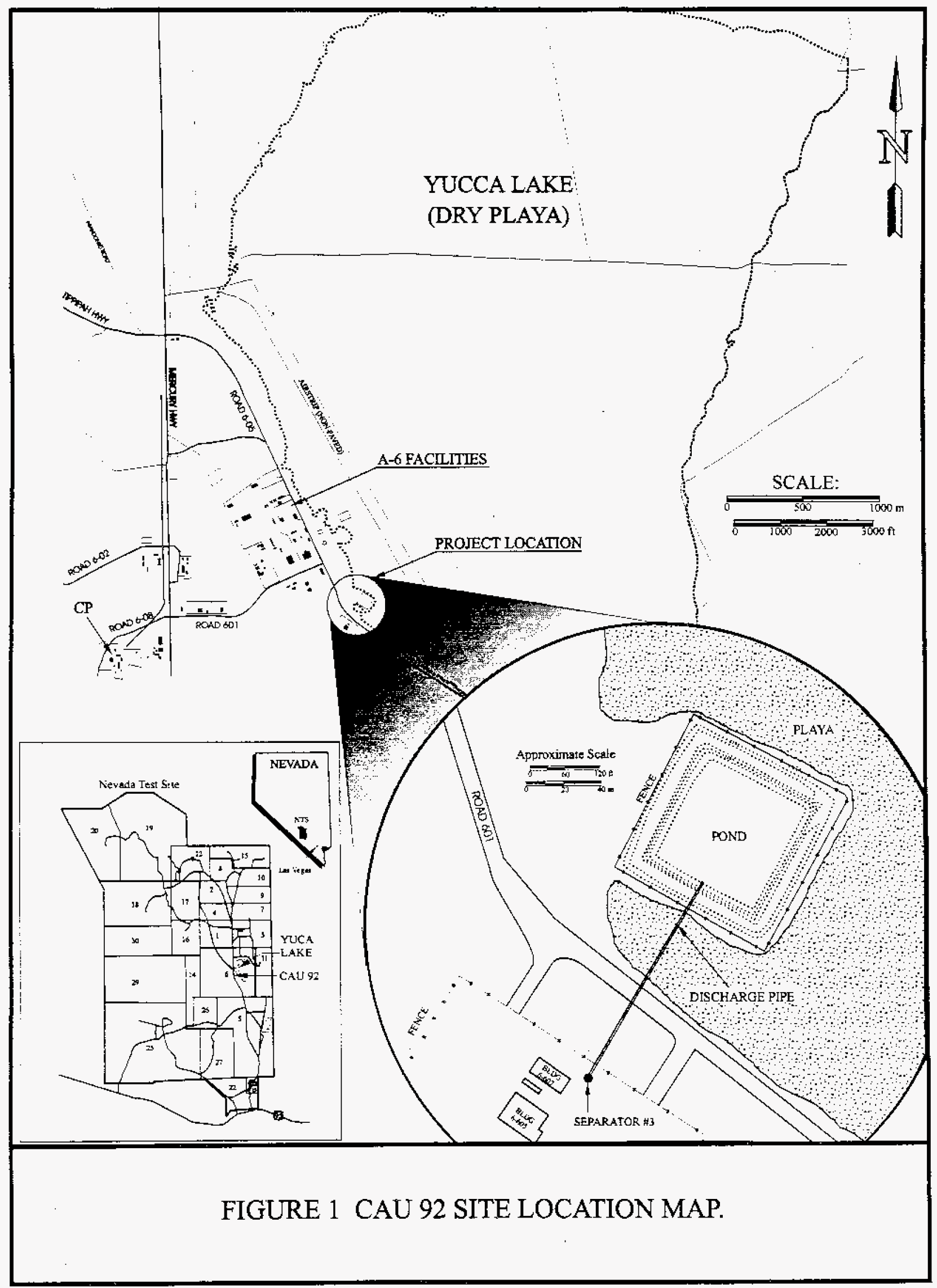




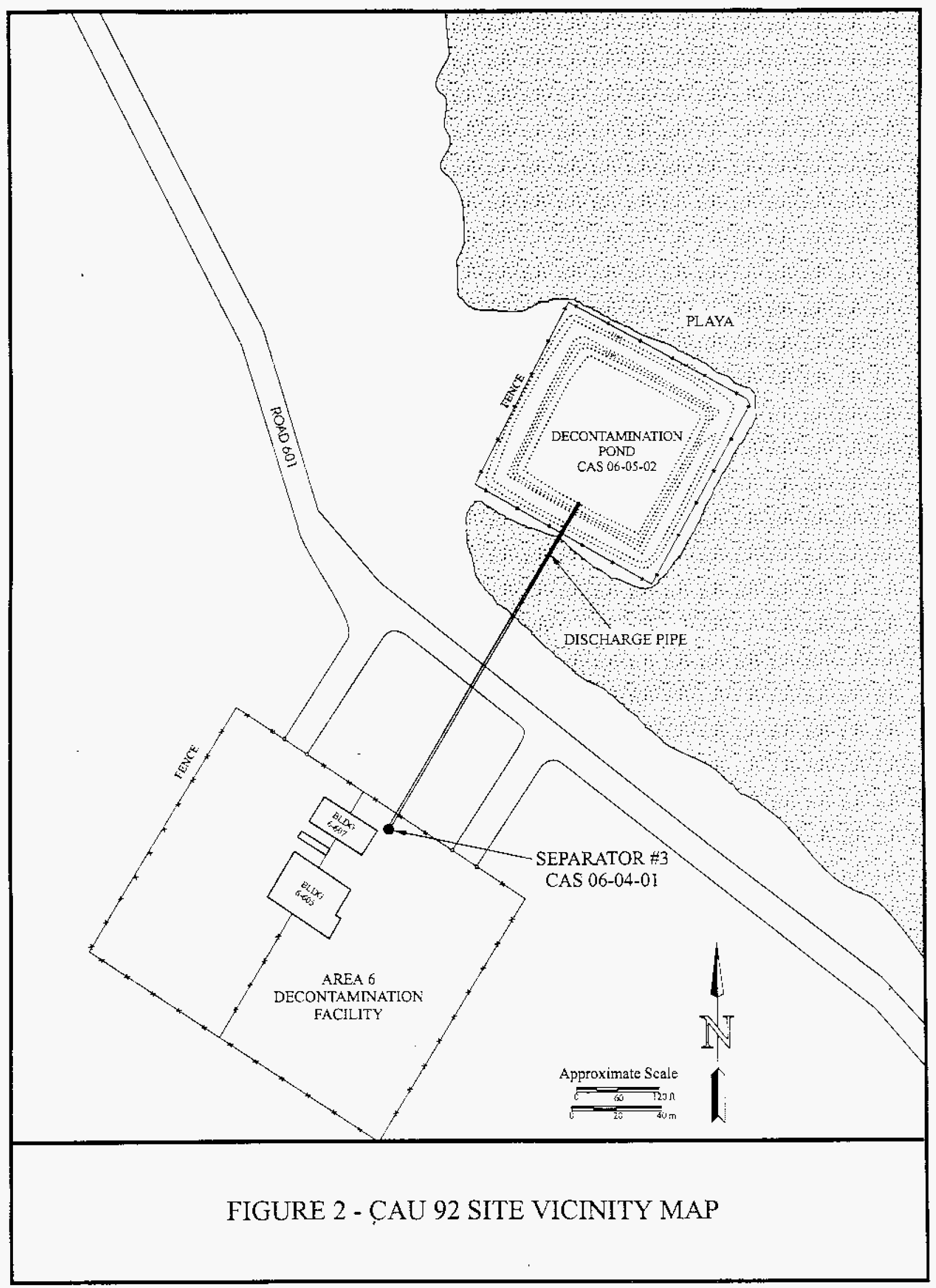


Decontamination Pond and the results are documented in the Resource Conservation and Recovery Act, Industrial Site Environmental Restoration Site Characterization Report, Area 6 Decontamination Pond Facility (DOE, 1997a). Characterization data indicates that the native soils in the floor of the Decontamination Pond are primarily impacted to a depth of $0.3 \mathrm{~m}$ ( $1 \mathrm{ft}$ ) below grade.

Suspected Resource Conservation and Recovery Act (RCRA) regulated hazardous waste or hazardous waste constituents released into the Decontamination Pond were degreasing solvents associated with equipment decontamination activities (chlorobenzene and 1,1,1-trichloroethane), petroleum hydrocarbon constituents (xylenes), and heavy metals (lead and arsenic). Releases of RCRA-regulated hazardous waste or hazardous waste constituents have not been discharged to the Decontamination Pond since 1988 (DOE, 1996a). The following radionuclides were detected: cobalt-60, cesium-137, plutonium-238, and tritium. A detailed discussion of the unit activities and analytical results can be found in the Characterization Report (DOE, 1997a).

\subsection{PURPOSE}

The purpose of this CR is to:

- Document the closure activities as proposed in the Closure Plan (DOE, 1998a).

- Obtain a Notice of Completion from the Nevada Division of Environmental Protection (NDEP).

- Recommend the movement of CAU 92 from Appendix III to Appendix IV of the FEACO.

\subsection{SCOPE}

The following is the scope of the closure actions implemented for CAU 92:

- The clay pipe that connected the Decontamination Pad oil/water separator and the Decontamination Pond was excavated up to within $6.1 \mathrm{~m}$ (20 ft) of the Decontamination Facility fence line (Figure 2). This pipe was disposed of in the Decontamination Pond. The associated impacted soil was excavated and disposed along with the pipe. Prior to disposal, waste characterization samples were collected from the pipe and associated soil (Section 4.0). The clay pipe between $20 \mathrm{ft}$ of the Decontamination Facility fence line and the separator was closed in place using an expandable cement grout installed under pressure.

- Excavations were backfilled with clean fill and compacted following accepted construction practices. 
- The Decontamination Pad oil/water separator (CAS 06-04-01) was closed in place. The soil around the separator was sampled (Section 4.0), and the separator filled with expanding cement grout.

- The Decontamination Pond (CAS 06-05-02) was closed by constructing an engineered cover. First the pipe, excavated soil from around the pipe (previously stored in a B-25 container), and characterization investigation derived waste (IDW) were added to the base of the pond. The berm walls were then pushed into the pond and compacted to meet the Construction Quality Assurance (CQA) Plan. Additional materials were imported to form subsequent layers following the CQA Plan (DOE, 1998a). This cover was designed to meet or exceed all requirements stated in 40 Code of Federal Regulations (CFR) $\$ 265.228(a)$ (2).

Closure of CAU 92 was completed using an NDEP-approved Closure Plan, Revision 2, dated November 1998 (DOE, 1998a).

\subsection{CONTENTS}

This $\mathrm{CR}$ is divided into the foliowing sections:

- Section 1.0 - Introduction

- Section 2.0 - Closure Activities

- Section 3.0- Waste Disposition

- Section 4.0 - Closure Verification Results

- Section 5.0 - Conclusions and Recommendations

- Section 6.0 - References

This report was developed using information and guidance from the following documents:

- Bechtel Nevada Performance Management Plan, B-A20/96.01, BN, 1997.

- Resource Conservation and Recovery Act, Industrial Sites, Environmental Restoration Site Characterization Plan, Area 6 Decontamination Pond Facility, Rev, 1. DOE, 1996 .

- Resource Conservation and Recovery Act, Industrial Sites Environmental Restoration Site Chatacterization Report, Area 6 Decontamination Pond Facility, Corrective Action Unit No. 92 Rev. 1, DOE, 1997a. 
- Resource Conservation and Recovery Act. Conective Measures Study: Area 6. Decontamination Pond Facility, Corrective Action Unit Jio. 92, Rev, 0, DOE, $1997 \mathrm{~b}$.

- Closure Plan for Corrective.Action Unit 92: Area 6 Decontamination Pond Nevada Test Site, Nevada, Revision 2, DOE, 1998a.

- Report of Activities and Geotechnical/Hydrological Results May and June 1997 Characterization Sampling_Area 6 Decontamination Pond, CAU 92, DOE, 1997c.

- Nevada EnvironmentaI Restoration Project.Industrial Sites, Quality Assurance Project Plan, Nevada Test Site, Nevada, Revision 1, DOE, 1996 b.

- Nevada Environmental Restoration Project, Health and Safety Plan, Revision 3, DOE, $1998 \mathrm{~b}$. 


\subsection{CLOSURE ACTIVITIES}

The closure activities associated with the Decontamination Pad oil/water separator were initiated on March 10,1998, with a health and safety kickoff meeting and mobilization. Excavation of the effluent pipe and soil sampling around the separator and pipe were performed in multiple phases. Final closure was completed on October 12, 1998, after the $19.8 \mathrm{~m}(65 \mathrm{ft})$ of pipe closest to the Decontamination Facility was grouted in place and the excavation backfilled. The specific activities associated with the Decontamination Pad oil/water separator and pipeline closure are described below. Appendix A includes digital images of selected activities throughout the duration of the project.

The closure activities associated with the Decontamination Pond were initiated on July 30,1998 , with a health and safety kickoff meeting and mobilization. Final closure was completed on February 16,1999, after the fencing around the cover was installed. The specific activities associated with the Decontamination Pond closure are described below. Appendix A includes digital images of selected activities throughout the duration of the project.

\subsection{DESCRIPTION OF CORRECTIVE ACTION ACTIVITIES}

Closure of CAL 92 was completed using the approved Closure Plan for Corrective Action Unit 22. Area 6 Decontamination Pond, Nevada Test Site, Nevada, Revision 2, dated November 1998 (DOE, 1998a). The closure plan was based on the recommendations presented in a Corrective Measures Study (CMS) (DOE, 1997b). The CMS was developed from the analytical results of three separate characterization studies (DOE, 1997a). Before the closure field activities began, the following prefield activities were completed:

- Preparation of National Environmental Protection Act documentation.

- Preparation of the Site-Specific Health \& Safety Plan.

- Preparation of the Field Management Plan.

- An As Low As Reasonably Achievable Committee review of the proposed activities.

The following is the scope of the closure actions implemented for CAL 92, separated by CAS number.

\subsubsection{Decontamination Pad Oil/Water Separator - CAS 06-04-01 Corrective Action}

The closure activities associated with the Decontamination Pad oil/water separator and effluent pipe were initiated on March 10, 1998, and carried out in multiple phases. These phases included excavation and pipe removal, verification sampling, and grouting and backfilling. 


\subsubsection{Excavation and Pipe Removal}

Before pipe excavation and removal activities began, an approved Excavating and Trenching Permit was obtained and the exclusion zone and hotline were established in the vicinity of Sump 3. The effluent pipeline connecting Sump 3 to the Decontamination Pond was excavated using a backhoe. The effluent pipeline was located at a depth of $1.8 \mathrm{~m}(6 \mathrm{ft})$ at Sump 3. The first $19.8 \mathrm{~m}(65 \mathrm{ft})$ of pipeline was cast iron and encased in concrete. The remainder of the pipe was made of clay. The pipeline was excavated in stages and the $145-\mathrm{m}$ (475-ft) portion which was not encased in concrete was completely removed. Each pipe section was separated at the joint. After the pipe joints were disconnected, a Radiological Control Technician surveyed the exterior and interior of the pipe using a NE Technology Model Electra for radiological activity levels. A MSA Photon Gas Detector was subsequently used to detect for the presence of Volatile Organic Compounds (VOCs) within the pipe. The pipe sections were removed using a sling connected to the backhoe. Once out of the excavation, the pipe sections were wrapped in plastic and stored on site in a SEALAND ${ }^{2}$ container.

Soils excavated from along the pipeline were stockpiled adjacent to the trench. During pipe excavation, soils that showed discoloration were separated and put into a B-25 container. Verification sampling indicated that the soils in one location along the pipeline contained Americium-241 activity levels above background. The soils from this sample location were over excavated and stored in the B-25 container.

\subsubsection{Soil Sampling}

Soil samples were collected from the soils surrounding Sump 3 and along the pipeline excavation. Three additional sludge samples were taken from the interior of the clay pipe. All sample locations were analyzed for VOCs, Semivolatile Organic Compounds (SVOCs), Toxicity Characteristic Leaching Potential (TCLP) metals, Polychlorinated biphenyls (PCBs), Total Petroleum Hydrocarbon (TPH), gamma scan, and gross alpha/beta. Unique sample identifications were given to each sample to distinguish sample locations. In addition, quality assurance samples were also collected. Blind duplicates and equipment blanks were collected for at least 20 percent of the samples, trip blanks were sent with every sample batch for VOC analysis, and matrix spike/matrix spike duplicate samples were designated in every sample batch.

Undisturbed grab samples from the vicinity of Sump 3 (Appendix B, Samples A6-SMP-E1 through A6-SMP-E4 and A6-SMP-W1 through A6-SMP-W4) were collected using a Geoprobe . All other samples were collected directly from the excavation adjacent to a pipe joint or within the pipe directly. Samples were placed in clean, glass or nalgene laboratory jars. All samples were labeled with a sample identification, date and time sampled, sampler's initials, and analysis to be preformed. A custody seal was placed across the lid of each sample container. Samples were placed in an ice chest and cooled to approximately 4 degrees Centigrade ( 39 degrees Fahrenheit). Samples were controlled under strict chain of custody until relinquished to the laboratory. Figure 3 provides the sample locations from which they were taken. 


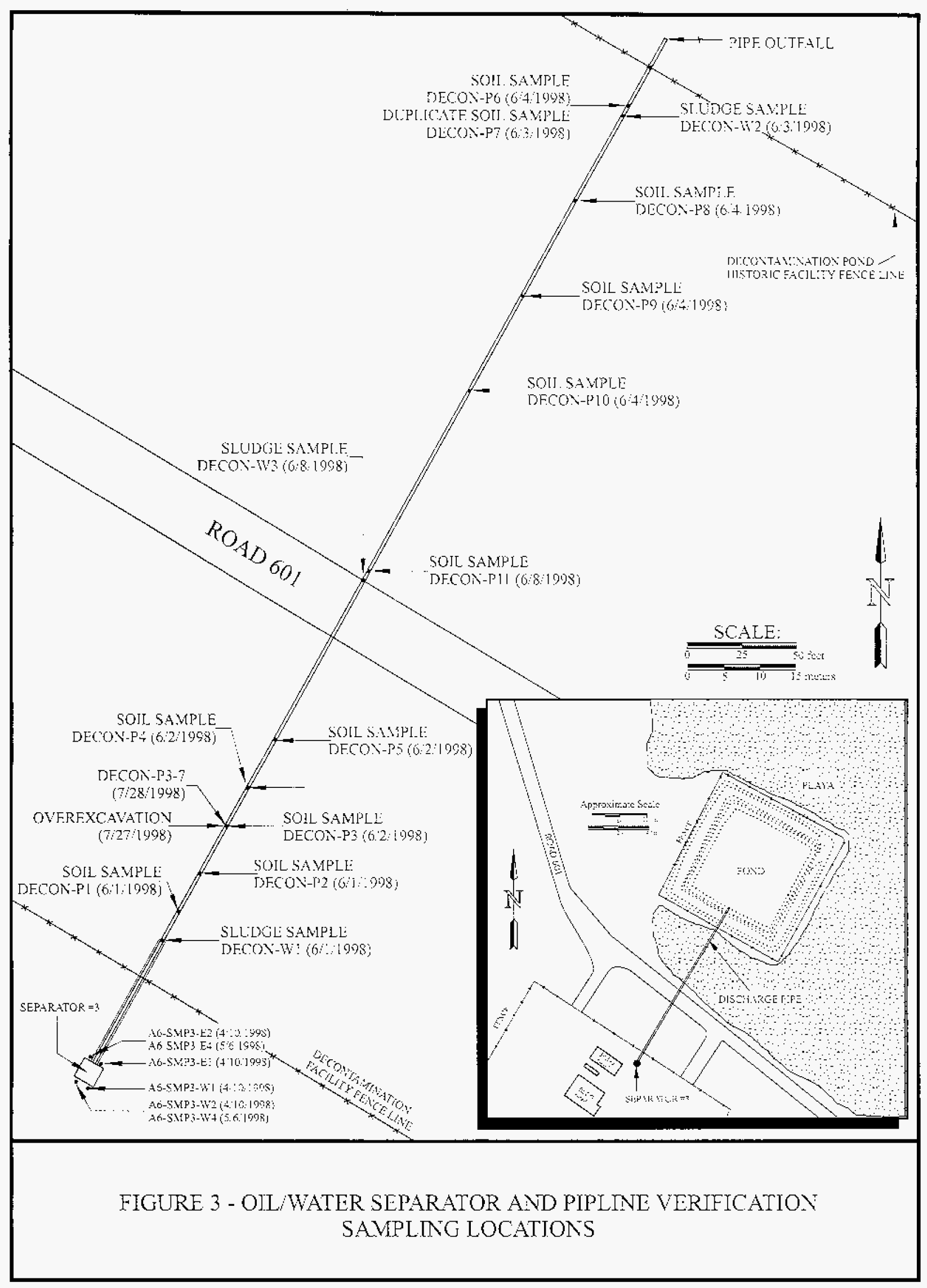




\subsubsection{Grouting and Backfilling}

During the closure of Sump 3 and the effluent pipeline, several different components were grouted closed. Sump 3 was filled in place with an expanding cenent grout using a boom pump truck. Sump 3 was inspected visually to confirm that it had been completely sealed. Prior to placement on the base of the Decontamination Pond, each end of the clay pipe sections were grouted closed. This was accomplished by first closing off each end of the pipe with plugs made up of rags. The grout was then injected into the space between the rag plug and the end of the pipe and allowed to set. Finally, the port: on of pipe encased in concrete, which extended $6.1 \mathrm{~m}$ (20 ft) north of the Decontamination Facility fence line, was groutcd in place following NDEP approval. An expanding cement grout was tremmied into the pipe form the north end back to Sump 3.

The pipe excavations were backfilfed with clean fill which had been stockpilled during the pipe removal. Excess clean fill was brought in to bring the pipe excavation to grade and compacted following accepted construction practices.

\subsubsection{Waste Disposal}

Waste generated during the closure of Sump 3 and the effluent pipeline connecting Sump 3 to the Decontamination Pond was placed on the base of the Decontamination Pond prior to cover construction. Waste included the pipe removed and grouted closed, the excavated soils placed into the B-25 container, and IDW.

\subsubsection{Decontamination Pond-CAS 06-05-02 Corrective Action}

The Decontamination Pond was closed by constructing an engineered cover. First, the pipe, excavated soil from around the pipe, and characterization IDW were added to the base of the pond. The berm walls were then pushed into the pond and compacted. Additional materials were imported to form subsequent layers following the CQA Plan (DOE, 1998a). The fitral layers include a bedding layer, Geosynthetic Clay Liner (GCL), buffer layer, and erosion control layer. This cover was designed to meet or exceed all requirements stated in $40 \mathrm{CFR}$ $\$ 265.228(\mathrm{a})(2)$. Following cover construction, monuments were placed on the cover to monitor subsidence. Finally, a chainlink security fence was built to limit access to the cover. Two-foot fiberglass screening strips were added to the chainlink fence to mitigate wave action on the cover should Yucca Playa become submerged. Specific details regarding the cover constnction are discussed below. 


\subsubsection{Berm Removal and Placement}

Characterization data previously collected from the Decontamination Pond indicated that the pond floor and berms were impacted with both radiological and hazardous constituents. The first phase of the Decontamination Pond cover construction was to push the berms into the pond. Before this was done the area was designated an exclusion zone and a hotline was set up. Access was limited to personnel with Radiological Worker II and Occupational Safety and Health Administration 40-hour training. The berms were then pushed in to establish the first lift using a front end loader. Once the original Decontamination Pond floor was completely covered and compacted a radiologic demarcation crew surveyed the new surface. The swipes were analyzed using a one-minute alpha/beta count and a 20-minute gamma count for radiological constituents. Results indicated that radiation levels were not above background ( 1260 disintegrations per minute, beta) and work proceeded without the requirement of a Radiological Work Permit.

The remaining berm materials were compacted in approximately $0.15-\mathrm{m}(0.5-\mathrm{ft})$ thick lifts to at or above 90 percent of maximum compaction with a moisture content dry of optimum as required by the CQA Plan. Each lift underwent density tests using Campbell MC-2/MC-3 Troxler nuclear density gage to confirm the specified compaction. Densities were confirmed at the $16 \mathrm{CQA}$ Plan-specified test locations. Once the density measurements indicated that the lift was compacted to CQA Plan specifications, the surface was surveyed to establish the elevation of the new pond surface. This process was verified by both a $\mathrm{BN}$ verification inspector and an independent registered professional engineer. The final layer of berm material was not sufficient to cover the entire pond surface. In order to complete the lift, fill materials, as designated for the shaping fill type, were used.

\subsubsection{Shaping Fill Placement}

Prior to placement, shaping fill materials were stockpilled at the site. Shaping fill materials were compacted in approximately $0.15-\mathrm{m}(0.5-\mathrm{ft})$ thick lifts to at or above 90 percent of maximum compaction with a moisture content dry of optimum as required by the CQA Plan. Each lift underwent density tests using Campbell MC-2/MC-3 Troxler nuclear density gage to confirm the specified compaction. Densities were confirmed at the 16 CQA Plan-specified test locations. Once the density measurements indicated that the lift was compacted to CQA Plan specifications, the surface was surveyed to establish the elevation of the new pond surface. This process was verified by both a $\mathrm{BN}$ verification inspector and an independent registered professional engineer. A total of 7 lifts were required to finish this portion of the cover. The final lift was verified using 5 density test locations because it was much smaller than the preceding layers.

\section{2,1.2.3 Bedding/GCL/Buffer Layer Placement}

Prior to placement, the bedding layer material was manufactured to CQA Plan specifications at the Area 3 shaker plant. This layer provided a low permeable surface free of material that might puncture the GCL. After the bedding layer was placed, the GCL was installed. The GCL was placed under the guidance of the manufacturer's representative and verified by an independent subcontractor specialist from Knight Piesold. The buffer layer was placed on top of the GCL as 
it was being laid. This layer was of the same type used for the bedding layer. The bedding and buffer layers both consisted of a single $0.15-\mathrm{m}(0.5-\mathrm{ft})$ thick lift. The bedding and buffer layers were compacted to at or above 90 percent of maximum compaction with a moisture content dry of optimum as required by the CQA Plan. Densities were confirmed at the 16 CQA Planspecified test locations using a Campbell MC-2/MC-3 Troxler nuclear density gage to confirm the specified compaction. Five additional density estimates using Sand Cone Density Method D1556-90 (ASTM, 1997) were acquired. Once the density measurements indicated that the lift was compacted to CQA Plan specifications, the surface was surveyed to establish the elevation of the new pond surface. This process was verified by both a BN verification inspector and an independent registered professional engineer.

\subsubsection{Erosion Control Layer Placement}

The final cover layer consisted of two $0.15-\mathrm{m}(0.5-\mathrm{ft})$ thick lifts of type II material. This material was placed to prevent cover erosion. This layer was compacted to at or above 90 percent of maximum compaction with a moisture content dry of optimum as required by the CQA Plan. Densities were confirmed at the $16 \mathrm{CQA}$ Plan-specified test locations using a Campbell MC$2 / \mathrm{MC}-3$ Troxler nuclear density gage to confirm the specified compaction. Once the density measurements indicated that the lift was compacted to CQA Plan specifications, the surface was surveyed to establish that the CQA Plan-specified slope of 3.5 percent was achieved on the final grade of the cover. Once this was confirmed, five survey monuments were placed on the cover to monitor subsidence. This process was verified by both a $\mathrm{BN}$ verification inspector and an independent registered professional engineer.

\subsubsection{Certification of Closure}

As required under 40 CFR 264.115, closure must be certified by an independent professional engineer. Dean Nelson of PEER Consultants P.C. acted as the Professional Engineer for this closure. He reviewed the Closure Plan (DOE, 1998a) and all aspects of the closure activities to reach the conclusion that closure was accomplished according to the Closure Plan (DOE, 1998a). The Certification of Closure is provided in Appendix C.

\subsection{DEVIATIONS FROM CLOSURE PLAN AS APPROVED}

The Final Corrective Action Plan (CAP) was approved by NDEP (NDEP, 1998) in November of 1998. One deviation from the Final CAP was approved on December 29, 1998. This change reduced the size of the area to be enclosed by chain-ink fencing to more accurately fit the size of the cover as constructed. Appendix D contains a copy of the signed Design Change Notice.

Survey data collected from lift \#9 (Drawing \# CAU-006-92-C9) indicates that two density tests were acquired from the same grid (points 216 and 217). The locations of each point which were tested for density were verified by five individuals in the field (Site Supervisor, Becthel Nevada Inspector, Independent Verifier, Laboratory Technician and the Surveyor). All five individuals concur that the density measurements for points 216 and 217 were taken in the proper grid 
location. However, after extensive review of the survey log book, no direct evidence can be found to support this. The survey $\log$, therefore, remains in error.

\subsection{CORRECTIVE ACTION SCHEDULE AS COMPLETED}

A detailed schedule as completed for the corrective action is provided in Table 1.

\subsection{SITE PLAN/SURVEY PLAT}

A composite of all of the layers which make up the cover is located in Figure 4. The as-built drawings for the engineered cover are located in Appendix $\mathrm{E}$. 
TABLE 2 - CAU 92 CIOSURE SCIIEDULE, AS COMPIATLD.

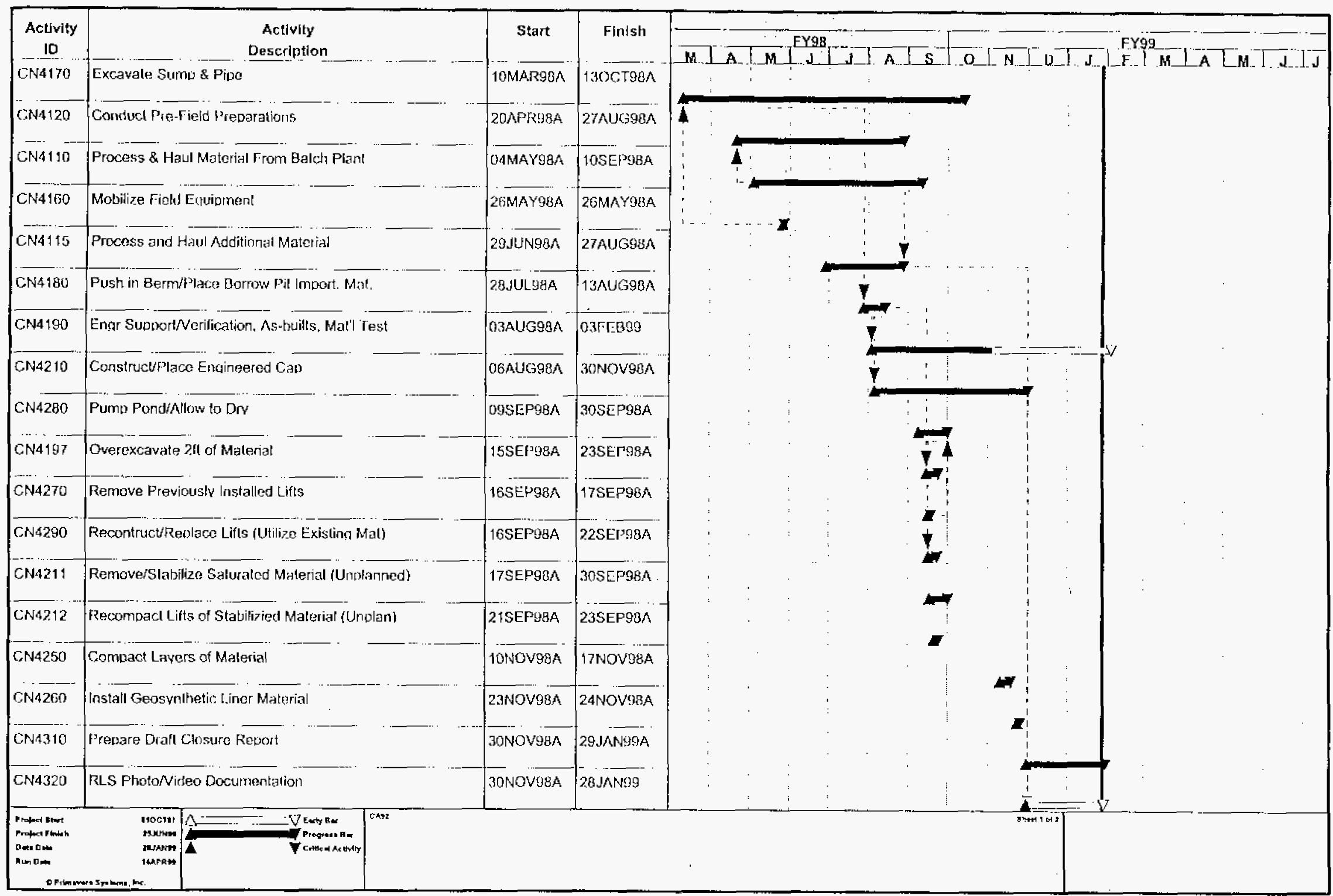


TABI.E 2 - CAU 92 CI,OSURE SCHEDUE AS COMPIETHD (CONTINUED).

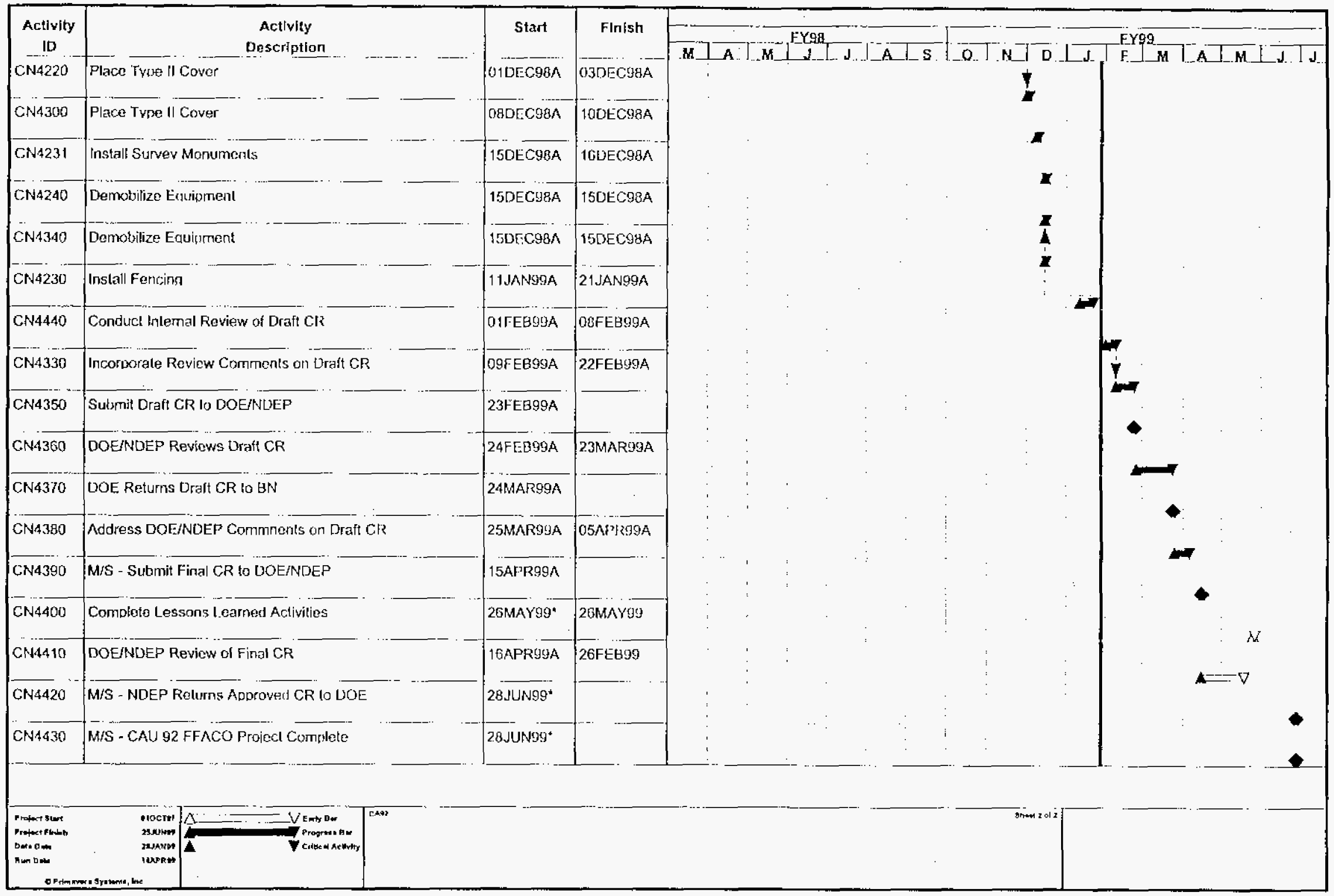




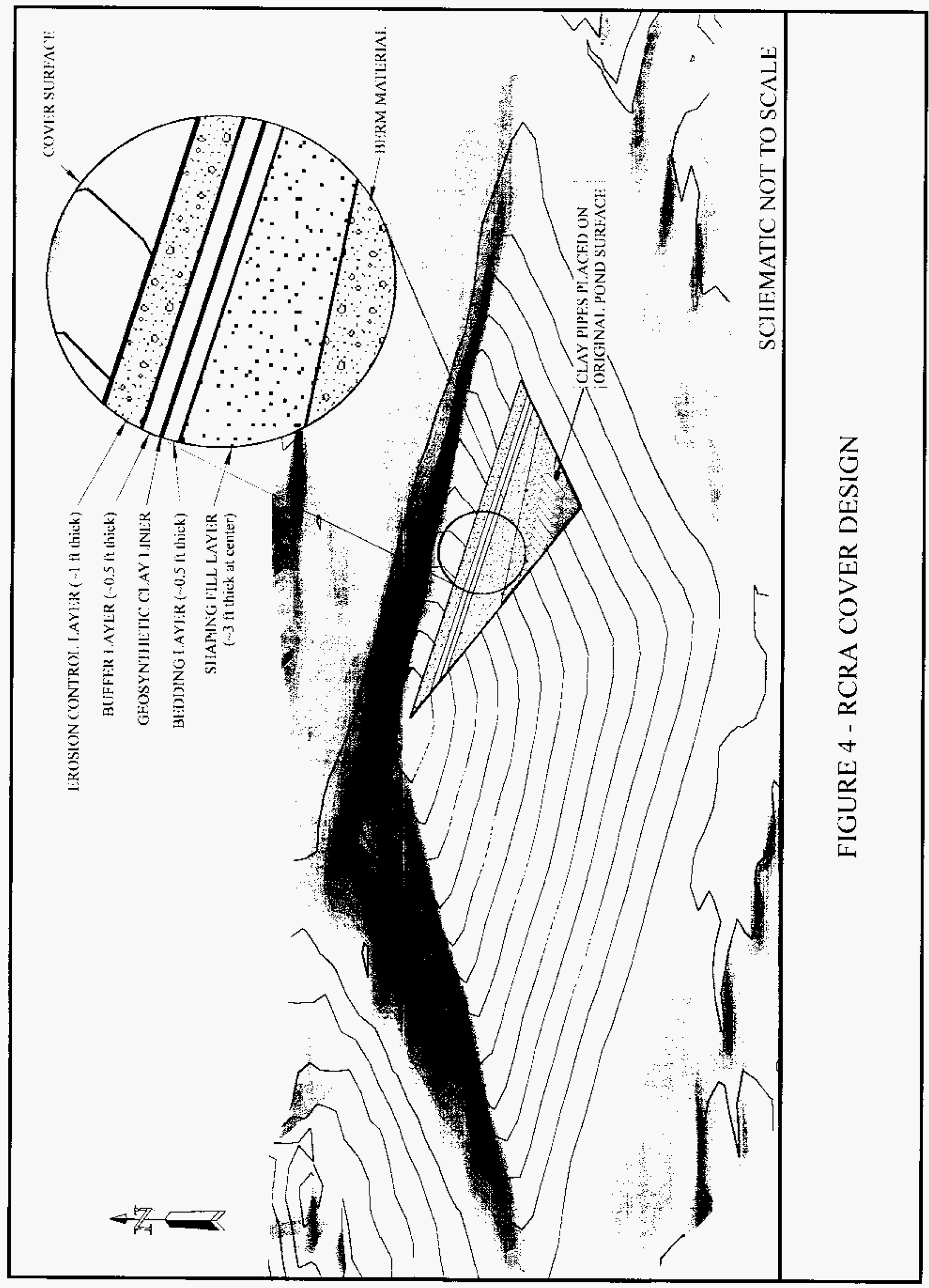


Waste generated from CAU 92 closure activities consisted of radiologically impacted soil and pipe, personal protective equipment, and sanitary trash. All waste (excluding sanitary trash) was placed into the pond prior to building the engineered cover. All sanitary trash was bagged and disposed of in the Decontamination Facility dumpster. 
THIS PAGE INTENTIONALLY LEFT BLANK 
Closure verification for the Decontamination Pad oiliwater separator and the influent pipe line connecting the separator to the Decontamination Pond was accomplished with confirmatory sampling for clean closure. The sampling was conducted following Section 2.4 of the approved Closure Plan (DOE, 1998a).

The Decontamination Pond was closed in place by constructing an engineered cover. Closure verification consists of nuclear density compaction measurements for each lift following the approved CQA Plan (Appendix F, DOE, 1998a). Detailed verification results are provided by CAS numbers below.

\subsection{DECONTAMINATION PAD OIL/WATER SEPARATOR- CAS 06-04-01 VERIFICATION RESULTS}

Site characterization data from the Area 6 Decontamination Pond indicated that radiological constituents (plutonium, strontium, cobalt, cesium, and americium), petroleum hydrocarbons, bis(2-ethylhexyl)phthalate, methylene chloride, xylene, PCBs, and lead are the constituents detected in the pond sediment and inner berm surfaces (DOE, 1997a). The Constituents of Concern (COC) identified during site characterization activities were used to establish verification sampling parameters.

Six soil samples were collected from the vicinity of the oil/water separator. This sample set is identified as A6-SMP-W1, A6-SMP-W2, A6-SMP-E1, A6-SMP-E2, A6-SMP-W4, and A6SMP-E4. Sample analytical results are found in Tabje 2 and Appendix B. Sample locations are shown in Figure 3. All RCRA-regulated VOCs and SVOCs were at or below the contract required detection limit. The TCLP metal analytical data were below the concentrations provided in Table 1 of 40 CFR $\$ 261.24$. All PCB isomers were reported at or below the contract required detection limit. The TPH concentrations did not exceed the Nevada Administrative Code Action Level of 100 milligram per kilogram (mg/kg). Radioactive isotopes were not detected above the minimal detectable activity.

Twelve soil samples (DECON-P1 through DECON-P 1 and DECON-P3-7) were collected at various locations along the clay pipeline (Figure 3). Analytical results from sample DECON-P3 indicated that Americium-241 was present. The soils in the vicinity of this sample location were over excavated and resampled (DECON-P3-7). This reverification sample did not detect any radioisotopes above the minimal detectable activity. Sample analytical results for the other 11 soil verification samples did not detect any COCs. Table 2 summarizes the analytical results for the 12 verification samples collected from the soil surrounding the pipe. 


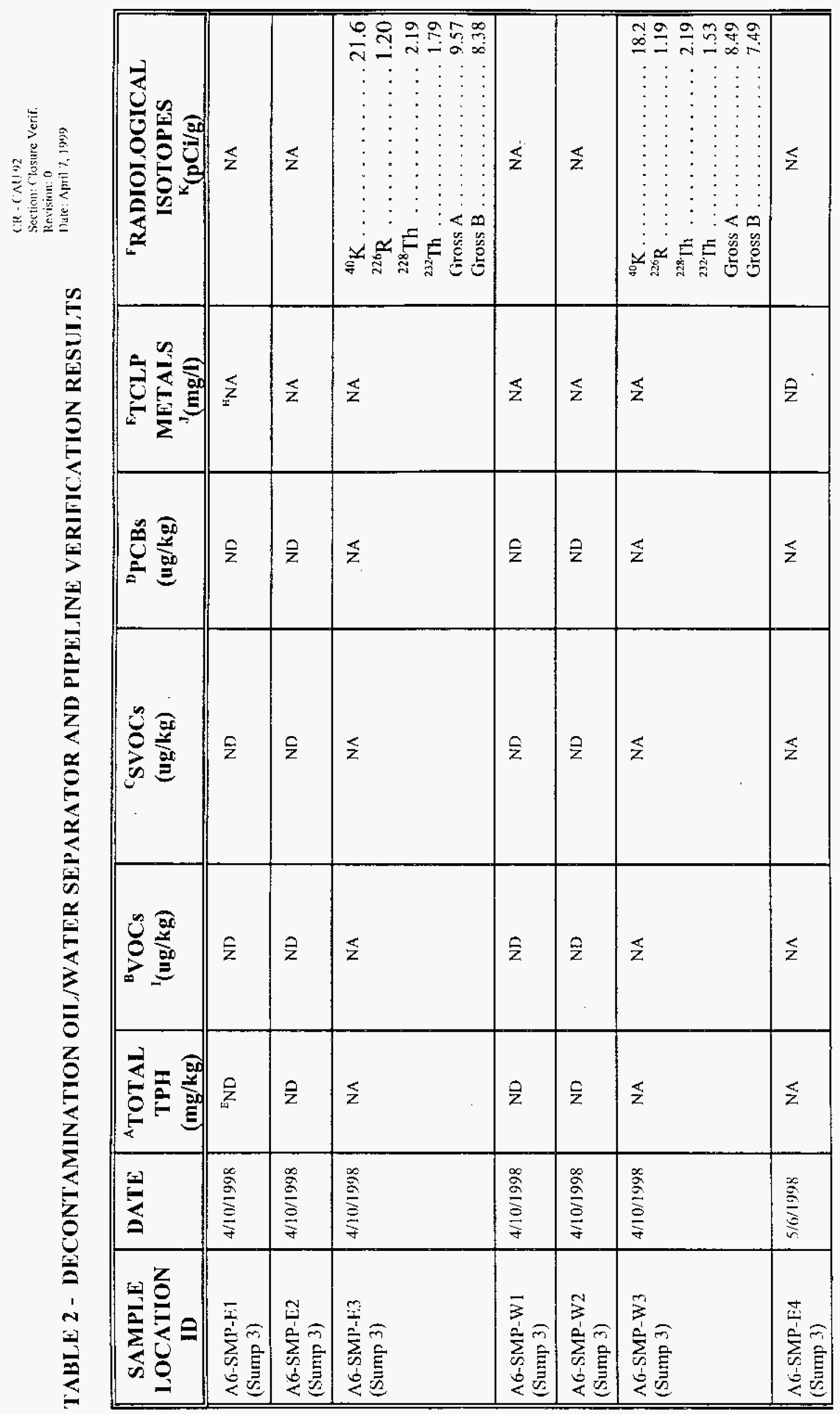


'TABLE 2 - DECONTAMINAIION OIL/WATER SEPARATOR AND PIPELINE VERIFICATION RESULTS (CONTINUED)

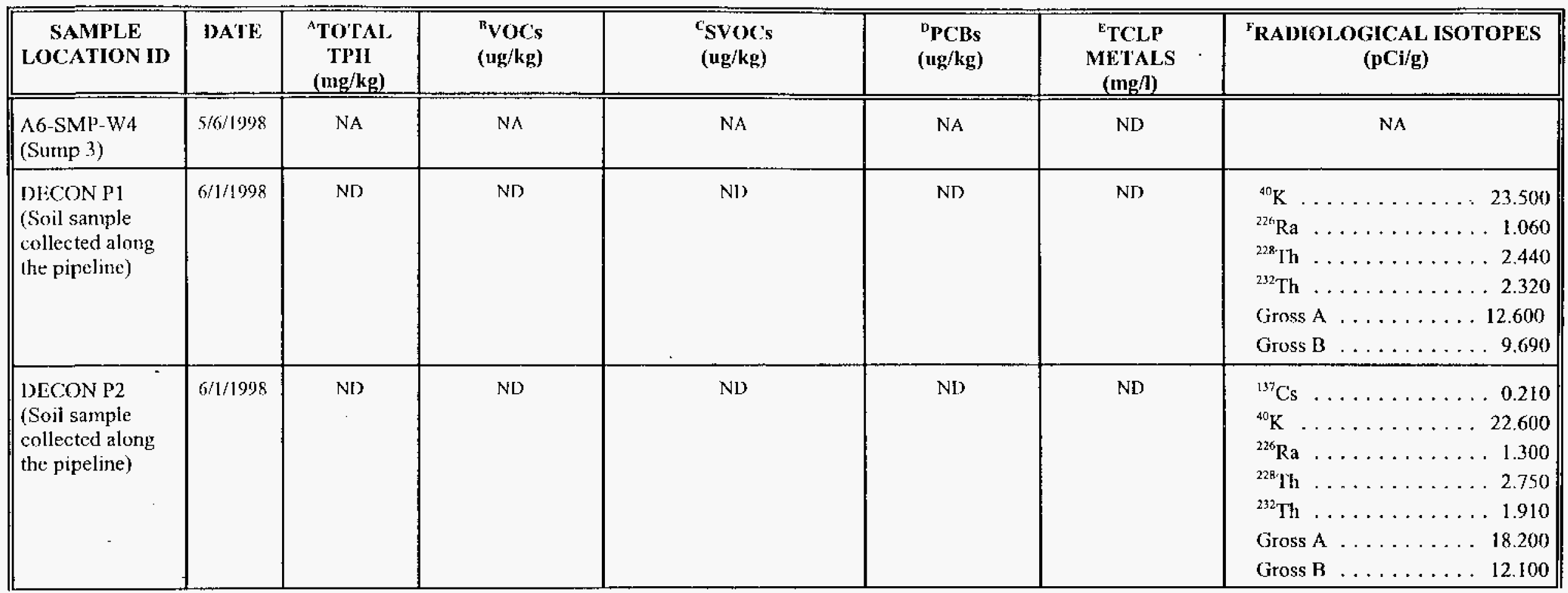


TABIE 2 - DECONTAMINAIION OII/WATER SEPARATOR AND PIPEIINE VERIFICATION RESUITS (CONTINUED).

\begin{tabular}{|c|c|c|c|c|c|c|c|}
\hline $\begin{array}{c}\text { SAMPLE } \\
\text { LOCATION } \\
\text { ID } \\
\end{array}$ & DATE & $\begin{array}{c}\text { 'TO'TAI. } \\
\text { TPH } \\
(\mathrm{mg} / \mathrm{kg}) \\
\end{array}$ & $\begin{array}{l}\text { "VOCs } \\
\text { (ug/kg) }\end{array}$ & $\begin{array}{c}\text { 'SVOCs } \\
\text { (ug/kg) }\end{array}$ & $\begin{array}{l}{ }^{D} \mathrm{PCBs} \\
(\mathrm{ug} / \mathrm{kg})\end{array}$ & $\begin{array}{c}\text { ETCLP } \\
\text { METALS } \\
(\mathrm{mg} / \mathrm{l}) \\
\end{array}$ & $\begin{array}{c}\text { FADIOIOOGICAI } \\
\text { ISOTOPES } \\
\text { (pCi/g) }\end{array}$ \\
\hline $\begin{array}{l}\text { DECON P3 } \\
\text { (Soil sample } \\
\text { collected along } \\
\text { the pipetine) }\end{array}$ & $6 / 2 / 1998$ & $\mathrm{ND}$ & ND & $\mathrm{ND}$ & ND & ND & 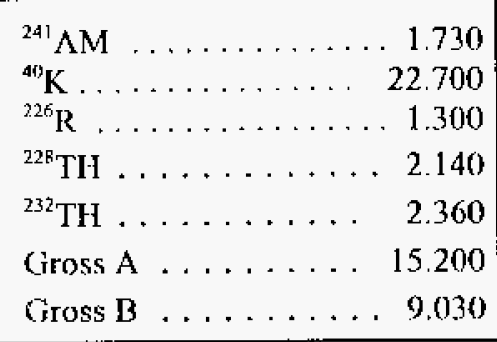 \\
\hline $\begin{array}{l}\text { DFCON P3-7 } \\
\text { (Vcrification } \\
\text { sample collected } \\
\text { within the } \\
\text { overexeavation) } \\
\end{array}$ & $7 / 28 / 1998$ & NA & NA & NA & NA & $\mathrm{NA}$ & 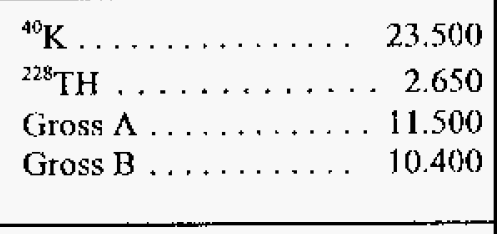 \\
\hline $\begin{array}{l}\text { DFCON P4 } \\
\text { (Soil sample } \\
\text { collected along } \\
\text { the pipcline) }\end{array}$ & $6 / 2 / 1998$ & ND & ND & ND & ND & ND & 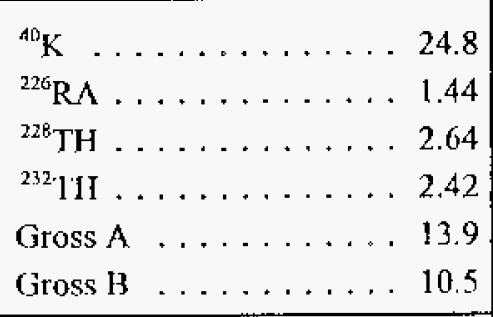 \\
\hline $\begin{array}{l}\text { DJCON P5 } \\
\text { (Soil sample } \\
\text { collected along } \\
\text { the pipcline) }\end{array}$ & $6 / 2 / 1998$ & $\mathrm{ND}$ & N1) & NI & $\mathrm{ND}$ & ND. & 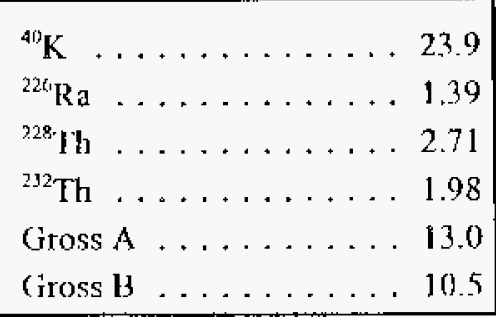 \\
\hline
\end{tabular}




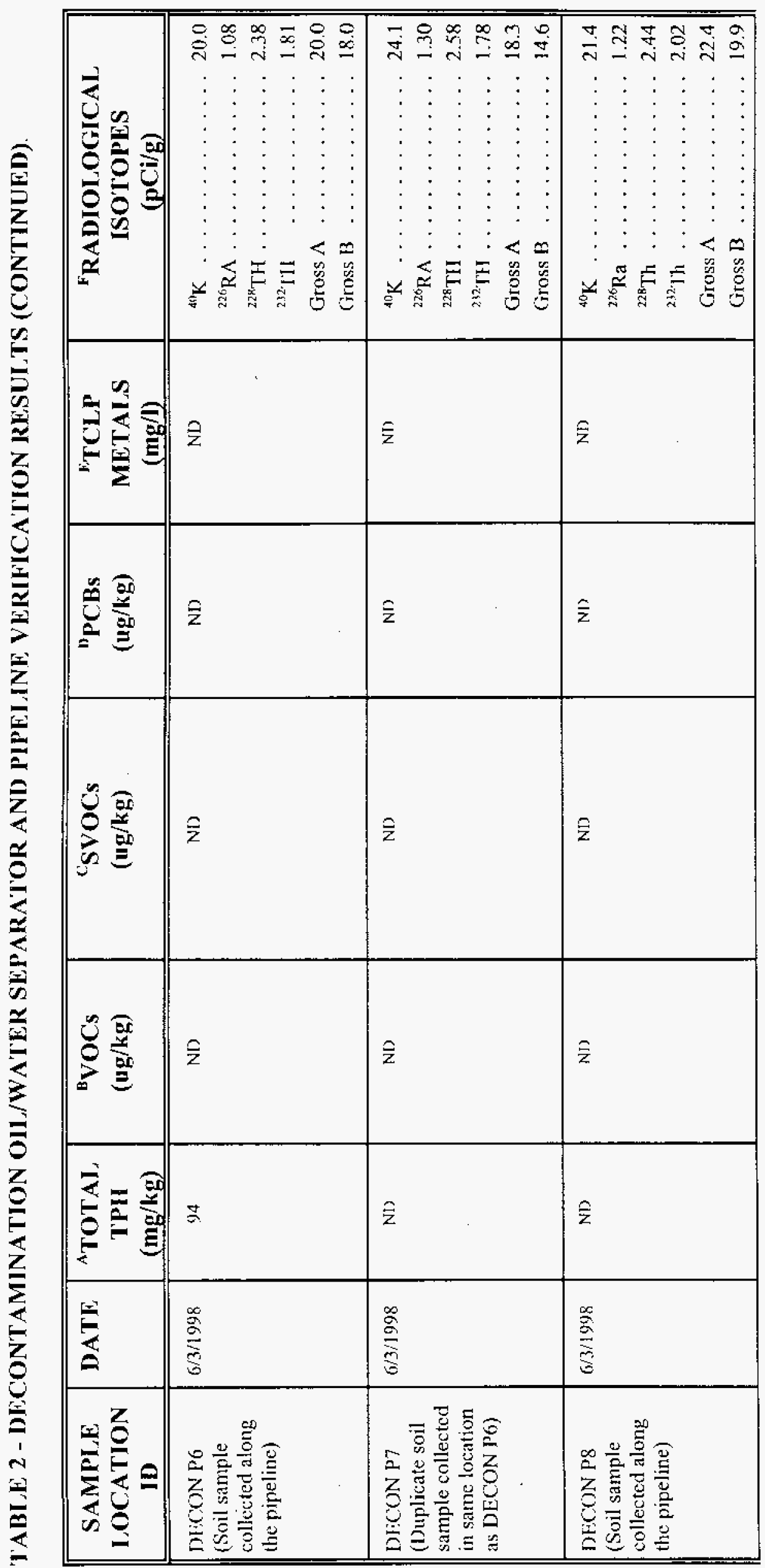


TABLE 2 - DECONIAMINATION OIL/WA'TER SFPARATOR AND PIPEI.INE VERIFICA'ION RESULIS (CONTINUED)

\begin{tabular}{|c|c|c|c|c|c|c|c|}
\hline $\begin{array}{c}\text { SAMPLE } \\
\text { LOCA'IION } \\
\text { II) } \\
\end{array}$ & DATE & $\begin{array}{c}\text { 'TOTAI } \\
\text { TPII } \\
(\mathrm{mg} / \mathrm{kg}) \\
\end{array}$ & $\begin{array}{l}{ }^{\mathrm{H}} \mathrm{VOCs} \\
(\mathrm{ug} / \mathrm{kg})\end{array}$ & $\begin{array}{c}\text { SVYOCs } \\
(\mathrm{ug} / \mathrm{kg})\end{array}$ & $\begin{array}{l}{ }^{D} \mathrm{PCBs} \\
\text { (ug/kg) }\end{array}$ & $\begin{array}{l}\text { TCCLP } \\
\text { METALS } \\
(\mathbf{m g} / \mathbf{l}) \\
\end{array}$ & $\begin{array}{c}\text { FRADIOLOGICAL } \\
\text { ISOTOPES } \\
(\mathrm{pCi} / \mathrm{g}) \\
\end{array}$ \\
\hline $\begin{array}{l}\text { DECON P9 } \\
\text { (Soil sample } \\
\text { collected along } \\
\text { the pipeline) }\end{array}$ & $6 / 4 / 1998$ & ND & ND) & ND & ND & ND & 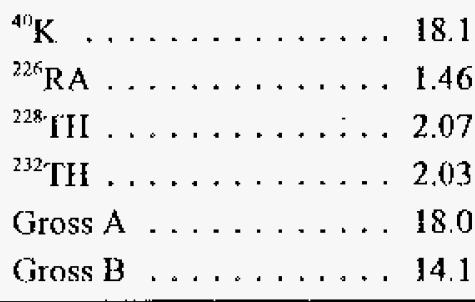 \\
\hline $\begin{array}{l}\text { DISON P10 } \\
\text { (Soil sample } \\
\text { collected along } \\
\text { the pipeline) }\end{array}$ & $6 / 4 / 1998$ & ND & ND & ND & ND & ND & 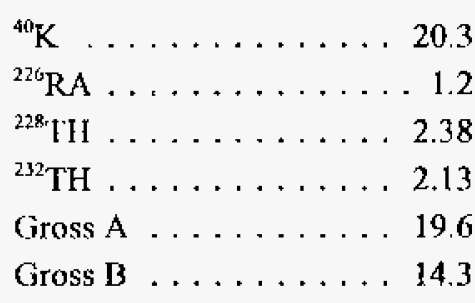 \\
\hline $\begin{array}{l}\text { DECON P11 } \\
\text { (Soil sample } \\
\text { collected along } \\
\text { the pipeline) }\end{array}$ & $6 / 8 / 1998$ & NII & ND & ND & NI & ND) & 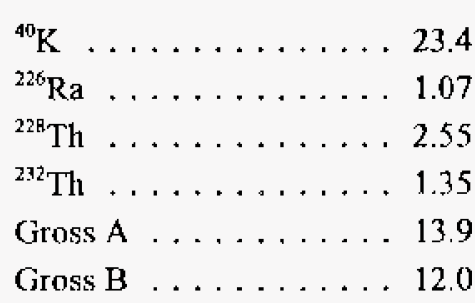 \\
\hline
\end{tabular}


TABLE 2 - DECONTAMINAIIION OII/WATER SEPARATOR AND PIPEI,INE VERIFICATION RESUITS (CONTINUED)

\begin{tabular}{|c|c|c|c|c|c|c|c|}
\hline $\begin{array}{l}\text { SAMPLE } \\
\text { LOCATION } \\
\text { ID } \\
\end{array}$ & DATE & $\begin{array}{c}\text { 'TOTAI. } \\
\text { TPH } \\
(\mathrm{mg} / \mathrm{kg}) \\
\end{array}$ & $\begin{array}{l}{ }^{\mathrm{B}} \mathrm{VOCs} \\
(\mathrm{ug} / \mathrm{kg})\end{array}$ & $\begin{array}{l}\text { CSVOCs } \\
\text { (ug/kg) }\end{array}$ & $\begin{array}{l}\text { "PCBs } \\
\text { (ug/kg) }\end{array}$ & $\begin{array}{c}\text { 'TCLP } \\
\text { METAISS } \\
(\text { mg/l) } \\
\end{array}$ & $\begin{array}{c}\text { RADIOLOGICAI } \\
\text { ISOTOPES } \\
(\mathrm{pCi} / \mathrm{g})\end{array}$ \\
\hline $\begin{array}{l}\text { JPCON W1 } \\
\text { (Sludge sample } \\
\text { froui inside } \\
\text { pipe) }\end{array}$ & $6 / 1 / 1998$ & 24010 & ND & Bis(2-ethylhexyl)phthatate 1600 & 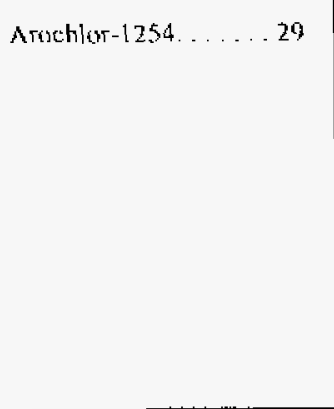 & $\begin{array}{l}\text { Cadmium ...0.014 } \\
\text { 1.ead ...... } 0.20\end{array}$ & 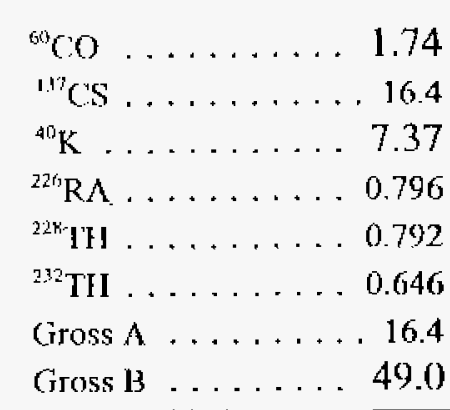 \\
\hline $\begin{array}{l}\text { DECON W2 } \\
\text { (Sludge sample } \\
\text { from inside } \\
\text { pipe) }\end{array}$ & $6 / 3 / 1998$ & 5500 & ND & ND & Arocblort-1254 $\ldots 450$ & Cadmiumr . . 0014 & 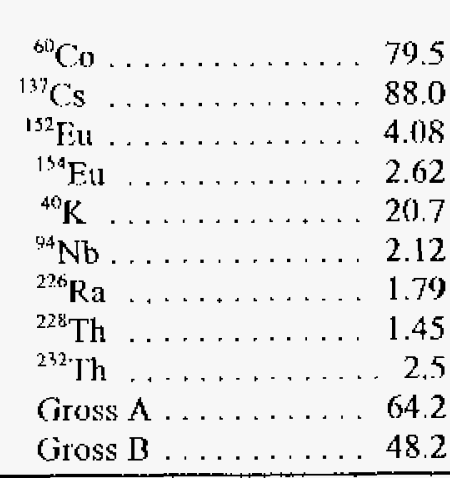 \\
\hline
\end{tabular}


TABLE 2 - DECONTAMINATION OIL/WATER SFPARATOR AND PIPELINE VERIFICATION RESULTS (CONTINUED)

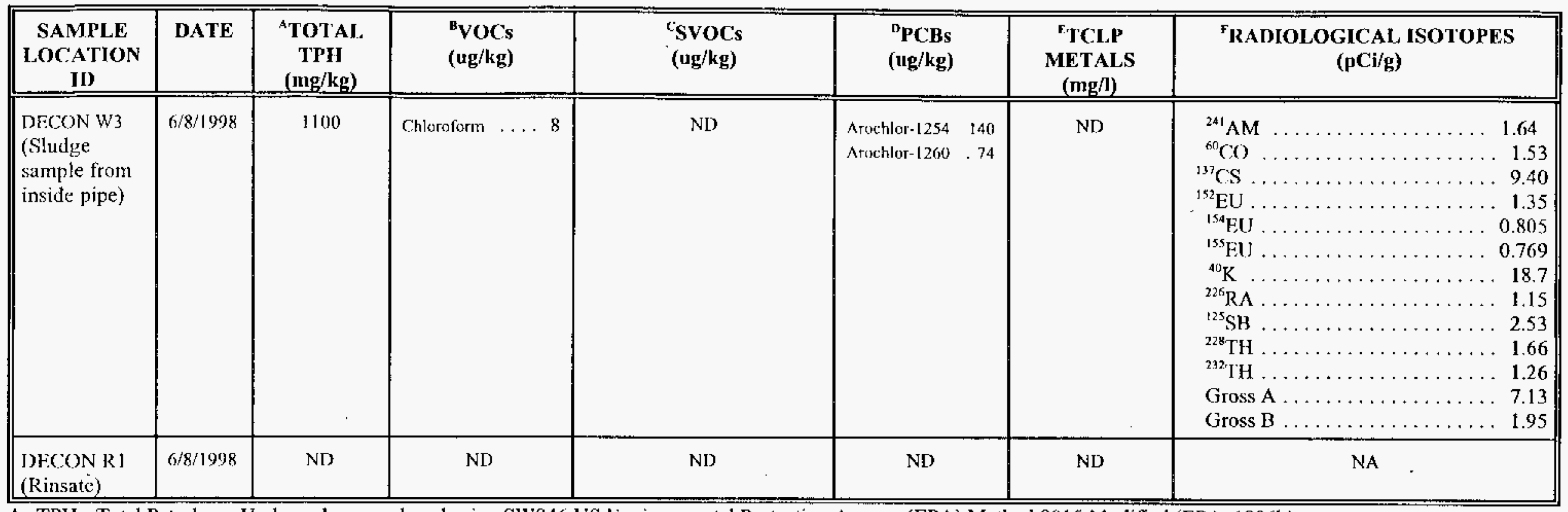

A- TPH - Total Petroleum Hydrocarbons analyzed using SW846 US Environmental Protection Agency (EPA) Method 8015 Modified (EPA, 1996b).

B- VOCs - Volatile Organic Compounds analyzed using SW846 US EPA Mctlod 8260H (HI'A, 1996c).

C- SVOCs - Scmivolatile Organic Compounds analyzed using SW846 US EPA Method 8270 (EPA, 1996d).

D- PCBs - Polychlorinated biphenyls analyzed using SW846 US EPA Method 8080A Modified (EPA, 1996a)

E- TCI.P metals - J'oxicity Characteristic I eaching Potential metals analyzed using EPA $1311 / 6010(1 \mathrm{PA}, 1996 \mathrm{e})$.

F- Radioactive Constituents (Gamma Scan and Gross AJpha/Beta) analyzed using L-E10.602 PC and L-E 10.612.1PJ.

(i- NI)- Not detected above reportable quantitics ( $\Lambda$ ppendix 13 ).

H- NA- Not tested.

I- ug/kg - microyrams per kilograms

J. Ing/l-milligrams per liter

$\mathrm{K}-\mathrm{pCi} / \mathrm{g}$ - picocutries pet gram 
In addition to the 12 verification samples, 3 sludge samples were collected from the interior of the pipes for waste characterization purposes. Sludge sample locations are shown in Figure 3. Table 2 summarizes the analytical results for the sludge samples (DECON W1, DECON W2 and DECON W3). The sludge sample waste characterization analytical results were consistent with previous waste characterization efforts for the Area 6 Decontamination Pond.

In addition, qualty assurance samples were also collected. Blind duplicates and equipment blanks were collected for at least 20 percent of the samples, trip blanks were sent with every sample batch for VOC analysis, and matrix spike/matrix spike duplicate samples were designated in every sample batch.

\subsection{DECONTAMINATION POND - CAS 06-05-02 VERIFICATION RESULTS}

The CQA Plan outlines the inspection methods and testing requirements for the Decontamination Pond closure cover (Appendix F). Inspection procedures outlined in the CQA Plan were performed by both a $\mathrm{BN}$ verification inspector and an independent registered professiona] engineer to certify that all closure activities were completed in accordance with the Closure Plan. Table 3 summarizes the nuclear density test results for each lift.

\subsection{LAND-USE RESTRICTIONS}

The oil/water separator and buried grouted pipe lies within the Decontamination Facility boundaries and have restricted access according to Decontamination Facility requirements. This area has been posted with signs reading "Subsurface Radiologic Contamination."

The remainder of the pipe line was completely removed. Following overexcavation, all hazardous COCs were below detection and radiological COCs were below the minimal detectable activity. Therefore, based upon the analytical results, this site is considered clean and available for unrestricted use.

Hazardous and radiological constituents within the Decontamination Pond were closed in place with an engineered cover. The engineered cover is fenced from general entry. Only authorized personnel may enter for post-closure monitoring requirements and repair. Post-closure monitoring will consist of quarterly inspections of the RCRA cover. Figure 5 summarizes the restricted access areas. 
TABLE 3 - NUCLEAR DENSITY TEST RESULTS.

\begin{tabular}{|c|c|c|c|c|}
\hline $\begin{array}{c}\text { LIFT } \\
\text { DESCRIPTION }\end{array}$ & $\begin{array}{c}{ }^{A} \text { GRID } \\
\text { LOCATION } \\
\#\end{array}$ & $\begin{array}{c}\text { PERCENT } \\
\text { COMPACTION }\end{array}$ & $\begin{array}{c}\text { MOISTURE } \\
\text { CONTENT } \\
\% \\
\end{array}$ & $\begin{array}{c}\text { DRY } \\
\text { DENSITY } \\
\text { lb/ft }^{3} \\
\end{array}$ \\
\hline $\begin{array}{l}\text { Berm material Lift } 1 \\
\text { Tested } 9-21-1998 \text { at a depth of } 0.15 \mathrm{~m} \\
(0.5 \mathrm{ft}) \\
\text { Average lift thickness }=0.366 \mathrm{~m} \\
(1.2 \mathrm{ft}) \\
\text { Modified Proctor Specs. } \\
\text { Optimum moisture content }=14.0 \% \\
\text { Maximum density } \text { lb/ft }^{3}=113.0 \\
\text { CQA Specs. } \\
\text { Required compaction }=90 \\
\text { Appendix E } \\
\text { Drawing } \# \text { SK-CAU-006-92-C1 }\end{array}$ & $\begin{array}{l}1 \\
2 \\
3 \\
4 \\
5 \\
6 \\
7 \\
8 \\
9 \\
10 \\
11 \\
12 \\
13 \\
14 \\
15 \\
16\end{array}$ & $\begin{array}{l}91.2 \\
94.0 \\
91.2 \\
94.9 \\
95.3 \\
95.6 \\
91.5 \\
93.7 \\
94.8 \\
91.9 \\
97.7 \\
96.4 \\
92.1 \\
95.9 \\
93.5 \\
90.5\end{array}$ & $\begin{array}{l}11.5 \\
12.1 \\
11.9 \\
11.1 \\
10.7 \\
11.5 \\
9.9 \\
9.2 \\
9.4 \\
9.0 \\
12.3 \\
13.0 \\
10.7 \\
7.3 \\
9.9 \\
9.7\end{array}$ & $\begin{array}{l}103.0 \\
106.2 \\
103.0 \\
107.2 \\
107.7 \\
108.0 \\
103.4 \\
105.9 \\
107.1 \\
103.9 \\
110.4 \\
108.9 \\
104.1 \\
108.4 \\
105.6 \\
102.3\end{array}$ \\
\hline $\begin{array}{l}\text { Berm material Lift } 1 \\
\text { Tested } 9-25-1998 \text { at a depth of } 0.30 \mathrm{~m} \\
(1.0 \mathrm{ft}) \\
\text { Average lift thickness }=0.366 \mathrm{~m} \\
(1.2 \mathrm{ft}) \\
\text { Modified Proctor Specs. } \\
\text { Optimum moisture content }=14.0 \% \\
\text { Maximum density } 1 b / f^{3}=1 / 3.0 \\
\text { CQA Specs. } \\
\text { Required compaction }=90 \\
\text { Appendix E } \\
\text { Drawing \# SK-CAL-006-92-C1 }\end{array}$ & $\begin{array}{l}1 \\
2 \\
3 \\
4 \\
5 \\
6 \\
7 \\
8 \\
9 \\
10 \\
11 \\
12 \\
13 \\
14 \\
15 \\
16\end{array}$ & $\begin{array}{l}93.0 \\
92.8 \\
95.3 \\
98.5 \\
97.6 \\
96.2 \\
93.7 \\
90.9 \\
91.8 \\
95.7 \\
93.7 \\
98.1 \\
90.0 \\
93.0 \\
91.3 \\
89.6\end{array}$ & $\begin{array}{c}9.1 \\
9.3 \\
8.2 \\
8.9 \\
13.7 \\
11.5 \\
11.6 \\
7.5 \\
9.6 \\
9.9 \\
11.1 \\
9.4 \\
15.7 \\
7.9 \\
11.1 \\
8.6\end{array}$ & $\begin{array}{l}105.1 \\
104.9 \\
107.7 \\
111.3 \\
110.3 \\
108.7 \\
105.9 \\
102.1 \\
103.7 \\
108.1 \\
105.9 \\
110.9 \\
101.7 \\
105.1 \\
103.2 \\
101.3\end{array}$ \\
\hline $\begin{array}{l}\text { Berm material Lift } 2 \\
\text { Tested } 9-29-1998 \\
\text { Ave. lift thickness }=0.159 \mathrm{~m}(0.52 \mathrm{ft}) \\
\text { Modified Proctor Specs. } \\
\text { Optimum moisture content }=14.0 \% \\
\text { Maximum density lb/ft }=113.0 \\
\text { CQA Specs. } \\
\text { Required compaction }=90 \\
\text { Appendix E } \\
\text { Drawing } \# \mathrm{SK}-\mathrm{CAL}-006-92-\mathrm{C} 2\end{array}$ & $\begin{array}{l}1 \\
2 \\
3 \\
4 \\
5 \\
6 \\
7 \\
8 \\
9 \\
10 \\
11 \\
12 \\
13 \\
14 \\
15 \\
16\end{array}$ & $\begin{array}{l}93.9 \\
90.2 \\
92.0 \\
95.0 \\
99.3 \\
93.8 \\
91.9 \\
91.9 \\
95.7 \\
91.1 \\
92.5 \\
92.5 \\
96.2 \\
94.2 \\
94.9 \\
93.8\end{array}$ & $\begin{array}{l}12.6 \\
10.3 \\
10.1 \\
10.3 \\
13.2 \\
12.0 \\
10.9 \\
11.0 \\
13.4 \\
10.1 \\
10.1 \\
11.1 \\
12.7 \\
12.6 \\
12.6 \\
10.4\end{array}$ & $\begin{array}{l}106.1 \\
101.9 \\
104.0 \\
107.4 \\
112.2 \\
106.0 \\
103.9 \\
103.9 \\
108.1 \\
102.9 \\
104.5 \\
104.5 \\
108.7 \\
106.4 \\
107.2 \\
106.0\end{array}$ \\
\hline
\end{tabular}


TABLE 3 - NUCLEAR DENSITY TEST RESULTS (CONTINUED).

\begin{tabular}{|c|c|c|c|c|}
\hline $\begin{array}{c}\text { LIFT } \\
\text { DESCRIPTION }\end{array}$ & $\begin{array}{c}\text { 'GRID } \\
\text { LOCATTON } \\
\# \\
\#\end{array}$ & $\begin{array}{c}\text { PERCENT } \\
\text { COMPACTION }\end{array}$ & $\begin{array}{c}\text { MOISTLRE } \\
\text { CONTENT } \\
\%\end{array}$ & $\begin{array}{c}\text { DRY } \\
\text { DENSITY } \\
\text { lb/ft }{ }^{3}\end{array}$ \\
\hline $\begin{array}{l}\text { Berm material Lift } 3 \\
\text { Tested } 9-30-1998 \\
\text { Ave. lift thickness }=0.107 \mathrm{~m}(0.35 \mathrm{ft}) \\
\text { Modified Proctor Specs. } \\
\text { Optimum moisture content }=14.0 \% \\
\text { Maximum density } \mathrm{lb} / \mathrm{ft}^{3}=113.0 \\
\text { CQA Specs. } \\
\text { Required compaction }=90 \\
\text { Appendix E } \\
\text { Drawing \# SK-CAL-006-92-C3 }\end{array}$ & $\begin{array}{c}1 \\
2 \\
3 \\
4 \\
5 \\
6 \\
7 \\
8 \\
9 \\
10 \\
11 \\
12 \\
13 \\
14 \\
15 \\
16\end{array}$ & $\begin{array}{l}93.9 \\
90.0 \\
92.1 \\
91.5 \\
95.3 \\
94.7 \\
94.9 \\
89.9 \\
97.3 \\
97.6 \\
95.6 \\
94.8 \\
98.3 \\
96.8 \\
90.1 \\
92.0\end{array}$ & $\begin{array}{l}11.7 \\
11.0 \\
11.5 \\
10.4 \\
12.8 \\
13.7 \\
12.3 \\
11.9 \\
11.8 \\
12.8 \\
11.7 \\
12.4 \\
12.5 \\
12.4 \\
12.9 \\
11.2\end{array}$ & $\begin{array}{l}106.1 \\
101.8 \\
104.1 \\
103.4 \\
107.7 \\
107.0 \\
107.3 \\
101.5 \\
109.9 \\
110.3 \\
108.0 \\
107.1 \\
111.1 \\
109.4 \\
101.8 \\
104.0\end{array}$ \\
\hline $\begin{array}{l}\text { Berm material Lift } 4 \\
\text { (Composite layer) } \\
\text { Tested } 10-12-1998 \\
\text { Ave. lift thickness }=0.165 \mathrm{~m}(0.54 \mathrm{ft}) \\
\text { Modified Proctor Specs. } \\
\text { Optimum moisture content } \\
\text { Grid \#'s } 8,12,13,14,15,16=7.9 \% \\
\text { Grid \#'s } 2,4,5,6,7,9=14.0 \% \\
\text { Grid \#'s } 1,3,10,11=20.0 \% \\
\text { Maximum density } 16 / f t^{3} \\
\text { Grid \#'s } 8,12,13,14,15,16=137.1 \\
\text { Grid \#'s } 2,4,5,6,7,9=113.0 \\
\text { Grid \#'s } 1,3,10,11=103.5 \\
\text { CQA Specs. } \\
\text { Required compaction }=90 \\
\text { Appendix E } \\
\text { Drawing \# SK-CAL-006-92-C4 }\end{array}$ & $\begin{array}{c}1 \\
2 \\
3 \\
4 \\
5 \\
6 \\
7 \\
8 \\
9 \\
10 \\
11 \\
12 \\
13 \\
24 \\
25 \\
26\end{array}$ & $\begin{array}{l}94.8 \\
96.5 \\
96.3 \\
92.7 \\
93.5 \\
95.8 \\
97.8 \\
94.9 \\
95.1 \\
99.9 \\
100.0 \\
93.2 \\
91.0 \\
96.6 \\
97.5 \\
96.8\end{array}$ & $\begin{array}{c}8.2 \\
9.0 \\
10.5 \\
13.1 \\
13.9 \\
13.9 \\
14.2 \\
5.3 \\
11.8 \\
13.0 \\
11.9 \\
5.3 \\
7.3 \\
5.3 \\
5.5 \\
5.3\end{array}$ & $\begin{array}{l}98.1 \\
109.1 \\
99.7 \\
104.7 \\
105.7 \\
108.3 \\
10.5 \\
130.1 \\
007.5 \\
103.4 \\
103.5 \\
127.8 \\
124.8 \\
132.4 \\
133.7 \\
132.7\end{array}$ \\
\hline
\end{tabular}


TABLE 3 - NUCLEAR DENSITY TEST RESULTS (CONTINUED).

\begin{tabular}{|c|c|c|c|c|}
\hline $\begin{array}{c}\text { LIFT } \\
\text { DESCRIPTION }\end{array}$ & $\begin{array}{c}{ }^{\text {A GRD }} \\
\text { LOCATION } \\
\#\end{array}$ & $\begin{array}{c}\text { PERCENT } \\
\text { COMPACTION }\end{array}$ & $\begin{array}{c}\text { MOISTURE } \\
\text { CONTENT } \\
\%\end{array}$ & $\begin{array}{c}\text { DRY } \\
\text { DENSITY } \\
{\text { Ib } / \mathrm{ft}^{3}}^{3}\end{array}$ \\
\hline $\begin{array}{l}\text { Perimeter Over Excavation } \\
\text { Tested 10-14-1998 } \\
\text { Ave. lift thickness }=0.173 \mathrm{~m}(0.57 \mathrm{ft}) \\
\text { Modified Proctor Specs. } \\
\text { Optimum moisture content }=21.5 \% \\
\text { Maximum density } 1 \text { lb/ft }=103.2 \\
\text { CQA Specs } \\
\text { Required compaction }=90 \\
\text { Appendix E } \\
\text { Drawing \# SK-CAU-006-92-COV }\end{array}$ & $\begin{array}{l}1 \\
2 \\
3 \\
4 \\
5 \\
6 \\
7 \\
8 \\
9 \\
10 \\
11 \\
12 \\
13 \\
14 \\
15 \\
16\end{array}$ & $\begin{array}{l}92.7 \\
93.4 \\
91.9 \\
94.3 \\
90.5 \\
90.2 \\
92.3 \\
94.9 \\
95.3 \\
95.7 \\
99.6 \\
95.4 \\
98.0 \\
94.9 \\
92.9 \\
96.3\end{array}$ & $\begin{array}{l}19.1 \\
13.5 \\
19.8 \\
17.5 \\
12.6 \\
13.1 \\
8.5 \\
13.8 \\
10.8 \\
7.3 \\
6.7 \\
6.1 \\
6.2 \\
8.7 \\
19.0 \\
19.0\end{array}$ & $\begin{array}{c}95.7 \\
96.4 \\
94.8 \\
97.3 \\
93.4 \\
93.1 \\
95.3 \\
97.9 \\
98.4 \\
98.8 \\
102.8 \\
98.5 \\
101.1 \\
97.9 \\
95.9 \\
99.4\end{array}$ \\
\hline $\begin{array}{l}\text { Shaping Fill Lift } 1 \\
\text { Tested } 10-20-98 \\
\text { Ave. lift thickness }=0.176 \mathrm{~m}(0.58 \mathrm{ft}) \\
\text { Modifted Proctor Specs. } \\
\text { Optimum moisture content }=8.0 \% \\
\text { Maximum density } l b / \mathrm{ft}^{3}=134.2 \\
\text { CQA Specs } \\
\text { Required compaction }=90 \\
\text { Appendix E } \\
\text { Drawing } \mathrm{SK} \text {.CAL-006-92-C5 }\end{array}$ & $\begin{array}{l}1 \\
2 \\
3 \\
4 \\
5 \\
6 \\
7 \\
8 \\
9 \\
10 \\
11 \\
12 \\
13 \\
14 \\
15 \\
16\end{array}$ & $\begin{array}{c}98.4 \\
95.2 \\
99.6 \\
99.6 \\
98.6 \\
95.1 \\
93.4 \\
100.9 \\
92.5 \\
97.1 \\
98.7 \\
102.5 \\
95.2 \\
95.7 \\
97.5 \\
101.4\end{array}$ & $\begin{array}{l}5.6 \\
5.5 \\
5.4 \\
5.1 \\
5.8 \\
5.3 \\
4.6 \\
5.3 \\
6.0 \\
4.6 \\
5.3 \\
4.7 \\
5.5 \\
5.1 \\
4.8 \\
4.5\end{array}$ & $\begin{array}{l}132.0 \\
127.7 \\
133.6 \\
133.7 \\
132.3 \\
127.6 \\
125.4 \\
135.4 \\
124.1 \\
130.3 \\
132.5 \\
137.6 \\
127.8 \\
128.4 \\
130.9 \\
136.1\end{array}$ \\
\hline $\begin{array}{l}\text { Shaping Fill Lift } 2 \\
\text { Tested } 10-22-1998 \\
\text { Ave. lift thickness }=0.18 \mathrm{~m}(0.59 \mathrm{ft}) \\
\text { Modified Proctor Specs. } \\
\text { Optimum moisture content }=8.0 \% \\
\text { Maximum density } l b / f t^{3}=134.2 \\
\text { CQA Specs } \\
\text { Required compaction }=90 \\
\text { Appendix E } \\
\text { Drawing \# SK-CAU-006-92-C6 }\end{array}$ & $\begin{array}{l}1 \\
2 \\
3 \\
4 \\
5 \\
6 \\
7 \\
8 \\
9 \\
10 \\
11 \\
12 \\
13 \\
14 \\
15 \\
16 \\
\end{array}$ & $\begin{array}{l}99.7 \\
99.9 \\
99.9 \\
96.9 \\
99.9 \\
93.3 \\
93.7 \\
97.5 \\
97.5 \\
96.8 \\
97.3 \\
97.2 \\
97.0 \\
95.5 \\
93.2 \\
97.6\end{array}$ & $\begin{array}{l}6.8 \\
7.0 \\
7.4 \\
7.0 \\
6.7 \\
6.6 \\
7.1 \\
6.9 \\
7.6 \\
6.5 \\
7.2 \\
8.2 \\
7.5 \\
6.9 \\
7.5 \\
6.1 \\
\end{array}$ & $\begin{array}{l}133.8 \\
134.0 \\
134.1 \\
130.0 \\
134.1 \\
125.2 \\
125.8 \\
130.8 \\
130.8 \\
129.9 \\
130.6 \\
130.4 \\
130.2 \\
128.1 \\
125.1 \\
131.0\end{array}$ \\
\hline
\end{tabular}


TABLE 3 - NUCLEAR DENSITY TEST RESULTS (CONTLNUED).

\begin{tabular}{|c|c|c|c|c|}
\hline $\begin{array}{c}\text { LIFT } \\
\text { DESCRIPTION }\end{array}$ & $\begin{array}{c}{ }^{\text {A GRTD }} \\
\text { LOCATION } \\
\#\end{array}$ & $\begin{array}{c}\text { PERCENT } \\
\text { COMPACTION }\end{array}$ & $\begin{array}{c}\text { MOISTURE } \\
\text { CONTENT } \\
\%\end{array}$ & $\begin{array}{c}\text { DRY } \\
\text { DENSITY } \\
\mathrm{lb} / \mathrm{ft}^{3}\end{array}$ \\
\hline $\begin{array}{l}\text { Shaping Fill Lift } 3 \\
\text { Tested } 10-27-1998 \\
\text { Ave. lift thickness }=0.16 \mathrm{~m}(0.52 \mathrm{ft}) \\
\text { Modified Proctor Specs. } \\
\text { Optimum moisture content }=8.0 \% \\
\text { Maximum density } 1 b / f^{3}=134.2 \\
\text { CQA Specs } \\
\text { Required compaction }=90 \\
\text { Appendix E } \\
\text { Drawing \# SK-CAU-006-92-C7 }\end{array}$ & $\begin{array}{c}1 \\
2 \\
3 \\
4 \\
5 \\
6 \\
7 \\
8 \\
9 \\
10 \\
11 \\
12 \\
13 \\
14 \\
15 \\
16\end{array}$ & $\begin{array}{c}96.9 \\
96.5 \\
95.8 \\
100.0 \\
99.8 \\
94.9 \\
97.8 \\
97.8 \\
96.0 \\
95.2 \\
95.6 \\
95.9 \\
97.4 \\
97.5 \\
96.3 \\
95.0\end{array}$ & $\begin{array}{l}6.5 \\
6.9 \\
5.7 \\
6.2 \\
6.3 \\
6.7 \\
5.8 \\
6.2 \\
6.5 \\
6.5 \\
6.9 \\
6.4 \\
7.1 \\
6.9 \\
6.6 \\
5.7\end{array}$ & $\begin{array}{l}130.0 \\
129.5 \\
128.5 \\
134.2 \\
133.9 \\
127.3 \\
131.2 \\
131.2 \\
128.8 \\
127.8 \\
128.3 \\
128.7 \\
130.7 \\
130.9 \\
129.3 \\
127.5\end{array}$ \\
\hline $\begin{array}{l}\text { Shaping Fill Lift } 4 \\
\text { Tested } 10-29-1998 \\
\text { Ave. lift thickness }=0.17 \mathrm{~m}(0.56 \mathrm{ft}) \\
\text { Modified Proctor Specs. } \\
\text { Optimum moisture content }=8.0 \% \\
\text { Maximum density } l b / \mathrm{ft}^{3}=134.2 \\
\text { CQA Specs } \\
\text { Required compaction }=90 \\
\text { Appendix E } \\
\text { Drawing }\end{array}$ & $\begin{array}{c}1 \\
2 \\
3 \\
4 \\
5 \\
6 \\
7 \\
8 \\
9 \\
10 \\
11 \\
12 \\
13 \\
14 \\
15 \\
16\end{array}$ & $\begin{array}{c}97.9 \\
98.3 \\
99.5 \\
98.7 \\
97.3 \\
97.2 \\
93.7 \\
95.8 \\
96.6 \\
100.0 \\
94.8 \\
98.7 \\
96.9 \\
98.8 \\
96.0 \\
92.6\end{array}$ & $\begin{array}{l}6.3 \\
6.1 \\
5.7 \\
6.1 \\
6.0 \\
6.4 \\
6.2 \\
5.7 \\
5.6 \\
6.3 \\
6.8 \\
6.3 \\
6.0 \\
6.5 \\
6.7 \\
6.8\end{array}$ & $\begin{array}{l}131.3 \\
131.9 \\
133.5 \\
132.4 \\
130.6 \\
130.4 \\
125.7 \\
128.6 \\
129.7 \\
134.2 \\
127.2 \\
132.5 \\
130.1 \\
132.6 \\
128.8 \\
124.3\end{array}$ \\
\hline $\begin{array}{l}\text { Shaping Fill Lift } 5 \\
\text { Tested } 11-2-1998 \\
\text { Ave. lift thickness }=0.16 \mathrm{~m}(0.52 \mathrm{ft}) \\
\text { Modified Proctor Specs. } \\
\text { Optimum moisture content }=8.0 \% \\
\text { Maximum density } \text { lb/ft }=134.2 \\
\text { CQA Specs } \\
\text { Required compaction }=90 \\
\text { Appendix E } \\
\text { Drawing } \# \text { SK-CAU-006-92-C9 }\end{array}$ & $\begin{array}{c}1 \\
2 \\
3 \\
4 \\
5 \\
6 \\
7 \\
8 \\
9 \\
10 \\
11 \\
12 \\
13 \\
14 \\
15 \\
16\end{array}$ & $\begin{array}{l}97.0 \\
95.5 \\
96.8 \\
99.0 \\
95.2 \\
95.4 \\
96.0 \\
94.1 \\
100.0 \\
95.1 \\
95.2 \\
98.1 \\
95.2 \\
96.2 \\
98.9 \\
94.0\end{array}$ & $\begin{array}{l}6.3 \\
5.7 \\
5.4 \\
5.3 \\
5.8 \\
5.9 \\
5.6 \\
5.8 \\
5.8 \\
5.4 \\
5.9 \\
5.5 \\
5.9 \\
5.8 \\
5.5 \\
5.7\end{array}$ & $\begin{array}{l}130.2 \\
128.2 \\
129.9 \\
132.9 \\
127.7 \\
128.0 \\
128.8 \\
126.3 \\
134.2 \\
127.6 \\
127.8 \\
131.6 \\
127.8 \\
129.1 \\
132.7 \\
126.1\end{array}$ \\
\hline
\end{tabular}


TABLE 3 - NUCLEAR DENSITY TEST RESULTS (CONTINUED).

\begin{tabular}{|c|c|c|c|c|}
\hline $\begin{array}{c}\text { LIFT } \\
\text { DESCRIPTION }\end{array}$ & $\begin{array}{c}{ }^{\mathrm{A}} \mathrm{GRID} \\
\text { LOCATION } \\
\# \\
\end{array}$ & $\begin{array}{c}\text { PERCENT } \\
\text { COMPACTION }\end{array}$ & $\begin{array}{c}\text { MOISTURE } \\
\text { CONTENT } \\
\% \\
\end{array}$ & $\begin{array}{c}\text { DRY } \\
\text { DENSITY } \\
\text { lb/ft }{ }^{3} \\
\end{array}$ \\
\hline $\begin{array}{l}\text { Shaping Fill Lift } 6 \\
\text { Tested } 11-4-1998 \\
\text { Ave. lift thickness }=0.18 \mathrm{~m}(0.59 \mathrm{ft}) \\
\text { Modified Proctor Specs. } \\
\text { Optimum moisture content }=8.0 \% \\
\text { Maximum density } l b / \mathrm{f}^{3}=134.2 \\
\text { CQA Specs } \\
\text { Required compaction }=90 \\
\text { Appendix E } \\
\text { Drawing \# SK-CAL-006-92-C10 }\end{array}$ & $\begin{array}{c}1 \\
2 \\
3 \\
4 \\
5 \\
6 \\
7 \\
8 \\
9 \\
10 \\
11 \\
12 \\
13 \\
14 \\
15 \\
16\end{array}$ & $\begin{array}{c}97.0 \\
95.5 \\
96.8 \\
99.0 \\
95.2 \\
95.4 \\
96.0 \\
94.1 \\
100.0 \\
95.1 \\
95.2 \\
98.1 \\
97.7 \\
96.2 \\
98.9 \\
94.0\end{array}$ & $\begin{array}{l}6.3 \\
5.7 \\
5.4 \\
5.3 \\
5.8 \\
5.9 \\
5.6 \\
5.8 \\
5.8 \\
5.4 \\
5.9 \\
5.5 \\
5.8 \\
5.8 \\
5.5 \\
5.7\end{array}$ & $\begin{array}{l}130.2 \\
128.2 \\
129.9 \\
132.9 \\
127.7 \\
128.0 \\
128.8 \\
126.3 \\
131.1 \\
127.6 \\
127.8 \\
131.6 \\
131.1 \\
129.1 \\
132.7 \\
126.1\end{array}$ \\
\hline $\begin{array}{l}\text { Shaping Fill Lift } 7 \\
\text { Tested } 11-4-1998 \\
\text { Ave. lift thickness }=0.18 \mathrm{~m}(0.59 \mathrm{ft}) \\
\text { Modified Proctor Specs. } \\
\text { Optimum moisture content }=8.0 \% \\
\text { Maximum density } / b / f^{3}=134.2 \\
\text { CQA Spec. } \\
\text { Required compaction }=90 \\
\text { Appendix E } \\
\text { Drawing \# SK-CAL-006- } 92-\mathrm{Cl}\end{array}$ & $\begin{array}{l}1 \\
2 \\
3 \\
4 \\
5\end{array}$ & $\begin{array}{l}97.2 \\
95.2 \\
93.4 \\
92.3 \\
93.3\end{array}$ & $\begin{array}{l}5.4 \\
5.4 \\
6.2 \\
6.0 \\
6.2\end{array}$ & $\begin{array}{l}130.5 \\
127.8 \\
125.4 \\
123.9 \\
125.2\end{array}$ \\
\hline $\begin{array}{l}\text { Shaping Fill Lift } 7 \text { (after reshaping) } \\
\text { Tested } 11-4-1998 \\
\text { Aye. lift thickness }=0.18 \mathrm{~m}(0.59 \mathrm{ft}) \\
\text { Modified Proctor Specs. } \\
\text { Optimum moisture content }=8.0 \% \\
\text { Maximum density lb/ft }=134.2 \\
\text { CQA Spec. } \\
\text { Required compaction }=90 \\
\text { Appendix E } \\
\text { Drawing } \# \text { SK-CAL-006-92-C11 }\end{array}$ & $\begin{array}{c}1 \\
2 \\
3 \\
4 \\
5 \\
6 \\
7 \\
8 \\
9 \\
10 \\
11 \\
12 \\
13 \\
14 \\
15 \\
16\end{array}$ & $\begin{array}{l}96.2 \\
95.8 \\
98.1 \\
96.1 \\
99.2 \\
99.8 \\
99.7 \\
94.0 \\
95.7 \\
96.5 \\
96.8 \\
95.8 \\
91.6 \\
97.3 \\
97.7 \\
99.1\end{array}$ & $\begin{array}{l}10.5 \\
5.6 \\
5.4 \\
5.5 \\
4.8 \\
6.3 \\
6.4 \\
5.8 \\
5.5 \\
6.7 \\
6.8 \\
5.7 \\
6.3 \\
6.7 \\
5.4 \\
4.1\end{array}$ & $\begin{array}{l}99.6 \\
128.5 \\
131.6 \\
128.9 \\
133.1 \\
133.9 \\
133.8 \\
126.2 \\
128.4 \\
129.5 \\
129.9 \\
128.5 \\
122.9 \\
130.6 \\
131.1 \\
133.0\end{array}$ \\
\hline
\end{tabular}


TABLE 3 - NUCLEAR DENSITY TEST RESULTS (CONTINUED)

\begin{tabular}{|c|c|c|c|c|}
\hline $\begin{array}{c}\text { LIFT } \\
\text { DESCRIPTION }\end{array}$ & $\begin{array}{c}{ }^{\mathrm{A}} \mathrm{GRTD} \\
\text { LOCATION } \\
\#\end{array}$ & $\begin{array}{c}\text { PERCENT } \\
\text { COMPACTION }\end{array}$ & $\begin{array}{c}\text { MOISTLRE } \\
\text { CONTENT } \\
\% \\
\end{array}$ & $\begin{array}{c}\text { DRY } \\
\text { DENSITY } \\
{\mathrm{Ib} / \mathrm{ft}^{3}}^{3}\end{array}$ \\
\hline $\begin{array}{l}\text { Bedding Layer Lift } \\
\text { Tested } 11-16-1998 \\
\text { Ave. lift thickness }=0.20 \mathrm{~m}(0.66 \mathrm{ft}) \\
\text { Modified Proctor Spec. } \\
\text { Optimum moisture content }=15.7 \% \\
\text { Maximum density lb/fit }=110.3 \\
\text { CQA Spec. } \\
\text { Required compaction }=90 \\
\text { Appendix E } \\
\text { Drawing } \# \text { SK-CAU-006-92-C12 }\end{array}$ & $\begin{array}{c}1 \\
2 \\
3 \\
4 \\
5 \\
6 \\
7 \\
8 \\
9 \\
10 \\
11 \\
12 \\
13 \\
14 \\
15 \\
16 \\
\text { A-1 } \\
\text { B-1 } \\
\text { C-1 } \\
\text { D-1 }\end{array}$ & $\begin{array}{l}99.7 \\
95.1 \\
98.1 \\
95.6 \\
95.8 \\
90.1 \\
93.9 \\
94.4 \\
94.9 \\
91.3 \\
95.4 \\
95.6 \\
98.7 \\
99.9 \\
97.4 \\
98.3 \\
{ }^{8} 99.5 \\
{ }^{8} 91.8 \\
{ }^{8} 97.4 \\
{ }^{8} 97.5\end{array}$ & $\begin{array}{l}10.6 \\
10.4 \\
10.3 \\
10.4 \\
11.2 \\
10.3 \\
10.2 \\
10.5 \\
11.9 \\
12.2 \\
13.5 \\
10.8 \\
11.2 \\
10.5 \\
11.9 \\
11.1 \\
10.6 \\
11.6 \\
11.1 \\
10.8\end{array}$ & $\begin{array}{c}110.0 \\
104.9 \\
108.2 \\
105.5 \\
105.7 \\
99.4 \\
103.6 \\
104.1 \\
104.7 \\
100.7 \\
105.2 \\
105.4 \\
108.9 \\
110.2 \\
107.4 \\
108.4 \\
\mathrm{NA} \\
\mathrm{NA} \\
\mathrm{NA} \\
\mathrm{NA}\end{array}$ \\
\hline $\begin{array}{l}\text { Buffer Layer Lift } \\
\text { Tested } 12-1-1998 \\
\text { Ave. lift thickness }=0.195 \mathrm{~m}(0.64 \mathrm{ft}) \\
\text { Modified Proctor Spec. } \\
\text { Optimum moisture content }=15.7 \% \\
\text { Maximum density } \text { lb/ft }=110.3 \\
\text { CQA Spec. } \\
\text { Required compaction }=90 \\
\text { Appendix E } \\
\text { Drawing \# SK-CAL-006-92-C13 }\end{array}$ & $\begin{array}{c}1 \\
2 \\
3 \\
4 \\
5 \\
6 \\
7 \\
8 \\
9 \\
10 \\
11 \\
12 \\
13 \\
14 \\
15 \\
16 \\
\text { A-2 } \\
\text { B-2 } \\
\text { C-2 } \\
\text { D-2 }\end{array}$ & $\begin{array}{l}93.5 \\
95.2 \\
94.8 \\
95.4 \\
91.1 \\
94.1 \\
93.8 \\
95.5 \\
92.7 \\
92.7 \\
94.5 \\
96.0 \\
91.5 \\
91.2 \\
93.3 \\
98.5 \\
{ }^{{ }^{8}} 95.0 \\
{ }_{9}^{8} 98.4 \\
{ }^{8} 90.7 \\
{ }^{{ }^{5}} 95.9\end{array}$ & $\begin{array}{l}11.4 \\
12.3 \\
12.8 \\
12.0 \\
12.6 \\
10.5 \\
11.1 \\
12.7 \\
10.5 \\
12.1 \\
11.4 \\
11.5 \\
11.5 \\
10.5 \\
10.5 \\
11.5 \\
10.3 \\
10.7 \\
9.5 \\
8.8\end{array}$ & $\begin{array}{l}103.1 \\
105.0 \\
104.6 \\
105.2 \\
100.5 \\
103.8 \\
103.5 \\
105.3 \\
102.3 \\
102.2 \\
104.2 \\
105.9 \\
1009 \\
100.6 \\
102.9 \\
108.6 \\
\mathrm{AA} \\
\mathrm{AA} \\
\mathrm{NA} \\
\mathrm{NA}\end{array}$ \\
\hline
\end{tabular}


TABLE 3 - NUCLEAR DENSITY TEST RESULTS (CONTINUED).

\begin{tabular}{|c|c|c|c|c|}
\hline $\begin{array}{c}\text { LIFT } \\
\text { DESCRIPTION }\end{array}$ & $\begin{array}{c}{ }^{A} \text { GRID } \\
\text { LOCATION } \\
\#\end{array}$ & $\begin{array}{c}\text { PERCENT } \\
\text { COMPACTION }\end{array}$ & $\begin{array}{c}\text { MOISTURE } \\
\text { CONTENT } \\
\% \\
\end{array}$ & $\begin{array}{c}\text { DRY } \\
\text { DENSITY } \\
\text { lb/ft } \\
\end{array}$ \\
\hline $\begin{array}{l}\text { Perimeter Backfill Lift } 1 \\
\text { Tested } 12-7-1998 \\
\text { Modified Proctor Spec. } \\
\text { Optimum moisture content }=8.0 \% \\
\text { Maximum density lb/ft }=134.2 \\
\text { CQA Spec. } \\
\text { Required compaction }=90 \\
\text { Appendix E } \\
\text { Drawing \# SK-CAU-006-92-C14 }\end{array}$ & $\begin{array}{c}1 \\
2 \\
3 \\
4 \\
5 \\
6 \\
7 \\
8 \\
9 \\
10 \\
11 \\
12 \\
13 \\
14 \\
15 \\
16\end{array}$ & $\begin{array}{l}97.7 \\
96.6 \\
95.7 \\
97.1 \\
99.6 \\
97.7 \\
99.0 \\
96.9 \\
99.6 \\
94.6 \\
96.9 \\
95.3 \\
97.5 \\
93.2 \\
98.5 \\
90.1\end{array}$ & $\begin{array}{l}5.4 \\
5.3 \\
5.8 \\
6.2 \\
5.0 \\
6.4 \\
6.9 \\
6.1 \\
5.4 \\
5.4 \\
5.2 \\
5.6 \\
5.7 \\
5.1 \\
7.6 \\
5.8\end{array}$ & $\begin{array}{l}131.1 \\
129.7 \\
128.4 \\
130.3 \\
133.7 \\
131.1 \\
132.9 \\
130.0 \\
133.6 \\
126.9 \\
130.0 \\
127.9 \\
130.8 \\
125.1 \\
132.2 \\
120.9\end{array}$ \\
\hline $\begin{array}{l}\text { Erosion Control Layer Lift } 1 \\
\text { Tested } 12-7-1998 \\
\text { Ave. lift thickness }=0.198 \mathrm{~m}(0.65 \mathrm{ft}) \\
\text { Modified Proctor Spec. } \\
\text { Optimum moisture content }=12.8 \% \\
\text { Maximum density lb/ft' }=120.0 \\
\text { CQA Spec. } \\
\text { Required compaction }=90 \\
\text { Appendix E } \\
\text { Drawing \# SK-CAU-006-92-C14 }\end{array}$ & $\begin{array}{l}1 \\
2 \\
3 \\
4 \\
5 \\
6 \\
7 \\
8 \\
9 \\
10 \\
11 \\
12 \\
13 \\
14 \\
15 \\
16\end{array}$ & $\begin{array}{l}92.3 \\
90.5 \\
93.2 \\
90.0 \\
90.1 \\
91.2 \\
90.6 \\
93.0 \\
90.8 \\
90.5 \\
90.1 \\
90.0 \\
91.9 \\
92.2 \\
91.1 \\
91.3\end{array}$ & $\begin{array}{c}9.4 \\
8.0 \\
8.2 \\
8.0 \\
8.0 \\
7.4 \\
11.7 \\
7.6 \\
7.2 \\
8.0 \\
7.4 \\
7.5 \\
8.0 \\
7.6 \\
7.9 \\
9.9\end{array}$ & $\begin{array}{l}110.7 \\
108.6 \\
111.8 \\
108.0 \\
108.1 \\
109.4 \\
108.7 \\
111.6 \\
109.0 \\
108.6 \\
108.1 \\
107.9 \\
110.3 \\
110.6 \\
109.3 \\
109.6\end{array}$ \\
\hline $\begin{array}{l}\text { Perimeter Backfill Lift } 2 \\
\text { Tested } 12-15-1998 \\
\text { Modified Proctor Spec. } \\
\text { Optimum moisture content }=8.0 \% \\
\text { Maximum density lb/ft }=134.2 \\
\text { CQA Spec. } \\
\text { Required compaction }=90 \\
\text { Appendix E } \\
\text { Drawing } \# \text { SK-CAU-006-92-C15 }\end{array}$ & $\begin{array}{l}1 \\
2 \\
3 \\
4 \\
5 \\
6 \\
7 \\
8 \\
9 \\
10 \\
11 \\
12 \\
13 \\
14 \\
15 \\
16\end{array}$ & $\begin{array}{l}94.7 \\
99.6 \\
97.0 \\
100.0 \\
100.0 \\
99.7 \\
100.0 \\
98.4 \\
96.3 \\
97.0 \\
98.7 \\
98.2 \\
97.8 \\
95.9 \\
94.3 \\
99.8\end{array}$ & $\begin{array}{l}7.9 \\
7.9 \\
6.6 \\
6.9 \\
6.6 \\
7.1 \\
6.9 \\
7.3 \\
7.6 \\
6.6 \\
6.6 \\
7.6 \\
7.9 \\
6.8 \\
7.2 \\
6.5\end{array}$ & $\begin{array}{l}127.1 \\
133.6 \\
130.2 \\
134.2 \\
134.2 \\
133.8 \\
134.2 \\
132.1 \\
129.2 \\
130.2 \\
132.5 \\
131.8 \\
131.2 \\
128.7 \\
126.5 \\
133.9\end{array}$ \\
\hline
\end{tabular}


TABLE 3 - NUCLEAR DENSITY TEST RESULTS (CONTINUED).

\begin{tabular}{||c|c|c|c|c||}
\hline $\begin{array}{c}\text { LIFT } \\
\text { DESCRIPTION }\end{array}$ & $\begin{array}{c}\text { AGRID } \\
\text { LOCATION } \\
\#\end{array}$ & $\begin{array}{c}\text { PERCENT } \\
\text { COMPACTION }\end{array}$ & $\begin{array}{c}\text { MOISTURE } \\
\text { CONTENT } \\
\%\end{array}$ & $\begin{array}{c}\text { DRY } \\
\text { DENSITY } \\
\text { [b/ft }^{3}\end{array}$ \\
\hline \hline Erosion Control Layer Lift 2 & 1 & 93.0 & 9.4 & 109.2 \\
Tested 12-15-1998 & 2 & 92.6 & 8.0 & 111.1 \\
Ave. lift thickness =0.188 m (0.62 ft) & 3 & 91.4 & 8.8 & 109.7 \\
& 4 & 90.8 & 7.4 & 109.0 \\
Modified Proctor Spec. & 5 & 91.3 & 8.8 & 109.5 \\
Optimum moisture content $=12.8 \%$ & 6 & 91.0 & 8.8 & 109.2 \\
Maximum density lb/ft $=120.0$ & 7 & 93.6 & 9.6 & 112.3 \\
& 8 & 93.8 & 8.9 & 112.5 \\
CQA Spec. & 9 & 93.7 & 8.7 & 112.4 \\
Required compaction $=90$ & 10 & 91.7 & 7.9 & 110.0 \\
Appendix E & 11 & 90.3 & 8.4 & 108.3 \\
Drawing \# SK-CAU-006-92-C15 & 12 & 91.8 & 8.8 & 110.2 \\
& 13 & 91.8 & 9.4 & 110.2 \\
& 15 & 91.5 & 9.3 & 109.8 \\
\hline
\end{tabular}

A: Grid locations are identified in Figure 6. Tests were taken near the center of the grid.

B: Density estimates are from Sand Cone Density ASTM D1556-90 (ASTM, 1997a). All other density measurements were estimated using ASTM D2922-96 (ASTM, 1997b) (Campbell MC-2/MC-3 Troxler).

C: NA=Not applicable. 


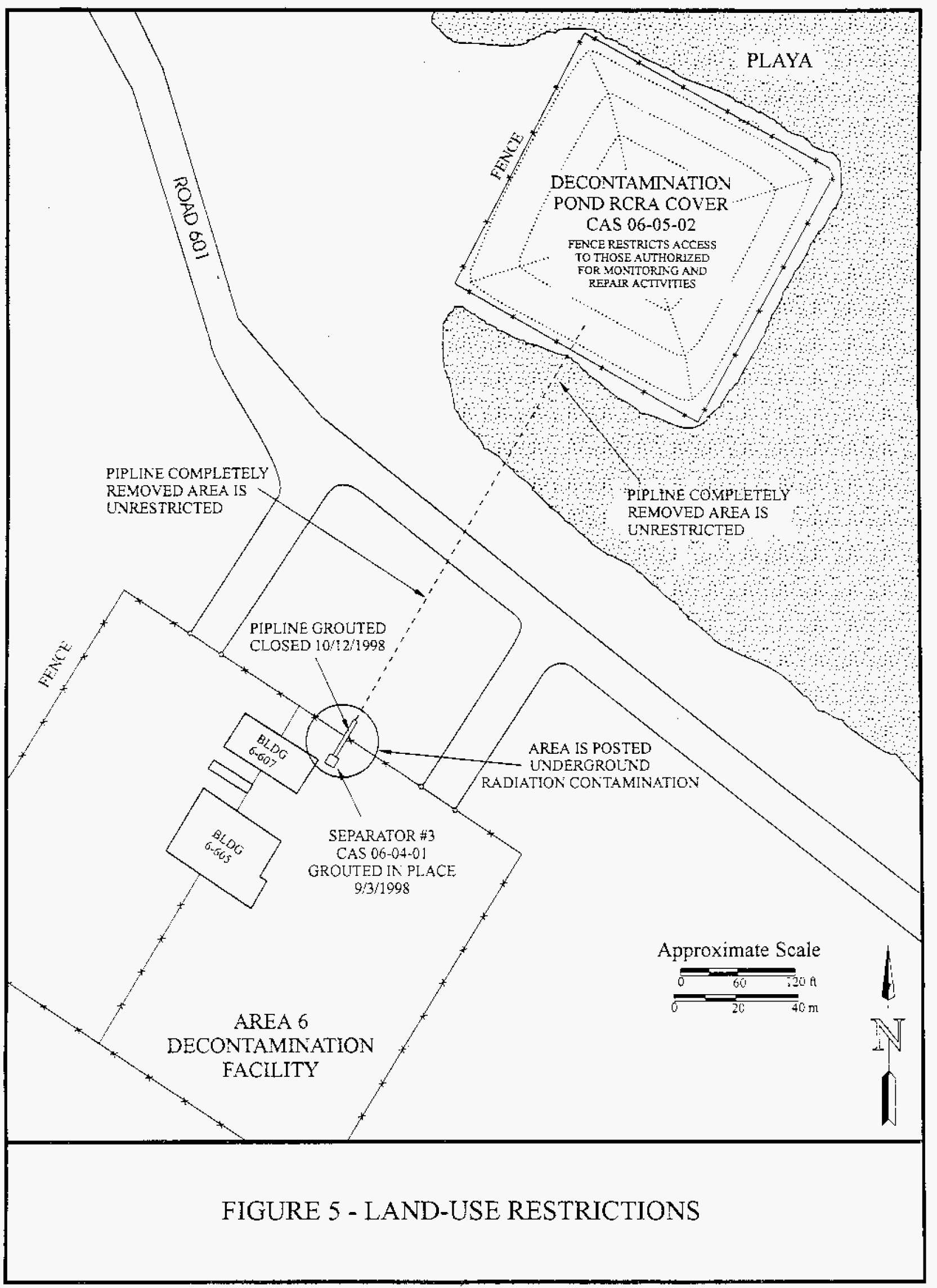


A-1 THROLGH D-1 ARE THE LOCATIONS FOR THE SANDCONE DENSITY TESTS FOR THE BEDDRNG LAYER

A-2 THROUGH D-2 ARE THE LOCATIONS FOR THE SANDCONE DENSITY TESTS FOR THE BLFFER LAYER.

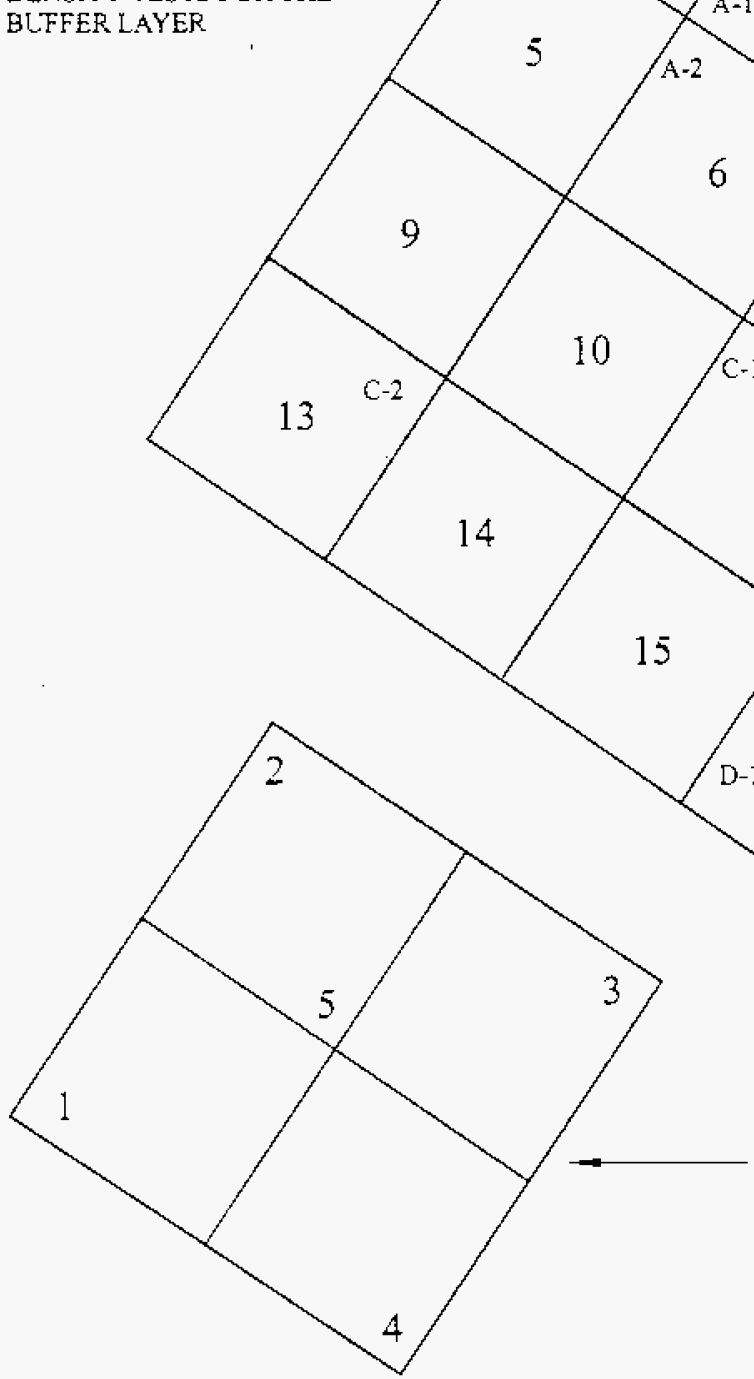

FINAL SHAPING FILL LIFT ( $(7)$ ). NLCLEAR DENSITY TESTS WERF. CONDLCTED IN THE LOCATIONS OF THE NUMBERS. THE GRDING WAS CHANGED BECAUSE THE FINAL SHAPING FILS LIFT WAS MLCH SMALLER THAN PRECEDING LIFTS

SCALE:

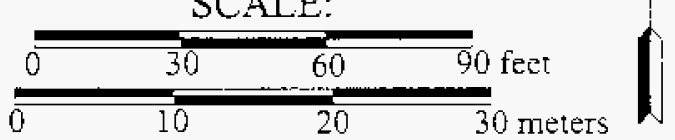

FIGURE 6 - LOCATIONS FOR RCRA COVER NUCLEAR DENSITY TESTS 
THIS PAGE INTENTIONALLY LEFT BLANK 


\subsection{CONCLUSIONS AND RECOMMENDATIONS}

\subsection{CONCLUSIONS}

Closure for this site was completed by the following:

\subsubsection{Decontamination Pad Oil/Water Separator - CAS 06-04-01}

- The Decontamination Pad oil/water separator was closed in place. The soil around the separator was sampled (Section 4.0), and the separator filled with expanding cement grout.

- The clay pipe that connected the Decontamination Pad oil/water separator and the Decontamination Pond was excavated up to $6.1 \mathrm{~m}(20 \mathrm{ft})$ north of the Decontamination Facility fence line. This pipe was disposed of in the Decontamination Pond. The associated impacted soil was excavated and disposed with the pipe. Prior to disposal, waste characterization samples were collected from the pipe and associated soil (Section 4.0). The clay pipe between the Decontamination Facility fence line and the separator were closed in place using an expandable cement grout installed under pressure.

- Excavations were backfilled with clean fill and compacted following accepted construction practices.

\subsubsection{Decontamination Pond - CAS 06-05-02}

- The Decontamination Pond (CAS 06-05-02) was closed by constructing an engineered cover. First the pipe, excavated soil from around the pipe, and characterization IDW were added to the base of the pond. The berm walls were then pushed into the pond and compacted following the CQA Plan. Additional materials were imported to form subsequent layers as per the CQA Plan (DOE, 1998a). The final layers included a GCL, buffer layer and erosion control layer. This cover was designed to meet or exceed all requirements stated in $40 \mathrm{CFR} \$ 265.228(\mathrm{a})(2)$.

\subsection{POST-CLOSURE MONITORING}

Closure of the clay pipe extending from the pond to within $6.1 \mathrm{~m}(20 \mathrm{ft})$ north of the Decontamination Facility fence line was completed by removal of all impacted material. Therefore, under the conditions of 40 CFR 270.1(c)(5) and (6), post-closure monitoring is not required for this portion of CAL 92.

The Decontamination $\mathrm{Pad}$ oil/water separator and the concrete encased pipe extending from the separator to $6.1 \mathrm{~m}(20 \mathrm{ft})$ north of the Decontamination Facility fence line were closed in place. Verification sampling indicated that no historic releases occurred from these components. 
Therefore, under agreement with NDEP by approval of the Closure Plan these components of CAU 92 require no post-closure monitoring.

Post-closure monitoring of the Area 6 Decontamination Pond urit is required under $40 \mathrm{CFR}$ 265.310 (b). Post-closure groundwater monitoring requirements specified in 40 CFR 265.310 have been waived based on 40 CFR 265.90. The post-closure monitoring will be confined to the Decontamination Pond RCRA Cover. Inspections will be conducted quarterly or if:

- Precipitation occurs in excess of 1.28 centimeters $(\mathrm{cm})(0.50$ inches [in]) in a 24-hour period, or

- Earthquake occurs with a magnitude exceeding 4.5 on the Richter scale within 100 kilometers (62 miles).

A post-closure inspection will consist of the following elements:

- A detailed inspection of the RCRA Cover fencing. The perimeter of the fencing will be walked by the inspector and the condition of the wave barrier (fiber glass slats laced through the bottom of the chain-link fencing), waming signs, entrance gate, and lock will be documented.

- The condition of the five survey subsidence monuments will be inspected. In addition, on an annual basis, all five survey monuments will be resurveyed to determine if the RCRA Cover has subsided.

- During each inspection, any changes in the condition of the cover or fenced area will be documented. In addition, the reason for the inspection (quarterly, excess precipitation, or earthquake) will be noted on the inspection report. Specific changes noted on the current condition of the cover include, but are not limited to, trash/debris within the fenced area, vegetation growing on the cover, animal burrows/nesting activity, or erosion of the cover.

- Small cracks or settling imperfections ( $<5 \mathrm{~cm}$ [2 in] deep) on the cover will be documented and will be scheduled for repair on an annual basis. Larger disruptions of the cover (vegetation, animal borings, or erosion) will be immediately evaluated and repaired within 60 days.

- All repair work should preserve the original RCRA Cover "as built" design. If the cover. repair requires the modification of the RCRA Cover design, DOE will present a formal design modification request to the NDEP prior to making the design modification. 


\subsection{CLOSURE PETITION}

Based upon the completion of site activities in accordance with the approved Closure Plan (DOE, 1998a), it is requested that a notice of completion be provided by the NDEP for CAU 92. Upon closure approval, CAU 92 will be promoted from Appendix III to Appendix IV of the FFACO, "Closed Corrective Action Units." 
THIS PAGE INTENTIONALLY LEFT BLANK 


\subsection{REFERENCES}

American Society for Testing Materials, see ASTM

ASTM, 1997a, Standard Test Method for Density and Unit Weight of Soil in Place by the SandCone Method, 1997 Annual Book of ASTM Standards, Volume 04.08, Soil and Rock (I): D 420- D 4914

ASTM, 1997b, Standard Test Method for Density of Soil and Soil-Aggregate in Place by Nuclear Methods, 1997 Annual Book of ASTM Standards, Volume 04.08, Soil and Rock (1): D 420- D 4914

Bechtel Nevada, 1997, Bechtel Nevada Performance Management Plan, B-A20/96.01, Las Vegas, NV.

Bechtel Nevada, 1998, Bechtel Nevada Analytical Services Laboratory Procedures Manual, L-E10.602.PC, Las Vegas, NV.

Bechtel Nevada, 1998, Bechtel Nevada Analytical Services Laboratory Procedures Manual, L-E10.612.PL, Las Vegas, NV.

BN, see Bechtel Nevada.

DOE, see U.S. Department of Energy.

EPA, see U.S. Environmental Protection Agency.

Nevada Division of Environmental Protection, 1995, Nevada Division of Environmental Protection Permit for a Hazardous Waste Management Facility, L.S. DOE, Vevada Test. Site, Permit Number NEV HW009.

Nevada Division of Environmental Protection, 1996, Federal Facilities Agreement and Consent Order (FEACO) of 1996 Prepared by Nevada Division of Environmental Protection, U.S. Department of Energy, and U.S. Department of Defense.

Nevada Division of Environmental Protection, 1998, Letter from Paul J. Liebendorfer regarding the final approval of the CAU 92 Corrective Action Plan. November 3, 1998, Carson City, Nevada.

U.S. Department of Energy, Nevada Operations Office, August 1996a, Resource Conservation and Recovery Act Industrial Site Environmental Restoration Site Characterization Report, Area 6 Decontamination Pond Facility, Rev. 1, DOE/NV-438. 
U.S. Department of Energy, Nevada Operations Office, 1996b, Nevada Environmental Restoration Project, Industrial Sites Quality Assurance Project Plan, Nevada Test Site Nevada, Rev 1, Las Vegas, NV.

U. S. Department of Energy, Nevada Operations Office, October 1997a, Resource Conservation and Recovery Act Industrial Site Environmental Restoration Site Characterization Report, Area 6 Decontamination Pond Facility, Comective Action Lnit No. 92, Rev, 1, DOE/NV470 .

U.S. Department of Energy, Nevada Operations Office, October 1997b, Resource Conservation and Recovery Act Corrective Measures Study: Area 6 Decontamination Pond Facility, Corrective Action Unit No. 92, Rev. 0, DOE/NV-493.

U.S. Department of Energy, Nevada Operations Office, September 1997c, Report of Activities and_Geotechnical/Hydrological_Results May and June 1997 Characterization Sampling Area 6 Decontamination Pond, CAU 92.

U.S. Department of Energy, Nevada Operations Office, November 1998a, Closure Plan for Conrective Action Unit 92; Area 6 Decontamination Pond Nevada Test Site, Nevada, Rev 2 , DOE/NV-11718-205 UC-700.

U.S. Department of Energy, Nevada Operations Office, July 1998b, Nevada Environmental Restoration Project, Health and Safety Plan. Revision 3, Las Vegas, NV.

U.S. Environmental Protection Agency, 1996a, Test Methods for Evaluating Solid Waste Physical/Chemical Methods (SW-846) Third Edition, Method 8080 A Modified, Washington, DC.

U.S. Environmental Protection Agency, 1996b, Test Methods for Evaluating Solid Waste Physical/Chemical Methods(SW-846) Third Edition, Method 8015 Modified, Washington, DC.

U.S. Environmental Protection Agency, 1996c, Test Methods for Evaluating Solid Waste Physical/Chemical Methods (SW-846) Third Edition, Method, Method 8260B Washington, DC.

U.S. Environmental Protection Agency, 1996d, Test Methods for Evaluating Solid Waste Physical/Chemical Methods (SW-846) Third Edition, Method 8270, Washington, DC.

U.S. Environmental Protection Agency, 1996e, Test Methods for Evaluating Solid Waste Physical/Chemical Methods (SW-846) Third Edition,Methods1311/6010, Washington, DC. 
U.S. Environmental Protection Agency, 1996f, 40 Code of Federal Regulations 261.24, Identification and Listing of Hazardous Waste, Toxicity Characteristics, Wảshington, DC.

U.S. Environmental Protection Agency, 1996g, 40 Code of Federal Regulations 264.115, Status Standards for Owners and Operators of Hazardous Waste Treatment, Certification of Closure, Washington, DC.

U.S. Environmental Protection Agency, 1996h, 40 Code of Federal Regulations 265.90, Interim. Status Standards for Owners and Operators of Hazardous Waste Treatment, Storage and Disposal Facilities Ground Water Monitoring, Washington, DC.

U.S. Environmental Protection Agency, 1996i, 40 Code of Federal Regulations 265.228, Interim Status Standards for Owners and Operators of Hazardous Waste Treatment, Storage and Disposal Facilities Closure and Post-Closure Care, Washington, DC.

U.S. Environmental Protection Agency, 1996j, 40 Code of Federal Regulations 265.310, Interim Status Standards for Owners and Operators of Hazardous.Waste Treatment, Storage and Disposal Facilities Closure and Post Closure_Care, Washington, DC.

U.S. Environmental Protection Agency, 1996k, 40 Code of Federal Regulations 270.1, The Hazardous Waste Permit Program, Purpose and Scope of These Regulations,-Washington, DC. 
APPENDIX A

PROJECT PHOTOGRAPHS 

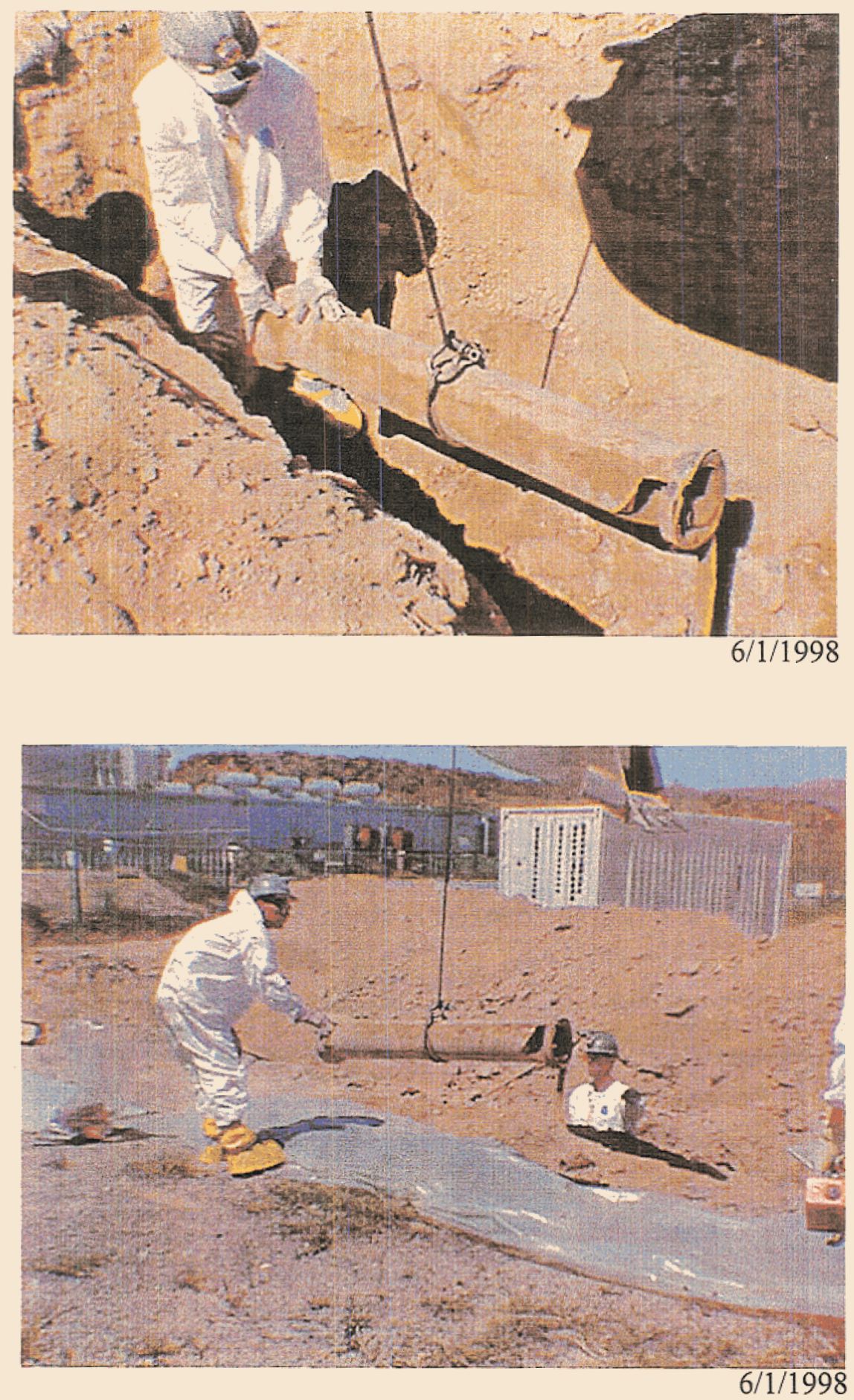

AREA 6 DECONTAMINATION POND INFLUENT PIPE CLOSURE EXCAVATION AND VERIFICATION SAMPLING 


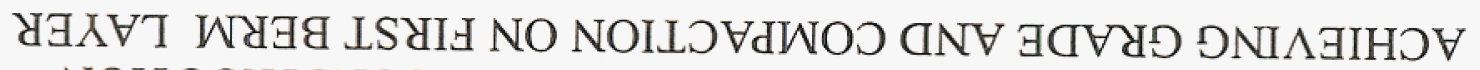

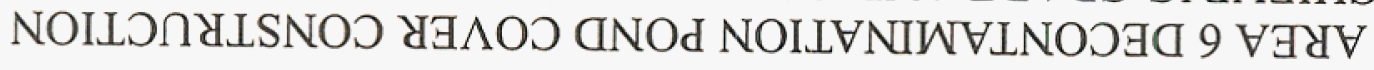

$866 \mathrm{I} / 8 / \mathrm{L}$

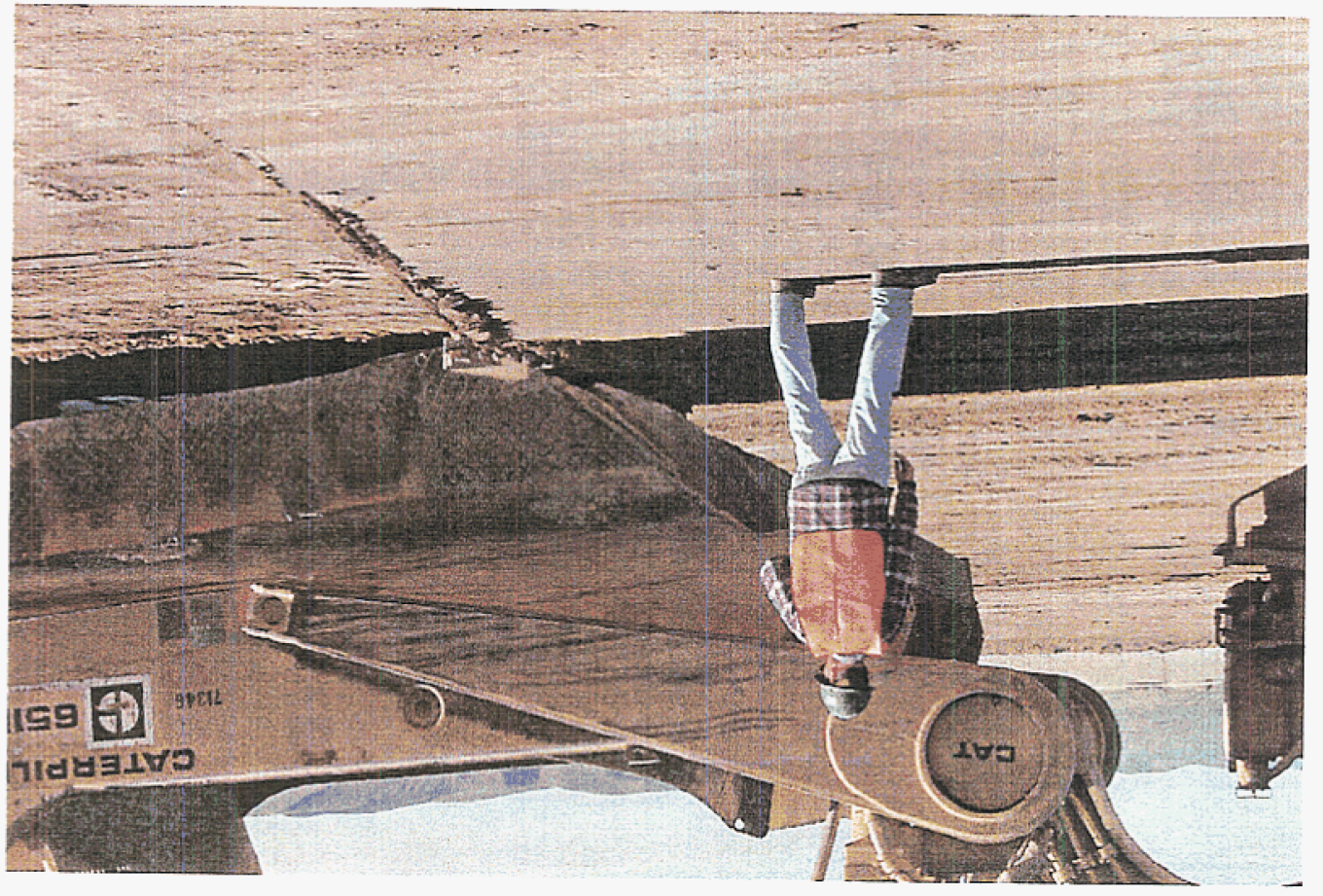

$866 \mathrm{I} / 8 / \mathrm{L}$

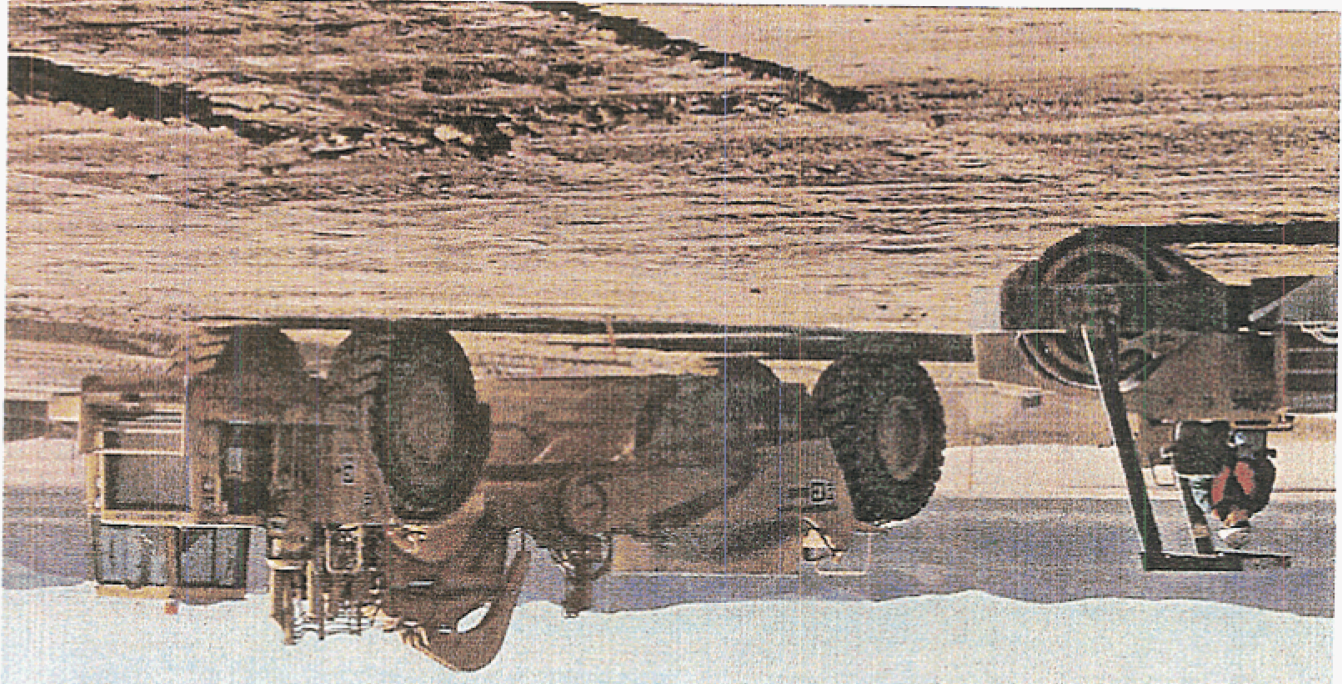



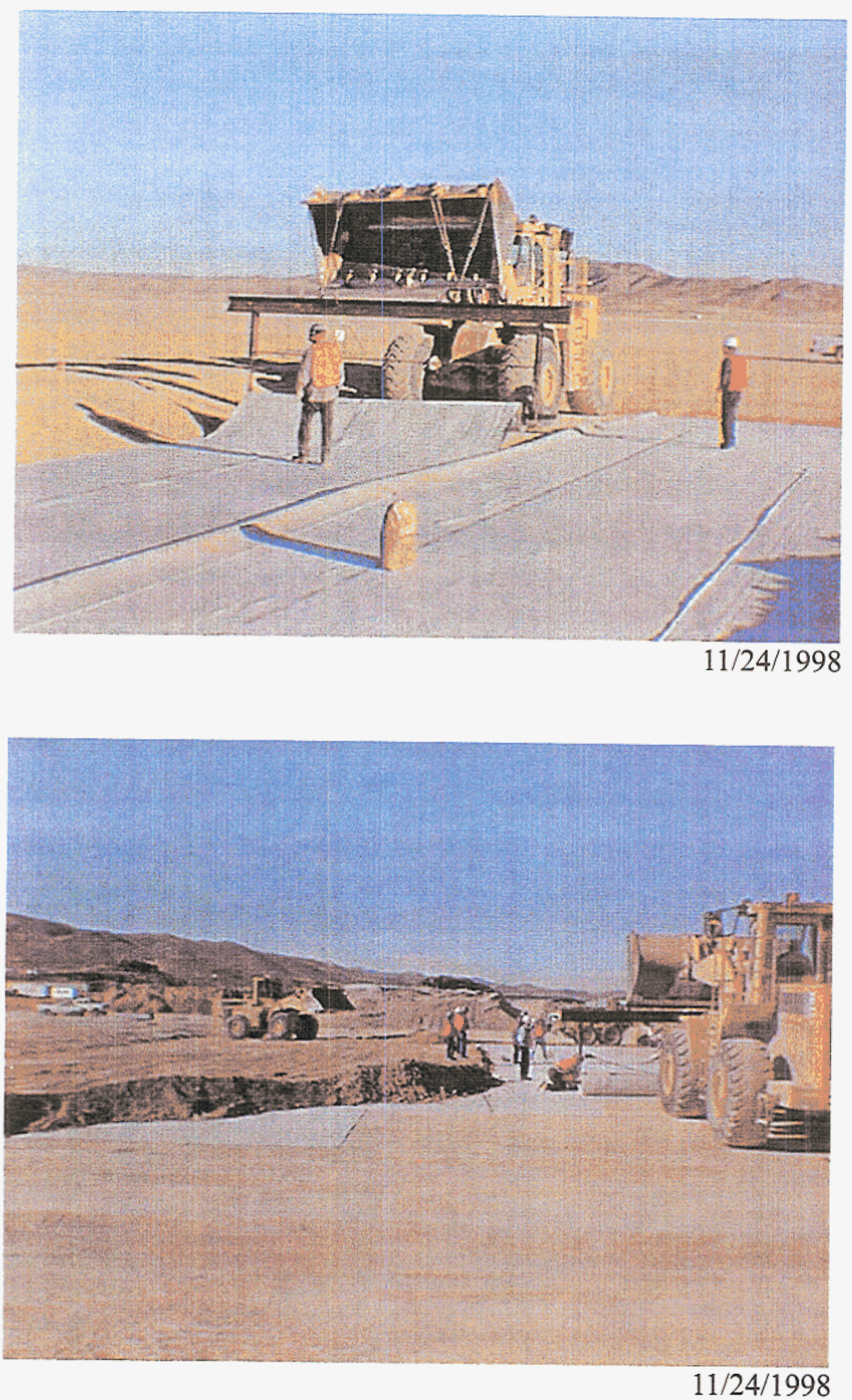

AREA 6 DECONTAMINATION POND CLOSURE GCL PLACEMENT 


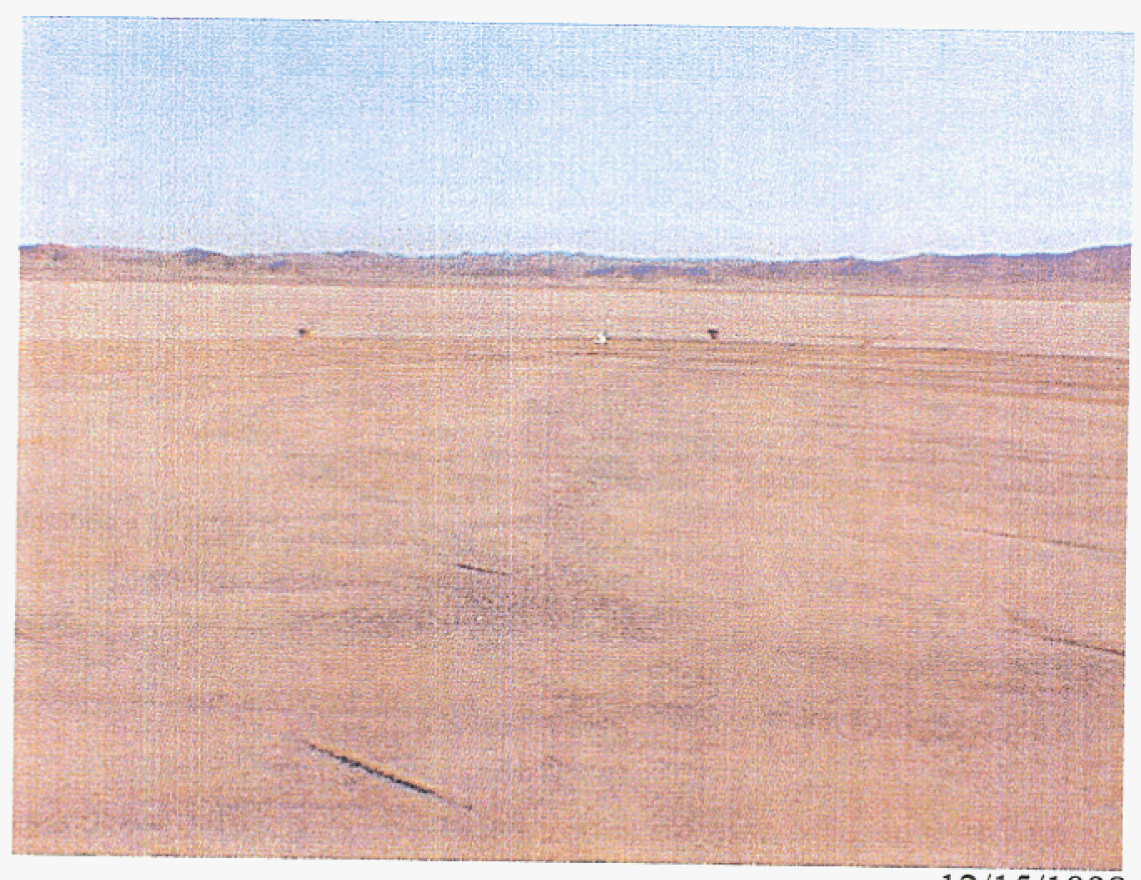

$12 / 15 / 1998$

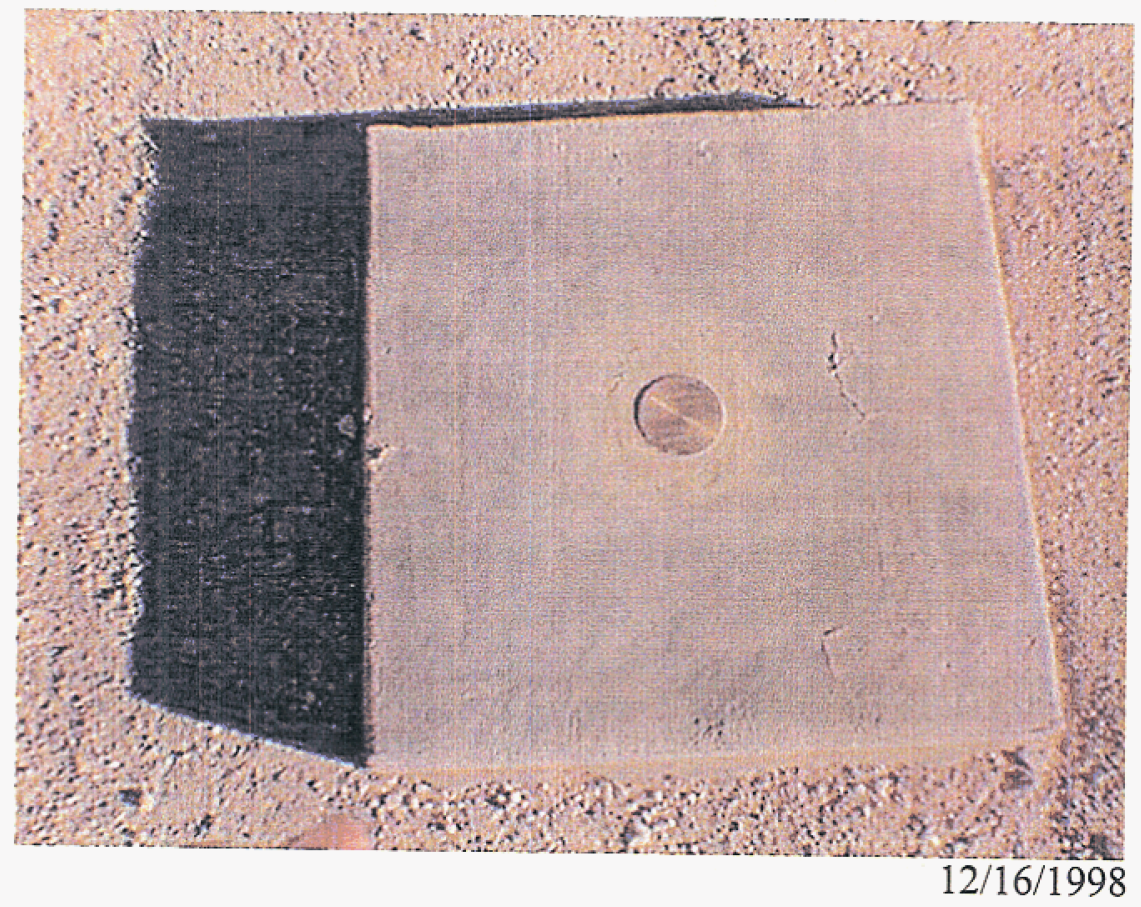

AREA 6 DECONTAMINATION POND CLOSURE COMPLETED COVER AND MONUMENT 


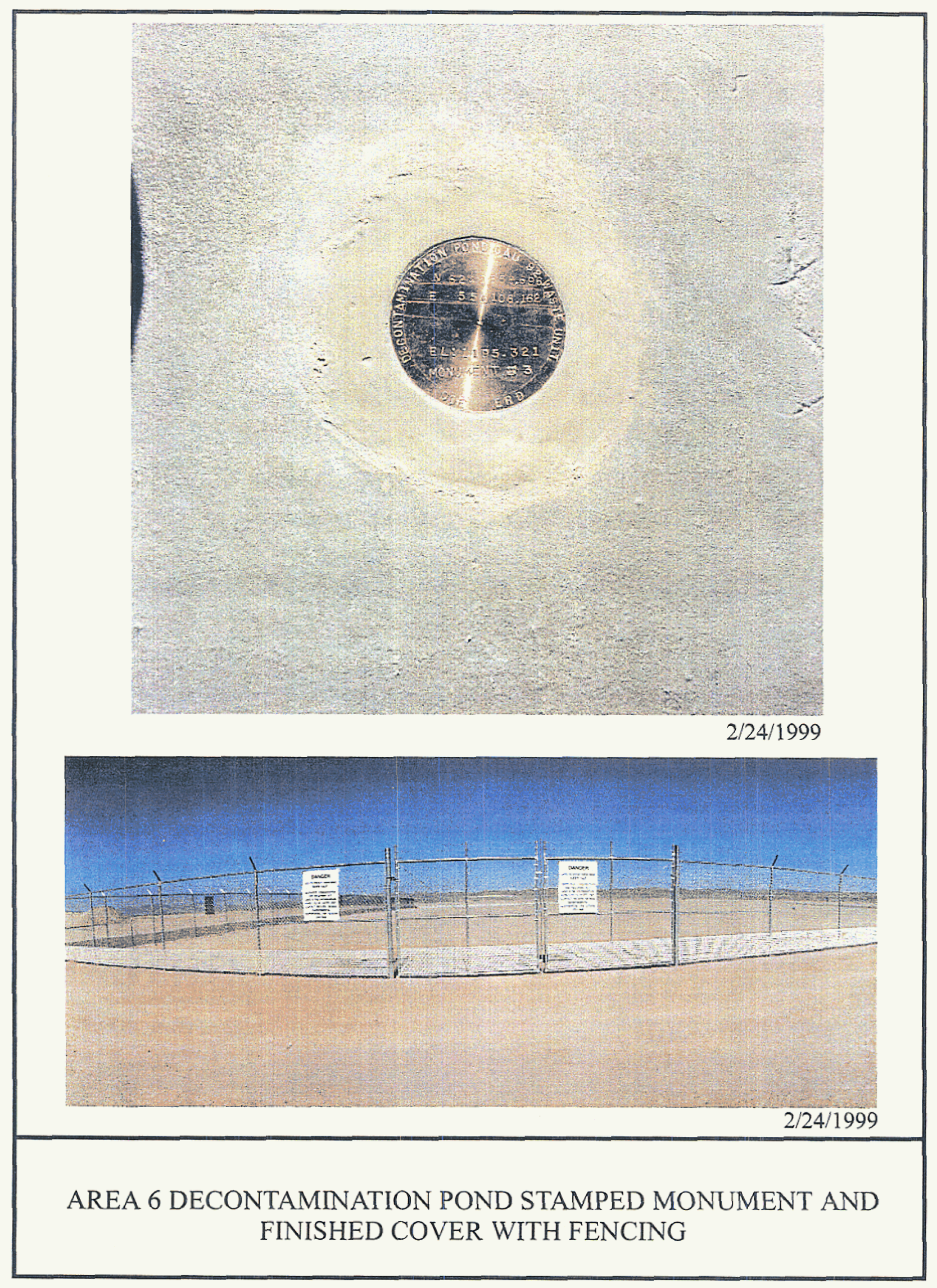




\section{APPENDIX B}

SAMPLE ANALYTICAL RESULTS 


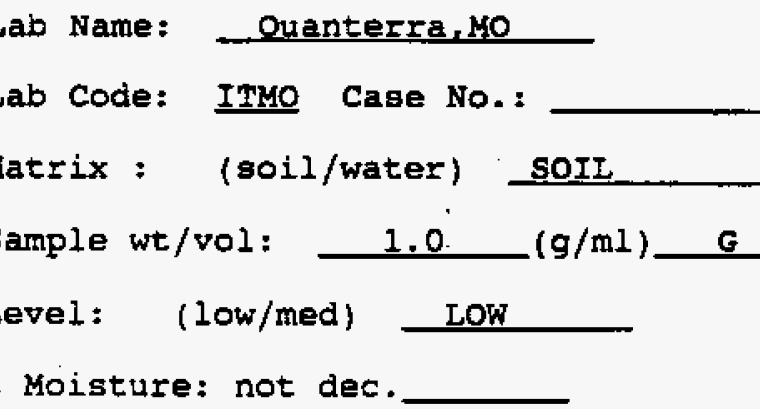

Concentration of analyte is less than the value given. Pattern reasonably matches gasoline Pattern appears to be multi-peaks, but does not match gasoline concentration is primarily from a single peak

(1): Low Boiling Hydrocarbon (LBH) is quantitated as if it is Gasoline. 


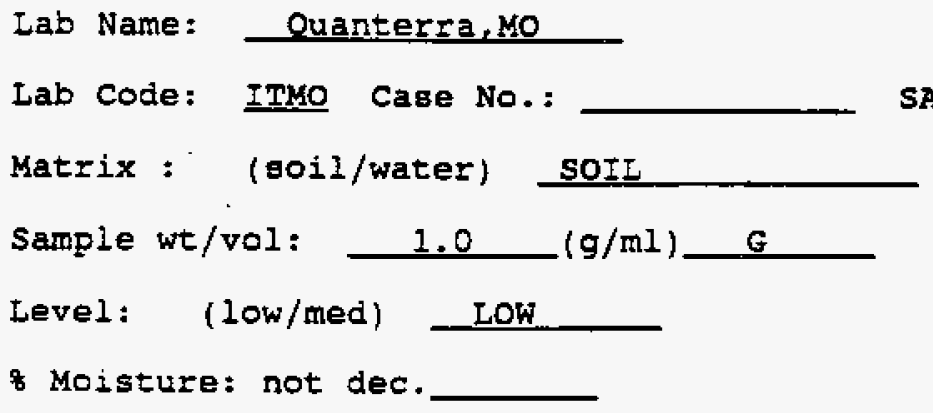

Contract: 625.01

SAS No.: SDG No.: V36B

Lab Sample ID: 17161-003

Lab File ID:

Date Sampled: $03-10-98$

Date Analyzed: $03-13-98$

Dilution Factor: 1.0
CAS NO. Compound LBH (1)
CONCENTRATION UNITS:

(ug/L or $u g / \mathrm{Kg}$ ) UG/Ka

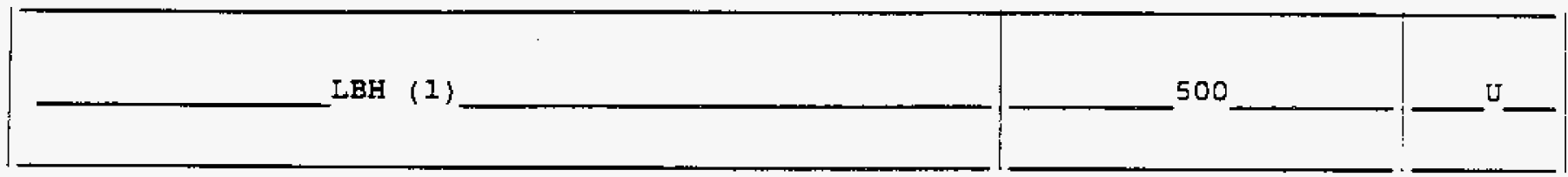

U: Concentration of analyte is less than the value given.

$\mathrm{X}$ : Pattern reasonably matches gasoline

$Y$ : Pattern appears to be multi-peaks, but does not match gasoline

$z$ : Concentration is primarily from a single peak

(1): Low Boiling Hydrocarbon (LBH) is quantitated as if it is Gasoline. 
iab Name: Ouanterra.Mo

bode: ITMO case No.: iatrix : (soil/water) sort

ample wt/vol:

1.0 (g/ml)

G

evel: (low $/ \mathrm{med})$ Low

Moigture: not dec.

CAS NO. contract: $\quad 625.01$

SAS No.: SDG NO.: V368

Lab sample ID: 17161-002

Lab File ID:

Date Sampled: $\quad 03-10-98$

Date Analyzed: $03-13-98$

Dilution Factor: 1.0

Concentration of analyte is less than the value given.

Pattern reasonably matches gasoline

Pattern appears to be multi-peaks, but does not match gasoline

Concentration is primarily from a single peak

(1): Low Boiling Hydrocarbon (LBH) is quantitated as if it is GagoIine. 
Lab Name: $\frac{\text { Ouanterra,MO }}{\text { Lab Code: ITMO Case No.: }}$
ITS

Matrix : (soil/water) soIL

Sample wt/vol:

1.0 $(\mathrm{g} / \mathrm{ml})$

G.

Level: (low/med) LoW

t Moisture: not dec.

CAS No.

Compound contract: 625.01

SAS Ḣo.: SDG NO.: $\mathrm{V} 368$

Lab sample ID: 17161-004

Lab File ID:

Date sampled: $\quad 03-10-98$

Date Analyzed: 03-13-98

Dilution Factor: 1.0

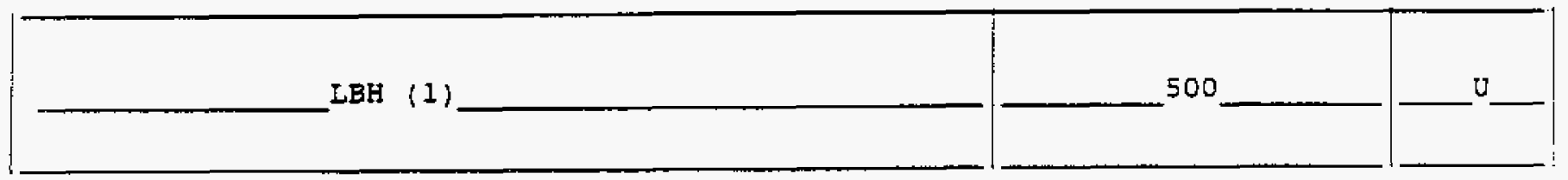

$U$ : Concentration of analyte is lesg than the value given.

$\mathrm{X}$ : Pattern reasonably matches gasoline

Y: Pattern appears to be multi-peaks, but does not match gasoline

2: Concentration is primarily from a single peak

(1): Low Boiling Hydrocarbon (LBH) is quantitated as if it is Gasoline. 
ID

LEH ANALYSIS DATA SHEET
EPA SAMPLE NO

\section{LBHLCSO1}

Lab Name:

ouanterra, $\mathbf{n O}$

Lab code: ITMO cage No.:

Matrix : (goil/water) SOIL

Sample wt/vol: 1. 0 $(g / m 1)$

G

Level: (low/med) LOW

* Moisture: not dec.

compound

LBH ( 1 )

U: Concentration of analyte is less than the value given.

x:- Pattern reasonably matches gasoline

$Y$ : Pattern appears to be multi-peaks, but does not match gasoline

Z: Concentration is primarily from a single peak

(1): Low Boiling Hydrocarbon (LBH) is quantitated as if it is Gasoline.
(ug/L of $\mathrm{ug} / \mathrm{Kg}$ ) UG/Ko 5400

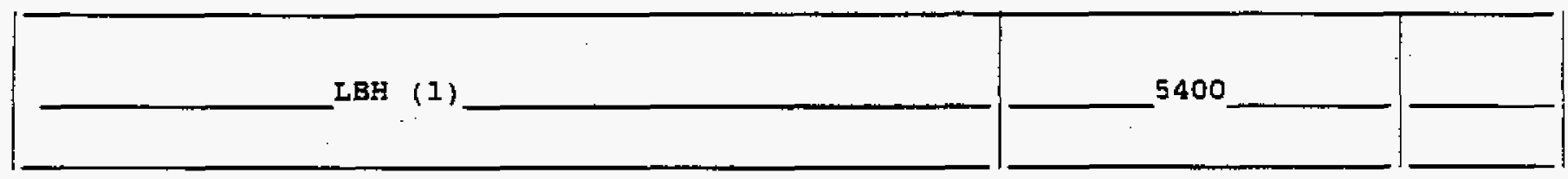

Contract: $\quad 625.01$

SDG No.: $\mathrm{V} 368$

Lab Sample ID: OCLCS167322

Lab File ID:

Date sampled:

Date Analyzed: 03-13-98

Dilution Factor: 1.0 
LBH SOIL SURROGATE RECOVERY

ab Name: Quanterra, Mo

ab code: ITHO Cage No.: contract: 625.01

SAS NO.:

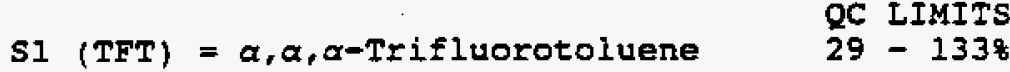

* Column to be used to flag recovery values

- Values outside of QC limits

D Surrogates diluted out

age 1 of 1

FORM II LBE 
30

LBH SPIKE BLANK (LCS) RECOVERY

Lab Name: Quanterra, MO

Lab Code: ITMO Case No.:

Spike Blank No.: LBHLCSO1
Contract: 625.01

SAS NO.:
SDG No. : $\mathrm{V} 368$

\begin{tabular}{|c|c|c|c|c|}
\hline $\begin{array}{l}\text { COMPOUND } \\
============= \pm=========0 \\
\text { Gasoline }\end{array}$ & $\begin{array}{l}\text { SPIKE } \\
\text { ADDED } \\
\text { (UG } / \mathrm{Kg}) \\
======= \\
5000\end{array}$ & $\begin{array}{c}\text { SPIKE } \\
\text { CONCENTRATION } \\
(\mathrm{UG} / \mathrm{Rg}) \\
=========== \\
5400\end{array}$ & \begin{tabular}{|c|} 
SPK \\
z \\
REC \\
$=====$ \\
108
\end{tabular} & $\begin{array}{c}\text { QC } \\
\text { LIMITS } \\
\text { REC. } \\
===== \\
76-118\end{array}$ \\
\hline & & & & \\
\hline
\end{tabular}

\# Column to be used to flag recovery values with an asterisk

* Valueg outside of QC limits

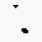

Spike Recovery: 0 out of 1 outside Iimits 
ab Name: Ouanterra, MO

ab code: ITMO Case No.:

ab Sample ID: OCBLK167322

atrix:(soil/water) sorL

ate Analyzed : 03-13-98

ime Analyzed : $\quad$ :B:20 contract: 625.01

SAS No.: SDG No : : V368

- Lab File ID:

Level ( low/med) LON

Instrument ID: GCF

cC column ID: $\mathrm{DB}=5$

THIS METHOD BLANK APPLIES TO THE FOLLONING SAMPLES, MS AND MSD:

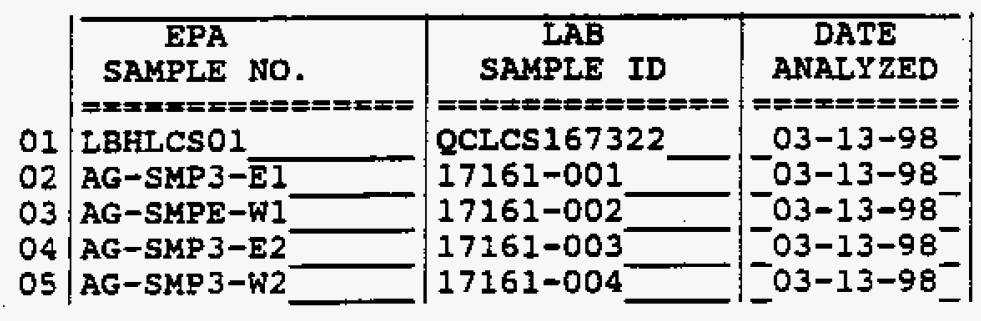

MMENTS :

Ige of 1 


\section{$6 F$ \\ LBH INITIAL CALIBRATION DATA}

Lab Name: Quanterra,

Lab Code: ITMO Cage No.:

Instrument ID: $\mathrm{GCH}$
Contract: 625.01

SAS No.: SDG No.: V368

Calibration Date (8): 03-12-98 to $03-12-98$

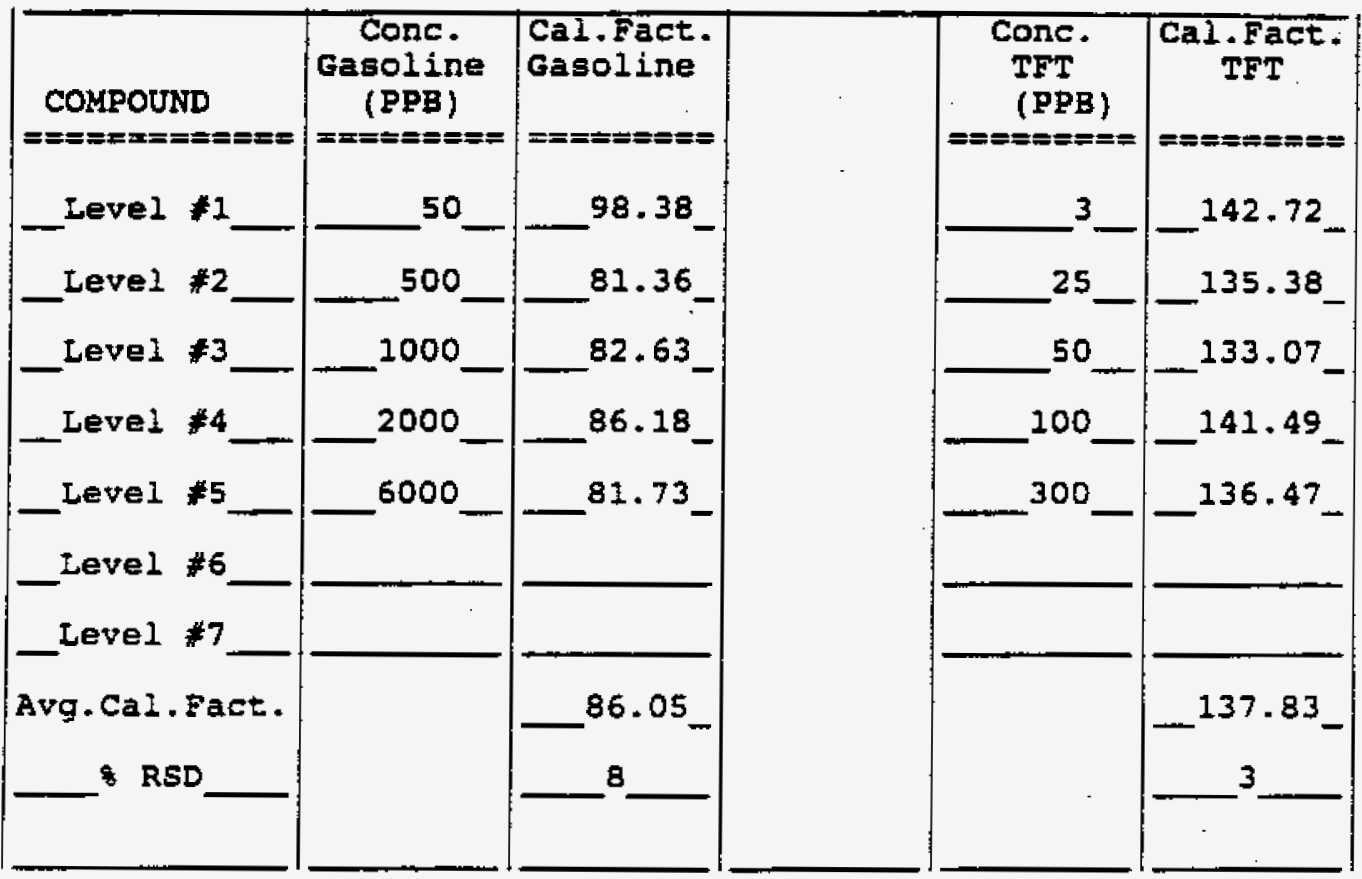

In AVERAGE CAIIBRATION FACTOR, enter the average of the all calibration factors.

The oRSD must be lesg than 208 for the average calibration factor to be used. If the 8RSD is $\geq 20 \%$, a point-to-point caibration must be uged. In this case, enter "Pt-to-Pt" in AVERAGE CALIBRATION FACTOR instead of the average calibration factor. 


\section{E \\ LBH EVALUATION STANDARDS SUMMARY}

ab Name: ouanterra.MO

ab code: ITMO Cage No.:

ingtrument ID: $\mathrm{GCH}$

tates of Analyses: $03=13-98$
Contract: 625.01

Sins No : SDG No.: V368

GC Column ID:

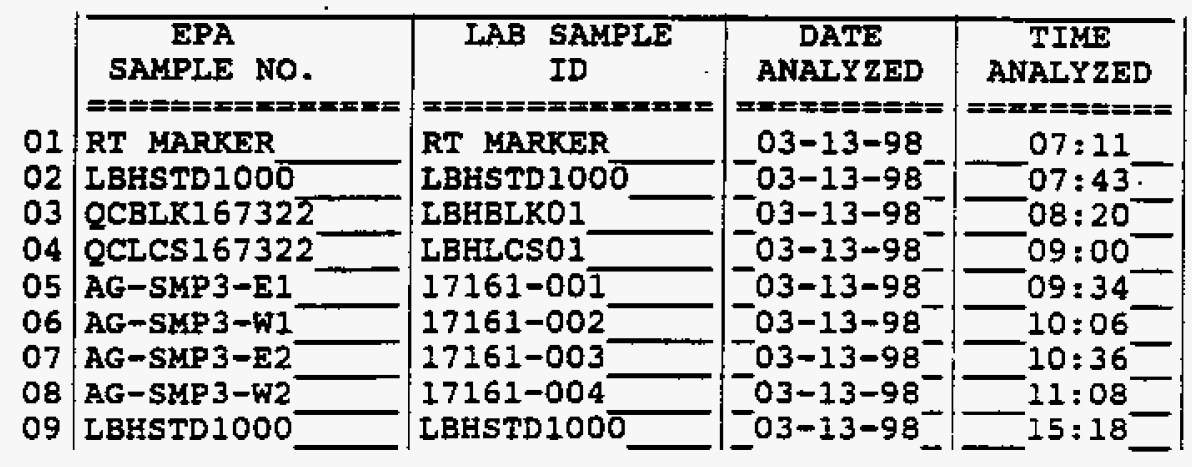

ige of 1 
9

LBH STANDARDS SUMMARY

Lab Name: Ouanterra, MO

Lab Code: Imso case No.:

Ingtrument ID: GCH
Contract: 625.01

SAS No. : SDG No.: V36B

GC Column ID: $\mathrm{DB}-5$

\begin{tabular}{|c|c|c|c|c|c|c|c|c|}
\hline & \multicolumn{4}{|c|}{$\begin{array}{ll}\text { DATE (S) OF FROK: } \frac{03-12-98}{0.12-98} \\
\text { ANALYSIS } & \text { TO: } \frac{03-12-98}{07: 42} \\
\text { TIME (S) OF FROK: } & 07: 52 \\
\text { ANALYSIS } & \text { TO: } 10: 53\end{array}$} & \multicolumn{2}{|c|}{$\begin{array}{l}\text { DATE OF ANALYSIS } \\
\text { TIME OF ANALYSIS } \\
\text { EPA SAMPLE NO. } \\
\text { (STANDARD) }\end{array}$} & \multicolumn{2}{|c|}{$\frac{03-13-98}{07: 43}$} \\
\hline \multirow{2}{*}{$\begin{array}{l}\text { COMPOUND } \\
\text { Gasoline } \\
\text { TFT }\end{array}$} & RT & $\begin{array}{l}\text { FROM } \\
\text { FII } \\
2.11\end{array}$ & $\begin{array}{l}\text { DOW } \\
\text { TO } \\
=== \\
11.98\end{array}$ & $\begin{array}{c}\text { CALIBRATION } \\
\text { FACTOR } \\
=86.05\end{array}$ & RT & $\begin{array}{c}\text { CAL IBRATION } \\
\text { FACTOA } \\
\text { =- } \\
90.72\end{array}$ & $\begin{array}{c}\mathrm{QNT} \\
\mathrm{Y} / \mathrm{N} \\
== \\
Y\end{array}$ & $\begin{array}{c}\frac{6 D}{8 D} \\
5 \\
5==-=\end{array}$ \\
\hline & 3.04 & 2.90 & 3.18 & 137.83 & 3.04 & & $Y$ & \\
\hline
\end{tabular}

Under QNT $Y / N$ : enter $Y$ if quantitation was performed, $N$ if not performed. 8D must be less than or equal to 15 for quantitation.

Note: Determining that no compounds were found above the CRDL is a form of quantitation, and therefore at least one column must meet the 15 criteria.

page 11 of 2 
Lab Name: Ouanterra, MO

tab code: ITHO Case No.: Instrument ID: $\mathrm{GCH}$
Contract: 625.01

SAS No.: SDE NO.: V368

GC Column ID:

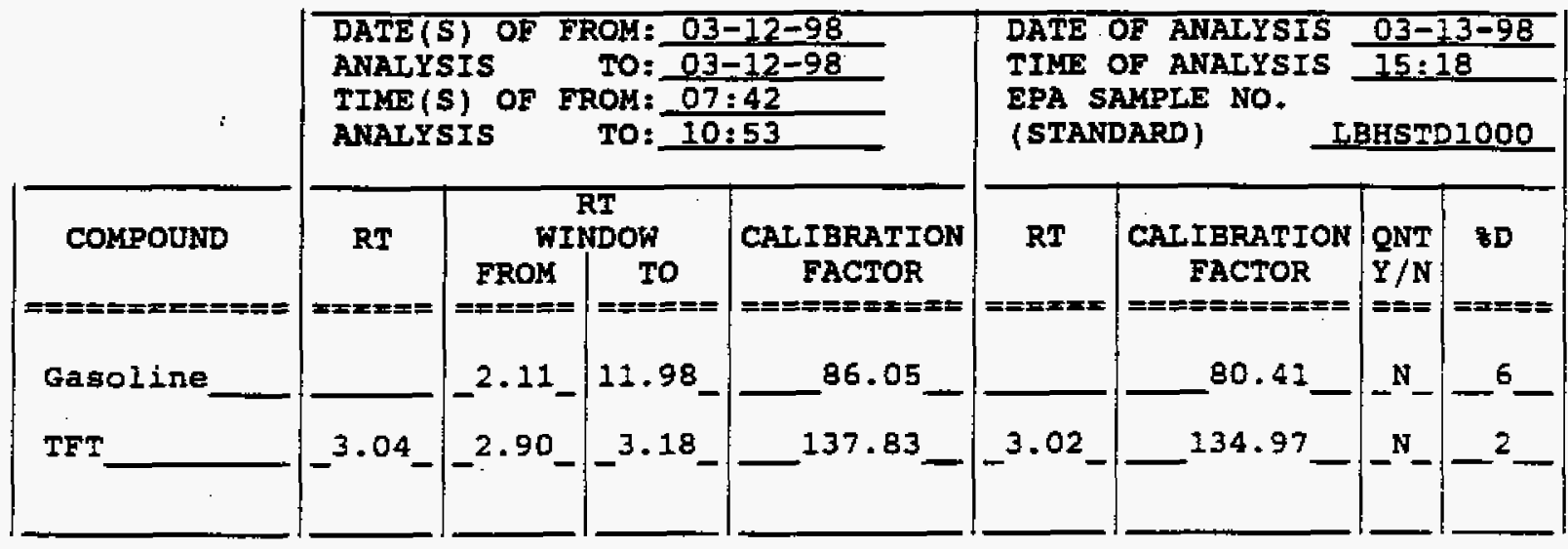

Under QNT $Y / N$ : enter $Y$ if quantitation was performed, $N$ if not performed.

1 mugt be less than or equal to 15 for quantitation.

Note: Determining that no compounds were found above the CRDL is a form of quantitation, and therefore at least one column mugt meet the 158 criteria.

je 1 of 2

FORM IX LBH 


\section{Bechtel Ievada}

PROJECTLCLIENT INFORMATION

AG DECONJ POND

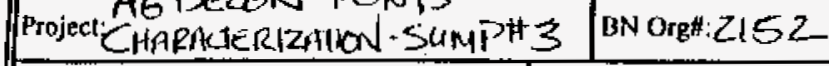
\begin{tabular}{|l|l|l|}
\hline Charge Number: C4C SAODE & ASL Prog: \\
\hline
\end{tabular} Project Manager: JEFF SHT1T-1

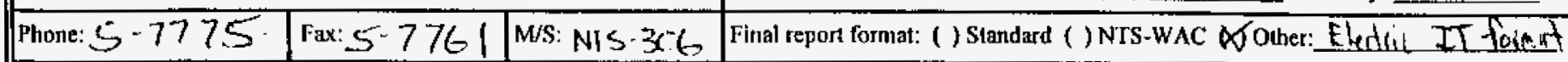

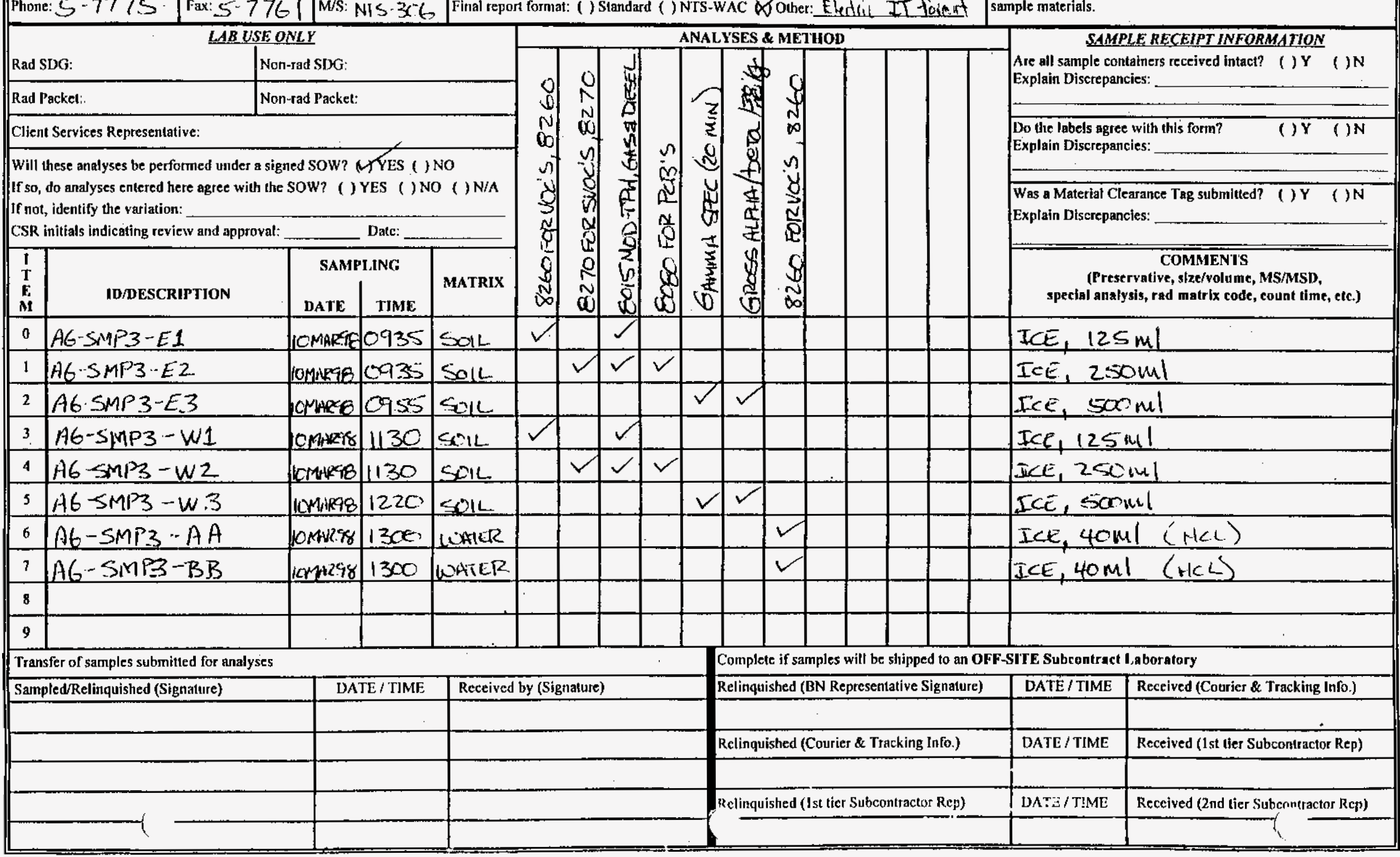

ANALYTICAL SER VICES LABORATORY SER VICES REQUEST FORM AND CHAIN OF CUSTODY REPORTINFORMATION

Send Report to: FEF SMMTH

\begin{tabular}{l|l|l|} 
Phone: $5-7775$ & Fax: $5-7761$ M/S:NI 206 \\
\hline
\end{tabular}
S- 1776 M/Sists Turnaround: () Standard [30 days Non-rad, 60 days (or negotiated) Rad]

6
Sampling Site: $\mathrm{G}$ DECON FACILAT The samples submitted contain (check);

C) Hazardous () Radloactive (1) Unknown contamination. If known, attach a brief narrative summary identifying conlaminants. This information will ensure compliance with applicable regulations and allow for the safc hardling of the sample materials.

Are all sample containers received intact? ( ) $Y$ ()N

Do the labels ggree with this form? () Y ( ) N

Explain Discrepancies:

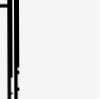


SAMPLE DELIVERY GROUP: V368 ABORATORY: QUANTERRA

FINAL REPORT RECEIVED: $4 / 7 / 98$

DATE REVIEWED: $5 / 5 / 98$

TPH - DIESEL / OIL:

MATRIX: SOIL
CLIENT: RP - JEFF SMITH

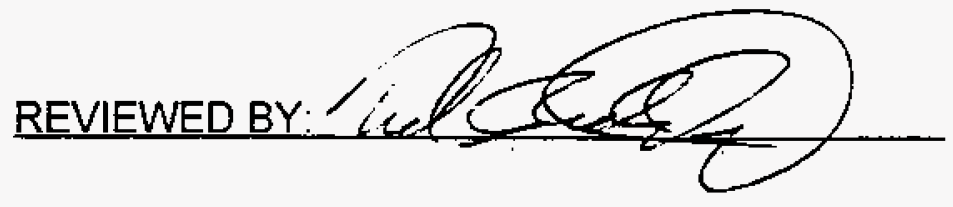

ARE TPH DIESEL/OIL DATA ACCEPTABLE: YES

ARE CRITERIA MET

1. QC SAMPLES ACCEPTABLE

2. MATRIX SPIKE ACCEPTABLE

3. METHOD BLANK ACCEPTABLE

4. HOLDING TIMES ACCEPTABLE

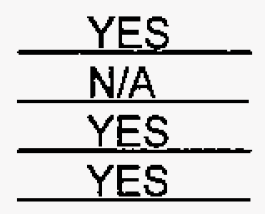

\# DAYS ACCEPTABLE

a. Date Sampled:

$\underline{3 / 10 / 98}$

0

0

b. Date ASL Received:

$\underline{3 / 10 / 98}$

$\underline{0}$

1

i. Date Contract Lab Received:

$\underline{3 / 12 / 98}$

$\underline{2}$

5

d. Date Extracted:

$\underline{3 / 17 / 98}$

7

14

e. Date Analyzed:

3/17/98

0

40

5. CALCULATIONS ACCEPTABLE:

6. CORRECT CONC. UNITS USED:

7. FORMS ACCEPTABLE:

8. CHAIN-OF-CUSTODY AGREES:

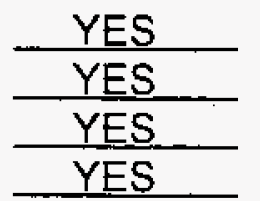

COMMENTS: Method associated quality assurance and control criteria were met. 
Lab Name:

OUANTERRA MO

Contract:

625.01

Lab Code: ITMO Case No.:

SAS NO.:

SDG No.: $\quad$ V368

Matrix : (soil/water)

SOIL

Sample wt/vol:

20.1 $(g / m 1)$

$G$

Level: (low/med $)$ LOW

o Moisture: not dec. dec .

Date Extracted:

$03-17-98$

Extraction:(SepF/Cont/Sonc/Shak) Sonc

GPC Cleanup: (Y/N)

Date Anaiyzed:

$03-17-98$

Dilution Facto:

CONCENTRATION UNITS:

$(\mathrm{mg} / \mathrm{L}$ or $\mathrm{mg} / \mathrm{Kg}) \mathrm{mg} / \mathrm{Kg}$

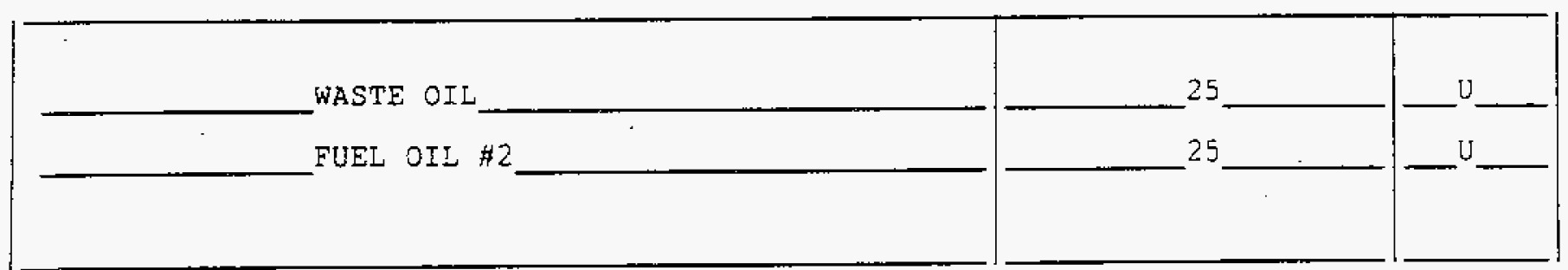

$U$ : Concentzation of anaiyte is less than the value given. 
Lab Name: QUANTERRA MO

Lab Code: ITMO Case No.:

Matrix : (soil/water)

SOIL

Sample wt/vol: 20.2 $(g / m)$

G Level: (low/med) LOW

Foisture: not dec. dec.

Extraction: (SepF/Cont/Sonc/Shak) Sonc GPC Cleanup: $(\mathrm{Y} / \mathrm{N}) \stackrel{\mathrm{N}}{\mathrm{pH}}$ CAS NO. Compound

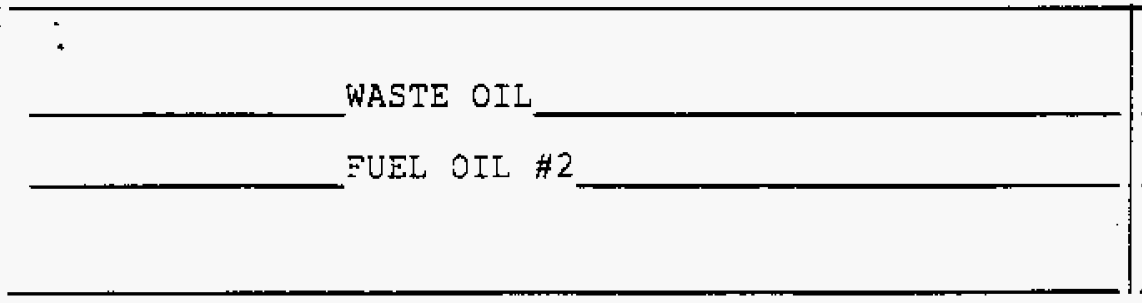

$\mathrm{U}$ : Concentration of analyte is less than the value given.
Contract:

625.01

SAS NO.: SDG No.:

Lab Sample ID: $\quad 17161-003$ Lab File ID:

Date Sampled:

$03-10-98$

Date Extracted:

$03-17-98$

Date Analyzed:

$03-17-98$

Dilution Factor:

1 CONCENTRATION UNITS: $(\mathrm{mg} / \mathrm{L}$ or $\mathrm{mg} / \mathrm{kg}) \mathrm{mg} / \mathrm{Kg}$

$\square^{25}-$
U

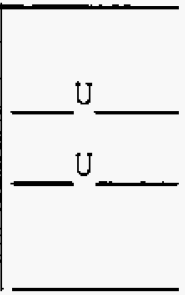

FORM I HBH 
Lab Name: OUANTERRA MO

Lab code: ITMO Case No.:

Matrix : (soil/water)

SOIL

Sample wt/vol:

20.

$(g / m 1)$

G

Levei: (low/med)

LOW

요의 Moisture: not dec. dec.

Extzaction: (SepF/Cont/Sonc/Shak)

Sonc

GPC Cleanup: $(Y / N)-\mathrm{N}$

$\mathrm{pH}:$
Contract:

625.01

SAS NO.:

SDG No.:

Lab Sample ID:

$1716 \div-002$

Lab File ID:

Date Sampled:

$03-10-98$

Date Extracted:

$03-17-98$

Date Analyzed:

$03-17-98$

Dilution Factor:

CONCENTRATION UNITS:

$(\mathrm{mg} / \mathrm{L}$ or $\mathrm{mg} / \mathrm{kg}) \mathrm{mg} / \mathrm{Kq}$
CAS NO.

Compound

-

WASTE OI:

EUEL OI: \#2

$\because$ Concentration of analyte is less than the value given.

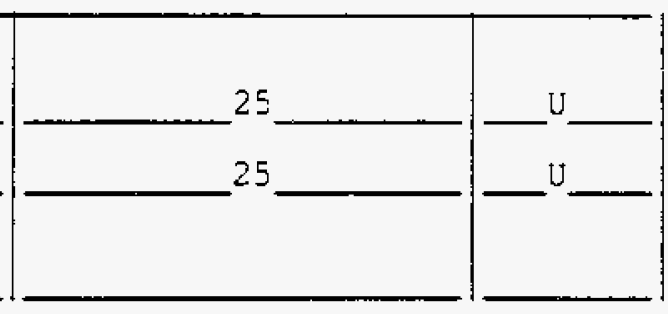


$1 \mathrm{D}$

HBH ANALYSIS DATA SHEET
EPA SAMPLE NO A6-SMP 3-W2
Lab Name: $\quad$ QUANTERRA MO

Lab Code: ITMO Case No.:

Matrix: (soil/water)

SOTL

Sample wt/vol:

20 $(g / m 1)$

$\mathrm{G}$

Leve i: $\quad$ (low/med)

LOW

\% Moisture: not dec. dec.

Extraction: (SepF/Cont/Sonc/Shak) Sonc

GPC CIEanup: (Y/N)

SAS NO.:

Contract:

625.01

SDG No.: $\quad$ V368

Lab Sample ID:

$1716 i-004$

Lab File ID:

Date Sampied: $\quad$ 03-10-98

Date Extractec:

Date Analyzed: $\quad$ 03-17-98

Dizution Factor:

CONCENTRATION UNITS:

$(\mathrm{mg} / \mathrm{L}$ or $\mathrm{mg} / \mathrm{Kg}) \mathrm{mg} / \mathrm{Kg}$

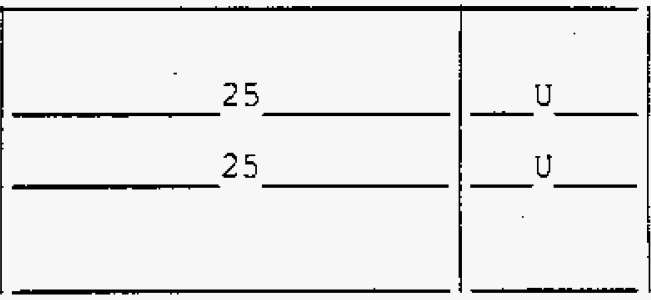

U: Concentration of analyte is less than the value given. 
Lab Name: $\quad$ QUANTERRA MO

Lab Code: ITMO Case No.:

Matrix : (soil/water)

SOIL

Sample wt/vol:

20.0
$(g / \mathrm{mI})$

G

Level: (low/med) LOW

\% Mo:sture: not dec. dec.

Extraction: (SepF/Cont/Sonc/Shak) Sonc GPC Cleanup: $(\mathrm{Y} / \mathrm{N})+\mathrm{N}$ Contract: SAS NO.: SDG No.: $\quad$ V368 Lab Sample ID: BLK 167396 Lab Fiie ID: Date Sampled: Date Extracted: $03-+7-98$ Date Analyzed: $03-17-98$ Dilution Factor: 1 CONCENTRATION UNITS: ( $\mathrm{mg} / \mathrm{L}$ or $\mathrm{mg} / \mathrm{kg}$ ) $\mathrm{mg} / \mathrm{Kg}$
Compound WASTE OIL FUEL OIL \#2

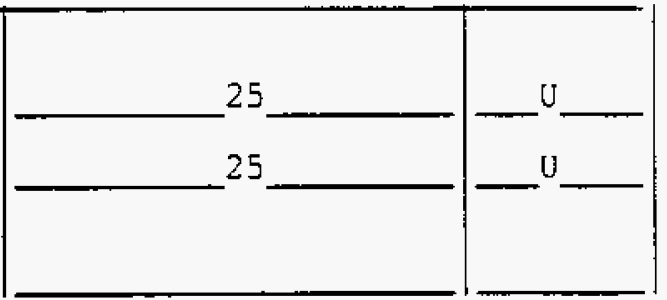

$\mathrm{U}$ : Concentration of analyte is less than the value given. 
Lab Code: ITMO Case No.:

Matrix : (soil/water)

SOIL

Sample wt/vol: $20.0(\mathrm{~g} / \mathrm{ml})$ G Level: (low/med) LOW

\% Morsture: not dec. dec. Extraction: (SepF/Cont/Sonc/Shak) Sonc GFC Cleanup: $(Y / N)$

pH: SAS NO.: SDG No.: Lab Sample ID: SPK $167396-1$ Lab File ID: Date Sampled:

Date Extracted: $03-17-98$

Date Anaiyzed: $03-17-98$

Dilution Factor: 1 CONCENTRAT:ON UNITS: $(\mathrm{mg} / \mathrm{L}$ or $\mathrm{mg} / \mathrm{kg}) \mathrm{mc} / \mathrm{kg}$
CAS No.

Compound

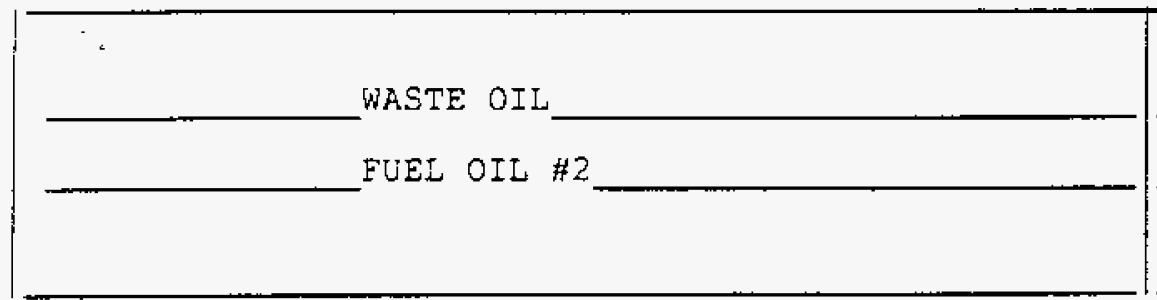

iJ: Concentration of analyte is iess than the value given.

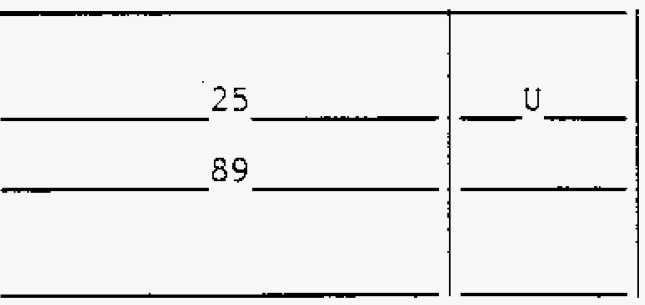


$1 \mathrm{D}$

HBH ANALYSIS DATA SHEET

EPA SAMPLE NO

TPHSPKOIB

Lab Name: QUANTERRA MO

Contract:

625.01

Lab code: ITMO Case No.:

SAS No.:

SDG No.: $-\frac{1}{6} 368$

Matrix : (soil/water)

SOIL

Lab Sample ID:

SPK 167396-2

Sample wt/vol:

$20.0 \quad(g / m 1)$

G

Lab File ID:

Level: (low/med)

LOW

Date Sampled:

․․․ Noisture: not dec. dec.

Date Extracted:

$03-\div 7-98$

Extraction:(SepF/Cont/Sonc/Shak) Sonc

Date Anaîyzed:

$03-17-98$

GPC Cleanup: (Y/N) $\mathrm{N} \quad \mathrm{pH}:$

Dilution Factor:

1

CONCENTRATION UNITS:

CAS NO.

Compound

$(\mathrm{mg} / \mathrm{L}$ or $\mathrm{mg} / \mathrm{kg})$

$\mathrm{mg} / \mathrm{Kg}$

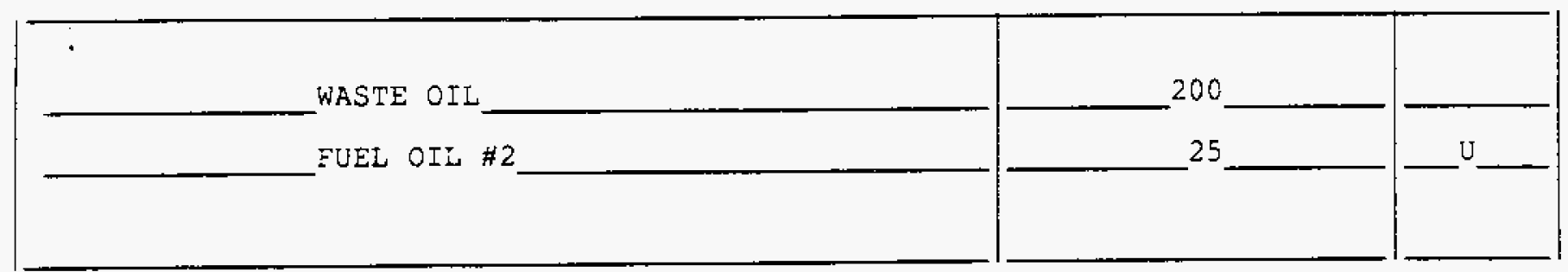

$u$ : Concentration of anaiyte is less than the value given.

FORM I HBH

000038 


\section{SOIL HBH SURROGATE RECOVERY}

Lab Name: QUANTERRA MO

Contract: 625.01

Lab Code: ITMO Case No.: SAS No.: SDG No : : V368

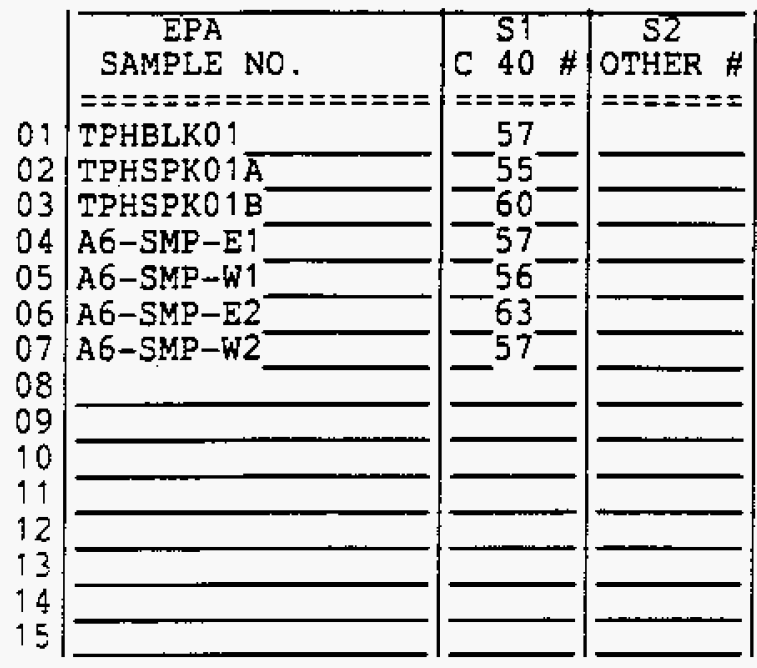

ADVISORY

S1 $($ C 40$)=$ N-Tetracontane

OC LIMITS

\# Colum to be used to flag recovery values

* Values outside of QC limits due to matrix interference

D Surrogates diluted out

page $i$ of 1

FORM II HBH 
Lab Code: ITMO Case No.:

SAS Nó. : SDG No. : V368

Spike Blank No.: SPK $167396-1$

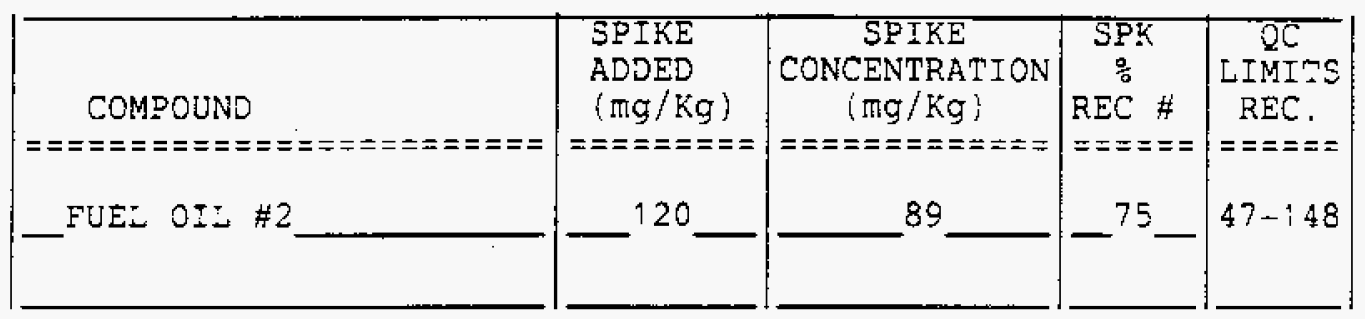

\# Colum to be used to flag recovery values with an asterisk

* Values outsiae of QC limits

Spike Recovery: $\ldots$ _ out of $\ldots 1$ outside limits

COMMENTS:

FORM III HBH-3 
30

SOIL HEH SPIKE BLANK (LCS) RECOVERY

Lab Name: OUANTERRA MO

Contract:

625.01

Lab Code: ITMO Case No.:

SÁS Nó. : SDG NO :

$\mathrm{v} 368$

Spike Blank No.:

SPK $\quad 167396-2$

\begin{tabular}{|c|c|c|c|c|}
\hline $\begin{array}{l}\text { COMPOUND } \\
========== \pm=========1 \\
\text { WASTE OIL }\end{array}$ & 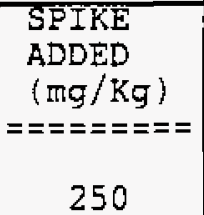 & $\begin{array}{c}\text { SPIKE } \\
\begin{array}{c}\text { CONCENTRATION } \\
(\mathrm{mg} / \mathrm{Kg})\end{array} \\
========== \\
200\end{array}$ & $\begin{array}{c}S P \vec{K} \\
\frac{9}{5} \\
\operatorname{REC} \\
===== \\
77\end{array}$ & $\begin{array}{c}\text { QC } \\
\text { LIMITS } \\
\text { REC. } \\
==== \\
47-148\end{array}$ \\
\hline & & & & \\
\hline
\end{tabular}

\# Column to be used to Eiag recovery values with an asterisk

* Vailies outside of oc Iimits

Sprke Recovery: 0 out of $\frac{1}{0}$ outside limits

COMMENTS :

FORM IIT HBH-3 
$4 \mathrm{C}$

HBH METHOD BLANK SUMMARY

Lab Name: OUANTERRA MO

Lab code: ITMO Case No. :

Lab Sample ID:

BLK 167396

Matrix:(soil/water) SOIL

Date Extracted : $03-17-98$

Date Analyzed $03-17-98$

Time Analyzed :
Contract: 625.01 SA'S No.: SDG No.: V368

Lab File ID:

- Level (low/med) LOW

Extraction: (SepF/Cont/Sonc) Sonc

Instrument ID : GC $F$

G C Column ID : $\mathrm{DB}-5 \mathrm{MS}$

THIS METHOD BLANK APPLIES TO THE FOLLOWING SAMPLES, MS AND MSD:

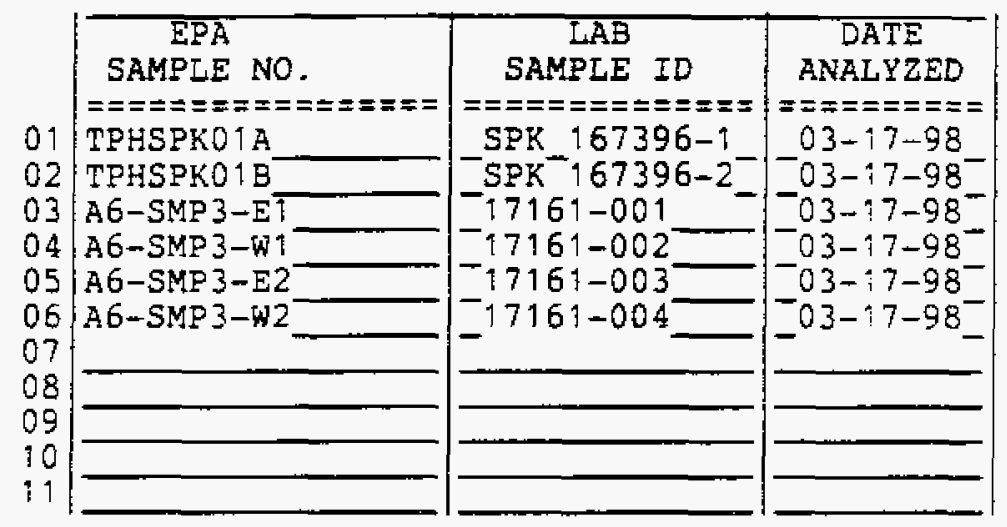

COMMENTS :

page 1 of 1 


\section{HBH INITIAL CALIBRATION DATA}

Lab Name: OUANTERRA MO

Lab code: ITMO Case No.:

Instrument ID: GC F GC Column ID: _ DB-5MS Calibration Date $(5):-09-27-97$ to $109-28-97$ Calibration Date $(5):-09-27-97$ to $109-28-97$
Contract: 625.01

SAS NO.: SDG NO.: v368

Injection Vol: i. Oul

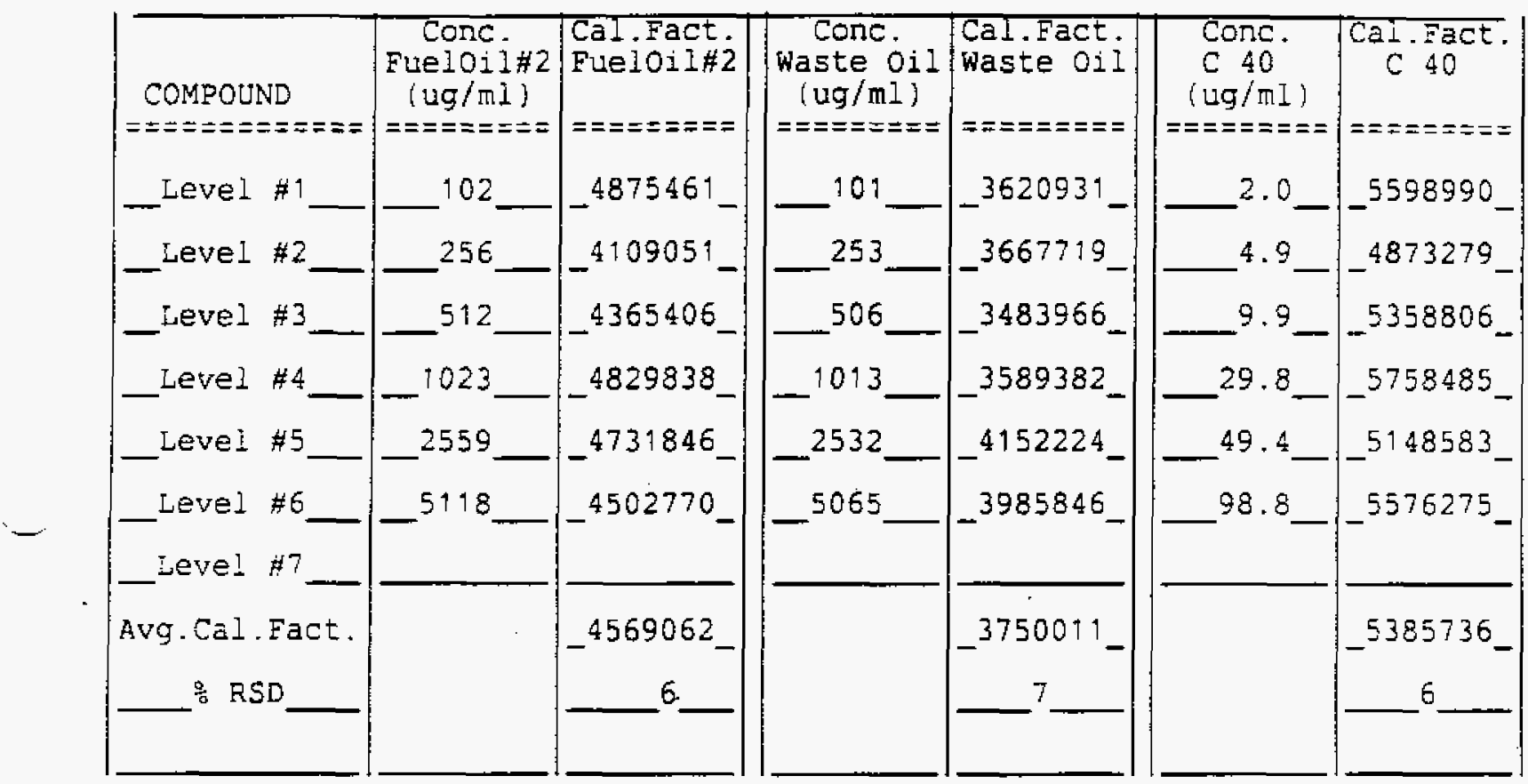

In AVERAGE CALIBRATION FACTOR, enter the average of the all caibration factors.

The oRSD must be less than $20 \%$ for the average calibration factor to be used. If the Rㅗ is $\geq 20 \%$, a calibration curve must be used. The R-squared value must be $\geq 0.990$ for the curve to be used. In this case, enter "Curve" in AVERAGE CALIBRATION FACTOR instead of the average calibration factor and the R-squared value instead of the 
$8 E$

HBH SEQUENCE SUMMARY

Lab Name: QUANTERRA MO

Lab Code: ITMO Case No.:

Instrument ID: GC $F$
Contract :

SDG No.:

GC Colum ID:
625.01

SAS NO. $03-17-98$ to $03-17-98$

Dates of Analyses

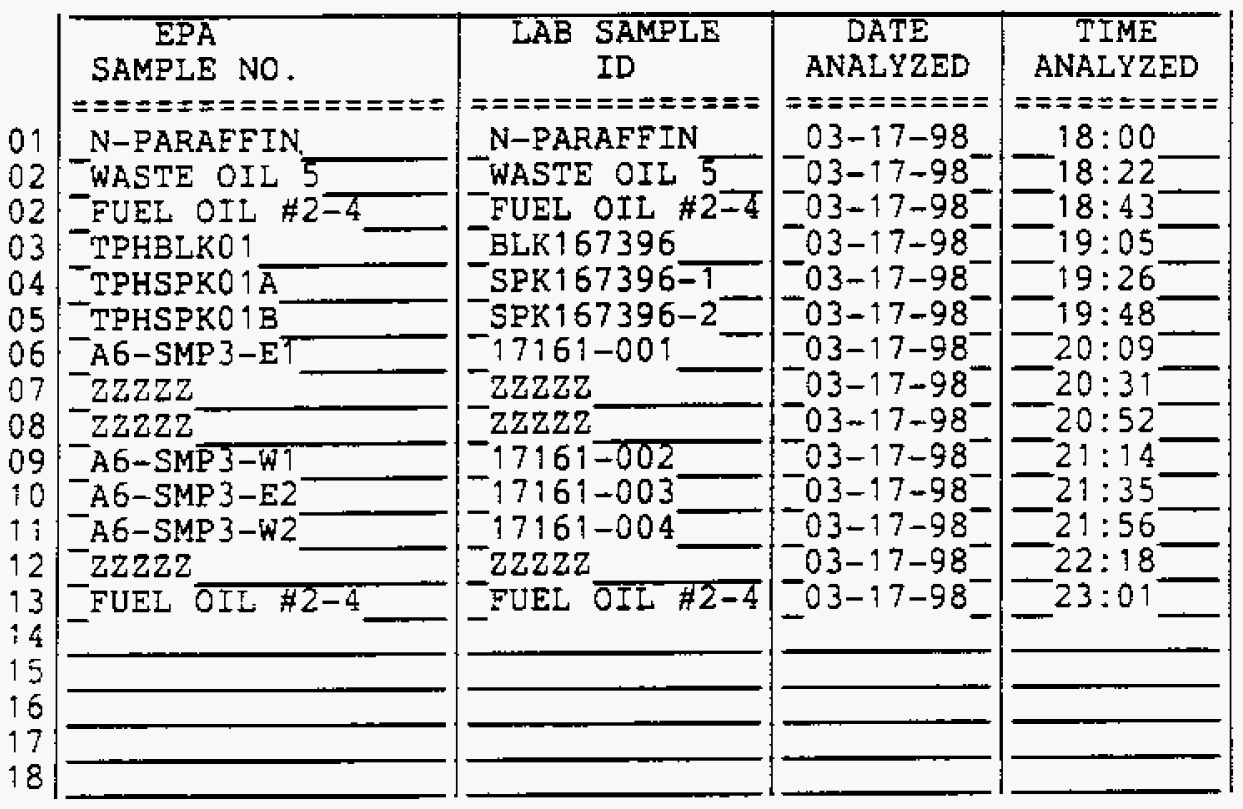

page $\underline{i}$ of 1 
9

HBH STANDARDS SUMMARY

$-$

Lab Name: QUANTERRA MO

Contract:

625.01

Lab Code: ITMO Case No.:

SAS NO.:

SDG NO.:

$\mathrm{V} 368$

Instrument ID:

GC F

GC Column ID:

$\mathrm{DB}-5 \mathrm{MS}$

\begin{tabular}{|c|c|c|c|c|c|c|c|c|}
\hline \multirow[b]{2}{*}{$\begin{array}{c}\text { COMPOUND } \\
==\text { = = = = = = = }\end{array}$} & \multicolumn{4}{|c|}{$\begin{array}{lr}\text { DATE (S) OF } & \text { FROM: } \\
\text { ANALYSIS } & \text { TO }: \frac{09-27-97}{09-28-97} \\
\text { TIME (S) OF } & \text { FROM: } \\
\text { ANALYSIS } & \text { TO: }\end{array}$} & \multicolumn{4}{|c|}{ 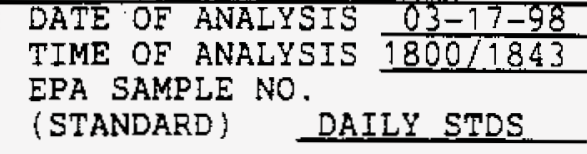 } \\
\hline & $\begin{array}{c}\text { RT } \\
== \pm===\end{array}$ & $\begin{array}{l}\text { WI } \\
\text { FROM } \\
==ニ==\end{array}$ & $\begin{array}{l}\mathrm{TOW} \\
\mathrm{TO} \\
==\end{array}$ & $\begin{array}{l}\text { CALIBRATION } \\
\text { FACTOR } \\
===ニ====+=\end{array}$ & $\begin{array}{c}\mathrm{RT} \\
======\end{array}$ & $\begin{array}{c}\text { CALIBRATION } \\
\text { EACTOR } \\
========\end{array}$ & $\begin{array}{l}\mathrm{QNT} \\
\mathrm{Y} / \mathrm{N} \\
===\end{array}$ & $\begin{array}{l}\frac{}{6} D \\
===\end{array}$ \\
\hline WASTE OIL & & -0.93 & -9.13 & -3750011 & -5.03 & 4096830 & $\mathrm{Y}_{-}$ & 9 \\
\hline FUEL OIL $\# 2$ & & -0.93 & -9.13 & -4569062 & -5.03 & ${ }_{-3826272}$ & $Y_{-}$ & $-^{2}$ \\
\hline
\end{tabular}

Under $O N T Y / N$ : enter $Y$ if quantitation was performed, $N$ if not performed.

\&D must be less than or equal to $15 \%$ for quantitation.

page 1 of 2

FORM IX HBH 
Lab Name: OUANTERRA MO

Contract:

625.01

Lab Code: ITMO Case No.:

SAS No. :

SDG No.:

V368

Instrument ID: $\quad$ GC F

GC Column ID:

$\mathrm{DB}-5 \mathrm{MS}$

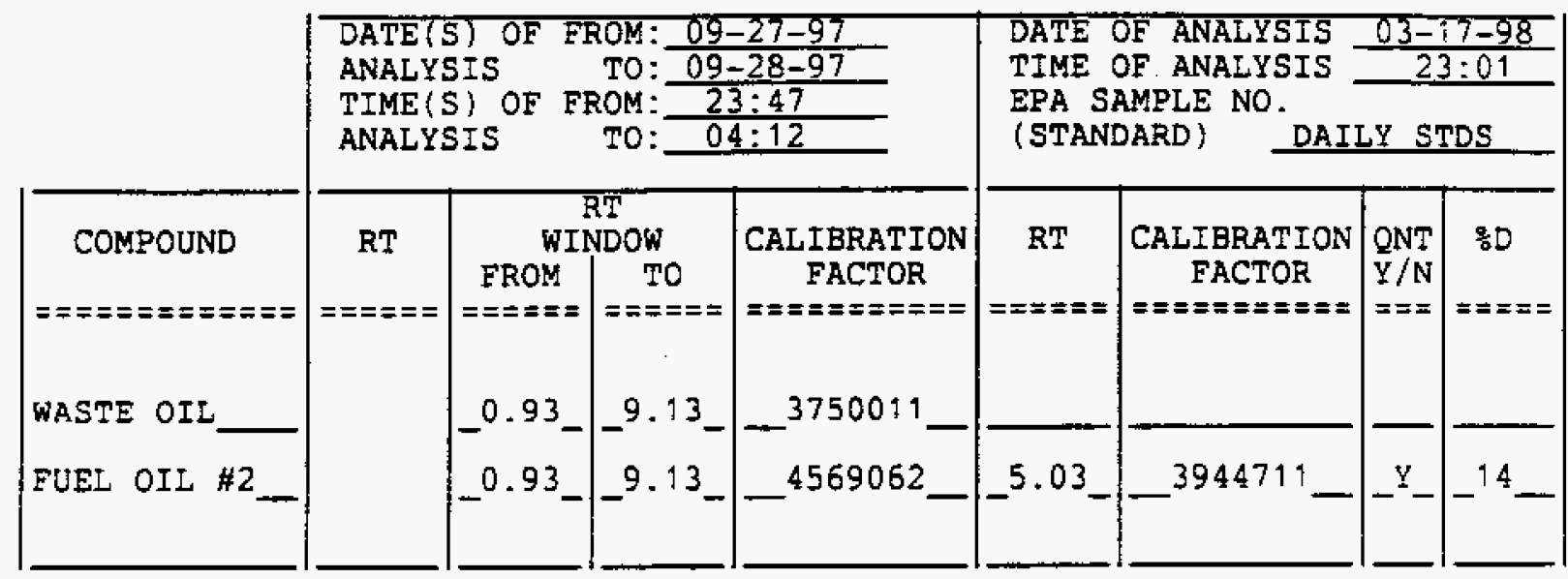

Under QNT $\mathrm{Y} / \mathrm{N}$ : enter $\mathrm{Y}$ if quantitation was performed, $\mathrm{N}$ if not performed. $\because D$ must be less than or equal to $15 \%$ for quantitation.

page $\underline{2}$ of 2

- FORM IX HBH 
ab Name : QUANTERRA MO

ab code: ITMO

\section{lacrix: (soil/water) SoIL}

ample wt/vol:

\section{evel: (low/med) Low}

Moisture: decanted: $(\mathrm{Y} / \mathrm{N}) \mathrm{N}$
Contract: $525-01$

SAS NO.:
$A 6+\operatorname{SMP} 3-E 2$ oncentrated Extract volume: 1000 (uL) njection volume:

FC Cleanup: $\quad(Y / N) N$

CAS NO.
2.0 (UL)

pH:

$30.00(\mathrm{~g} / \mathrm{mL}) \mathrm{G}$

COMDOUND
Lab Sample ID: 17161-003

Lab File ID: $\quad H 6312$

Date Received: $03 / 12 / 98$

Date Extracted: $03 / 23 / 98$

Date Anaiyzed: $03 / 31 / 98$

Dilution Factor: 1.0

CONCENTRATEON UNITS:

(ug/L or $\mathrm{ug} / \mathrm{Kg}$ ) UG/KG

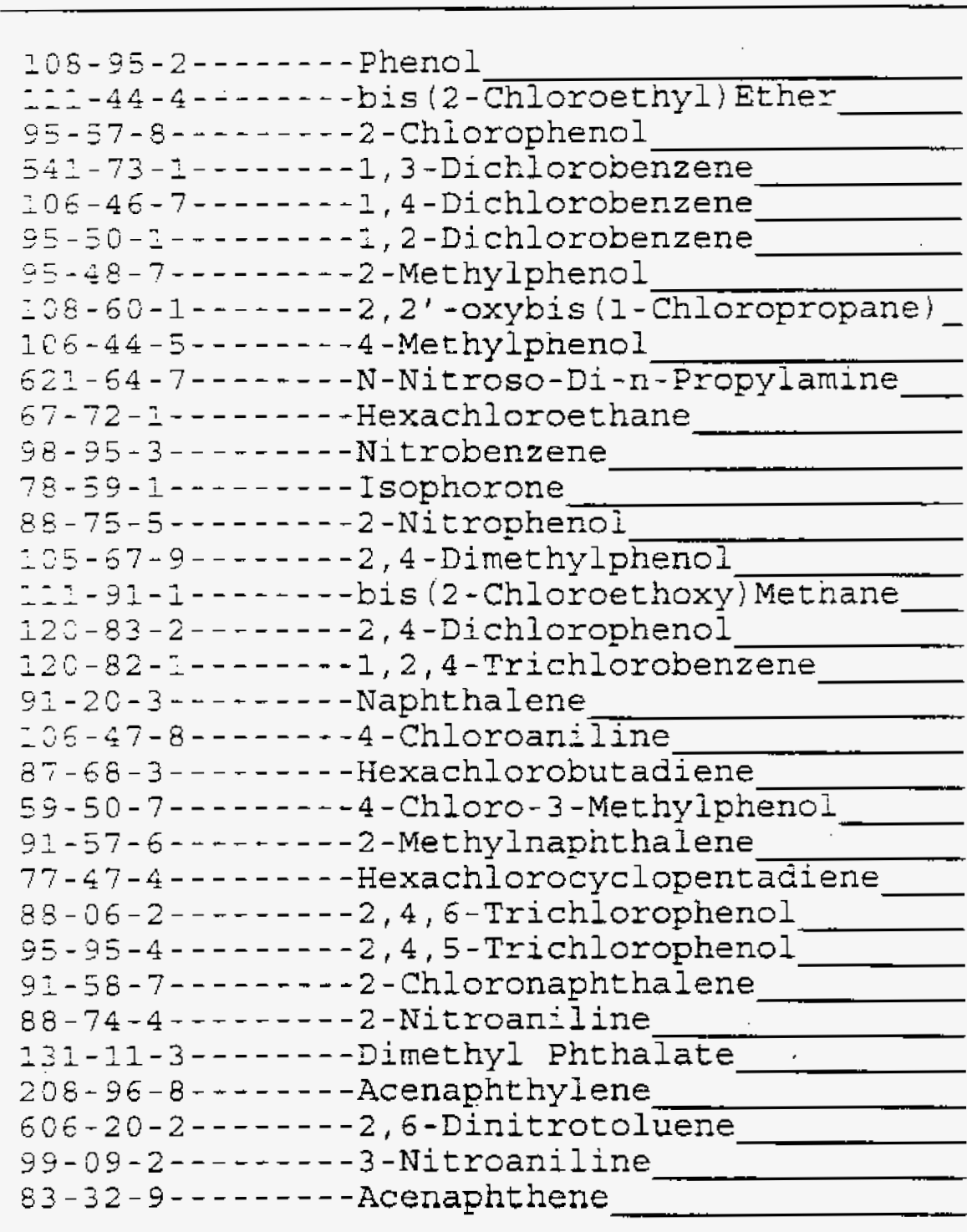


Lab Name: QUANTERRA MO

Lab Code: ITMO

Matrix: (soil/water) soIL

Sample wt/vol:

Level:

(low/med)

음 Moisture:

decanted: $(Y / N) N$

Concentrated Extract Volume: 1000 (uL)

Injection volume:

2.0 (uL)

GPC Cleanup: $\quad(Y / N) N$

pF:
Contract: $625-01$

SAS NO.:

SDG No.: $\mathrm{V} 368$

Lab Sample ID: 17161-003

Lab File ID: H6312

Date Received: 03/12/98

Date Extracted: $03 / 23 / 98$

Date Analyzed: $03 / 31 / 98$

Dilution Factor:

1.0

CAS NO.

COMPOUND

CONCENTRATION UNITS:

(ug/L or $\mathrm{ug} / \mathrm{Kg}$ ) UG $/ \mathrm{KG}$

\begin{tabular}{|c|}
\hline 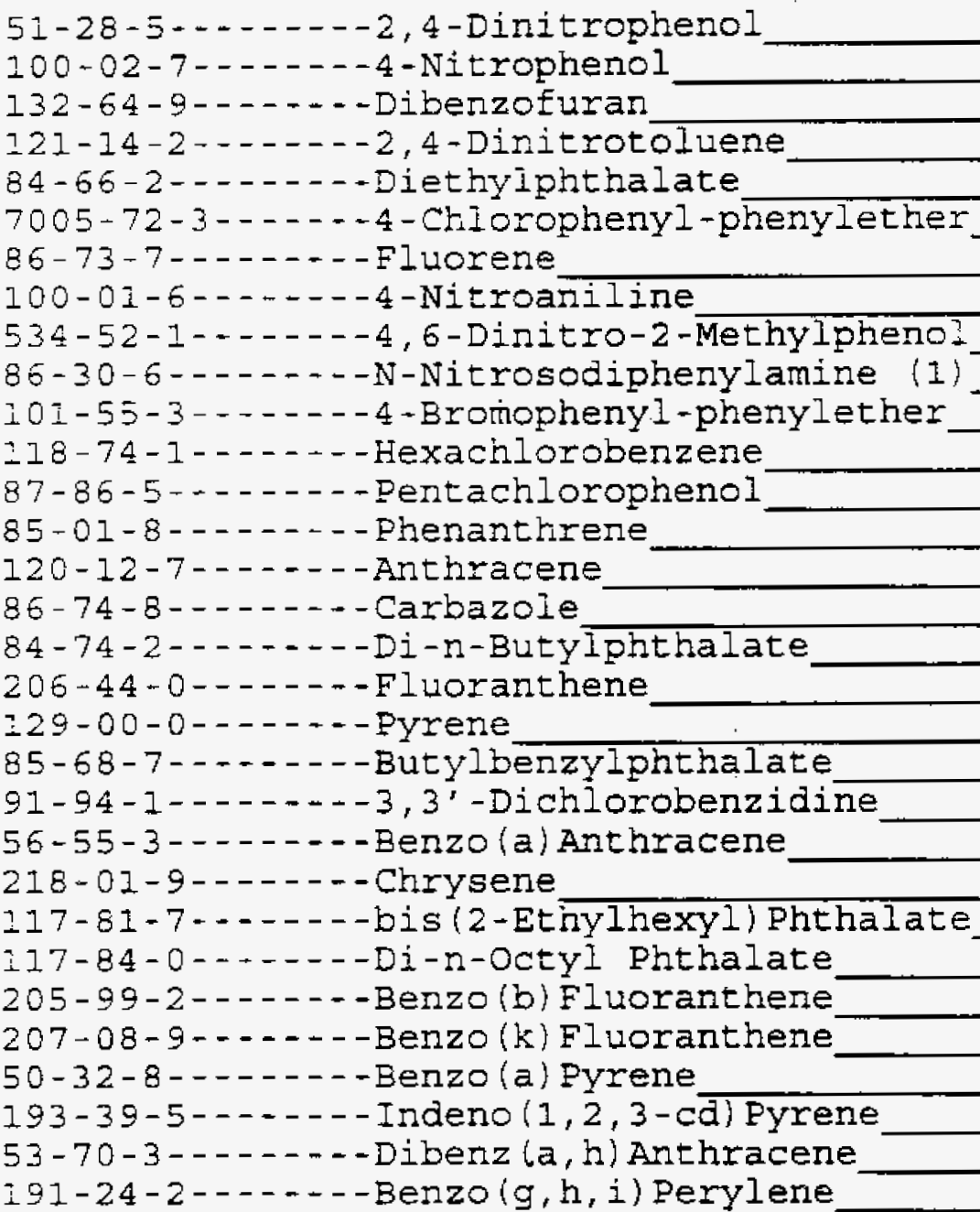 \\
\hline
\end{tabular}

(1) - Cannot be separated from Diphenylamine 
aD Name: QUANTERRA MO

ab coce: ITMO

atrix: (soil/water) SoIL

ample wt/vol:

evel: (low $/$ med) Low

Moisture:

decanted: $(\mathrm{Y} / \mathrm{N}\rangle \mathrm{N}$

Contract: $625-01$

SAS NO.:

Lab Sample ID: 17161-004

Lab File ID:

H6313

Date Received: $03 / 12 / 98$

Date Extracted: $03 / 23 / 98$

Dace Analyzed: $03 / 31 / 98$

Dilution Factor:

1.0

CONCENTRATION UNITS:

(ug/I or $\mathrm{ug} / \mathrm{Kg}$ ) UG/KG

GAS NO.

COMPOUND

PC Gleanup: (Y/N) N $\mathrm{NH}$ :
2. O (UL)
PC Eleanup: $(\mathrm{Y} / \mathrm{N}) \mathrm{N} \quad \mathrm{pF}$ :

$A 6-\operatorname{SMP} 3-W 2$

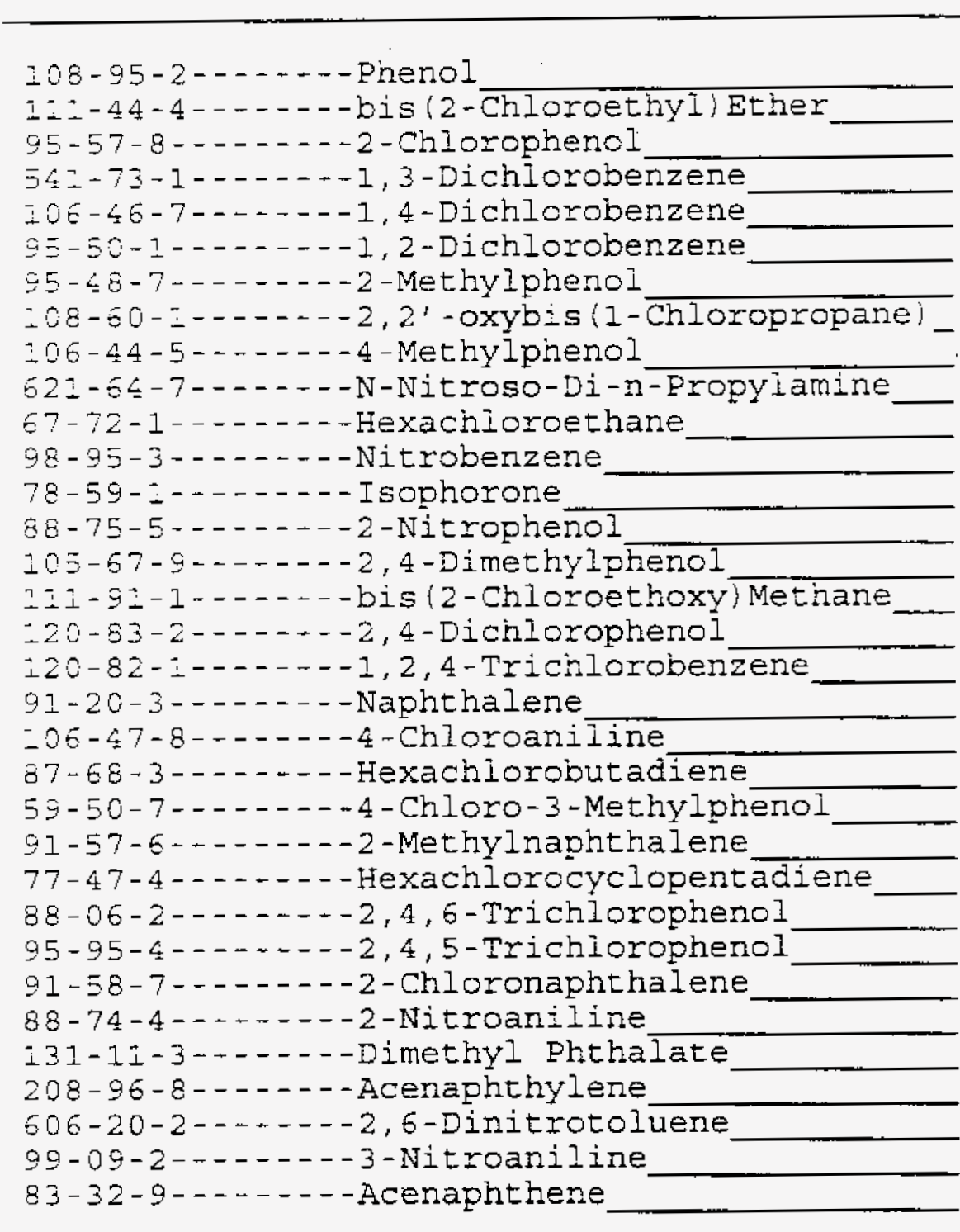


Lab Name: QUANTERRA MO

Lab code: ITMO
Case No.: S16103
Contract: $625-01$

SAS NO.:

A6-SMP $3-$ W2

Matrix: (soil/watex) Soİ

Sample wt/voi:

Leve1:

$(10 w /$ med $)$

$30.10(\mathrm{~g} / \mathrm{mL}) \mathrm{G}$

LOW

: Moisture:

decanted: ( $Y / N) \mathrm{N}$

Concenzrated Extract Volume: 1000 (uL)

Injection volume:

2.0 (UL)

pH:

\section{$(Y / N) \quad N$}

COMPOUND
Lab Sample ID: 27161-004

Lab File ID:

H6313

Date Received: 03/12/98

Date Extracted: 03/23/98

Date Aralyzed: 03/3i/98

Dilution Factor:

1.0

CAS NO.

$5:-28-5-\cdots--2,4-D i n i t r o p h e n o l$
$100-02-7 \ldots \ldots-1$

$\pm 32-64-9-----2-D i b e n z o$ furan

$122-14-2-\cdots-\cdots 2,4-$ Dinitrotoluene

84-66-2--.---Diethylphthalate

$7095-72-3-\cdots--4-$ Chlorophenyl-phenylether

$86-73-7-\ldots . .-51$-... Erene

$100-01-6-\ldots---4-N i$ troaniline

$534-52-1-\cdots---4,6-D i n i t r o-2-$ Methylphenol

$86-30-6-2---N-N i t r o s o d i p h e n y l a m i n e$ ( 1 )

$101-55-3-----4-$ - - romophenyl-phenylether

II8-74-1-.----Hexachlorobenzene

87-86-5-------Pentachlorophenol

85-01-8-...-. Phenanthrene

-20-12-7------Anthracene

$86-74-8-\cdots---$ Carbazole

8 $\leq-74-2------D i=n-B u t y$ phthalate

206-44-0-----Eluoranthene

129-00-0---.--Pyrene

85-68-7-------Butylberzyiphthalate

9 i-94-i--.--- 3, 3'-Dichlorobenzidine

56-55-3-...---Benzo (a) Arthracene

$218-01-9-------C h r y s e n e$

$117-81-7-\ldots--b i s(2-$ Ethylhexyl) Phthalate

1:7-84-0--..--Di-n-octyl Phthalate

205-99-2-...--Benzo(b) Fluoranthene

207-08-9------Benzo(k) Fluoranthene

$50-32-8-\ldots-$ - - Benzo (a) Pyrene

193-39-5---- - - Indeno $(1,2,3-c$ d) Pyrene

53-70-3-...--Dibenz $(a, h)$ Anthracene I $91-24-2-----$-Benzo $(g, h, i)$ Perylene

- Cannot be separated from Diphenylamine
CONCENTRATION UNITS:

( $\mathrm{ug} / \mathrm{L}$ or $\mathrm{ug} / \mathrm{Kg}$ ) UG/KG 
Name: QUANTERRA MO

Code : ITMO

rix: (soil/water) SoIL

ole wt/vol:

el: $\quad$ (low $/ \mathrm{med})$

sisture :

centrated Extract Volume: 1000 (UL)

ection Volume:

$2.0(u L)$

$30.00(\mathrm{~g} / \mathrm{mL}) \mathrm{G}$

Contract: $625-01$

SBLK01

SAS NO.:

SDG No.: $\mathrm{V} 368$
Lab Sample ID: QCBLK167889

Lab File ID: H6315

Date Received:

Date Extracted: $03 / 23 / 98$

Date Analyzed: $03 / 31 / 98$

Dilution Factor: 1.0
Cleanup: $(\mathrm{Y} / \mathrm{N}) \mathrm{N} \quad \mathrm{pH}$ :

CAS NO.
COMPOUND

CONCENTRATION UNITS:

$(\mathrm{ug} / \mathrm{L}$ or $\mathrm{ug} / \mathrm{Kg})$ UG/KG $\mathrm{Q}$

108-95-2-. - - - Phenol

$111-44-4 \ldots-\cdots-$ bis (2-Chloroethyl) Ether

95-57-8-...-2-Chlorophenol

541-73-1------1, 3-Dichlorobenzene

106-46-7-....1,4-Dichlorobenzene

$95-50-1 \ldots-\ldots-1,2-D i c h l o r o b e n z e n e$

$95-48-7-----2-$ Methylphenol

$108-60-1 \ldots-\cdots-2,2^{\prime}$-oxybis (1-Chloropropane)

$106-44-5-\ldots-\ldots 4-$ Methylphenol

$621-64-7-----N-N i t r o s o-D i-n-$ Propylamine

67-72-1-..---Hexachloroethane

$98-95-3-\cdots---N i t r o b e n z e n e$

78-59-1---1--- Isophorone

$88-75-5-\ldots-2-\mathrm{Ni}$ tropheñl

$105-67-9-----2,4-$ Dimethylphenol

111-91-1--.---bis (2-Chloroethoxy) Mechane

$120-83-2 \ldots-\ldots-2,4-D i c h l o r o p h e n o l$

120-82-1-..---1,2,4-Trichlorobenzene

91-20-3-..---Naphthalene

106-47-8-----4-Chioroaniline

87-68-3----1--Hexachlorobutadiene

59-50-7---.--4-Chloro-3-Methylphenol

$91-57-6-\ldots-\cdots-2-$ Methylnaphthalene

$77-47-4-\ldots-$ - - Hexachlorocyclopent adiene

88-06-2-------2, 4, 6-Trichlorophenol

95-95-4-------2,4,5-Trichlorophenol

$91-58-7 \ldots----2-C h l o r o n a p h t h a l e n e$

$88-74-4------2-N i t r o a n i l i n e$

$131-11-3---2-0$ - Dimethyl Phthaiate

208-96-8-----Acenaphthylene

$606-20-2-\ldots---2,6-$ Dinitrotoluene

99-09-2----.--3-Nitroaniline

83-32-9------Acenaphthene

330

330

330

330

330

330

330

330

330

330

330

330

330

330

330

330

330

330

330

330

330

330

330

1600

330

330

330

1600

330

330

330

1600

330 
Lab Name: QUANTERRA MO

zab Code: ItMo Case No.: S16103

Matrix: (soil/water) soIL

Sample wt/vol:

Level: (low $/$ med) Low

\% Moisture:

decanted: $(\mathrm{Y} / \mathrm{N}) \mathrm{N}$

Concentrated Extract Volume: 1000 (UL)

Injection volume:

$2.0(L I)$

Contract: $625-01$

SBLKOI

SAS NO.:

Lab Sample ID: QCBLK167889

Lab File ID: H63I5

Date Received:

Date Extracted: $03 / 23 / 98$

Date Aralyzed: 03/31/98

Dilution Factor:

1.0

GPC Cleanup: $(\mathrm{Y} / \mathrm{N}) \mathrm{N} \quad \mathrm{pH}$ :

CAS NO.

COMPOUND

CONCENTRATION UNITS:

$(\mathrm{ug} / \mathrm{L}$ or $\mathrm{ug} / \mathrm{Kg}) \mathrm{UG} / \mathrm{KG}$

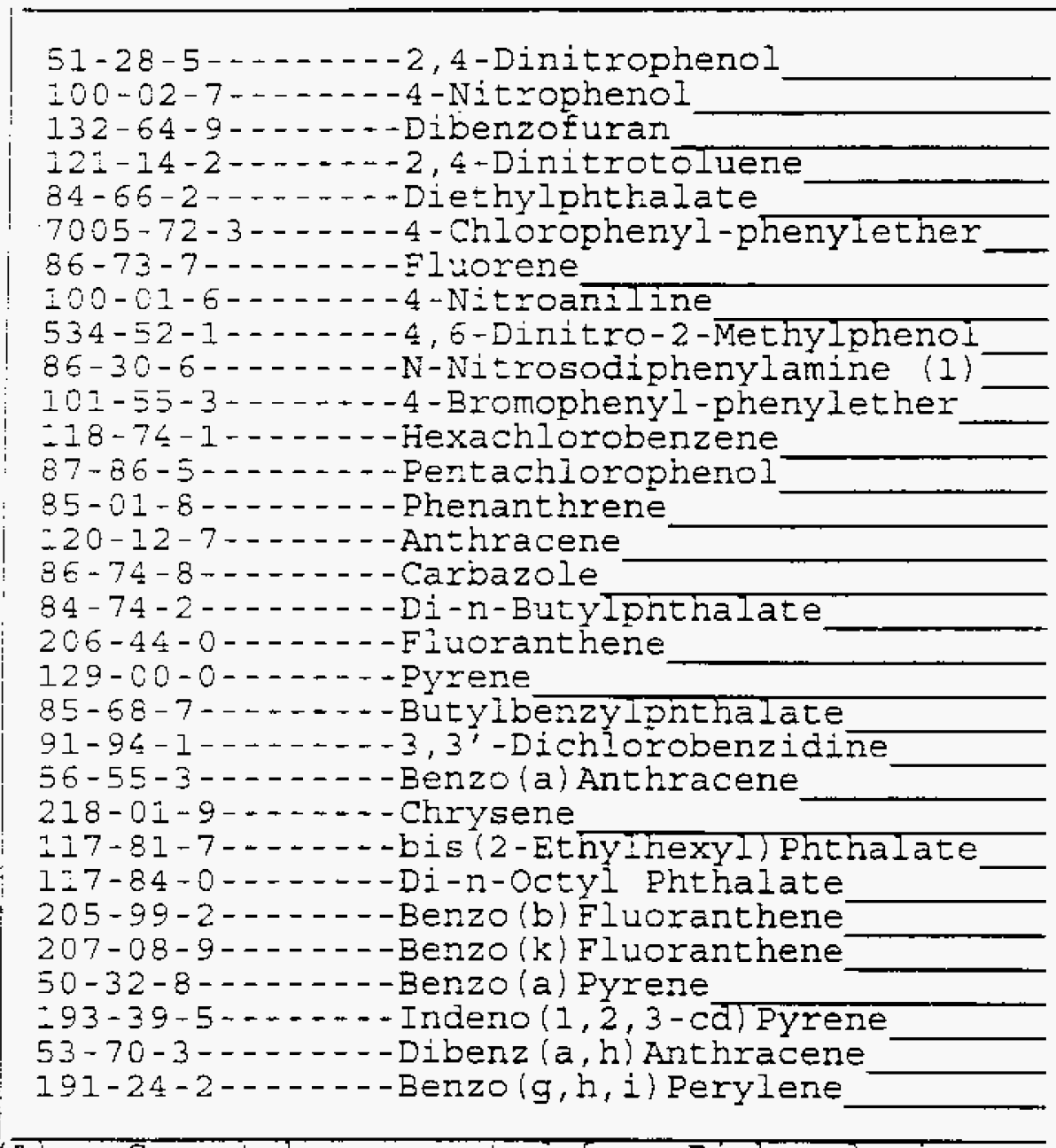

(I) - Cannot be separated from Diphenyiamine

1600

1600

330

330

330

330

330

1600

1600

330

330

330

1600

330

330

330

330

330

330

330

1600

330

330

330

330

330

330

330

330

330

330 
ab Name: QUANTERRA MO

ab Code: ITMO Case No.: S16103. SAS No.:
SSPKOLA atrix: (soil/water) soIL

ample wt/vol:

evel: $\quad(10 w /$ med $\}$ Low

Moisture:

decanted: $(\mathrm{Y} / \mathrm{N}) \mathrm{N}$

sncentrated Extract VoIume: 1000 (uL)

ijection voiume:

$(\mathrm{Y} / \mathrm{N}) \quad \mathrm{N}$

2.0 (UL)

$\mathrm{pH}$ :

CAS NO.

COMPOUND
Lab Sample ID: QCSPK167889-1

Lab File ID: H6310

Date Received:

Date Extracted: 03/23/98

Date Analyzed: $03 / 31 / 98$

Dilution Factor:

1.0

CONCENTRATION UNITS:

(ug/L or $\mathrm{ug} / \mathrm{Kg}$ ) UG/KG
$108-95-2------$ Pheno1

I11-44-4--..--bis (2-Chloroethyl) Ether

95-57-8-...-.-2-Chlorophenol

$541-73-1-\ldots-1,3-$ Dichlorobenzene

$106-46-7-\ldots---1,4-D i c h l o r o b e n z e n e$

95-50-:-..---1,2-Dichlorobenzene

$95-48-7-\ldots-\cdots-2-$ Methylpheno?

208-60-1------2, 2' - oxybis (1 - Chloropropane)

$106-44-5-\cdots---4-$ Methylphenol

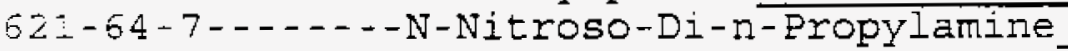

$67-72-1-\ldots-\ldots-$ Hexachloroethane

$98-95-3-\cdots----N i t r o b e n z e n e$

$78-59-i-\ldots-\ldots-$ Isophorone

$88-75-5 \cdots----2-\mathrm{Ni}$ trophenol

$105-67-9-\ldots--2,4-D i m e t h y l$ phenol

$111-91-1-\ldots$ - . bis (2-Chloroethoxy) Methane

-20-83-2-...--2, 4-Dichlorophenol

120-82-I----.-1,2,4-Trichlorobenzene

$91-20-3--\cdots--N a p h t h a l e n e$

$106-47-8-\ldots-2-$-Chloroaniline

87-68-3-...-.-. Hexachlorobutadiene

59-50-7--.-.-4-Chloro-3-Methylphenol

91-57-6------2-Methylnaphthalene

$77-47-4-----$ - Hexachlorocyclopentadiene

$88-06-2-\ldots-\ldots-2,4,6-$ Trichlorophenol

$95-95-4-\cdots---2,4,5-$ Trichlorophenol

91-58-7------2-Chloronaphthalene

88-74-4-...--2-Nitroaniline

131-11-3-----Dimethyl Phthalate

208-95-8--..-Acenaphthylene

$606-20-2 \ldots---2,6-$ Dinitrotoluene

99-09-2--..--3-Nitroaniline

83-32-9------Acenaphthene $\mp 400$

330

1500

330

1100

330

330

330

330

1400

330

330

330

330

330

330

330

1100

330

330

330

1700

330

1600

330

330

330

1600

3.30

330

330

1600

1100 
Jab Name: QUANTERRA MO

Lab Code: ITMO

Case No.: S16103

Matrix: (soil/water) SOIL

Sample wt/vol:

$30.00(\mathrm{~g} / \mathrm{mL}) \mathrm{G}$

Level:

(low/med)

LOW

\% Moisture:

decanted: $(\mathrm{Y} / \mathrm{N}) \mathrm{N}$

Concentrated Extract Volume: 1000 (uL)

Injection volume:

$2.0(\mathrm{UL})$

GFC Cleanup: $(Y / N) \mathrm{N} \quad \mathrm{pH}$ :

GFC Cleanup: $(Y / N) \mathrm{N} \quad \mathrm{pH}$ :

CAS NO.

COMPOUND
Contract: $625-01$

SAS NO.:

SDG No.: V3 68
SSPKO1A

Lab Sample ID: QCSPK167889-I

Lab File ID: H6310

Date Received:

Date Extracted: 03/23/98

Date Analyzed: 03/31/98

Dilution Factor:

1.0

CONCENTRATION UNITS:

$(\mathrm{ug} / \mathrm{L}$ or $\mathrm{ug} / \mathrm{Kg}) \mathrm{UG} / \mathrm{KG}$

Q

$51-28-5-----2,4-$ Dinitrophenol

200-02-7------4-Nitrophenol

$132-64-9-\ldots-\cdots$ Dibenzofuran

$121-14-2 \cdots---2,4-D i n i t r o t o l u e n e$

84-66-2--.-.-Diethylphthalate

$7005-72-3-\cdots--4-$ Chlorophenyl -phenylether

86-73-7------Fluorene

$100-01-6-\ldots--4-N i t r o a n i l i n e$

$534-52-2-\cdots---4,6$-Dinitro-2-Methylphenol

$86-30-6-\ldots . .-N-N i$ trosodiphenylamine (1)

i $01-55-3-----4$ - - - romophenyl-phenylether

:18-74-1---... Fexachlorobenzene

$87-86-5-\ldots---$ - Pentachlorophenol

85-0I-8-------Phenanthrene

120-12-7--.--Anthracene

$86-74-8-\ldots-\ldots$ - Carbazole

84-74-2-...--Di-n-Buty lphthalate

206-44-0-------Fluoranthene

$129-00-0-\cdots-\cdots-$ - Pyrene

85-68-7---- - - Butylbenzylphthalate

$91-94-1-\ldots----3,3^{\prime}-$ Dichlorobenzidine

56-55-3--...-Berzo (a) Anthracene

218-01-9------Chrysene

$1: 7-81-7-\ldots--$ - bis (2-Ethylhexyl) Phthalate

117-84-0------Di-n-Octyl Phthalate

205-99-2---.--Benzo (b) Fluoranthene

207-08-9------Benzo(k) Fluoranthene

50-32-8---.-.-Benzo (a) Pyrene

$193-39-5-\ldots-.-$ - Indeno $(1,2,3$-cd) Pyrene

53-70-3-..--Dibenz $(a, h)$ Anthracene

19I-24-2------Benzo $(g, h, i)$ Perylene

-

(1) - Cannot be separated from Diphenylamine

\begin{tabular}{r|l|l}
1600 & $\mathrm{U}$ \\
1600 & & \\
330 & $\mathrm{U}$ & \\
650 & & \\
330 & $\mathrm{U}$ & \\
330 & $\mathrm{U}$ & \\
330 & $\mathrm{U}$ \\
1600 & $\mathrm{U}$ \\
1500 & $\mathrm{U}$ \\
330 & $\mathrm{U}$ \\
330 & $\mathrm{U}$ \\
330 & $\mathrm{U}$ \\
1300 & $\mathrm{~J}$ \\
330 & $\mathrm{U}$ \\
330 & $\mathrm{U}$ \\
330 & $\mathrm{U}$ \\
330 & $\mathrm{U}$ \\
330 & $\mathrm{U}$ \\
1000 & \\
330 & $\mathrm{U}$ \\
1600 & $\mathrm{U}$ \\
330 & $\mathrm{U}$ \\
330 & $\mathrm{U}$ \\
320 & $\mathrm{~J}$ \\
330 & $\mathrm{U}$ \\
330 & $\mathrm{U}$ \\
330 & $\mathrm{U}$ \\
330 & $\mathrm{U}$ \\
330 & $\mathrm{U}$ \\
330 & $\mathrm{U}$ \\
330 & $\mathrm{U}$ \\
& \\
&
\end{tabular}


ab Name: QUANTERRA MO

ab code: ITMO

latrix: (soil/water) soIL

ampie wt/vol:

evel: (low/med) Low

Moisture:

decanted: $(Y / N) N$

Contract: $625-01$

SSPKO1B

oncentratea Extract volume: 1000 (UL) njecrion Volume:

$2.0(L L)$

PC Cleanup: $\{\mathrm{Y} / \mathrm{N}\} \mathrm{N} \quad \mathrm{pH}$ :

PC Cleanup: $\{\mathrm{Y} / \mathrm{N}\} \mathrm{N} \quad \mathrm{pH}$ :

CAS NO.

COMPOUND

CONCENTRATION UNITS:

$(\mathrm{ug} / \mathrm{L}$ or $\mathrm{ug} / \mathrm{kg})$ UG/KG

Lab Sample ID: QCSPK167889-2

Lab Eile ID: H6311

Date Received:

Date Extracted: $03 / 23 / 98$

Date Analyzed: 03/31/98

Dilution Factor: $\quad 1.0$

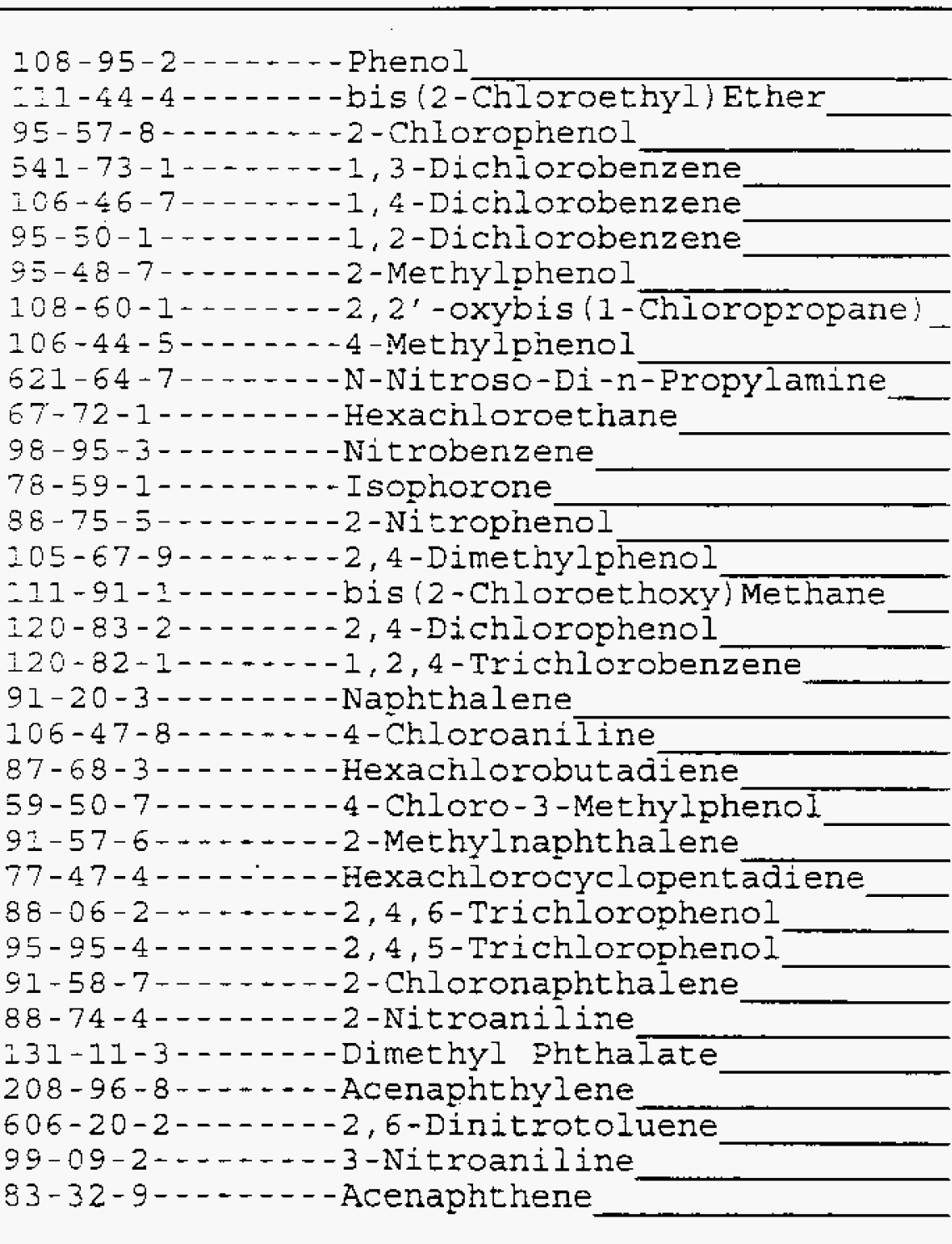

1700

330

1800

330

1300

330

330

330

330

1600

330

330

330

330

330

330

330

1300

330

330

330

2100

330

1600

330

330

330

1600

330

330

330

1600

1400

000053 
Lab Name: QUANTERRA MO

Iab Code: ITMO Case No.: S16103. SAS No.:
SSPKOIB

Contract: $625-01$

SDG No.: V368
Matrix: (soil/water) SoIL

Sample wt/vol:

$30.00(\mathrm{~g} / \mathrm{mL}) \mathrm{G}$

Level: $\quad$ (low/med) LOW

兴 Moisture:

decanted: $(\mathrm{Y} / \mathrm{N}) \mathrm{N}$

Concentrated Extract volume: 1000 (UL)

Injection Volume:

2.0 (LI)
Lab Sampie ID: QCSPK167889-2

Lab Fize ID: H6311

Date Received:

Date Extracted: $03 / 23 / 98$

Date Analyzed: $03 / 31 / 98$

Dilution Factor: $\quad 1.0$
GPC Cleanup: (Y/N) N $\quad \mathrm{NH}$ :

GPC Cleanup: $(\mathrm{Y} / \mathrm{N}) \mathrm{N} \quad \mathrm{pH}$ :

CAS NO.
COMPOUND
CONCENTRATION UNITS:

(ug/L or $\mathrm{ug} / \mathrm{Kg}$ ) UG/KG

\begin{tabular}{|c|}
\hline 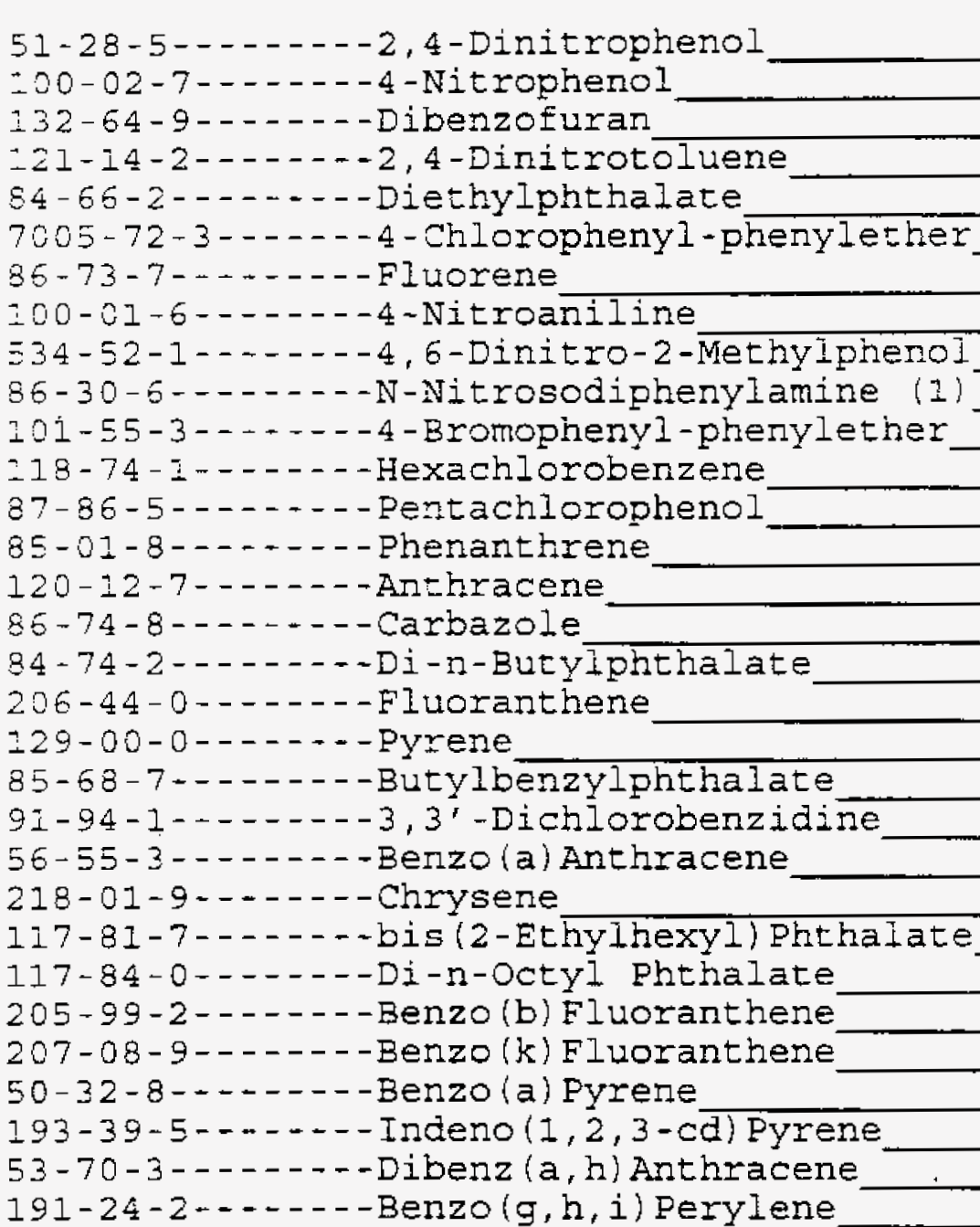 \\
\hline
\end{tabular}

a! - Camnot be separated from Diphenylamine

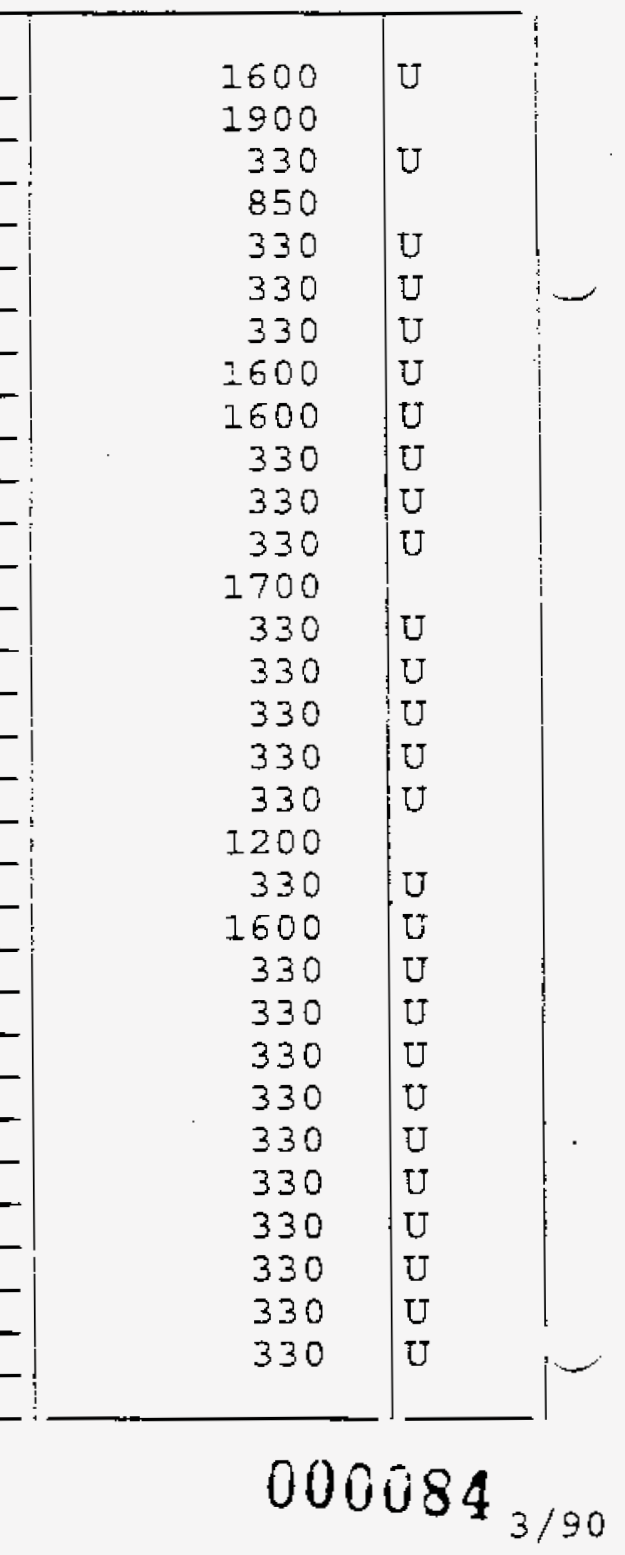


2D

SOIL SEMIVOLATILE SURROGATE RECOVERY

ab Name: QUANTERRA MO

ab Code: ITMO

Contract: 625-01

evel: (low/med) Low

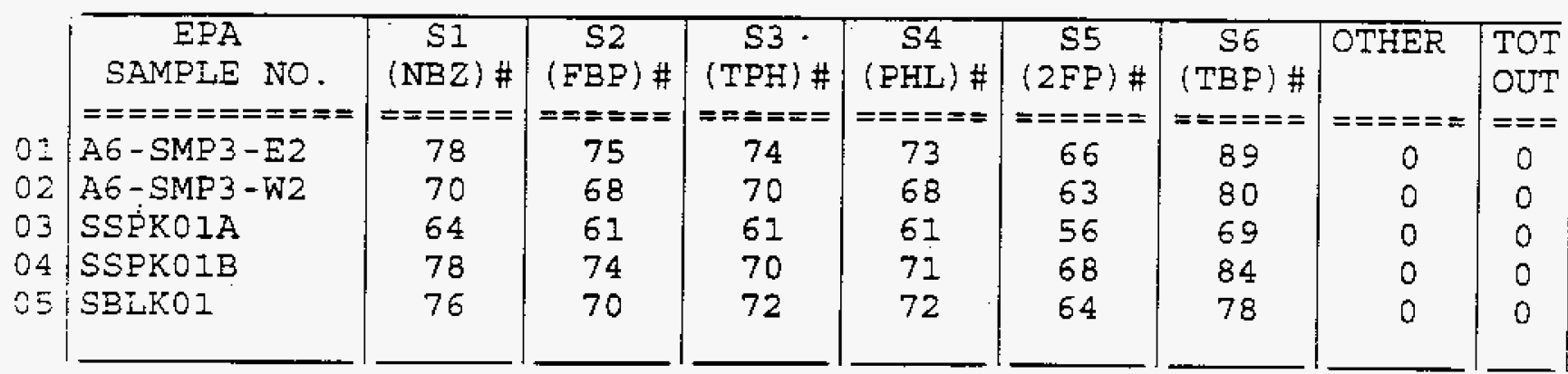

S1 (NBZ) = Nitrobenzene-d5

S2 $(\mathrm{FBP})=2-$ Fluorobiphenyl

S3 $(\mathrm{TPH})=$ Terphenyl

S4 $($ PHL $)=$ Phenol-d5

S5 $(2 F P)=2-F$ luorophenol

S6 $(\mathrm{TBP})=2,4,6$-Tribromophenol

\# Column to be used to flag recovery values

* Values outside of contract required QC limits

D Surrogates diluted out
QC LIMITS

$(23-120)$

$(30-115)$

$(18-137)$

$(24-113)$

$(25-121)$

(19-122) 
Lâb Name: QUANTERRA MO

Lab Code: ITMO

Case No.: S16103

Lab File ID:

H6315

Instrument ID:

MSH

Marvix: (soil/water) soIL

Leve: : (low/med)

LOW
Contract: $625-01$

SAS NO.:
EPA SAMPLE NC

SBLKOI

SDG No.: V368

THIS METHOD BLANK APPLIES TO THE FOLLOWING SAMPLES, MS AND MSD:

\begin{tabular}{|c|c|c|c|}
\hline$E P A$ & $I A B$ & $\overline{I A B}$ & $\overline{D A T E}$ \\
\hline SAMPLE NO. & SAMPLE ID & FILE ID & ANALYZED \\
\hline$======0==$ & $====0==0==0$ & 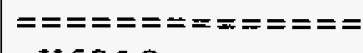 & $=========$ \\
\hline$A 6-S M P 3-E 2$ & $17161-003$ & H6312 & $03 / 31 / 98$ \\
\hline$A 6-S M P 3-W 2$ & $17161-004$ & H63i3 & $03 / 31 / 98$ \\
\hline SSDK01A & OCSPK167889-1 & H6 310 & $03 / 31 / 98$ \\
\hline SSEKOIB & QCSPK167889-2 & $\mathrm{H} 6311$ & $03 / 31 / 98$ \\
\hline
\end{tabular}

COMMENTS: BLK 167889

ZNST\#MSH; JJB; 550.260 
ab Name: QUANTERRA MO

ab Code: ITMO

ab File ID: HHO97

nstrument ID: MSH
Contract: $625-01$

Case No.: $\$ 16103$ SAS No.:

SDG NO.: $\mathrm{V} 368$

DFTPP Injection Date: 03/26/98

DFTPP Injection. Time: 1613 $\mathrm{m} / \mathrm{e}$ ION ABUNDANCE CRITERIA

5

68

69

70

$\perp 27$

$\div 97$

198

$\div 99$

275

365

$\div 4 \div$

442

$\pm 43$

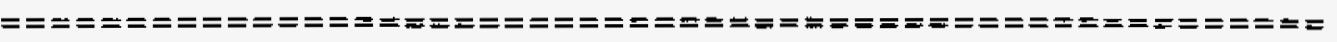

$30.0-60.0 \%$ of mass 198

iess than $2.0 \%$ of mass $6 \overline{9}$

Mass 69 relative abundance

Less than $2.0 \%$ of mass 69

$40.0-60.0 \%$ of mass 198

iess than $1.0 \%$ of mass $1 \overline{98}$

Base peak, $100 \%$ relative abundance

5.0 to $9.0 \%$ of mass 198

-0.0 - 30.0\% of mass $19 \overline{8}$

Greater than $1.00 \%$ of mass I98

Fresent, but less than mass $44 \overline{3}$

Greater thar $40.0 \%$ of mass 198

$17.0-23$. O高 of mass 442

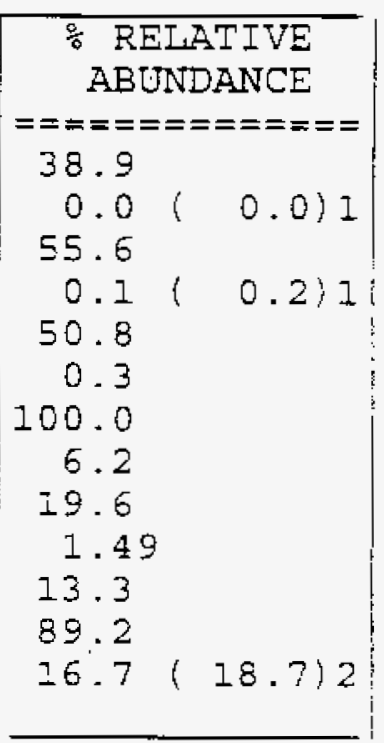

2-VaIue is \% mass 442

:

\begin{tabular}{|c|c|c|c|c|}
\hline$E P A$ & $\overline{L A B}$ & $\overline{\mathrm{IAB}}$ & $\overline{D A T E}$ & TIME \\
\hline SAMPIE NO. & SAMPIE ID & EILE ID & ANALYZED & ANALYZED \\
\hline 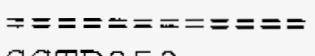 & 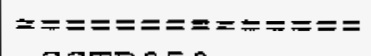 & 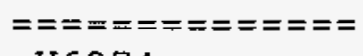 & $==-=F===0=$ & $\Rightarrow x=======$ \\
\hline $\operatorname{SSTD0} 50$ & SSTD050 & $\mathrm{H} 6274$ & $03 / 26 / 98$ & 1642 \\
\hline$S S T D 020$ & SSTDO20 & H6275 & $03 / 26 / 98$ & 1728 \\
\hline SSED080 & SSTDO80 & H6276 & $03 / 26 / 98$ & 1808 \\
\hline SSTग120 & SSID120 & $\mathrm{H} 6277$ & $03 / 26 / 98$ & 1847 \\
\hline SSTDI60 & SSTD160 & $\mathrm{H} 6278$ & $03 / 26 / 98$ & 1927 \\
\hline
\end{tabular}


Lab Name: QUANTERRA MO

Lab Code: ITMO

Lab File ID: HH100

Case No.: $\$ 16103$.

Contract : $625-01$

Instrument ID: MSH
SAS No.:

SDG NO.: V368

DFTPP Injection Date: 03/31/98

- DFTPP Injection Time: 0815

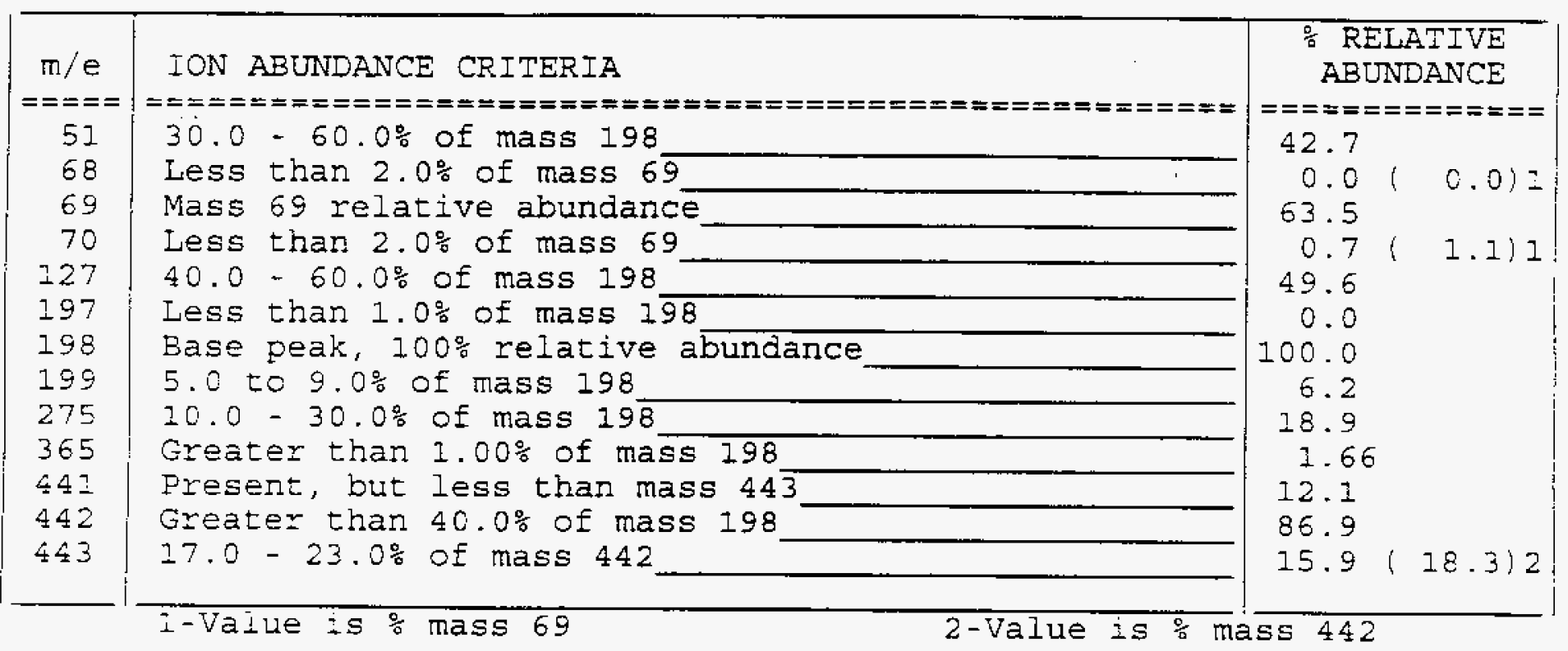

THIS TUNE APPLIES TO THE FOLIOWING SAMPLES, MS, MSD, BLANKS, AND STANDARDS:

\begin{tabular}{|c|c|c|c|c|}
\hline$\overline{E D A}$ & $\overline{\mathrm{IAB}}$ & $\overline{L A B}$ & $\overline{\mathrm{DATE}}$ & TIME \\
\hline SAMPLE NO. & SAMPLE ID & FILE ID & ANALYZED & ANALYZED \\
\hline $\begin{array}{l}=========== \\
\text { SSTD050 }\end{array}$ & $\begin{aligned}== & ===i===== \\
& \text { SSTD0 } 50\end{aligned}$ & $\begin{array}{c}=====0===\equiv=== \\
H 6307\end{array}$ & $=== \pm=====$ & $========$ \\
\hline SSPKOIA & OCSPKI 67889-1 & H6310 & $\begin{array}{l}03731 / 98 \\
03 / 31 / 98\end{array}$ & 0829 \\
\hline SSPKO1E & QCSPK167889-2 & H6311 & $03 / 31 / 98$ & 1115 \\
\hline$A 6-S M P 3-\Xi 2$ & $17161-003$ & H6312 & $03 / 31 / 98$ & 1154 \\
\hline$A 6-S M P 3-W 2$ & $17161-004$ & H6313 & $03 / 31 / 98$ & 1234 \\
\hline SBLKOI & QCBLK167889 & $\mathrm{H} 6315$ & $03 / 31 / 98$ & 1401 \\
\hline
\end{tabular}


ab Name: QUANTERRA MO

ab code: ITMO

nstrument ID: MSH
Contract: $625-01$

Case No.: S16103 SAS No.:

SDG NO.: V368
Calibration Date(s): 03/26/98 03/26/98

Calibration Times: $\quad 1642 \quad 1927$ $\triangle A B \quad F I L E=2$

$\mathrm{RRFBO}=\mathrm{H} 6276$
RRF20 $=H 6275$

RRF $120=\mathrm{H} 6277$
RRF50 = H6274

RRF160 $=\mathrm{H} 6278$
COMPOUND

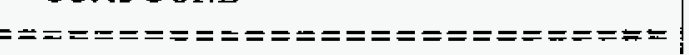
herol

ois (2 - Chloroethyl) Ether

- Chloropineriol

3-Dichlorobenzene

4-Dichiorobenzene

, 2-Dicinorobenzene

- Methylpisenoi

2 '-oxyis (1-Chloropropane

- Nethyiphenol

J-Nitzoss-Di-n-Propy-amine \#

iexachloroetrane

J trobenzene

sophorone

- Nitropienol

-4-Dimetsyipherol

is (2-Choroethoxy) Methane

, 4-DisioropinenoI

2, 4-mriniorobenzene

laphisaíne

- Chioroanjīne

exachlorobutadiene

- Chioro- 3 - Methylphenol.

- Methy lraphthalene.

exachlorocyciopentadiene

, 4,6-Trichlorophenol

, 4, 5- Trichiorophenoi

- Chioronaprthalene

- Nitroaniline

inethyl Dhtha late

cenaphthyiene

, E-Dinitrotoluene

- Nitroaniline

ceraphthene

4-Dinitrophenol

- Nitropinenol

¿benzofuran

,4-Dini zrotoluene
RRF20

$== \pm==$

1.682

1.306

1.467

1. 437

1.402

1. 362

1.137

0.908

1. 252

0.703

0.581

0.315

0.640

0.241

0.327

0.459

0.338

0.341

1. 071

0.352

0.143

0.279

0.661

0.268

0.425

0.466

1.194

0.298

1. 326

1. 824

0.332

0.336

I. 771

0.077

0.218

1. 729

0.452
RRF50

$====$

1.649

1. 319

1.370

1. 442

1. 433

I. 367

1.123

0.856

1.177

0.714

0.593

0.310

0.613

0.243

0.311

0.433

0.325

0.332

‥ 033

0.475

0.153

0.294

0.658

0.312

0.418

0.454

1. 175

0.330

1. 287

1. 786

0.342

0.420

1. 092

0.161

0.204

1. 642

0.453
RRFBO

$====$

I. 720

I. 478

1.430

1.377

1. 339

1. 296

1. 143

1.007

1. 251

0.725

0.561

0.284

0.602

0.234

0.304

0.422

0.310

0.303

0.942

0.310

0.135

0.280

0.624

0.291

0.406

0.450

1.123

0.300

1. 260

1.719

0.327

0.329

1. 080

0.196

0.204

1. 612

0.437
RRF120 =ニニニニ

I. 662

1. 425

1. 392

1.308

1. 274

1. 258

1. 130

1.009

1.259

0.642

0.549

0.309

0.632

0.247

0.318

0.439

0.324

$0.31 i$

0.989

0.410

0.135

0.284

0.540

0.294

0.427

0.474

1. . 364

0.321

土. 284

1. 735

0.341

0.334

1.074

0.222

0.208

1.622

0.445
RRE 160

$====$

1. 650

I. 432

1. 362

1. 261

1. 268

1. 206

3. 159

0.981

1. 214

0.523

0.517

0.307

0.620

0.25 I

0.309

0.444

0.324

0. 304

0.950

0.425

0.129

0.286

0.645

0.287

0.408

0.444

1. 116

0.308

1. 246

I. 665

0.339

0.340

1. 029

0.234

0.213

1. 543

0.409

$\overline{\mathrm{RRF}}$

$=== \pm=$

1.573

1. 392

‥ 404

1. 365

‥ 343

1. 298

1. 138

0.952

1. 231

0.661

0.560

0.305

0.621

0.243

0.314

0.439

0.324

0.318

0.997

0.394

0.139

0.285 
$6 \mathrm{C}$

SEMIVOLATILE ORGANICS INITIAL CALIBRATION DATA

Lab Name: QUANTERRA MO

Lab Code: ITMO

Instrument ID: MSH
Contract: $625-01$

SAS NO.:
SDG NO : $\mathrm{V3} 68$

Calibration Date(s): $03 / 26 / 98$

$03 / 26 / 98$

Calibration Times: 1642

\begin{tabular}{|c|c|c|c|c|c|c|c|}
\hline \multicolumn{3}{|l|}{$\begin{array}{l}\text { LAB FILE ID: } \\
\text { RRF } 80=\$ 6276\end{array}$} & \multicolumn{4}{|c|}{$\begin{array}{l}\text { RRF50 = H6274 } \\
\text { RRF160 }=\mathrm{H} 6278\end{array}$} & \multirow[b]{2}{*}{$R S D$} \\
\hline COMPOUND & RRF20 & RRF50 & RRF80 & RRF120 & RRF 160 & $\overline{\mathrm{RRF}}$ & \\
\hline 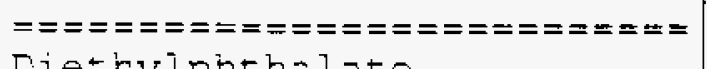 & $=====$ & $=====$ & $==== \pm=$ & $=====$ & & $====$ & $====$ \\
\hline Diethylphthal & 1.414 & 14 & 1.251 & I. 306 & 1.220 & 1.301 & 5.7 \\
\hline 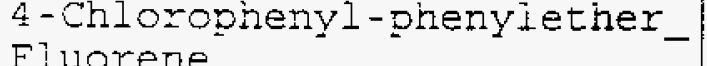 & 0.629 & & 0.565 & 0.578 & & 0.585 & 5.7 \\
\hline Fluorene & 1.308 & 1.257 & 1.781 & 1.198 & 1.112 & 1.211 & 6.2 \\
\hline 4-Nitroaniline & 0.335 & 0.434 & 0.380 & 0.387 & 0.362 & 0.380 & 9.6 \\
\hline 4,6-Dinitro-2-Meth & 0.126 & 0.155 & 0.164 & 0.168 & 0.179 & 0.158 & 12.7 \\
\hline $\mathrm{N}-\mathrm{Ni}=$ rosodipher & 0.470 & 0.474 & 0.456 & 0.443 & 0.432 & 0.455 & $3.9 *$ \\
\hline ६- $\Xi$ romopheny i-phen & 0.178 & 0.184 & 0.181 & 0.286 & 0.178 & 0.181 & $2.0:$ \\
\hline Hexachlorodenz & 0.248 & 0.252 & 0.238 & 0.238 & 0.226 & 0.240 & 4.2 \\
\hline Pertacijorophe & 0.047 & 0.080 & 0.096 & 0.102 & 0.116 & 0.088 & $30.0 \star$ \\
\hline Phenanthren & 1.084 & 1.074 & 1.029 & 1.041 & i. 018 & $\mp .049$ & 2.7 \\
\hline$n$ thracene & 1.110 & 1.094 & 1.015 & 1. .029 & 0.978 & 1.045 & 5.3 \\
\hline Carbazole & 0.894 & I.051 & 0.721 & 0.741 & 0.758 & 0.833 & 158 \\
\hline $5:-n-3 u=y$ lpnthalate & 1.403 & 1.388 & 2.317 & 1.353 & 1.343 & 1.361 & \\
\hline Fluoranthene & 1.102 & 1.109 & 1.047 & $1.05 I$ & I.034 & 1.067 & 3.3 \\
\hline Pyrene & 1.205 & 1.250 & 1.477 & \pm .251 & 1.786 & 1.394 & 27.5 \\
\hline Butylbenzylphthalate & 0.681 & 0.711 & 0.854 & 0.740 & 1.1 & 0.819 & $21.3 !$ \\
\hline $3,3^{\prime}$ - Dichlorobenzid & 0.278 & 0.408 & 0.293 & 0.275 & 0.415 & 0.334 & $2 i .4 i$ \\
\hline Benzo (a) Anthracene & 1.190 & 1.123 & 1.365 & $i .175$ & 1. & 1.302 & $16.7 !$ \\
\hline Cincysene & 0.944 & 1.025 & 1.051 & 0.939 & 1.303 & 1.052 & $14 .=$ \\
\hline bis $(2-E t h y i h e x y I) P h$ & 0.970 & 0.994 & 1.124 & 0.985 & $=.418$ & 1.098 & 17.2 \\
\hline Di-roctyl Phthala & 1.890 & 1.953 & 2.013 & 2.074 & $2-124$ & 2.011 & $4.6 *$ \\
\hline Berzo (b) Fluoranzhen & 1.229 & 1.198 & \pm .306 & 1.458 & I. 524 & 1.343 & 10.6 \\
\hline Benzo (k) Eluoranther & 1.084 & 1.029 & 0.937 & 0.822 & 0.717 & 0.918 & 16.3 \\
\hline Eenzo (a) Pyrene & 1.005 & 1.021 & 1.001 & 1.020 & 1.023 & 1.012 & 0.9 \\
\hline Endeno $(1,2,3$-cd) Pyrene & 0.933 & 0.9 & 0.958 & 0.989 & $1.1 \pm 6$ & 0.982 & 8.2 \\
\hline Dibenz (a, b) Anthrac & 0.924 & 0. & 0.915 & 0.922 & 0.892 & 0.911 & \\
\hline Benzo $(g, h, i)$ Peryiene. & 0.912 & 0.911 & 0.928 & 0.921 & 0.920 & 0.918 & 0.8 \\
\hline \multicolumn{8}{|c|}{ 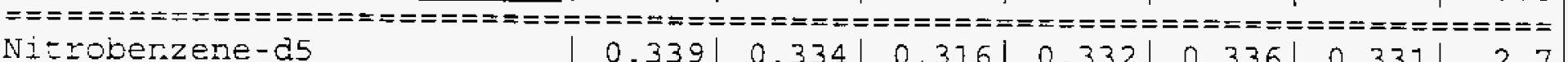 } \\
\hline Ni Eroberzene-d5 & 0.339 & 0.334 & 0.316 & 0.332 & 0.336 & 0.331 & 2.7 \\
\hline 2-Eluorobipheny $\bar{l}$ & 1.348 & 1.320 & 1.226 & 1.254 & $1 . \mp 77$ & 1.265 & 5.5 \\
\hline Terphenyl - d14 & 0.838 & 0.875 & 1.023 & 0.857 & 1.206 & 0.960 & 16.2 \\
\hline Phenol-d5 & 1.489 & 1.408 & 1.499 & $I .446$ & 1.496 & 1.468 & 2.7 \\
\hline 2-Fliorophenol & 1.318 & 1.257 & 1.308 & 1. .256 & 1.266 & 1.281 & $2: 3$ \\
\hline $2,4,6-\operatorname{Trib}$ & 0.221 & 0.228 & 0.210 & 0.211 & 0.199 & 0.214 & 5.2 \\
\hline
\end{tabular}

* Compounds with required minimum RRF and maximum $: R S D$ values.

All other compounds must meet a minimum RRF of 0.010 . 
ab Name: QUANTERRA MO

ab code: ITMO

nstrument ID: MSH
Contract: $625-01$

Case No.: \$16I03 SAS No.:

SDG No.: V368

Calibration Times: $1642 \quad 1927$

$\begin{array}{lll}\text { LAB FILE ID: } & \text { RRF20 }=H 6275 & \text { RRF50 }=\text { H6274 } \\ \text { RRF } 80=H 6276 & \text { RRF120 }=H 6277 & \text { RRF160 }=H 6278\end{array}$

COMPOUND

$=====$
Phenol

bis (2-Chioroethyl) Ether

2 -Chlorophenol

1,3-Dichlorobenzene

I, 4-Dichlorobenzene

, 2-Dichiorobenzene

- Methylpheno?

2, $2^{\prime}$-oxybis 11 -Chioropropane 1 -Methylphenol

$v$-Nitroso-Di-n-Propylamine_\# Hexachloroethane

vitrobenzerie

Isophorone.

2-Nitropheñl

2,4 -Dimethylphenol

is (2-Chloroethoxy) Methane

, i-Dictiorophenol

.,2,4-Trichlorobenzene.

Japhthalene

- Chloroani ine

iexachiorobutadiene

-Chloro-3-Methylphenol.

- Methylnaph halene.

lexachlorocyclopentadiene

$\therefore$ 4,6-Trichlorophenol

, 4,5-Trichlorophenoi

- Chloronaphthalene.

- Nitroaniline

imethy 1 Phthalate

cenaphthylene

,6-Dinitrotoluene

-Nitroaniline

cenaphthene

, 4-Dinitrophenol

- Nitrophenol

ibenzofuran

,4-Dinitrotoluene . - $x^{\wedge} 2$ Coefficient

$===\boldsymbol{=}=\mathbf{=}=\boldsymbol{=}=\mathrm{x=}=$

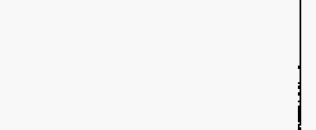

$\mathrm{X}^{\wedge} \mathrm{I}$ Coefficient

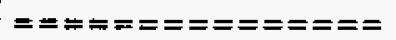
0.60650949

0.68512219

0.73633290

0.81322157

0.81230097

0.84576527

0.86365006

0.98150065

0.81258113

1. 86296099

1.96155120

3. 24876542

1.60142482

3.94734843

3. 22168341

2. 25273702

3.09007223

3. 34815361

1. 06385930

2.27627627

7.93761439

3.51018073

1. 55928486

3.47018052

2.42801756

2. 21462553

0.89608730

3.20828495

0.80391943

0.60480805

2. 93912682

3. 02033877

0.98177996

3.85341694

4.70187044

0.65154913

2.43663394

Intercept $==== \pm==$

$-0.025 \star$

0.059

$-0.049$

$-0.167$

$-0.135$

$-0.148$

0.028

0.091

$-0.001$

-0.350 \#

$-0.152$

0.027

0.014

0.067 *

$-0.009$

0.029

$0.010 *$

$-0.088$

$+0.079$

0.184

$-0.155 *$

0.0 *

$-0.002$

$-0.027 \#$

-0.015 *

$-0.020$

$-0.049$

$-0.004$

$-0.041$

$-0.081$

0.019

$-0.093$

-0.098 *

$0.429 \#$

$0.037 \#$

$-0.089$

$-0.109$ 
Lai Name: QUANmERRA MO

Lab Code: ITMO

Instrument ID: MSH
Contract: $625-01$

Case No.: S16103 SAS No.:
SDG NO.: V368

Calibration Date(s):

$03 / 26 / 98$

$03 / 26 / 98$

Calibration Times: 1642

1927

\begin{tabular}{|c|c|c|c|}
\hline $\begin{array}{l}\text { LAE FIEE ID: } \\
\text { RRF80 = H6276 }\end{array}$ & $\begin{array}{l}=\mathrm{H} 6275 \\
0=\mathrm{H} 6277\end{array}$ & $\begin{array}{l}\text { RRF50 = H6274 } \\
\text { RRF160 }=H 6278\end{array}$ & \\
\hline COMPOUND & $x^{\wedge} 2$ Coefficient & $x^{\wedge} 1$ CoefEicient & Intercept \\
\hline 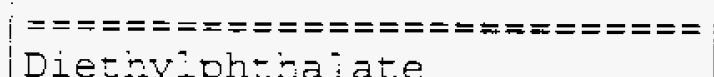 & 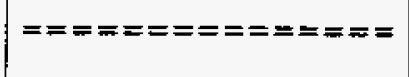 & $==============$ & $========$ \\
\hline 4-Chl crophenyl-phenylether & & 0.82283612 & -0.094 \\
\hline 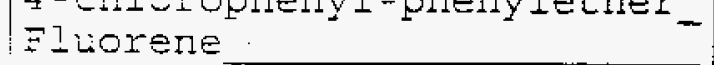 & & $\begin{array}{l}1.85737778 \\
0.90906400\end{array}$ & $\begin{array}{l}-0.722 \\
-0.146\end{array}$ \\
\hline$\leq-N i t r o a n i$ ine & & 2.73990161 & $\begin{array}{l}-0.086 \\
-0.08\end{array}$ \\
\hline 4,6-Dinitro-2-Methylphenol & & 5.39536402 & 0.202 \\
\hline N-Nitrosodiphenylamine (1) & & 2.35451460 & $-0.108 *$ \\
\hline 4-Bromophenyl-phenylether & & 5.55523153 & -0.020 \\
\hline Hexachlorobenzene_ & & 4.45917457 & -0.112 \\
\hline Pentactiorophenol & & 7.99719951 & $0.418 *$ \\
\hline Enenanthrene_ & & 0.98964634 & -0.054 \\
\hline An:hracene & & $I .03680362$ & -0.121 \\
\hline Carbazole & & 1.39829821 & -0.216 \\
\hline D-n-Butyiphthalate & & 0.74910265 & -0.023 \\
\hline Fiuoranzinen & & 0.97927991 & $-0.064 *$ \\
\hline Pyrene & & 0.53514898 & 0.436 \\
\hline Buty-oenzylphthalate & & 0.84666250 & 0.527 \\
\hline 3,3'-Dichorobenzidine & & 2.32046156 & 0.424 \\
\hline Benzo:aiAnthracene & & 0.57753597 & 0.432 \\
\hline Corysene & & 0.74508030 & 0.379 \\
\hline bis $(2-E$ thy hexyz) Phthalate & & 0.67307367 & 0.454 \\
\hline Di-n-octyl Phthalate. & & 0.46230539 & $0.104 *$ \\
\hline Benzo bi Eluoranthene & & 0.62599099 & 0.248 \\
\hline Eerzoik: Fluoranthene. & & I. 47558223 & -0.516 \\
\hline Benzola Pyrene. & & 0.98426957 & $0.005 *$ \\
\hline Indero $(1,2,3-c \bar{d}$ Pyrene & & 0.87663119 & 0.229 \\
\hline Dibenz (a,h) Anthracene, & & 1.11521021 & -0.025 \\
\hline Benzolg, h, i) Perylene & & ב. 08497534 & 0.004 \\
\hline 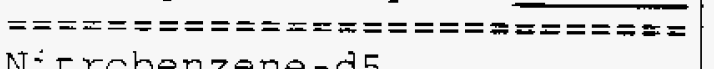 & $== \pm===$ & 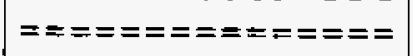 & $=======\equiv=$ \\
\hline $\begin{array}{l}\mathrm{N} \text { - crobenzene-d5 } \\
z-F \text {. }\end{array}$ & & 2.97606862 & 0.031 \\
\hline $\begin{array}{l}\text {-Finorobiphenyl } \\
\text { merphenyl-dat }\end{array}$ & & 0.85843953 & -0.124 \\
\hline Phenol-d5 & & 0.79726898 & 0.403 \\
\hline 2-Fluoropherol & & $\begin{array}{l}0.66912547 \\
0.79562169\end{array}$ & $\begin{array}{r}0.032 \\
-0.026\end{array}$ \\
\hline ribrome & & 5.09441080 & -0.136 \\
\hline
\end{tabular}


Jab Name: QUANTERRA MO

ab Code: ITMO

instrument ID: $\mathrm{MSH}$

ab File ID: H6307
Contract: $625-01$

Case No.: S16103 SAS No.:
SDG No.: $V 368$

Calibration date: 03/31/98 Time: 0829

Init. Calib. Date(s): 03/26/98

$03 / 26 / 98$

Init. Calib. Times: 1642

1927

\begin{tabular}{|c|c|c|c|c|c|}
\hline & MIN & TRUE & ANALYZ & & MAX \\
\hline RRF & RRF & CONC & CONC & $\because D$ & $\because D$ \\
\hline$=====$ & $=====$ & $=====$ & $== \pm==$ & $=== \pm=$ & $F===$ \\
\hline $\begin{array}{l}1.646 \\
7.284\end{array}$ & & 25.0 & $\begin{array}{l}24 \cdot 5 \\
23\end{array}$ & $2 \cdot \frac{1}{7}$ & 20.0 \\
\hline $\begin{array}{l}1.284 \\
1.406\end{array}$ & & $\begin{array}{l}25.0 \\
25.0\end{array}$ & $\begin{array}{l}23.2 \\
24.9\end{array}$ & $7 \cdot 3$ & \\
\hline 1.461 & & 25.0 & 26.4 & 5.5 & \\
\hline 1.453 & & 25.0 & 26.8 & 7.2 & 20.0 \\
\hline 1.394 & & 25.0 & 26.5 & 6.1 & \\
\hline 1.176 & & 25.0 & 26.0 & 3.8 & \\
\hline 1.080 & & 25.0 & 28.3 & 13.3 & \\
\hline 1.261 & & 25.0 & 25.6 & 2.4 & \\
\hline 0.795 & 0.050 & 25.0 & 30.0 & 20.1 & \\
\hline 0.615 & & 25.0 & 27.1 & 8.5 & \\
\hline 0.344 & & 25.0 & 28.5 & 14.1 & \\
\hline 0.679 & & 25.0 & 27.5 & 9.9 & \\
\hline 0.244 & & 25.0 & 25.5 & 1.9 & 20.0 \\
\hline 0.333 & & 25.0 & 26.7 & 6.6 & \\
\hline 0.462 & & 25.0 & 26.6 & 6.4 & \\
\hline 0.333 & & 25.0 & 25.9 & 3.6 & 20.0 \\
\hline 0.335 & & 25.0 & 26.3 & 5.0 & \\
\hline 1.083 & & 25.0 & 27.2 & 8.8 & \\
\hline 0.495 & & 25.0 & 31.8 & 27.3 & \\
\hline 0.156 & & 25.0 & 27.8 & 11.3 & 20.0 \\
\hline 0.310 & & 25.0 & 27.2 & 8.7 & 20.0 \\
\hline 0.665 & & 25.0 & 25.9 & 3.5 & \\
\hline 0.310 & 0.050 & 25.0 & $26 \cdot 3$ & 5.4 & \\
\hline 0.418 & & 25.0 & 25.1 & .3 & 20.0 \\
\hline 0.443 & & 25.0 & 24.1 & 3.4 & \\
\hline 1.209 & & 25.0 & 26.1 & 4.5 & \\
\hline 0.349 & & 25.0 & 27.9 & 11.5 & \\
\hline 1.227 & & 25.0 & 23.8 & 4.6 & \\
\hline 1.833 & & 25.0 & 26.1 & 4.4 & \\
\hline 0.328 & & 25.0 & 24.5 & 2.2 & \\
\hline 0.415 & & 25.0 & 29.5 & 17.8 & \\
\hline 1.117 & & 25.0 & 25.5 & 1.8 & 20.0 \\
\hline 0.158 & 0.050 & 25.0 & 23.8 & 4.7 & \\
\hline 0.214 & 0.050 & 25.0 & 25.9 & 3.5 & \\
\hline 1.688 & & 25.0 & 25.7 & 2.9 & \\
\hline 0.439 & & 25.0 & 24.6 & 1.6 & \\
\hline
\end{tabular}

FORM VII BNA 
Lab Name: QUANTERRA MO

Lab Code: ITMO

Instrument ID: MSH

Lab File ID: H6307
Case No.: S16103 SAS No.:

Calibration date: 03/31/98 Time: 0829

Init. Calib. Date(s): $03 / 26 / 98$

$03 / 26 / 98$

Init. Calib. Times: 1642

1927

\begin{tabular}{|c|c|c|c|c|c|c|}
\hline COMPOUND & RRF & $\begin{array}{l}\text { MIN } \\
\text { RRF }\end{array}$ & $\begin{array}{l}\text { TRUE } \\
\text { CONC }\end{array}$ & $\begin{array}{c}\text { ANALYZ } \\
\text { CONC }\end{array}$ & $\because \mathrm{D}$ & $\begin{array}{l}\text { MAX } \\
\div D\end{array}$ \\
\hline 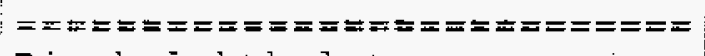 & $== \pm==$ & $====$ & $===\equiv==$ & $=====$ & $====$ & $====$ \\
\hline Diethyiphthalate & 1.326 & & 25.0 & 25.4 & 1.4 & \\
\hline 4-Chlorophenyl-phenylether & 0.597 & & 25.0 & 25.3 & 1.1 & \\
\hline Ficiorerle & 1.264 & & 25.0 & 25.8 & 3.2 & \\
\hline 4 -Nitroaniline & 0.416 & & 25.0 & 26.8 & 7.1 & \\
\hline 4,6-Dinitro-2-Methylphenol. & 0.166 & & 25.0 & 26.4 & 5.5 & \\
\hline N-Nitrosodiphenylamine (I). & 0.520 & & 25.0 & 28.5 & 13.8 & 20.0 \\
\hline 4-Bromophenyl-phenylether & 0.196 & & 25.0 & 26.8 & 7.2 & \\
\hline Hexachlorobenzene & 0.255 & & 25.0 & 26.3 & 5.0 & \\
\hline PentachIorophenol. & 0.077 & & 25.0 & 23.7 & 5.3 & 20.0 \\
\hline Shenanthrene. & 1.129 & & 25.0 & 26.9 & 7.4 & \\
\hline Anthracene & 1.142 & & 25.0 & 27.2 & 8.6 & \\
\hline Carbazole. & 1.004 & & 25.0 & 30.8 & 23.2 & \\
\hline Di-n-Butyiphthalate. & 1.365 & & 25.0 & 25.1 & .4 & \\
\hline Eluoranthene. & 1.118 & & 25.0 & 26.1 & 4.4 & 20.0 \\
\hline Pyrene & 1.276 & & 25.0 & 25.8 & 3.2 & \\
\hline Butylbenzylphthalate & 0.760 & & 25.0 & 26.6 & 6.5 & \\
\hline $3,3^{\prime}$-Dichlorobenzidine & 0.403 & & 25.0 & 31.9 & 27.5 & \\
\hline Benzo (a) Anthracene & 1.156 & & 25.0 & 25.3 & I. 3 & \\
\hline Chrysene & 1.074 & & 25.0 & 27.6 & 10.3 & \\
\hline bis (2-Ethylhexyl) Phthalate & 1.030 & & 25.0 & 26.4 & 5.6 & \\
\hline Di-n-octyl Phthalate & 2.157 & & 25.0 & 27.0 & 8.0 & 20.0 \\
\hline Benzo (b) Fluoranthene & 1.268 & & 25.0 & 24.8 & .8 & \\
\hline Benzo (k) Fluoranthene. & 1.128 & & 25.0 & 30.9 & 23.7 & \\
\hline Benzo (a) Pyrene. & I. 075 & & 25.0 & 26.6 & 6.2 & 20.0 \\
\hline Indeno $(1,2,3$-coj) Pyrene & 0.932 & & 25.0 & 25.0 & 0.0 & \\
\hline Dibenz (a, h) Anthracene & 0.896 & & 25.0 & 24.5 & 2.0 & \\
\hline Benzo (g, h, i) Perylene. & 0.892 & & 25.0 & 24.3 & 2.9 & \\
\hline 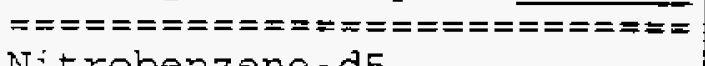 & $=====$ & $==\approx=\neq$ & $=====$ & $===-\approx \neq$ & $====$ & $== \pm=$ \\
\hline Nitrobenzene-d5 & 0.378 & & 25.0 & 28.7 & 14.9 & \\
\hline 2-Fluorobiphenyl & 1.294 & & 25.0 & 25.3 & 1.2 & \\
\hline TerphenyI-dI4 & 0.872 & & 25.0 & 25.5 & 1.8 & \\
\hline Phenol-d5 & 1.482 & & 25.0 & 25.4 & 1.8 & \\
\hline 2-Eluorophenol & 1.228 & & 25.0 & 23.9 & 4.3 & \\
\hline $2,4,6$-Tribromophenol. & 0.217 & & 25.0 & 24.9 & .2 & \\
\hline
\end{tabular}


Jab Name: QUANTERRA MO

ab Code: ITMO Case No.: $\$ 16103$ SAS No.:

uab File ID (Standard): $\mathbf{H 6 3 0 7}$

nstrument. ID: MSH
Contract : $625-01$

SDG No.: V368

Date Analyzed: $03 / 31 / 98$

Time Analyzed: 0829

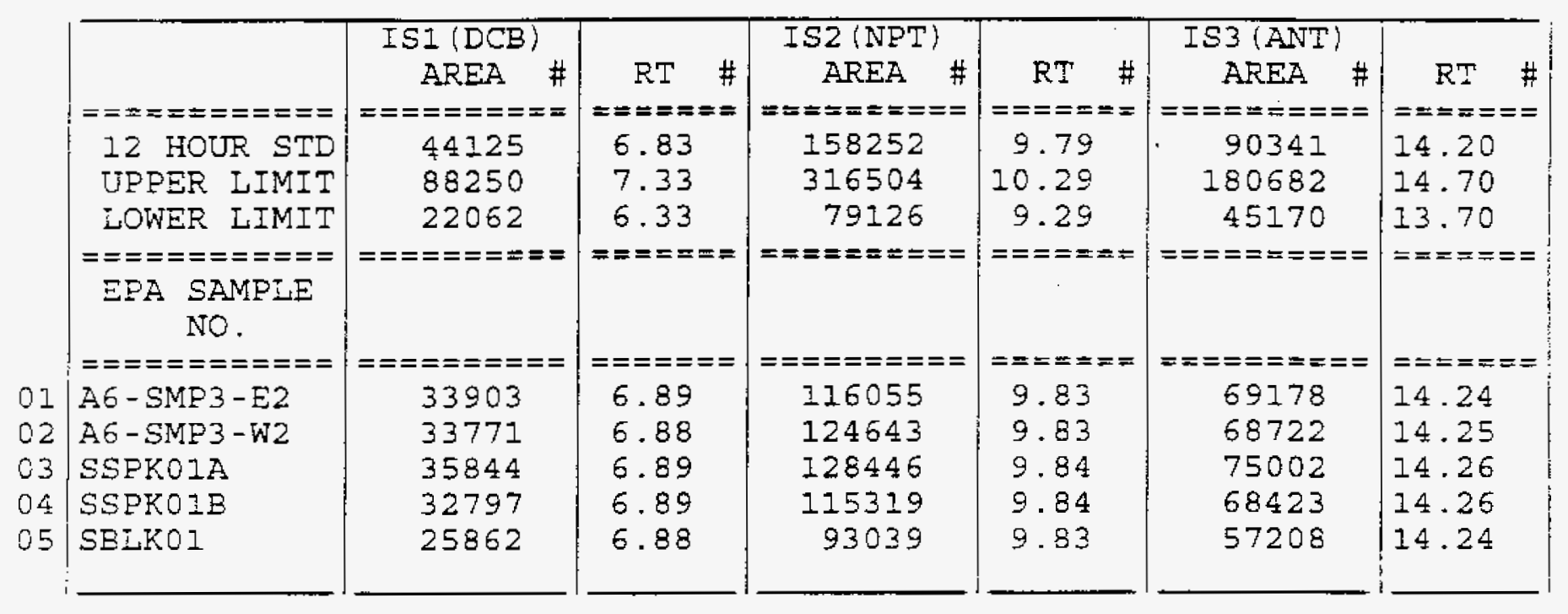

IS: $(D C B)=1,4-$ Dichlorobenzene-d4

IS2 $\left(\mathrm{NP}_{-}^{\mathrm{m}}\right)=$ Naphthalene-d8

IS3 $($ ANT $)=$ Acenaphthene-dio

AREA UPDER LIMIT $=+100 \%$ of internal standard area.

AREA LOWER LIMIT $=-50 \%$ of internal standard area.

RT JPPER LIMIT $=\div 0.50$ minutes of internal standard RT.

$\mathrm{R}$. LOWER LIMIT $=-0.50$ minutes of internal standard RT.

\# Column used to flag internal standard area values with an asterisk.

* Values outside of QC limits. 
Lab Name: QUANTERRA MO

Lab Code: ITMO

Case No.: S16103

Lab File ID (Standard): H6307

Instrument ID: MSH
Contract: $625-01$

SAS NO.:

SDG No.: V368

Date Analyzed: 03/31/98

Time Analyzed: 0829

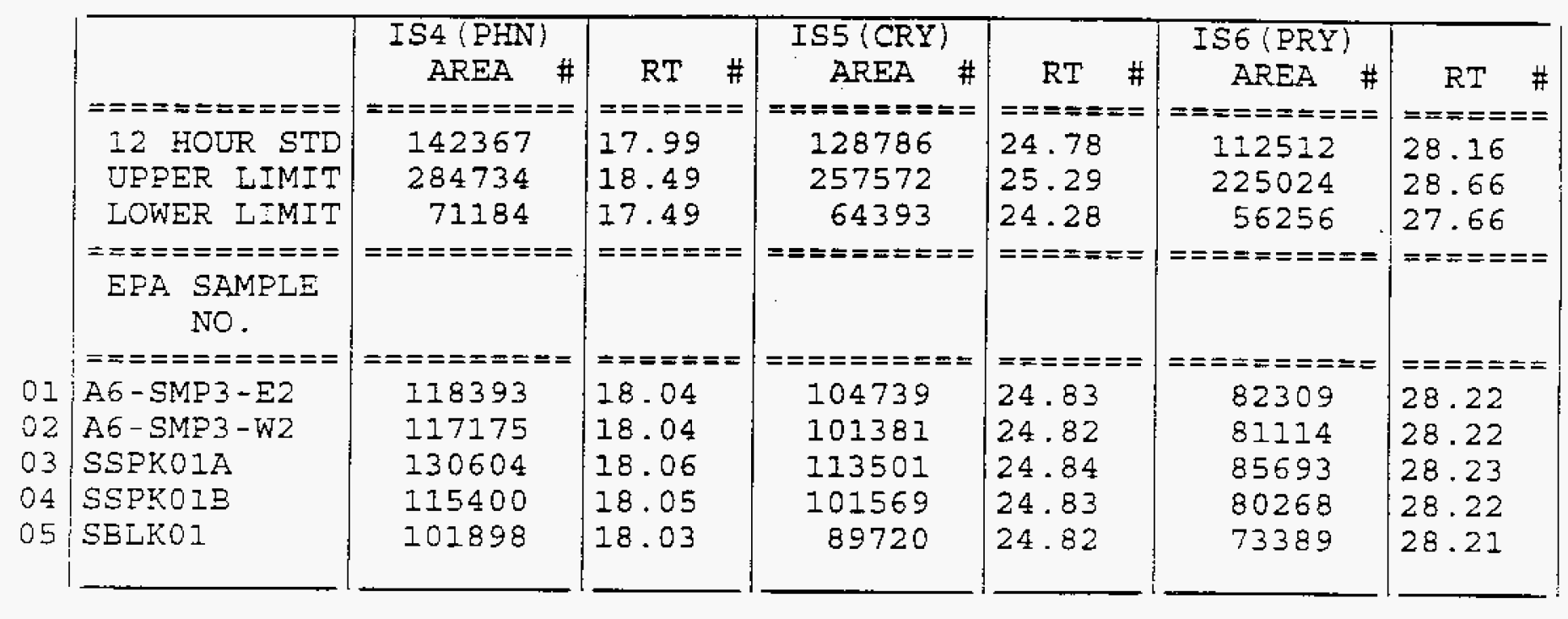

IS4 (FHN) = Phenanthrene-dlo

ISE (CRY) = Chrysene-dI2

IS6 $($ PRY $)=$ Peryiene-diz

AREA UPFER I IMIT $=+100 \%$ of internal standard area.

AREA LOWER LIMIT = - 50\% of internaI standard area.

RT UPPER LIMIT $=+0.50$ minutes of internal standard RT.

RT LOWER LIMIT $=-0.50$ minutes of internal standard RT.

\# Columr used to flag internal standard area values with an asterisk.

* Values outside of QC limits. 
$9 C-\operatorname{LCS}$

SOIL SEMIVOLATILE LCS RECOVERY

ab Name: ITAS-ST.LOUIS

ab code: ITSL

IPA Sample No.; SSPKO1A
Contract: $625-01$

SAS NO.:

Level: (Iow/med) LOW

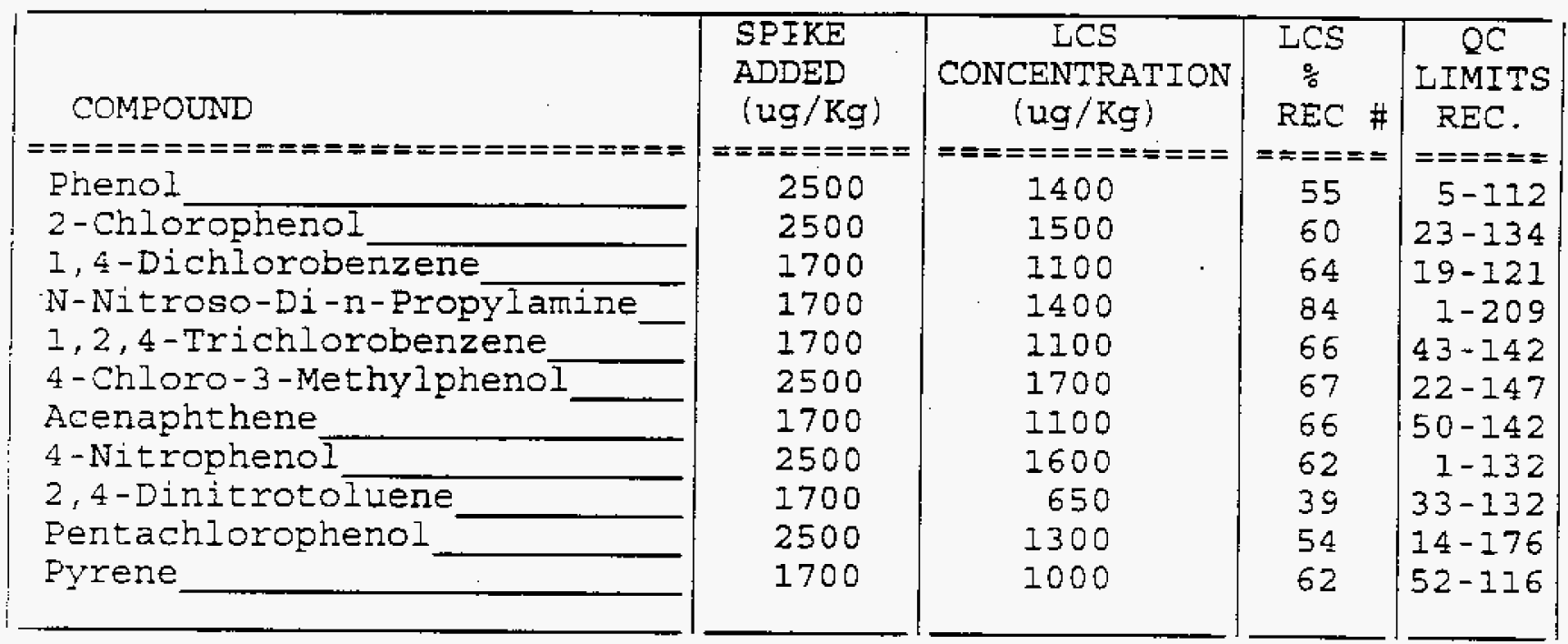

\# Columr to be used to flag recovery values with an asterisk.

* Values outside of QC limits.

D Detected. 
9C-ICS

SOIL SEMIVOLATILE LCS RECOVERY

Lab Name: ITAS-ST.LOUIS

Contract: $625-01$

Lab Code: ITSI

Case No.: 516103

SAS NO. :

SDG No.: V368

EPA Sample No.: SSPKOIB

Level: (low/med) LOW

\begin{tabular}{|c|c|c|c|c|}
\hline COMPOUND & $\begin{array}{l}\text { SPFKE } \\
\text { ADDED } \\
(\mathrm{ug} / \mathrm{Kg})\end{array}$ & $\begin{array}{c}\text { LCS } \\
\text { CONCENTRAT ION } \\
(\mathrm{ug} / \mathrm{Kg})\end{array}$ & $\begin{array}{l}\text { LCS } \\
\frac{\circ}{1} \\
\text { REC \# }\end{array}$ & $\begin{array}{c}Q C \\
\text { LIMITS } \\
\text { REC. }\end{array}$ \\
\hline$==========$ & $=\mathrm{x}=\mathrm{=}=\mathrm{=}=\mathrm{x}=$ & 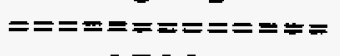 & $=== \pm=x$ & $=====$ \\
\hline Phenol & 2500 & 1700 & 67 & $5-112$ \\
\hline 2-Chlorophenol & 2500 & 1800 & 72 & $23-134$ \\
\hline chlorobenzene & 1700 & 1300 & 77 & $19-121$ \\
\hline N-Nitroso-Di-n-Propylamine & 1700 & 1600 & 98 & $1-209$ \\
\hline - Trichlorobenzene & 1700 & 1300 & 80 & $43-142$ \\
\hline 4-Chloro-3-Methylpl & 2500 & 2100 & 84 & $22-147$ \\
\hline ohthene & 1700 & 1400 & 81 & $50-i 42$ \\
\hline 4-Nitrophenol. & 2500 & 1900 & 75 & $1-132$ \\
\hline 2,4-Dinitrotoluene & 1700 & 850 & $5 I$ & $33-132$ \\
\hline Pentachlo & 2500 & 1700 & 66 & $14-176$ \\
\hline Pyrer & \pm 700 & 1200 & 73 & $52-116$ \\
\hline
\end{tabular}

\# Column to be used to flag recovery values with an asterisk.

- Values outside of QC limits.

D Detected. 
$1 \mathrm{D}$

PCB ORGANICS ANALYSIS DATA SHEET
EPA SAMPLE NO.

A6-SMP 3-W2

Lab Name:
Lab Code: ITMO Case No.:

Matrix: (soil/water) soli

Sampie $w t / v o i: \quad 30.2\left(g / \mathrm{ml}^{2}\right) \mathrm{G}$

Level: (low/med) Low

․ Moisture: not dec.

dec.

Extraction: (SepF/Cont/Sonc)

Contract: 625.01

SAS NO.:

SDG No.: V368

GPC Cleanup: $(\mathrm{Y} / \mathrm{N})$

pH:

SONC Date Analyzed:

$17161-004$

Lab Sample ID:

Lab File ID:

Date Samplec:

$03-10-98$

Date Extracted:

$03-17-98$

Date Analyzed: $\quad 03-20-98$

CAS NO.

Compound

Dilution Factor:

1

CONCENTRATION UNITS:

( $\mathrm{ug} / \mathrm{L}$ oI $\mathrm{ug} / \mathrm{kg}) \mathrm{JG} / \mathrm{KG}$

$Q$

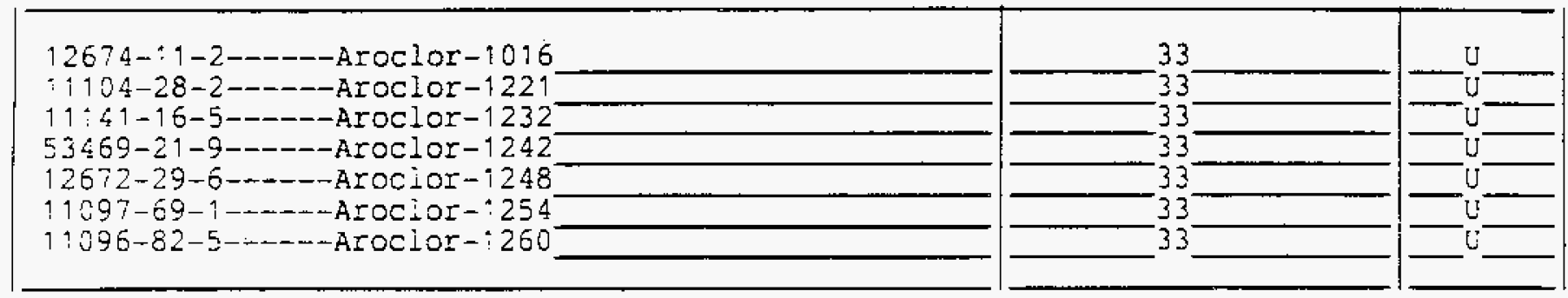

U: Concentration of analyte is less than the value given.

FORM I PEST 
ab Name: $\quad$ OUANTERRA.MO Contract: ab Code: ITMO Case No.: atrix: (soil/water) SOIL ample wt/vol: $30.0(\mathrm{~g} / \mathrm{ml})$ eve1: (low/med) Low Moisture: not dec. xtraction: (SepF/Cont/Sonc) PC cleanup: $(Y / N)$ CAS $\mathrm{NO}$ Compound dec.

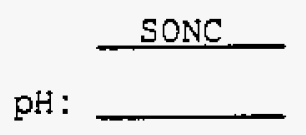

\section{PBLKO 1}

\section{SAS NO. :}

SDG NO.: V368

Lab Sample ID: $\quad$ BLK :67391

Lab File ID:

Date Sampied:

Dete Extracted:

$03-17-98$

Date Aralyzed:

$03-20-98$

Dilution Factor: 1 CONCENTRATION UNITS:

(Ug/L or $u g / \mathrm{Kg}) \mathrm{UG} / \mathrm{KG} \quad Q$

$12674-11-2------A I$ OClOr-10 6

i $1104-28-2------A I O C$ OI -1221

$: 1141-16-5-----A$ IOC 1 OI- 1232

$53469-21-9----\infty A I O C+$ OI 1242

$12672-29-6------A r o c l$ or -1248

$11097-69-i-----A I O C i$ OI -1254

i $1096-82-5-----A r O C l o r-1260$.

Concentration of analyte is less than the vaiue given.

FORM I PEST 


\section{ID \\ PCB ORGANICS ANALYSIS DATA SHEET}

EPA SAMPLE NO.

Lab Name: QUANTERRA, MO

Contract: $\quad 625.01$

\section{PSPK01A}

Lab Code: ITMO Case No.:

SAS NO.:

SDG No.: $\mathrm{V} 368$

Matrix: (soil/water) SOLL

Sample wt/vol: $\quad 30 . \dot{0}(\mathrm{~g} / \mathrm{ml}) \mathrm{G}$

\section{Level: (low/med) LOW}

: Moisture: not dec.

Extraction: (SepF/Cont/Sonc)

GPC Cleanup: (Y/N)

CAS NO.

Compound dec.

SONC
Lab Sample ID:

Lab File ID:

Date Sampled:

Date Extracted: - 03-17-98

Date Analyzed: 03-20-98

\section{SPK 167391-1}

Dilution Factor:
CONCENTRATION UNITS:

(ug/L or $u g / \mathrm{kg}$ ) UG/KG
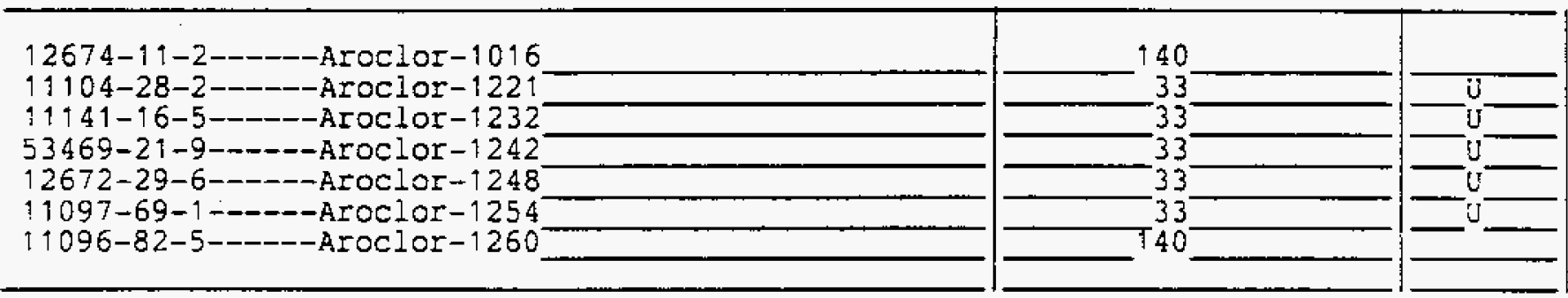

$\mathrm{U}$ : - Concentration of analyte is less than the value given. 
10

PCB ORGANICS ANALYSIS DATA SHEET
EPA SAMPLE NO.

PSPKO1B

Dame: QUANTERRA,MO Contract: 625.01

PSPKO1B

ab Code: ITMO Case No.:

SAS NO.:

SDG NO.: $\vee 368$

at =ix: (soil/water) So:L

impie $w t / v o l: \quad 30.0(\mathrm{~g} / \mathrm{mI}) \mathrm{G}$

svel : (low/med) Low

Moisture: not dec.

dec.

:tracrion: (SepF/Cont/Sonc)

'C Cieanup: $(\mathrm{Y} / \mathrm{N})$

$\mathrm{pH}:$

SONC Date Analyzed:

SPK $\quad 16739 i-2$

tab Sample ID:

Lab File ID:

Date Sampled:

Date Extracted:

$03-17-98$

CAS NO.

Compound

Dilution Factor:

$03-20-98$

$\mathrm{oH}:$

CONCENTRATION UNITS:

(ug/L or $\mathrm{ug} / \mathrm{Kg}) \mathrm{UG} / \mathrm{KG}$

$Q$

i $2674-1$ i-2--D-Aroclor-1016

$11 \uparrow 04-28-2-m---A r o c l o r-1221$

$11141-16-5$-...-Aroc lor-1232

$53469-21-9-\ldots-$-Aroclor- $: 242$

12672-29-6-----Aroclor-1248

$11097-69-1$-_-Aroclor-1254

$11095-82-5-\ldots-$ Aroclor- $\$ 260$

Concentracion of anaiyte is less than the value given.

FORM I PEST 
SOIL PCB SURROGATE RECOVERY

Lab Name: OUANTERRA, MO

Contract: 625.01

Lab Code: ITMO Case No.

SAS NO. : SDG NO.: $\mathrm{V} 368$

Level: (low/med) LOW

\begin{tabular}{|c|c|c|c|}
\hline & $\begin{array}{l}\text { EPA } \\
\text { SAMPLE NO. } \\
=====\equiv==== \pm=====\end{array}$ & $\begin{array}{c}S 1 \\
(\mathrm{DCB}) \\
=====\end{array}$ & $\begin{array}{c}\mathrm{S2} \\
(\mathrm{TCMX}) \# \\
=\approx \approx===\end{array}$ \\
\hline 1 & PBLKO1 & 90 & 110 \\
\hline & DSPKO1 & 86 & $109^{-}$ \\
\hline & PSPKOIB & $-86=$ & 二112 \\
\hline & A6-SMP $3-\overrightarrow{E 2}$ & $83-$ & $-109-$ \\
\hline & $A 6-S M P 3-W 2$ & $-{ }^{86}$ & -108 \\
\hline & & & \\
\hline & & & \\
\hline
\end{tabular}

S1 $(D C B)=$ Decachlorobiphenyl

ADVISORY

QC LIMITS

S2 $($ TCMX) = Tetrachlorom-m-xylene

$(6 \uparrow-135)$

$(61-153)$

\# Columr to be used to flag recovery values

* Values outside of QC Iimits

D Surrogates diluted out

M Matrix interference

page 1 of 1 
ab Name: OUANTERRA, MO

ab code: ITMO Case No.:
Contract:

625.01 SDG No. :

$\mathrm{V} 368$

pike Blank No.:

SPK $167391-1$

SAS NO. : $+2$

\begin{tabular}{|c|c|c|c|c|}
\hline $\begin{array}{l}\text { COMPOUND } \\
===================== \\
\text { Aroclor }-1016 \\
\text { Arocior }-1260\end{array}$ & $\begin{array}{l}\text { SPIKE } \\
\text { ADDED } \\
(\mathrm{ug} / \mathrm{Kg}) \\
====== \pm \\
170 \\
\end{array}$ & $\begin{array}{c}\text { SPIKE } \\
\text { CONCENTRARION } \\
\text { (ug } / \mathrm{Kg} \text { ) } \\
========== \\
140 \\
140\end{array}$ & $\begin{array}{c}\text { SPK } \\
\text { REC \# } \\
\text { REC \# } \\
===== \\
86 \\
84\end{array}$ & $\begin{array}{l}\text { QC } \\
\text { LIMITS } \\
\text { REC. } \\
===== \\
80-139 \\
59-142\end{array}$ \\
\hline
\end{tabular}

\# Column to be used to flag recovery and RPD values with an asterisk

* Values outside of QC limits

ND: not determined

Spike Recovery:

out of 2 outside limits COMMENTS :

FORM III PEST-2 
SOIL PCB SPIKE BLANK(LCS) RECOVERY

Lab Name: QUANTERRA, MO

Lab Code: ITMO Case No.:

Spike Blank No.:

SPK $167391-2$
Contract : 625.01

SAS NO. : SDG NO.: $\quad$ V368

\begin{tabular}{|c|c|c|c|c|}
\hline $\begin{array}{l}\text { COMPOUND } \\
======================= \\
\text { Aroctor } 1016 \\
\text { Aroclor }-1260\end{array}$ & $\begin{array}{l}\text { SPIKE } \\
\text { ADDED } \\
(\mathrm{wg} / \mathrm{Kg}) \\
==== \pm== \\
170 \\
170\end{array}$ & $\begin{array}{c}\text { SPIKE } \\
\text { CONCENTRATION } \\
(1 \mathrm{~g} / \mathrm{Kg}) \\
========== \pm \\
\uparrow 40 \\
150\end{array}$ & $\begin{array}{c}\text { SPK } \\
5 \\
\text { REC \# } \\
===== \\
85 \\
93\end{array}$ & $\begin{array}{c}\text { QC } \\
\text { LIMITS } \\
\text { FEC. } \\
== \pm= \\
80-139 \\
59-142\end{array}$ \\
\hline
\end{tabular}

\# Column to be used to flag recovery and RPD vaiues with an asterisk

* Vaiues cutside of QC limits

ND: not determined

Spike Recovery: 0 out of 2 outside Imits COMMENTS: 
ab Name: OUANTERRA, MO

ab Code: ITMO Case No.:

ab Sample ID: BLK 167391

atrix:(soil/water)

SOIL

ate Extracted:

$03-17-98$

ate Analyze

$03-20-98$

ime Analyze

nstiment I

C Column ID

Contract: 625.01

SAS NO. :

SDG No : V 368
Lab File ID:
Level (low/med) Low
Date Analyzed (2):
Time Aralyzed (2):
Instiunent ID (2):
GC Colunn ID (2):

Extraction: (SepF/Cont/Sonc)

SONC

THIS METHOD BLANK APPLIES TO THE FOLLOWING SAMPLES, MS AND MSD:

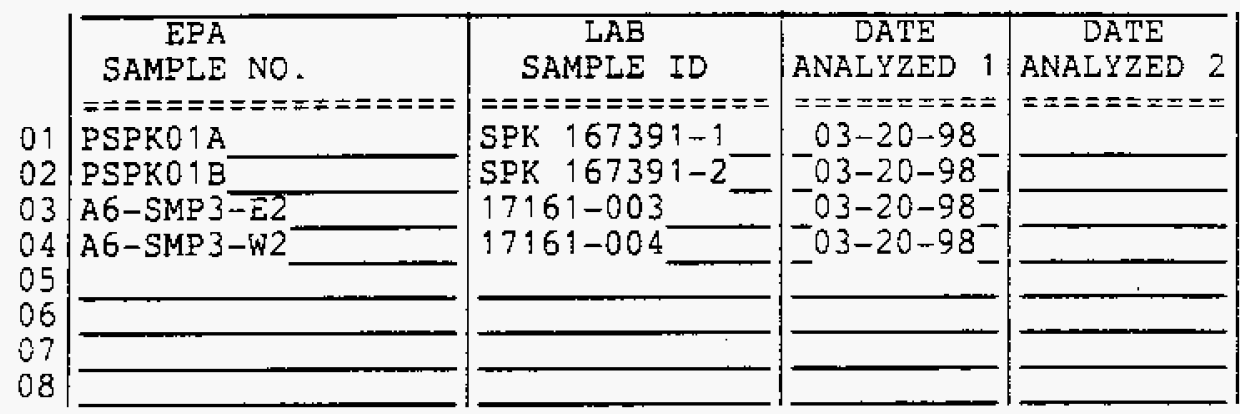

DMMENTS:

ge 1 of 1 
Lab Name: QUANTERRA, MO

Lab Code: ITMO Case No.:

SAS NO.:

Contract: 625.01

Instrument ID: GCE

Calibration Date(s): 02-05-98

to

to
SDG No.: V368

GC Column ID: DB-5MS

\begin{tabular}{|c|c|c|c|c|c|c|}
\hline $\begin{array}{l}\text { COMPOUND } \\
==\approx========== \\
\text { AR } 1221 \\
\text { AR } 1232 \\
\text { AR } 1248 \\
\text { AR } 1254 \\
\text { AR } 1250 \\
\text { AR:016/AR } 1242 \\
\text { TCMX } \\
\text { DCB }\end{array}$ & $\begin{array}{l}\text { CALIBRAT. } \\
\text { FACTOR } \\
\text { LEVEL } \# 1 \\
======= \\
-563120^{-} \\
-1526460^{-} \\
-1676160^{-} \\
-1130860^{-} \\
-2470050^{-} \\
T 1732400^{-} \\
9610000^{-}\end{array}$ & $\begin{array}{l}\text { CALIBRAT. } \\
\text { FACTOR } \\
\text { LEVEL } \$ 2 \\
======= \\
588260^{-} \\
-1457295 \\
-1689425^{-} \\
-1094980^{-} \\
-2187500^{-} \\
11046600^{-} \\
9458300^{-}\end{array}$ & \begin{tabular}{|c|} 
CALIBRAT \\
FACTOR \\
LEVEL \#3 \\
$========$ \\
$492152-$ \\
1300234 \\
$-1393066^{-}$ \\
$-909842^{-}$ \\
$1797960^{-}$ \\
T0503480- \\
$8404520^{-}$
\end{tabular} & \begin{tabular}{|l|} 
CALIBRAT - \\
FACTOR \\
LEVEL \#4 \\
$=======$ \\
$-434461-$ \\
T193467 \\
$-1256121^{-}$ \\
$-850514^{-}$ \\
-1654048 \\
$-9182220^{-}$ \\
$-7630280^{-}$
\end{tabular} & $\begin{array}{l}\text { CALIBRAT. } \\
\text { FACTOR } \\
\text { LEVEL \#5 } \\
======== \\
383662 \\
7295264- \\
-710093- \\
-782664- \\
-778504^{-}\end{array}$ & $\begin{array}{l}\text { CALIBRAT. } \\
\text { FACTOR } \\
\text { LEVEL \#6 } \\
==x===== \\
966789- \\
670099- \\
-6966745- \\
-5850890^{-6}-\end{array}$ \\
\hline $\begin{array}{l}\text { COMPOUND } \\
============ \\
\text { AR } 1221 \\
\text { AR } 1232 \\
\text { AR } 1248 \\
\text { AR } 1254 \\
\text { AR } 1260 \\
\text { AR } 1016 / \mathrm{AR} 1242 \\
\text { TGMX } \\
\text { SCB }\end{array}$ & $\begin{array}{l}\text { CALIBRAT. } \\
\text { FACTOR } \\
\text { LEVEL } \# 7 \\
========\end{array}$ & 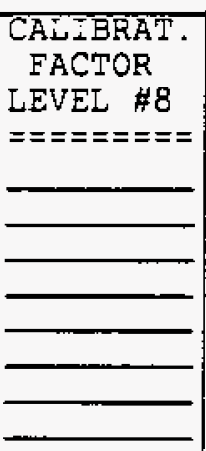 & $\begin{array}{l}\text { CAL ERAT. } \\
\text { FACTOR } \\
\text { LEVEL \#9 } \\
========\end{array}$ & \begin{tabular}{|l|} 
CALIBRAT - \\
FACTOR \\
LEVEL $\# 10$ \\
$========$
\end{tabular} & \begin{tabular}{|c|} 
AVERAGE \\
CALBRAT. \\
FACTOR \\
$========$ \\
492331 \\
${ }^{1289918}$ \\
-1424973 \\
-906493 \\
CURVE \\
8190798 \\
-9886169
\end{tabular} & 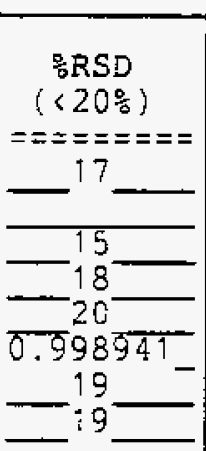 \\
\hline
\end{tabular}

Under AVERAGE CAIIBRATION FACTOR, enter the average of the calibration factors.

The :RSD nust be iess than $20 \%$ for the average calibration factor to be used. If the FRSD is $220 \%$, a Curve calibration must be used. In this case, enter "CuRve" under AVERAGE CALIBRATION FACTOR instead of the average cailibration factor.

* Only low ievel was anaiyzed.

FORM VI PCB-1 
$8 \mathrm{E}$

PCB ANALYTICAL SEQUENCE SUMMARY

ab Name: OUANTERRA. MO

ab code: ITMO Case No.: nstrument ID: GCE ates of Analyses: $03-20-98$
Contract: 625.01

SAS No. SDG NO.: $\mathrm{V} 368$ GC Column ID: DB-5MS to $03-20-98$

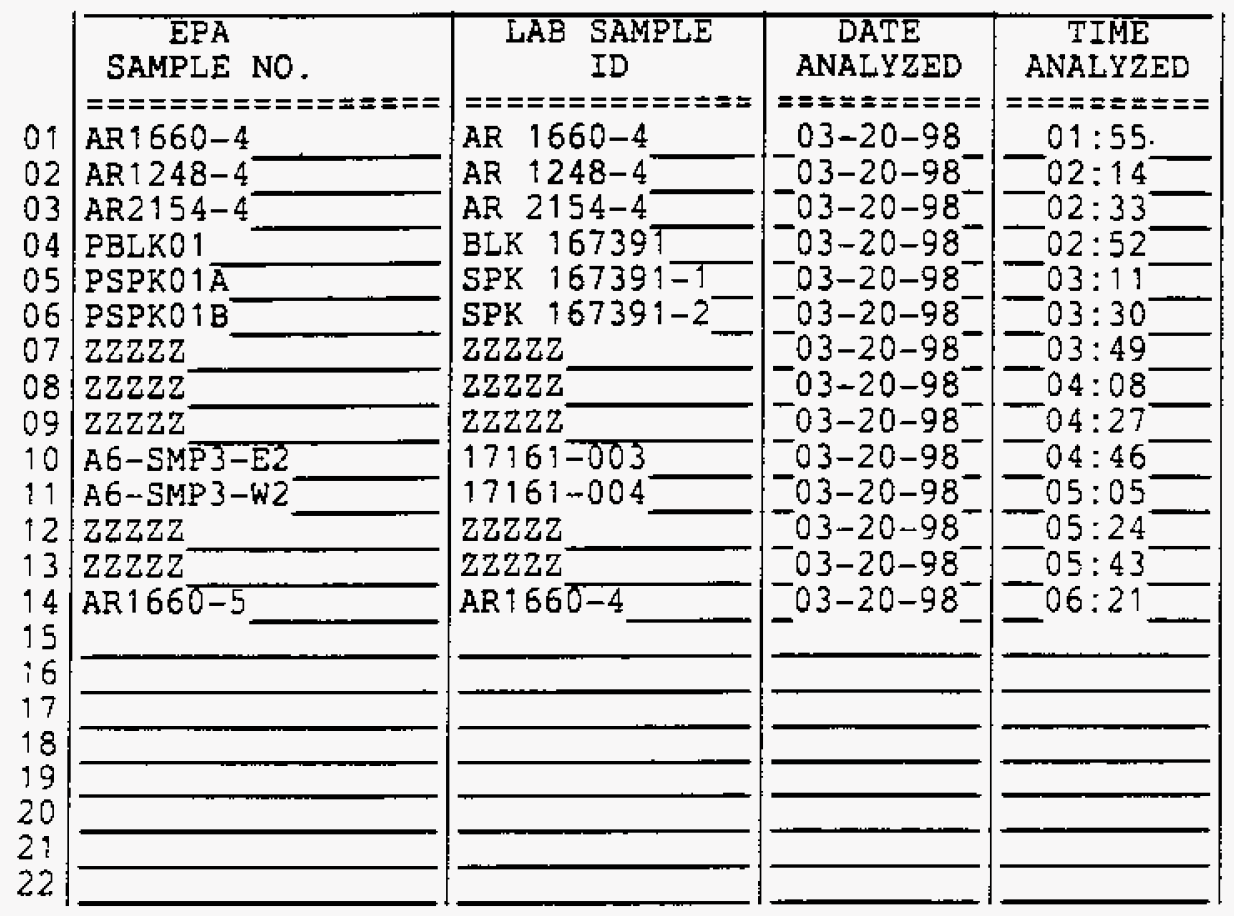

FORM VIII PEST-3 


\section{9 \\ PCB STANDARDS SUMMARY}

Lab Name: QUANTERRA, MO

Lab Code: ITMO Case No.: Instrument ID: GCE
Contract : 625.01

SAS No. SDG NO.:

GC COlumn ID: DB-5MS
DATE OF ANALYSIS
TIME OF ANALYSIS
$0155-0233$

EPA SAMPLE NO.

(STANDARD)

DAILY STDS

\begin{tabular}{|c|c|c|c|}
\hline $\begin{array}{c}\mathrm{RT} \\
====== \\
-3.63 \\
-5.72^{-} \\
-9.79^{-} \\
-9.45^{-} \\
\mathrm{T} 3.03^{-} \\
2.98^{-} \\
T 4.93^{-}\end{array}$ & $\begin{array}{c}\text { CALIBRATION } \\
\text { FACTOR } \\
========== \\
498927 \\
-304415 \\
-300403 \\
\text { CURVE } \\
849635 \\
331260 \\
838360\end{array}$ & $\begin{array}{c}\mathrm{QNT} \\
\mathrm{Y} / \mathrm{N} \\
=== \\
\mathrm{Y}\end{array}$ & $\begin{array}{l}\square \mathrm{D} \\
==== \\
1 \\
-9 \\
-7 \\
-6 \\
-6\end{array}$ \\
\hline
\end{tabular}

Uñer AVERAGE CALIBRATION FACTOR, encer the average calibration factor from the multi-point calibration curve (FORM VI) if the average calibration factor was used for calculations. If curve calculations were performed, enter "CURVE":

Under QNT $Y / N$ : enter $Y$ if guantitation was performed, $N$ if not performed. $\therefore D$ must be less than or equal to $15 \%$ for quantitation.

For multicomponent analytes, a single peak that is characteristic of the component should be used to establish retention time and $: 0$. identification of such analytes is based primarily on pattern recognition. 
PCE CALIBRATION STANDARD CONCENTRATIONS

Lab Name: OUANTERRA, MO

Contract :

625.01

Lab Code: ITMO Case No.:

SAS NO.:

SDG No. :

V368

Instrument ID: $\mathrm{GCE}$

GC Column ID:

$D E-5 M S$

Calibration Date(s):

$02-05-98$

to $02-06-98$

\begin{tabular}{|c|c|c|c|c|c|}
\hline $\begin{array}{l}\text { COMPOUD } \\
============= \\
\text { AR } 1221 \\
\text { AR } 1232 \\
\text { AR } 1242 \\
\text { AR } 1248 \\
\text { AR } 1254 \\
\text { AR } 1016 \\
\text { AR } 1260 \\
\text { TCMX } \\
\text { DCB }\end{array}$ & $\begin{array}{c}\text { CONCENTRATION } \\
\text { (ng/mi) } \\
\text { LEVEL \#1 } \\
=====0====== \\
100 \\
100 \\
100 \\
100 \\
100 \\
100 \\
\\
\end{array}$ & $\begin{array}{c}\text { CONCENTRATION } \\
(\mathrm{ng} / \mathrm{ml}) \\
\text { LEVEL \#2 } \\
========== \\
200 \\
200 \\
200 \\
200 \\
200 \\
200 \\
200 \\
10 \\
\end{array}$ & $\begin{array}{c}\text { CONCENTRATION } \\
(\mathrm{ng} / \mathrm{mi}) \\
\text { LEVEL } \# 3 \\
============ \\
500 \\
500 \\
500 \\
500 \\
500 \\
500 \\
500 \\
25 \\
\end{array}$ & $\begin{array}{c}\text { CONCENTRATION } \\
(\mathrm{ng} / \mathrm{mI}) \\
\text { LEVEL } \$ 4 \\
============ \\
1000 \\
1000 \\
1000 \\
1000 \\
1000 \\
1000 \\
1000 \\
50 \\
\end{array}$ & $\begin{array}{c}\text { CONCENTRATION } \\
\text { (ng/ml } \\
\text { LEVEL } \# 5 \\
=========== \\
2000 \\
2000 \\
2000 \\
2000 \\
2000 \\
2000 \\
2000 \\
100 \\
\end{array} 00$ \\
\hline
\end{tabular}

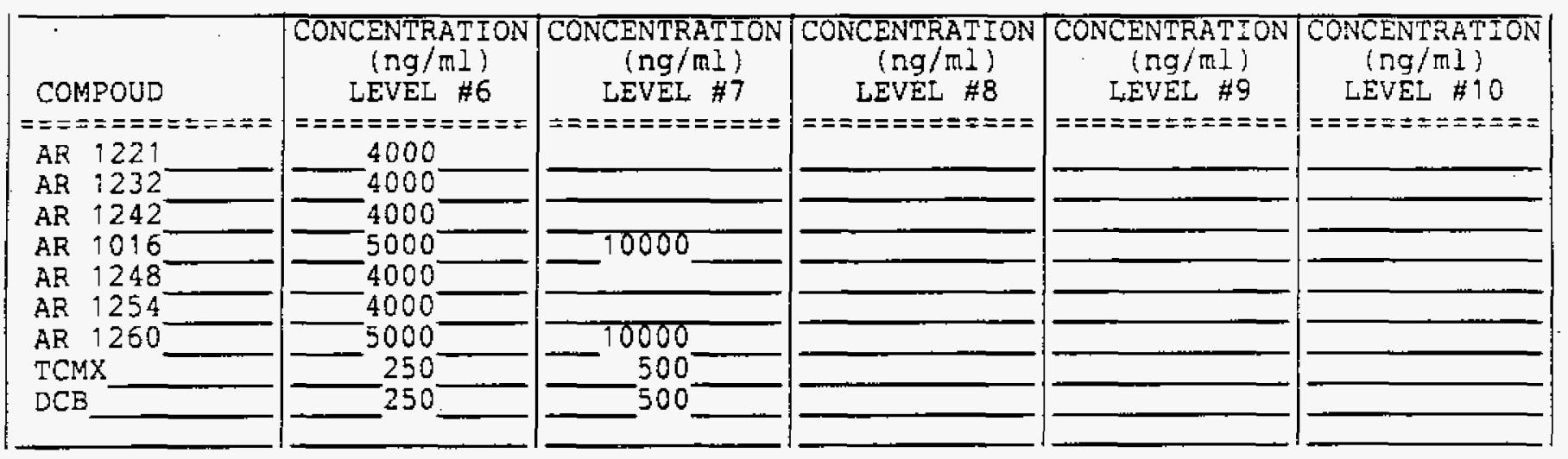

FORM XI PEST-2 
Lab Name: QUANTERRA MO

Lab Code: ITMO Case No.:

Matrix: (soil/water) SOIL

Sample wt/vol:

$5.0(g / \pi L) G$

Level: (low/med) LOW

은 Moisture: not dec.

Colunn: (pack/cap) CAP
Contract: 625.01

SAS NO.:

A6SMP3EI

SDG No.: V368

Lab Sample ID: 17161-001

Lab File ID: BSMP1918

Date Received: 03/12/98

Date Aralyzed: 03/24/98

Dilution Factor: 1.0

CONCENIRATION UNITS:

CAS NO.

COMPOUND

$(\mathrm{ug} / \mathrm{L}$ or $\mathrm{ug} / \mathrm{Kg}) \mathrm{ug} / \mathrm{Kg}$

$Q$

74-87-3--1---Chloromethane

74-83-9-.....-Bromomethane

75-01-4-......-Vinyl Chloride

75-00-3....---Chloroethane

75-09-2-1----Methylene Chloride

67-64-1-...-Acetone

75-15-0------Carbon Disulfide

75-35-4-------1,1-Dichloroethene

75-34-3-------1, 1-Dichloroethane

540-59-0-...-1,2-Dichloroethene (total)

67-66-3--.-.-Chloroform

107-06-2-..--1,2-Dichloroethane

$78-93-3-\ldots-c-2$-Butanone

$71-55-6-\cdots \cdots-1,1$-Trichloroethane

56-23-5 -----Carbon Tetrachloride

75-27-4-1-2--Bromodichloromethane

78-87-5-..---1, 2-Dichloropropane

10061-01-5-----cis-1,3-Dichloropropene

79-01-6-.-.-.--Trichloroethene

124-48-1-------Chlorodibromome thane

79-00-5------1, 1,2-Trichloroethane

71-43-2-------Benzene

10061-02-6-----trans-1,3-Dichloropropene

75-25-2-1..--Bromoform

108-10-1------4-Methyl-2-pentanone

591-78-6-------2-Hexanone

127-18-4-..-..-Tetrachloroethene

108-88-3---.---Toluene

$79-34-5-\cdots-\cdots 1,1,2,2$-Tetrachloroethane

108-90-7-...-Chlorobenzene

100-41-4-------Ethylbenzene

100-42-5--.----styrene

1330-20-7-----Xylenes (total)

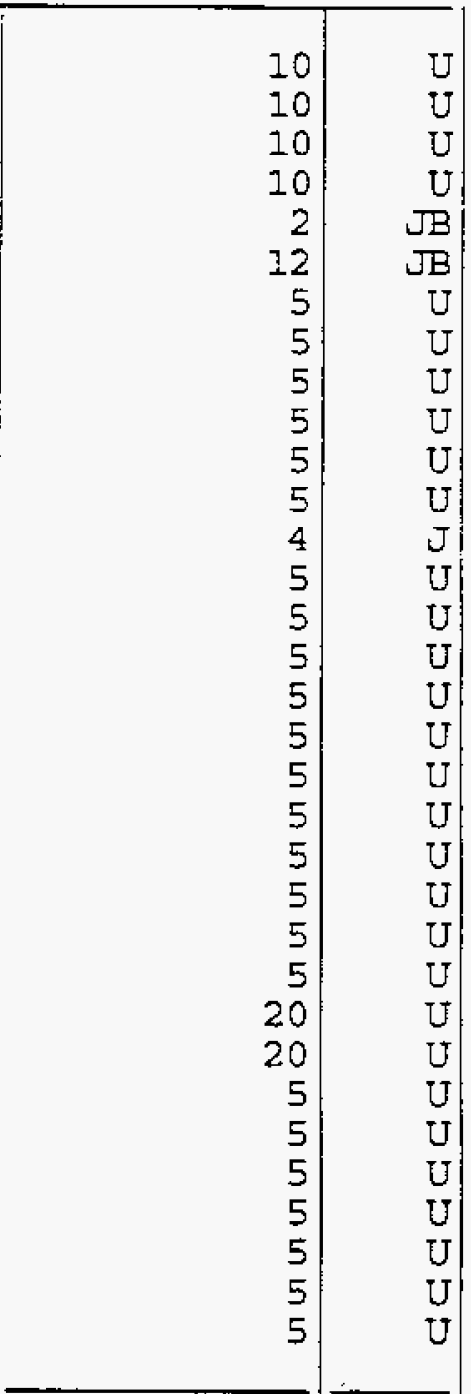


Lab Name: QUANTERRA MO

Iab Code: ITMO

Case No.:

Matrix: (soil/water) soIL

Sarmle wt/vol:

$5.0(\mathrm{~g} / \mathrm{mL}) \mathrm{G}$

Level: (low/med) LOW

\& Moisture: not dec.

Columt : (pack/cap) CAP
Contract: 625.01

SAS NO. :
EPA SAMPLE NO.

A6SMP3W1

SDG No. : V368

Lab Sample ID: 17161-002

Iab File ID: BSMP1893

Date Received: 03/I2/98

Date Analyzed: 03/20/98

Dilution Factor: 1.0

CONCENIRATION UNITS :

$(\mathrm{ug} / \mathrm{L}$ or $\mathrm{ug} / \mathrm{Kg}) \mathrm{ug} / \mathrm{Kg}$

CAS NO.

COMPOUND

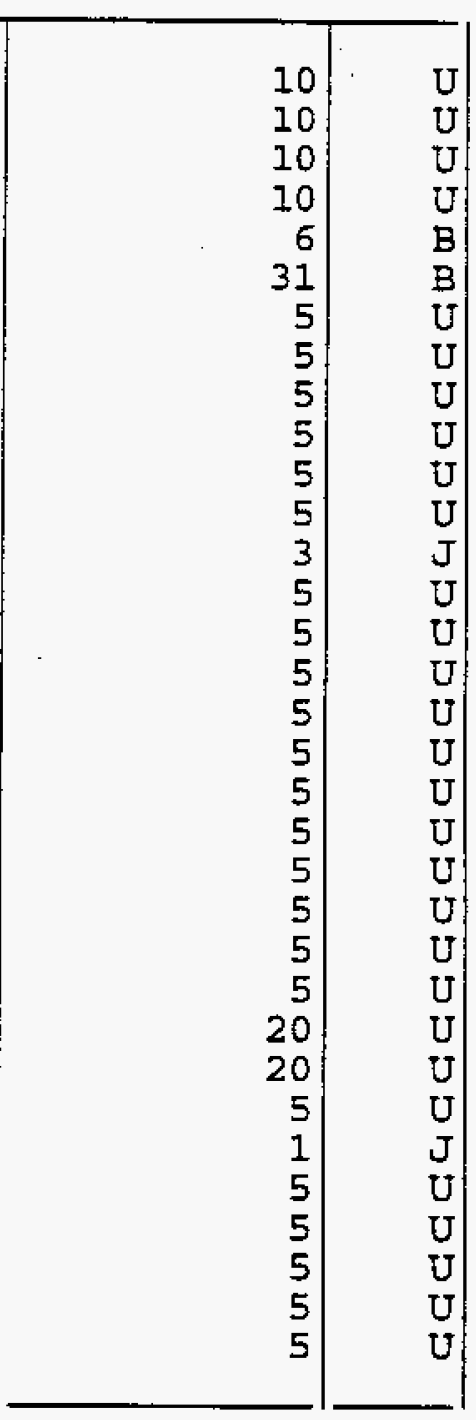


Lab Name: QUANTERRA MO

Lab code: ITMO

Case No.:
Contract: 625.01

SAS NO.:
SDG No.: V368

EPA Sample No.: VLCSB079A

\begin{tabular}{|l}
\hline SPIKE COMPOUND \\
\hline 11 1,1-Dichloroethere \\
42 Trichloroethene \\
40 Benzene \\
52 Toluene \\
62 Chlorobenzene \\
\hline
\end{tabular}

\begin{tabular}{l} 
CONC \\
ADDED \\
$u g / L$ \\
\hline 50.00 \\
50.00 \\
50.00 \\
50.00 \\
50.00
\end{tabular}

\begin{tabular}{|}
$\begin{array}{c}\text { CONC } \\
\text { RECOVERED } \\
\text { ug/L }\end{array}$ \\
\hline 61.12 \\
47.79 \\
48.49 \\
48.94 \\
46.52 \\
\hline
\end{tabular}

\begin{tabular}{r|r|}
\multicolumn{1}{c|}{ RECOVERED } & LIMITS \\
\hline 122.25 & \\
95.59 & $71-234$ \\
96.98 & $37-157$ \\
97.89 & $47-150$ \\
93.05 & $37-160$ \\
\hline
\end{tabular}

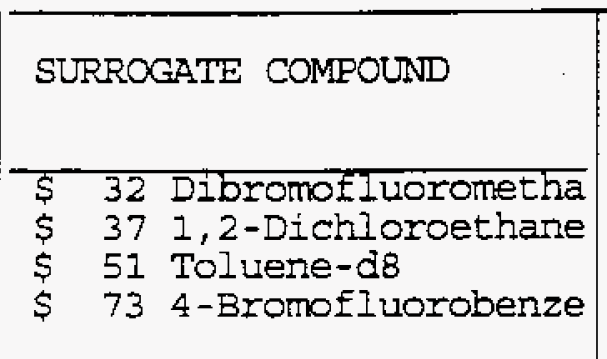

\begin{tabular}{|l|}
$\begin{array}{c}\text { AMOUNT } \\
\text { ADDED } \\
\text { ug/ }\end{array}$ \\
\hline 50.00 \\
50.00 \\
50.00 \\
50.00 \\
\hline
\end{tabular}

\begin{tabular}{|c|}
$\begin{array}{c}\text { AMOUNT } \\
\text { RECOVERED } \\
u g / L\end{array}$ \\
\hline 50.09 \\
48.35 \\
49.28 \\
48.94 \\
\hline
\end{tabular}

\begin{tabular}{|r|r|} 
RECOVERED & LIMITS \\
& \\
\hline 100.19 & $80-120$ \\
96.69 & $80-120$ \\
98.56 & $80-120$ \\
97.89 & $80-120$ \\
\hline
\end{tabular}


Lab Name: QUANTERRA MO

Lab code: ITMO

Case No. :
Contract: 625.01

SAS NO.:
EPA SAMPIE NO;

VBLKBO79A
Matrix: (soil/water) WATER

Sample wt/vol: $\quad 5.000(\mathrm{~g} / \mathrm{ml}) \mathrm{ML}$

Level: (low/med) LOW

\& Moisture: not dec.

Column: (pack/cap) CAP
Lab Sample ID: QCBLK167948

Lab File ID: BBLK1889

Date Received:

Date Analyzec: 03/20/98

Dilution Factor: 1.0

CAS NO.

COMPOUND

CONCENTRATION UNITS: (ug/L or $\mathrm{ug} / \mathrm{kg}$ ) $\mathrm{ug} / \mathrm{L}$

$Q$

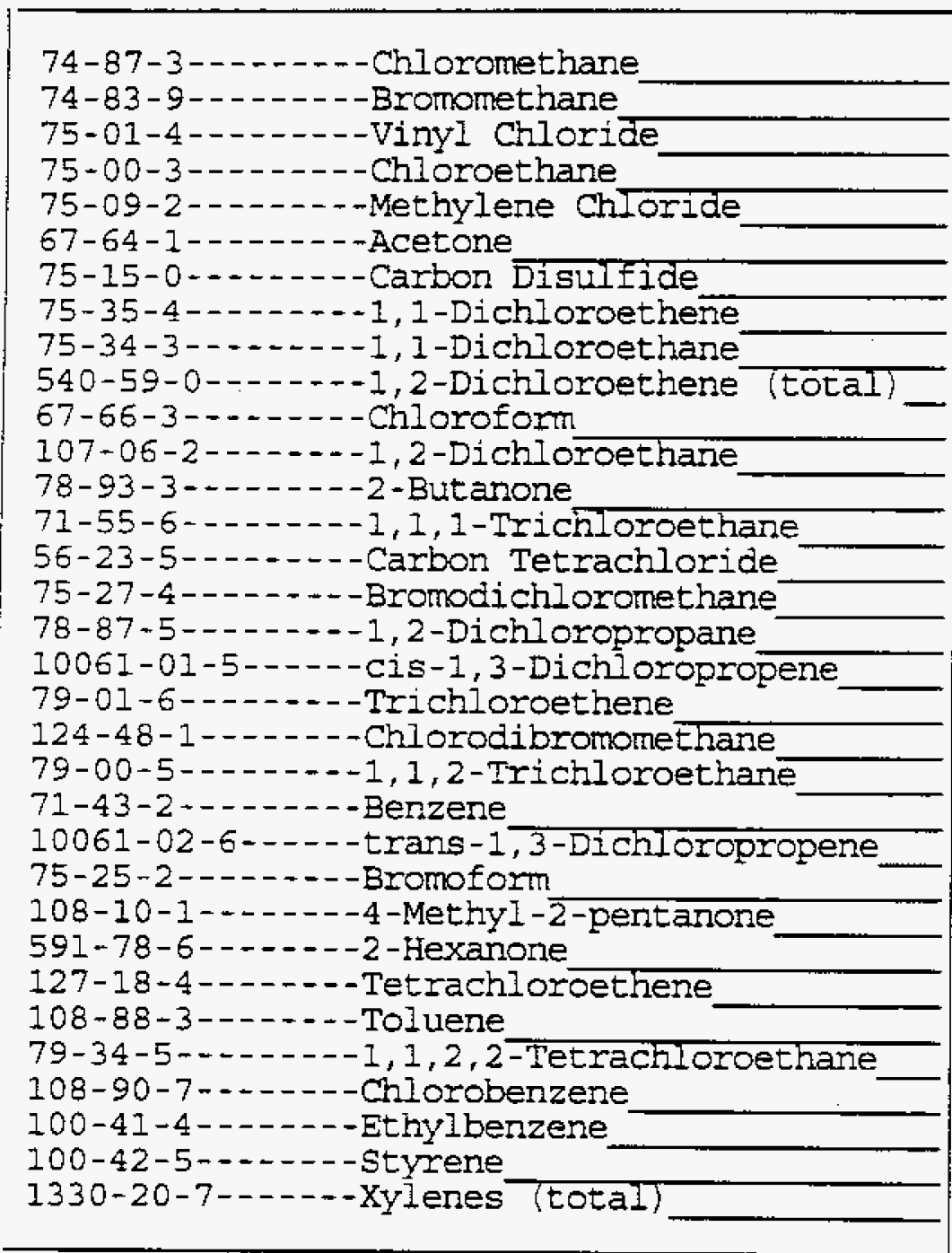

\begin{tabular}{|r|r|}
10 & \\
10 & $\mathrm{U}$ \\
10 & $\mathrm{U}$ \\
10 & $\mathrm{U}$ \\
2 & $\mathrm{~J}$ \\
14 & $\mathrm{~J}$ \\
5 & $\mathrm{U}$ \\
5 & $\mathrm{U}$ \\
5 & $\mathrm{U}$ \\
5 & $\mathrm{U}$ \\
5 & $\mathrm{U}$ \\
5 & $\mathrm{U}$ \\
20 & $\mathrm{U}$ \\
5 & $\mathrm{U}$ \\
5 & $\mathrm{U}$ \\
5 & $\mathrm{U}$ \\
5 & $\mathrm{U}$ \\
5 & $\mathrm{U}$ \\
5 & $\mathrm{U}$ \\
5 & $\mathrm{U}$ \\
5 & $\mathrm{U}$ \\
5 & $\mathrm{U}$ \\
5 & $\mathrm{U}$ \\
5 & $\mathrm{U}$ \\
20 & $\mathrm{U}$ \\
20 & $\mathrm{U}$ \\
5 & $\mathrm{U}$ \\
5 & $\mathrm{U}$ \\
5 & $\mathrm{U}$ \\
5 & $\mathrm{U}$ \\
5 & $\mathrm{U}$ \\
5 & $\mathrm{U}$ \\
5 & $\mathrm{U}$ \\
\hline & \\
\hline
\end{tabular}

FORM I VOA

1/87 Rev. 
Lab Name: QUANTERRA MO

Lab code: ITMO Case No.:

Lab File ID (Standard) : BCAL1888

Instrument ID: MSB

Matrix:(soll/water) SOIL Level:(Iow/med) LOW Colum: (pack/cap) CAP
Contract: 625.01

SAS NO.:

SDG No. : $\sqrt{3} 68$

Date Analyzed: $03 / 20 / 98$

Time Analyzed: 0551

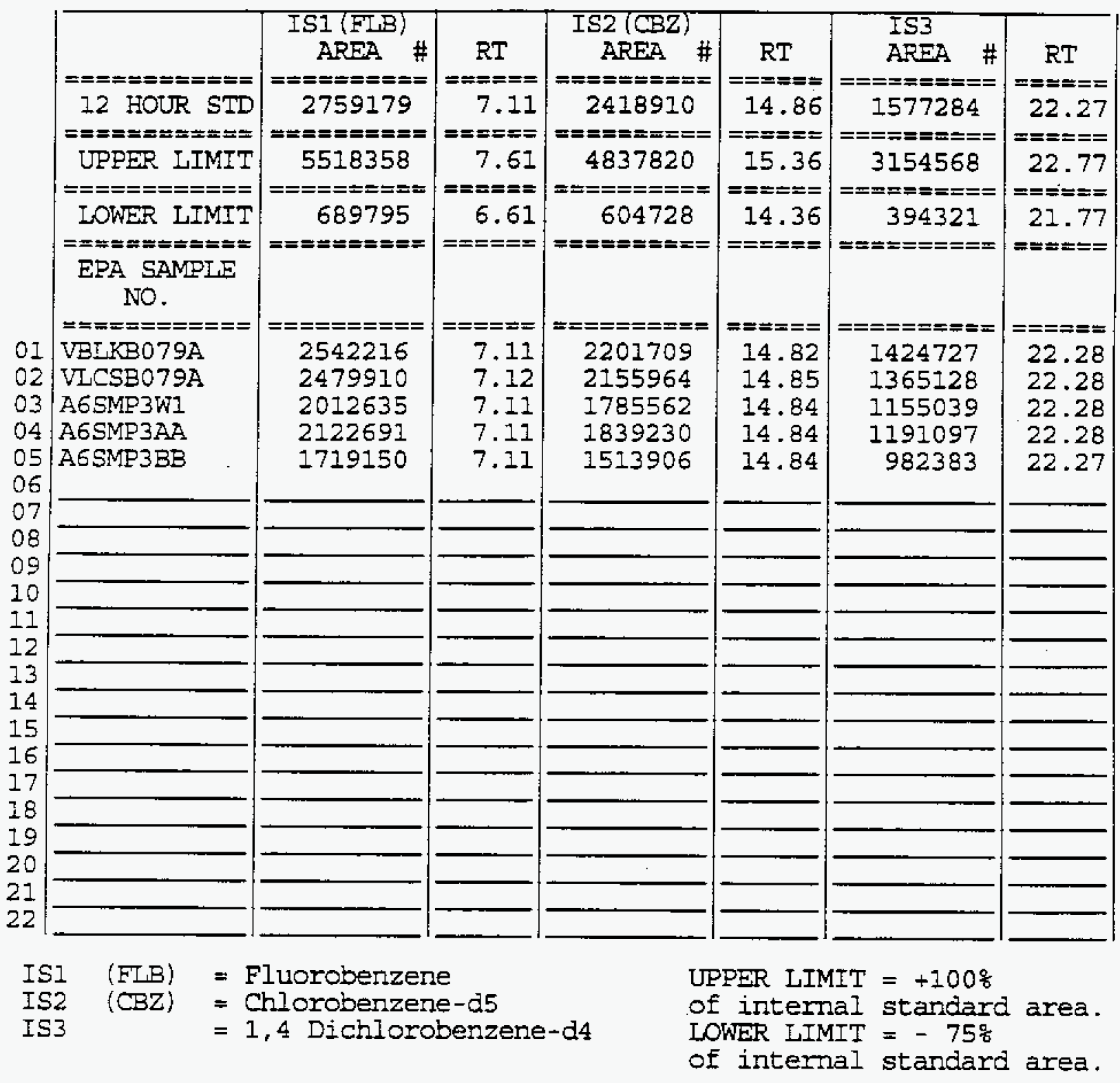

\# Colum used to flag internal standard area values with an asterisk.

page 1 of 1 
Lab Name: QUANTERRA MO

Lab Code: ITMO Case No.:

Matrix: (soil/water) WATER

Sample wt/vol: $\quad 5.000(\mathrm{~g} / \mathrm{ml}) \mathrm{ML}$

Level: (low/med) LOW

\& Moisture: not dec.

Colunn: (pack/cap) CAP
Contract: 625.01

SAS NO.:
VLCSB079A

SDG No.: $\mathrm{V} 368$

Lab Sample ID: QCLCS167948

Lab File ID: BLCS1890

Date Received:

Date Analyzed: 03/20/98

Dilution Factor: 1.0

CONCENTRATION UNITS:
(ug/L or $\mathrm{ug} / \mathrm{Kg}$ ) ug/L

$Q$

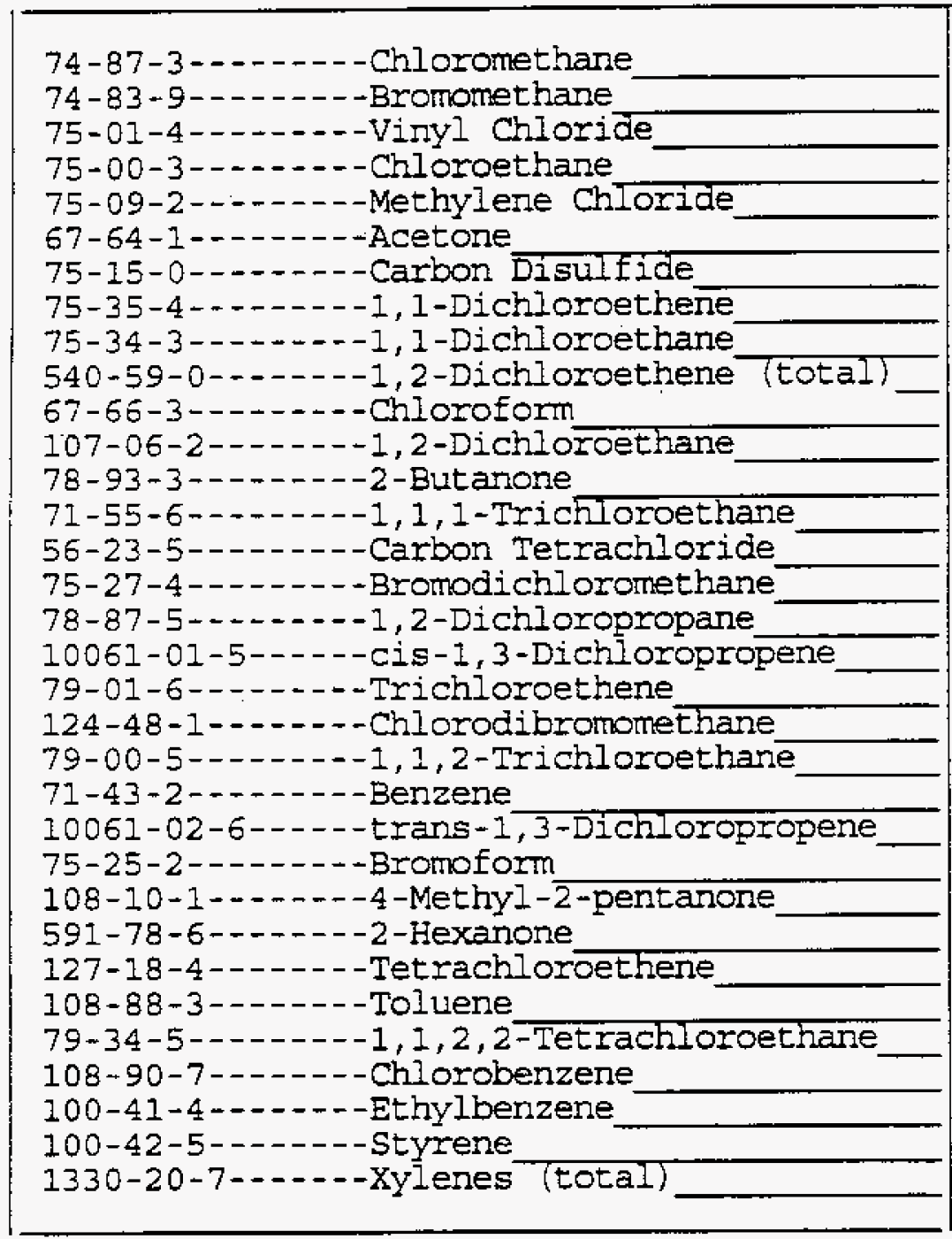


VOLA: 2 ORGANICS INITIAL CALIBRAT: DATA

Lab Name: QUANTERRA MO

Lab Code: ITMO Case No.:
Contract : 625.01

SAS NO.:
SDG No.: $\mathrm{V3} 68$
Instrument ID: MSB

Matrix: (soil/water) SOII
Calibration Date(s) : 03/24/98

Level: (low/med) LOW Colum: (pack/cap) CAP

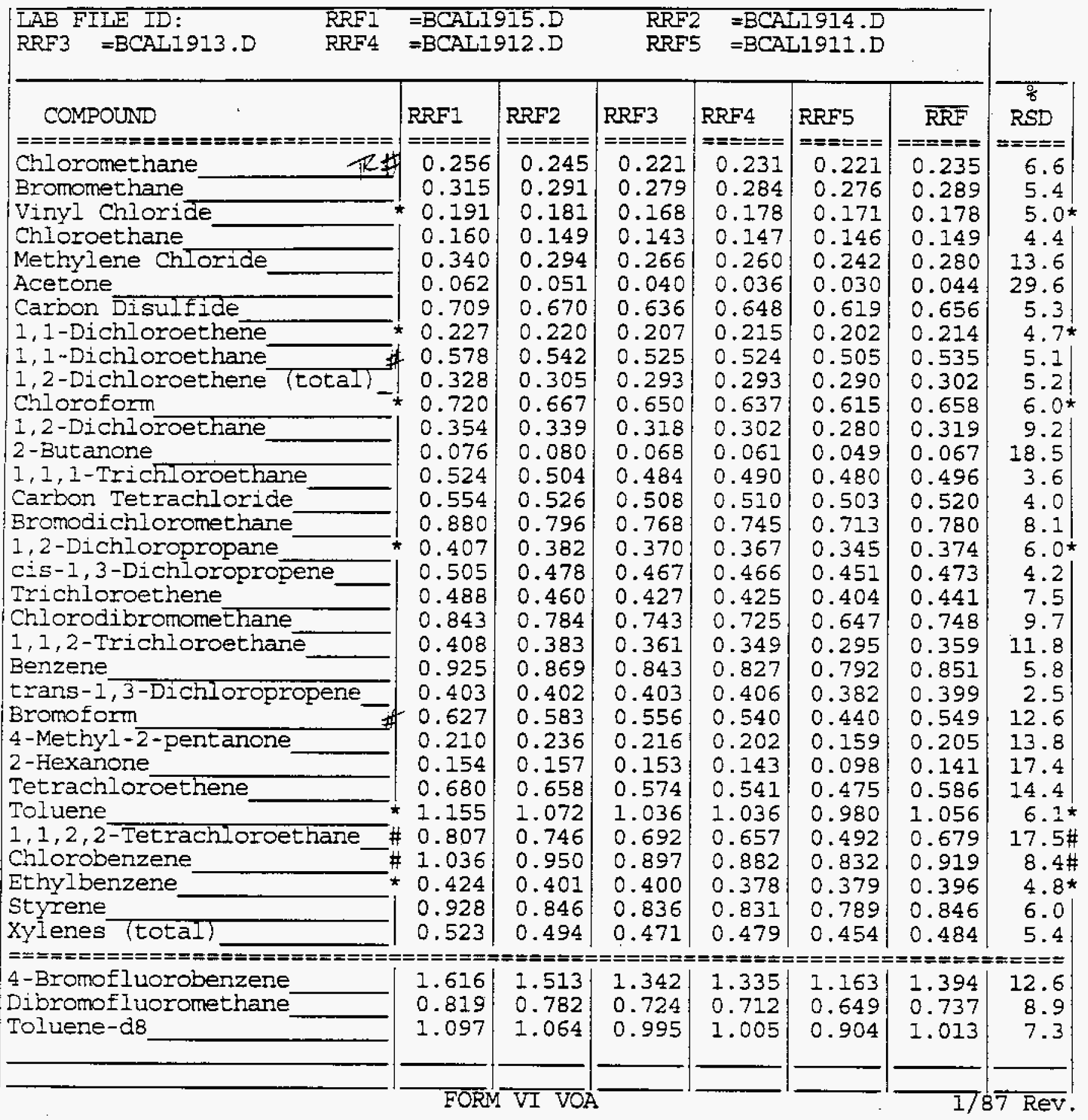


WA: VOIATILE SURROGATE RECOVER:

Iab Name: QUANTERRA MO

Iab code: ITMO
Case No.:
Contract: 625.01

SAS No.:
SDG NO.: V368

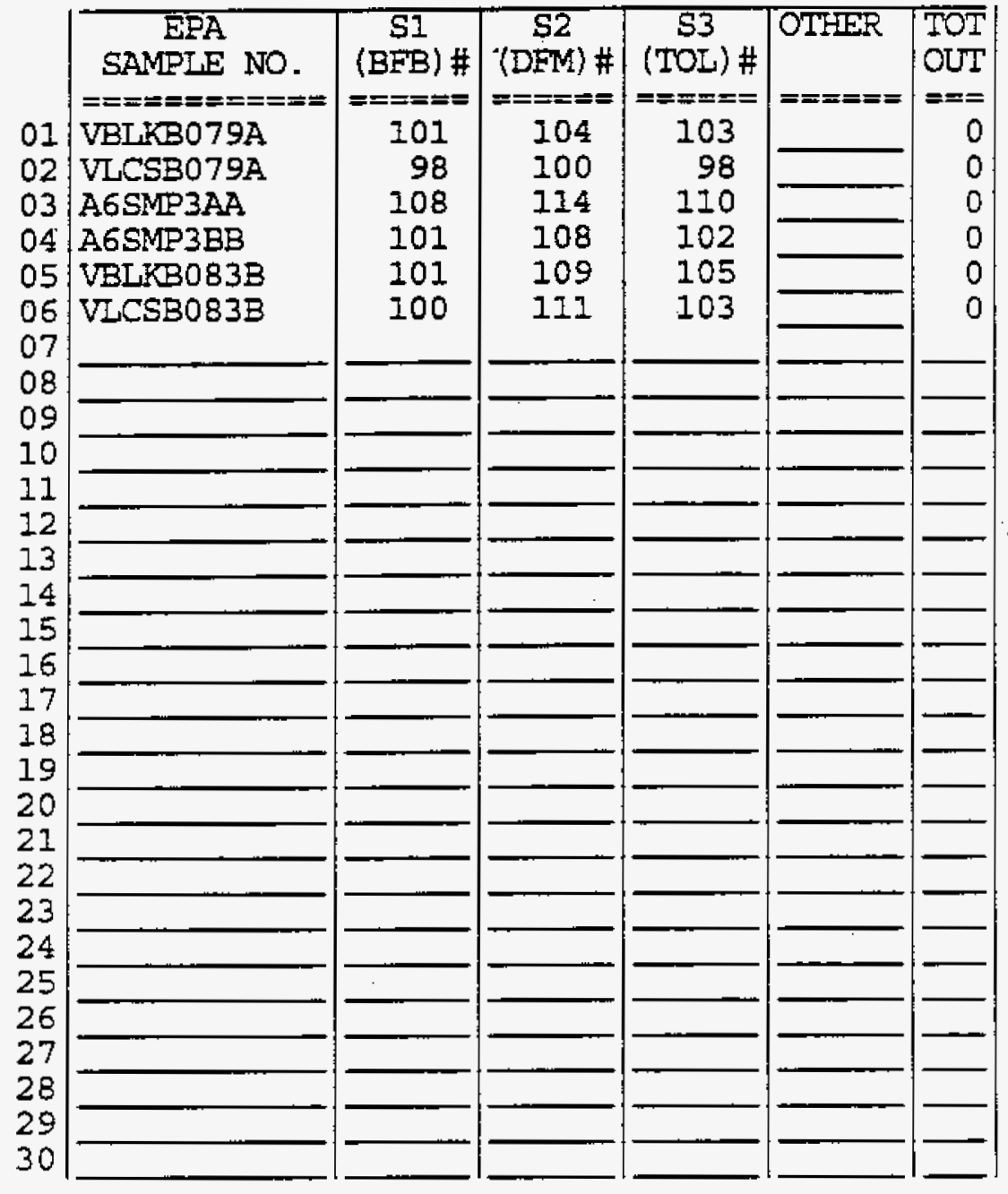

\footnotetext{
S1 (BFB) = 4-Bromofluorobenzene

$(80-120)$

$(80-120)$

$(80-120)$

S3 $($ TOL $)=$ Toluene-d8

\# Colurn to be used to flag recovery values

* Values outside of contract required QC limits

D Surrogates diluted out
}

QC LIMITS

page 1 of 1 
VOLATILF RGANIC GC/MS TUNING AND MASE

CALIBRATIUN - BROMOFLUOROBENZENE (BFB)

Lab Name: QUANTERRA MO

Lab Code: ITMO Case No.:

Lab File ID: BBFB1910

Instrument ID: MSB

Matrix: (soil/water) WATER Level:(low/med) LOW Column:(pack/cap) CAP
Contract: 625.01

SAS NO. :

SDG No.: $\mathrm{V} 368$

BFB Injection Date: $03 / 24 / 98$

BFB Injection Time: 0932

\begin{tabular}{|c|c|c|}
\hline $\begin{array}{c}m / e \\
===== \\
50 \\
75 \\
95 \\
96 \\
173 \\
174 \\
175 \\
176 \\
177\end{array}$ & $\begin{array}{l}\text { ION ABUNDANCE CRITERIA } \\
============================================ \\
15.0-40.0 \% \text { of mass } 95 \\
30.0-60.0 \% \text { of mass } 95 \text { } \\
\text { Base Peak, } 100 \% \text { relative abundance } \\
5.0-9.0 \% \text { of mass } 95 \\
\text { Less than } 2.0 \% \text { of mass } 174 \\
\text { Greater than } 50.0 \% \text { of mass } 95 \\
4.0-9.0 \% \text { of mass } 174 \\
\text { Greater than } 95.0 \% \text { but less than } 101.0 \% \text { of mass } 174 \\
5.0-9.0 \% \text { of mass } 176\end{array}$ & 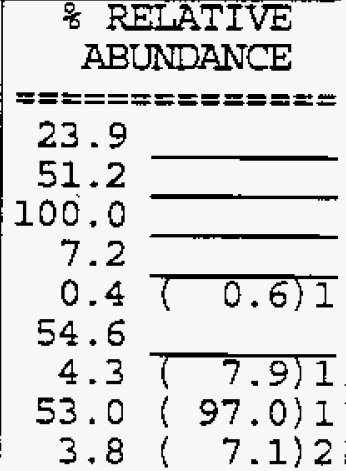 \\
\hline
\end{tabular}

THIS TUNE APPLIES TO THE FOLLOWING SAMPLES, MS, MSD, BLANKS, AND STANDARDS:

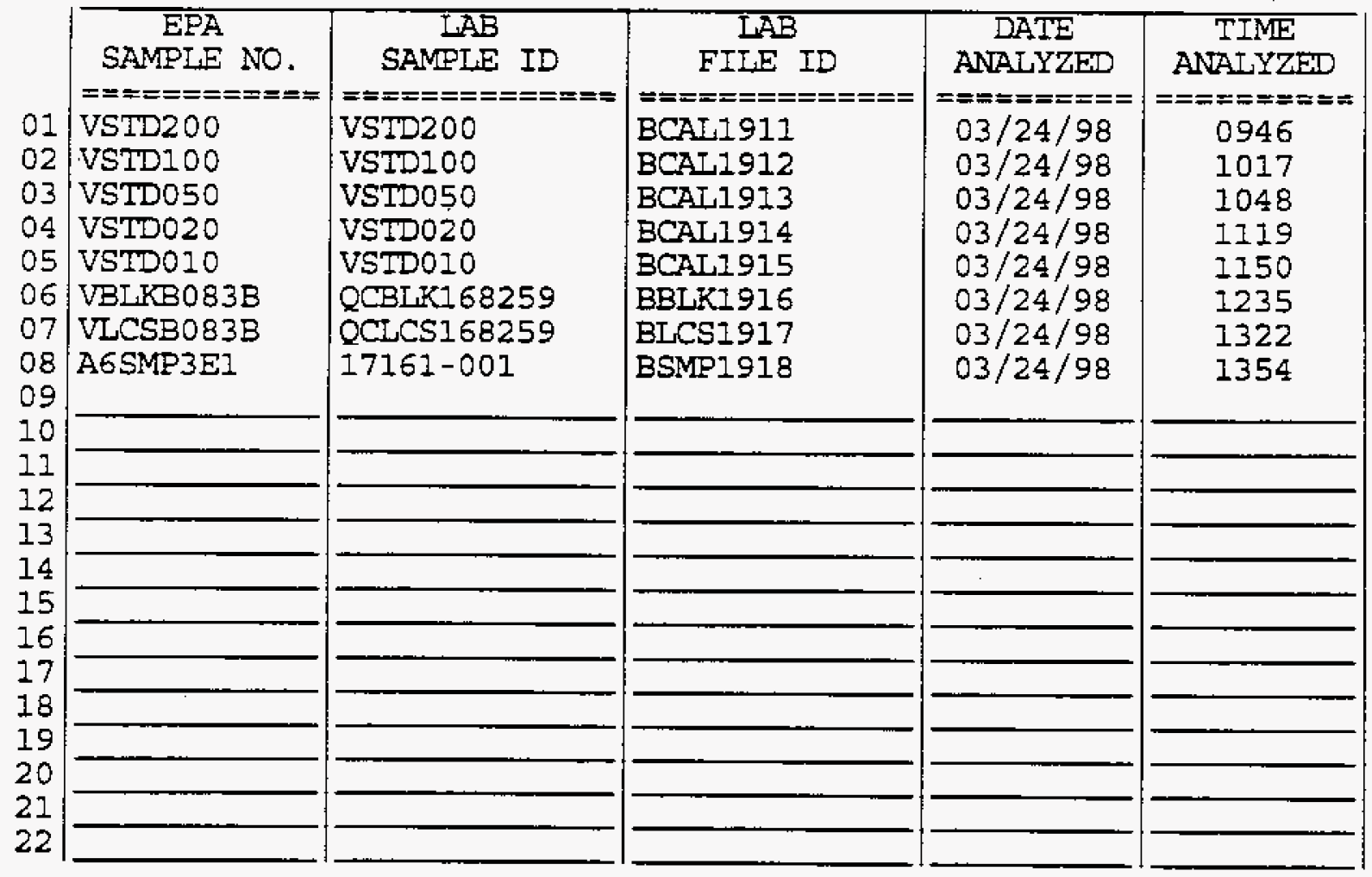

page 1 of 1 
VOIJ LE METHOD BLANK SUMMARY

Lab Name: QUANTERRA MO

Lab code: ITMO

Case No. :

Lab File ID:

BBLK1889

Date Analyzed:

Matrix: (soil/water) WATER

Instrument ID:

MSB
Contract: 625.01

SAS NO.:

SDG No.: V368

Lab Sample ID: QCBLK167948

Time Analyzed: $\quad 0635$

Level : (low/med) IOW

THIS METHOD BIANK APPLIES TO THE FOLIOWING SAMPLES, MS and MSD:

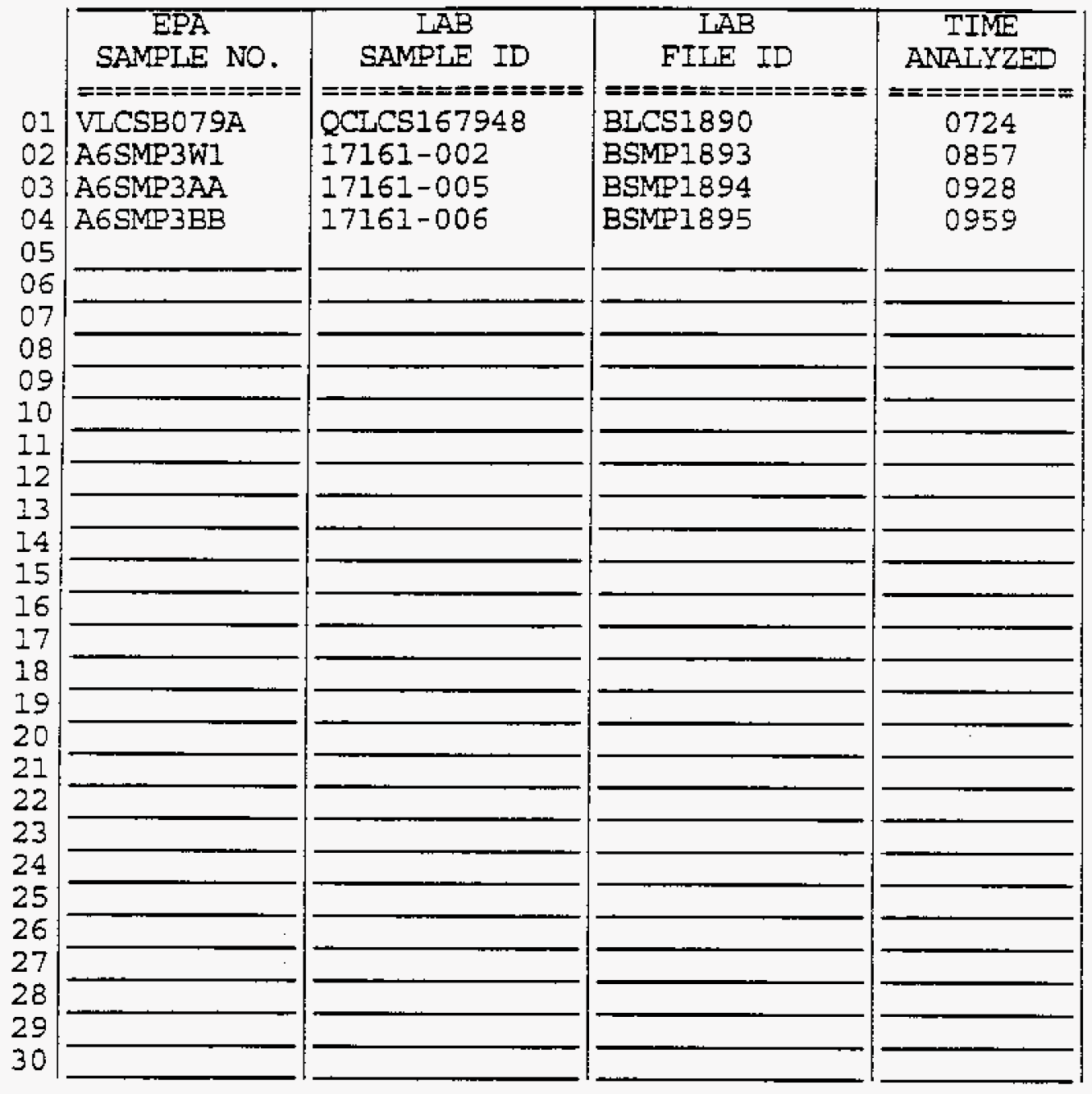

COMMENTS : 
VOLATILE RGANIC GC/MS TUNING AND MASS

CALIBRATIUN - BROMOFLUOROBENZENE (BFB)

Lab Name: QUANTERRA MO

Lab Code: ITMO Case No.:

Lab File ID: BBFB1842

Instrument ID: MSB

Matrix: (soil/water) WATER Level:(low/med) LOW Colunn: (pack/cap) CAP
Contract: 625.01

SAS NO. :

SDG No.: $\mathrm{V} 368$

BFB Injection Date: $03 / 18 / 98$

BFB Injection Time: 0558

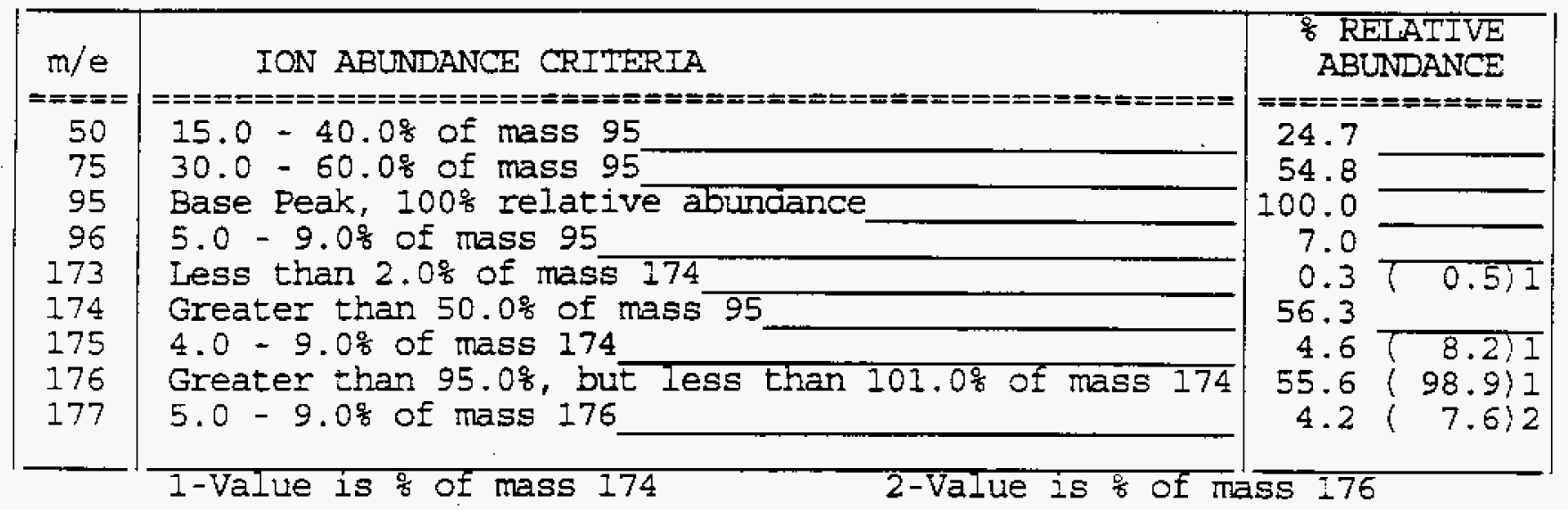

THIS TUNE APPLIES TO THE FOLIOWING SAMPLES, MS, MSD, BLANKS, AND STANDARDS:

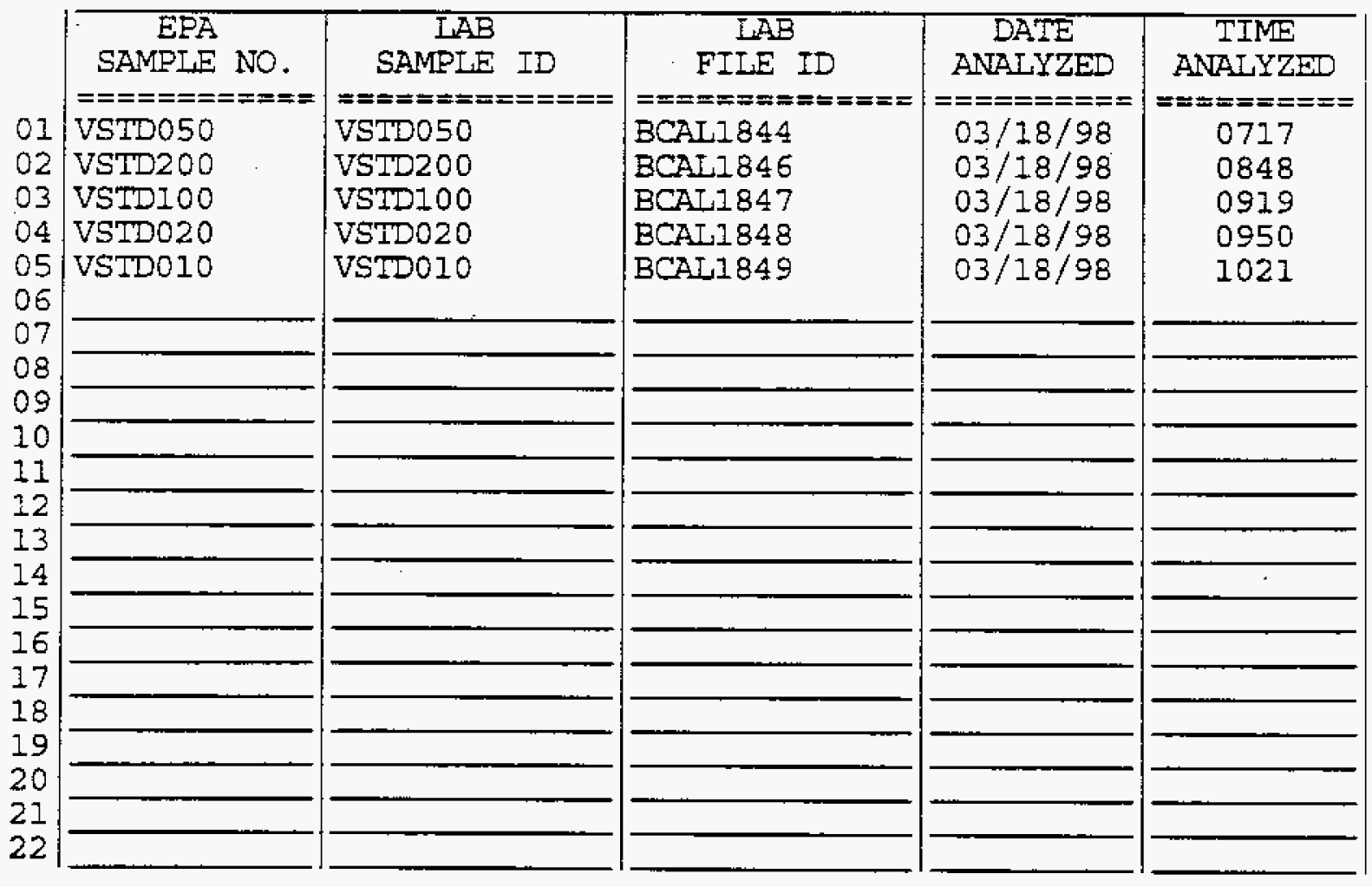

page 1 of 1 
Lab Name: QUANTERRA MO

Lab Code: ITMO Case No.:

Matrix: (soil/water) WATER

Sample wt/vol: $\quad 5.000(\mathrm{~g} / \mathrm{ml})$ ML

Level: (low/med) LOW

\%oisture: not dec.

Column: (pack/cap) CAP
Contract: 625.01

SAS NO.:
EPA SAMPLE NO.

A6SMP3AA

SDG No.: V368

Lab Sample ID: 17161-005

Lab File ID: BSMP1894

Date Received: 03/12/98

Date Analyzed: 03/20/98

Dilution Factor: 1.0

CONCENTRATION UNITS:
CAS NO.
COMPOUND
(ug/L or $\mathrm{ug} / \mathrm{Kg}) \mathrm{ug} / \mathrm{L}$
$Q$

\begin{tabular}{|c|c|c|}
\hline 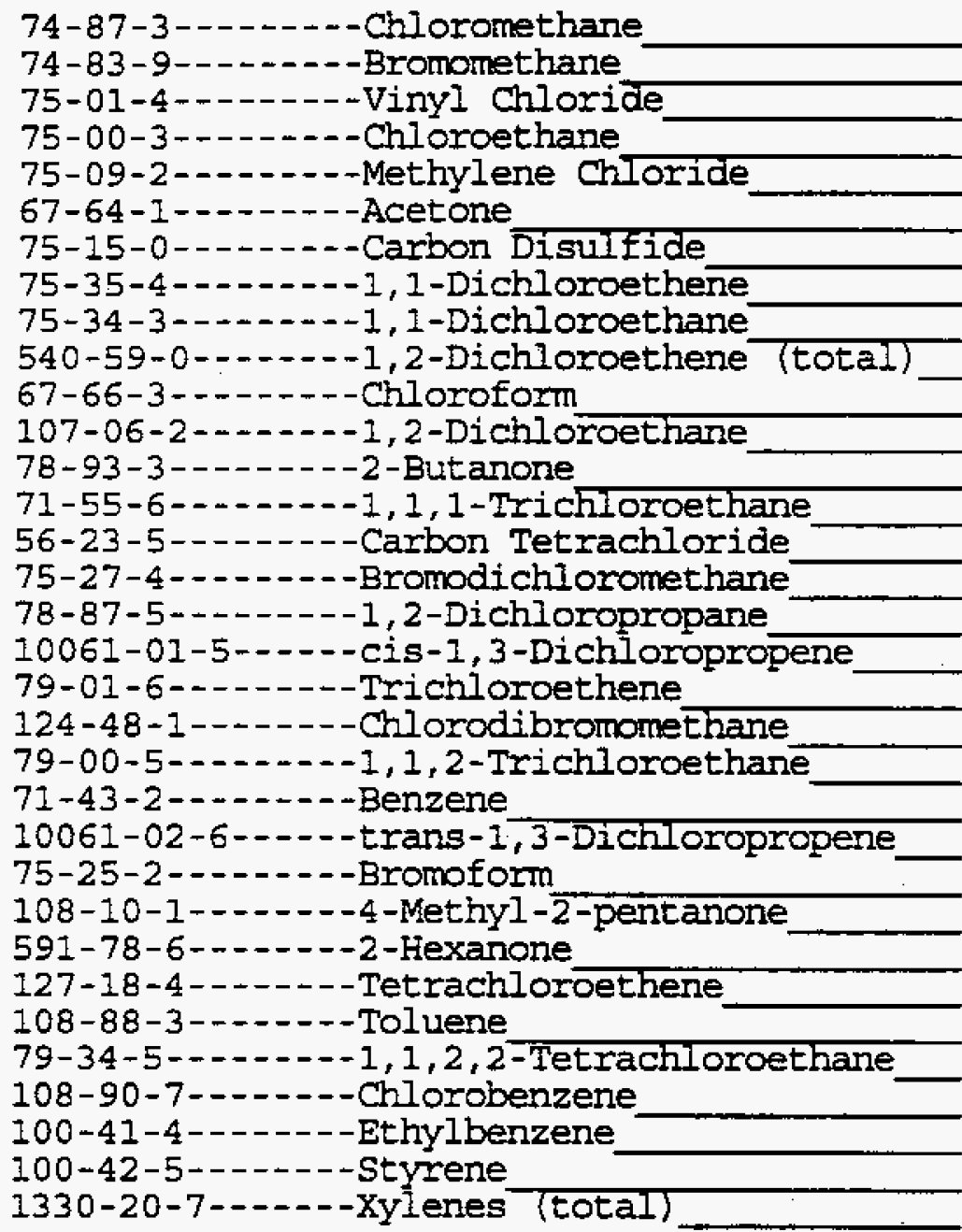 & $\begin{array}{r}10 \\
10 \\
10 \\
10 \\
4 \\
11 \\
5 \\
5 \\
5 \\
5 \\
5 \\
5 \\
5 \\
5 \\
5 \\
5 \\
5 \\
5 \\
5 \\
5 \\
5 \\
5 \\
5 \\
5 \\
20 \\
20 \\
5 \\
5 \\
5 \\
5 \\
5 \\
5 \\
5\end{array}$ & $\begin{array}{c}\mathrm{U} \\
\mathrm{U} \\
\mathrm{U} \\
\mathrm{U} \\
\mathrm{JB} \\
\mathrm{JB} \\
\mathrm{U} \\
\mathrm{U} \\
\mathrm{U} \\
\mathrm{U} \\
\mathrm{U} \\
\mathrm{U} \\
\mathrm{J} \\
\mathrm{U} \\
\mathrm{U} \\
\mathrm{U} \\
\mathrm{U} \\
\mathrm{U} \\
\mathrm{U} \\
\mathrm{U} \\
\mathrm{U} \\
\mathrm{U} \\
\mathrm{U} \\
\mathrm{U} \\
\mathrm{U} \\
\mathrm{U} \\
\mathrm{U} \\
\mathrm{U} \\
\mathrm{U} \\
\mathrm{U} \\
\mathrm{U} \\
\mathrm{U} \\
\mathrm{U}\end{array}$ \\
\hline
\end{tabular}


Lab Name: QUANTERRA MO

Lab Code: ITMO ' Case No.:
Contract: 625.01

SAS NO.:
A6SMP3 $3 B$
Matrix: (soil/water) WATER

Sample wt/vol: $\quad 5.000(\mathrm{~g} / \mathrm{ml}) \mathrm{ML}$

Level: (low/med) LOW

o Moisture: not dec.

Colum: (pack/cap) CAP
Lab Sample ID: 17161-006

Lab File ID: BSMP1895

Date Received: $03 / 12 / 98$

Date Analyzed: $03 / 20 / 98$

Dilution Factor: 1.0

CAS NO.

COMPOUND

CONCENTRATION UNITS:

$(\mathrm{ug} / \mathrm{L}$ or $\mathrm{ug} / \mathrm{Kg}) \mathrm{ug} / \mathrm{L}$

Q

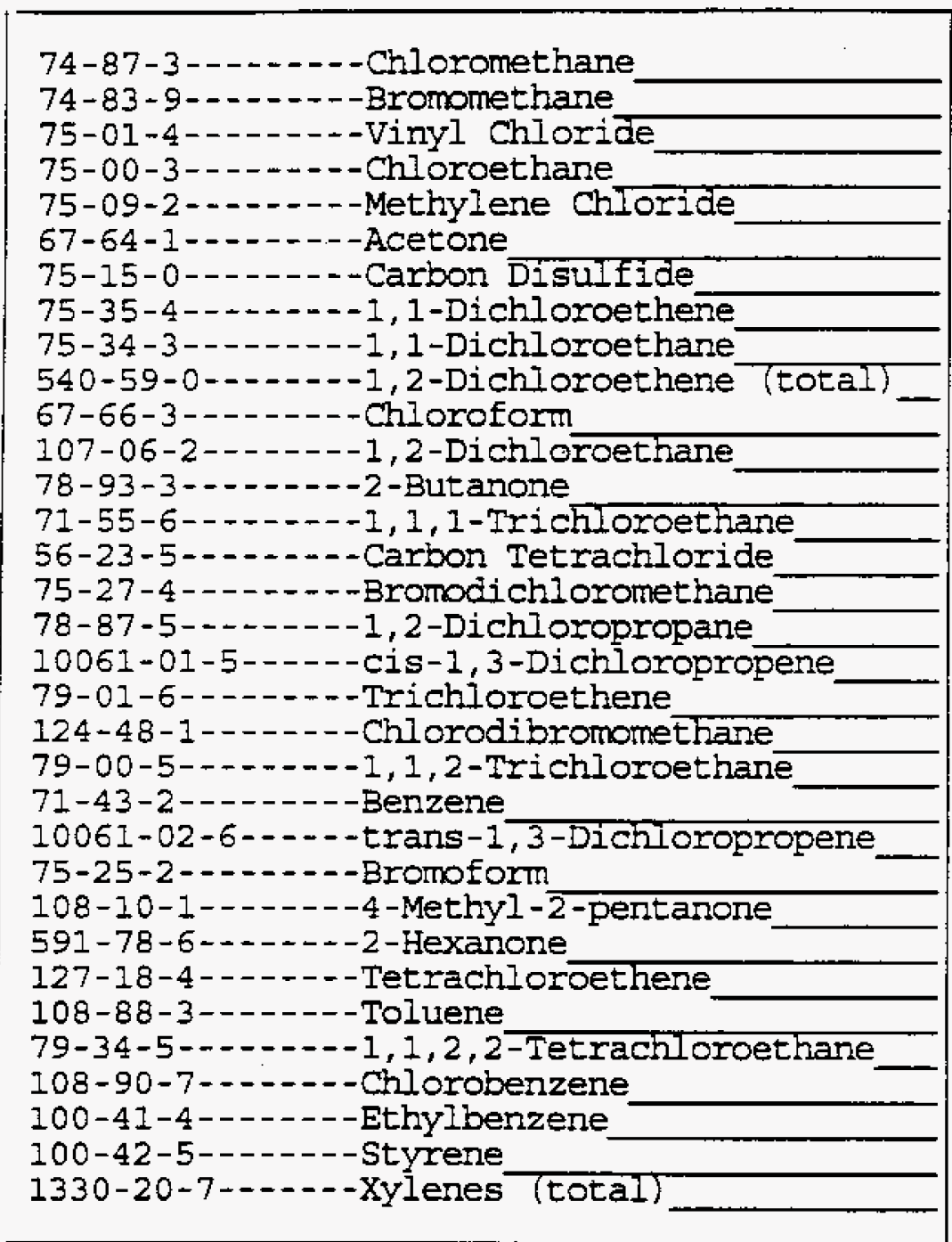


Lab Name: QUANTERRA MO

Lab Code: ITMO Case No.:

Matrix: (soil/water) WATER

Sample wt/vol: $\quad 5.000(\mathrm{~g} / \mathrm{mL}) \mathrm{ML}$

Level: (low/med) LOW

号 Moisture: not dec.

Colunn: (pack/cap) CAP
Contract: 625.01

SAS NO. :
EPA SAMPLE NO;

VBLKB079A

CAS NO.

COMPOUND

CONCENTRATION UNITS:

(ug/L or $u g / \mathrm{kg}$ ) ug/L

SDG No.: V368

Lab Sample ID: QCBLK167948

Lab File ID: BBLK1889

Date Received:

Date Analyzed; 03/20/98

Dilution Factor: 1.0

\begin{tabular}{|c|c|c|}
\hline 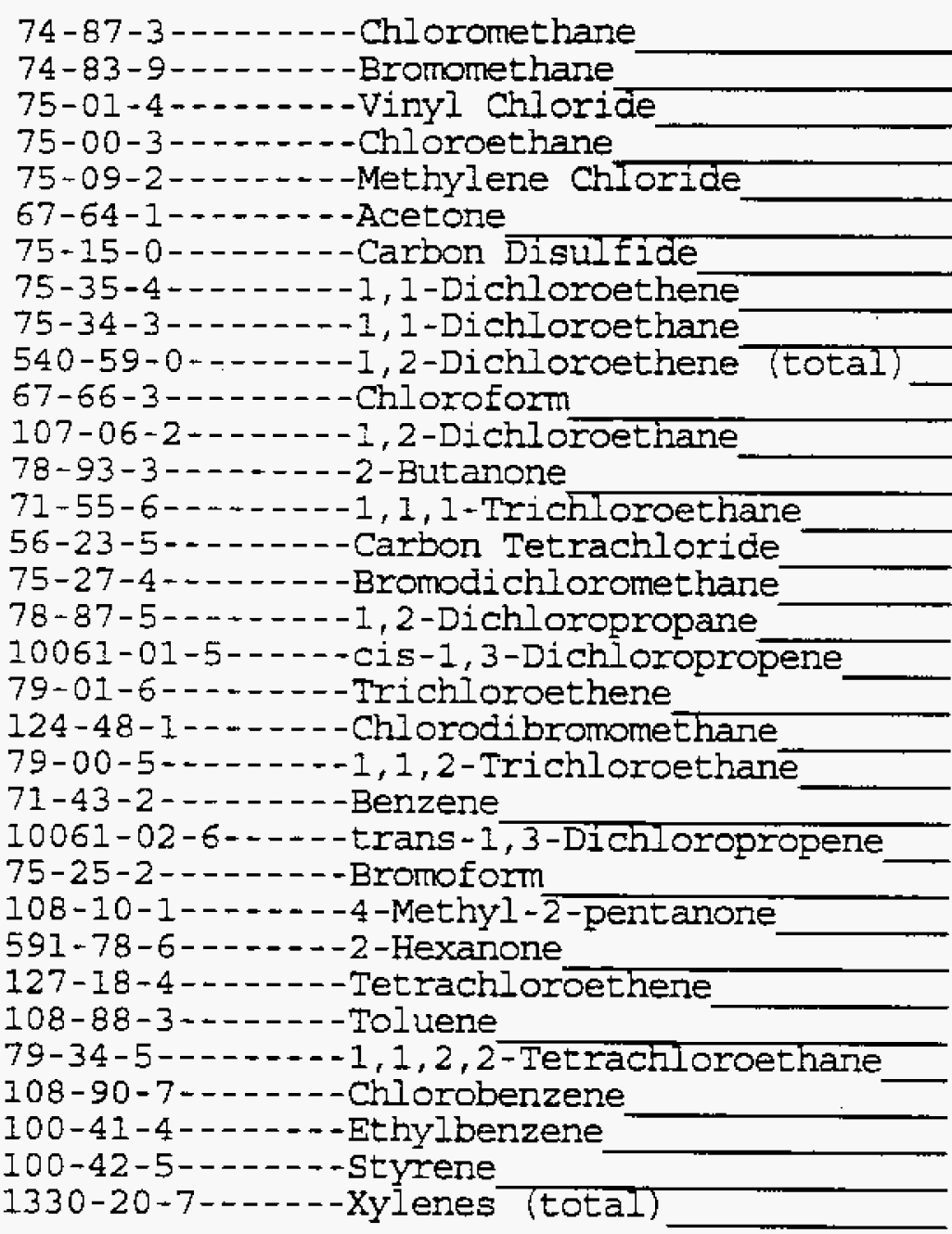 & $\begin{array}{r}10 \\
10 \\
10 \\
10 \\
2 \\
14 \\
5 \\
5 \\
5 \\
5 \\
5 \\
5 \\
20 \\
5 \\
5 \\
5 \\
5 \\
5 \\
5 \\
5 \\
5 \\
5 \\
5 \\
5 \\
20 \\
20 \\
5 \\
5 \\
5 \\
5 \\
5 \\
5 \\
5\end{array}$ & $\begin{array}{l}\mathrm{U} \\
\mathrm{U} \\
\mathrm{U} \\
\mathrm{U} \\
\mathrm{U} \\
\mathrm{J} \\
\mathrm{U} \\
\mathrm{U} \\
\mathrm{U} \\
\mathrm{U} \\
\mathrm{U} \\
\mathrm{U} \\
\mathrm{U} \\
\mathrm{U} \\
\mathrm{U} \\
\mathrm{U} \\
\mathrm{U} \\
\mathrm{U} \\
\mathrm{U} \\
\mathrm{U} \\
\mathrm{U} \\
\mathrm{U} \\
\mathrm{U} \\
\mathrm{U} \\
\mathrm{U} \\
\mathrm{U} \\
\mathrm{U} \\
\mathrm{U} \\
\mathrm{U} \\
\mathrm{U} \\
\mathrm{U} \\
\mathrm{U}\end{array}$ \\
\hline
\end{tabular}


Lab Name: QUANTERRA MO

Lab Code: ITMO Case No.:

Matrix: (soil/water) WATER

Sample wt/vol: $\quad 5.000(\mathrm{~g} / \mathrm{ml}) \mathrm{ML}$

Level: (low/med) LOW

\& Moisture: not dec.

Column: (pack/cap) CAP
Contract: 625.01

SAS NO. :

SDG No.: $\sqrt{368}$

Iab Sanple ID: CCBLK168259

Lab File ID: BBLK1916

Date Received:

Date Analyzed: $03 / 24 / 98$

Dilution Factor: 1.0 CONCENTRATION UNITS : (ug/L or $u g / \mathrm{Kg}$ ) $u g / L$.

$74-87-3---1-93-----$ - Bromomethane

75-01-4--.----Vinyl Chloride

75-00-3-..---Chloroethane

75-09-2-...--Methylene Chloride

67-64-1-------Acetone

75-15-0--.----Carbon Disulfide

75-35-4-1.--1,1-Dichloroethene

75-34-3-...-1,1-Dichloroethane

540-59-0-..--1,2-Dichloroethene (total)

67-66-3-n---chioroform

107-06-2------1,2-Dichloroethane

78-93-3-2----2-Butanone

71-55-6-..---1, 1, 1-Trichloroethane

55-23-5----2--Carbon Tetrachloride

75-27-4--1----Bromodichloromethane

78-87-5-----1,2-Dichloropropane

10061-01-5-----cis-1, 3-Dichloropropene

79-01-6-------Trichloroethene

124-48-1-..--Chlorodibromomethane

79-00-5--.--1,1,2-Trichloroethane

71-43-2--.-----Benzene

10061-02-6-2.--trans-1, 3-Dichloropropene

75-25-2--------Bromoform

108-10-1------4-Methyl-2-pentanone

591-78-6------2-Hexanone

127-18-4 - - -- Tetrachloroethene

108-88-3---.--Toluene

79-34-5--.---1, 1,2, 2-Tetrachloroethane

108-90-7-...--Chlorobenzene

100-41-4-...--Ethylbenzene

100-42-5-------Styrene

$1330-20-7-\ldots--$ Xylenes (total)

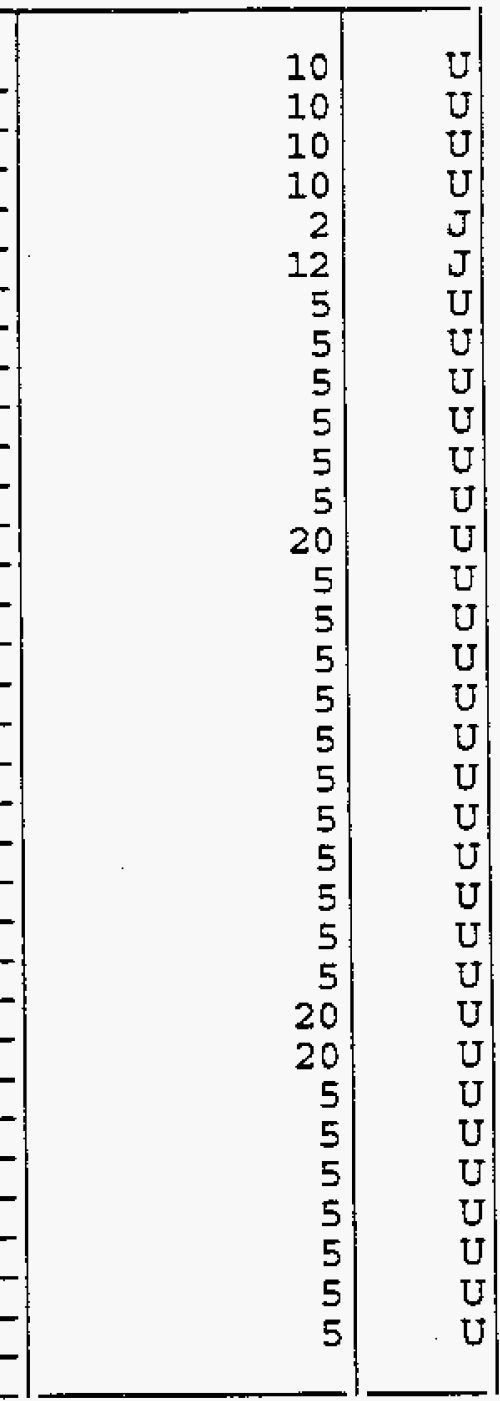


Lab Name: QUANTERRA MO

Lab Code: ITMO

Matrix: (soil/water) WATER

Sample wt/vol:

$5.000(\mathrm{~g} / \mathrm{mI}) \mathrm{ML}$

Level: (low/med) LOW

응 Moisture: not dec.

Colum: (pack/cap) CAP
Contract: 625.01

SAS NO. :
VLCSBO79A

SDG No.: V368

Lab Sample ID: QCLCS167948

Lab File ID: BLCS1890

Date Received:

Date Analyzed: 03/20/98

Dilution Factor: 1.0

CONCENTRATION UNITS:

CAS NO. COMPOUND $\quad(u g / L$ or $u g / \mathrm{kg}) u g / \tau$

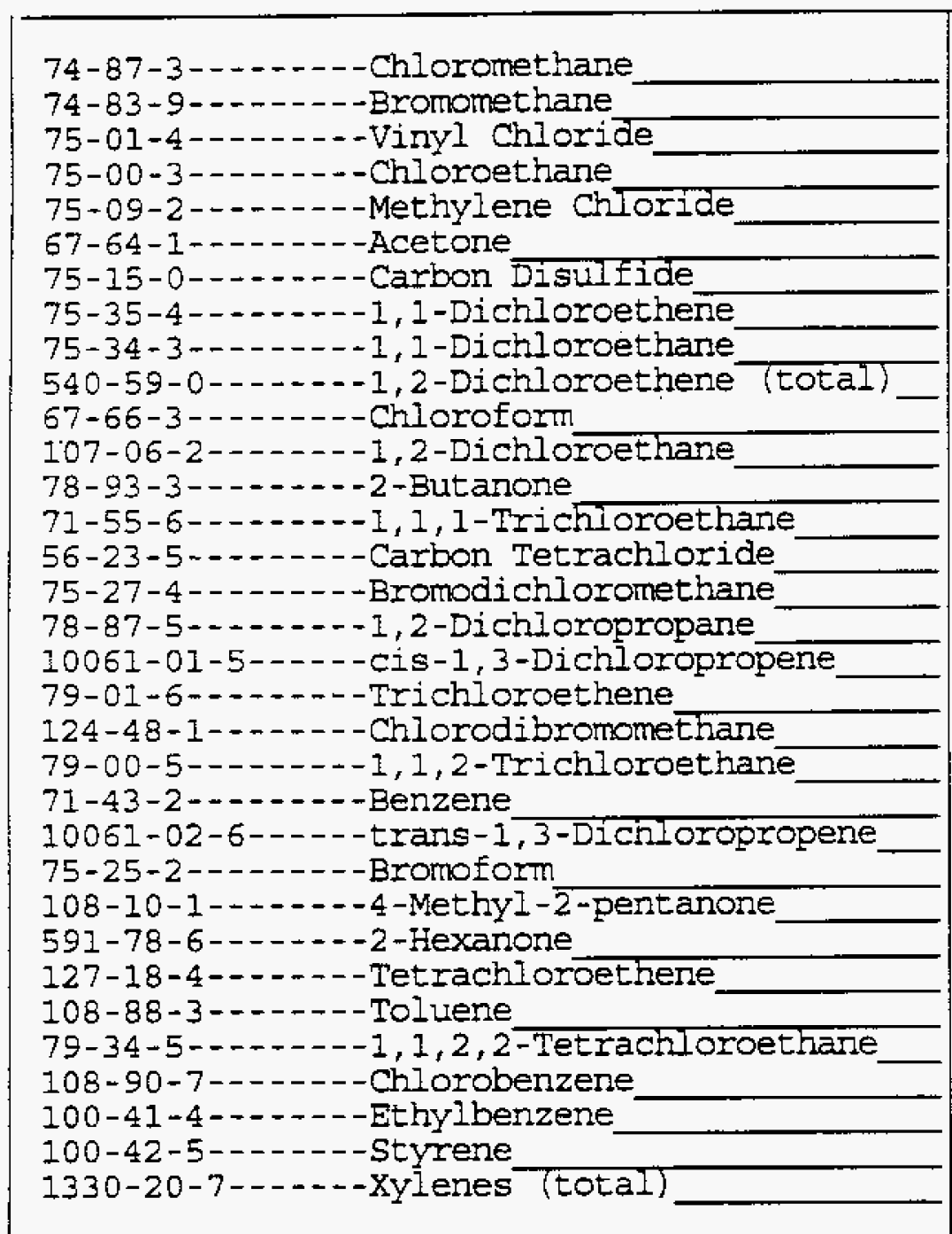

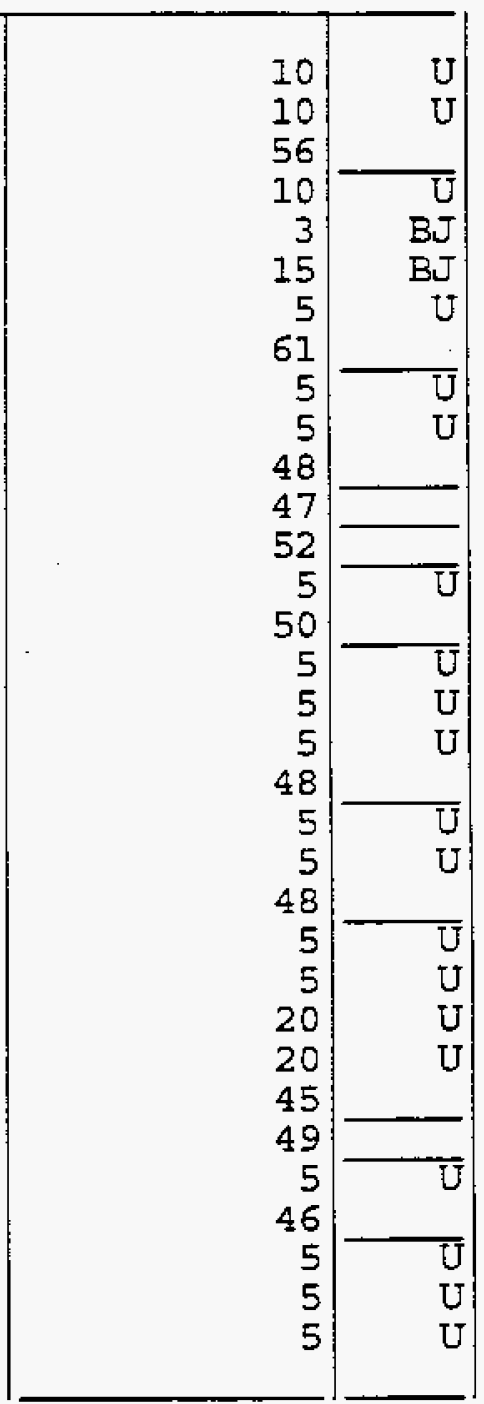

I/87 Rev. 
Lab Name: QUANTERRA MO

Lab Code: IIMO Case No.:

Matrix: (soil/water) WATER

Sample wt/vol:

$5.000(\mathrm{~g} / \mathrm{ml}) \mathrm{ML}$

Level: (low/med) LOW

음 Moisture: not dec.

Columan: (pack/cap) CAP
Contract: 625.01

SAS NO.:

VLCSB083B

SDG No.: $\sqrt{3} 68$

Lab Sample ID: QCLCS168259

Lab File ID: BLCS1917

Date Received:

Date Analyzed: 03/24/98

Dilution Factor: 1.0

CONCENIRATION UNITS:

$(\mathrm{ug} / \mathrm{L}$ or $\mathrm{ug} / \mathrm{Kg}) \mathrm{ug} / \mathrm{L}$

Q

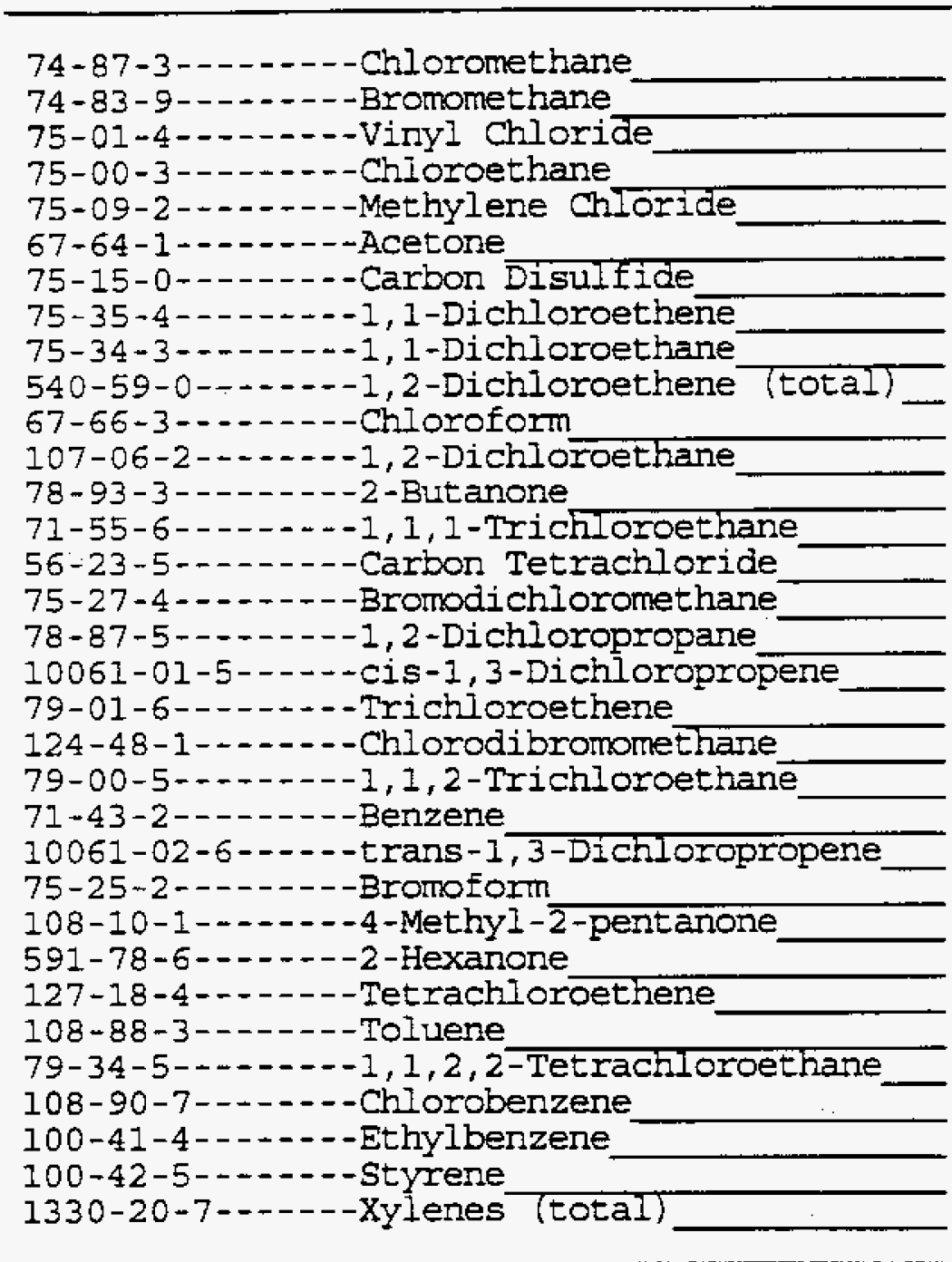

74-87-3------Chloromethane

74-83-9-2.----Bromomethane

75-01-4-------vinyl Chloride

75-00-3--..--Chioroethane

75-09-2--.---Methylene Chloride

67-64-1--.---Acetone

75-15-0-------Carbon Disulfide

75-35-4---n--1, 1-Dichloroethene

75-34-3--.---1, 1-Dichloroethane

540-59-0-...-1,2-Dichloroethene (total)

67-66-3--.---Chloroform

107-06-2-...-1,2-Dichloroethane

$78-93-3------2-$-Butanone

71-55-6--..--1, 1, 1-Trichloroethane

56-23-5-------Carbon Tetrachloride

75-27-4-------Bromodichloromethane

78-87-5-------1,2-Dichloropropane

79-01-6-...-Trichloroethene

124-48-1-- - - - - Chlorodibromomethane

79-00-5-..---1, 1, 2-Trichloroethane

$71-43-2--------$ Benzene

10061-02-6-----trans-1,3-Dichloropropene

$75-25-2--------B r o m o$ form

108-10-1-.----4-Methyl-2-pentanone

591-78-6---.---2-Hexanone

127-18-4-----Tetrachloroethene

108-88-3------ Toluene

79-34-5-..---1, 1, 2, 2-Tetrachloroetnane

- Chlorobenzene

$100-42-5-\ldots--$-Styrene

\begin{tabular}{|r|r}
10 & $\mathrm{U}$ \\
10 & $\mathrm{U}$ \\
53 & \\
10 & $\mathrm{U}$ \\
2 & $\mathrm{UB}$ \\
14 & $\mathrm{UB}$ \\
5 & $\mathrm{U}$ \\
68 & \\
5 & $\mathrm{U}$ \\
5 & $\mathrm{U}$ \\
56 & \\
61 & \\
57 & \\
5 & $\mathrm{U}$ \\
56 & \\
5 & $\mathrm{U}$ \\
5 & $\mathrm{U}$ \\
5 & $\mathrm{U}$ \\
55 & \\
5 & $\mathrm{U}$ \\
5 & $\mathrm{U}$ \\
55 & \\
\hline 5 & $\mathrm{U}$ \\
5 & $\mathrm{U}$ \\
20 & $\mathrm{U}$ \\
20 & $\mathrm{U}$ \\
47 & \\
55 & $\mathrm{U}$ \\
5 & \\
55 & $\mathrm{U}$ \\
5 & $\mathrm{U}$ \\
5 & $\mathrm{U}$ \\
5 & \\
\hline & \\
\hline
\end{tabular}


WA: VOLATIIE SURROGATE RECOVER:

Iab Name: QUANTERRA MO

Iab Code: ITMO
Case No.:
Contract: 625.01

SAS NO. :
SDG NO.: $\sqrt{368}$

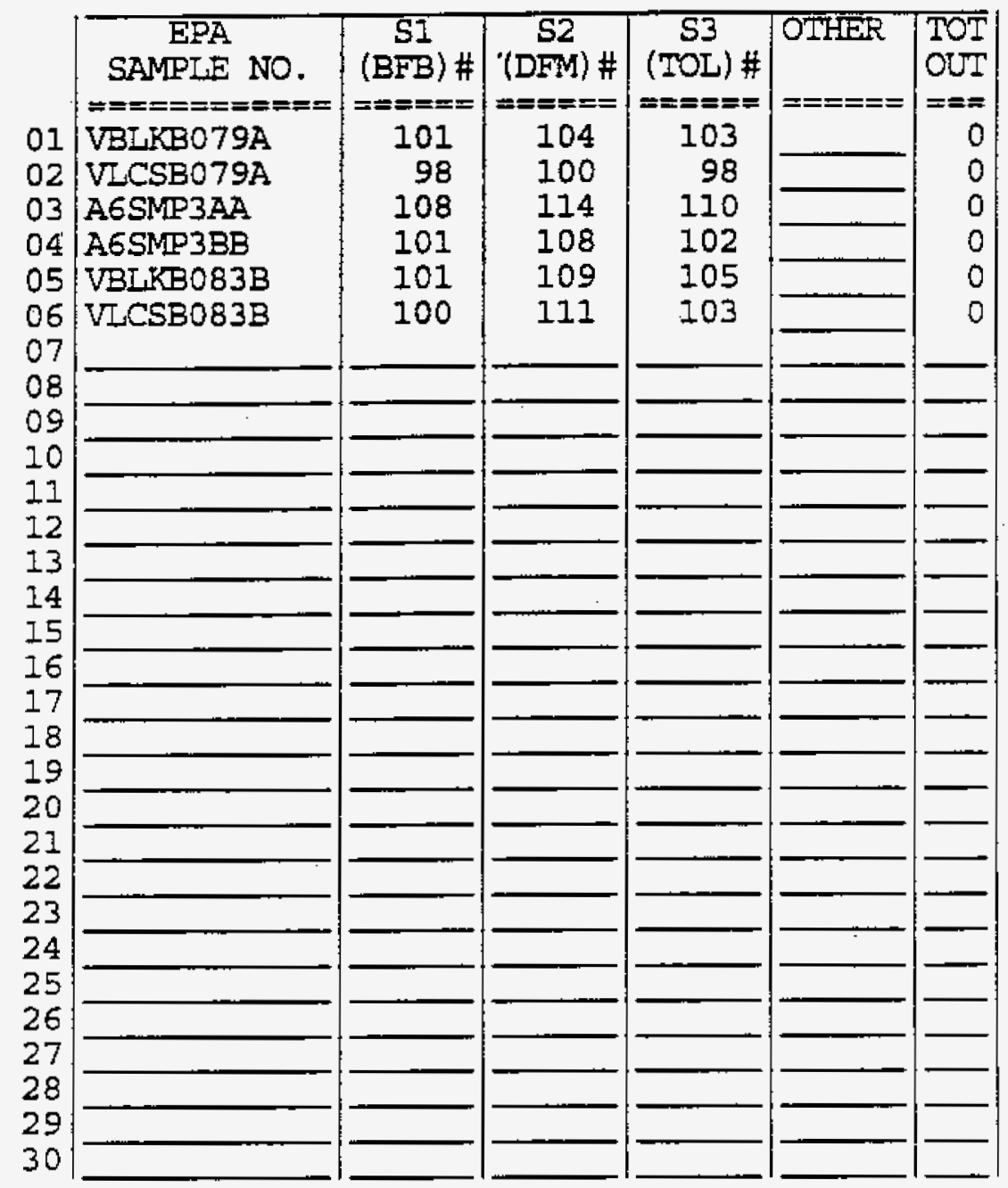

\footnotetext{
S1 (BFB) $=4$-Bromofluorobenzene

S2 (DFM) = Dibromofluoromethane

$(80-120)$

$(80-120)$

S3 (TOL) = Toluene-d8

$(80-120)$

\# Colurm to be used to flag recovery values

* Values outside of contract required $Q C$ limits

D Surrogates diluted out
}

OC LIMITS

page 1 of 1 
SC VOLATILE SURROGATE RECOVER:

Lab Name: QUANTERRA MO

Lab Code: ITMO Case No.:
Contract: 625.01

SAS NO.:

Level : (low/med) LOW

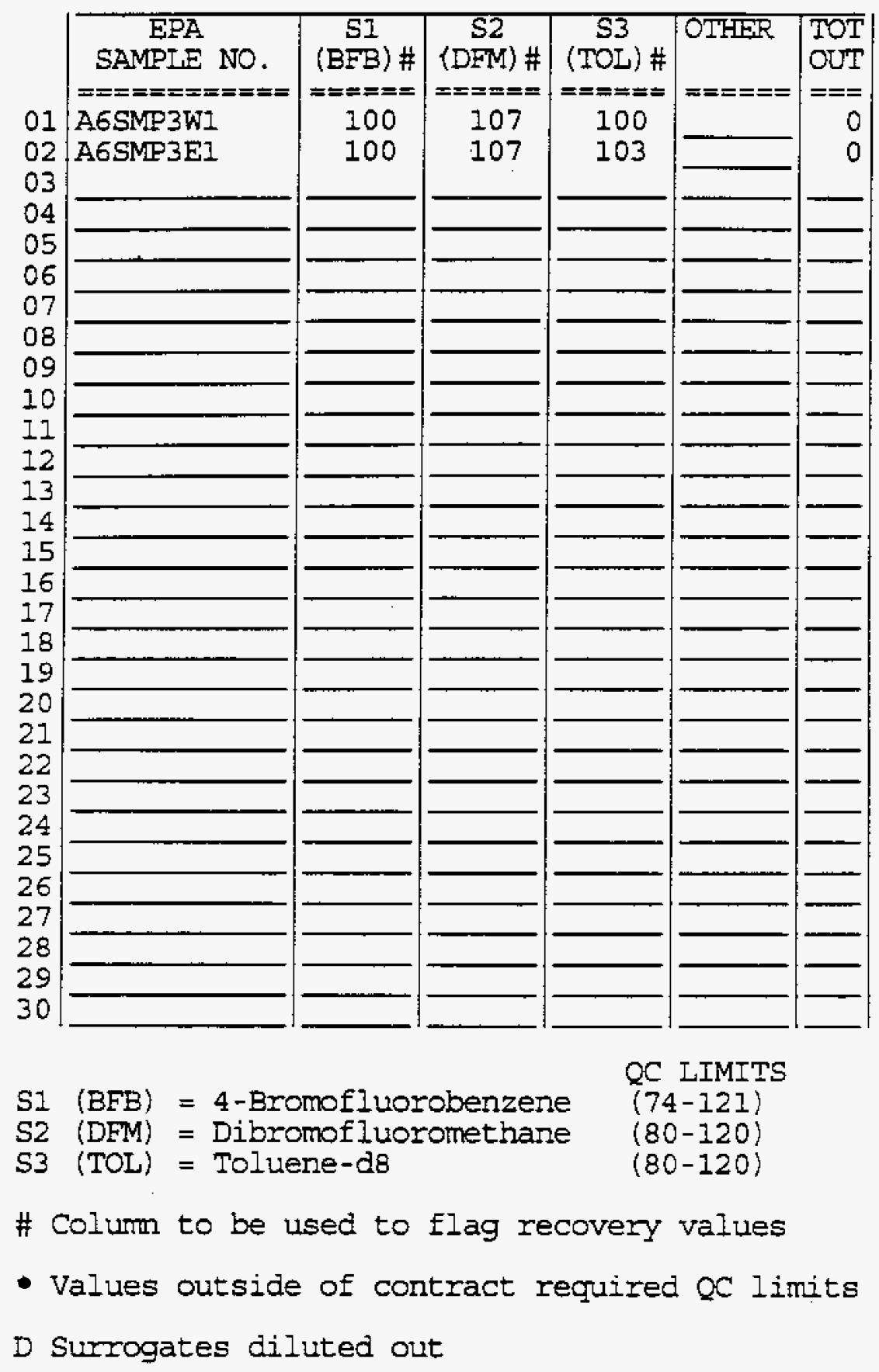

page 1 of 1 
Lab Name: QUANTERRA MO

Lab Code: ITMO

Case No.:

Lab File ID:

BBLK1889

Date Analyzed:

$03 / 20 / 98$

Matrix: (soil/water) WATER

Instrument ID:

MSB
Contract: 625.01

SAS NO. :

SDG No.: $\sqrt{3} 68$

Lab Sarple ID: QCBLK167948

Time Analyzed: $\quad 0635$

Level : (low/med) LOW

THIS METHOD BLANK APPLIES TO THE FOIIOWING SAMPLES, MS and MSD:

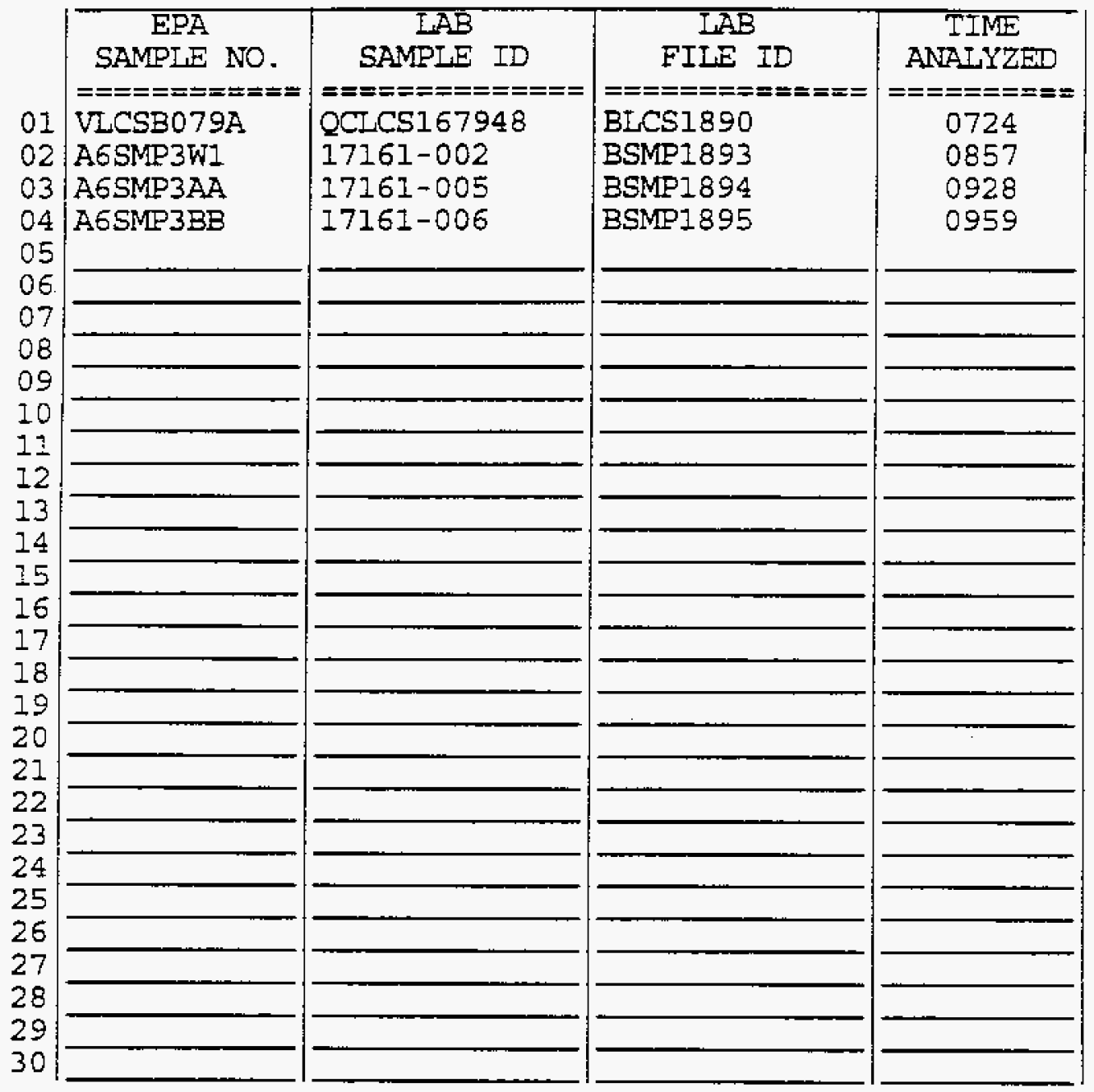

COMMENTS : 
VOLi IE METHOD BLANK SUMMARY

Lab Name: QUANTERRA MO

Lab Code: ITMO

Case No.:

Lab File ID:

BBLKI916

Date Analyzed:

Matrix: (soil/water) WATER

Instrument ID:

MSB
Contract : 625.01

SAS NO.:

SDG NO.: V368

Lab Sample ID: QCBLK168259

Time Analyzed: $\quad 1235$

Level: (low/med) LOW

THIS METHOD BLANK APPLIES TO THE FOLLOWING SAMPLES, MS and MSD:

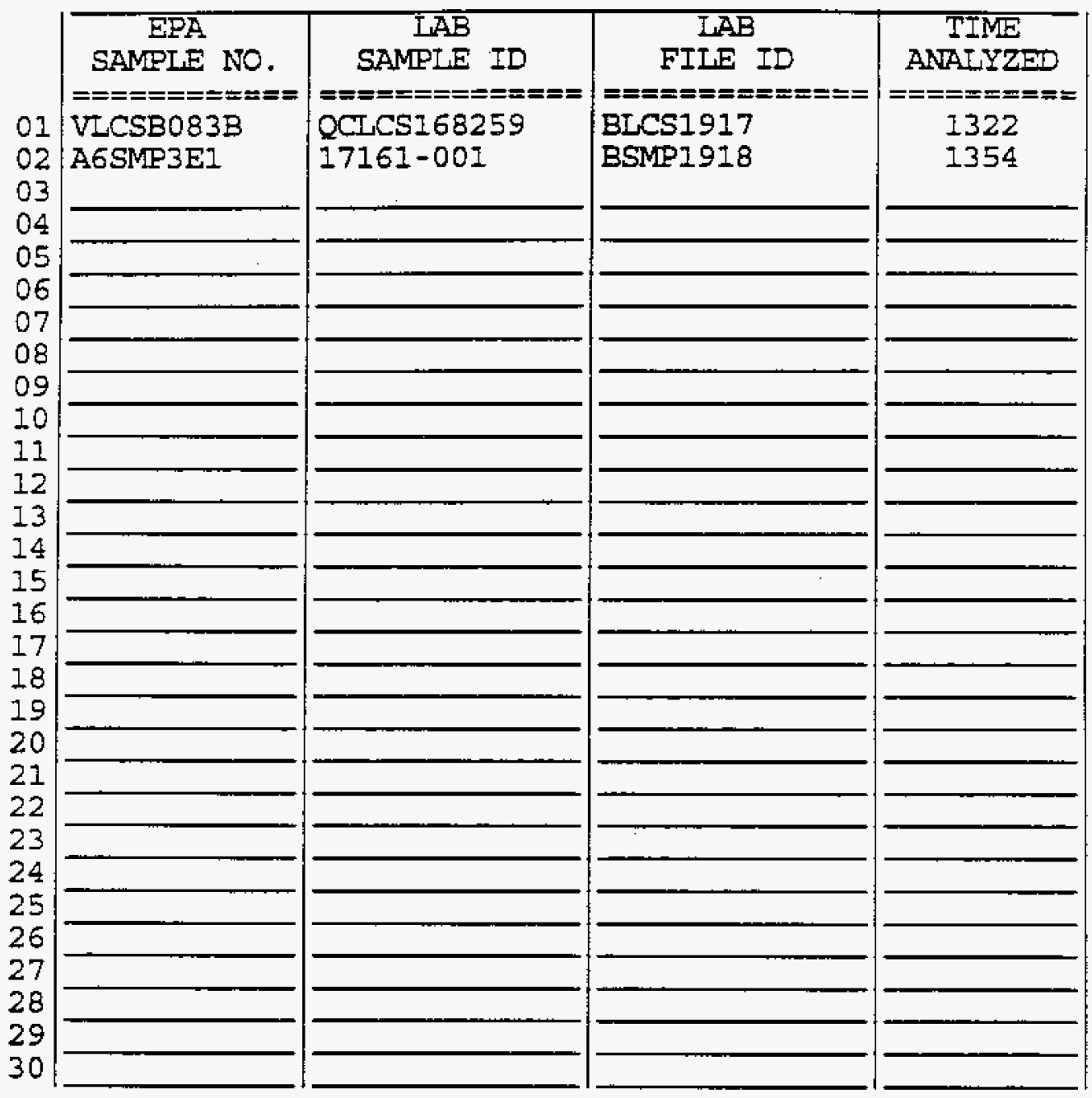

COMMENTS : 
VOLATILE RGANIC GC/MS TUNING AND MASE

CAIIBRATIUN - BROMOFLUOROBENZENE (BFB)

Lab Name: QUANTERRA MO

Lab code: ITMO Case No.:

Lab File ID: BEFB1842

Instrument ID: MSB

Matrix: (soil/water) WATER Level:(low/med) LOW Column:(pack/cap) CAP
Contract: 625.01

SAS NO. :

SDG No.: $\sqrt{ } 368$

BFB Injection Date: 03/18/98

BFB Injection Time: 0558

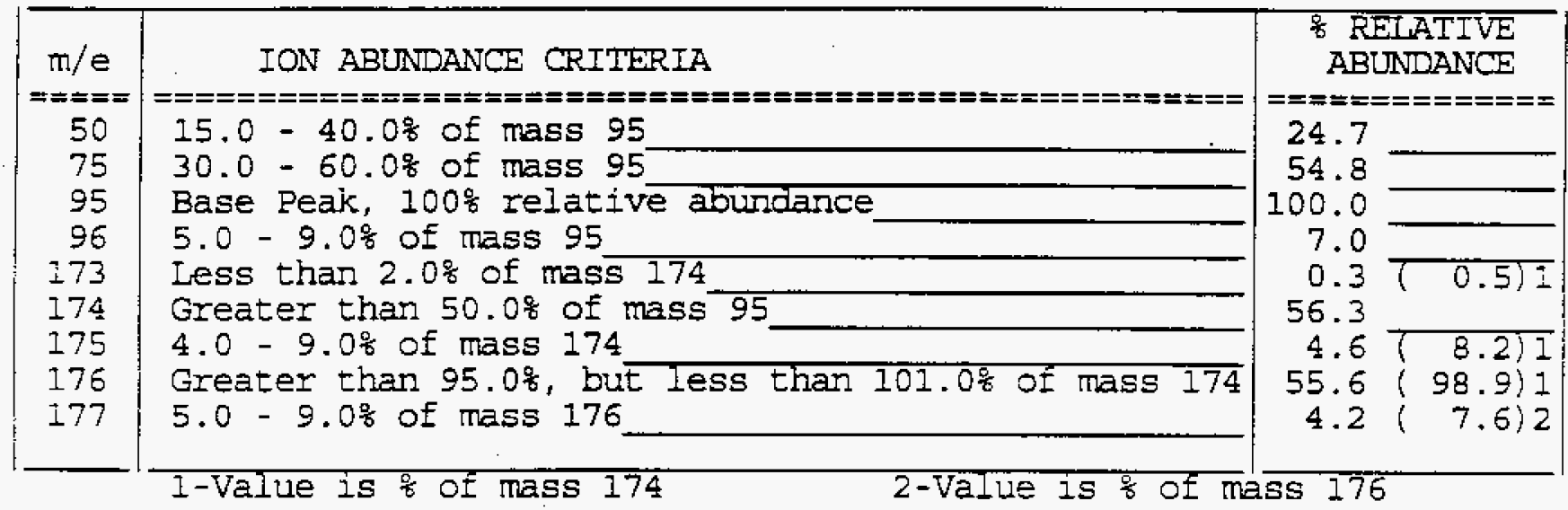

THIS TUNE APPLIES TO THE FOLLOWING SAMPIES, MS, MSD, BLANKS, AND STANDARDS:

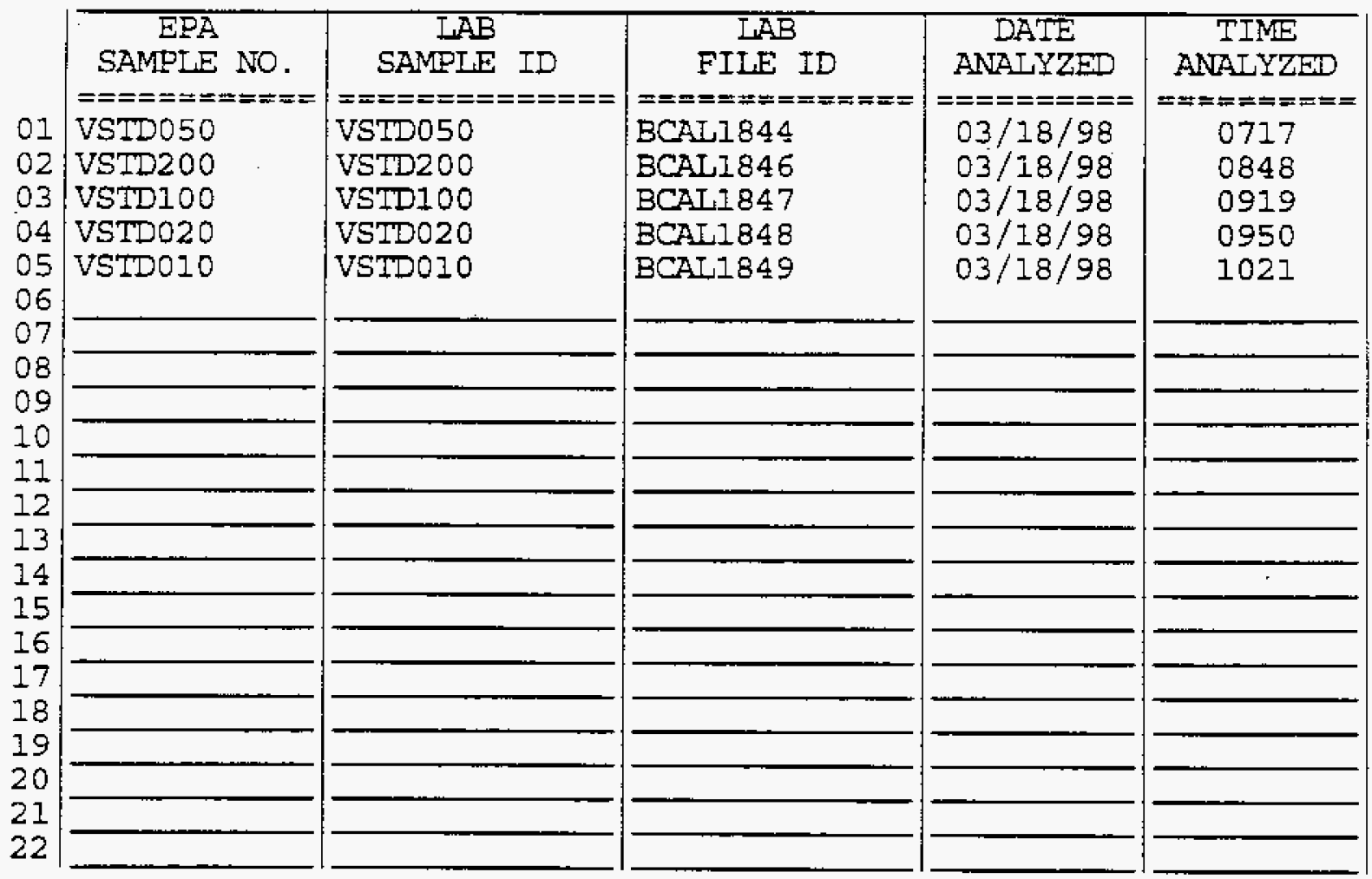

page 1 of 1 
VOLATILI RGANIC GC/MS TUNING AND MAS! CALIBRATION - BROMOFLUOROBENZENE (BFB)

Lab Name: QUANTERRA MO

Lab Code: ITMO Case No.:

Lab File ID: BBFBI886

Instrument ID: MSB

Matrix: (soil/water) WATER Level:(low/med) LOW Column:(pack/cap) CAP
Contract: 625.01

SAS No. :

SDG No.: V368

BFB Injection Date: 03/20/98

BFB Injection Time: 0444

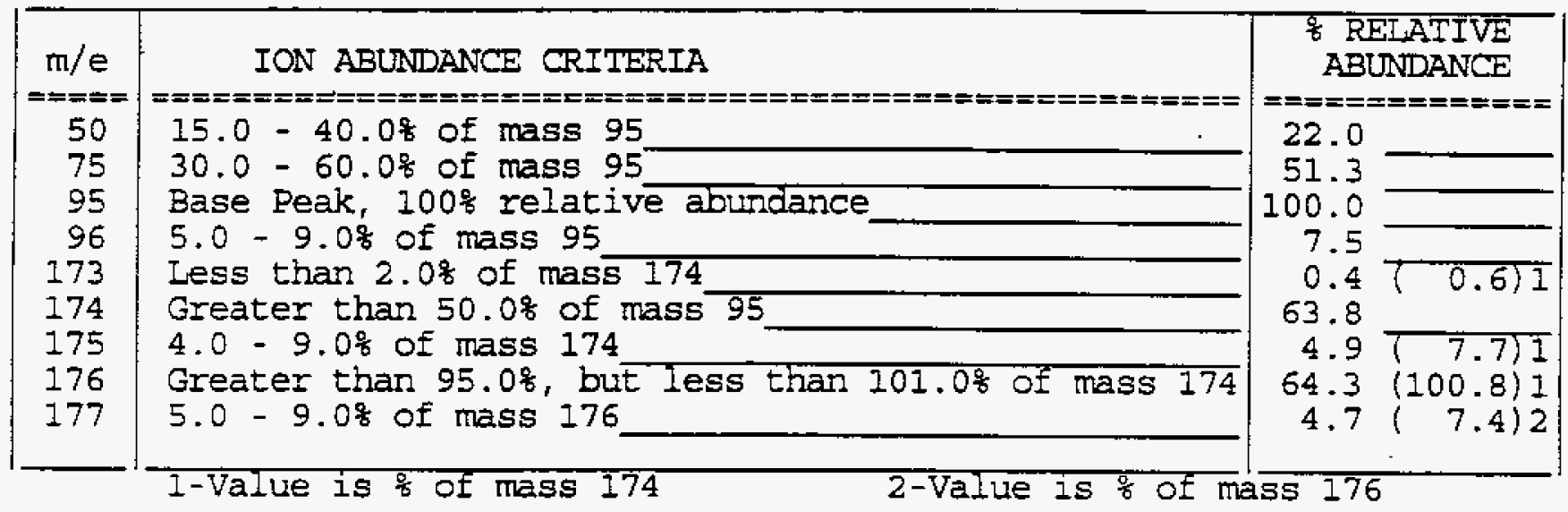

THIS TUNE APPLIES TO THE FOLIOWING SAMPLES, MS, MSD, BLANKS, AND STANDARDS:

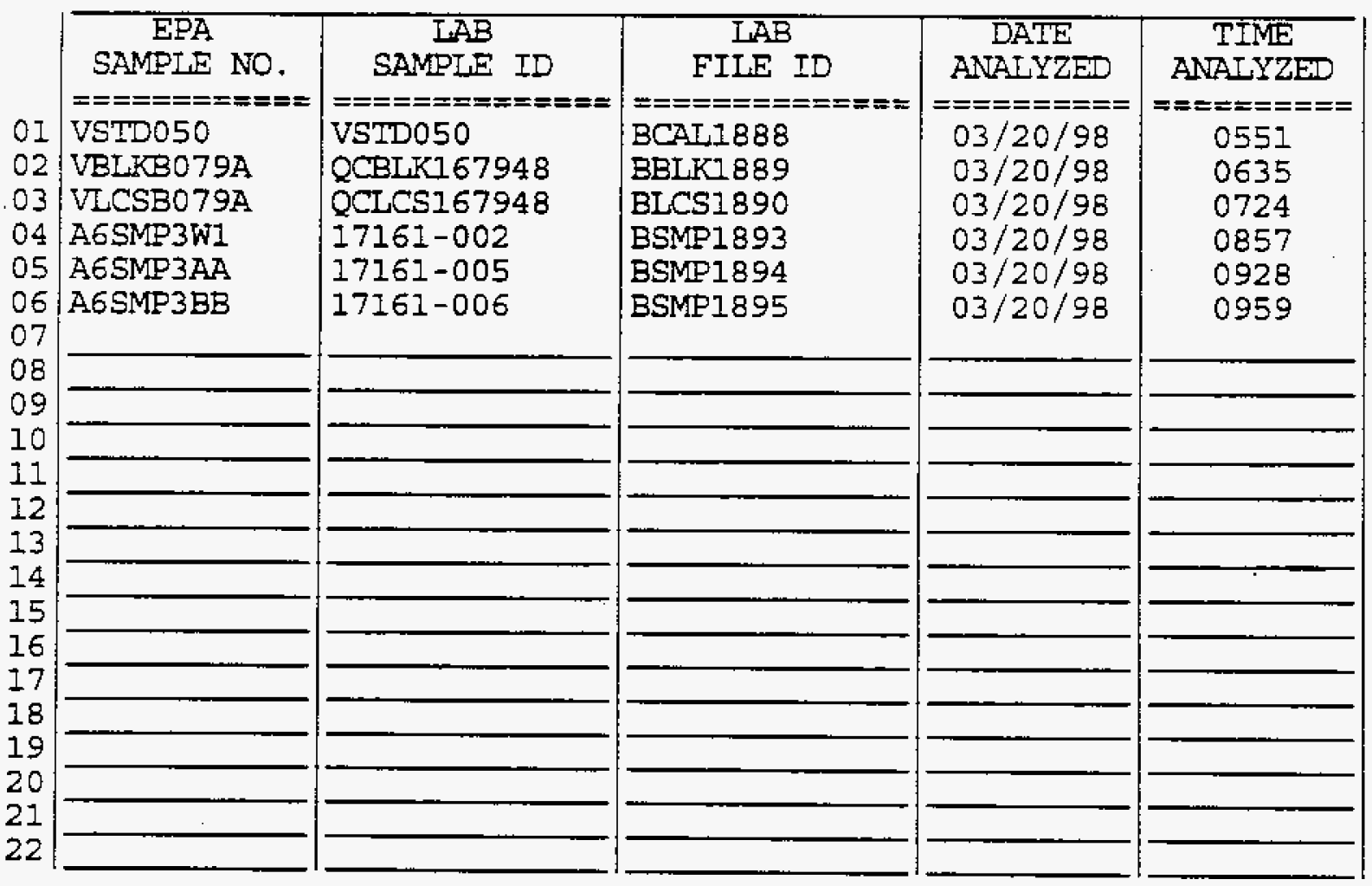

page 1 of 1 
VOLATIIE RGANIC GC/MS TUNING AND MASE

CALIBRATION - BROMOFLUOROBENZENE (BFB)

Lab Name: QUANIERRA MO

Lab Code: ITMO ' Case No.:

Lab File ID: BBFB1910

Instrument ID: MSB

Matrix: (soil/water) WATER Level:(low/med) LOW Colunn: (pack/cap) CAP
Contract: 625.01

SAS NO.:

SDG No.: V368

BFB Injection Date: $03 / 24 / 98$

BFB Injection Time: 0932

\begin{tabular}{|c|c|c|}
\hline $\mathrm{m} / \mathrm{e}$ & ION ABUNDANCE CRITERIA & $\begin{array}{l}\% \text { RELATIVE } \\
\text { ABUNDANCE }\end{array}$ \\
\hline$===x=$ & 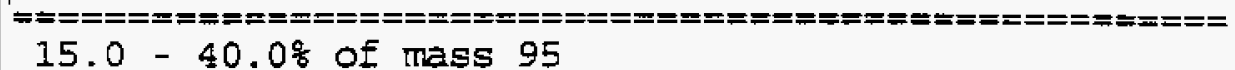 & $=============$ \\
\hline 75 & $30.0-60.0 \%$ of mass 95 & 51.2 \\
\hline 95 & Base Peak, $100 \%$ relative abundance & 100.0 \\
\hline 96 & $5.0-9.0 \%$ of mass 95 & 7.2 \\
\hline 173 & Less than $2.0 \%$ of mass 174 & $0 . 4 \longdiv { ( 0 . 6 ) 1 }$ \\
\hline 174 & Greater than $50.0 \%$ of mass 95 & 54.6 \\
\hline 175 & $4.0-9.08$ of mass 174 & $4 . 3 \longdiv { 7 . 9 ) 1 }$ \\
\hline 176 & Greater than $95.0 \%$, but less than $101.0 \%$ of mass 174 & $53.0(97.0) 1$ \\
\hline 177 & $5.0-9.0 \%$ of mass 176 & $3.8(7.1) 2$ \\
\hline
\end{tabular}

THIS TUNE APPLIES TO THE FOLLOWING SAMPLES, MS, MSD, BLANKS, AND STANDARDS:

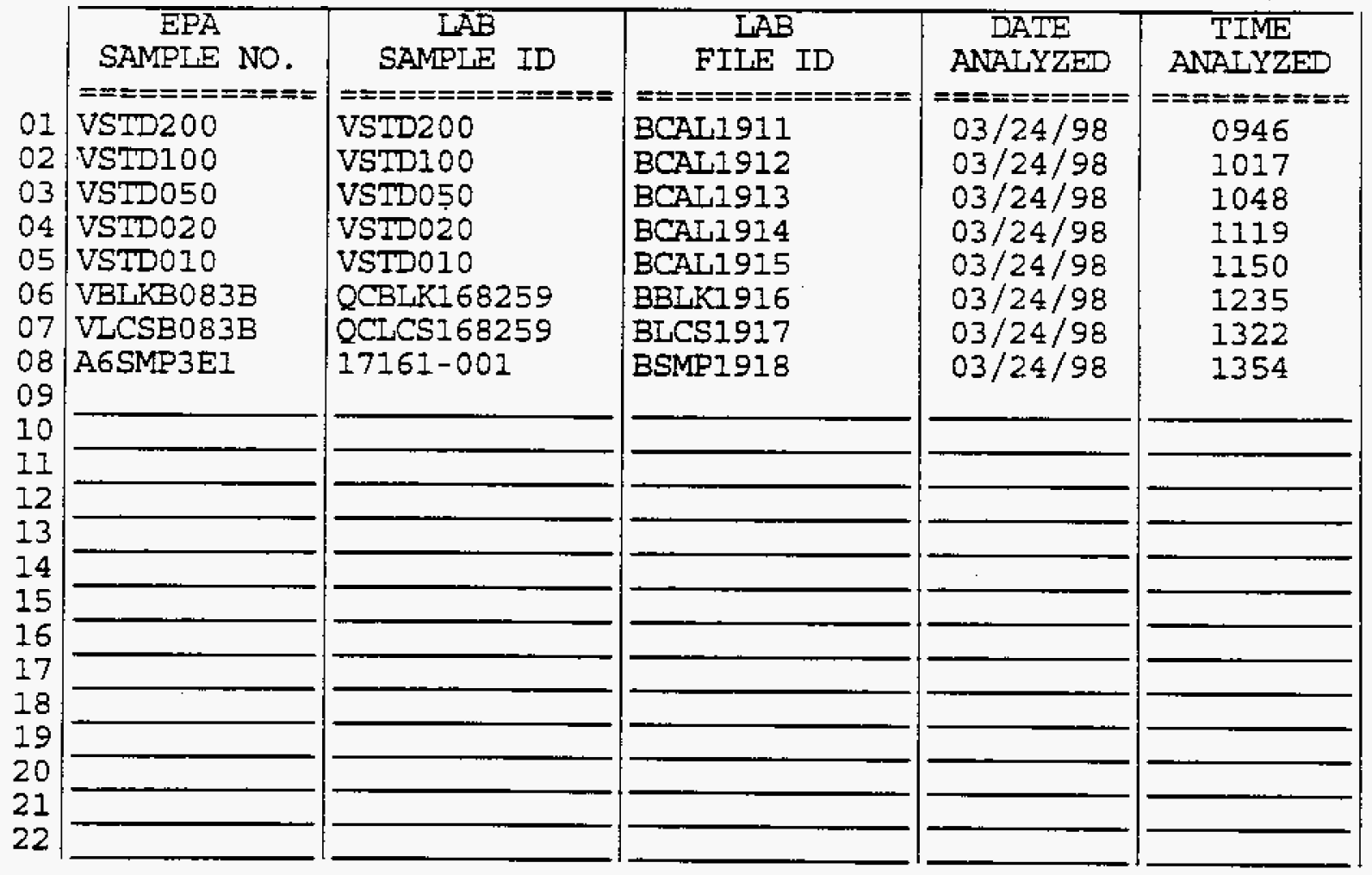

page 1 of 1 
VOLA: $\quad 3$ ORGANICS INITLAL CALIBRAT: 'DATA

Lab Name: QUANTERRA MO

Iab Code: ITMO

Case No.:
Contract: 625.01

SAS NO.:
SDG No. : V368

Instrument ID: MSB

Calibration Date (s) : 03/18/98

Matrix: (soil/water) SOII Level:(low/med) LOW Colunn: (pack/cap) CAP

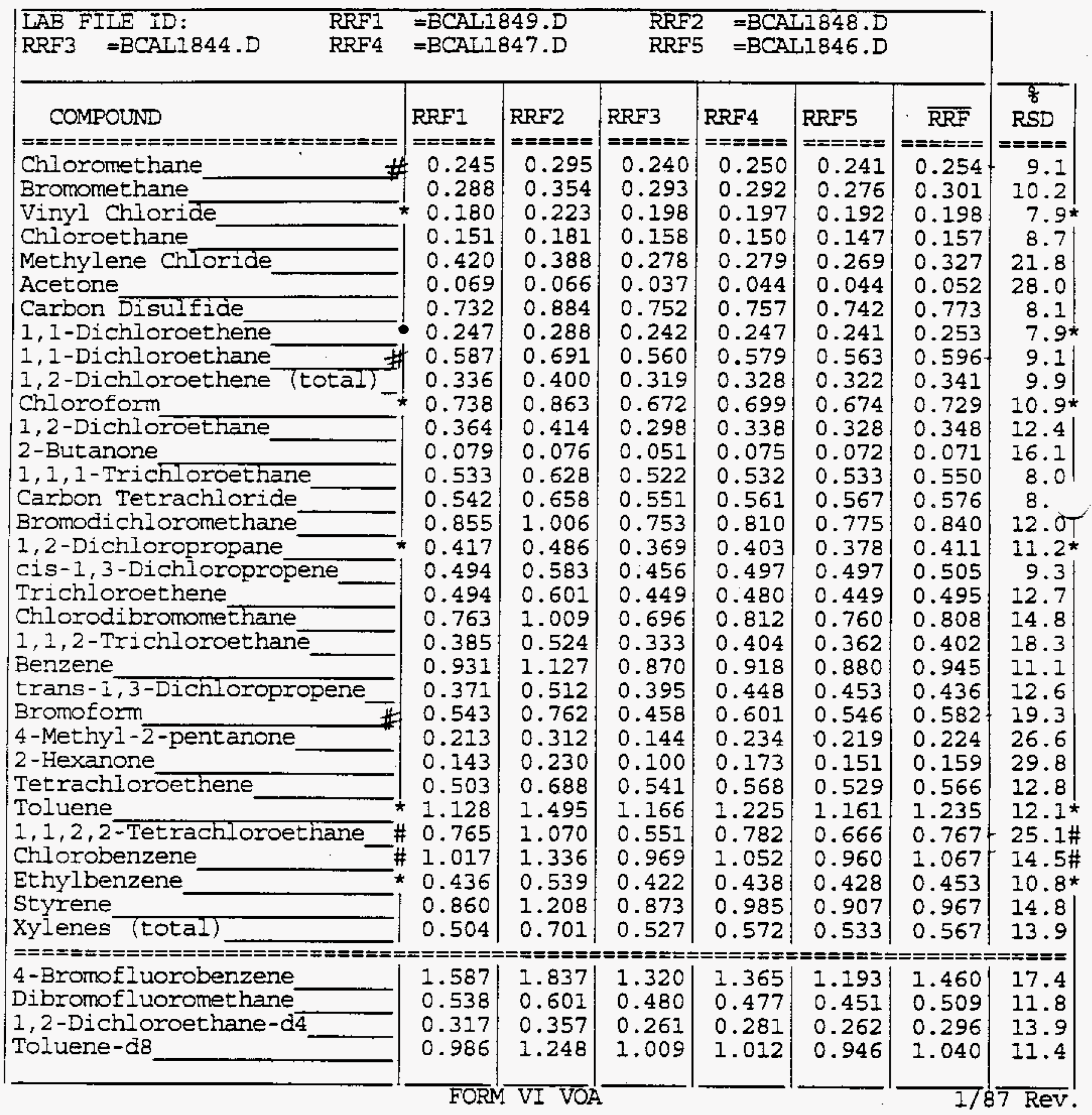


VOLA: E ORGANICS INITTAL CALIBRAT: DATA

Lab Name: QUANTERRA MO

Lab code: ITMO Case No.:
Contract: 625.01

SAS NO.:
SDG No.: $\mathrm{V} 368$

Instrument ID: MSB

Calibration Date(s): 03/24/98

Matrix: (soil/water) SOIL Level:(low/med) IOW Column: (pack/cap) CAP

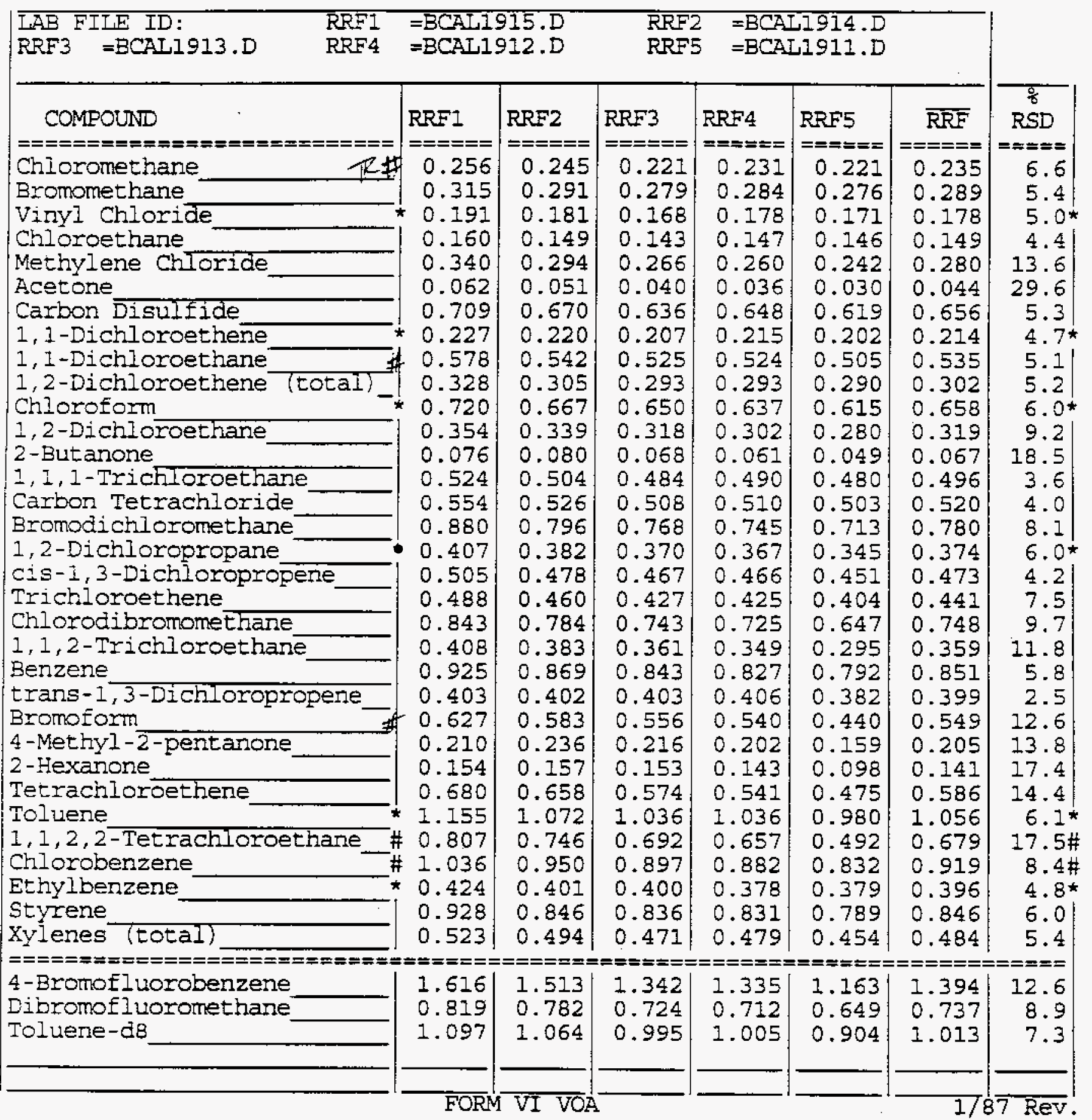


Lab Name: QUANTERRA MO

Contract: 625.01

Lab code: ITMO' Case No.: SAS No.:

SDG No. : $\sqrt{368}$

Instrument ID: MSB

Calibration Date: $03 / 20 / 98$

Time: 0551

Lab File ID: BCAL1888

Init. Calib. Date (s) : 03/18/98

Matrix: (soil/water) SOIL Level:(low/med) IOW Colum: (pack/cap) CAP

Min RRE50 for SPCC $(\#)=0.300(0.250$ for Bromoform) Max \&D for CCC $(*)=20.0 \%$

\begin{tabular}{|c|c|c|c|c|c|}
\hline $\begin{array}{l}\text { COMPOUND } \\
=========1\end{array}$ & $\begin{array}{l}\text { SAMPLE } \\
\text { AMOUNT } \\
=====\end{array}$ & $\begin{array}{l}\text { CAL50 } \\
\text { AMOUNT } \\
=====\end{array}$ & $\begin{array}{l}\text { CURVE } \\
=====\end{array}$ & $\mathrm{SD}$ & $R R F$ \\
\hline Chiloromethane & \multirow{2}{*}{$\begin{array}{r}\# 42.387 \\
-46.314\end{array}$} & \multirow{2}{*}{$\begin{array}{l}50.000 \\
50.000\end{array}$} & \multirow{2}{*}{$\begin{array}{l}\text { LINR } \\
\text { LINR }\end{array}$} & \multirow{4}{*}{$\begin{array}{l}15.226 \\
7.3720 \\
14.962 \\
6.1800\end{array}$} & \multirow[t]{2}{*}{0.20677} \\
\hline Bromomethane - & & & & & \\
\hline Vinyl chloride. & \multirow{4}{*}{$-\begin{array}{l}42.519 \\
46.910 \\
47.229 \\
42.015\end{array}$} & \multirow{4}{*}{$\begin{array}{l}50.000 \\
50.000 \\
50.000 \\
50.000\end{array}$} & \multirow{4}{*}{$\begin{array}{l}\text { LINR } \\
\text { LINR } \\
\text { LINR } \\
\text { LINR }\end{array}$} & & \\
\hline Chloroethane & & & & & \\
\hline Methylene Chloride. & & & & 5.5420 & \\
\hline Acetone & & & & 15.970 & \\
\hline Carbon Disulfide & 46.198 & 50.000 & LINR & 7.6040 & \\
\hline $\begin{array}{l}\text { 1, 1-Dichloroethene } \\
1,1 \text {-Dichloroethane }\end{array}$ & $\begin{array}{l}\star 46.553 \\
\# 42.361\end{array}$ & $\left|\begin{array}{l}50.000 \\
50.000\end{array}\right|$ & $\begin{array}{l}\text { LINR } \\
\text { LINR }\end{array}$ & $\begin{array}{l}6.8940^{\star} \\
15.278^{*}\end{array}$ & 0.481 \\
\hline 1,2 -Dichloroethene (total) & 91.474 & 100.00 & LINR & 2601 & \\
\hline Chloroform & 45.799 & 50.000 & LINR & $8.4020^{\star}$ & \\
\hline 1,2-Dichloroethane. & 42.745 & 50.000 & LINR & 14.510 & \\
\hline 2-Butanone & 34.703 & 50.000 & LINR & 30.594 & \\
\hline $\begin{array}{l}\text { 1, 1, 1-Trichloroethane } \\
\text { Carbon Tetrachloride }\end{array}$ & 44.881 & 50.000 & LINR & .238 & \\
\hline Bromodichloromethane & 44.780 & 50.000 & LINR & 10.440 & \\
\hline 1,2-Dichloropropane & 44.659 & 50.000 & LINR & $10.682 *$ & \\
\hline cis-1,3-Dichloropropene & 42.790 & 50.000 & LINR & 14.420 & \\
\hline Trichloroethene & 45.743 & 50.000 & LINR & 8.5140 & \\
\hline Chlorodibromomethane & 42.444 & 50.000 & LINR & 15.112 & \\
\hline 1, 1,2-Trichloroethane & 41.863 & 50.000 & LINR & 16.274 & \\
\hline trans-1,3-Dichloroprop & 40.075 & 50.000 & $\begin{array}{l}\operatorname{LiNR} \\
\mathrm{LINR}\end{array}$ & 19.850 & \\
\hline Bromoform & $\# 39.297$ & 50.000 & LINR & $21.406 \#$ & 0. \\
\hline 4 -Methy $1-2$-pertanone & 33.331 & 50.000 & LINR & 33.338 & \\
\hline 2-Hexanone & 32.992 & 50.000 & LINR & 34.016 & \\
\hline Tetrachloroethene & 44.973 & 50.000 & LINR & 10.054 & \\
\hline Toluene & $\$ 44.307$ & 50.000 & LINR & $11.386 *$ & 0,537 \\
\hline $\begin{array}{l}1,1,2,2 \text {-Tetrachloroethane } \\
\text { Chlorobenzene }\end{array}$ & $\begin{array}{l}\# 38.840 \\
\# 44.991\end{array}$ & $\begin{array}{l}50.000 \\
50.000\end{array}$ & $\begin{array}{l}\text { IINR } \\
\text { IINR }\end{array}$ & $\begin{array}{l}22.320 \# \\
10.018 \#\end{array}$ & 0.357 \\
\hline Ethylbenzene & 254 & 50.000 & LINR & $11.492^{\star}$ & \\
\hline Styrene & 43.706 & 50.000 & LINR & 12.588 & \\
\hline Xylenes (total) & 133.01 & 150.00 & LINR & 11.327 & \\
\hline 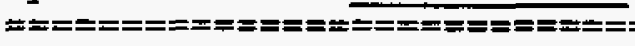 & $==$ & & $E= \pm=$ & & \\
\hline 4-Bromofluorobenzene & 54.667 & 50.000 & LINR & -9.334 & \\
\hline Dibromofluoromethane & 52.875 & 50.000 & LINR & -5.750 & \\
\hline Tolvene-d8 & 51.387 & 50.000 & LINR & -2.774 & \\
\hline
\end{tabular}


Lab Name: QUANTERRA MO

Lab Code: ITMO Case No.:

Lab File ID (Standard): BCALI888

Instrument ID: MSB

Matrix: (soil/water) SOII Level: (Iow/med) LOW
Contract: 625.01

SAS NO. :

SDG No.: $\mathrm{V} 368$

Date Analyzed: 03/20/98

Time Analyzed: 0551

Colum: (pack/cap) CAP

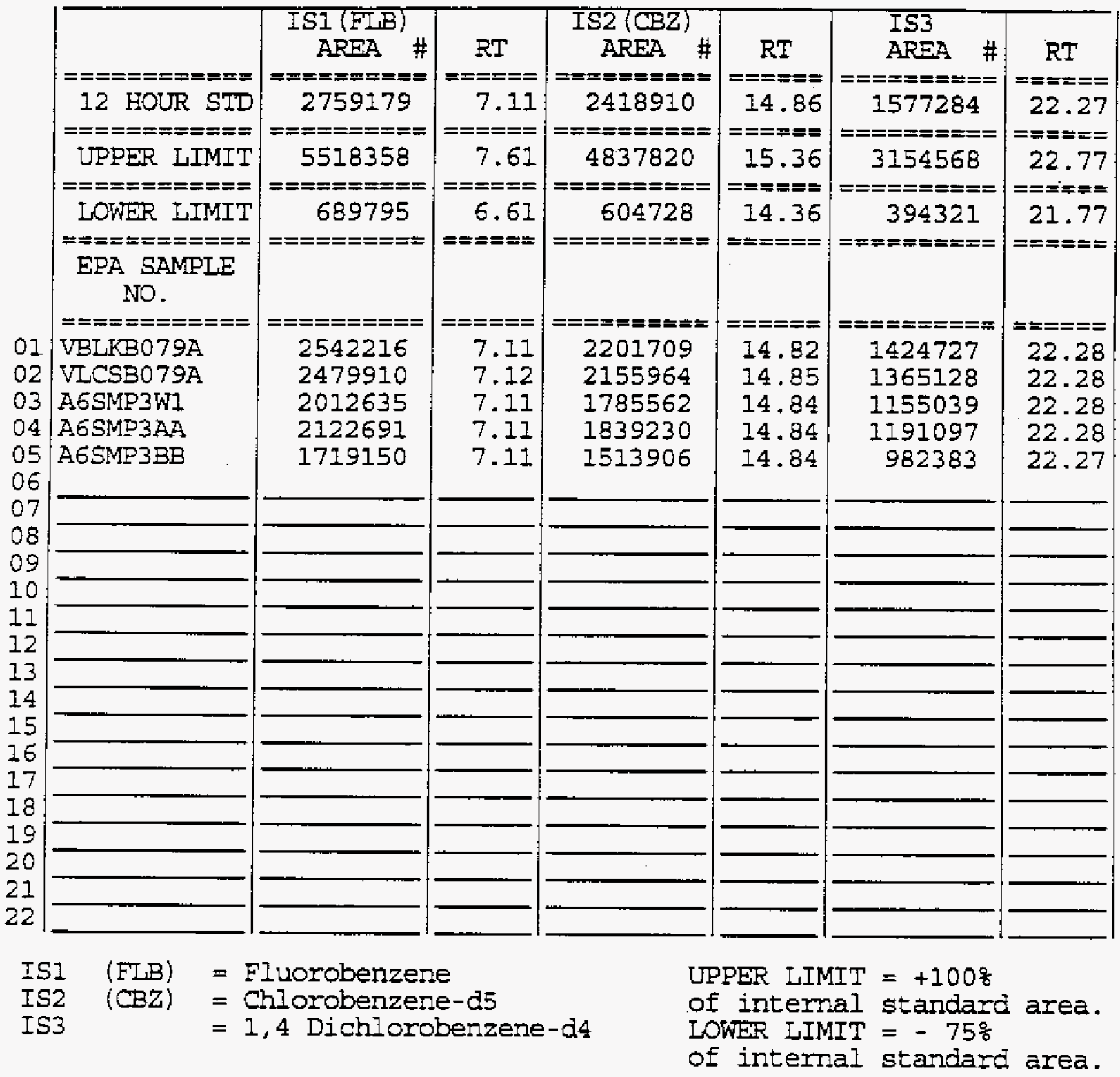

\# Colum used to flag internal standard area values with an asterisk.

page 1 of 1

FORM VIII VOA

$1 / 87$ Rev. 
Lab Name: QUANTERRA MO

Lab Code: ITMO Case No.:

Lab File ID (Standard): BCAI1915

Instrument ID: MSB

Matrix: (soil/water) SOIL Level:(low/med) LOW Colurn: (pack/cap) CAP
Contract: 625.01

SAS NO.:

SDG No.: $\mathrm{V} 368$

Date Analyzed: 03/24/98

Time Analyzed: 1150 .

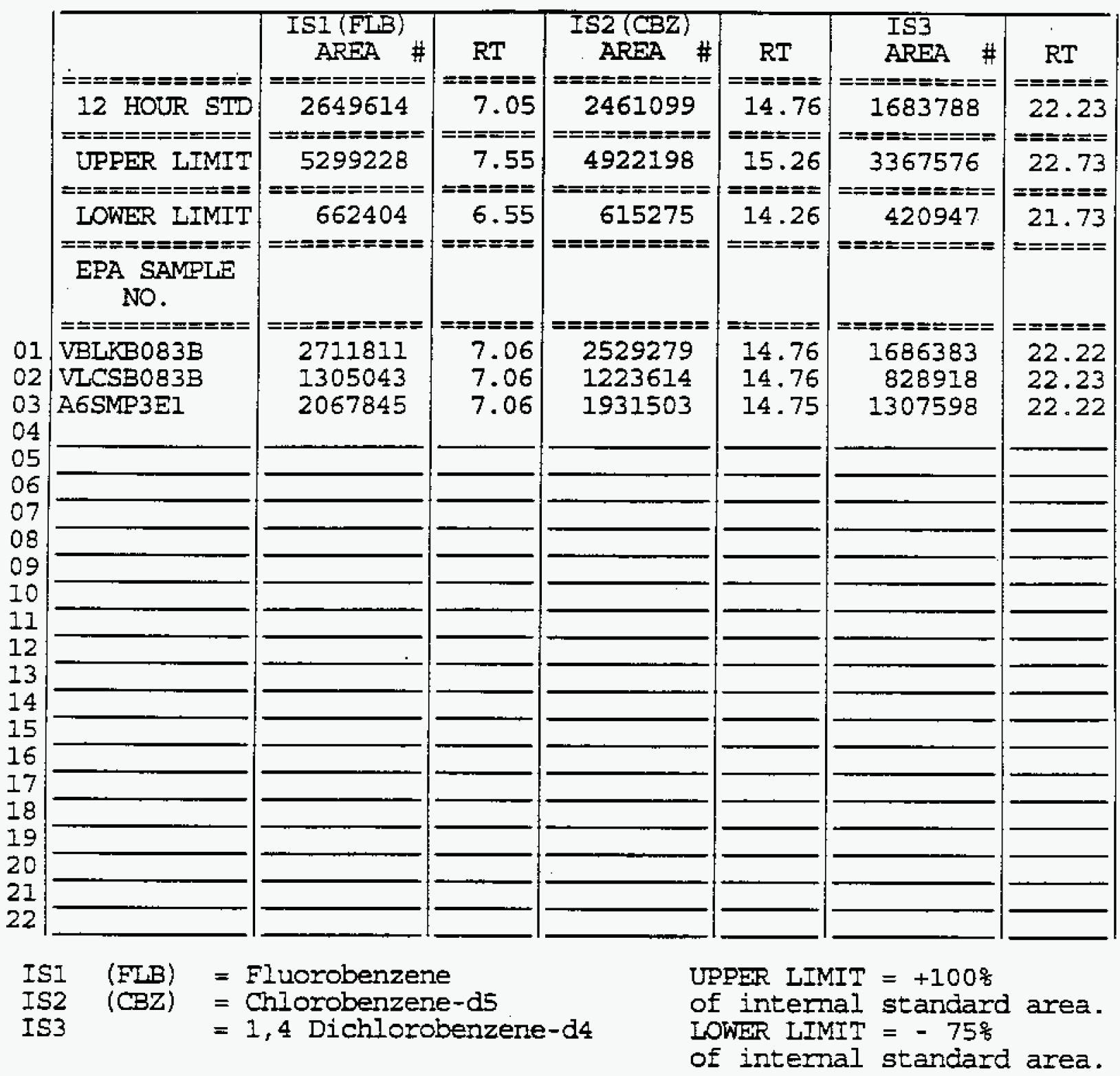

\# Colum used to flag internal standard area values with an asterisk.

page 1 of 1 
9A

VOLATILE SPIKE RECOVERY

Lab Name: QUANTERRA MO

Lab Code: ITMO

Case No.:
Contract: 625.01

SAS NO.:

SDG No.: V368

EPA Sample No.: VLCSBO79A

\begin{tabular}{|ll}
\hline SPIKE COMPOUND \\
\hline 11 1,1-Dichloroethene \\
42 Trichloroethene \\
40 Benzene \\
52 Toluene \\
62 Chlorobenzene \\
\hline
\end{tabular}

\begin{tabular}{|l|} 
CONC \\
ADDED \\
$u g / I$ \\
\hline 50.00 \\
50.00 \\
50.00 \\
50.00 \\
50.00 \\
\hline
\end{tabular}

\begin{tabular}{|}
$\begin{array}{c}\text { CONC } \\
\text { RECOVERED } \\
\text { ug/L }\end{array}$ \\
\hline 61.12 \\
47.79 \\
48.49 \\
48.94 \\
46.52 \\
\hline
\end{tabular}

\begin{tabular}{|r|r|}
\hline \multicolumn{1}{|c|}{ RECOVERED } & IIMITS \\
\hline 122.25 & \\
95.59 & $71-157$ \\
96.98 & $37-151$ \\
97.89 & $47-150$ \\
93.05 & $37-160$ \\
\hline
\end{tabular}

\begin{tabular}{|ll|}
\hline SURROGATE COMPOUND \\
\end{tabular}

\begin{tabular}{|}
$\begin{array}{c}\text { AMOUNT } \\
\text { ADDED } \\
u g / L\end{array}$ \\
\hline 50.00 \\
50.00 \\
50.00 \\
50.00 \\
\hline
\end{tabular}

\begin{tabular}{|}
$\begin{array}{c}\text { AMOUNT } \\
\text { RECOVERED } \\
\text { Ug/I }\end{array}$ \\
\hline 50.09 \\
48.35 \\
49.28 \\
48.94 \\
\hline
\end{tabular}

\begin{tabular}{|r|r|}
\multicolumn{1}{c|}{$\frac{\text { co }}{\text { RECOVERED }}$} & LIMITS \\
\hline 100.19 & $80-120$ \\
96.69 & $80-120$ \\
98.56 & $80-120$ \\
97.89 & $80-120$ \\
\hline
\end{tabular}


$9 A$

VOLATILE SPIKE RECOVERY

Lab Name: QUANTERRA MO

Lab Code: ITMO Case No.:
Contract: 625.01

SAS NO.:

SDG No.: V368

EPA Sample No.: VLCSB083B

\begin{tabular}{|c|c|c|c|c|}
\hline SPIKE COMPOUND & $\begin{array}{l}\text { CONC } \\
\text { ADDED } \\
u g / L\end{array}$ & $\begin{array}{c}\text { CONC } \\
\text { RECOVERED } \\
u g / L\end{array}$ & RECOVERED & IIMITS \\
\hline $\begin{array}{l}11 \text { 1, 1-Dichioroethene } \\
42 \text { Trichloroethene } \\
40 \text { Berzene } \\
52 \text { Toluene } \\
62 \text { Chlorobenzene }\end{array}$ & $\begin{array}{l}50.00 \\
50.00 \\
50.00 \\
50.00 \\
50.00\end{array}$ & $\begin{array}{l}67.71 \\
54.92 \\
54.65 \\
55.05 \\
54.90\end{array}$ & $\begin{array}{l}135.42 \\
109.84 \\
109.30 \\
110.10 \\
109.80\end{array}$ & $\begin{array}{r}1-234 \\
71-157 \\
37-151 \\
47-150 \\
37-160\end{array}$ \\
\hline
\end{tabular}

\begin{tabular}{|c|c|c|c|c|}
\hline SURROGATE COMPOUND & $\begin{array}{l}\text { AMOUNT } \\
\text { ADDED } \\
\mathrm{ug} / \mathrm{L}\end{array}$ & $\begin{array}{c}\text { AMOUNT } \\
\text { RECOVERED } \\
u g / L\end{array}$ & $\begin{array}{c}\text { : } \\
\text { RECOVERED }\end{array}$ & LIMITS \\
\hline $\begin{array}{lll}\$ & 32 & \text { Dibromofluorometha } \\
\$ & 37 & 1 \\
\$ & \text { 2-Dichloroethane } \\
\$ & 51 & \text { Toluene-d8 } \\
\$ & 73 & 4 \text {-Bromofluorobenze }\end{array}$ & $\begin{array}{l}50.00 \\
50.00 \\
50.00 \\
50.00\end{array}$ & $\begin{array}{l}55.32 \\
59.02 \\
51.70 \\
50.11\end{array}$ & $\begin{array}{l}110.64 \\
118.04 \\
103.40 \\
100.22\end{array}$ & $\begin{array}{l}80-120 \\
80-120 \\
80-120 \\
74-121\end{array}$ \\
\hline
\end{tabular}


SAMPLE DELIVERY GROUP: V368

ABORATORY: QUANTERRA

FINAL REPORT RECEIVED: $4 / 7 / 98$

DATE REVIEWED: $5 / 5 / 98$
CLIENT: RP - JEFF SMITH

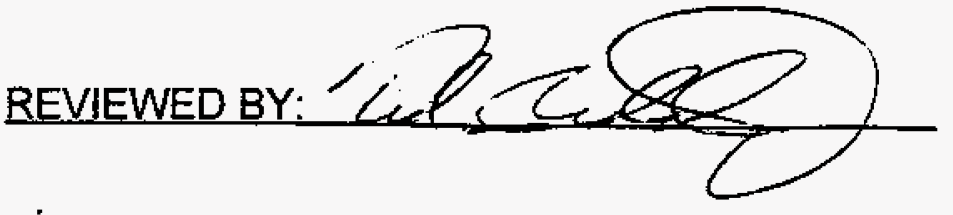

VOLATILES:

MATRIX: SOIL

1. TUNE

\section{CALIBRATION INITIAL \\ VSTD050 \\ INITIAL}

3. METHOD BLANK $\underline{\text { QCBLK167948 }}$

4. EXT. BLANK

5. LAB CONTROL

6. TRIP BLANK

7. SAMPLES QCBLK168259

\section{SAMPLE ID} BFB

$B F B$

N/A

QCLCS167948

QCLCS168259

N/A

A6-SMP3-W1

A6-SMP3-AA

A6-SMP3-BB

A6-SMP3-E1

8. SURROGATE RECOVERY ACCEPTABLE:

ARE VOA DATA ACCEPTABLE: YES

\section{INSTRUMENT ID}

MSB

MSB

MSB

MSB

MSB

MSB

MSB

$\underline{M S B}$

N/A

MSB

MSB

N/A

MSB

MSB

MSB

MSB
DATE TIME

$\underline{3 / 18 / 98} \quad \underline{0558}$

$\underline{3 / 20 / 98} \quad \underline{0444}$

$\underline{3 / 24 / 98} \quad \underline{0932}$

$3 / 18 / 98$

$3 / 20 / 98$

$3 / 24 / 98$

$3 / 20 / 98$

$\underline{3 / 24 / 98}$

N/A

$3 / 20 / 98$

$3 / 24 / 98$

$\mathrm{N} / \mathrm{A}$

$3 / 20 / 98$

$3 / 20 / 98$

$3 / 20 / 98$

$3 / 24 / 98$ $\underline{0551}$

$\underline{0635}$

1235

N/A

$\underline{0724}$

1322

N/A

$\underline{0857}$

$\underline{0928}$

$\underline{0959}$

1354
ACCEPTABLE?

YES

YES

YES

YES

YES

YES

YES

YES

N/A

YES

YES

N/A

YES

YES

YES

YES

9. MS/MSD RECOVERY ACCEPTABLE:

10. METHOD BLANK ACCEPTABLE:

11. HOLDING TIMES ACCEPTABLE:
a. Date Sampled
b. Date ASL Received:
c. Date Contract Lab Received:
d. Date Extracted:
e. Date Analyzed:

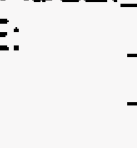

$\frac{\text { YES }}{\frac{N / A}{\text { YES }}}$

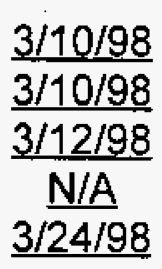

\#DAYS

ACCEPTABLE

$\frac{\frac{0}{1}}{\frac{5}{N / A}} \frac{14}{1}$

12. Correct Conc units used:

13. CALCULATIONS ACCEPTABLE:

14. ARE AREAS ACCEPTABLE:

15. RRT IS ACCEPTABLE:

16. FORMS ACCEPTABLE:

17. Chain-of-Custody agrees:

\begin{tabular}{c} 
YES \\
\hline YES \\
\hline YES \\
\hline YES \\
\hline YES \\
\hline YES \\
\hline
\end{tabular}

SAMPLE: Continuing Calibration (VSTD050)

Analyzed: 3/20/98@0551

\begin{tabular}{|l|c|c|c|c|c|}
\hline PCC & AREA & IS AREA & RRF & MinimLm RRF & ACCEPTABLE? \\
\hline Chloromethane & 569556 & 2759179 & 0.206 & 0.100 & YES \\
\hline 1,1-Dichloroethane & 1326048 & 2759179 & 0.481 & 0.100 & YES \\
\hline
\end{tabular}




\begin{tabular}{|c|c|c|c|c|c|}
\hline SPCC & AREA & IS AREA & RRF & Minimum RRF & ACCEPTABLE? \\
\hline Bromoform & 1057704 & 2418910 & 0.437 & 0.100 & YES \\
\hline 1,1,2,2-Tetrachloroethane & 1299177 & 2418910 & 0.537 & 0.300 & YES \\
\hline Chlorobenzene & 2139740 & 2418910 & 0.885 & 0.300 & YES \\
\hline $\operatorname{ccc}$ & True Conc. & Analyzed Conc. & $\%$ Difference & \multicolumn{2}{|c|}{$\begin{array}{c}\text { ACCEPTABLE? } \\
(w / i n \pm 20 \%)\end{array}$} \\
\hline Vinyl chloride & 50.0 & 42.5 & -15.0 & \multicolumn{2}{|r|}{ YES } \\
\hline 1,1-Dichloroethene & 50.0 & 46.6 & -6.9 & \multicolumn{2}{|r|}{ YES } \\
\hline Chloroform & 50.0 & 45.8 & -8.4 & \multicolumn{2}{|r|}{ YES } \\
\hline 1,2-Dichloropropane & 50.0 & 44.7 & -10.7 & \multicolumn{2}{|r|}{$\underline{\text { YES }}$} \\
\hline Toluene & 50.0 & 44.3 & -11.4 & \multicolumn{2}{|r|}{ YES } \\
\hline Ethylbenzene & 50.0 & 44.3 & -11.5 & \multicolumn{2}{|r|}{ YES } \\
\hline
\end{tabular}

COMMENTS: Method associated quality assurance and control criteria were met. Data qualified with: 1) a "J" indicates the component was detected below the method detection limit, and 2) a " $\mathrm{B}$ " indicates the component was detected in the sample's associated method blank. Amounts of acetone and methylene chloride were found in the samples and associated method blanks. The data have ber flagged accordingly 
SAMPLEDELIVERY GROUP: V368 ABORATORY: QUANTERRA

EINAL REPORT RECEIVED: $4 / 7 / 98$

DATE REVIEWED: $5 / 5 / 98$

SEMIVOLATILES:

MATRIX: SOIL

1. TUNE

2. CALIBRATION

3. METHOD BLANK

4. LAB CONTROL

5. SAMPLES

\section{SAMPLE ID}

DFTPP

DFTPP

INITIAL

SSTD050

QCBLK167889

QCSPK167889-1

QCSPK167889-2

A6-SMP3-E2

A6-SMP3-W2
CLIENT: RP - JEFF SMITH

REVIEWED BY:

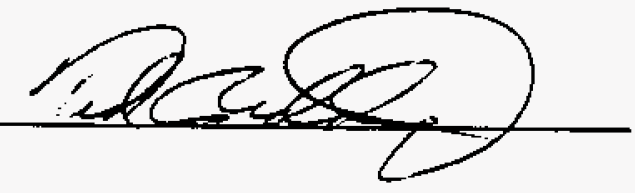

ARE BNA DATA ACCEPTABLE: YES

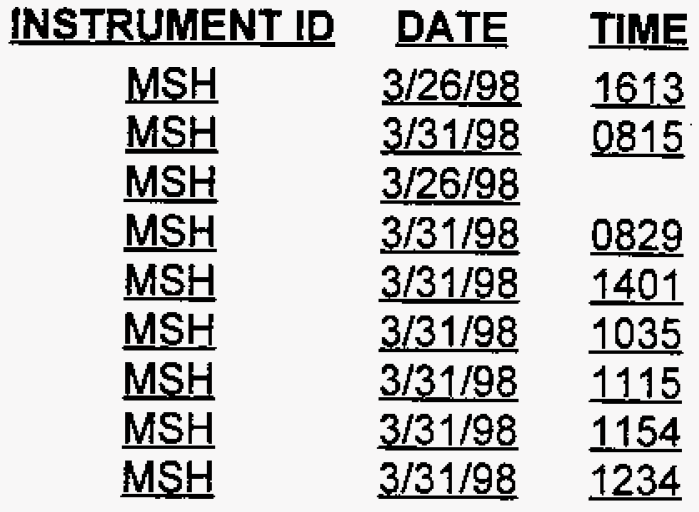

ACCEPTABLE?

\begin{tabular}{c} 
YES \\
\hline YES \\
\hline YES \\
\hline YES \\
\hline YES \\
\hline YES \\
\hline YES \\
\hline YES \\
\hline YES \\
\hline
\end{tabular}

6. SURROGATE RECOVERY ACCEPTABLE:

7. MS/MSD RECOVERY ACCEPTABLE:

8. METHOD BLANK ACCEPTABLE:

- HOLDING TIMES ACCEPTABLE:

\begin{tabular}{|c|}
\hline YES \\
\hline $\mathrm{N} / \mathrm{A}$ \\
\hline YES \\
\hline YES \\
\hline
\end{tabular}

a. Date Sampled:

b. Date ASL Received:

c. Date Contract Lab Received:

d. Date TCLP Extraction:

e. Date Preparative Extraction:

f. Date Analyzed:

\begin{tabular}{|c|c|}
\hline $3 / 10 / 98$ & 0 \\
\hline $3 / 10 / 98$ & 0 \\
\hline $3 / 12 / 98$ & 2 \\
\hline N/A & N/A \\
\hline $3 / 23 / 98$ & 13 \\
\hline $3 / 31 / 98$ & 8 \\
\hline
\end{tabular}

ACCEPTABLE

10. Correct conc units used:

11. CALCULATIONS ACCEPTABLE:

12. ARE AREAS ACCEPTABLE:

13. RRT IS ACCEPTABLE:

14. FORMS ACCEPTABLE:

15. Chain-of-Custody agrees:

\begin{tabular}{l} 
YES \\
\hline YES \\
\hline YES \\
\hline YES \\
\hline YES \\
\hline YES \\
\hline
\end{tabular}

SAMPLE: Continuing Calibration (SSTD050)

Analyzed: 3/31/98@0829

\begin{tabular}{|l|c|c|c|c|c|}
\hline SPCC & AREA & IS AREA & RRF & Minimum RRF & ACCEPTABLE? \\
\hline N-nitroso-di-n-propylamine & 43853 & 44125 & 0.795 & 0.050 & YES \\
\hline Hexachlorocyclopentadiene & 34981 & 90341 & 0.310 & 0.050 & YES \\
\hline H-Dinitrophenol & 17876 & 90341 & 0.158 & 0.050 & YES \\
\hline 4-Nitrophenol & 24146 & 90341 & 0.214 & 0.050 & YES \\
\hline
\end{tabular}




\begin{tabular}{|l|c|c|c|c|}
\hline CCC & True Conc. & Analyzed Conc. & $\%$ Difference & $\begin{array}{c}\text { ACCEPTABLE? } \\
(\text { W/in } \pm 20 \%)\end{array}$ \\
\hline Phenol & 25.0 & 24.5 & -2.1 & YES \\
\hline 1,4-Dichlorobenzene & 25.0 & 26.8 & 7.2 & YES \\
\hline 2-Nitrophenol & 25.0 & 25.5 & 1.9 & YES \\
\hline 2.4-Dichtorophenol & 25.0 & 25.9 & 3.6 & YES \\
\hline Hexachlorobutadiene & 25.0 & 27.8 & 11.3 & YES \\
\hline 4-Chioro-3-methylphenol & 25.0 & 27.2 & 8.7 & YES \\
\hline 2.4,6-Trichlorophenol & 25.0 & 25.1 & 0.3 & YES \\
\hline Acenaphthene & 25.0 & 25.5 & 1.8 & YES. \\
\hline N-Nitrosodiphenylamine & 25.0 & 28.5 & 13.8 & YES \\
\hline Pentachlorophenol & 25.0 & 23.7 & -5.3 & YES \\
\hline Fluoranthene & 25.0 & 26.1 & 4.4 & YES \\
\hline Di-n-octyl phthalate & 25.0 & 27.0 & 8.0 & YES \\
\hline Benzo (a) pyrene & 25.0 & 26.6 & 6.2 & YES \\
\hline
\end{tabular}

COMMENTS: Method associated quality assurance and control criteria were met. Data qualified wi ${ }^{*}$ a " $\mathrm{J}$ " indicates the component was detected below the method detection limit. 
SAMPLE DELIVERY GROUP: V368 ABORATORY: QUANTERRA

FINAL REPORT RECEIVED: $4 / 7 / 98$

DATE REVIEWED: $5 / 5 / 98$
CLIENT: RP - JEFF SMITH

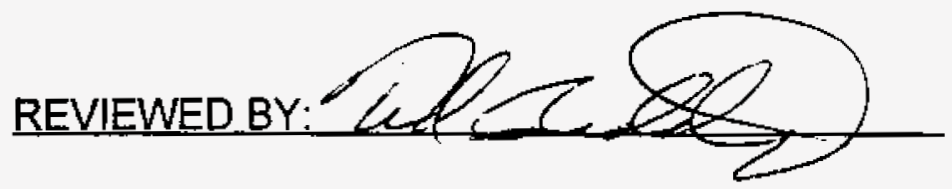

PCB:

MATRIX: SOIL

1. ANALYSIS SEQUENCE

2. METHOD BLANK

3. MS/MSD RESULTS

4. SURROGATE RECOVERY

5. HOLDING TIMES
ARE PCB DATA ACCEPTABLE: YES

ARE CRITERIA MET

\begin{tabular}{c}
\hline YES \\
\hline YES \\
\hline N/A \\
\hline YES \\
\hline YES
\end{tabular}

\# DAYS

ACCEPTABLE

a. Date Sampled:

$\underline{3 / 10 / 98}$

0

0

3/10/98

0

1

$3 / 12 / 98$

2

5

3/17/98

7

3

40

6. CONFIRMED BY SECOND COLUMN: CONFIRMED BY GC/MS:

7. CALCULATIONS:

8. Correct Conc. units used:

9. FORMS ACCEPTABLE:

10. Chain-of-Custody agrees:

\begin{tabular}{|c|}
\hline NO \\
\hline $\mathrm{NO}$ \\
\hline YES \\
\hline YES \\
\hline YES \\
\hline YES \\
\hline
\end{tabular}

COMMENTS: Method associated quality assurance and control criteria were met. 


\section{Bechtel Nevada Corporation}

\section{ANALYTICAL SERVICES LABORATORY}

P.O.Box 3936, N. Las Vegas, NV 89036

Reported to: Remediation Projects

$$
\text { J. L. Smith }
$$

M/S NTS306

Report Date: 29-APR-98

Sample Delivery Group: B228

Batch: 0789

Program: 720

Report No.

\begin{tabular}{|c|c|c|c|c|c|c|c|c|c|c|c|c|c|c|c|c|}
\hline $\begin{array}{c}\text { Sampla } \\
\text {...... Idertification ...... }\end{array}$ & Isotope & Analysis & Rasult & $\begin{array}{l}\text { Error } \\
\%\end{array}$ & $\begin{array}{l}\text { Ougl } \\
\text { Flag }\end{array}$ & MDA & $\begin{array}{l}\text { Result } \\
\text { Units }\end{array}$ & $\begin{array}{c}\text { Analysis } \\
\text { Date }\end{array}$ & $\begin{array}{l}\text { Sarmple } \\
\text { Coll Date }\end{array}$ & Size & $\begin{array}{l}\text { Size } \\
\text { Units }\end{array}$ & $\left|\begin{array}{c}\text { Type } \\
\text { Matrix }\end{array}\right|$ & $\begin{array}{l}\text { Tracer } \\
\text { Yield \% }\end{array}$ & $\begin{array}{l}\text { Spike } \\
\text { Recr } \%\end{array}$ & $\begin{array}{l}\text { System } \\
\text { Detector }\end{array}$ & $\begin{array}{l}\text { Packet-Item } \\
\text { Sample }\end{array}$ \\
\hline A6-SMP3-E3 & K 40 & GAM20 & $2.16 E+01$ & $2.1 E+01$ & & $1.6 E+\infty 0$ & $\mathrm{pCi} / \theta$ & 03-11-98 & 03-10-98 & $4.57 \mathrm{E}+02 \mid$ & $\mathrm{gm}$ & soll G & & & 05.01 & G8692-0-75026 \\
\hline A6-SMP3-E3 & RA22B & GAM20 & $1.20 E+\infty$ & $2.8 \mathrm{E}+01$ & & $1.8 \mathrm{E}-01$ & $\mathrm{pCi} / \mathrm{g}$ & 03-11-98 & 03-10-98 & $4,57 E+02$ & on & soll, G & & & $05-01$ & $68692-0.75028$ \\
\hline A6-SMP3-E3 & TH228 & GAM20 & $2.19 \mathrm{E}+00$ & $1.9 E+01$ & & $1.4 \mathrm{E}-09$ & pCilg & $03-11-98$ & 03-10-98 & $4.57 \mathrm{E}+02$ & $\mathrm{gm}$ & sott G & & & 05.01 & G8692-0-75026 \\
\hline A6-SMP3-E3 & TH232 & GAM20 & $1.79 \mathrm{E}+00$ & $3.6 E+01$ & & $2.9 \mathrm{E}-01$ & pCilg & |03-11.98 & 03-10-98 & $|4.57 \mathrm{E}+02|$ & $\mathrm{gm}$ & SOIL G & & & 05-01 & G8692-0-75026 \\
\hline A6-5MP3-W3 & K 40 & GAM20 & $4.82 E+01$ & $2.2 E+01$ & & $1.6 \mathrm{E}+\infty$ & pCilg & $|03-11-98|$ & $03-10-98$ & $|4.40 E+02|$ & gm & soll G & & & 05.01 & 68692-1-75027 \\
\hline A6-SMP3-W3 & RA226 & GAM20 & $1.19 E+00$ & $2.6 E+01$ & & $1.4 E-01$ & $\mathrm{pCi} / \mathrm{g}$ & 03-11-98 & $03-10-98$ & $4.40 \mathrm{E}+02$ & $g m$ & soll G & & & 05.01 & G8692-1-76027 \\
\hline A6-5MP3-W3 & TH228 & GAM20 & $2.19 E+00$ & $1.9 E+01$ & & $1.4 \mathrm{E}-01$ & $\mathrm{pCi} / \mathrm{g}$ & |03-11-98 & $03-10-98$ & $|4.40 E+02|$ & $\operatorname{gm}$ & soll G & & & 05.01 & G8692-1.75027 \\
\hline A6-SMP3-W3 & TH232 & GAM2O & $1.53 \mathrm{E}+00$ & $3.8 E+01$ & & $2.9 \mathrm{E}-01$ & $\mathrm{pCi} / \mathrm{g}$ & $03-11-98$ & $03-10-98$ & $4.40 E+02$ & $\mathrm{gm}$ & soll G & & & $05-01$ & G8692-1-75027 \\
\hline OA BKG EMPTY BTL UO5 & No Nuc1 Dot & GAM20 & $0.00 E+00$ & $0.0 E+00$ & $\mathrm{U}$ & $0.0 E+00$ & NA & $03-11-98$ & $07-01-95$ & $1.00 E+00$ & $g m$ & QUAL & & & $05-01$ & $00539-0.00125$ \\
\hline QA BKG EMPTY BTL U05 & AM241 & GAM 20 & $0.00 E+00$ & $0.0 E+\infty 0 \mid$ & u & $1.8 \mathrm{E}+00$ & $\mathrm{pCl}$ & |03-11-98 & $07-01-95$ & $|1.00 E+00|$ & $\mathbf{s m}$ & QuAL & & & 05.01 & $00539-0-00125$ \\
\hline
\end{tabular}

\section{Comment:}

A small portion of the sample was used for the gross alpha and gross beta determinations. Accordingly, the data may not be representative of the sample as a whole.

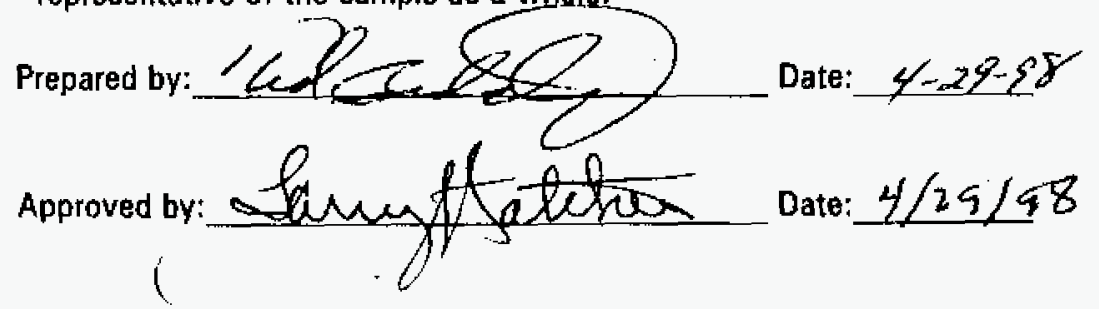

Qualification Flags:

E = Estimated Quantity

$H=$ High Recovery for Sample

$J=$ Result is less than the RDL

$L=$ Low Recovery for Sample

$P=$ Preliminary Results

$\mathrm{O}=$ Bad Instrument Quality Control, Result is OK

$\mathbf{A}=$ Results are Unusable, Resampling is Necessary

$\mathrm{U}=$ Result is less than Minimum Detectable Activity
Note: \% Error is the 2.0 Sigma Error 


\section{Bechtel Nevada Corporation}

\section{ANALYTICAL SERVICES LABORATORY}

P.O.Box 3936, N. Las Vegas, NV 89036

Reported to: Remediation Projects

Report Date: 29-APR-98

M/S NTS306

Semple Delivery Group: B228

Batch: 0789

Program: 500

Feport No. :

\begin{tabular}{|c|c|c|c|c|c|c|c|c|c|c|c|c|c|c|c|c|}
\hline $\begin{array}{c}\text { Sample } \\
\ldots . . . .\end{array}$ & Isotope & Analysis & Result & $\begin{array}{c}\text { Error } \\
\%\end{array}$ & $\begin{array}{l}\text { Qusl } \\
\text { Flag }\end{array}$ & MDA & $\begin{array}{l}\text { Result } \\
\text { Units }\end{array}$ & $\begin{array}{c}\text { Analysis } \\
\text { Date }\end{array}$ & $\begin{array}{l}\text { Sample } \\
\text { Coll Dato }\end{array}$ & Size & $\begin{array}{l}\text { Size } \\
\text { Units }\end{array}$ & \begin{tabular}{|c} 
Type \\
Matrix
\end{tabular} & $\begin{array}{l}\text { Tracer } \\
\text { Yield \% }\end{array}$ & $\begin{array}{l}\text { Spike } \\
\text { Recv \% }\end{array}$ & $\begin{array}{l}\text { System } \\
\text { Dotector }\end{array}$ & $\begin{array}{l}\text { Packet-then } \\
\text { Samplo }\end{array}$ \\
\hline QA BKG EMPTY BTL U05 & $\mathrm{CO60}$ & GAM20 & $0.00 E+\infty 0$ & $0.0 E+00$ & u & 2.7E-01 & $\mathrm{pCi}$ & 03-11-98 & 07.01.95 & $1.00 E+00$ & $\mathrm{sm}$ & QUAL & & & 05-01 & 00539-0-00125 \\
\hline QA BKG EMPTY BTL U05 & $\operatorname{cs} 137$ & GAM20 & $0.00 E+00$ & $0.0 E+\infty$ & $\dot{U}$ & $3.2 \mathrm{E}-01$ & $\mathrm{pCi}$ & 03-11-98 & 07-01-95 & $|1.00 E+\infty|$ & $8 m$ & QUAL & & & 05.01 & $00539-0.00125$ \\
\hline QA SP:NAS-A0271 & AM241 & GAM20 & $1.79 \mathrm{E}+05$ & B. $8 E+\infty$ & & $3.1 E+02$ & $\mathrm{pCi}$ & 03-11-98 & $04-01-92$ & $1.00 E+00$ & $8 \pi$ & QUAL & & 110.3 & 05.01 & $00534-3-12681$ \\
\hline QA SP:NAS-A0271 & $\mathrm{CO60}$ & GAM20 & $3.01 E+05$ & $8.5 E+\infty$ & & $2.2 E+02$ & $\mathrm{pCi}$ & $03-11-98$ & $04-01-92$ & $1.00 E+00$ & $\mathrm{sm}$ & OUAL & & 101.0 & 05.01 & $00534-3 \cdot 12681$ \\
\hline QA SP:NAS-A0271 & $\operatorname{cs} 137$ & GAM20 & $2.07 E+05$ & $8.1 E+00$ & & $7.4 E+01$ & $p C \mathrm{Ci}$ & $03-11-98$ & $04-01-92$ & $|1.00 E+00|$ & $\mathrm{sm}$ & GUAL. & & 101.8 . & $05-01$ & $00534-3-12681$ \\
\hline
\end{tabular}

\section{Comment:}

A small portion of the sample was used for the gross alpha and gross beta determinations. Accordingly, the data may not be representative of the sample as a whole.

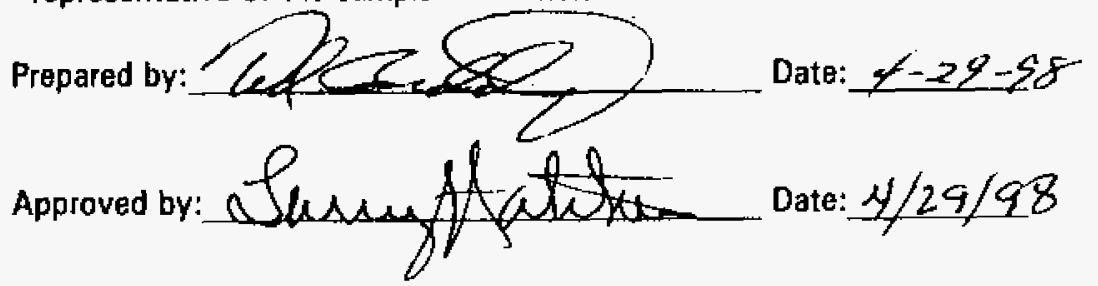

Qualification Flags:

Note: \% Error is the 2.0 Sigma Error

$\mathbf{E}=$ Estimated Quantity

$H=$ High Recovery for Sample

$\mathrm{J}=$ Result is less than the $\mathrm{FDL}$

$L=$ Low Recovery for Sample

$\mathrm{P}=$ Preliminary Results

$\mathrm{Q}=$ Bad Instrument Quality Control, Result is OK

$R=$ Results are Unusable, Resampling is Necessary

$\mathrm{U}=$ Result is less than Minimum Detectable Activity 


\section{Bechtel Nevada Corporation}

\section{ANALYTICAL SERVICES LABORATORY}

P.O.Box 3936, N. Las Vegas, NV 89036

Reported to: Remediation Projects

J. L. Smith

M/S NTS306

Heport Date: 29-APR-98

Sample Delivery Group: B228

Batch: M459

Program: $\mathbf{7 2 0}$

Report No. :

\begin{tabular}{|c|c|c|c|c|c|c|c|c|c|c|c|c|c|c|c|c|}
\hline 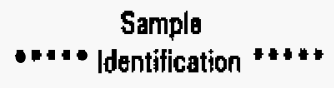 & Isotope & Analysis & Pesult & $\begin{array}{c}\text { Error } \\
\%\end{array}$ & $\begin{array}{l}\text { Qual } \\
\text { Flag }\end{array}$ & MDA & $\begin{array}{l}\text { Result } \\
\text { Units }\end{array}$ & $\begin{array}{c}\text { Analysis } \\
\text { Dato }\end{array}$ & $\begin{array}{l}\text { Sampla } \\
\text { Coll Date }\end{array}$ & Size & $\begin{array}{r}\text { Size } \\
\text { Units }\end{array}$ & \begin{tabular}{|l} 
Type \\
Matrix
\end{tabular} & $\begin{array}{l}\text { Tracer } \\
\text { Yield } \%\end{array}$ & $\begin{array}{l}\text { Spike } \\
\text { Racv } \%\end{array}$ & $\begin{array}{l}\text { Systom } \\
\text { Detector }\end{array}$ & $\begin{array}{l}\text { Packet-ltem } \\
\text { Sample }\end{array}$ \\
\hline A6-SMP3-E3 & Gross Alpha & Gross A & $9.57 \mathrm{E}+00$ & $1,7 E+01$ & & $1.4 E+\infty 0$ & $\mathrm{pCl} / \mathrm{g}$ & 03-27-98 & 03-10-98 & $1.00 E+00$ & $\mathrm{gm}$ & SOIL G & & & 14-01 & G8692-0-75028 \\
\hline A6-SMP3-W3 & Gross Alpha & Gross A & 8. $49 \mathrm{E}+00$ & $1.9 E+01$ & & $1.4 E+00$ & pCi/g & $03-27-98$ & 03-10-98 & $1.00 E+00$ & $\mathrm{gm}$ & SOIL $\mathrm{G}$ & & & $14-01$ & G8692-1.75029 \\
\hline QA REA & Gross Alpha & Gross A & $1.65 E-02$ & $1.4 E+03$ & $u$ & $4.2 \mathrm{E}-01$ & $\mathrm{pCi}$ & 03-27-98 & 03-11-98 & $1.00 E+00$ & $\operatorname{sm}$ & QUAL & & & 1401 & G8693-0-06772 \\
\hline QA SP:GROSSAB 04 & Gross Alpha & Gross A & $3.46 \mathrm{E}+00$ & $1.5 \mathrm{E}+01$ & & 4.2E-01 & $\mathrm{pCi}$ & $03.27-98$ & 02-07-92 & $1.00 \mathrm{E}+00$ & $\sin$ & QUAL & & 101.1 & 14-01 & G8693-1-06773 \\
\hline A6-SMP3-E3 & Gross Beta & Gross B & $8.38 E+\infty 0$ & $1.0 \mathrm{E}+01$ & & $1.1 E+00$ & pCilg & $03-27-98$ & 03-10-98 & $1.00 E+\infty$ & $\operatorname{grn}$ & soll $G$ & & & $14-01$ & $68692-0-75028$ \\
\hline A6-5MP3-W3 & Gross Beta & Gross B & $7.49 E+\infty$ & $1.1 E+01$ & & $1.1 E+\infty$ & pCilg & 03-27.98 & 03-10-98 & $1.00 E+00$ & $\mathrm{gm}$ & soll G & & & 1401 & $68692-1-76029$ \\
\hline QA REA & Gross Bata & Gross B & $-1.94 E-01$ & $2.9 E+02$ & $\mathbf{u}$ & 9.7E-01 & $\mathrm{pCi}$ & $03-27-98$ & 03-11-98 & $1.00 E+00 \mid$ & $\mathrm{sm}$ & OUAL & & & 14.01 & G8693-0-06772 \\
\hline OA SP:GAOSSAB & Gross Bata & Gross B & $4.82 E+00$ & $1.6 E+01$ & & $9.7 \mathrm{E}-01$ & $\mathrm{pCi}$ & $03-27-98$ & 02-07-92 & $1.00 E+00$ & $\mathrm{sm}$ & OUAL & & 94.5 & $14-01$ & 68693-1.06773 \\
\hline
\end{tabular}

\section{Comment:}

A small portion of the sample was used for the gross alpha and gross beta determinations. Accordingly, the data may not be representative of the sample as a whole.

\section{Prepared by:}

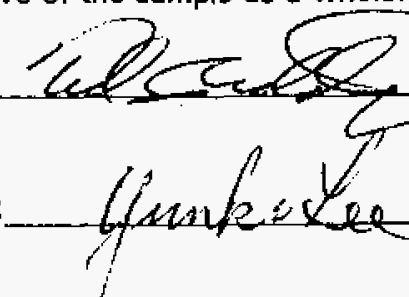

Dato: $4-29-95$ Date: $4 / 29 / 41$
Qualification Flags:

$E$ = Estimated Quantity

$\mathrm{H}=$ High Recovery for Sample

$\mathrm{J}=$ Result is less than the RDL

$L=$ Low Recovery for Sample

$\mathbf{P}=$ Preliminary Results

$a=$ Bad Instrument Quality Control, Result is OK

$R=$ Results are Unusable, Resampling is Necessary

$\mathbf{U}=$ Result is less than Minimum Detectable Activity
Note: \% Error is the 2.0 Sigma Error ( 
SAMPLE DELIVERY GROUP: V368

ABORATORY: QUANTERRA

FINAL REPORT RECEIVED: $4 / 7 / 98$

DATE REVIEWED: $5 / 6 / 98$

TPH - GASOLINE:

MATRIX: SOIL

1. QC SAMPLES ACCEPTABLE

2. MATRIX SPIKE ACCEPTABLE

3. METHOD BLANK ACCEPTABLE

4. HOLDING TIMES ACCEPTABLE
CLIENT: RP - JEFF SMITH

REVIEWED BY:

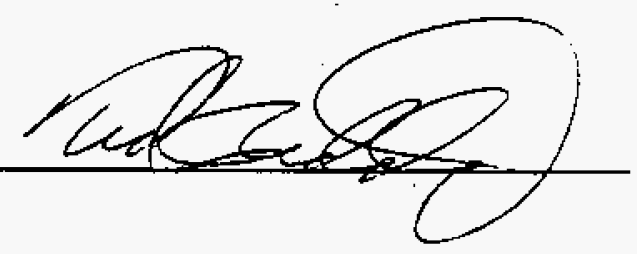

ARE TPH GASOLINE DATA ACCEPTABLE: YES

ARE CRITERIA MET

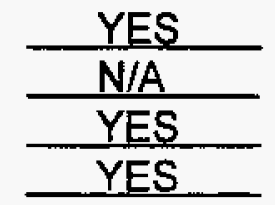

\#DAYS ACCEPTABLE

a. Date Sampled:

$3 / 10 / 98$

Q

0

b. Date ASL Received:

$3 / 10 / 98$

0

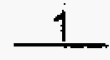

$3 / 12 / 98$

2

5

$\underline{3 / 13 / 98}$

3

14

5. CALCULATIONS ACCEPTABLE:

6. CORRECT CONC. UNITS USED:

7. FORMS ACCEPTABLE:

8. CHAIN-OF-CUSTODY AGREES:

\begin{tabular}{|c|}
\hline YES \\
\hline YES \\
\hline YES \\
\hline YES \\
\hline
\end{tabular}

COMMENTS: Method associated quality assurance and control criteria were met. 


\section{CASE NARRATIVE}

Bechtel Nevada Corporation

May 27, 1998

MAII STOP NTS273

2621 Losee Road

Building A-2, Receiving

North Las Vegas, NV 89030-4129

Attention: Ted Redding

Quanterra Project Number

Date Received 625.01

Number of Samples

SDG Name

May 8, 1998

Bechtel Report No

Two (2)

V411

Sample type

W1441

Soil

\section{INTRODUCTION}

Two (2) soil samples from Bechtel Nevada were received at Quanterra Environmental Services, Earth City, MO for TCLP Metais analyses. The analytical test performed, as well as date of receipt and analysis, can be found in the enclosed data package and corresponding summary report.

The results were transmitted by fax on May 27, 1998

SDG V41 I was transmitted previously.

Reviewed and Approved

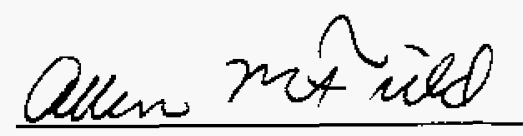

Allen M. Field

Quanterra Project Manager 
PAGE 2 of 3

May 27, 1998

Quanterra Project Number: 625.01

The sample was labeled as follows:

\section{CLIENT ID}

AG-SMP3-E4

AG-SMP3-W4 $\underline{\text { LAB ID }}$

17767-001

$17767-002$

\section{MATRIX}

Soil

Soil

\section{ANALYTICAL RESULTS/METHODOLOGY}

The analytical results are presented by analytical test in the attached data packages. This report includes information on client identification numbers, lab identification numbers, preparation date, analysis date, results, units, and results for quality control samples.

The following table is a list of the analyses and the methods used for the above samples:

\section{Analysis}

TCLP Metals

\section{Method}

EPA $1311 / 6010 / 7470$

\section{QUALITY CONTROL}

'A method blank and a laboratory control sample was anaiyzed with the samples listed above per parameter.

\section{NONCONFORMANCES}

No nonconformances were observed with the analysis of these samples.

\section{COMMENTS}

\section{TCLP Metals}

Both the ICP and Mercury digestions were prepped $25 \mathrm{mls}$ to $100 \mathrm{mls}$ for all samples including the extraction blank.

\section{$\underline{\text { Receipt }}$}

Login 17767 was received in one (1) cooler at a temperature of $4^{\circ} \mathrm{C}$. 
PAGE 3 of 3

May 27, 1998

Quanterra Project Number: 625.01

\section{Analytical Notes}

\section{QUALIFIERS/DEFINITIONS}

$\begin{array}{ll}* & =\text { Reported value outside the QC iimits } \\ \text { NC } & =\text { No criteria at this time } \\ \text { NA } & =\text { Not applicable } \\ \text { ND } & =\text { Non - detect } \\ \text { MG/L } & =\text { Milligrams per liter } \\ \text { UG/L } & =\text { Micrograms per liter } \\ \text { \%REC } & =\text { Percent Recovery } \\ \text { QCBLK } & =\text { Method Blank } \\ \text { QCLCS } & =\text { Laboratory Control Sample } \\ \text { CRDL } & =\text { Customer Required Detection Limit } \\ \text { RPD } & =\text { Relative Percent Difference } \\ \text { IDL } & =\text { Instrument detection limit } \\ \text { DL } & =\text { PQL } \\ \text { J } & =\text { Estimated Value (Organics only) } \\ \text { D } & =\text { Diluted } \\ \text { U } & =\text { Non - Detect } \\ \text { B } & =\text { Value greater than IDL but less than CRDL (Metals only) } \\ \text { B } & =\text { Analyte found in the blank (Organics only) } \\ \text { E } & =\text { Exceeds calibration }\end{array}$


TCLP

METALS

DATA 
Bechtel Nevada

NTS273, 2621 Losee Road

Buitding A-2, Receiving

North Las Vegas, HV 89030-4129

Project: 625.01
Cotegory : TCLP Metals

Matrix : Soil

Client ID: AG-SHP3-E4
Environmental Services

Sample Date $=05 / 06 / 98$

Receipt Dete : 05/08/98

Report Date : 05/27/98

Quanterra ID : 17767-009

\begin{tabular}{|c|c|c|c|c|c|c|c|c|c|c|}
\hline Analyte & Method & $\begin{array}{l}\text { Extract } \\
\text { Date }\end{array}$ & $\begin{array}{l}\text { Prep } \\
\text { Date }\end{array}$ & $\begin{array}{l}\text { Analyses } \\
\text { Date }\end{array}$ & Result & Units & Qual. & $\begin{array}{c}\text { Detection } \\
\text { Limit }\end{array}$ & $\begin{array}{l}\text { Regulatory } \\
\text { Level }\end{array}$ & Dilution \\
\hline Mercury & EPA 7470 & $05 / 18 / 98$ & $05 / 20 / 98$ & $05 / 20 / 98$ & 0.0004 & $\mathrm{MG} / \mathrm{L}$ & $u$ & 0.0008 & 0.20 & 4 \\
\hline Arsenic & EPA $6010^{\circ}$ & $05 / 18 / 98$ & $05 / 20 / 98$ & $05 / 20 / 98$ & 0.020 & $M G / L$ & B & 1.2 & 5.0 & 4 \\
\hline Barium & EPA $6010=$ & $05 / 18 / 98$ & $05 / 20 / 98$ & $05 / 20 / 98$ & 0.37 & MG/L & B & 0.80 & 100 & 4 \\
\hline Cadmitun & EPA 6010 - & $05 / 18 / 98$ & $05 / 20 / 98$ & $05 / 20 / 98$ & 0.0024 & $M G / L$ & $\mathbf{u}$ & 0.020 & 1.0 & 4 \\
\hline Chromiun & EPA 6010 - & $05 / 18 / 98$ & $05 / 20 / 98$ & $05 / 20 / 98$ & 0.012 & $\mathrm{MG} / \mathrm{L}$ & B & 0.040 & 5.0 & 4 \\
\hline Lead & EPA 6010 . & $05 / 18 / 98$ & $05 / 20 / 98$ & $05 / 20 / 98$ & 0.0080 & $M G / L$ & B & 0.40 & 5.0 & 4 \\
\hline Selenium & EPA 6010 - & $05 / 18 / 98$ & $05 / 20 / 98$ & $05 / 20 / 98$ & 0.012 & $\mathrm{MG} / \mathrm{L}$ & $\mathrm{u}$ & 1.0 & 1.0 & 4 \\
\hline Silver & EPA $6010^{\circ}$ & $05 / 18 / 98$ & $05 / 20 / 98$ & $05 / 20 / 98$ & 0.0049 & $\mathrm{HG} / \mathrm{L}$ & B & 0.040 & 5.0 & 4 \\
\hline
\end{tabular}


Bechtel Nevada

NTS273, 2621 Losee Road

Building A-2, Receiving

North Las Vegas, NV $89030-4129$

Project: 625.01
Category : TCLP Metals

Matrix : Soil

Client ID: AG-SMP3-W4
Sample Date : 05/06/98

Receipt Date : 05/08/98

Report Date : 05/27/98

Quanterra ID : 17767-002

\begin{tabular}{|c|c|c|c|c|c|c|c|c|c|c|}
\hline Analyte & Method & $\begin{array}{l}\text { Extract } \\
\text { Date }\end{array}$ & $\begin{array}{l}\text { Prep } \\
\text { Date }\end{array}$ & $\begin{array}{l}\text { Analyses } \\
\text { Date }\end{array}$ & Result & Units & Quat. & $\begin{array}{c}\text { Detection } \\
\text { Limit }\end{array}$ & $\begin{array}{l}\text { Regulatory } \\
\text { Level }\end{array}$ & Dilution \\
\hline Mercury & EPA 7470 & $05 / 18 / 98$ & $05 / 20 / 98$ & $05 / 20 / 98$ & 0.0004 & MG/L & $u$ & 0.0008 & 0.20 & 4 \\
\hline Arsenic & EPA 6010 & $05 / 18 / 98$ & $05 / 20 / 98$ & $05 / 20 / 98$ & 0.024 & $M G / L$ & B & 1.2 & 5.0 & 4 \\
\hline Barium & EPA 6010 - & $05 / 18 / 98$ & $05 / 20 / 98$ & $05 / 20 / 98$ & 0.18 & MG/L & B & 0.80 & 100 & 4 \\
\hline Cadmium & EPA 6010 & $05 / 18 / 98$ & $05 / 20 / 98$ & $05 / 20 / 98$ & 0.0024 & MG/L & $\mathbf{u}$ & 0.020 & 1.0 & 4 \\
\hline Chromium & EPA 6010 - & $05 / 18 / 98$ & $05 / 20 / 98$ & $05 / 20 / 98$ & 0.0051 & $M G / L$ & $B$ & 0.040 & 5.0 & 4 \\
\hline Lead & EPA 6010 - & $05 / 18 / 98$ & $05 / 20 / 98$ & $05 / 20 / 98$ & 0.0044 & $M G / L$ & $u$ & 0.40 & 5.0 & 4 \\
\hline Selenium & EPA 6010 - & $05 / 18 / 98$ & $05 / 20 / 98$ & $05 / 20 / 98$ & 0.012 & $M G / L$ & $u$ & 1.0 & 1.0 & 4 \\
\hline silver & EPA 6010 - & $05 / 18 / 98$ & $05 / 20 / 98$ & $05 / 20 / 98$ & 0.0028 & $M G / L$ & $u$ & 0.040 & 5.0 & 4 \\
\hline
\end{tabular}


Bechtel Mevada

NTS273, 2621 Losee Road

Building A-2, Receiving

North Las Vegas, HV 89030-4129

Project: 625.01
Category : TCLP Metals

Matrix : Soil

Client ID: HA
Environmenta! Services

Sample Date : NA

Receipt Date : NA

Report Date : 05/27/98

Quanterra ID : EXTBLK172739-1

\begin{tabular}{|c|c|c|c|c|c|c|c|c|c|c|}
\hline Analyte & Method & $\begin{array}{l}\text { Extract } \\
\text { Date }\end{array}$ & $\begin{array}{l}\text { Prep } \\
\text { Date }\end{array}$ & $\begin{array}{l}\text { Analyses } \\
\text { Date }\end{array}$ & Result & Units & Oual. & $\begin{array}{l}\text { Detection } \\
\text { Limit }\end{array}$ & $\begin{array}{c}\text { Regulatory } \\
\text { Level }\end{array}$ & Dilution \\
\hline егcury & EPA 7470 & $05 / 18 / 98$ & $05 / 20 / 98$ & $05 / 20 / 98$ & 0.0004 & HG/L & $\mathrm{U}$ & 0.0008 & 0.20 & 4 \\
\hline Arsenic & EPA 6010 & $05 / 18 / 98$ & $05 / 20 / 98$ & $05 / 20 / 98$ & 0.0076 & $M G / L$ & $W$ & .1 .2 & 5.0 & 4 \\
\hline lar ium & EPA 6010 & $05 / 18 / 98$ & $05 / 20 / 98$ & $05 / 20 / 98$ & 0.0040 & $M G / L$ & $\mathbf{U}$ & 0.80 & 100 & 4 \\
\hline admi um & EPA 6010 & $05 / 18 / 98$ & $05 / 20 / 98$ & $05 / 20 / 98$ & 0.0024 & $M G / L$ & $\mathbf{U}$ & 0.020 & 1.0 & 4 \\
\hline hromiun & EPA 6010 & $05 / 18 / 98$ & $05 / 20 / 98$ & $05 / 20 / 98$ & 0.0076 & $M G / 1$ & $\mathbf{B}$ & 0.040 & 5.0 & 4 \\
\hline ad & EPA 6010 & $05 / 18 / 98$ & $05 / 20 / 98$ & $05 / 20 / 98$ & 0.0044 & $M G / L$ & $\mathbf{U}$ & 0.40 & 5.0 & 4 \\
\hline elenium & EPA 6010 & $05 / 18 / 98$ & $05 / 20 / 98$ & $05 / 20 / 98$ & 0.012 & $M G / L$ & $\mathbf{u}$ & 1.0 & 1.0 & 4 \\
\hline I ver & EPA 6010 & $05 / 18 / 98$ & $05 / 20 / 98$ & $05 / 20 / 98$ & 0.0028 & $M G / L$ & U & 0.040 & 5.0 & 4 \\
\hline
\end{tabular}


Bechtel Nevada

NTS273, 2621 Losee Road

Building A-2, Receiving

North Las Vegas, NV $89030-4129$

Project: $\quad 625.01$
Category : TCLP 1CAP

Hatrix : soil

Client 10: NA

Sample Date : HA

Receipt Date : $\mathrm{HA}$

Report Date : 05/27/98

Quanterra ID : QCBLK17295B-1

\begin{tabular}{|c|c|c|c|c|c|c|c|c|c|c|}
\hline Analyte & Method & $\begin{array}{l}\text { Extract } \\
\text { Date }\end{array}$ & $\begin{array}{l}\text { Prep } \\
\text { Date }\end{array}$ & $\begin{array}{l}\text { Analyses } \\
\text { Date }\end{array}$ & Result & Units & Dual. & $\begin{array}{l}\text { Detection } \\
\text { Limit }\end{array}$ & $\begin{array}{l}\text { Regulatory } \\
\text { Level }\end{array}$ & Di lution \\
\hline Arsenic & EPA 6010 & WA & $05 / 20 / 98$ & $05 / 20 / 98$ & 0.0019 & $M G / L$ & $\mathbf{U}$ & 0.30 & 5.0 & 1 \\
\hline Barium & EPA 6010 & NA & $05 / 20 / 98$ & $05 / 20 / 98$ & 0.0050 & $M G / L$ & $\mathbf{U}$ & 0.20 & 400 & 1 \\
\hline Cadmiun & EPA 6010 & NA & $05 / 20 / 98$ & $05 / 20 / 98$ & $0.000 s$ & $M G / L$ & $\mathbf{B}$ & 0.005 & 1.0 & 1 \\
\hline Chromium & EPA 6010 & NA & $05 / 20 / 9 \mathrm{~B}$ & $05 / 20 / 98$ & 0.0008 & MG/L & $\mathbf{U}$ & 0.010 & $5.0^{\circ}$ & 1 \\
\hline Lead & EPA 6010 & HA & $05 / 20 / 98$ & $05 / 20 / 98$ & 0.0028 & $\mathrm{MG} / \mathrm{L}$ & 8 & 0.10 & 5.0 & 1 \\
\hline Seleniun & EPA 6010 & NA & $05 / 20 / 98$ & $05 / 20 / 98$ & 0.0031 & $M G / L$ & U & 0.25 & 1.0 & 1 \\
\hline silver & EPA 6010 & HA & $05 / 20 / 98$ & $05 / 20 / 98$ & 0.0007 & $M G / L$ & $U$ & 0.010 & 5.0 & 1 \\
\hline
\end{tabular}


Bechtel Hevada

NTS273, 2621 Losee Road

Building A-2, Receiving

North Las Vegas, NV $89030-4129$

Project: 625.01
Huanterra

Envinonmental

Services

Sample Date : WA

Receipt Date : wh

Report Date : 05/27/98

Client ID: $\mathrm{KA}$

Category : TCLP Metals

Hatrix : Soil
Quanterra ID : DCBLK1T2908-1

\begin{tabular}{|c|c|c|c|c|c|c|c|c|c|c|}
\hline Analyte & Method & $\begin{array}{l}\text { Extract } \\
\text { Date }\end{array}$ & $\begin{array}{l}\text { Prep } \\
\text { Date }\end{array}$ & $\begin{array}{l}\text { Analyses } \\
\text { Date }\end{array}$ & Resut $t$ & Units & oual. & $\begin{array}{l}\text { Detection } \\
\text { Limit }\end{array}$ & $\begin{array}{l}\text { Regulatory } \\
\text { Level }\end{array}$ & Dilution \\
\hline Nertury & EPA 7470 & NA & $05 / 20 / 98$ & $05 / 20 / 98$ & 0.0001 & Mo/L & u & 0.0002 & 0.20 & 1 \\
\hline
\end{tabular}


Bechtel Nevada

wTS273, 2621 Losee Road

Euilding A-2, Receiving

North Las Vegas, NY $89030-4129$

Project: 625.01
Category : TCLP Metals

tetrix:50il

Client ID: NA

Sample Date : NA

Receipt Dete: NA

Report Date : 05/27/98

Quanterra 10 : aCLCS172908-1

Detection Regulatory

$\begin{array}{llll}\text { Extract } & \text { Prep } & \text { Analyses } & \\ \text { Date } & \text { Date } & \text { Date } & \text { Result units qual. Limit Regulatory }\end{array}$

Analyte

Method

Date

$05 / 20 / 98$

$05 / 20 / 98$

105

XREC

1 
Bechtel Nevada

NTS273, 2621 Losee Road

Building A-2, Receiving

North Las Vegas, NV 89030-4129

Project: 625.01

Category : TCLP ICAP

Sample Date : NA

Receipt Date : MA

Report Date : 05/27/98

Client ID: NA

EuBnterta ID : acLCS172958-

\begin{tabular}{|c|c|c|c|c|c|}
\hline Analyte & Method & $\begin{array}{l}\text { Extract } \\
\text { Date }\end{array}$ & $\begin{array}{l}\text { Prep } \\
\text { Date }\end{array}$ & $\begin{array}{l}\text { Ansilyses } \\
\text { Date }\end{array}$ & Resul $\mathrm{x}$ \\
\hline Arsenic & EPA $6010^{\circ}$ & MA & $05 / 20 / 98$ & $05 / 20 / 98$ & 100 \\
\hline Barium & EPA 6010 & MA & $05 / 20 / 98$ & $05 / 20 / 98$ & 97 \\
\hline Cadmi un & EPA 6010 & MA & $05 / 20 / 98$ & $05 / 20 / 98$ & 98 \\
\hline Chromiun & EPA 6010 & $\mathrm{HA}$ & $05 / 20 / 98$ & $05 / 20 / 98$ & 98 \\
\hline Lead & EPA 6010 & NA & $05 / 20 / 98$ & $05 / 20 / 98$ & 97 \\
\hline Seleniun & EPA 6010 & NA & $05 / 20 / 98$ & $05 / 20 / 98$ & 99 \\
\hline
\end{tabular}

Detection Regulatory

Limit Level Dilution

6010

$05 / 20 / 98$

$05 / 20 / 98$

1


Bechtel Hevada

N士\$273, 2621 Losee Road

Building A-2, Receiving

Harth Las Vegas, WV $89030-4129$

Project: 625.01

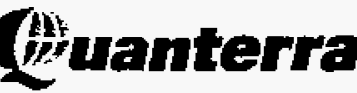

Environtuental

Services
Cotegory : TCLP ICAP

Matrix : Soil

C: ient ID: NA
Sample Date : NA

Receipt Date : MA

Report Dete : 05/27/98

Ouanterra ID : OCLCS172958-2

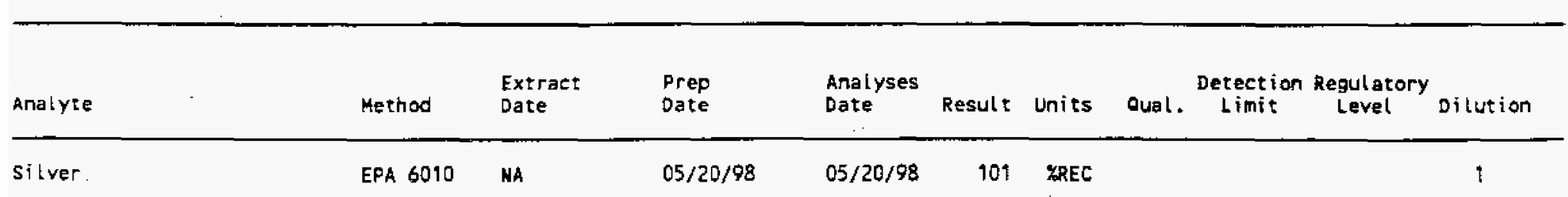




\section{U.S. EPA - CLP $\leadsto$}

\section{COVER PAGE - INORGANIC ANALYSES DATA PACKAGE}

Lab Name: QUANTERRA_MO

Lat code: ITMO__ Case No.: SOW No.: SW846
Contract: 625.01

SAS NO.:

SDG No.: V411
EPA Sample No.

AG-SMP $3-E 4$

AG-SMP3-W4

PBTI 72739
Lab Sample ID

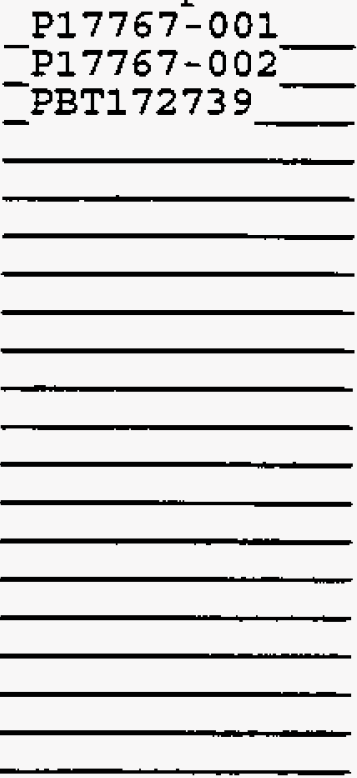

Were ICP interelement corrections applied ?

Yes/No YES

were

ICP background corrections applied ?

If yes - were raw data generated before application of background corrections?

Yes/No YES

Yes/No No

I certify that this data package is in compliance with the terms and conditions of the contract, both technically and for completeness, for other than the conditions detailed above. Release of the data contained in this hardcopy data package and in the computer-readable data submitted on floppy diskette has been authorized by the Laboratory Manager or the Manager's designee, as verified by the following signature.

Signature:

Date:
Name :

Title:

COVER PAGE - IN

TCLP 
Lab Name: QUANTERRA_MO

Lab Code: ITMO Case No.:

Matrix (soil/water): WATER

Level (low $/ \mathrm{med}$ ): LOW : Solids :
Contract: 625.01

AG-SMP3 - E4
SAS NO.:

SDG No.: V411

Lab Sample ID: P17767-001

Date Received: 05/08/98

Concentration Units (ug/L or $\mathrm{mg} / \mathrm{kg}$ dry weight) : UG/L_

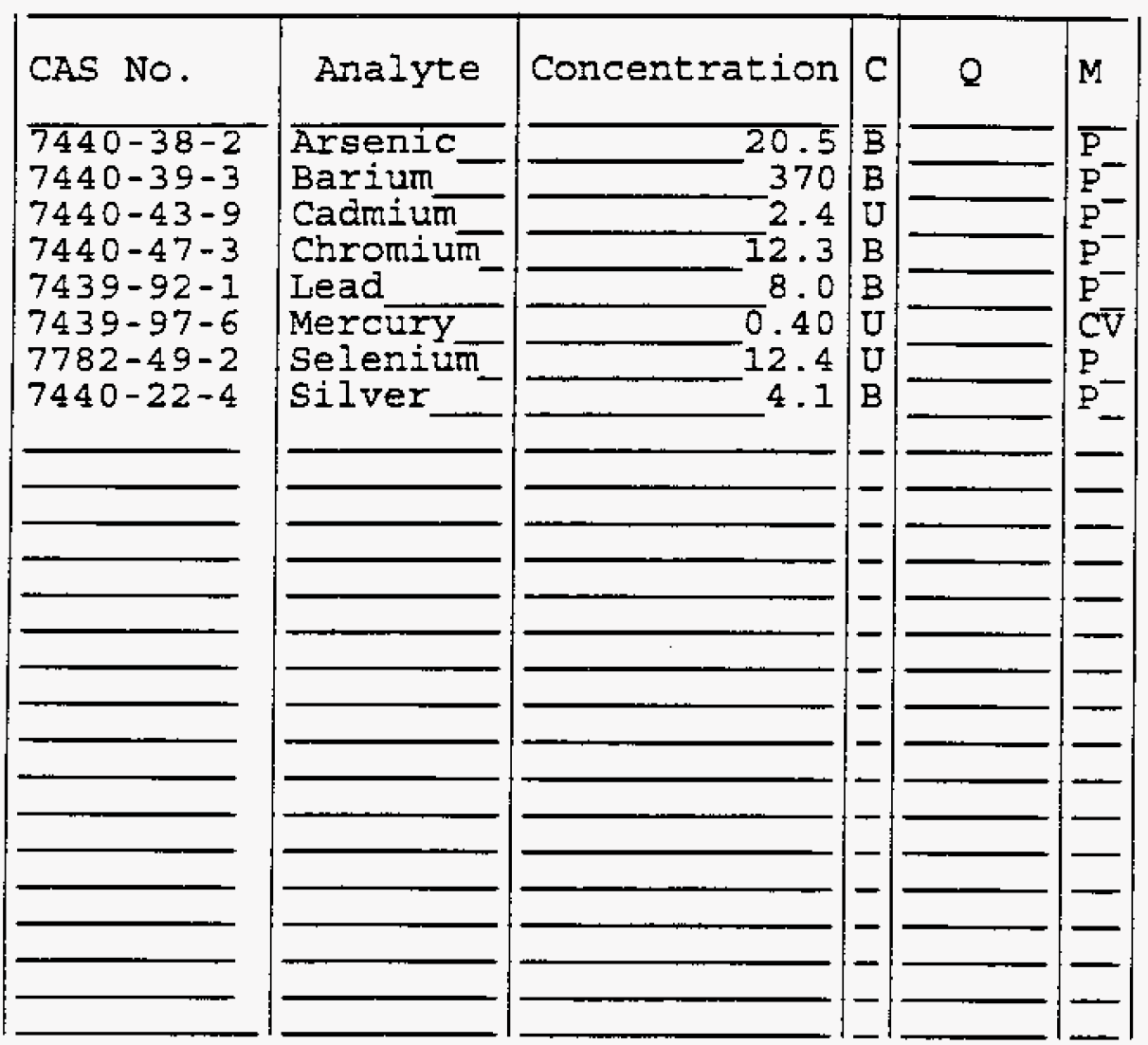

Color Before:

Clarity Before:

Texture:

Clarity After:

Artifacts : 
LA Name: QUANTERRA_MO

Lab Code: ITMO_ Case No.:

Matrix (soil/water) : WATER

Level (low/med) : LOW

: Solids:

$-0.0$
Contract: 625.01

SAS NO.:

SDG No.: V4II

Lab Sample ID: P17767-002

Date Received: 05/08/98

AG-SMP3 - W4

Concentration Units (ug/L or $\mathrm{mg} / \mathrm{kg}$ dry weight): UG/L_

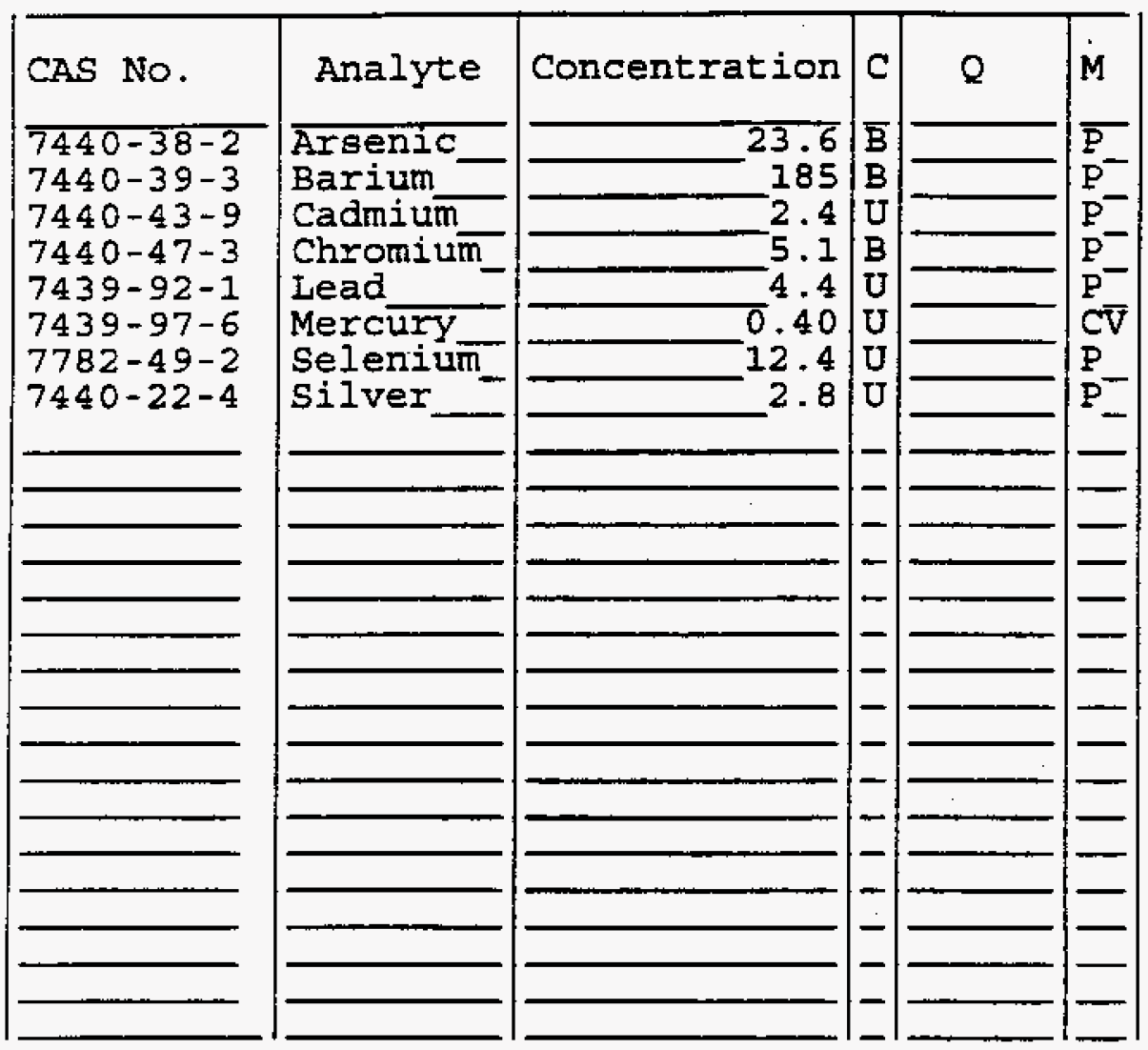

Color Before:

Clarity Before:

Texture:

Color After:

Clarity After:

Artifacts :

Comments :

FORM I - IN

TCLP 


\section{U.S. EPA - CLP}

1

INORGANIC ANALYSES DATA SHEET
Lab Name: QUANTERRA_MO

Lab Code: ITMO_ Case No.:

Matrix (soil/water): WATER

Level (low/med): LOW

: Solids: $-0.0$
Contract: 625.01 SAS No.:

SDG No.: V4I1

Lab Sample ID: PBT172739

Date Received: 05/08/98
EPA SAMPLE NO.

PBT172739 r

Concentration Units (ug/L or $\mathrm{mg} / \mathrm{kg}$ dry weight): UG/L_

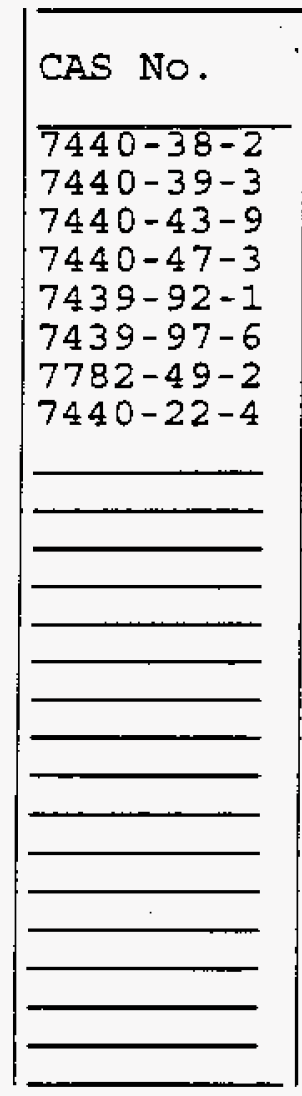

Color Before: Color After: Comments:

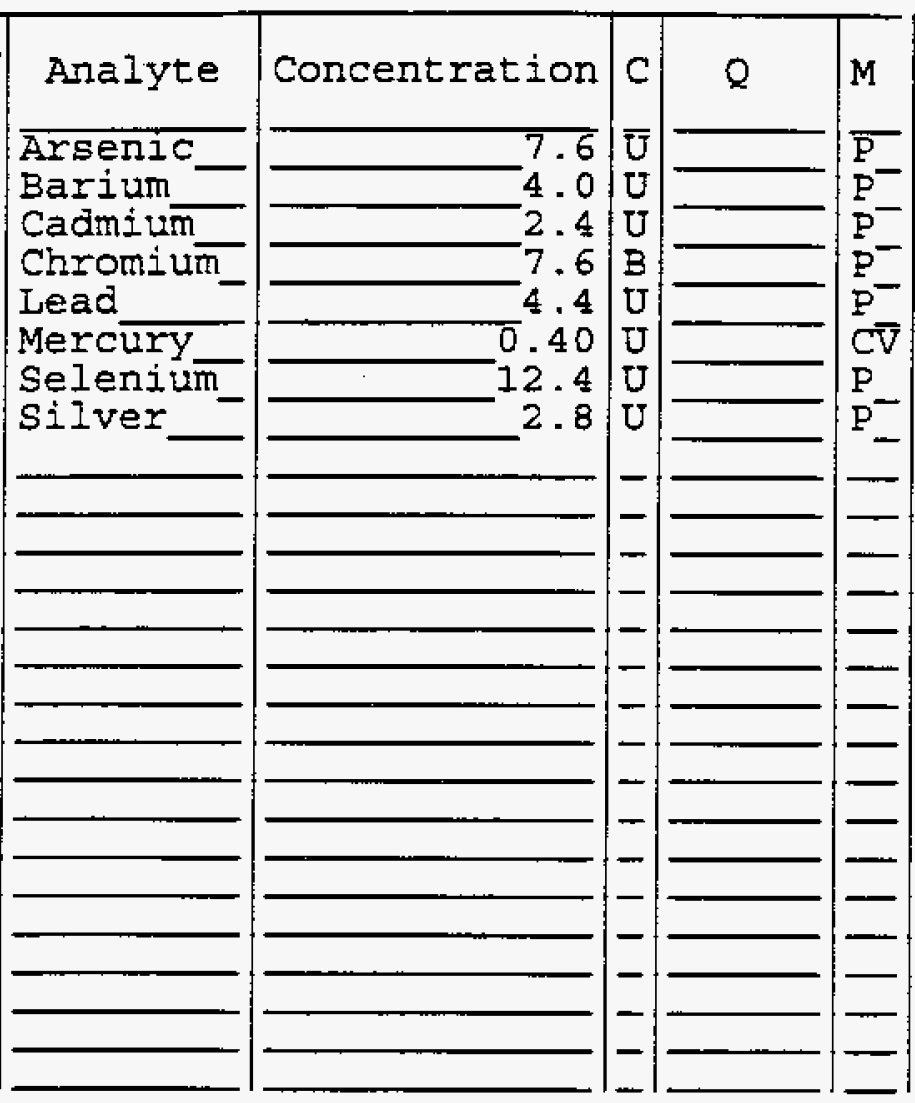

Clarity Before:

Texture:

Clarity After:

Artifacts : 
U.S. EPA - CLP

INITIAL AND CONTINUING CALIBRATION VERIFICATION

Lab Name: QUANTERRA_MO

Contract : 625.01

Lab Code: ITMO_ Case No.:

SAS NO.:

SDG No : V411

Initial Calibration Source: SOL/I/SPX/IC

Continuing Calibration Source: SOL+/LL/SPX_

Concentration Units: ug/L

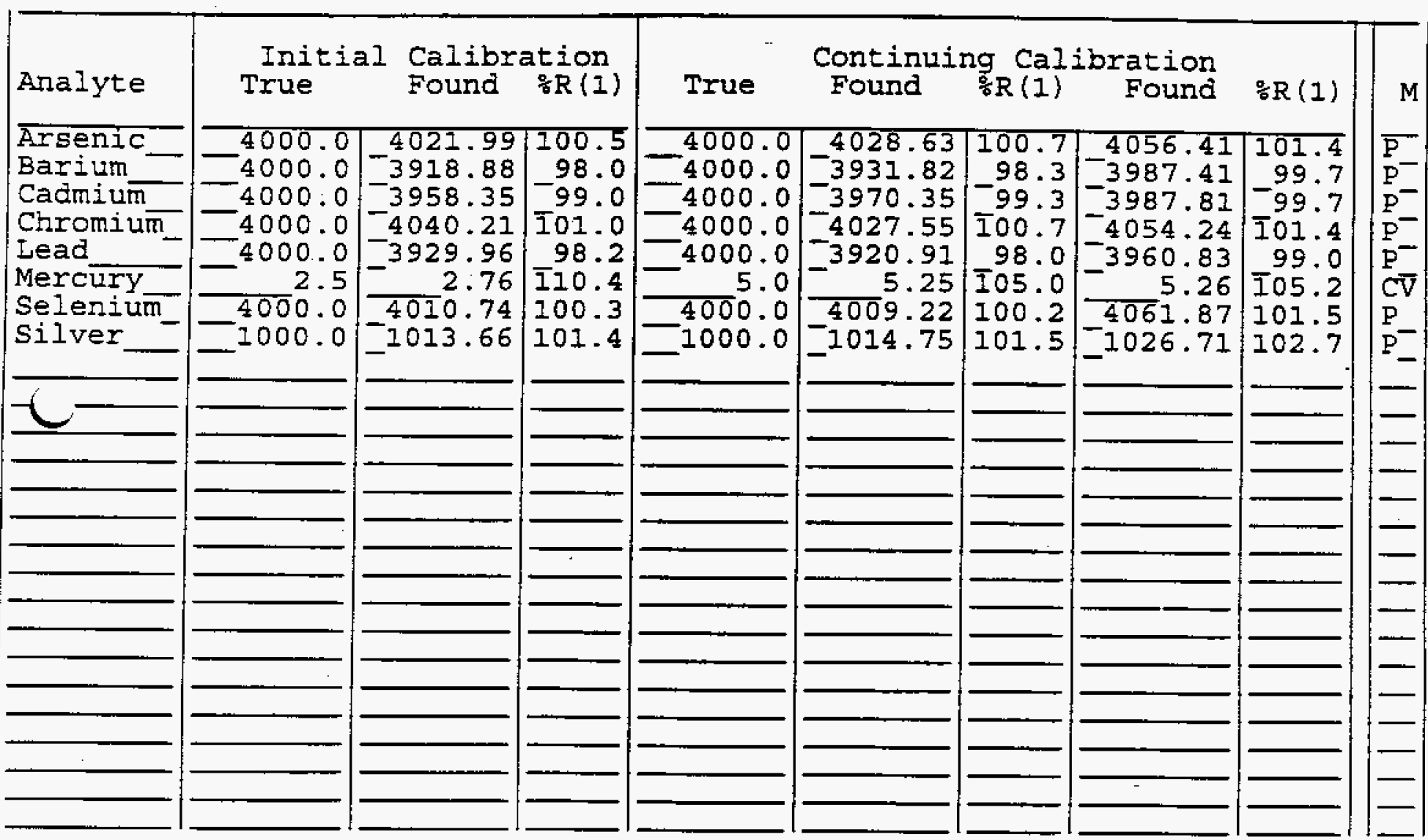

(1) Control Limits: Mercury 80-120; Other Metals 90-110; Cyanide 85-115

FORM II (PART I) - IN

TCLF

000006 
Lab Name: QUANTERRA_MO

Contract: 625.01

Lab Code: ITMO_ Case No.: SAS NO.:

SDG NO.: V411

Initial Calibration Source: SOL/L/SPX/IC

Continuing Calibration Source: SOL+/LL/SPX_

Concentration Units: ug/L

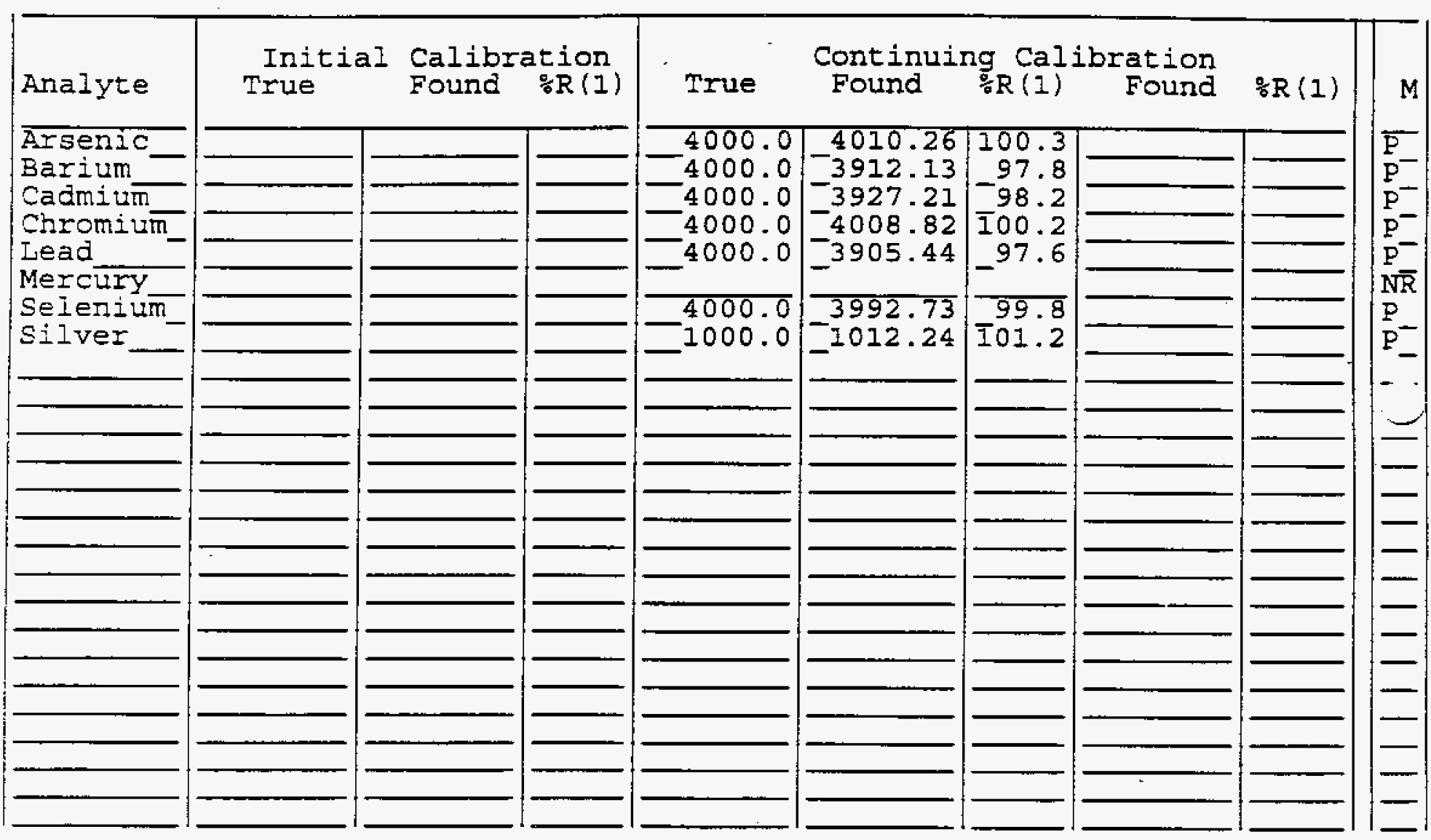

(1) Control Limits: Mercury 80-120; Other Metals 90-110; Cyanide 85-115 
Lab Name: QUANTERRA_MO

Lab Code: ITMO_ Case No.::

AA CRDL Standard Source: SPX/SOL+/LI_

ICP CRDL Standard Source: SOL+/SPX

Concentration Units: ug/L

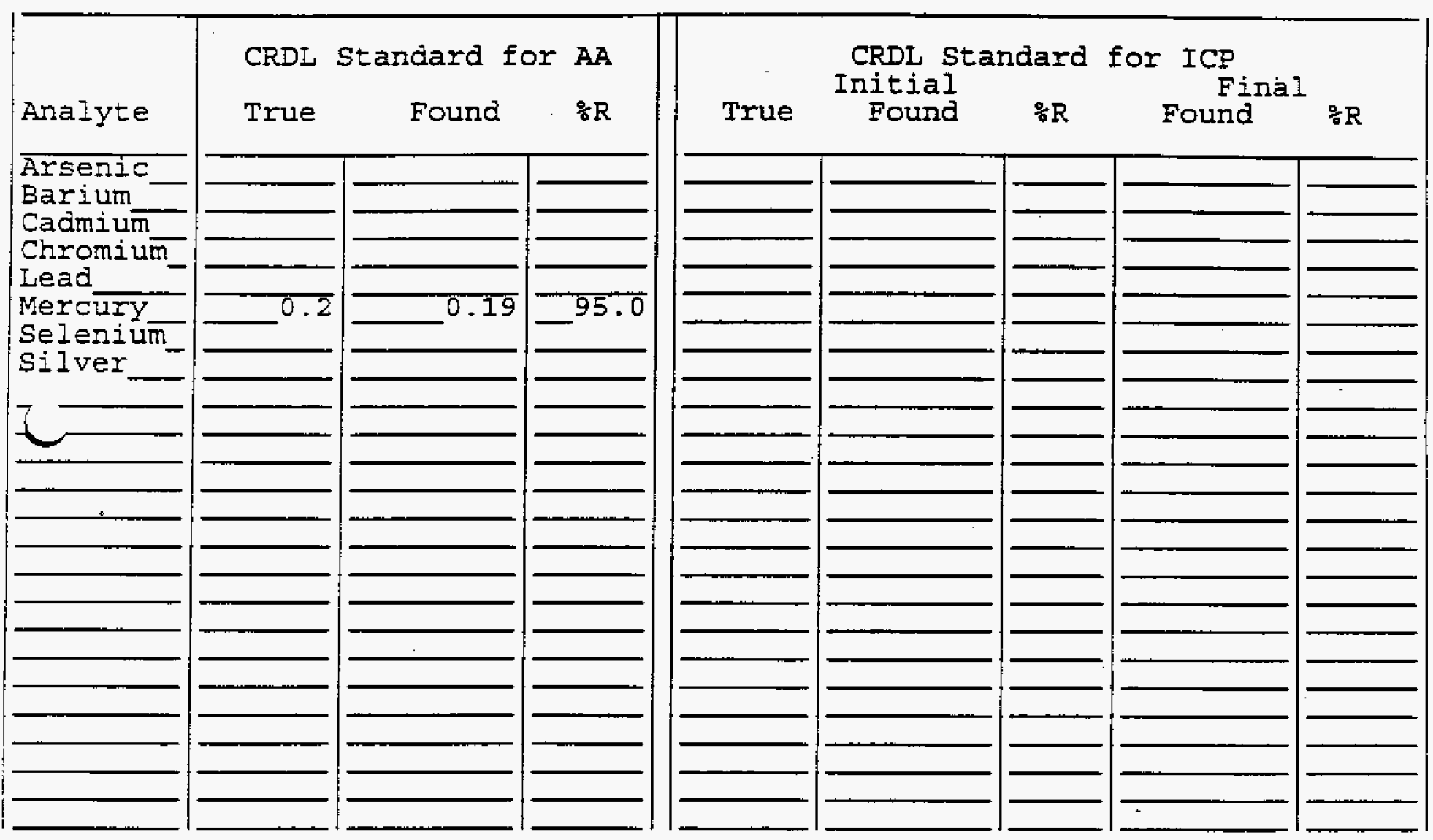

FORM II' (PART 2) - IN 
U.S. EPA - CLP

BIANKS

Lab Name: QUANTERRA_MO

Contract: 625.01

Lab Code: ITMO_ Case No.:

SAS NO. :

SDG NO.: V411

Preparation Blank Matrix (soil/water): WATER

Preparation Blank Concentration Units (ug/I or $\mathrm{mg} / \mathrm{kg}$ ): UG/L_

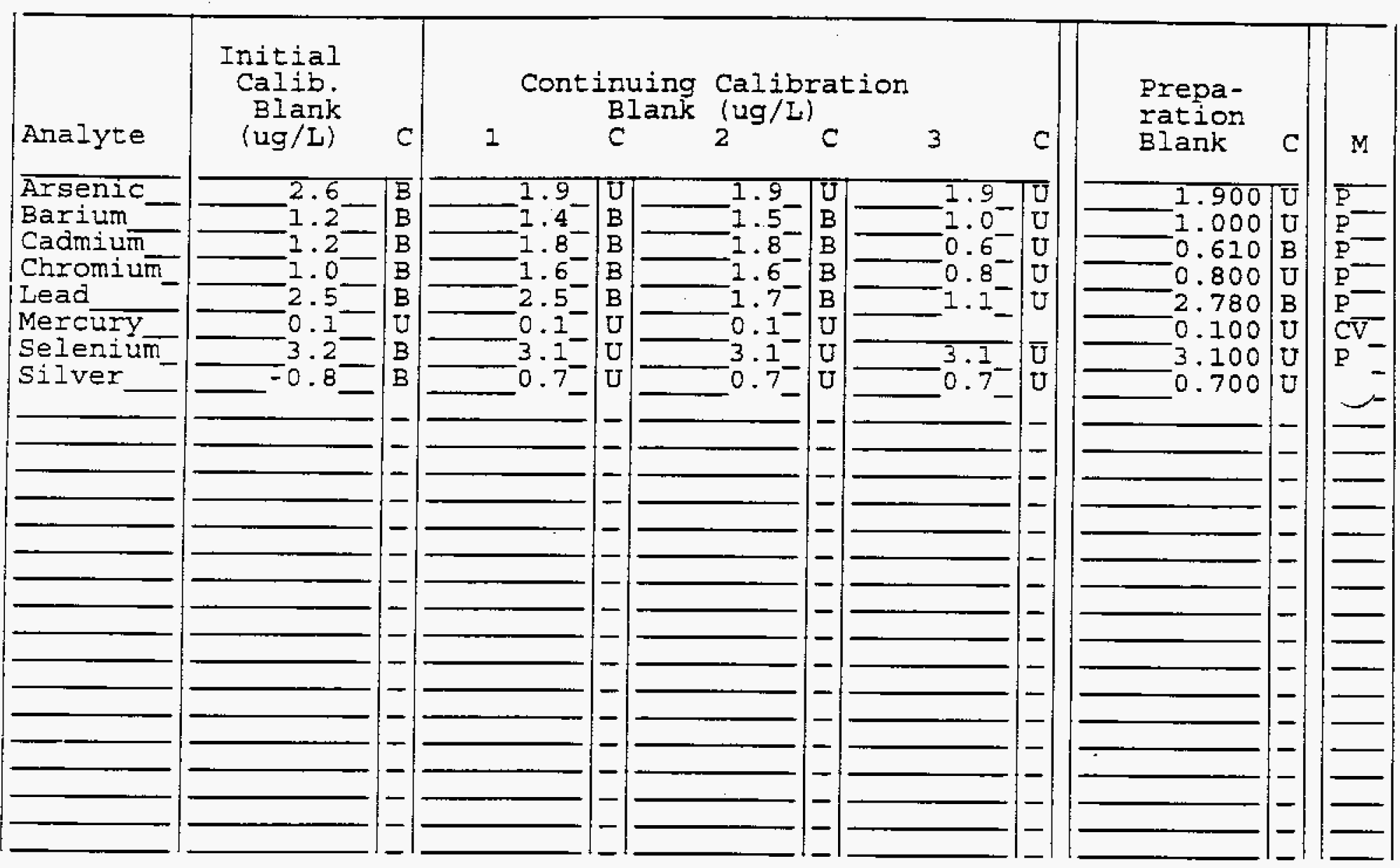


U.S. EPA - CLP
ICP INTERFERENCE CHECK SAMPLE

Lab Name: QUANTERRA_MO

Contract: 625.01

Lab code: ITMO Case No.:

SAS NO:

SDG NO.: V411

ICP ID Number: TJA61E

ICS Source: SOL+/SPX

Concentration Units: ug/L

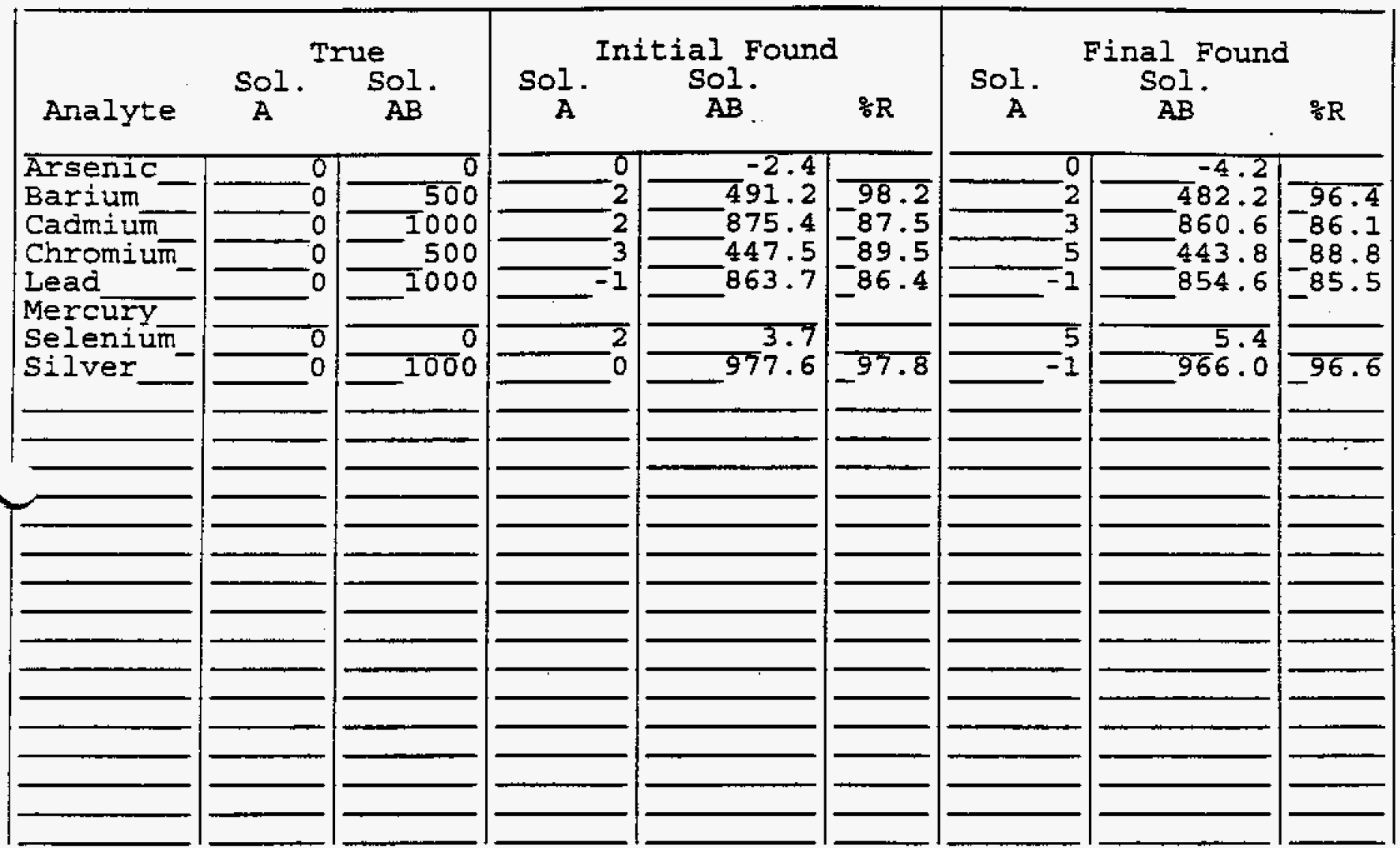




\section{U.S. EPA - CLP \\ 7 \\ IABORATORY CONTROL SAMPIE}

Lab Name: QUANTERRA_MO

Lab Code: ITMO_ Case No.:

Solid LCS Source:

Aqueous LCS Source: SOL+/CHEMPUR
Contract: 625.01

SAS NO.:

SDG No.: V411

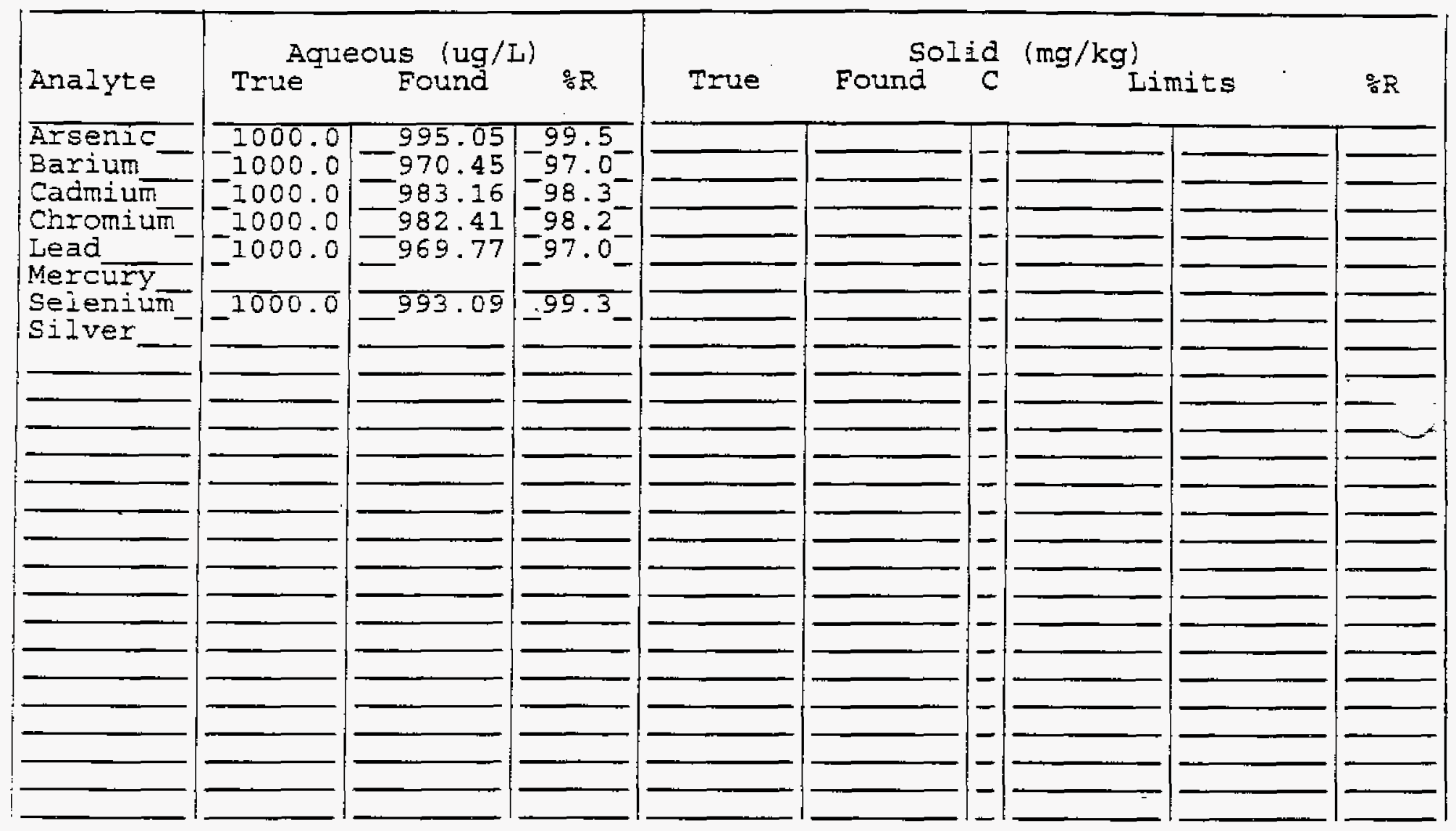




\section{IABORATORY CONTROL SAMPLE}

i

Lab Name: QUANTERRA_MO

Contract: 625.01

Lab code: ITMO_ Case No.:

SAS NO.:

SDE NO.: V4II

Solid LCS Source:

Aqueous LCS Source: SOLt/CHEMPUR

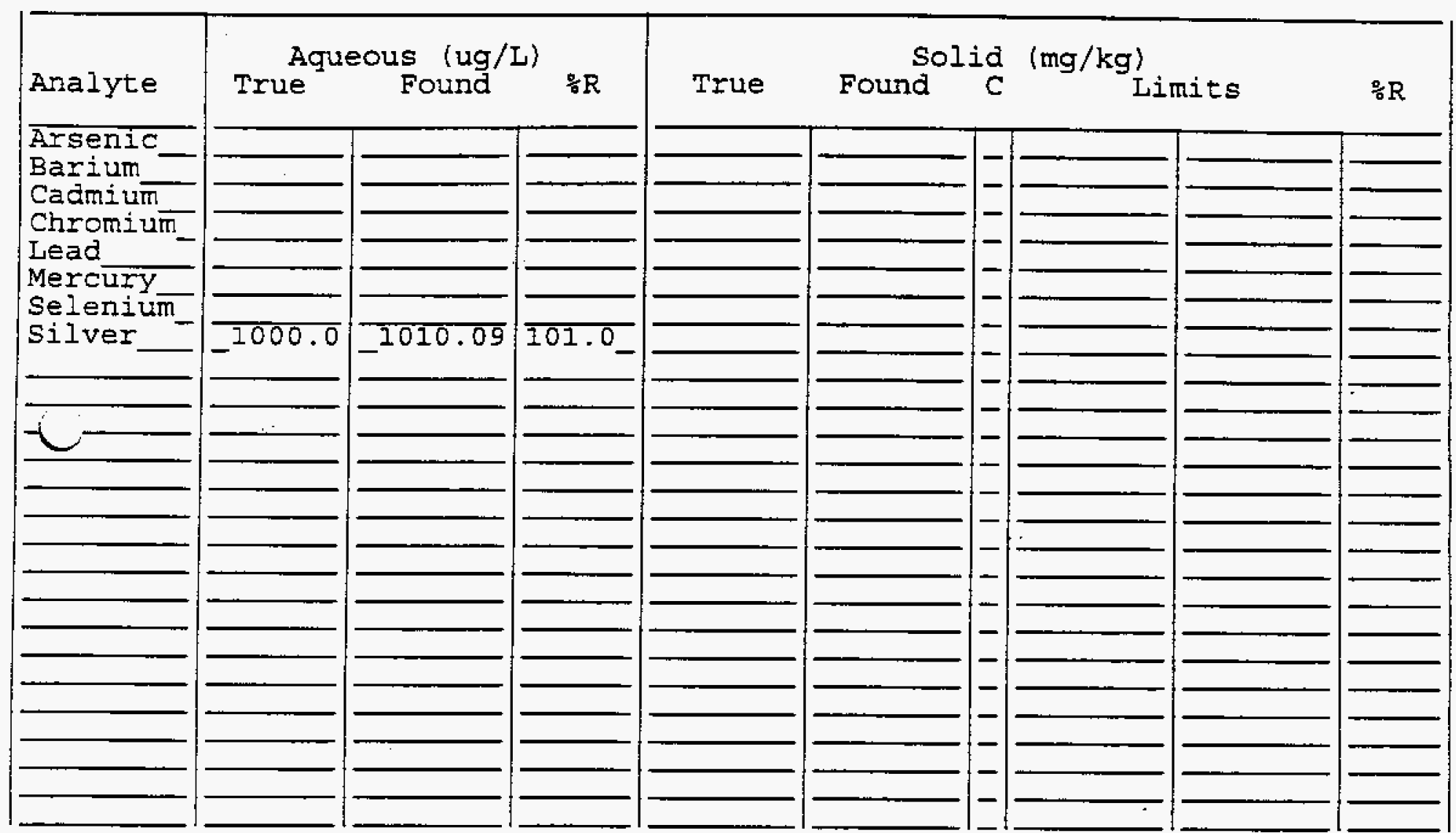




\section{U.S. EPA - CLP \\ LABORATORY CONTROL SAMPLE}

Lab Name: QUANTERRA_MO

Lab Code: ITMO_ Case No.:

Solid LCS Source:

Aqueous LCS Source: SOL+/CHEMPUR
Contract: 625.01

SAS No.: —_ SDG No.: V411

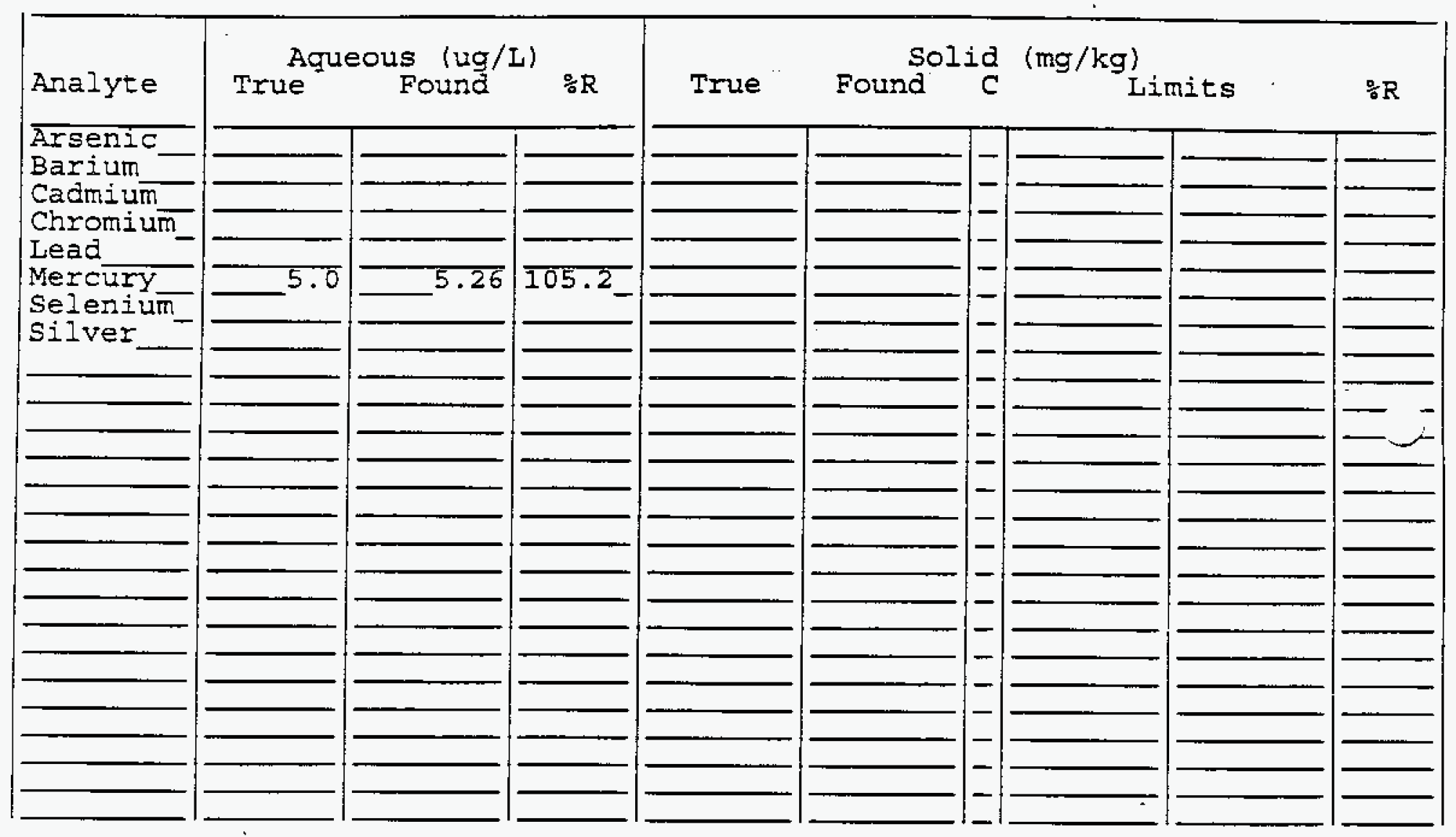

FORM VII - IN

TCLP 


\section{U.S. EPA - CLP A...}

\section{Instrument Detection Limits (Quarterly)}

L: Name: QUANTERRA_MO

Lab Code: ITMO_ Case No. :

ICP ID Number:

Flame AA ID Number : PS200

Furnace AA ID Number :
Contract: 625.01

SAS NO.:

SDG No.: V4II

Date: $\quad 04 / 01 / 98$

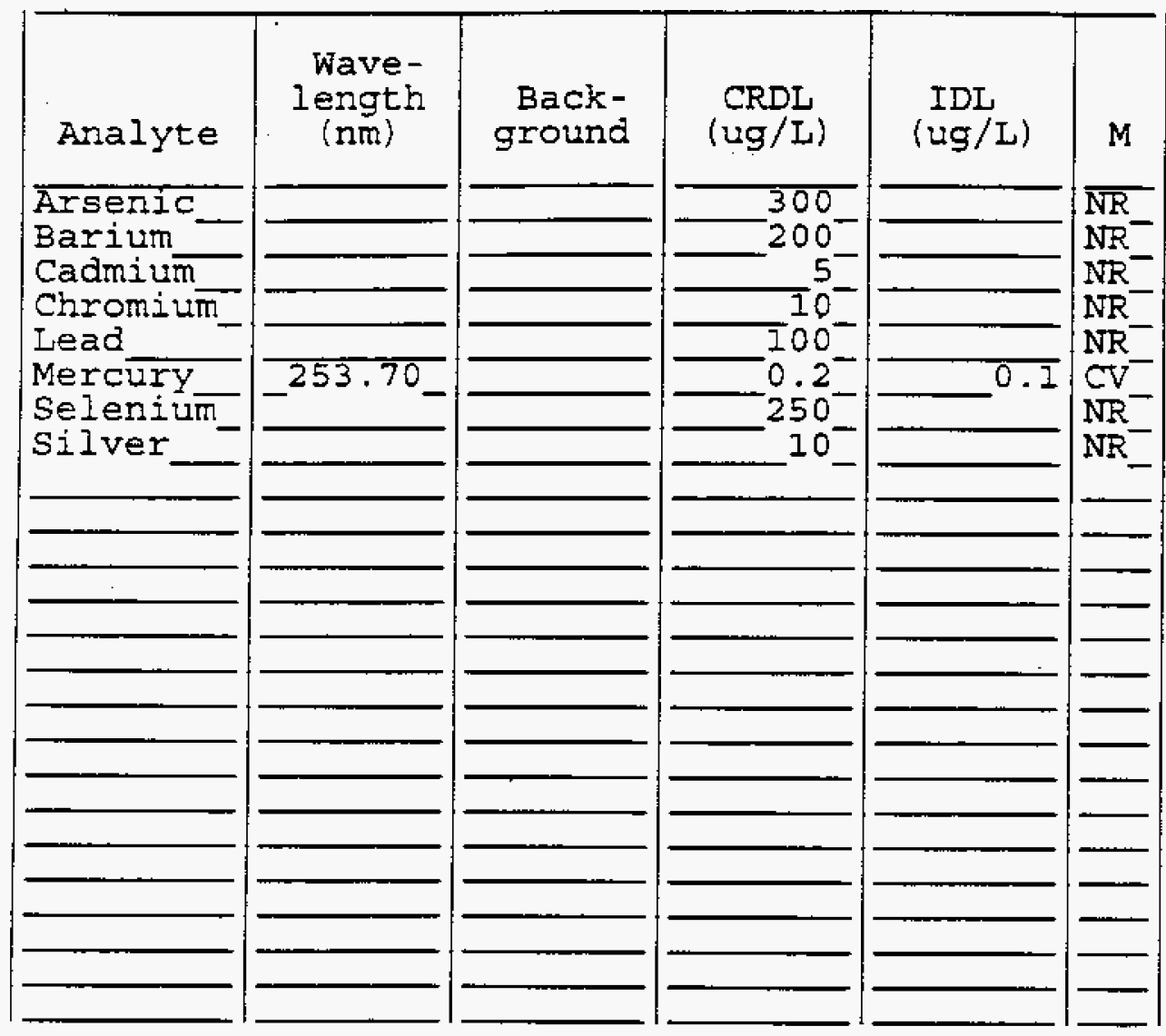

Comments :

FORM X - IN 


\section{U.S. EPA - CLP A}

Instrument Detection Limits (Quarterly)

Lab Name: QUANTERRA_MO

Lab Code: ITMO_Case No.:

TJAEIE

ICP ID Number:

Flame AA ID Number :

Furnace AA ID Number :
Contract: 625.01

SAS No.:

SDG NO.: V411

Date: $\quad 04 / 01 / 98$

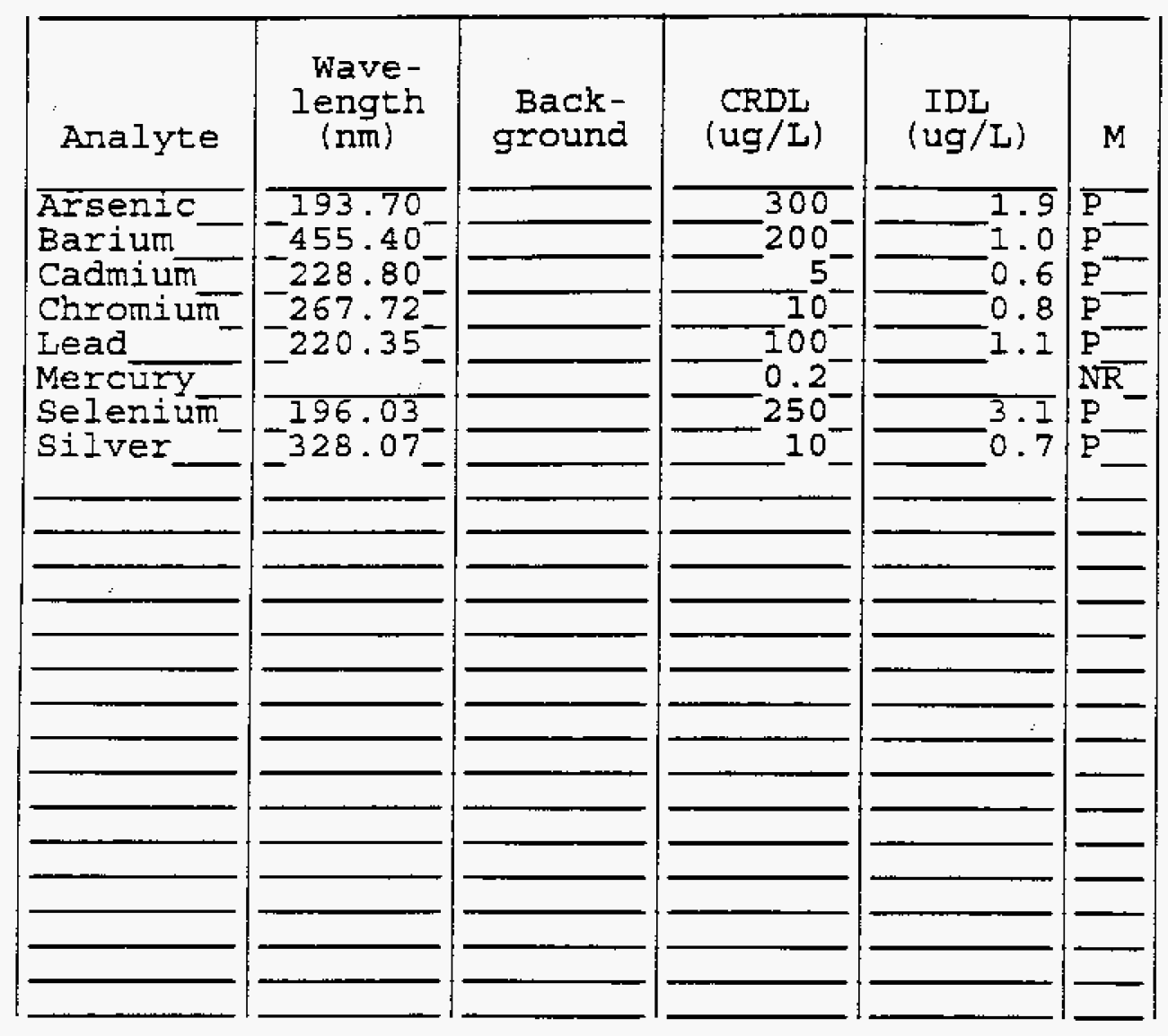

Comments : 


\section{U.S. EPA - CLP \\ $I I A$ \\ ICP INTERELEMENT CORRECTION FACTORS (ANNUALLY)}

Là Name: QUANTERRA_MO

Lab Code: ITMO_ Case No.:

ICP ID Number: TJA61E
Contract: 625.01

SAS NO.:

SDG NO.: V411

Date: $\quad 10 / 01 / 97$

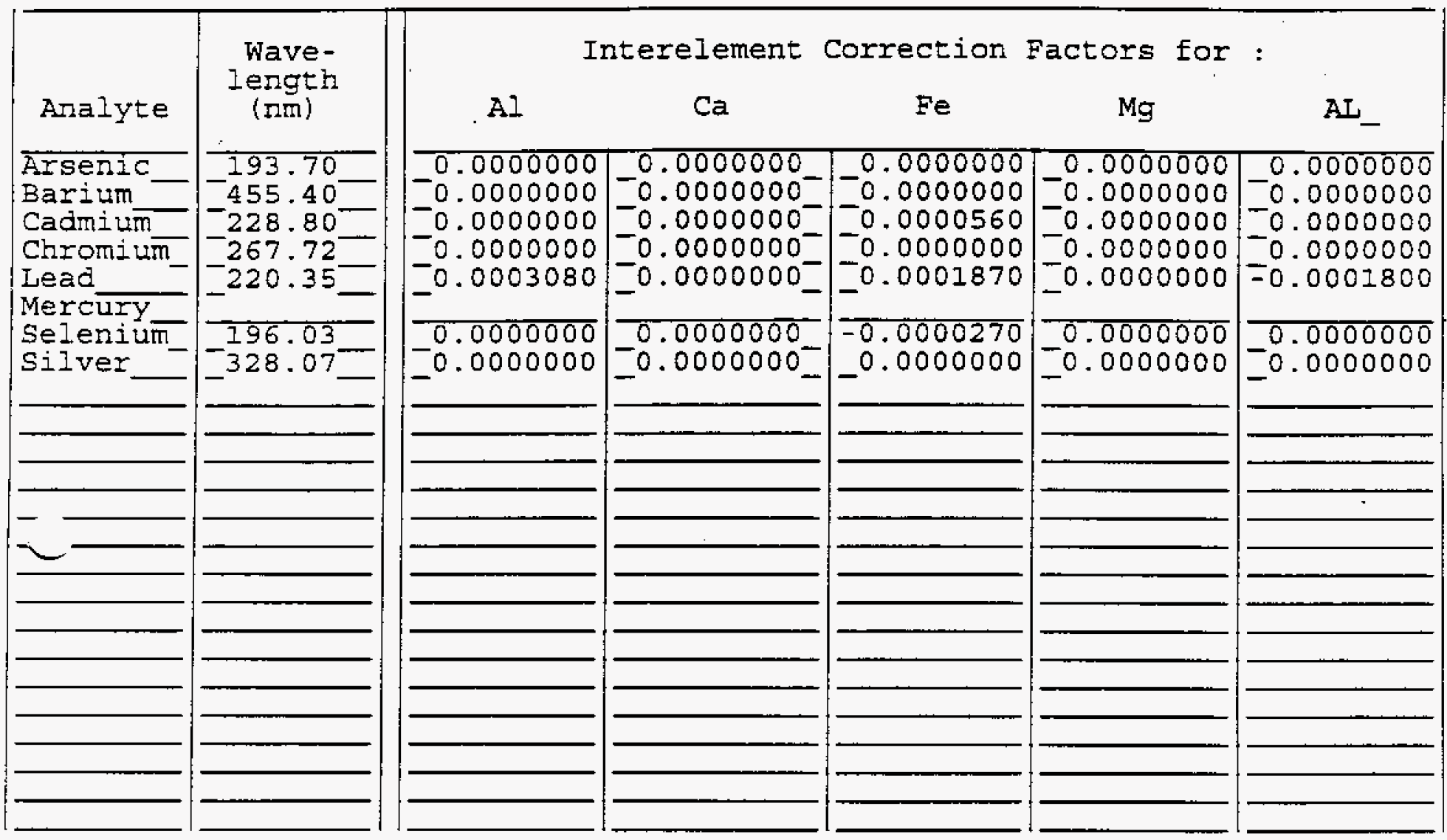

Comments:

FORM XI. (Part 1) - IN

TCIP 


\section{U.S. EPA - CLP 4.}

\section{IIB \\ ICP INTERELEMENT CORRECTION FACTORS (ANNUALIY)}

Lab Name: QUANTERRA_MO

Lab Code: ITMO_ Case No.:

ICP ID Number: TJA6IE
Contract: 625.01

SAS No.:

SDG No.: V4II

Date: $10 / 01 / 97$

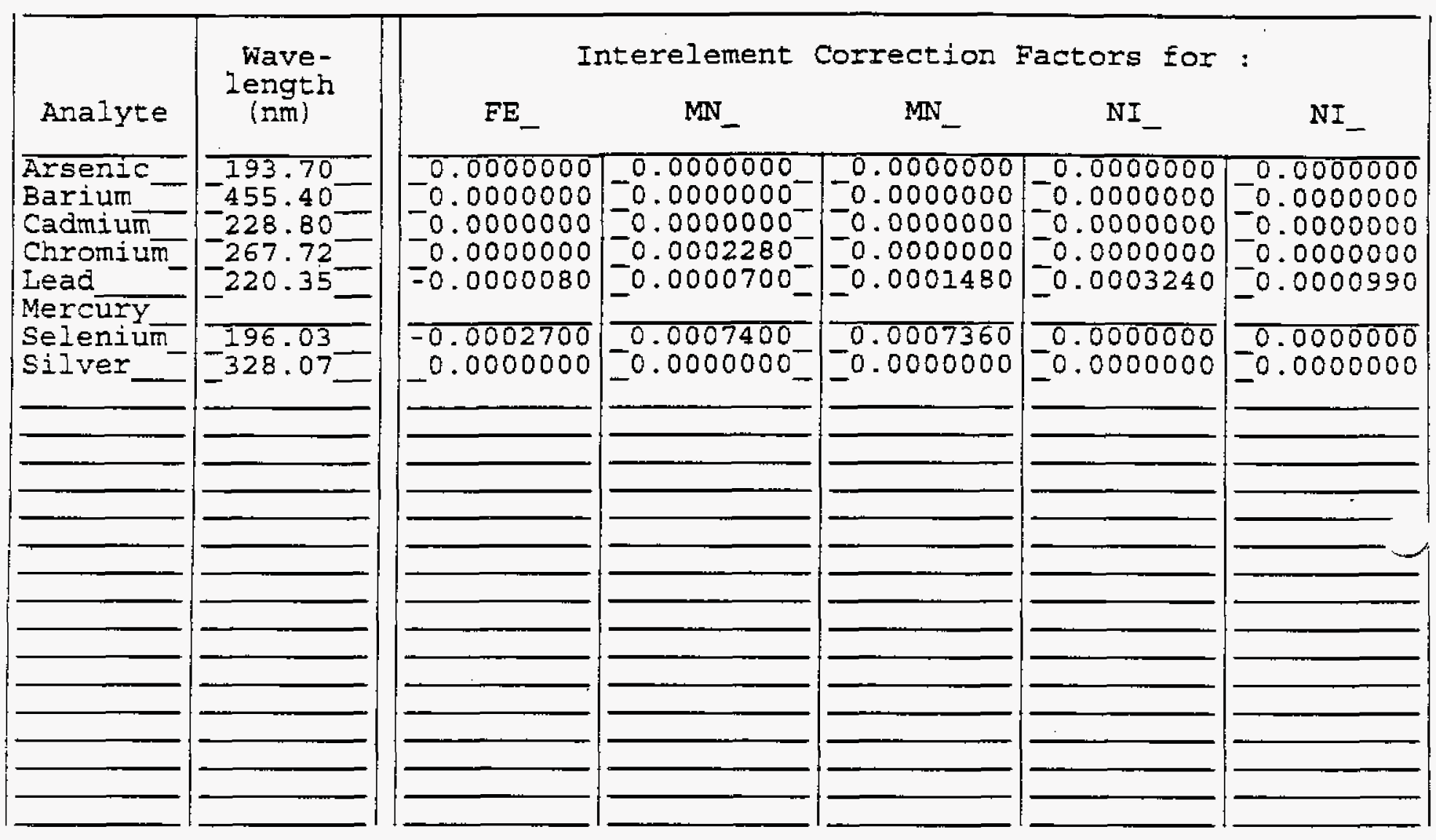

Comments :

FORM XI . (Part 2) - IN

TCLP' 


\section{U.S. EPA - CLP \\ $11 B$ \\ ICP INTERELEMENT CORRECTION FACTORS (ANNUALLY)}

In Name: QUANTERRA_MO

Lab Code: ITMO_ Case No.:

ICP ID Number: TJA6IE
Contract: 625.01

SAS NO.:

Date: 10/01/97

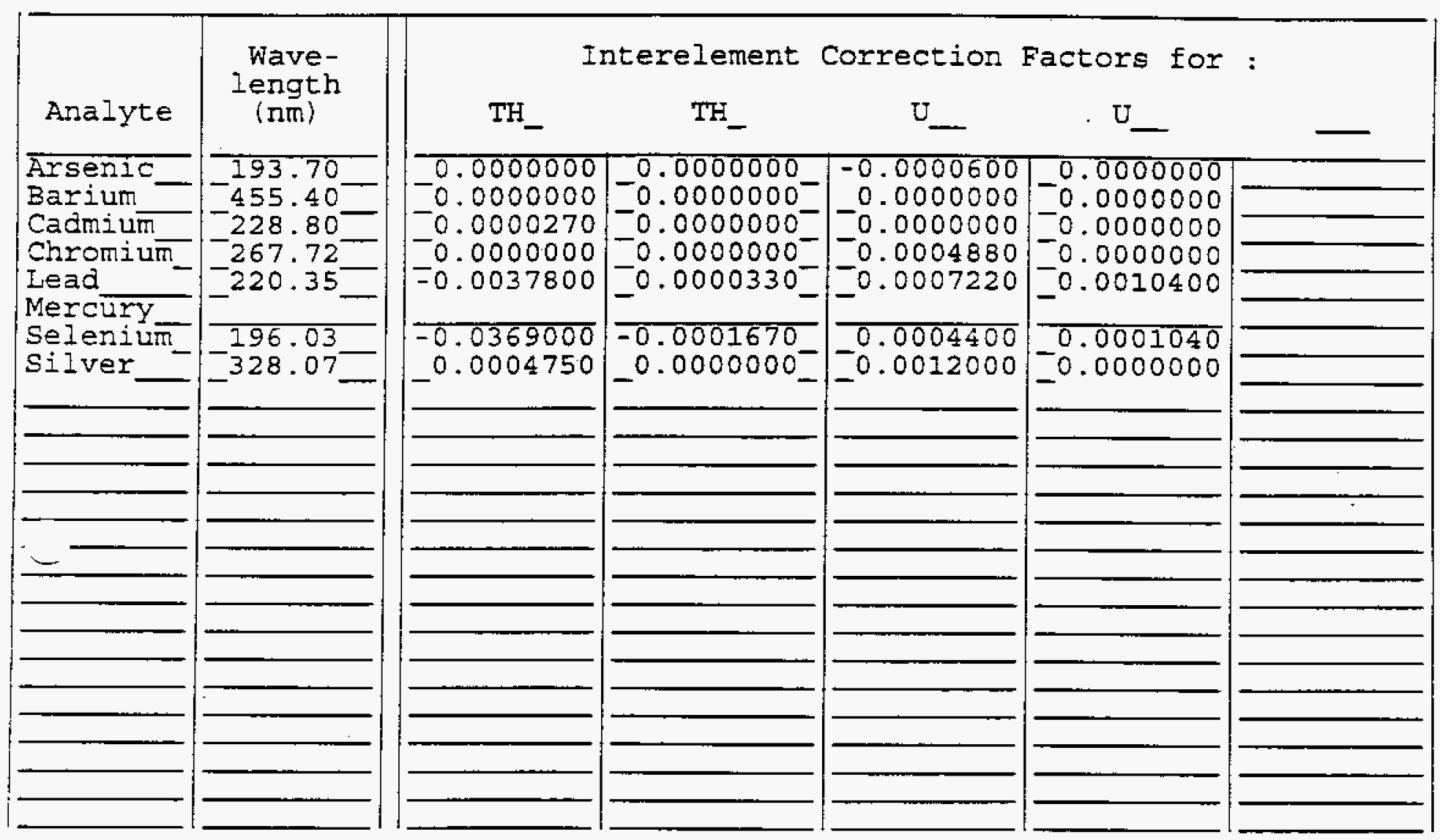

Comments:

FORM XI. (Part 2) - IN

TCLP 
U.S. EPA - CLP -

ICP LINEAR RANGES (QUARTERLY)

Lab Name: QUANTERRA_MO

Lab Code: ITMO_ Case No.:

ICP ID Number: TJA6IE
Contract: 625.01

SAS NO.:

SDG No.: V411

Date: $\quad 04 / 01 / 98$

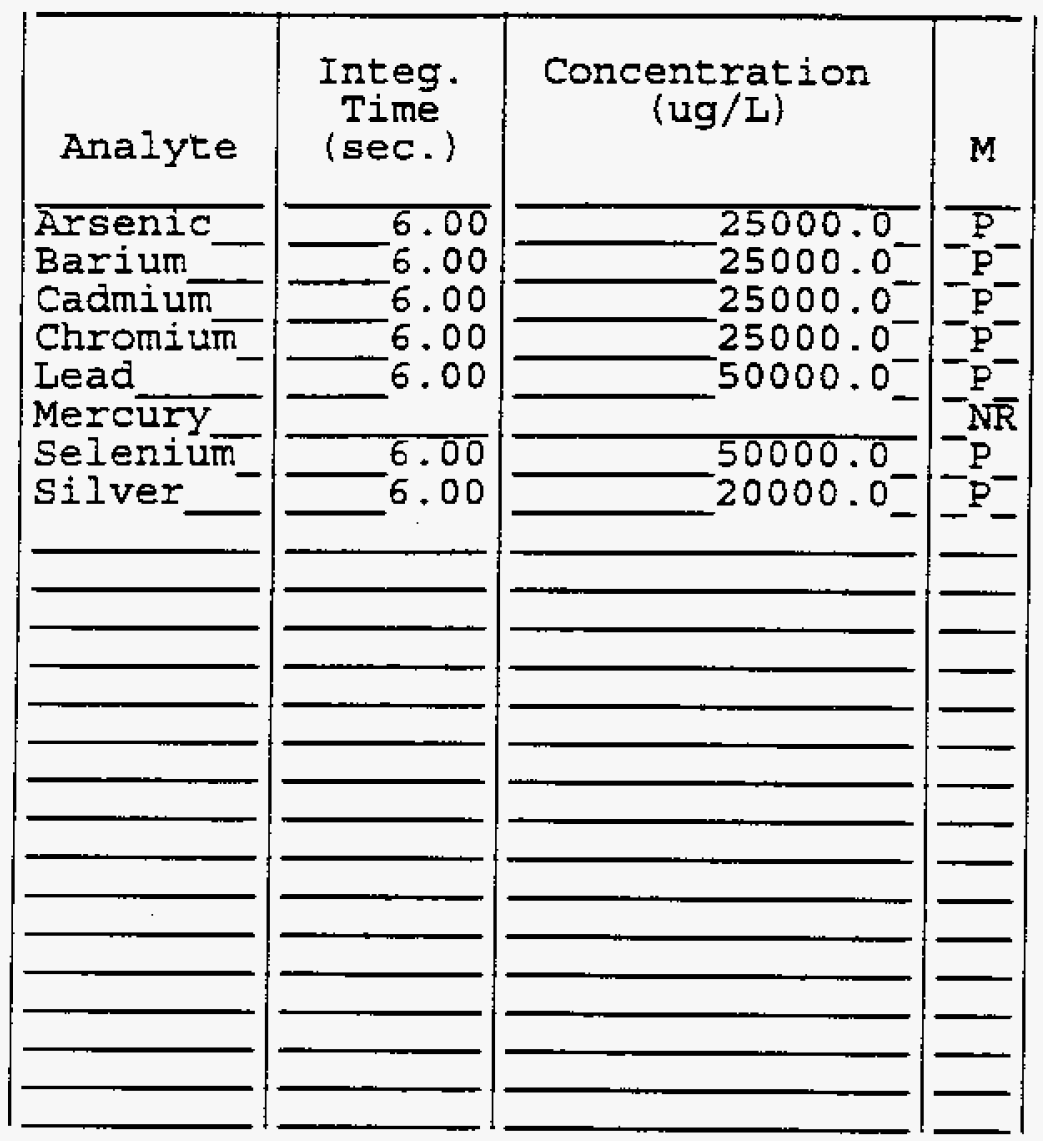

Comments:

FORM XII - IN

TCLP 


\section{U.S. EPA - CLP}

13

PREPARATION LOG

La Name: QUANTERRA_MO

Contract: 625.01

Lab Code: ITMO _ Case No.: SAS No.:

SDG No.: V4II Method: $P_{-}$

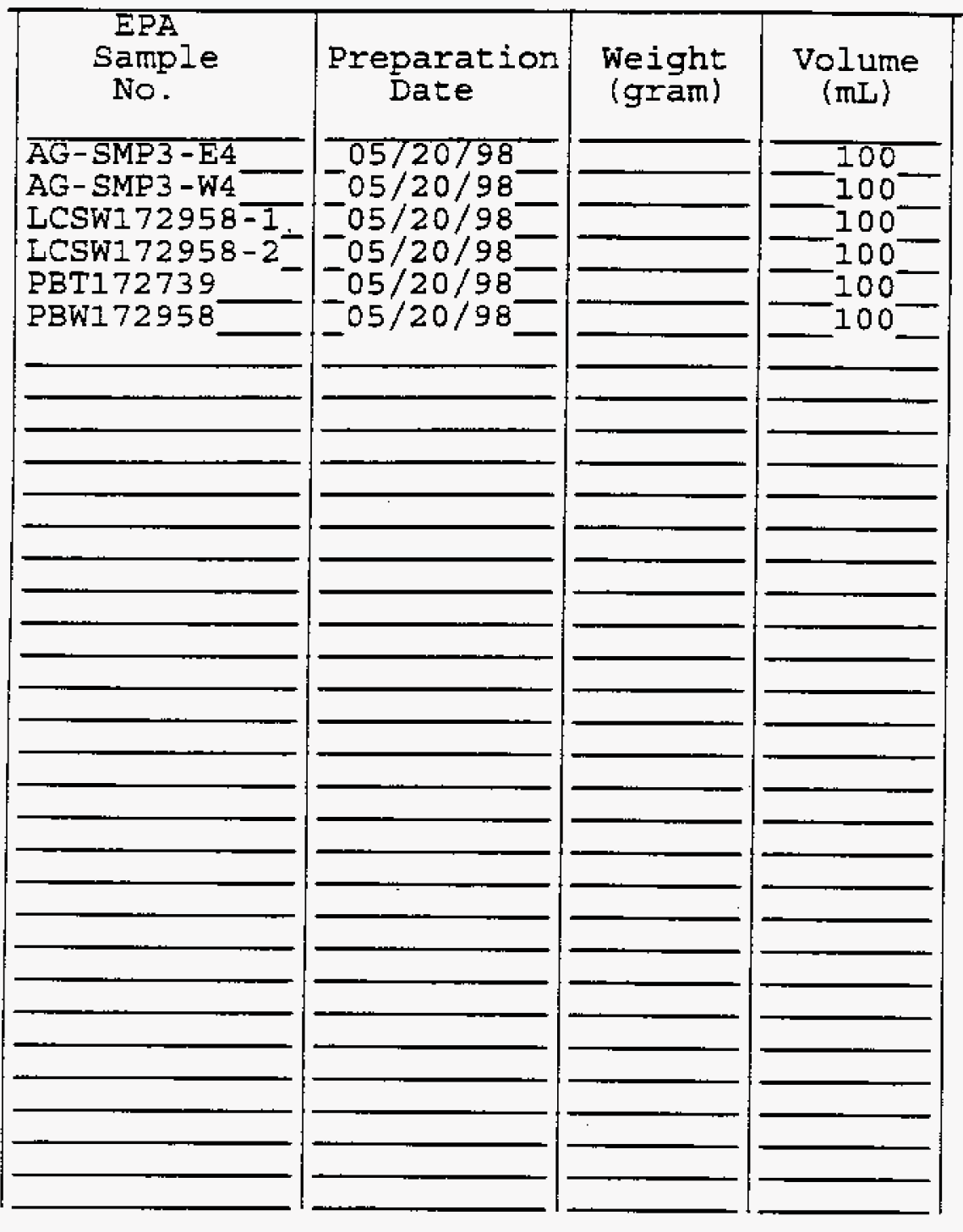

FORM XIII - IN 
Lab Name: QUANTERRA_MO

Lab code: ITMO

Case No.:

Method: CV
Contract: 625.01

SAS NO.:

SDG No.: V4II

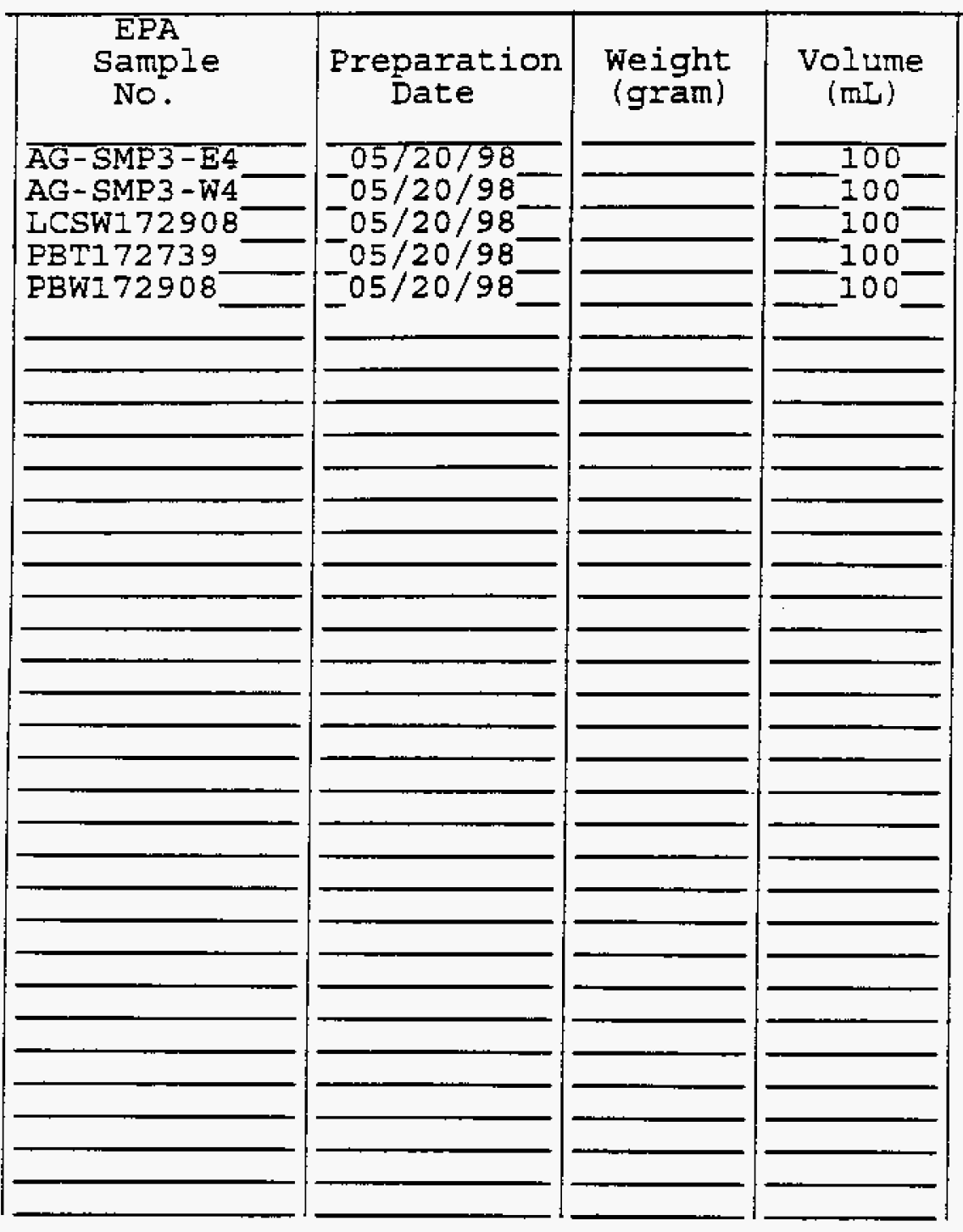

FORM XIII - IN

TCLP 


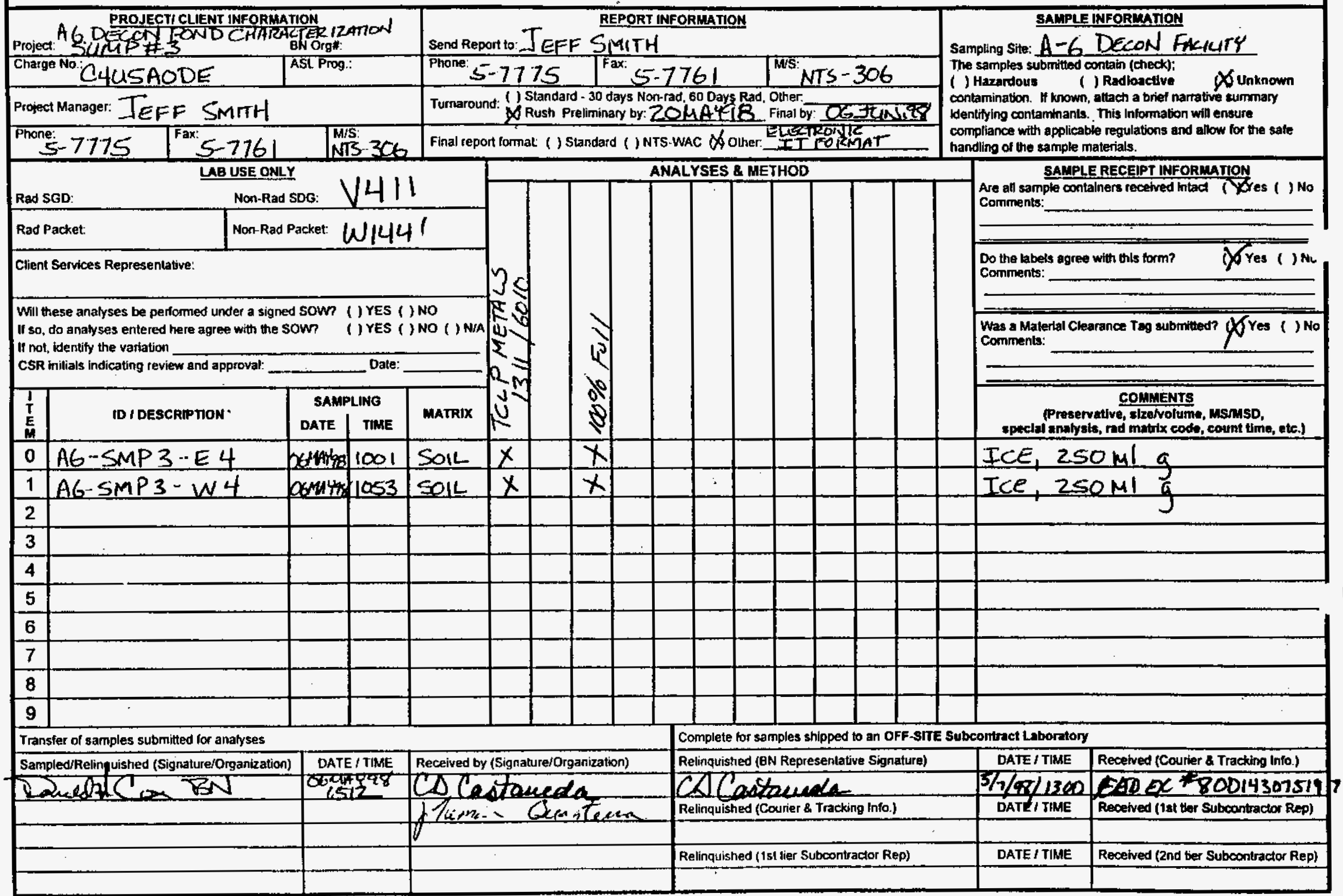




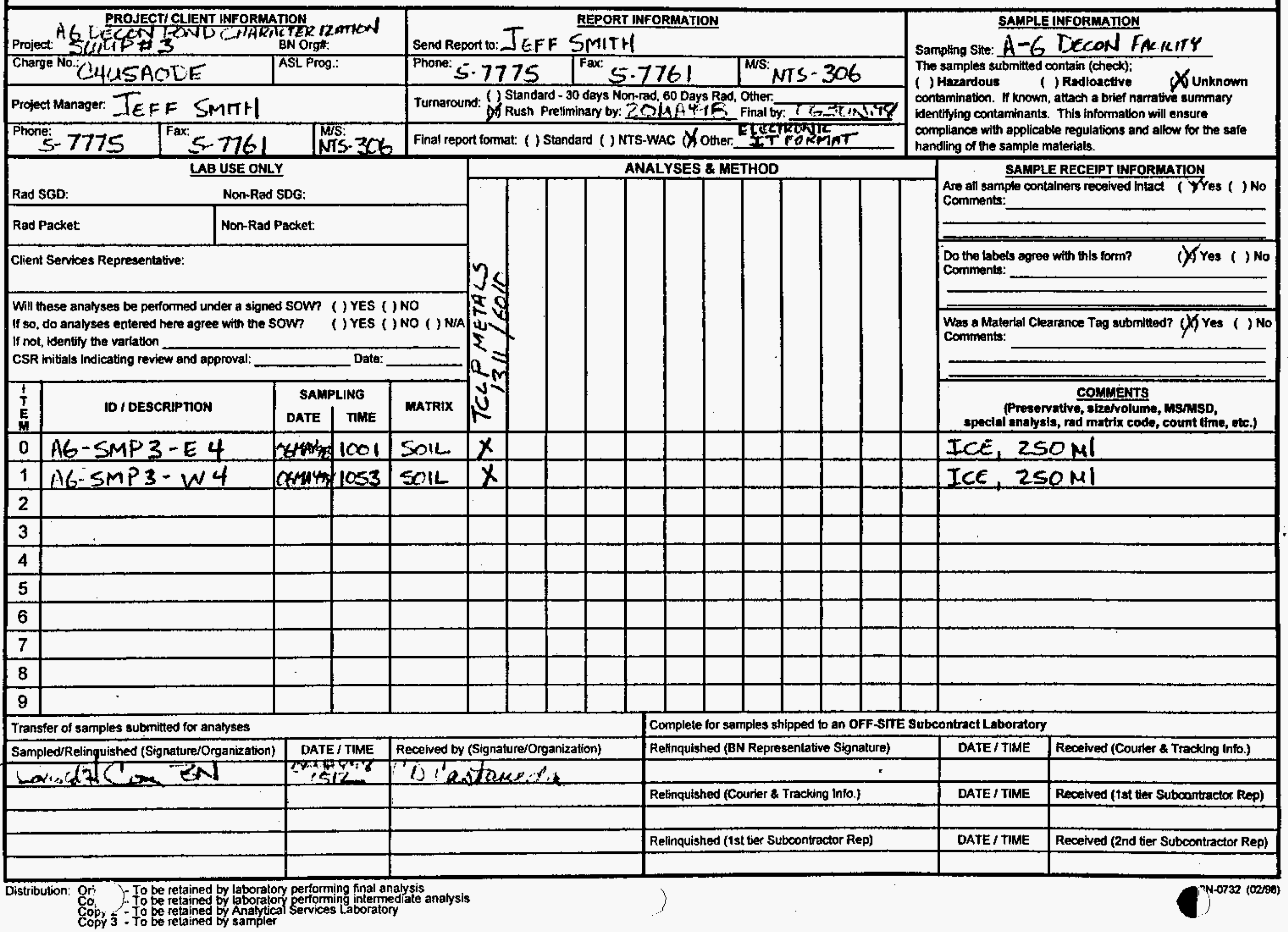


$c$

VOLATILE

ORGANICS

DATA 
NEL LABORATORIES

CLIENT: Bechtel Nevada

PROJECT NAME: V427

JECT NUMBER: 17777
CLIENT ID: $\quad$ Decon W1-1 TB

DATE SAMPLED: $6 / 1 / 98$

NEL SAMPLE ID: L9806040-01

TEST: Volatile Organic Compounds by EPA SW846 Method 8260B, Dec. 1996

MATRIX: Aqueous $\quad$ EXTRACTED: $6 / 4 / 98$ DILUTION: 1

ANALYZED: $6 / 4 / 98$

ANALYST: Suzanne

\begin{tabular}{|c|c|c|c|c|c|}
\hline PARAMETER & $\begin{array}{l}\text { Result } \\
\mu \mathrm{g} / \mathrm{L}\end{array}$ & $\begin{array}{c}\text { Reporting } \\
\text { Limit } \\
\end{array}$ & PARAMETER & $\begin{array}{l}\text { Result } \\
\mu \mathrm{g} / \mathrm{L}\end{array}$ & $\begin{array}{c}\text { Reporting } \\
\text { Limit } \\
\end{array}$ \\
\hline Acetone & $\overline{\mathrm{ND}}$ & $25 . \mu \mathrm{g} / \mathrm{L}$ & & & \\
\hline Benzene & $\mathrm{ND}$ & 5. $\mu g / L$ & & & \\
\hline Bromodichloromethane & ND & 5. $\mu g / L$ & & & \\
\hline Bromofomm & ND & 5. $\mu g / L$ & & & \\
\hline Bromomethane & ND & 5. $\mu \mathrm{g} / \mathrm{L}$ & & & \\
\hline 2-Butanone & ND & 25. $\mu \mathrm{g} / \mathrm{L}$ & & & \\
\hline Carbon disulfide & $\mathrm{ND}$ & 5. $\mu g / L$ & & & \\
\hline Carbon tetrachloride & ND & 5. $\mu g / L$ & & & \\
\hline Chlorobenzene & ND & 5. $\mu \mathrm{g} / \mathrm{L}$ & & & \\
\hline Chloroethane & ND & 5. $\mu \mathrm{g} / \mathrm{L}$ & & & \\
\hline Chloroform & ND & 5. $\mu g / L$ & & & \\
\hline Chloromethane & ND & 5. $\mu g / L$ & & & \\
\hline Dibromochloromethane & ND & 5. $\mu g / L$ & & & \\
\hline 1,1-Dichloroethane (1,1-DCA) & ND & 5. $\mu \mathrm{g} / \mathrm{L}$ & & & \\
\hline 1,2-Dichloroethane (1,2-DCA) & ND & 5. $\mu g / \mathrm{L}$ & & & \\
\hline 1,1-Dichloroethene (1,1-DCE) & ND & 5. $\mu \mathrm{g} / \mathrm{L}$ & & & \\
\hline cis-1,2-Dichloroethene & ND & 5. $\mu \mathrm{g} / \mathrm{L}$ & & & $\cdot$ \\
\hline 1,2-Dichloroethene & ND & 5. $\mu g / L$ & & & \\
\hline 1)-oichloropropane & ND & 5. $\mu \mathrm{g} / \mathrm{L}$ & & & \\
\hline cis-1,3-Dichioropropene & ND & 5. $\mu \mathrm{g} / \mathrm{L}$ & & & \\
\hline trans-1,3-Dichloropropene & ND & 5. $\mu \mathrm{g} / \mathrm{L}$ & & & \\
\hline Ethylbenzene & ND & 5. $\mu g / L$ & & & \\
\hline 2-Hexanone & ND & 25. $\mu \mathrm{g} / \mathrm{L}$ & & & \\
\hline Methylene chloride (Dichioromethane) & ND & 5. $\mu \mathrm{g} / \mathrm{L}$ & & & \\
\hline 4-Methyl-2-pentanone & ND & 25. $\mu g / L$ & & & \\
\hline Styrene & ND & 5. $\mu g / L$ & & & \\
\hline 1, 1,2,2-Tetrachloroethane & ND & 5. $\mu g / \mathrm{L}$ & & & \\
\hline Tetrachloroethene (PCE) & $\mathrm{ND}$ & 5. $\mu g / L$ & & & \\
\hline Toluene & ND & 5. $\mu g / L$ & & & \\
\hline t.1,1-Trichloroethane (1,1,1-TCA) & ND & 5. $\mu \mathrm{g} / \mathrm{L}$ & & & \\
\hline 1,1,2-Trichioroethane (1,1,2-TCA) & ND & 5. $\mu \mathrm{g} / \mathrm{L}$ & & & \\
\hline Trichloroethene (TCE) & $\mathrm{ND}$ & 5. $\mu \mathrm{g} / \mathrm{L}$ & & & \\
\hline Vinyl chioride & ND & 5. $\mu g / \mathrm{L}$ & & & \\
\hline o-Xylene & ND & 5. $\mu \mathrm{g} / \mathrm{L}$ & & & \\
\hline $\mathrm{m}, \mathrm{p}$-Xyiene & ND & 5. $\mu g / L$ & & & \\
\hline
\end{tabular}

ND - Not Detected

QUALITY CONTROL DATA:

Surrogate

4-Bromofluorabenzene

Dibromofluoromethane

Te ne-d8

\section{\% Recovery}

103

104

105
Acceptable Range

$70-130$

$70-130$

$70-130$ 
NEL LABORATORIES

CLIENT: Bechtel Nevada

PROJECT NAME: V427

PROIECT NUMBER: 17777
CLIENT ID: Decon W1-1 A\&B

DATE SAMPLED: $6 / 1 / 98$

NEL SAMPLE ID: $\quad$ L9806040-02

TEST: Volatile Organic Compounds by EPA SW846 Method 8260B, Dec. 1996
MATRLX: Solid
EXTRACTED: $6 / 4 / 98$
ANALYST: Suzanne
DILUTION; 1
ANALYZED: $6 / 4 / 98$

\begin{tabular}{|c|c|c|c|c|c|c|}
\hline PARAMETER & $\begin{array}{l}\text { Result } \\
\mu \mathrm{g} / \mathrm{kg}\end{array}$ & & $\begin{array}{c}\text { Reporting } \\
\text { Limit }\end{array}$ & PARAMETER & $\begin{array}{l}\text { Result } \\
\mu \mathrm{g} / \mathrm{kg}\end{array}$ & $\begin{array}{c}\text { Reporting } \\
\text { Limit }\end{array}$ \\
\hline Acetone & $\overline{\mathrm{ND}}$ & & $25 . \mu g / \mathrm{kg}$ & & & \\
\hline Benzene & ND & Js & 5. $\mu g / \mathrm{kg}$ & & & \\
\hline Bromodichloromethane & $\mathrm{ND}$ & Is & 5. $11 \mathrm{~g} / \mathrm{kg}$ & & & \\
\hline Bromoform & ND & Is & 5. $11 \mathrm{~g} / \mathrm{kg}$ & & & \\
\hline Bromomethane & ND & & 5. $4 \mathrm{gg} / \mathrm{kg}$ & & & \\
\hline 2-Butanone & ND & & 25. $\mu \mathrm{g} / \mathrm{kg}$ & & & \\
\hline Carbon disulfide & ND & & 5. $\mu g / k g$ & & & - \\
\hline Carbon tetrachloride & ND & & 5. $\mu \mathrm{g} / \mathrm{kg}$ & & & \\
\hline Chlorobenzene & 6 & Js & 5. $\mu \mathrm{g} / \mathrm{kg}$ & & & \\
\hline Chloroethane & ND & & 5. $\mu \mathrm{g} / \mathrm{kg}$ & & & \\
\hline Chloroform & ND & & 5. $\mu g / \mathrm{kg}$ & & & \\
\hline Chloromethane & ND & & 5. $\mu g / \mathrm{kg}$ & & & \\
\hline Dibromochloromethane & ND & Js & 5. $\mu \mathrm{g} / \mathrm{kg}$ & & & \\
\hline 1,1-Dichloroethane (1,1-DCA) & ND & & 5. $\mu g / k g$ & & & \\
\hline 1,2-Dichloroethane (1,2-DCA) & $\mathrm{ND}$ & Js & 5. $\mu \mathrm{g} / \mathrm{kg}$ & & & \\
\hline 1,1-Dichloroethene (1,1-DCE) & ND & & 5. $\mu g / \mathrm{kg}$ & & & \\
\hline cis-1,2-Dichloroethene & $\mathrm{ND}$ & & 5. $\mu \mathrm{g} / \mathrm{kg}$ & & & - \\
\hline trans-1,2-Dichloroethene & ND & & 5. $\mu g / \mathrm{kg}$ & & & \\
\hline 1,2-Dichioropropane & ND & Js & 5. $\mu \mathrm{g} / \mathrm{kg}$ & & & \\
\hline cis-1,3-Dichloropropene & ND & Js & 5. $\mu g / \mathrm{kg}$ & & & \\
\hline trans-1,3-Dichloropropene & ND & Js & 5. $\mu g / \mathrm{kg}$ & & & \\
\hline Ethylbenzene & ND & Js & 5. $\mu g / \mathrm{kg}$ & & & \\
\hline 2-Hexanone & ND & Js & 25. $\mu g / \mathrm{kg}$ & & & \\
\hline Methylene chioride (Dichloromethane) & ND & & 5. $\mu \mathrm{g} / \mathrm{kg}$ & & & \\
\hline 4-Methy]-2-pentanone & ND & Js & 25. $\mu \mathrm{g} / \mathrm{kg}$ & & & \\
\hline Styrene & ND & Is & 5. $\mu \mathrm{g} / \mathrm{kg}$ & & & \\
\hline 1,1,2,2-Tetrachloroethane & ND & Is & 5. $\mu g / \mathrm{kg}$ & & & \\
\hline Tetrachloroethene (PCE) & ND & Js & 5. $\mu \mathrm{g} / \mathrm{kg}$ & & & \\
\hline Toluene & $\mathrm{ND}$ & Js & 5. $\mu g / \mathrm{kg}$ & & & \\
\hline 1,1,1-Trichloroethane $(1,1,1-\mathrm{TCA})$ & ND & & 5. $\mu \mathrm{gg} / \mathrm{kg}$ & & & \\
\hline 1,1,2-Trichloroethane $(1,1,2-$ TCA $)$ & $\mathrm{ND}$ & Js & 5. $\mu g / \mathrm{kg}$ & & & \\
\hline Trichloroethene (TCE) & $\mathrm{ND}$ & Js & 5. $\mu g / \mathrm{kg}$ & & & \\
\hline Vinyl chloride & ND & Js & 5. $\mu g / \mathrm{kg}$ & & & . \\
\hline o-Xyiene & ND & js & 5. $\mu \mathrm{g} / \mathrm{kg}$ & & & \\
\hline $\mathrm{m}, \mathrm{p}$-Xylene & ND & Js & 5. $\mu \mathrm{g} / \mathrm{kg}$ & & & \\
\hline
\end{tabular}

ND - Not Detected

\section{QUALITY CONTROL DATA:}

\section{Surrogate}

4-Bromofluorobenzene

Dibromofluoromethane

Toluene-d8
\% Recovery

67

110

80
Acceptable Range

$$
\begin{aligned}
& 74-121 \\
& 80-120 \\
& 81-117
\end{aligned}
$$


CLIENT: Bechtel Nevada

PPOJECT NAME: V427

\JECT NUMBER: 17777
CLIENT ID: Decon P1-1 A\&B

DATE SAMPLED: $6 / 1 / 98$

NEL SAMPLE ID: L9806040 -07

TEST: Volatile Organic Compounds by EPA SW846 Method 8260B, Dec. 1996

\section{MATRIX: Solid} DILUTION: 1
EXTRACTED: $6 / 4 / 98$

ANALYZED: $6 / 4 / 98$

ANALYST: Suzanne

\begin{tabular}{|c|c|c|c|c|c|}
\hline PARAMETER & $\begin{array}{l}\text { Result } \\
\mu \mathrm{g} / \mathrm{kg}\end{array}$ & $\begin{array}{c}\text { Reporting } \\
\text { Limit }\end{array}$ & PARAMETER & $\begin{array}{l}\text { Result } \\
\mu \mathrm{g} / \mathrm{kg}\end{array}$ & $\begin{array}{c}\text { Reporting } \\
\text { Limit }\end{array}$ \\
\hline$\overline{\text { Acetone }}$ & $\overline{\mathrm{ND}}$ & 25. $\mu \mathrm{g} / \mathrm{kg}$ & & & \\
\hline Benzene & ND & 5. $\mu \mathrm{g} / \mathrm{kg}$ & & & . \\
\hline Bromodichloromethane & $\mathrm{ND}$ & 5. $\mu g / \mathrm{kg}$ & & & \\
\hline Bromoform & $\mathrm{ND}$ & 5. $\mu g / \mathrm{kg}$ & & & \\
\hline Bromomethane & ND & 5. $\mu g / \mathrm{kg}$ & & & \\
\hline 2-Butanone & ND & 25. $\mu \mathrm{g} / \mathrm{kg}$ & & & \\
\hline Carbon disulfide & ND & 5. $\mu \mathrm{gg} / \mathrm{kg}$ & & & \\
\hline Carbon tetrachloride & ND & 5. $\mu g / \mathrm{kg}$ & & & \\
\hline Chlorobenzene & ND & 5. $\mu \mathrm{g} / \mathrm{kg}$ & & & \\
\hline Chloroethane & $\mathrm{ND}$ & 5. $\mu g / \mathrm{kg}$ & & & \\
\hline Chloroform & ND & 5. $\mu g / k g$ & & & \\
\hline Chloromethane & ND & 5. $\mu g / \mathrm{kg}$ & & & \\
\hline Dibromochloromethane & ND & 5. $\mu g / \mathrm{kg}$ & & & \\
\hline 1,1-Dichloroethane (1,1-DCA) & ND & 5. $\mu g / \mathrm{kg}$ & & & \\
\hline 1.2-Dichloroethane (1,2-DCA) & $\mathrm{ND}$ & 5. $\mu g / \mathrm{kg}$ & & & \\
\hline 1.1-Dichloroethene (1,1-DCE) & ND & 5. $\mu \mathrm{g} / \mathrm{kg}$ & & & \\
\hline cis-1,2-Dichloroecthene & ND & 5. $\mu g / \mathrm{kg}$ & & & - \\
\hline tI 1,2-Dichloroethene & $\mathrm{ND}$ & 5. $\mu \mathrm{g} / \mathrm{kg}$ & & & \\
\hline 1. ofichloropropane & $\mathrm{ND}$ & 5. $\mu \mathrm{g} / \mathrm{kg}$ & & & \\
\hline cis-1,3-Dichloropropene & ND & 5. $\mu g / \mathrm{kg}$ & & & \\
\hline trans-I,3-Dichloropropene & $\mathrm{ND}$ & 5. $\mu g / \mathrm{kg}$ & & & \\
\hline Ethylbenzene & ND & 5. $\mu \mathrm{g} / \mathrm{kg}$ & & & \\
\hline 2-Hexanone & $\mathrm{ND}$ & 25. $\mathrm{Hg} / \mathrm{kg}$ & & & \\
\hline Methylene chloride (Dichloromethane) & ND & 5. $\mu \mathrm{g} / \mathrm{kg}$ & & & \\
\hline 4-Methyl-2-pentanone & $\mathrm{ND}$ & 25. $\mu \mathrm{g} / \mathrm{kg}$ & & & \\
\hline Styrene & ND & 5. $\mu \mathrm{g} / \mathrm{kg}$ & & & \\
\hline 1,1,2,2-Tetrachlotoethane & ND & 5. $\mu \mathrm{g} / \mathrm{kg}$ & & & \\
\hline Tetrachloroethene (PCE) & ND & 5. $\mu \mathrm{g} / \mathrm{kg}$ & & & \\
\hline Toluene & ND & 5. $\mu \mathrm{g} / \mathrm{kg}$ & & & \\
\hline 1,1,1-Trichloroethane (1,1,1-TCA) & ND & 5. $\mu \mathrm{g} / \mathrm{kg}$ & & & \\
\hline 1,1,2-Trichloroethane $(1,1,2-\mathrm{TCA})$ & ND & 5. $\mu g / \mathrm{kg}$ & & & \\
\hline Trichloroethene (TCE) & ND & 5. $\mu g / \mathrm{kg}$ & & & \\
\hline Vinyi chloride & ND & 5. $\mu \mathrm{g} / \mathrm{kg}$ & & & \\
\hline o-Xylene & $\mathrm{ND}$ & 5. $\mu \mathrm{g} / \mathrm{kg}$ & & & \\
\hline m,p-Xylene & $\mathrm{ND}$ & 5. $\mu \mathrm{g} / \mathrm{kg}$ & & & \\
\hline
\end{tabular}

ND - Not Detected

QUALITY CONTROL DATA:

\section{Surrogate}

4-Bromofluorobenzene

Dibromofluoromethane

Te: e-d8

\section{$\%$ Recovery}

96

85

97

\section{Acceptable Range}

$74-121$

. $80-120$

$81-117$

This report shall not be reproduced except in full, without the written approval of the laboratory. 
NEL LABORATORIES

CLIENT: Bechtel Nevada

PROJECT NAME: V427

PROJECT NUMBER: 17777
CLIENT ID: Decon P2-1 A\&B

DATE SAMPLED: $6 / 1 / 98$

NEL SAMPLE ID: L9806040-12

TEST: Volatile Organic Compounds by EPA SW846 Method 8260B, Dec. 1996

MATRIX: Solid

DILUTION: 1
EXTRACTED: 6/4/98

ANALYZED: $6 / 4 / 98$

ANALYST: Suzanne

\begin{tabular}{|c|c|c|c|c|c|}
\hline PARAMETER & $\begin{array}{l}\text { Result } \\
\mu \mathrm{g} / \mathrm{kg}\end{array}$ & $\begin{array}{c}\text { Reporting } \\
\text { Limit } \\
\end{array}$ & PARAMETER & $\begin{array}{l}\text { Result } \\
\mu \mathrm{g} / \mathrm{kg}\end{array}$ & $\begin{array}{c}\text { Reporting } \\
\text { Limit } \\
\end{array}$ \\
\hline$\overline{\text { Acetone }}$ & $\overline{\mathrm{ND}}$ & $\overline{25 .} \mu \mathrm{g} / \mathrm{kg}$ & & & \\
\hline Benzene & ND & 5. $\mu g / \mathrm{kg}$ & & & \\
\hline Bromodichloromethane & ND & 5. $\mu \mathrm{g} / \mathrm{kg}$ & & & \\
\hline Bromoform & ND & 5. $\mu \mathrm{g} / \mathrm{kg}$ & & & \\
\hline Bromomethane & ND & 5. $\mu \mathrm{g} / \mathrm{kg}$ & & & \\
\hline 2-Butanone & ND & 25. $\mu g / \mathrm{kg}$ & & & \\
\hline Carbon disulfide & ND & 5. $\mu \mathrm{g} / \mathrm{kg}$ & & & \\
\hline Carbon tetrachioride & $\mathrm{ND}$ & 5. $\mu \mathrm{g} / \mathrm{kg}$ & & & \\
\hline Chlorobenzene & $\mathrm{ND}$ & 5. $\mu g / \mathrm{kg}$ & & & \\
\hline Chloroethane & ND & 5. $\mu \mathrm{g} / \mathrm{kg}$ & & & \\
\hline Chloroform & ND & 5. $\mu \mathrm{g} / \mathrm{kg}$ & & & \\
\hline Chloromethane & ND & 5. $\mu \mathrm{g} / \mathrm{kg}$ & & & \\
\hline Dibromochloromethane & ND & 5. $\mu g / \mathrm{kg}$ & & & \\
\hline 1,1-Dichloroethane (1,1-DCA) & $\mathrm{ND}$ & 5. $\mu g / \mathrm{kg}$ & & & \\
\hline 1,2-Dichloroethane (1,2-DCA) & $\mathrm{ND}$ & 5. $\mu \mathrm{g} / \mathrm{kg}$ & & & \\
\hline 1,1-Dichloroethene (1,1-DCE) & $\mathrm{ND}$ & 5. $\mu g / \mathrm{kg}$ & & & \\
\hline cis-1,2-Dichloroethene & ND & 5. $\mu \mathrm{g} / \mathrm{kg}$ & & & - \\
\hline trans-1,2-Dichioroethene & ND & 5. $\mu \mathrm{g} / \mathrm{kg}$ & & & \\
\hline 1,2-Dichloropropane & ND & 5. $\mu \mathrm{g} / \mathrm{kg}$ & & & \\
\hline cis-1,3-Dichloropropene & ND & 5. $\mu g / \mathrm{kg}$ & & & \\
\hline trans-1,3-Dichloropropene & ND & 5. $\mu g / \mathrm{kg}$ & & & \\
\hline Ethylbenzene & ND & 5. $\mu \mathrm{g} / \mathrm{kg}$ & & & \\
\hline 2-Hexanone & ND & 25. $\mu \mathrm{g} / \mathrm{kg}$ & & & \\
\hline Methylene chioride (Dichloromethane) & ND & 5. $\mu \mathrm{g} / \mathrm{kg}$ & & & \\
\hline 4-Methyl-2-pentanone & ND & 25. $\mu \mathrm{g} / \mathrm{kg}$ & & & \\
\hline Styrene & ND & 5. $\mu \mathrm{g} / \mathrm{kg}$ & & & \\
\hline 1,1,2,2-Tetrachloroethane & ND & 5. $\mu g / \mathrm{kg}$ & & & \\
\hline Tetrachloroethene (PCE) & ND & 5. $\mu \mathrm{g} / \mathrm{kg}$ & & & \\
\hline Toluene & ND & 5. $\mu g / \mathrm{kg}$ & & & \\
\hline 1,1,1-Trichloroethane (1,1,1-TCA) & $\mathrm{ND}$ & 5. $\mu g / \mathrm{kg}$ & & & \\
\hline $\mathrm{I}, 1,2-$ Trichloroethane $(\mathrm{I}, 1,2-\mathrm{TCA})$ & ND & 5. $\mu g / \mathrm{kg}$ & & & \\
\hline Trichloroethene (TCE) & $\mathrm{ND}$ & 5. $\mu \mathrm{g} / \mathrm{kg}$ & & & \\
\hline Vinyl chlonide & ND & 5. $\mu g / \mathrm{kg}$ & & & \\
\hline o-Xyiene & $\mathrm{ND}$ & 5. $\mu \mathrm{g} / \mathrm{kg}$ & & & \\
\hline m,p-Xylene & $\mathrm{ND}$ & 5. $\mu g / \mathrm{kg}$ & & & \\
\hline
\end{tabular}

ND - Not Detected

QUALITY CONTROL DATA:

Surrogate

4-Bromofluorobenzene

Dibromofluoromethane

Toluene-d8

\section{\% Recovery}

100

94

101
Acceptable Range

$74-121$

$80-120$

$81-117$

This report shall not be reproduced except in full, without the written approval of the laboratory. 
CLIENT: Bechtel Nevada

PROJECT NAME: V427

JJECT NUMBER: 17777
CLIENT ID: Method Blank

DATE SAMPLED: NA

NEL SAMPLE ID: 9806042W60-BLK

TEST: $\quad$ Volatile Organic Compounds by EPA SW846 Method 8260B, Dec. 1996

MATRIX: Aqueous

EXTRACTED: $\quad 6 / 4 / 98$

ANALYZED: $\quad 6 / 4 / 98$

\begin{tabular}{|c|c|c|c|c|c|}
\hline PARAMETER & $\begin{array}{l}\text { Result } \\
\mu \mathrm{g} / \mathrm{L}\end{array}$ & $\begin{array}{c}\text { Reporting } \\
\text { Limit }\end{array}$ & PARAMETER & $\begin{array}{l}\text { Result } \\
\mu \mathrm{g} / \mathrm{L}\end{array}$ & $\begin{array}{c}\text { Reporting } \\
\text { Limit }\end{array}$ \\
\hline$\overline{\text { Agetone }}$ & ND & $25 \mu \mathrm{g} / \mathrm{L}$ & & & \\
\hline Benzene & ND & $5 \mu \mathrm{g} / \mathrm{L}$ & & & \\
\hline Bromodichloromethane & ND & $5 \mu \mathrm{g} / \mathrm{L}$ & & & \\
\hline Bromoform & ND & $5 \mu \mathrm{g} / \mathrm{L}$ & & & \\
\hline Bromomethane & ND & $5 \mu g / L$ & & & \\
\hline 2-Butanone & ND & $25 \mu g / L$ & & & \\
\hline Carbon disulfide & ND & $5 \mu \mathrm{g} / \mathrm{L}$ & & & \\
\hline Carbon tetrachloride & ND & $5 \mu \mathrm{g} / \mathrm{L}$ & & & \\
\hline Chlorobenzene & ND & $5 \mu \mathrm{g} / \mathrm{L}$ & & & \\
\hline Chloroethane & ND & $5 \mu \mathrm{g} / \mathrm{L}$ & & & \\
\hline Chloroform & ND & $5 \mu g / L$ & & & \\
\hline Chloromethane & ND & $5 \mu \mathrm{g} / \mathrm{L}$ & & & \\
\hline Dibromochloromethane & ND & $5 \mu \mathrm{g} / \mathrm{L}$ & & & \\
\hline 1,1-Dichloroethane (1,1-DCA) & ND & $5 \mu \mathrm{g} / \mathrm{L}$ & & & \\
\hline 1,2-Dichloroethane (1,2-DCA) & ND & $5 \mu \mathrm{g} / \mathrm{L}$ & & & \\
\hline 1,1-Dichloroethene (1,1-DCE) & ND & $5 \mu g / L$ & & & \\
\hline cis-1,2-Dichloroethene & ND & $5 \mu \mathrm{g} / \mathrm{L}$ & & & - \\
\hline trngs-1,2-Dichloroethene & ND & $5 \mu g / L$ & & & \\
\hline Sichloropropane & ND & $5 \mu \mathrm{g} / \mathrm{L}$ & & & \\
\hline cis-1,3-Dichloropropene & ND & $5 \mu \mathrm{g} / \mathrm{L}$ & & & \\
\hline trans-1,3-Dichloropropene & ND & $5 \mu \mathrm{g} / \mathrm{L}$ & & & \\
\hline Ethylbenzene & ND & $5 \mu \mathrm{g} / \mathrm{L}$ & & & \\
\hline 2-Hexanone & $\mathrm{ND}$ & $25 \mu \mathrm{g} / \mathrm{L}$ & & & \\
\hline Methylene chioride (Dichloromethane & ND & $5 \mu g / L$ & & & \\
\hline 4-Methyl-2-pentanone & ND & $25 \mu \mathrm{g} / \mathrm{L}$ & & & \\
\hline Styrene & ND & $5 \mu \mathrm{g} / \mathrm{L}$ & & & \\
\hline 1,1,2,2-Tetrachloroethane & ND & $5 \mu \mathrm{g} / \mathrm{L}$ & & & \\
\hline Tetrachloroethene (PCE) & ND & $5 \mu \mathrm{g} / \mathrm{L}$ & & & \\
\hline Toluene & ND & $5 \mu \mathrm{g} / \mathrm{L}$ & & & \\
\hline 1,1,1-Trichioroethane $(1,1,1-\mathrm{TCA})$ & $\mathrm{ND}$ & $5 \mu \mathrm{g} / \mathrm{L}$ & & & \\
\hline 1,1,2-Trichloroethane $(1,1,2-T C A)$ & $\mathrm{ND}$ & $5 \mu g / L$ & & & \\
\hline Trichloroethene (TCE) & ND & $5 \mu \mathrm{g} / \mathrm{L}$ & & & \\
\hline Vinyl chloride & ND & $5 \mu \mathrm{g} / \mathrm{L}$ & & & \\
\hline o-Xylene & ND & $5 \mu \mathrm{g} / \mathrm{L}$ & & & \\
\hline $\mathrm{m}, \mathrm{p}$-Xyiene & $\mathrm{ND}$ & $5 \mu \mathrm{g} / \mathrm{L}$ & & & \\
\hline
\end{tabular}

ND - Not Detected

QUALITY CONTROL DATA:

\section{Surrogate}

4-Bromofluorobenzene

Dibromofiuoromethane

Toluene-d8
\% Recovery

95

101

102
Acceptable Range

$70-130$

$70-130$

$70-130$

This report shall not be reproduced except in full, without the written approval of the laboratory. 
CLIENT: Bechtel Nevada

PROJECT NAME: $\quad$ V427

PROJECT NUMBER: 17777
CLIENT ID: Method Blank

DATE SAMPLED: NA

NEL SAMPLE ID: 980604 S160B-BLK

TEST: $\quad$ Volatile Organic Compounds by EPA SW846 Method 8260B, Dec 1996

MATRLX: Solid

EXTRACTED: $\quad 6 / 4 / 98$

ANALYZED: $\quad 6 / 4 / 98$

\begin{tabular}{|c|c|c|c|c|c|}
\hline PARAMETER & $\begin{array}{l}\text { Result } \\
\mu \mathrm{g} / \mathrm{kg}\end{array}$ & $\begin{array}{c}\text { Reporting } \\
\text { Limit } \\
\end{array}$ & PARAMETER & $\begin{array}{l}\text { Result } \\
\mu \mathrm{g} / \mathrm{kg}\end{array}$ & $\begin{array}{c}\text { Reporting } \\
\text { Limit }\end{array}$ \\
\hline Acetone & ND & $25 \mu \mathrm{g} / \mathrm{kg}$ & & & \\
\hline Benzene & ND & $5 \mu g / k g$ & & & \\
\hline Bromodichioromethane & ND & $5 \mu g / \mathrm{kg}$ & & & \\
\hline Bromoform & ND & $5 \mu g / \mathrm{kg}$ & & & \\
\hline Bromomethane & ND & 5 मg/kg & & & \\
\hline 2-Butanone & ND & $25 \mu \mathrm{g} / \mathrm{kg}$ & & & \\
\hline Carbon disulfide & ND & $5 \mu g / \mathrm{kg}$ & & & \\
\hline Carbon tetrachloride & ND & $5 \mu \mathrm{g} / \mathrm{kg}$ & & & \\
\hline Chlorobenzene & ND & $5 \mu \mathrm{g} / \mathrm{kg}$ & & & \\
\hline Chloroethane & ND & $5 \mu g / k g$ & & & \\
\hline Chloroform & ND & $5 \mu \mathrm{g} / \mathrm{kg}$ & & & \\
\hline Chloromethane & ND & $5 \mu \mathrm{g} / \mathrm{kg}$ & & & \\
\hline Dibromochloromethane & $\mathrm{ND}$ & $5 \mu \mathrm{g} / \mathrm{kg}$ & & & \\
\hline 1,1-Dichloroethane (1,1-DCA) & ND & $5 \mu \mathrm{g} / \mathrm{kg}$ & & & \\
\hline 1,2-Dichloroethane (1,2-DCA) & $\mathrm{ND}$ & $5 \mu \mathrm{g} / \mathrm{kg}$ & & & \\
\hline 1,1-Dichloroethene (1,1-DCE) & ND & $5 \mu g / k g$ & & & \\
\hline cis-1,2-Dichloroethene & ND & $5 \mu \mathrm{g} / \mathrm{kg}$ & & & - \\
\hline trans-1,2-Dichloroethene & ND & $5 \mu \mathrm{g} / \mathrm{kg}$ & & & \\
\hline 1.2-Dichioropropane & ND & $5 \mu \mathrm{g} / \mathrm{kg}$ & & & \\
\hline cis-1,3-Dichloropropene & ND & $5 \mu g / k g$ & & & \\
\hline trans-1,3-Dichloropropene & ND & $5 \mu g / \mathrm{kg}$ & & & \\
\hline Ethylbenzene & ND & $5 \mu \mathrm{g} / \mathrm{kg}$ & & & \\
\hline 2-Hexanone & ND & $25 \mu g / k g$ & & & \\
\hline Methylene chloride (Dichloromethane & ND & $5 \mu g / \mathrm{kg}$ & & & \\
\hline 4-Methyl-2-pentanone & ND & $25 \mu \mathrm{g} / \mathrm{kg}$ & & & \\
\hline Styrene & $\mathrm{ND}$ & $5 \mu \mathrm{g} / \mathrm{kg}$ & & & \\
\hline 1.1,2,2-Tetrachloroethane & ND & $5 \mu \mathrm{g} / \mathrm{kg}$ & & & \\
\hline Tetrachloroethene (PCE) & ND & $S_{11 \mathrm{~g} / \mathrm{kg}}$ & & & \\
\hline Toluene & ND & $5 \mu \mathrm{g} / \mathrm{kg}$ & & & \\
\hline $1,1,1$-Trichloroethane $(1,1,1-\mathrm{TCA})$ & $\mathrm{ND}$ & $5 \mu g / \mathrm{kg}$ & & & \\
\hline 1,1,2-Trichloroethane $(1,1,2-\mathrm{TCA})$ & ND & $5 \mu \mathrm{g} / \mathrm{kg}$ & & & \\
\hline Trichloroethene (TCE) & ND & $5 \mu \mathrm{g} / \mathrm{kg}$ & & & \\
\hline Vinyl chioride & ND & $5 \mu \mathrm{g} / \mathrm{kg}$ & & & \\
\hline 0 -Xylene & $\mathrm{ND}$ & $5 \mu \mathrm{g} / \mathrm{kg}$ & & & \\
\hline m.p-xylene & ND & $5 \mu \mathrm{g} / \mathrm{kg}$ & & & \\
\hline
\end{tabular}

ND - Not Detected

OUALITY CONTROL DATA:

Surrogate

4-Bromofluorobenzene

Dibromofluoromethane

Toluene-d8
\% Recovery

100

93

91
Acceptable Range

$74-121$

$80-120$

$81-117$

This report shall not be reproduced except in full, without the written approval of the laboratory. 
- 1.

$C$

SEMI-VOLATILE

ORGANICS

DATA 
CLIENT: Bechtel Nevada

PROJECT NAME: V427

JJECT NUMBER: 17777
CLIENT ID: Decon W1-2

DATE SAMPLED: $6 / 1 / 98$

NEL SAMPLE ID: L9806040-03

TEST: Semi-Volatile Organic Compounds by EPA 8270C, Dec. 1996

MATRIX: Solid

DILUTION: 10
EXTRACTED: $6 / 8 / 98$

ANALYZED: $6 / 9 / 98$

ANALYST: JDG

\begin{tabular}{|c|c|c|c|c|c|}
\hline PARAMETER & $\begin{array}{l}\text { Result } \\
\mu \mathrm{g} / \mathrm{Kg}\end{array}$ & $\begin{array}{c}\text { Reporting } \\
\text { Limit }\end{array}$ & PARAMETER & $\begin{array}{l}\text { Result } \\
\boldsymbol{\mu g} / \mathbf{K g}\end{array}$ & $\begin{array}{c}\text { Reporting } \\
\text { Limit }\end{array}$ \\
\hline Acenaphthene & $\mathrm{ND}$ & 5000. $\mu \mathrm{g} / \mathrm{Kg}$ & 4,6-Dinitro-2-methyl phenol & $N D^{-}$ & $\overline{25000 . \mu \mathrm{g} / \mathrm{Kg}}$ \\
\hline Acenaphthylene & ND & 5000. $\mu g / \mathrm{Kg}$ & 2,4-Dinitrotoluene (DNT) & ND & 5000. $\mu g / \mathrm{Kg}$ \\
\hline Anthracene & $\mathrm{ND}$ & $5000 . \mu \mathrm{g} / \mathrm{Kg}$ & 2,6-Dinitrotoluene (DNT) & ND & 5000. $\mu \mathrm{g} / \mathrm{Kg}$ \\
\hline Benzo (a) anthracene & ND & $5000 . \mu \mathrm{g} / \mathrm{Kg}$ & 2,4-Dinitrophenol & ND & $25000 . \mu \mathrm{g} / \mathrm{Kg}$ \\
\hline Benzo (b\&k) fluoranthene & $\mathrm{ND}$ & $5000 . \mu g / \mathrm{Kg}$ & Di-n-octyl phthalate & ND & $5000 . \mu g / \mathrm{Kg}$ \\
\hline Benzo (b) fluoranthene & ND & 500. $\mu \mathrm{g} / \mathrm{kg}$ & Fluoranthene & ND & $5000 . \mu \mathrm{g} / \mathrm{Kg}$ \\
\hline Benzo (k) fluoranthene & ND & 500. $\mu g / \mathrm{kg}$ & Fiuorene & ND & $5000 . \mu \mathrm{g} / \mathrm{Kg}$ \\
\hline Benzoic Acid & ND & 2500. $\mu \mathrm{g} / \mathrm{kg}$ & Hexachlorobenzene & ND & 5000. $\mu \mathrm{g} / \mathrm{Kg}$ \\
\hline Benzo $(\mathrm{g}, \mathrm{h}, \mathrm{i})$ perylene & ND & 5000. $\mu \mathrm{g} / \mathrm{Kg}$ & Hexachlorobutadiene & ND & $5000 . \mu \mathrm{g} / \mathrm{Kg}$ \\
\hline Benzo (a) pyrene & $\mathrm{ND}$ & $5000 . \mu \mathrm{g} / \mathrm{Kg}$ & Hexachlorocyclopentadiene & $\mathrm{ND}$ & $5000 . \mu \mathrm{g} / \mathrm{Kg}$ \\
\hline Benzyl alcohol & $\mathrm{ND}$ & $1000 . \mu \mathrm{g} / \mathrm{kg}$ & Hexachloroethane & ND & $5000 . \mu \mathrm{g} / \mathrm{Kg}$ \\
\hline bis (2-Chloroethyl) ether & ND & 5000. $\mu \mathrm{g} / \mathrm{Kg}$ & Indeno $(1,2,3-c, d)$ pyrene & $\mathrm{ND}$ & $5000 . \mu \mathrm{g} / \mathrm{Kg}$ \\
\hline bis (2-Chloroethoxy) methane & ND & $5000 . \mu \mathrm{g} / \mathrm{Kg}$ & Isophorone & ND & $5000 . \mu \mathrm{g} / \mathrm{Kg}$ \\
\hline bis (2-chloroisopropyl) ether & ND & $5000 . \mu \mathrm{g} / \mathrm{Kg}_{\mathrm{g}}$ & 2-Methyinaphthalene & ND & 5000. $\mu \mathrm{g} / \mathrm{Kg}$ \\
\hline bis (2-Ethylhexyl)phthabate & 1600 & 5000. $\mu \mathrm{g} / \mathrm{Kg}$ & 2-Methylphenol & ND & $5000 . \mu \mathrm{g} / \mathrm{Kg}$ \\
\hline Butylbenzylphthalate & $\mathrm{ND}$ & 5000. $\mu g / \mathrm{Kg}$ & 4-Methylphenol & ND & $5000 . \mu g / \mathrm{Kg}$ \\
\hline 4-Bromophenyl phenyl ether & ND & $5000 . \mu \mathrm{g} / \mathrm{Kg}$ & Naphthalene & ND & $5000 . \mu \mathrm{g} / \mathrm{Kg}$ \\
\hline C zole & ND & 5000. $\mu \mathrm{g} / \mathrm{Kg}$ & 2-Nitroaniline & ND & $25000 . \mu \mathrm{g} / \mathrm{Kg}$ \\
\hline 4 noroanaline & ND & $10000 . \mu \mathrm{g} / \mathrm{Kg}$ & 3-Nitroaniline & ND & $25000 . \mu \mathrm{g} / \mathrm{Kg}$ \\
\hline 4-Chloro-3-methyl phenol & ND & 10000. $\mu \mathrm{g} / \mathrm{Kg}$ & 4-Nitroaniline & ND & $10000 . \mu \mathrm{g} / \mathrm{Kg}$ \\
\hline 2-Chloronaphthalene & ND & $5000 . \mu \mathrm{g} / \mathrm{Kg}$ & Nitrobenzene & ND & $5000 . \mu \mathrm{g} / \mathrm{Kg}$ \\
\hline 2-Chlorophenol & ND & 5000. $\mu \mathrm{g} / \mathrm{Kg}$ & 2-Nitrophenol & ND & $5000 . \mu \mathrm{g} / \mathrm{Kg}$ \\
\hline 4-Chiorophenyl phenyl ether & ND & $5000 . \mu \mathrm{g} / \mathrm{Kg}$ & 4-Nitrophenol & ND & $25000 . \mu \mathrm{g} / \mathrm{Kg}$ \\
\hline Chrysene & ND & $5000 . \mu \mathrm{g} / \mathrm{Kg}$ & N-Nitrosodi-n-propylamine & ND & $5000 . \mu \mathrm{g} / \mathrm{Kg}$ \\
\hline Dibenzo $(a, h)$ anthracene & ND & $5000 . \mu g / K g$ & N-Nitroso-Diethylamine & ND & $5000 . \mu \mathrm{g} / \mathrm{Kg}$ \\
\hline Dibenzofuran & ND & 5000. $\mu \mathrm{g} / \mathrm{Kg}$ & N-Nitrosodiphenylamine & ND & $5000 . \mu \mathrm{g} / \mathrm{Kg}$ \\
\hline Di-n-buty] phthalate & ND & 5000. $\mu \mathrm{g} / \mathrm{Kg}$ & Pentachlorophenol & ND & $25000 . \mu \mathrm{g} / \mathrm{Kg}$ \\
\hline 1,2-Dichiorobenzene (o-DCB) & ND & 5000. $\mu \mathrm{g} / \mathrm{Kg}$ & Phenol & ND & 5000. $\mu \mathrm{g} / \mathrm{Kg}$ \\
\hline 1,3-Dichlorobenzene (m-DCB) & ND & $5000 . \mu \mathrm{g} / \mathrm{Kg}$ & Phenanthrene & ND & 5000. $\mu \mathrm{g} / \mathrm{Kg}$ \\
\hline t.4-Dichlorobenzene (p-DCB) & ND & $5000 . \mu g / K g$ & Pyrene & ND & $5000 . \mu \mathrm{g} / \mathrm{Kg}$ \\
\hline 2,4-Dichlorophenol & ND & $5000 . \mu \mathrm{g} / \mathrm{Kg}$ & 1,2,4-Trichlorobenzene & ND & $5000 . \mu \mathrm{g} / \mathrm{Kg}$ \\
\hline 3.3'-Dichlorobenzidine & ND & $10000 . \mu g / \mathrm{Kg}$ & 2,4,5-Trichlorophenol & ND & $5000 . \mu \mathrm{g} / \mathrm{Kg}$ \\
\hline Diethylphthalate & ND & $5000 . \mu \mathrm{g} / \mathrm{Kg}$ & 2,4,6-Trichlorophenol & ND & 5000. $\mu \mathrm{g} / \mathrm{Kg}$ \\
\hline 2,4-Dimethylphenol & ND & $5000 . \mu \mathrm{g} / \mathrm{Kg}$ & & & \\
\hline Dimethylphthalate & ND & $5000 . \mu \mathrm{g} / \mathrm{Kg}$ & & & \\
\hline
\end{tabular}

ND - Not Detected

QUALITY CONTROL DATA.

Surrogate

2,4,6-Tribromophenol

2-Fluorobipheny]

2-F' rophenol

Nh-tenzene-d5

p-Terphenyi-d 14

Phenol-ds
$\%$ Recovery

89

77

70

85

63

87
Acceptable Range

19 - 122

$30-115$

$25-121$

23 - 120

18 - 137

$24-113$

This report shall not be reproduced except in full, without the written approval of the laboratory. 
CLIENT: Bechtel Nevada

PROJECT NAME: V427

PROJECT NUMBER: 17777
CLIENT ID:

Decon P1-2

DATE SAMPLED: $6 / 1 / 98$

NEL SAMPLE ID: L9806040-08

TEST: Semi-Volatile Organic Compounds by EPA 8270C, Dec. 1996

MATRLX: Solid

DILUTION: 1
EXTRACTED: $6 / 8 / 98$

ANALYZED: $6 / 9 / 98$

ANALYST: JDG

\begin{tabular}{|c|c|c|c|c|c|}
\hline PARAMETER & $\begin{array}{l}\text { Result } \\
\mu \mathrm{g} / \mathrm{Kg}\end{array}$ & $\begin{array}{c}\text { Reporting } \\
\text { Limit } \\
\end{array}$ & PARAMETER & $\begin{array}{l}\text { Result } \\
\mu \mathrm{g} / \mathrm{Kg}\end{array}$ & $\begin{array}{c}\text { Reporting } \\
\text { Limit }\end{array}$ \\
\hline Acenaphthene & $\overline{\mathrm{ND}}$ & $500 . \mu g / \mathrm{Kg}$ & 4,6-Dinitro-2-methyl phenol & $N D^{-}$ & $2500 . \mu \mathrm{g} / \mathrm{Kg}$ \\
\hline Acenaphthylene & ND & $500 . \mu \mathrm{g} / \mathrm{Kg}$ & 2,4-Dinitrotoluene (DNT) & ND & $500 . \mu \mathrm{g} / \mathrm{Kg}$ \\
\hline Anthracene & ND & 500. $\mu \mathrm{g} / \mathrm{Kg}$ & 2,6-Dinitrotoluene (DNT) & ND & 500. $\mu g / \mathrm{Kg}$ \\
\hline Benzo (a) anthracene & ND & $500 . \mu g / K g$ & 2,4-Dinitrophenol & ND & $2500 . \mu \mathrm{g} / \mathrm{Kg}$ \\
\hline Benzo (b\&k) fluoranthene & ND & $500 . \mu g / \mathrm{Kg}$ & Di-n-octyl phthalate & ND & 500. $\mu \mathrm{g} / \mathrm{Kg}$ \\
\hline Benzo (b) fluoranthene & ND & 500. $\mu \mathrm{g} / \mathrm{kg}$ & Fluoranthene & ND & 500. $\mu g / \mathrm{Kg}$ \\
\hline Benzo (k) fluoranthene & ND & $500 . \mu \mathrm{g} / \mathrm{kg}$ & Fiuorene & $\mathrm{ND}$ & 500. $\mu \mathrm{g} / \mathrm{Kg}$ \\
\hline Benzoic Acid & ND & 2500. $\mu \mathrm{g} / \mathrm{kg}$ & Hexachlorobenzene & ND & 500. $\mu \mathrm{g} / \mathrm{Kg}$ \\
\hline Benzo $(\mathrm{g}, \mathrm{h}, \mathrm{i})$ perylene & ND & 500. $\mu \mathrm{g} / \mathrm{Kg}$ & Hexachlorobutadiene & ND & 500. $\mu g / \mathrm{Kg}$ \\
\hline Benzo (a) pyrene & $\mathrm{ND}$ & 500. $\mu \mathrm{g} / \mathrm{Kg}$ & Hexachlorocyciopentadiene & $\mathrm{ND}$ & 500. $\mu \mathrm{g} / \mathrm{KE}$ \\
\hline Benzyl alcohol & ND & 1000. $\mu \mathrm{g} / \mathrm{kg}$ & Hexachloroethane & ND & $500 . \mu \mathrm{g} / \mathrm{Kg}$ \\
\hline bis (2-Chloroethyl) ether & $\mathrm{ND}$ & $500 . \mu g / \mathrm{Kg}$ & Indeno $(1,2,3-c, d)$ pyrene & ND & $500 . \mu \mathrm{g} / \mathrm{Kg}$ \\
\hline bis (2-Chloroethoxy) methane & ND & 500. $\mu \mathrm{g} / \mathrm{Kg}$ & Isophorone & ND & $500 . \mu \mathrm{g} / \mathrm{Kg}$ \\
\hline bis (2-chloroisopropyl) ether & $\mathrm{ND}$ & $500 . \mu \mathrm{g} / \mathrm{Kg}$ & 2-Methylnaphthalene & ND & 500. $\mu \mathrm{g} / \mathrm{Kg}$ \\
\hline bis (2-Ethylhexyl)phthalate & ND & $500 . \mu g / \mathrm{Kg}$ & 2-Methylphenol & ND & $500 . \mu \mathrm{g} / \mathrm{Kg}$ \\
\hline Butylbenzylphthalate & ND & 500. $\mu \mathrm{g} / \mathrm{Kg}$ & 4-Methylphenol & ND & 500. $\mu \mathrm{g} / \mathrm{Kg}$ \\
\hline 4-Bromophenyl phenyl ether & ND & $500 . \mu g / \mathrm{Kg}$ & Naphthalene & ND & $500 . \mu \mathrm{g} / \mathrm{Kg}$ \\
\hline Carbazole & ND & $500 . \mu \mathrm{g} / \mathrm{Kg}$ & 2-Nitroaniline & ND & $2500 . \mu \mathrm{g} / \mathrm{K}_{\mathrm{K}}$ \\
\hline 4-Chloroanaline & ND & 1000. $\mu \mathrm{g} / \mathrm{Kg}$ & 3-Nitroaniline & ND & $2500 . \mu \mathrm{g} / \mathrm{Kg}$ \\
\hline 4-Chloro-3-methyl phenol & ND & 1000. $\mu \mathrm{g} / \mathrm{Kg}$ & 4-Nitroaniline & $\mathrm{ND}$ & $1000 . \mu \mathrm{g} / \mathrm{Kg}$ \\
\hline 2-Chloronaphthalene & ND & $500 . \mu \mathrm{g} / \mathrm{Kg}$ & Nitrobenzene & $\mathrm{ND}$ & 500. $\mu \mathrm{g} / \mathrm{Kg}$ \\
\hline 2-Chlorophenol & ND & 500. $\mu \mathrm{g} / \mathrm{Kg}$ & 2-Nitrophenol & ND & $500 . \mu \mathrm{g} / \mathrm{Kg}$ \\
\hline 4-Chlorophenyl phenyl ether & ND & $500 . \mu \mathrm{g} / \mathrm{Kg}$ & 4-Nitrophenol & ND & $2500 . \mu \mathrm{g} / \mathrm{Kg}$ \\
\hline Chrysene & ND & 500. $\mathrm{Hg} / \mathrm{Kg}$ & N-Nitrosodi-n-propylamine & ND & 500. $\mu \mathrm{g} / \mathrm{Kg}$ \\
\hline Dibenzo $(a, h)$ anthracene & $\mathrm{ND}$ & $500 . \mu \mathrm{g} / \mathrm{Kg}$ & N-Nitroso-Diethylamine & $\mathrm{ND}$ & 500. $\mu \mathrm{g} / \mathrm{Kg}$ \\
\hline Dibenzofuran & ND & $500 . \mu \mathrm{g} / \mathrm{Kg}$ & N-Nitrosodiphenylamine & ND & 500. $\mu \mathrm{g} / \mathrm{Kg}$ \\
\hline Di-n-butyl phthalate & ND & $500 . \mu \mathrm{g} / \mathrm{Kg}$ & Pentachlorophenol & ND & 2500. $\mu \mathrm{g} / \mathrm{Kg}$ \\
\hline 1,2-Dichlorobenzene (o-DCB) & ND & 500. $\mu / \mathrm{Kg}$ & Phenol & $\mathrm{ND}$ & $500 . \mu \mathrm{g} / \mathrm{Kg}$ \\
\hline 1,3-Dichlorobenzene (m-DCB) & $\mathrm{ND}$ & 500. $\mu \mathrm{g} / \mathrm{Kg}$ & Phenanthrene & $\mathrm{ND}$ & 500. $\mu \mathrm{g} / \mathrm{Kg}$ \\
\hline 1,4-Dichjorobenzene (p-DCB) & ND & 500. $\mu g / \mathrm{Kg}$ & Pyrene & ND & 500. $\mu \mathrm{g} / \mathrm{Kg}$ \\
\hline 2,4-Dichlorophenol & ND & 500. $\mu \mathrm{g} / \mathrm{Kg}$ & 1,2,4-Trichlorobenzene & ND & $500 . \mu \mathrm{g} / \mathrm{Kg}$ \\
\hline $3,3^{\prime}$-Dichlorobenzidine & ND & 1000. $\mu \mathrm{g} / \mathrm{Kg}$ & 2,4,5-Trichlorophenol & ND & 500. $\mu \mathrm{g} / \mathrm{Kg}$ \\
\hline Diethylphthalate & ND & $500 . \mu \mathrm{g} / \mathrm{Kg}$ & 2,4,6-Trichjorophenol & ND & $500 . \mu \mathrm{g} / \mathrm{Kg}$ \\
\hline 2,4-Dimethylphenol & ND & 500. $\mu \mathrm{g} / \mathrm{Kg}$ & & & \\
\hline Dimethylphthalate & ND & 500. $\mu \mathrm{g} / \mathrm{Kg}$ & & & \\
\hline
\end{tabular}

ND - Not Detected

\section{QUALITY CONTROL DATA:}

\section{Surrogate}

2,4,6-Tribromophenol

2-Fluorobiphenyl

2-Fiuorophenol

Nitrobenzene-d5

p-Terphenyl-d14

Phenol-d5

\begin{tabular}{c}
$\%$ Recovery \\
\hline 72 \\
64 \\
62 \\
66 \\
80 \\
71
\end{tabular}

Acceptable Range

$19-1.22$

$30-1.15$

$25-121$

$23-120$

$18-137$

$24-113$

This report shall not be reproduced except in full, without the written approval of the laboratory. 
CLIENT: Bechtel Nevada

PROJECT NAME: V427

JJECT NUMBER: 17777
CLIENT ID: $\quad$ Decon P2-2

DATE SAMPLED: $6 / 1 / 98$

NEL SAMPLE ID: L9806040-I3

TEST: Semi-Volatile Organic Compounds by EPA 8270C, Dec. 1996
MATRIX: Solid
EXTRACTED: $6 / 8 / 98$
ANALYST: JDG
DILUTION: 1
ANALYZED: $6 / 9 / 98$

\begin{tabular}{|c|c|c|c|c|c|}
\hline PARAMETER & $\begin{array}{l}\text { Result } \\
\mu \mathrm{g} / \mathrm{Kg}\end{array}$ & $\begin{array}{l}\text { Reporting } \\
\text { Limit }\end{array}$ & PARAMETER & $\begin{array}{c}\text { Result } \\
\mu \mathrm{g} / \mathrm{Kg}\end{array}$ & $\begin{array}{c}\text { Reporting } \\
\text { Limit }\end{array}$ \\
\hline Acenaphthene & $\mathrm{ND}$ & $\overline{500 . \mu g / K g}$ & $\overline{\text { 4,6-Dinitro-2-methyl phenol }}$ & $\mathrm{ND}$ & $\overline{2500 . \mu \mathrm{g} K g}$ \\
\hline Acenaphthylene & ND & $500 . \mu \mathrm{g} / \mathrm{Kg}$ & 2,4-Dinitrotoluene (DNT) & ND & 500. $\mu \mathrm{g} / \mathrm{Kg}$ \\
\hline Anthracene & ND & 500. $\mu \mathrm{g} / \mathrm{Kg}$ & 2,6-Dinitrotoluene (DNT) & ND & 500. $\mu \mathrm{g} / \mathrm{Kg}$ \\
\hline Benzo (a) anthracene & ND & $500 . \mu g / \mathrm{Kg}$ & 2,4-Dinitrophenol & ND & $2500 . \mu \mathrm{g} / \mathrm{Kg}$ \\
\hline Benzo (b\&k) fluoranthene & $\mathrm{ND}$ & $500 . \mu g / \mathrm{Kg}$ & Di-n-octyl phthalate & ND & 500. $\mu \mathrm{g} / \mathrm{Kg}$ \\
\hline Benzo (b) fluoranthene & ND & 500. $\mu g / \mathrm{kg}$ & Fluoranthene & ND & 500. $\mu \mathrm{g} / \mathrm{Kg}$ \\
\hline Benzo (k) fluoranthene & ND & 500. $\mu \mathrm{g} / \mathrm{kg}$ & Fluorene & ND & $500 . \mu \mathrm{g} / \mathrm{Kg}$ \\
\hline Benzoic Acid & $\mathrm{ND}$ & $2500 . \mu \mathrm{g} / \mathrm{kg}$ & Hexachlorobenzene & ND & 500. $\mu \mathrm{g} / \mathrm{Kg}$ \\
\hline Benzo $(g, h, i)$ perylene & $\mathrm{ND}$ & 500. $\mu \mathrm{g} / \mathrm{Kg}$ & Hexachlorobutadiene & ND & 500. $\mu \mathrm{g} / \mathrm{Kg}$ \\
\hline Benzo (a) pyrene & ND & $500 . \mu g / \mathrm{Kg}$ & Hexachlorocyclopentadiene & ND & 500. $1 \mathrm{Hg} / \mathrm{Kg}$ \\
\hline Benzyl alcohol & $\mathrm{ND}$ & 1000. $\mathrm{\mu g} / \mathrm{kg}$ & Hexachloroethane & ND & 500. $\mu \mathrm{g} / \mathrm{Kg}$ \\
\hline bis (2-Chloroethyl) ether & $\mathrm{ND}$ & $500 . \mu g / \mathrm{Kg}$ & Indeno $(1,2,3-c, d)$ pyrene & ND & 500. $\mu \mathrm{g} / \mathrm{Kg}$ \\
\hline bis (2-Chloroethoxy) methane & ND & $500 . \mu \mathrm{g} / \mathrm{Kg}$ & Isophorone & ND & 500. $\mu \mathrm{g} / \mathrm{Kg}$ \\
\hline bis (2-chloroisopropyl) ether & ND & 500. $\mu \mathrm{g} / \mathrm{Kg}$ & 2-Methylnaphthalene & ND & 500. $\mu \mathrm{g} / \mathrm{Kg}$ \\
\hline bis (2-Ethylhexyl)phthalate & ND & $500 . \mu g / \mathrm{Kg}$ & 2-Methylphenol & ND & 500. $\mu \mathrm{g} / \mathrm{Kg}$ \\
\hline Butylbenzylphthalate & ND & 500. $\mu g / \mathrm{Kg}$ & 4-Methylphenol & ND & 500. $\mu \mathrm{g} / \mathrm{Kg}$ \\
\hline 4-Bromophenyl phenyl ether & ND & 500. $\mu g / \mathrm{Kg}$ & Naphthalene & ND & $500.1 \mathrm{~g} / \mathrm{Kg}$ \\
\hline$C^{-} \cdot$ zole & ND & 500. $\mu \mathrm{g} / \mathrm{Kg}$ & 2-Nitroaniline & ND & 2500. $\mathrm{Hg} / \mathrm{Kg}$ \\
\hline 4toroanaline & ND & 1000. $\mu \mathrm{g} / \mathrm{Kg}$ & 3-Nitroaniline & ND & 2500. $\mathrm{\mu g} / \mathrm{Kg}$ \\
\hline 4-Chloro-3-methy] phenol & ND & 1000, $\mu g / \mathrm{Kg}$ & 4-Nitroaniline & ND & 1000. $\mu \mathrm{g} / \mathrm{Kg}$ \\
\hline 2-Chloronaphthalene & ND & $500 . \mu g / K g$ & Nitrobenzene & ND & 500. $\mu \mathrm{g} / \mathrm{Kg}$ \\
\hline 2-Chlorophenol & ND & 500. $\mu \mathrm{g} / \mathrm{Kg}$ & 2-Nitrophenol & ND & 500. $\mathrm{Hg} / \mathrm{Kg}$ \\
\hline 4-Chlorophenyl phenyl ether & ND & $500 . \mu g / \mathrm{Kg}$ & 4-Nitrophenol & ND & 2500. $\mu \mathrm{g} / \mathrm{Kg}$ \\
\hline Chrysene & ND & 500. $\mu g / \mathrm{Kg}$ & N-Nitrosodi-n-propylamine & ND & $500 . \mu \mathrm{g} / \mathrm{Kg}$ \\
\hline Dibenzo (a,h) anthracene & ND & 500. $\mathrm{Hg} / \mathrm{Kg}$ & N-Nitroso-Diethylamine & ND & 500. $\mu \mathrm{g} / \mathrm{Kg}$ \\
\hline Dibenzofuran & ND & $500 . \mu g / \mathrm{Kg}$ & N-Nitrosodiphenylamine & ND & $500, \mu \mathrm{g} / \mathrm{Kg}$ \\
\hline Di-n-butyl phthalate & ND & $500 . \mu \mathrm{g} / \mathrm{Kg}$ & Pentachlorophenol & ND & 2500. $\mu \mathrm{g} / \mathrm{Kg}$ \\
\hline 1.2-Dichlorobenzene (o-DCB) & ND & $500 . \mu \mathrm{g} / \mathrm{Kg}$ & Phenol & $\mathrm{ND}$ & $500 . \mu \mathrm{g} / \mathrm{Kg}$ \\
\hline 1,3-Dichlorobenzene (m-DCB) & ND & 500. $\mu g / \mathrm{Kg}$ & Phenanthrene & ND & 500. $\mu \mathrm{g} / \mathrm{Kg}$ \\
\hline 1,4-Dichlorobenzene (p-DCB) & ND & 500. $\mu g / \mathrm{Kg}$ & Pyrene & ND & $500 . \mu \mathrm{g} / \mathrm{Kg}$ \\
\hline 2,4-Dichlorophenol & ND & $500 . \mu g / \mathrm{Kg}$ & 1,2,4-Trichlorobenzene & ND & 500. $\mu \mathrm{g} / \mathrm{Kg}$ \\
\hline 3,3'-Dichlorobenzidine & ND & 1000. $\mu g / \mathrm{Kg}$ & 2,4,5-Trichlorophenol & ND & $500 . \mu \mathrm{g} / \mathrm{Kg}$ \\
\hline Diethylphthalate & ND & 500. $\mu \mathrm{g} / \mathrm{Kg}$ & 2,4,6-Trichlorophenol & ND & 500. $\mu g / \mathrm{Kg}$ \\
\hline 2,4-Dimethylphenol & ND & 500. $\mu \mathrm{g} / \mathrm{Kg}$ & & & \\
\hline Dimethylphthalate & ND & 500. $\mu \mathrm{g} / \mathrm{Kg}$ & & & \\
\hline
\end{tabular}

ND - Not Detected

QUALITY CONTROL DATA:

Surrogate

2,4,6-Tribromophenol

2-Fluorobiphenyl

2-Fr-- ropheno]

Nik enzene-d5

p-Terphenyl-d 14

Phenol-d5

\begin{tabular}{c}
$\%$ Recovery \\
\hline 86 \\
78 \\
68 \\
75 \\
93 \\
78
\end{tabular}

\section{Acceptable Range}

$19-122$

$30-115$

$25-121$

$23-120$

$18-137$

$24-113$

This report shall not be reproduced except in full, without the written approval of the laboratory. 
CLIENT: Bechtel Nevada

PROJECT NAME: $\quad$ V427

PROJECT NUMBER: 17777
CLIENT ID: Method Blank

DATE SAMPLED: NA

NEL SAMPLE ID; $\quad 060898-8270-B L K$

TEST:

Semi-Volatile Organic Compounds by EPA 8270C, Dec. 1996

MATRIX: Solid

EXTRACTED: $\quad 6 / 8 / 98$

ANALYZED: $\quad 6 / 9 / 98$

\begin{tabular}{|c|c|c|c|c|c|}
\hline PARAMETER & $\begin{array}{c}\text { Result } \\
\mu \mathrm{g} / \mathrm{Kg}\end{array}$ & $\begin{array}{c}\text { Reporting } \\
\text { Limit } \\
\end{array}$ & PARAMETER & $\begin{array}{c}\text { Result } \\
\mu \mathrm{H} / \mathrm{Kg} \\
\end{array}$ & $\begin{array}{c}\text { Reporting } \\
\text { Limit } \\
\end{array}$ \\
\hline Acenaphthene & $\mathrm{ND}$ & $500 \mu g / \mathrm{Kg}$ & $\overline{\mathrm{Di}-\mathrm{n} \text {-octyl phthalate }}$ & $\overline{N D}$ & $500 \mu \mathrm{g} / \mathrm{Kg}$ \\
\hline Acenaphthylene & ND & $500 \mu g / \mathrm{Kg}$ & Fluoranthene & ND & $500 \mu \mathrm{g} / \mathrm{k}$ \\
\hline Anthracene & $\mathrm{ND}$ & $500 \mu \mathrm{g} / \mathrm{Kg}$ & Fluorene & $\mathrm{ND}$ & $500 \mu \mathrm{g} / \mathrm{t}$ \\
\hline Benzo (a) anthracenè & ND & $500 \mu \mathrm{g} / \mathrm{Kg}$ & Hexachlorobenzene & ND & $500 \mu g / \mathrm{t}$ \\
\hline Benzo (b\&k) fluoranthene & ND & $500 \mu g / K g$ & Hexachlorobutadiene & ND & $500 \mu \mathrm{g} / \mathrm{K}$ \\
\hline Benzo $(g, h, i)$ perylene & ND & $500 \mu \mathrm{g} / \mathrm{Kg}$ & Hexachlorocyclopentadiene & ND & $500 \mu \mathrm{g} / \mathrm{Kg}$ \\
\hline Benzo (a) pyrene & ND & $500 \mu \mathrm{g} / \mathrm{Kg}$ & Hexachloroethane & $\mathrm{ND}$ & $500 \mu \mathrm{g} / \mathrm{Kg}$ \\
\hline bis (2-Chloroethyl) ether & ND & $500 \mu g / \mathrm{Kg}$ & Indeno $(1,2,3-c, d)$ pyrene & ND & $500 \mu \mathrm{g} / \mathrm{Kg}$ \\
\hline bis (2-Chloroethoxy) methane & ND & $500 \mu g / \mathrm{Kg}$ & Isophorone & ND & $500 \mu \mathrm{g} / \mathrm{Kg}$ \\
\hline bis (2-chloroisopropyl) ether & $\mathrm{ND}$ & $500 \mu \mathrm{g} / \mathrm{Kg}$ & 2-Methylnaphthaiene & ND & $500 \mu \mathrm{g} / \mathrm{Kg}$ \\
\hline bis (2-Ethylhexyl)phthalate & $\mathrm{ND}$ & $500 \mu g / \mathrm{Kg}$ & 2-Methylphenol & ND & $500 \mu \mathrm{g} / \mathrm{k}$ \\
\hline Butylbenzylphthalate & $\mathrm{ND}$ & $500 \mu \mathrm{g} / \mathrm{Kg}$ & 4-Methylphenol & ND & $500 \mu \mathrm{g} / \mathrm{k}$ \\
\hline 4-Bromophenyi phenyi ether & $\mathrm{ND}$ & $500 \mu \mathrm{g} / \mathrm{Kg}$ & Naphthatene & ND & $500 \mu \mathrm{g} / \mathrm{k}$ \\
\hline Carbazole & $\mathrm{ND}$ & $500 \mu \mathrm{g} / \mathrm{Kg}$ & 2-Nitroaniline & ND & $2500 \mu \mathrm{g} / \mathrm{K}$ \\
\hline 4-Chloroanaline & ND & $1000 \mu \mathrm{g} / \mathrm{Kg}$ & 3-Nitroaniline & $\mathrm{ND}$ & $2500 \mu \mathrm{g} / \mathrm{Kg}$ \\
\hline 4-Chloro-3-methyl phenol & ND & $1000 \mu g / K g$ & 4-Nitroaniline & ND & $1000 \mu \mathrm{g} / \mathrm{Kg}$ \\
\hline 2-Chioronaphthalene & ND & $500 \mu \mathrm{g} / \mathrm{Kg}$ & Nitrobenzene & ND & $500 \mu \mathrm{g} / \mathrm{K}$ \\
\hline 2-Chlorophenol & $\mathrm{ND}$ & $500 \mu \mathrm{Kg} / \mathrm{Kg}$ & 2-Nitrophenol & ND & $500 \mu \mathrm{g} / \mathrm{K}$ \\
\hline 4-Chlorophenyl phenyl ether & $\mathrm{ND}$ & $500 \mu \mathrm{g} / \mathrm{Kg}$ & 4-Nitrophenol & ND & $2500 \mu \mathrm{g} / \mathrm{K}$ \\
\hline Chrysene & ND & $500 \mu \mathrm{g} / \mathrm{Kg}$ & N-Nitrosodi-n-propylamine & ND & $500 \mu \mathrm{g} / \mathrm{K}$ \\
\hline Dibenzo $(a, h)$ anthracene & ND & $500 \mu \mathrm{g} / \mathrm{Kg}$ & N-Nitroso-Diethylamine & ND & $500 \mu \mathrm{g} / \mathrm{K}$ \\
\hline Dibenzofuras & ND & $500 \mu g / \mathrm{Kg}$ & N-Nitrosodiphenylamine & ND & $500 \mu \mathrm{g} / \mathrm{K}$ \\
\hline Di-n-butyl phthaiate & $\mathrm{ND}$ & $500 \mu g / K g$ & Pentachlorophenol & ND & $2500 \mu \mathrm{g} / \mathrm{K}$ \\
\hline 1.2-Dichlorobenzene (o-DCB) & ND & $500 \mu \mathrm{g} / \mathrm{Kg}$ & Phenol & ND & $500 \mu g / \mathrm{k}$ \\
\hline 1,3-Dichlorobenzene (m-DCB) & ND & $500 \mu g / \mathrm{Kg}$ & Phenanthrene & ND & $500 \mu \mathrm{g} / \mathrm{k}$ \\
\hline 1.4-Dichlotobenzene ( $\mathrm{p}-\mathrm{DCB}$ ) & ND & $500 \mu \mathrm{g} / \mathrm{Kg}$ & Pyrene & ND & $500 \mu \mathrm{g} / \mathrm{K}$ \\
\hline 2.4-Dichlorophenol & ND & $500 \mu \mathrm{g} / \mathrm{Kg}$ & 1,2,4-Trichlorobenzene & $\mathrm{ND}$ & $500 \mu \mathrm{g} / \mathrm{K}$ \\
\hline 3,3'-Dichlorobenzidine & ND & $1000 \mu g / \mathrm{Kg}$ & 2,4,5-Trichlorophenol & ND & $500 \mu \mathrm{g} / \mathrm{k}$ \\
\hline Diethylphthalate & ND & $500 \mu \mathrm{g} / \mathrm{Kg}$ & 2,4,6-Trichlorophenol & ND & $500 \mu \mathrm{g} / \mathrm{K}$ \\
\hline 2.4-Dimethylphenol & ND & $500 \mu \mathrm{g} / \mathrm{Kg}$ & & & \\
\hline Dimethyiphthalate & ND & $500 \mu \mathrm{g} / \mathrm{Kg}$ & & & \\
\hline 4.6-Dinitso-2-methyl phenol & $\mathrm{ND}$ & $2500 \mu \mathrm{g} / \mathrm{Kg}$ & & & \\
\hline 2,4-Dinitrotoluene (DNT) & ND & $500 \mu g / \mathrm{Kg}$ & & & \\
\hline 2.6-Dinitrotoluene (DNT) & $\mathrm{ND}$ & $500 \mu g / K g$ & & & \\
\hline 2.4-Dinitrophenol & $\mathrm{ND}$ & $2500 \mu \mathrm{g} / \mathrm{Kg}$ & & & \\
\hline
\end{tabular}

ND - Not Detected

QUALITY CONTROL DATA:

\section{Surrogate}

2,4,6-Tribromophenol

2-Fluorobiphenyl

2-Fluorophenol

Nitrobenzene-d5

p-Terphenyl-d14

Phenol-d5
\% Recovery

85

67

60

66

85

70
Acceptable Range

$19-12 \%$

$30-115$

25- 121

$23-120$

$18-137$

$24-113$

This report shall not be reproduced except in full, without the written approval of the laboratory. 

POLYCHLORINATED

BIPHENYLS

DATA 
NEL LABORATORIES

$\begin{array}{ll}\text { CLIENT: Bechtel Nevada } & \text { CLIENT ID: Decon W1-3 } \\ \text { PROJECT NAME: V427 } & \text { DATE SAMPLED: } 6 / 1 / 98 \\ \text { JECT NUMBER: } 17777 & \text { NEL SAMPLE ID: } \text { L9806040-04 }\end{array}$

TEST: Polychlorinated Biphenyl's (PCB's) by EPA 8082, Dec. 1996

MATRIX: Solid

EXTRACTED: $6 / 10 / 98$

DILUTION: 1

ANALYZED: $6 / 12 / 98$

ANALYST: John

PARAMETER

Arochlor-1016

Arochlor-1221

Arochlor-1232

Arochlor-1242

Arochlor-1248

Arochlor-1254

Arochlor -1260

ND - Not Detected

QUALITY CONTROL DATA:

\section{Surrogate}

Decachlorobiphenyl

Tetrachloro-m-xylene

\begin{tabular}{|c|c|}
\hline Result & $\begin{array}{c}\text { Reporting } \\
\text { Limit }\end{array}$ \\
\hline ND & 20. $\mu \mathrm{g} / \mathrm{kg}$ \\
\hline ND & 20. $\mu \mathrm{g} / \mathrm{kg}$ \\
\hline ND & 20. $\mu \mathrm{g} / \mathrm{kg}$ \\
\hline ND & 20. $\mu \mathrm{g} / \mathrm{kg}$ \\
\hline ND & 20. $\mu \mathrm{g} / \mathrm{kg}$ \\
\hline$\mu \mathrm{g} / \mathrm{kg}$ & 20. $\mu \mathrm{g} / \mathrm{kg}$ \\
\hline ND & 20. $\mu \mathrm{g} / \mathrm{kg}$ \\
\hline
\end{tabular}

\%.Recovery

102

9]
Acceptable Range

$70-130$

$70-130$ 
NEL LABORATORIES

CLIENT: Bechtel Nevada

PROJECT NAME: V427

PROJECT NUMBER: 17777
CLIENT ID: Decon P1-3

DATE SAMPLED: $6 / 1 / 98$

NEL SAMPLE ID: L9806040-09

TEST: Polychlorinated Biphenyl's (PCB's) by EPA 8082, Dec. 1996

MATRIX: Solid

DILUTION: 1
EXTRACTED: 6/10/98

ANALYZED: $6 / 12 / 98$
ANALYST: John

\begin{tabular}{c}
$\begin{array}{c}\text { Reporting } \\
\text { Limit }\end{array}$ \\
\hline $20 . \mu \mathrm{g} / \mathrm{kg}$ \\
20. $\mu \mathrm{g} / \mathrm{kg}$ \\
20. $\mu \mathrm{g} / \mathrm{kg}$ \\
20. $\mu \mathrm{g} / \mathrm{kg}$ \\
20. $\mu \mathrm{g} / \mathrm{kg}$ \\
$20 . \mu \mathrm{g} / \mathrm{kg}$ \\
$20 . \mu g / \mathrm{kg}$ \\
\hline
\end{tabular}

PARAMETER

Arochlor-1016

Arochlor-1221

Arochlor-1232

Arochlor- 1242

Arochlor- 1248

Arochlor- 1254

Arochlor-1260

\begin{tabular}{l} 
Result \\
\hline $\mathrm{ND}$ \\
$\mathrm{ND}$ \\
$\mathrm{ND}$ \\
$\mathrm{ND}$ \\
$\mathrm{ND}$ \\
$\mathrm{ND}$ \\
$\mathrm{ND}$
\end{tabular}

$\mathrm{ND}$ - Not Detected

QUALITY CONTROL DATA:

Surrogate

Decachiorobiphenyl

\% Recovery

112

104

\section{Acceptabie Range}

$70-130$

$70-130$ 
NEL LABORATORIES

$\begin{array}{ll}\text { CLIENT: Bechtel Nevada } & \text { CLIENT ID: Decon P2-3 } \\ \text { PROJECT NAME: V427 } & \text { DATE SAMPLED: } 6 / 1 / 98 \\ \text { IJECT NUMBER: } 17777 & \text { NEL SAMPLE ID: L9806040-14 }\end{array}$

TEST: Polychlorinated Biphenyl's (PCB's) by EPA 8082, Dec. 1996

MATRIX: Solid

EXTRACTED: $6 / 10 / 98$

DILUTION: 1

ANALYZED: $6 / 12 / 98$

ANALYST: John

PARAMETER

Arochlor-1016

Arochlor-1221

Arochior-1232

Arochlor-1242

Arochlor-1248

Arochlor-1254

Arochlor- 1260

ND - Not Detected

QUALITY CONTROL DATA:

Surrogate

Decachlorobiphenyl

Tetrachioro-m-xylene
$\%$ Recovery

113

106

\section{Acceptable Range}

$70-130$

$70-130$ 
NEL LABORATORIES

CLIENT: Bechtel Nevada

PROJECT NAME: V427

PROJECT NUMBER: 17777
CLIENT ID: Method Blank

DATE SAMPLED: NA

NEL SAMPLE ID: 980610s8082-BK

TEST: Polychlorinated Biphenyl's (PCB's) by EPA 8082, Dec. 1996

MATRIX: Solid

EXTRACTED: $6 / 10 / 98$

ANALYZED: $6 / 12 / 98$

\section{PARAMETER}

Arochlor-1016

Arochlor-1221

Arochlor-1232

Arochlor-1242

Arochlor-1248

Arochlor-1254

Arochlor- 1260

ND - Not Detected

QUALITY CONTROL DATA:

Surrogate

Decachlorobiphenyl

Tetrachloro-m-xylene
$\%$ Recovery

107

92
ANALYST: John

\begin{tabular}{ll} 
Result \\
\hline ND \\
ND \\
ND \\
ND \\
ND \\
ND \\
ND
\end{tabular}

\section{Acceptable Range}

$70-130$

$70-130$ 


\section{TOTAL}

PETROLEUM

HYDROCARBON

DATA 
NEL LABORATORIES

CLIENT: Bechtel Nevada

PROJECT NAME: V427

JUECT NUMBER: 17777

TEST:

Total Extractable Petroleum Hydrocarbons by EPA Method 8015M, July 1992

METHOD: EPA 8015M

MATRLX: Solid ANALYST: Suzanne

\begin{tabular}{|c|c|c|c|c|c|c|}
\hline $\begin{array}{l}\text { CLIENT } \\
\text { SAMPLE ID }\end{array}$ & $\begin{array}{l}\text { SAMPLE } \\
\text { DATE }\end{array}$ & $\begin{array}{c}\text { NEL } \\
\text { SAMPLE ID }\end{array}$ & $\begin{array}{c}\text { RESULT } \\
\mathrm{mg} / \mathrm{kg}\end{array}$ & C.R. & $\begin{array}{l}\text { eporting } \\
\text { Limit }\end{array}$ & $\begin{array}{l}\text { Surrogate } \\
\text { Recovery }{ }^{\star} \text { EXTRA }\end{array}$ \\
\hline $\operatorname{con} W 1-4 A \& B$ & $6 / 1 / 98$ & L9806040-05 & 2400 & O & $30 . \mathrm{mg} / \mathrm{kg}$ & $6 / 10 / 98$ \\
\hline
\end{tabular}

\section{CR: Carbon Range}

O Oil Range Organics ( $\mathrm{C} 12$ to $\mathrm{C} 38$ ).

Note: The reporting limit for Oil Range Organics in soil is $\mathbf{5 0} \mathbf{m g} / \mathbf{k g}$.

QUALITY CONTROL DATA (Total for Gas and Diesel Range):"

Sample ID

Blank, 980610TPH-BLK

L. $\quad 980610 \mathrm{TP}-$ LCS
Result Acceptable Range Surrogate Recovery ${ }^{\star}$ Sample Number

ND $<10 . \mathrm{mg} / \mathrm{kg} \quad 109 \%$

$82 \% \quad 55-102 \% \quad 104 \%$
NA

NA

ND - Not Detected

tSurrogate used was Octacosane, acceptance limits 54-130\% for solids, 60-121\% for aqueous samples

This report shall not be reproduced except in full, without the written approval of the laboratory. 
NELL LABORATORIES

CLIENT: Bechtel Nevada

PROJECT NAME: V427

PROJECT NUMBER: 17777

TEST: $\quad$ Total Extractable Petroleum Hydrocarbons by EPA Method 8015M, July 1992

METHOD: EPA 8015M

MATRIX: Solid

ANALYST: Suzanne

CLIENT

SAMPLE ID

Decon P1-4 A\&B

Decon P2-4 A\&B
SAMPLE NEL RESULT

DATE SAMPLE ID $\mathrm{mg} / \mathrm{kg}$

6/1/98 L9806040-10

ND

6/1/98 L9806040-15 ND

C.R. $\frac{\begin{array}{l}\text { Reporting } \\ \text { Limit }\end{array}}{10 . \mathrm{mg} / \mathrm{kg}}$

10. $\mathrm{mg} / \mathrm{kg}$

\section{Surrogate}

Recovery* EXTRACTED ANALYZED

$70 \% \quad 6 / 9 / 98$

$.601986 / 1 / 98$

$80 \quad \% \quad 6 / 9 / 98$

$640986 / 11 / 98 \pi$

\section{CR: Carbon Range}

$\mathrm{O}$ Oil Range Organics ( $\mathrm{Cl} 2$ to $\mathrm{C} 38$ ).

Note: The reporting limit for Oil Range Organics in soil is $50 \mathrm{mg} / \mathrm{kg}$. QUALITY CONTROL DATA (Total for Gas and Diesel Range):"

Sample ID

Blank, 980609TPH-BLK

LCS, $980609 \mathrm{TP}-$ LCS
Result Acceptable Range Surrogate Recovery* Sample Number

$\begin{array}{lccccc}\mathrm{ND} & < & 10 . \mathrm{mg} / \mathrm{kg} & 67 & \% & \mathrm{NA} \\ 57 & \% & 55 & -102 \% & 69 & \%\end{array}$

ND - Not Detected

*Surrogate used was Octacosane, acceptance limits $54-130 \%$ for solids, 60-121\% for aqueous samples

This report shall not be reproduced except in full, without the written approval of the laboratory. 
TCLP

METALS

(minus Mercury)

DATA 
NEL LABORATORIES

CLIENT: Bechtel Nevada

TJECT NAME: V427

_.JJECT NUMBER: 17777

\section{CLIENT ID: $\quad$ Decon WI-5}

DATE SAMPLED: $6 / 1 / 98$

NEL SAMPLE ID: L9806040-06

TEST: $\quad$ TCLP by EPA 1311, July 1992 \& 7 Metals by EPA 6010A, July 1992

MATRLX: Solid

\begin{tabular}{|c|c|c|c|c|c|c|c|}
\hline PARAMETER & $\begin{array}{c}\text { RESULT } \\
\mathrm{mg} / \mathrm{L} \\
\end{array}$ & $\begin{array}{c}\text { REPORTING } \\
\text { LIMIT } \\
\end{array}$ & D. $\mathbf{F}$. & METHOD & $\begin{array}{c}\text { TCLP/STLC } \\
\text { EXTRACTION } \\
\text { DATE } \\
\end{array}$ & DIGESTED & ANALYZED \\
\hline Arsenic & $\mathrm{ND}$ & $0.1 \mathrm{mg} / \mathrm{L}$ & 1 & EPA 6010A & $6 / 4 / 98$ & $6 / 5 / 98$ & $6 / 5 / 98$ \\
\hline Barium & $\mathrm{ND}$ & 1. $\mathrm{mg} / \mathrm{L}$ & 1 & EPA $6010 A$ & $6 / 4 / 98$ & $6 / 5 / 98$ & $6 / 5 / 98$ \\
\hline Cadmium & 0.014 & $0.01 \mathrm{mg} / \mathrm{L}$ & 1 & EPA 6010A & $6 / 4 / 98$ & $6 / 5 / 98$ & $6 / 5 / 98$ \\
\hline Chromium & ND & $0.01 \mathrm{mg} / \mathrm{L}$ & 1 & EPA $6010 \mathrm{~A}$ & $6 / 4 / 98$ & $6 / 5 / 98$ & $6 / 5 / 98$ \\
\hline Lead & 0.20 & $0.05 \mathrm{mg} / \mathrm{L}$ & 1 & EPA $6010 \mathrm{~A}$ & $6 / 4 / 98$ & $6 / 5 / 98$ & $6 / 5 / 98$ \\
\hline Selenium & ND & $0.1 \mathrm{mg} / \mathrm{L}$ & 1 & EPA $6010 \mathrm{~A}$ & $6 / 4 / 98$ & $6 / 5 / 98$ & $6 / 5 / 98$ \\
\hline Silver & $\mathrm{ND}$ & $0.02 \mathrm{mg} / \mathrm{L}$ & 1 & EPA $6010 \mathrm{~A}$ & $6 / 4 / 98$ & $6 / 5 / 98$ & $6 / 5 / 98$ \\
\hline
\end{tabular}

D.F. - Dilution Factor

ND - Not Detected

This report shall not be reproduced except in full, without the written approval of the laboratory. 
CLIENT: Bechtel Nevada

PROJECT NAME: V427

PROJECT NUMBER: 17777
CLIENT ID: $\quad$ Decon PI-5

DATE SAMPLED: $6 / 1 / 98$

NEL SAMPLE ID: L9806040-11

TEST: $\quad$ TCLP by EPA 1311, July 1992 \& 7 Metals by EPA 6010A, July 1992 MATRIX: Solid

\begin{tabular}{|c|c|c|c|c|c|c|c|}
\hline PARAMETER & $\begin{array}{c}\text { RESULT } \\
\mathrm{mg} / \mathrm{L} \\
\end{array}$ & $\begin{array}{c}\text { REPORTING } \\
\text { LIMIT } \\
\end{array}$ & D. $\mathbf{F}$. & METHOD & $\begin{array}{c}\text { TCLP/STLC } \\
\text { EXTRACTION } \\
\text { DATE } \\
\end{array}$ & DIGESTED & ANALYZED \\
\hline Arsenic & ND & $0.1 \mathrm{mg} / \mathrm{L}$ & 1 & EPA 6010A & $6 / 4 / 98$ & $6 / 5 / 98$ & $6 / 5 / 98$ \\
\hline Barium & ND & 1. $\mathrm{mg} / \mathrm{L}$ & 1 & EPA 6010A & $6 / 4 / 98$ & $6 / 5 / 98$ & $6 / 5 / 98$ \\
\hline Cadmium & ND & $0.01 \mathrm{mg} / \mathrm{L}$ & 1 & EPA $6010 \mathrm{~A}$ & $6 / 4 / 98$ & $6 / 5 / 98$ & $6 / 5 / 98$ \\
\hline Chromium & ND & $.0 .01 \mathrm{mg} / \mathrm{L}$ & 1 & EPA 6010A & $6 / 4 / 98$ & $6 / 5 / 98$ & $6 / 5 / 98$ \\
\hline Lead & ND & $0.05 \mathrm{mg} / \mathrm{L}$ & 1 & EPA $6010 \mathrm{~A}$ & $6 / 4 / 98$ & $6 / 5 / 98$ & $6 / 5 / 98$ \\
\hline Selenium & $\mathrm{ND}$ & $0.1 \mathrm{mg} / \mathrm{L}$ & 1 & EPA $6010 \mathrm{~A}$ & $6 / 4 / 98$ & $6 / 5 / 98$ & $6 / 5 / 98$ \\
\hline Silver & ND & $0.02 \mathrm{mg} / \mathrm{L}$ & 1 & EPA $6010 A$ & $6 / 4 / 98$ & $6 / 5 / 98$ & $6 / 5 / 98$ \\
\hline
\end{tabular}

D.F. - Dilution Factor

ND - Not Detected

This report shall not be reproduced except in full, without the written approval of the laboratory. 
NEL LABORATORIES

CLIENT: Bechtel Nevada

PD OJECT NAME: V427

JJECT NUMBER: 17777
CLIENT ID: $\quad$ Decon P2-5

DATE SAMPLED: $6 / 1 / 98$

NEL SAMPLE ID: L9806040-16

TEST: $\quad$ TCLP by EPA 1311, July 1992 \& 7 Metals by EPA 6010A, July 1992

MATRIX: Solid

PARAMETER

Arsenic

Barium

Cadmium

Chromium

Lead

Selenium

Silver

\section{RESULT REPORTING}

$\mathrm{mg} / \mathrm{L}$

ND

ND

ND

ND

ND

ND

ND

LIMIT

$0.1 \mathrm{mg} / \mathrm{L}$

$1 . \mathrm{mg} / \mathrm{L}$

$0.01 \mathrm{mg} / \mathrm{L}$

$0.01 \mathrm{mg} / \mathrm{L}$

$0.05 \mathrm{mg} / \mathrm{L}$

$0.1 \mathrm{mg} / \mathrm{L}$

$0.02 \mathrm{mg} / \mathrm{L}$
TCLP/STLC

EXTRACTION

D. F. METHOD

I. EPA 6010A

EPA 6010A

EPA 6010A

EPA 6010A

EPA 6010A

EPA 6010A

EPA 6010A
DATE

$6 / 4 / 98$

$6 / 4 / 98$

$6 / 4 / 98$

$6 / 4 / 98$

$6 / 4 / 98$

$6 / 4 / 98$

$6 / 4 / 98$
DIGESTED ANALYZED

$6 / 5 / 98 \quad 6 / 5 / 98$

$6 / 5 / 98 \quad 6 / 5 / 98$

$6 / 5 / 98 \quad 6 / 5 / 98$

$6 / 5 / 98 \quad 6 / 5 / 98$

$6 / 5 / 98 \quad 6 / 5 / 98$

$6 / 5 / 98 \quad 6 / 5 / 98$

$6 / 5 / 98 \quad 6 / 5 / 98$

D.F. - Dilution Factor

ND - Not Detected

This report shall not be reproduced except in full, without the written approval of the laboratory. 
NEL LABORATORIES

CLIENT: Bechtel Nevada

PROJECT NAME: V427

PROJECT NUMBER: 17777
CLIENT ID: Method Blank

DATE SAMPLED: NA

NEL SAMPLE ID: L06040I-BLK

TEST: $\quad$ TCLP by EPA 1311, July 1992 \& 7 Metals by EPA 6010A, July 1992

MATRIX: TCLP Extract

\begin{tabular}{|c|c|c|c|c|c|c|c|}
\hline \multirow[b]{2}{*}{ PARAMETER } & \multirow[b]{2}{*}{ RESULT } & \multirow{2}{*}{$\begin{array}{c}\text { REPORTING } \\
\text { LIMIT } \\
\end{array}$} & \multicolumn{5}{|c|}{$\begin{array}{l}\text { TCLP/STLC } \\
\text { EXTRACTION }\end{array}$} \\
\hline & & & D. $\mathbf{F}$. & METHOD & DATE & DIGESTED & ANALYZED \\
\hline Arsenic & ND & $0.1 \mathrm{mg} / \mathrm{L}$ & 1 & EPA $6010 \mathrm{~A}$ & $6 / 4 / 98$ & $6 / 5 / 98$ & $6 / 5 / 98$ \\
\hline Barium & ND & $1 \mathrm{mg} / \mathrm{L}$ & 1 & EPA $6010 \mathrm{~A}$ & $6 / 4 / 98$ & $6 / 5 / 98$ & $6 / 5 / 98$ \\
\hline Cadmium & ND & $0.01 \mathrm{mg} / \mathrm{L}$ & 1 & EPA $6010 A$ & $6 / 4 / 98$ & $6 / 5 / 98$ & $6 / 5 / 98$ \\
\hline Chromium & $\mathrm{ND}$ & $0.01 \mathrm{mg} / \mathrm{L}$ & 1 & EPA $6010 A$ & $6 / 4 / 98$ & $6 / 5 / 98$ & $6 / 5 / 98$ \\
\hline Lead & $\mathrm{ND}$ & $0.05 \mathrm{mg} / \mathrm{L}$ & 1 & EPA 6010A & $6 / 4 / 98$ & $6 / 5 / 98$ & $6 / 5 / 98$ \\
\hline Selenium & ND & $0.1 \mathrm{mg} / \mathrm{L}$ & 1 & EPA $6010 A$ & $6 / 4 / 98$ & $6 / 5 / 98$ & $6 / 5 / 98$ \\
\hline Silvet & ND & $0.02 \mathrm{mg} / \mathrm{L}$ & 1 & EPA $6010 \mathrm{~A}$ & $6 / 4 / 98$ & $6 / 5 / 98$ & $6 / 5 / 98$ \\
\hline
\end{tabular}

D.F. - Dilution Factor

ND - Not Detected

This report shall not be reproduced except in full, without the written approval of the laboratory. 


\section{SDG B429 \\ RADIOANALYTICAL \\ RESULTS}




\section{Bechtel Nevada Corporation}

\section{ANALYTICAL SERVICES LABORATORY}

P.O.Box 3936, N. Las Vegas, NV 89036

Reported to: Area 6 Decon Pond Closure

Remediation Projects

Report Date: 6-JUL-98

J. L. Smith, M/S NTS306

Sample Delivery Group: B429

Batch: 0816

Program: 720

Report No. :

\begin{tabular}{|c|c|c|c|c|c|c|c|c|c|c|c|c|c|c|c|c|}
\hline 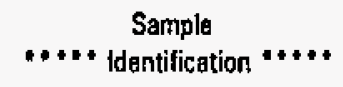 & Isotope & Analysis & Result & $\begin{array}{c}\text { Error } \\
*\end{array}$ & $\begin{array}{l}\text { Qual } \\
\text { Flag }\end{array}$ & MDA & \begin{tabular}{|l} 
Result \\
Units
\end{tabular} & $\begin{array}{c}\text { Analysis } \\
\text { Date }\end{array}$ & $\begin{array}{l}\text { Sample } \\
\text { Coll Dato }\end{array}$ & Size & \begin{tabular}{|l|} 
Size \\
Units
\end{tabular} & \begin{tabular}{|c|} 
Type \\
Matrix
\end{tabular} & $\begin{array}{l}\text { Tracer } \\
\text { Yield \% }\end{array}$ & $\begin{array}{l}\text { Spikg } \\
\text { Recv \% }\end{array}$ & \begin{tabular}{|l|} 
System \\
Detector
\end{tabular} & $\begin{array}{l}\text { Packet-Item } \\
\text { Sample }\end{array}$ \\
\hline DECON P1-6 & $\mathrm{K} 40$ & GAM20 & $2.35 E+01$ & $2,0 E+01$ & & 6.6E-01 & $\mathrm{pCi} / \mathrm{g}$ & 06-02-98 & 06-01-98 & $|5.95 E+02|$ & gm & SOIL G & & & 05-01 & G9099-1-75071 \\
\hline DECON P1.6 ${ }^{-}$ & AA226 & GAM20 & $1.06 E+00$ & $3.2 \mathrm{E}+01$ & & $1.5 \mathrm{E}-01$ & $\mathrm{pClig}$ & 06-02-98 & $06-01-98$ & $5.95 \mathrm{E}+02$ & on & SOlL G & & & 05-01 & G9099-1-75071 \\
\hline DECON P1-6 & TH228 & GAM20 & $2.44 E+00$ & $1.9 \mathrm{E}+01$ & & 1.JE-01 & pCilg & 06-02-98 & $06-01-98$ & $5.95 E+02$ & $\mathrm{gm}$ & SOIL $G$ & & & 05-01 & G9099-1-75071 \\
\hline DECON P1-6 & TH232 & GAM20 & $2.32 E+00$ & $3.0 E+01$ & & 3.2E-01 & pCilg & $06-02-98$ & $06-01-98$ & $5.95 E+02$ & $\mathrm{gm}$ & SolL G & & & 05-01 & G9099-1-76071 \\
\hline DECON P2-6 & $\operatorname{cs} 137$ & GAM20 & 2.10E-01 & $4.9 E+01$ & & B.4E-02 & pCilo & $06-02-98$ & 06-01-98 & $6.70 E+02$ & $\mathrm{gm}$ & soll G & & & 05.01 & G9099-2-75073 \\
\hline DECON P2-6 & K 40 & GAM2O & $2.26 E+01$ & $2.0 E+01$ & & B.3E-01 & pCilo & $06-02-98$ & $06-01-98$ & $6.70 E+02$ & $\mathrm{gm}$ & SOIL G & & & 05-01 & G9099-2-76073 \\
\hline DECON P2-6 & RA226 & GAM20 & $1.30 E+00$ & $2,8 E+01$ & & 1.7E-01 & pCilg & $06-02-98$ & $06-01-98$ & $6.70 E+02$ & gim & SOIL $\mathrm{G}$ & & & $05-01$ & G9099-2-76073 \\
\hline DECON P2-6 & TH228 & GAM20 & $2.75 E+00$ & $1.7 \mathrm{E}+01$ & & $1.6 \mathrm{E}-01$ & $\mathrm{pCilo}$ & $06-02-98$ & 06-01-98 & $6.70 E+02$ & gm & soll G & & & $05-01$ & G9099-2-75073 \\
\hline DECON P2-6 & TH232 & GAM20 & $1.91 E+00$ & $3.4 E+01$ & & $3.1 E-01$ & pCilg & 06-02-98 & $06-01-98$ & $6.70 E+02$ & $\mathrm{gm}$ & soll G & & & $05-01$ & G9099-2-75073 \\
\hline DECON W1-6 & $\mathrm{CO60}$ & GAM20 & $1.74 \mathrm{E}+00$ & $2.2 \mathrm{E}+01$ & & 9.9E-02 & pCilg & 06-02-98 & $06-01-98$ & $|3.80 \mathrm{E}+02|$ & $g m$ & soll $G \mid$ & & & 05.01 & G9099-0.75069 \\
\hline
\end{tabular}

Comment:

Data generated from analyses of samples submitted on $6 / 2 / 98$.

Gross alpha and gross beta determinations are performed on

small aliquots. Data may not represent the sample as a whole.

Prepared by:

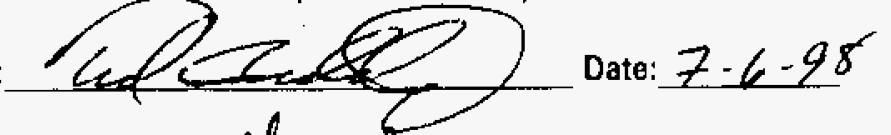

Approved by: Sasurfateros Date: $7 / 6 / 98$
Qualification Flags:

$E=$ Estimated Quantity

$H=$ High Recovery for Sample

$\mathrm{J}=$ Result is less than the RDL

$\mathrm{L}=$ Low Recovery for Sample

$\mathbf{P}=$ Preliminary Results

$\mathbf{Q}=$ Bad Instrument Quality Control, Rosult is OK

$R=$ Results are Unusable, Resampling is Necessary

$\mathrm{U}=$ Result is less than Minimum Detectable Activity

Note: \% Error is the 2.0 Sigma Error 


\section{Bechtel Nevada Corporation}

\section{ANALYTICAL SERVICES LABORATORY}

P.O.Box 3936, N. Las Vegas, NV 89036

Reported to: Area 6 Decon Pond Closure

Remediation Projects

J. L. Smith, M/S NTS306

Report Date: 6-JUL-98

Sample Delivery Group: 8429

Batch: 0816

Program: 720

Report No.:

\begin{tabular}{|c|c|c|c|c|c|c|c|c|c|c|c|c|c|c|c|c|}
\hline .* . * * Identification *...* & Sotope & Analysis & Result & $\begin{array}{c}\text { Error } \\
\%\end{array}$ & $\begin{array}{l}\text { Oual } \\
\text { Flag }\end{array}$ & MDA & $\begin{array}{l}\text { Result } \\
\text { Units }\end{array}$ & $\begin{array}{l}\text { Analysis } \\
\text { Date }\end{array}$ & $\begin{array}{l}\text { Sample } \\
\text { Coll Date }\end{array}$ & Size & \begin{tabular}{|c|} 
Size \\
Units
\end{tabular} & $\begin{array}{c}\text { Typo } \\
\text { Matrix }\end{array}$ & \begin{tabular}{|l|} 
Tracer \\
Yield $\%$
\end{tabular} & \begin{tabular}{|l|} 
Spike \\
Recu $\%$
\end{tabular} & \begin{tabular}{|l|} 
Srstem \\
Detector
\end{tabular} & $\begin{array}{l}\text { Packat-Itemt } \\
\text { Samplo }\end{array}$ \\
\hline DECON W1-6 & $\operatorname{cs} 137$ & GAM20 & $1.64 E+01$ & $1.1 \mathrm{E}+01$ & & $9.0 \mathrm{E}-02$ & pCiig & $06-02-98$ & $06.01-98$ & $3.80 F+02$ & gm & solt 6 & & & $05-01$ & G9099.0-75069 \\
\hline DECON W1-6 & $K 40$ & GAM20 & $7.37 E+00$ & $3.2 E+01$ & & 9.2E-01 & $\mathrm{pCl} / \mathrm{g}$ & $06-02-98$ & $06-01-98$ & $3.80 \mathrm{E}+02$ & $g m$ & SOIL G & & & $05-01$ & G9099-0-75069 \\
\hline DECON W1-6 & AA226 & GAM20 & 7.96E-01 & $3.4 \mathrm{E}+01$ & & 1.6E-01 & $p \mathrm{Ci} / \mathrm{g}$ & $06-02-98$ & 06-01-98 & 3. $80 \mathrm{E}+02$ & gm & SOIL G & & & $05-01$ & G9099-0-75069 \\
\hline DECON W1-6 & $\mathrm{TH} 228$ & GAM20 & $7.92 \mathrm{E}-01$ & $4.3 E+01$ & & $1.4 \mathrm{E}-01$ & pCils & $06-02-98$ & $06-01-98$ & $3.80 E+02$ & $\mathrm{gm}$ & SOL G & & & $05-01$ & G9099-0-75069 \\
\hline DECON W1-6 & TH232 & GAM20 & $6.46 \mathrm{E}-01$ & $7.7 \mathrm{E}+01$ & & $2.6 \mathrm{E}-01$ & $\mathrm{pCi} / \mathrm{g}$ & 06-02-98 & $06-01-98$ & $3.80 \mathrm{E}+02$ & gm & SOIL G & & & $05-01$ & G9099-0-75069 \\
\hline OA BKG EMPTY BTL U05 & No Nucl Det & GAM20 & $0.00 \mathrm{E}+00$ & $0.0 E+00$ & U & $0.0 \mathrm{E}+00$ & NA & $06-02-98$ & $07-01.95$ & $1.00 E+00$ & $\frac{\sin }{\sin }$ & QUAL & & & $06-01$ & $00539-0-00200$ \\
\hline OA BKG ENPTY BTL U05 & AM241 & GAM20 & $0.00 E+00$ & $0.0 E+00$ & u & $2.4 E+01$ & $\mathrm{pCi}$ & $06-02-98$ & $07-01-95$ & $1.00 E+00$ & $\mathrm{sm}$ & QUAL & & & $05-01$ & $00539-0-00200$ \\
\hline OA BKG EMPTY BTL U05 & $\mathrm{Co60}$ & GAM20 & $0.00 E+00$ & $0.0 \mathrm{E}+00$ & u & $9.9 \mathrm{E}+00$ & $\mathrm{pCi}$ & $06 \cdot 02-98$ & $07-01-95$ & $1.00 E+00$ & $\mathrm{sm}$ & QUAL & & & $05-01$ & $00539-0-00200$ \\
\hline OA BKG EMPTY BTL U05 & $\operatorname{cs} 137$ & GAM20 & $0.00 \mathrm{E}+00$ & $0.0 E+00$ & $\mathbf{u}$ & $8.0 E+00$ & $p \mathrm{Ci}$ & $06-02-98$ & $07-01-95$ & $1.00 E+00$ & $5 m$ & QUAL & & & $05-01$ & $00539-0-00200$ \\
\hline OA SP:NAS-A0271 & AN241 & GAM20 & $1.82 E+05$ & $8.6 \mathrm{E}+\infty$ & & $3.0 E+02$ & $\mathrm{pCi}$ & $06-02-98$ & 04-01-92 & $1.00 E+00$ & $\mathrm{sm}$ & QUAL & & 112.1 & 05.01 & $00534-3-14370$ \\
\hline
\end{tabular}

\section{Comment:}

Data generated from analyses of samples submitted on $6 / 2 / 98$

Gross alpha and gross beta determinations are performed on

small aliquots. Data may not represent the sample as a whole.

Prepared by:<smiles>C1CC[Te]C1</smiles>

approved by: faury/Agthes
Date: $7-6-s f$ Date: $7 / 6 / 98$
Qualification Flags:

$\mathrm{E}=$ Estimated Quantity

$H=$ High Recovery for Sample

$\mathrm{J}=$ Result is less than the RDL

$L=$ Low Recovery for Sample

$P=$ Preliminary Results

$\mathbf{Q}=$ Bad Instrument Quality Control, Result is OK

$\mathbf{R}=$ Results are Unusable, Resampling is Necessary

$\mathbf{U}=$ Result is less than Minimum Detectable Activity 


\section{Becintel Nevada Corporation}

ANALYTICAL SERVICES LABORATORY

P.O.Box 3936, N. Las Vegas, NV 89036

Page 3

Reported to: Area 6 Decon Pond Closure

Remediation Projects

Report Date: 6-JUL-9B

J. L. Smith, M/S NTS306

Sample Delivery Group: B429

Batch: 0816

Program: 117

Report No. :

\begin{tabular}{|c|c|c|c|c|c|c|c|c|c|c|c|c|c|c|c|c|}
\hline $\begin{array}{c}\text { Sample } \\
* \cdots \cdots \\
\text { *..... Identification *.. }\end{array}$ & Isotope & Analysis & Hesult & $\begin{array}{c}\text { Error } \\
\%\end{array}$ & $\begin{array}{l}\text { Oual } \\
\text { Flag }\end{array}$ & MDA & \begin{tabular}{|l|} 
Result \\
Units
\end{tabular} & $\begin{array}{l}\text { Analysis } \\
\text { Date }\end{array}$ & $\begin{array}{l}\text { Sample } \\
\text { Coll Date }\end{array}$ & Size & \begin{tabular}{|l|} 
Size \\
Units
\end{tabular} & \begin{tabular}{|l|} 
Type \\
Matrix
\end{tabular} & \begin{tabular}{|l|} 
Tracer \\
Yield $\%_{6}$
\end{tabular} & \begin{tabular}{|l|} 
Spikg \\
Recv $\%$
\end{tabular} & $\begin{array}{l}\text { System } \\
\text { Detector }\end{array}$ & $\begin{array}{l}\text { Packet-Item } \\
\text { Sample }\end{array}$ \\
\hline QA SP:NAS-A0271 & $\operatorname{Co60}$ & GAM20 & $3.03 E+05$ & $8.5 E+00$ & & $1.8 E+02$ & $\mathrm{pCi}$ & $06-02-98$ & $04-01-92$ & $1.00 E+00 \mid$ & $\mathrm{sm}$ & QUAL & & 101.6 & $05-01$ & $00534-3-14370$ \\
\hline QA SP:NAS-A0271 & CS137 & GAM2O & $2.06 \mathrm{E}+05$ & B. $1 E+\infty$ & & $8.7 E+01$ & ${ }_{\mathrm{pCi}}$ & $06-0298$ & $04-01-92$ & $1.00 E+\infty 0$ & sm & QUAL & & 101.5 & $05-01$ & $00534 \cdot 3 \cdot 14370$ \\
\hline
\end{tabular}

\section{Comment:}

Data generated from analyses of samples submitted on 6/2/98.

Gross alpha and gross beta determinations are performed on

small aliquots. Data may not represent the sample as a whole.

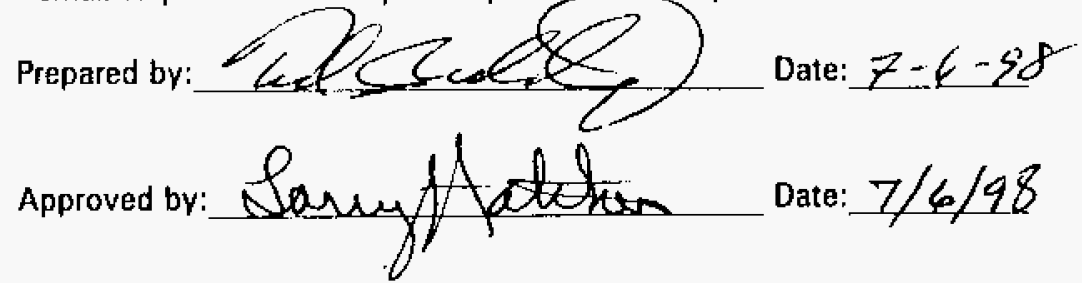

Qualification Flags:

$E=$ Estimated Quantity

Note: \% Error is the 2.0 Sigma Error

$\mathrm{H}=$ High Recovery for Sample

$J=$ Result is less than the RDL

$L=$ Low Recovery for Sample

$P=$ Preliminary Results

$\mathrm{Q}=$ Bad Instrument Quality Control, Result is $\mathrm{OK}$

$\mathrm{R}=$ Results are Unusable, Resampling is Necessary

$\mathrm{U}=$ Result is less than Minimum Detectable Activity 


\section{Bechtel Nevada Corporation}

\section{ANALYTICAL SERVICES LABORATORY}

P.O.Box 3936, N. Las Vegas, NV 89036

Reported to: Area 6 Decon Pond Closure

Remediation Projects

J. L. Smith, M/S NTS306

Report Date: 6-JUL-98

Sample Delivery Group: 8429

Batch: M559

Program: 720

Report No. :

\begin{tabular}{|c|c|c|c|c|c|c|c|c|c|c|c|c|c|c|c|c|}
\hline $\begin{array}{c}\text { Sample } \\
\ldots . . . \\
\text {..... Identification .... }\end{array}$ & isotope & Analysis & Result & $\begin{array}{l}\text { Error } \\
\%\end{array}$ & $\begin{array}{l}\text { Qual } \\
\text { Flag }\end{array}$ & MDA & $\begin{array}{l}\text { Result } \\
\text { Units }\end{array}$ & $\begin{array}{c}\text { Analysis } \\
\text { Date }\end{array}$ & $\begin{array}{l}\text { Sample } \\
\text { Coll Date }\end{array}$ & Sizo & $\begin{array}{r}\text { Size } \\
\text { Units }\end{array}$ & \begin{tabular}{|c|} 
Type \\
Matrix
\end{tabular} & $\begin{array}{l}\text { Tracer } \\
\text { Yibld } \%\end{array}$ & \begin{tabular}{|l|} 
Spike \\
Recr \%
\end{tabular} & $\left|\begin{array}{l}\text { System } \\
\text { Detector }\end{array}\right|$ & $\begin{array}{l}\text { Packet-Item } \\
\text { Sample }\end{array}$ \\
\hline DECON P1-6 & Gross Alpha & Gross A & $1.26 \mathrm{E}+01$ & $1.9 E+01$ & & $2.3 E+00$ & pCilg & 07-03-98 & $06-01.98$ & 5.00E-01 & $\mathrm{gm}$ & soll o & & & 14-01 & G9099-9.7507 \\
\hline DECON P2-6 & Gross Alpha & Gross A & $1.82 \mathrm{E}+01$ & $1.6 \mathrm{E}+01$ & & $2.5 E+\infty$ & $\mathrm{pCi} / \mathrm{g}$ & 07-03-98 & $06-01-98$ & $5.00 E-01$ & gm & solt G & & & 1401 & G9099-2-75072 \\
\hline DECONWT-6 & Gross Alpha & Grosa A & $1.64 E+01$ & $1.6 E+01$ & & $2.2 \mathrm{E}+\mathrm{OD}$ & pCilg & 07-02-98 & $06-01-98$ & 5.00E-01 & $\mathrm{gm}$ & soll G & & & $14-01$ & G9099-0.75068 \\
\hline OA REA & Gross Alpha & Gross A & $1.65 \mathrm{E}-02$ & $1.4 E+03$ & $u$ & $4.2 \mathrm{E}-01$ & $\mathrm{pCi}$ & 07.02 .98 & $06-02-98$ & $|1.00 E+\infty|$ & $8 m$ & QUAL & & & $14-01$ & $69100.0-06776$ \\
\hline OA SP:GROSSAB $\quad 05$ & Gross Alpha & Gross A & $3.38 E+00$ & $1.6 \mathrm{E}+01$ & & $4.2 \mathrm{E}-01$ & $\mathrm{pCi}$ & $07-02-98$ & $01-08-98$ & $1.00 E+00$ & $\mathrm{sm}$ & OUAL & & 98.6 & $14-01$ & G9100-1-06777 \\
\hline DECON P1-6 & Gross Bata & Gross B & $9.69 \mathrm{E}+00$ & $1.6 \mathrm{E}+01$ & & $2.2 E+\infty$ & $\mathrm{pCi} / \mathrm{g}$ & $07-03-98$ & $06-01-98$ & 5.00E-01 & gm & soll g & & & $14-01$ & G9099-1-75070 \\
\hline DECON $22-6$ & Gross Bata & Gross B & $1.21 E+01$ & $1.3 E+01$ & & $2.2 \mathrm{E}+00$ & $\mathrm{pCil} / \mathrm{g}$ & 07-03-98 & $06-01-98$ & $5.00 \mathrm{E}-01$ & $\mathrm{gm}$ & solt G & & & 14-01 & G9099-2-75072 \\
\hline DECON W1-6 & Gross Beta & Gross B & $4.90 E+01$ & $|4.7 E+\infty|$ & & $2.2 E+\infty$ & $\mathrm{pCi} / \mathrm{g}$ & $07-02.98$ & $06-01-98$ & $5.00 E-01$ & $\mathrm{gm}$ & Son G & & & $14-01$ & G9099-0-75068 \\
\hline QA AEA & Gross Beta & Gross B & $-2.67 E \cdot 01$ & $2.1 \mathrm{E}+02$ & $\mathbf{U}$ & $9.8 \mathrm{E}-01$ & $\mathrm{pCi}$ & $07-02-98$ & $06-02-98$ & $|1.00 E+\infty 0|$ & $8 \mathrm{~m}$ & QUAL & & & 14.01 & $69100-0-06776$ \\
\hline OA SP:GROSSAB 05 & Gross Beta & Gross B & $4.65 E+00$ & $1.5 E+01$ & & $9.8 E \cdot 01$ & $\rho \mathrm{Ci}$ & 07-02-98 & $01-06-98$ & $1.00 E+00$ & $\mathrm{sm}$ & QUAL & & 89.0 & 14.01 & G9100-1-06777 \\
\hline
\end{tabular}

\section{Comment:}

Data generated from analyses of samples submitted on 6/2/98.

Gross alpha and gross beta determinations are performed on

small aliquots. Data may not represent the sample as a whole.

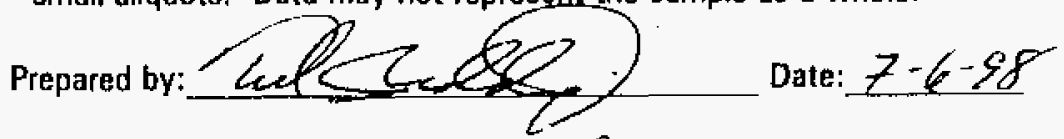

Approved by: Date: $7 / 6 / 98$
Qualification Flags:

Note: \% Error is the 2.0 Sigma Error

$E=$ Estimated Quantity

$H=$ High Recovery for Sample

$J=$ Result is less than the RDL

$L=$ Low Recovery for Sample

$\mathrm{P}=$ Preliminary Results

$\mathrm{O}=$ Bad Instrument Quality Control, Result is OK

$\mathbf{R}=$ Results are Unusable, Resampling is Necessary

$\mathrm{U}=$ Result is less than Minimum Detectablo Activity 


\section{SDG B435 \\ RADIOANALYTICAL \\ RESULTS}




\section{Beuhtel Nevada Corporation}

\section{ANALYTICAL SERVICES LABORATORY}

P.O.Box 3936, N. Las Vegas, NV 89036

Reported to: Area 6 Decon Pond Closure

Remediation Projects

Report Date: 6-JUL-98

J. L. Smith, M/S NTS306

Sample Delivery Group: 8435

Batch: 0818

Program: 720

Report No. :

\begin{tabular}{|c|c|c|c|c|c|c|c|c|c|c|c|c|c|c|c|c|}
\hline 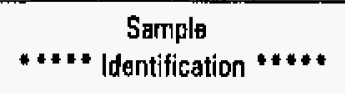 & Isotope & Analysis & Result & $\begin{array}{c}\text { Error } \\
\%\end{array}$ & $\begin{array}{l}\text { Qual } \\
\text { Flag }\end{array}$ & MDA & $\begin{array}{l}\text { Result } \\
\text { Units }\end{array}$ & $\begin{array}{c}\text { Analysis } \\
\text { Date }\end{array}$ & \begin{tabular}{|l} 
Sampla \\
Coll Date
\end{tabular} & Sizo & \begin{tabular}{|l|} 
Size \\
Units
\end{tabular} & \begin{tabular}{|c|} 
Type \\
Matrix
\end{tabular} & \begin{tabular}{|l|} 
Tracer \\
Yield \%
\end{tabular} & $\begin{array}{l}\text { Spike } \\
\text { Hecv \% }\end{array}$ & $\begin{array}{l}\text { System } \\
\text { Detector }\end{array}$ & $\begin{array}{l}\text { Packat-Item } \\
\text { Sample }\end{array}$ \\
\hline DECON P3-6 & AM241 & GAM20 & $1.73 E+00$ & $3.6 \mathrm{E}+01$ & & $2.9 \mathrm{E}-01$ & pCilg & 06-03-98 & 06-02-98 & $6.42 E+02$ & gm & soll G & & & $05 \cdot 01$ & G9115-0-75077 \\
\hline DECON P3-6 & K 40 & GAM20 & $2.27 E+01$ & $2.0 E+01$ & & $1.3 E+\infty 0$ & $\mathrm{pCi} / \mathrm{g}$ & $06-03-98$ & 06-02-98 & $6.42 E+02$ & gm & soll G & & & 05-01 & $69115-0-75077$ \\
\hline DECON P3-6 & RA226 & GAM20 & $1.30 \mathrm{E}+00$ & $2.7 \mathrm{E}+01$ & & $1.8 \mathrm{E}-01$ & $\mathrm{pCl} / \mathrm{g}$ & 06-03-98 & $06-02-98$ & $6.42 E+02$ & gm & soll G & & & 05.01 & $69115-0-75077$ \\
\hline DECON P3-6 & $\mathrm{TH} 228$ & GAM20 & $2.14 \mathrm{E}+00$ & $2.0 \mathrm{E}+01$ & & 1.5E-01 & $\mathrm{pCl} / \mathrm{g}$ & 06-03-98 & 06-02-98 & $6.42 E+02$ & $\mathrm{gm}$ & soll G & & & 05-01 & G9115-0-75077 \\
\hline DECON P3-6 & TH232 & GAM2O & $2.36 E+00$ & $3.0 E+01$ & & $2.4 E-01$ & DCi/g & 06.03.98 & 06-02-98 & $6.42 E+02$ & $\mathrm{gm}$ & soll G & & & $05-01$ & G9115-0.76077 \\
\hline DECON P4-6 & $K 40$ & GAM20 & $2.4 \mathrm{BE}+01$ & $1.9 E+01$ & & $1.2 \mathrm{E}+\infty$ & $\mathrm{pCl} / \mathrm{g}$ & 06-03-98 & $06-02-98$ & $6.61 E+02$ & $\mathrm{gm}$ & soll. G & & & 05-01 & G9116-1-75079 \\
\hline DECON P4-6 & HA226 & GAM20 & $1.44 E+00$ & $2.7 \mathrm{E}+01$ & & 1.6E-01 & $\mathrm{pCi} / \mathrm{\theta}$ & 06-03-98 & $06-02-98$ & $6.61 E+02$ & $\mathrm{gm}$ & solt G & & & $05-01$ & G9115-1-75079 \\
\hline DECON P4-6 & $\mathrm{TH} 228$ & GAM20 & $2.64 \mathrm{E}+00$ & $1.8 E+01$ & & 1.5E-01 & $\mathrm{pCi} / \mathrm{g}$ & 06-03-98 & $06-02-98$ & $6.61 E+02$ & gm & soll G & & & 05-01 & $69115-1-75079$ \\
\hline DECON P4-6 & TH232 & GAM20 & $2.42 E+00$ & $3,0 E+01$ & & $2.8 \mathrm{E}-01$ & $\mathrm{pCi} / \mathrm{g}$ & $06.03-98$ & $06-02-98$ & $6.61 E+02$ & $\mathrm{gm}$ & soll 6 & & & $05-01$ & G9116-1.75079 \\
\hline DECON P4-6 MS/MSD & $K 40$ & GAM20 & $2.13 \mathrm{E}+01$ & $2.0 \mathrm{E}+01$ & & $1.2 E+00$ & $\mathrm{pC}$ Ci/g & $06-03-98$ & $06-02-98$ & $6.61 E+02$ & am & solL G & & & 05-01 & G9115-2-75081 \\
\hline
\end{tabular}

Comment:

Data generated from analyses of samples submitted on 6/3/98.

Gross alpha/beta determinations are performed on small sample

aliqquots. Data may not represent the sample as a whole.

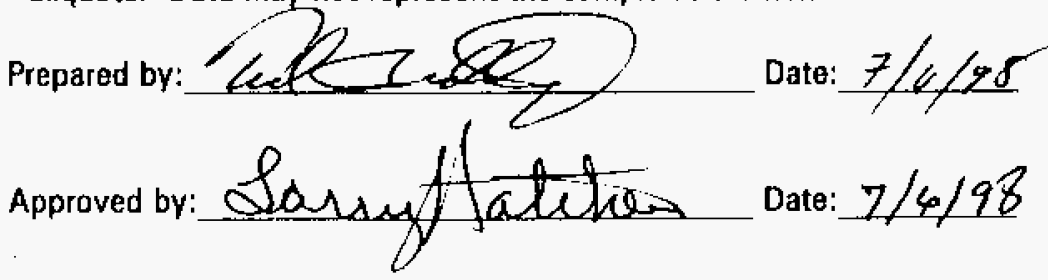

Qualification Flags:

$E=$ Estimated Quantity

$\mathrm{H}=$ High Recovery for Sample

$\mathrm{J}=$ Result is less than the RDL

$\mathrm{L}=$ Low Recovery for Sample

$P=$ Preliminary Results

$\mathrm{Q}=$ Bad Instrument Quality Control, Result is OK

$\mathrm{R}=$ Results are Unusable, Resampling is Necessary

$\mathrm{U}=$ Result is less than Minimum Detectable Activity

Note: \% Error is the 2.0 Sigma Error 


\section{Bechtel Nevada Corporation}

ANALYTICAL SERVICES LABORATORY

P.O.Box 3936, N. Las Vegas, NV 89036

Reported to: Area 6 Decon Pond Closure

Remediation Projects

Report Date: 6-JUL-98

J. L. Smith, M/S NTS306

Batch: 0818

Program: 720

Report No.:

\begin{tabular}{|c|c|c|c|c|c|c|c|c|c|c|c|c|c|c|c|c|}
\hline $\begin{array}{c}\text { Sample } \\
\text {..... Identification }\end{array}$ & Isotope & Antylysis & Result & $\begin{array}{l}\text { Error } \\
\%\end{array}$ & $\begin{array}{l}\text { Qual } \\
\text { Flag }\end{array}$ & MDA & $\begin{array}{l}\text { Result } \\
\text { Units }\end{array}$ & $\begin{array}{c}\text { Analysis } \\
\text { Date }\end{array}$ & \begin{tabular}{|c} 
Sampla \\
Coll Date
\end{tabular} & Size & \begin{tabular}{|c|} 
Sizg \\
Units
\end{tabular} & \begin{tabular}{|l|} 
Type \\
Matrix
\end{tabular} & $\begin{array}{l}\text { Tracer } \\
\text { Yield \& }\end{array}$ & $\begin{array}{l}\text { Spikg } \\
\text { Hecv } \%\end{array}$ & $\mid \begin{array}{l}\text { System } \\
\text { Detertor }\end{array}$ & \begin{tabular}{|l} 
Packet-Item \\
Sample
\end{tabular} \\
\hline DECON P4-6 MS/MSD & RA226 & GAM20 & $1.29 \mathrm{E} \uparrow 00$ & $2.9 \mathrm{E}+01$ & & $1.6 \mathrm{E}-01$ & pCilg & $06-03.98$ & $06-02-98$ & $6.61 \mathrm{E}+02$ & gn & soll $\mathrm{G}$ & & & 05.01 & G9115-2-75081 \\
\hline DECON P4-6 MS/MSD & TH228 & GAM20 & $2.09 E+\infty$ & $2.0 E+01$ & & $1,5 \mathrm{E}-01$ & $\mathrm{pCi} / \mathrm{g}$ & 06-03-98 & $06-02-99$ & $|6.61 E+02|$ & $g m$ & SOIL G & & & 05.01 & G9115-2-75081 \\
\hline DECON P4-6 MS/MSD & TH232 & GAM20 & $1.59 \mathrm{E}+00$ & $3.7 E+01$ & & $2.4 \mathrm{E}-01$ & $p$ Cigg & 06-03.98 & $06-02-98$ & $6.61 E+02 \mid$ & $\mathrm{gm}$ & SOll G & & & $05-01$ & G9115-2-75081 \\
\hline DECON P5-6 & $K 40$ & GAM20 & $2.39 \mathrm{E}+\mathrm{OT}$ & $1.9 \mathrm{E}+01$ & & $1.2 E+00$ & $\mathrm{pClig}$ & 06-03-98 & 06-02-98 & $6.73 E+02$ & gm & SOll G & & & $05-01$ & G91 15-3-75083 \\
\hline DECON P5-6 & AA226 & GAM20 & $1.39 E+00$ & $2.6 E+01$ & & $1.6 \mathrm{E}-01$ & $\mathrm{pCl} / \mathrm{g}$ & 06-03-98 & 06-02-98 & $6.73 \mathrm{E}+02$ & $\mathrm{gm}$ & SOIL G & & & 05.01 & G9115-3-75083 \\
\hline DECON P5-6 & TH228 & GAM20 & $2.71 E+00$ & $1.8 E+01$ & & $1,5 \mathrm{E}-01$ & $\mathrm{pCi} / \mathrm{g}$ & 06-03.98 & 06-02-98 & $6.73 E+02$ & $\mathrm{gm}$ & SOIL G & & & 05.01 & G9115-3-75083 \\
\hline DECON P5-6 & TH232 & GAM20 & $1.98 E+00$ & $3.3 E+01$ & & 2.8E-01 & pCilg & 06-03-98 & 06-02.9B & $6.73 E+02$ & am & solt G & & & 05.04 & G9115-3-76083 \\
\hline QA BKG EMPTY BTL U05 & No Nucl Det & GAM20 & $0.00 E+00$ & $0.0 E+00$ & U & $0.0 \mathrm{E}+00$ & NA & |06-03-98 & 07-01-95 & $1,000+00 \mid$ & 艘 & QUAL & & & 05-01 & $00539-0.00201$ \\
\hline QA BKG EMPTY BTL U05 & AM241 & GAM20 & $0.00 E+\infty 0$ & $0.0 \mathrm{E}+00$ & $u$ & $2.2 E+01$ & $\mathrm{pCi}$ & $08-03-98$ & 07.01-95 & $1.00 E+00$ & $\mathrm{~mm}$ & QUAL & & & 05.01 & $00539-0-00201$ \\
\hline OA BKG EMPTY BTL U05 & $\mathrm{CO} 60$ & GAM20 & $0.00 E+00$ & $0.0 E+00$ & U & $1.3 E+01$ & $\mathrm{pCl}$ & 06-03-98 & 07-01-95 & $|1.00 E+00|$ & $s m$ & OUAL & & & 05-01 & $00539-0-00201$ \\
\hline
\end{tabular}

\section{Comment:}

Data generated from analyses of samples submitted on $6 / 3 / 98$.

Gross alpha/beta determinations are performed on small sample

aliquots. Data may not represent the sample as a whole.

Prepared by:

Approved by: Sousy Aativon Date: $7 / 6 / 98$
Qualification Flags:

Note: \% Error is the 2.0 Sigma Error

$E$ = Estimated Quantity

$\mathrm{H}=$ High Recovery for Sample

$J=$ Result is less than the RDL

$L=$ Low Recovery for Sample

$P=$ Preliminary Results

$\mathrm{Q}=$ Bad Instrument Quality Control, Result is OK

$R=$ Results are Unusable, Resampling is Necessary

$\mathbf{U}=$ Result is less than Minimum Detectable Activity 


\section{Bechtel Nevada Corporation}

ANALYTICAL SERVICES LABORATORY

P.O.Box 3936, N. Las Vegas, NV 89036

Reported to: Area 6 Decon Pond Closure

Remediation Projects

Report Date: 6-JUL-98

J. L. Smith, M/S NTS306

Sample Delivery Group: $\mathbf{8 4 3 5}$

Batch: 0818

Program: 500

Report No. :

\begin{tabular}{|c|c|c|c|c|c|c|c|c|c|c|c|c|c|c|c|c|}
\hline $\begin{array}{c}\text { Sample } \\
\text {..... Identification } \\
\text {...... }\end{array}$ & Isotope & Analysis & Result & $\begin{array}{l}\text { Error } \\
\%\end{array}$ & $\begin{array}{l}\text { Oual } \\
\text { Flag }\end{array}$ & MDA & $\begin{array}{l}\text { Result } \\
\text { Units }\end{array}$ & $\begin{array}{c}\text { Analysis } \\
\text { Date }\end{array}$ & \begin{tabular}{|} 
Sample \\
Coll Date
\end{tabular} & Size & $\begin{array}{r}\text { Size } \\
\text { Units }\end{array}$ & \begin{tabular}{|l} 
Typs \\
Matrix
\end{tabular} & $\left|\begin{array}{l}\text { Tracer } \\
\text { Yield } \%\end{array}\right|$ & $\begin{array}{l}\text { Spikg } \\
\text { Recy \% }\end{array}$ & $\mid \begin{array}{l}\text { System } \\
\text { Detector }\end{array}$ & $\begin{array}{l}\text { Packet-Item } \\
\text { Sample }\end{array}$ \\
\hline OA BKG EMPTY BTL UO5 & $\operatorname{cs} 137$ & GAM20 & $0.00 E+00$ & $0.0 E+\infty$ & v & $1.0 E+01$ & $\mathrm{pCi}$ & $06-03-98$ & $07-01-95$ & $1.00 E+\infty \mid$ & $8 m$ & QUAL & & & $05-01$ & $00539-0-00201$ \\
\hline QA SP:NAS-A0271 & AM241 & GAM20 & $1.71 E+05$ & $8.8 E+00$ & & $2.7 E+02$ & $\mathrm{pCi}$ & |06.03.98 & 04-01-92 & $|1.00 E+00|$ & $8 m$ & QUAL & & 105.4 & $05-01$ & $00534-3-14425$ \\
\hline QA SP:NAS-A0271 & $\mathrm{C} 060$ & GAM20 & $3.04 E+05$ & $8.5 \mathrm{E}+00$ & & $2.0 E+02$ & $\mathrm{pCi}$ & |06-03-98 & $04-01-92$ & $|1.00 E+\infty 0|$ & $\mathbf{s m}$ & QUAL & & 102.0 & $05-01$ & $00534-3-14425$ \\
\hline OA SP:NAS-A0271 & $\operatorname{cs} 137$ & GAM20 & $2.07 E+05$ & $8.1 E+\infty$ & & $9.2 E+01$ & $\mathrm{pCi}$ & |06-03-9B & $0401-92$ & $1.00 E+00 \mid$ & $\operatorname{sm}$ & QUAL & & 102.1 & 05.01 & $00534-3-14425$ \\
\hline
\end{tabular}

Comment:

Data generated from analyses of samples submitted on $6 / 3 / 98$.

Gross alpha/beta determinations are performed on small sample

aliquots. Data may not represent the sample as a whole.

Prepared by:

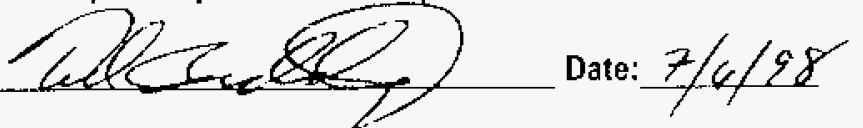

Approved by
Qualification Flags:

$\mathbf{E}=$ Estimated Quantity

$H=$ High Recovery for Sample

$\mathrm{J}=$ Result is less than the RDL

$L=$ Low Recovery for Sample

$\mathrm{P}=$ Preliminary Results

Q = Bad Instrument Quality Control, Result is OK

$R=$ Results are Unusable, Resampling is Necessary

$\mathrm{U}=$ Result is less than Minirnum Detectable Activity
Note: \% Error is the 2.0 Sigma Error 


\section{Bechtel Nevada Corporation}

\section{ANALYTICAL SERVICES LABORATORY}

P.O.Box 3936, N. Las Vegas, NV 89036

Reported to: Area 6 Decon Pond Closure

Remediation Projects

Report Date: 6-JUL-98

J. L. Smith, M/S NTS306

Sample Delivery Group: B435

Batch: M559

Program: 720

Report No.:

\begin{tabular}{|c|c|c|c|c|c|c|c|c|c|c|c|c|c|c|c|c|}
\hline $\begin{array}{c}\text { Sample } \\
\ldots . . . * \text { lớntification .....* }\end{array}$ & Isotope & Analysis & Result & $\begin{array}{c}\text { Error } \\
\%\end{array}$ & $\begin{array}{l}\text { Qual } \\
\text { Flag }\end{array}$ & MDA & $\begin{array}{l}\text { Restlt } \\
\text { Units }\end{array}$ & $\begin{array}{c}\text { Analysis } \\
\text { Date }\end{array}$ & $\begin{array}{c}\text { Sample } \\
\text { Co!l Date }\end{array}$ & Size & $\begin{array}{l}\text { Size } \\
\text { Units }\end{array}$ & $\begin{array}{c}\text { Type } \\
\text { Matrix }\end{array}$ & \begin{tabular}{|l|} 
Tracer \\
Yiald कo
\end{tabular} & $\begin{array}{l}\text { Spiko } \\
\text { Pecv \% }\end{array}$ & \begin{tabular}{|l|} 
System \\
Detector
\end{tabular} & $\begin{array}{l}\text { Packet-Item } \\
\text { Sample }\end{array}$ \\
\hline DECON P3-6 & Gross Alpha & Gross A & $1.52 \mathrm{E}+01$ & $1.6 E+01$ & & $1.9 E+\infty$ & $\mathrm{pCilg}$ & 07.03.98 & $06-02-98$ & $5.00 E-01$ & gm & soll G & & & $14-01$ & G9115.0-75076 \\
\hline DECON P4-6 & Gross Alpha & Gross A & $1.39 E+01$ & $1.8 E+01$ & & $2.2 E+\infty$ & pCirg & 07-03-98 & $06-02-98$ & $5.00 \mathrm{E}-01$ & $\operatorname{gm}$ & Soll G & & & $14-01$ & G9115-1-75078 \\
\hline DECON P4-6 MS/MSD & Gross Alphs & Gross A & $1.60 \mathrm{E}+01$ & $1.8 E+01$ & & $2.6 E+00$ & $\mathrm{pCl} / \mathrm{g}$ & 07-03-98 & $06-02-98$ & $5.00 \mathrm{E}-01$ & $\mathrm{gm}$ & SOIL G & & & 14-01 & G9115-2-75080 \\
\hline DECON P5-6 & Gross Alpha & Gross A & $1.30 E+01$ & $1.8 E+01$ & & $2.0 \mathrm{E}+00$ & pCil/g & 07-03-98 & $06-02-98$ & $5.00 \mathrm{E}-01$ & $\mathrm{gm}$ & SOIL G & & & $14-01$ & G9715-3-75082 \\
\hline QA REA & Gross Alpha & Gross A & $1.65 \mathrm{E}-02$ & $1.4 E+03$ & $\mathbf{u}$ & $4,2 \mathrm{E}-01$ & $\mathrm{pCi}$ & 07-02-98 & 06-02-98 & $1.00 E+\infty 0$ & $\mathrm{sm}$ & QUAL & & & $14-01$ & G9100-0.06776 \\
\hline QA SP:GROSSAB $\quad 05$ & Gross Alphe & Gross A & $3.3 B E+\infty 0$ & $1,6 E+01$ & & 4.2E-01 & $\mathrm{pCi}$ & $07.02-98$ & 01-06-98 & $1,00 E+00$ & $\mathrm{sm}$ & OUAL & & 98.6 & $14-01$ & $69100-1-06777$ \\
\hline DECON P3-6 & Gross Beta & Gross B & $9.03 E+00$ & $1.7 E+01$ & & $2.2 E+00$ & $\mathrm{pCilg}$ & 07-03-98 & $06-02-98$ & $5.00 E-01$ & $\mathrm{gm}$ & soll $G$ & & & $14-01$ & G9115-0-75076 \\
\hline DECON P4-6 & Gross Beta & Gross B & $1.05 E+01$ & $1.5 E+01$ & & $2.2 E+00$ & pCilg & 07.03-98 & 06-02-98 & $5.00 \mathrm{E}-01$ & am & soll G & & & $14-01$ & G9115-1-75078 \\
\hline DECON P4-6 MS/MSD & Gross Beta & Gross B & $1.06 E+01$ & $1.5 E+01$ & & $2.2 \mathrm{E}+00$ & pCi/g & 07-03-98 & $06-02-98$ & $5.00 \mathrm{E}-01$ & $\mathrm{gm}$ & soll g & & & $14-01$ & G9115-2-75080 \\
\hline DECON P5-6 & Gross Beta & Gross B & $1.05 E+01$ & $1.5 E+01$ & & $2.2 \mathrm{E}+00$ & $\mathrm{pCilg}$ & 07.03-98 & $06-02.98$ & $5.00 \mathrm{E}-01$ & $\mathrm{gm}$ & soll G] & & & 14-01 & G9115-3-75082 \\
\hline
\end{tabular}

Comment:

Data generated from analyses of samples submitted on 6/3/98.

Gross alpha/beta determinations are performed on small sample

aliquots. Data may not represent the sample as a whole.

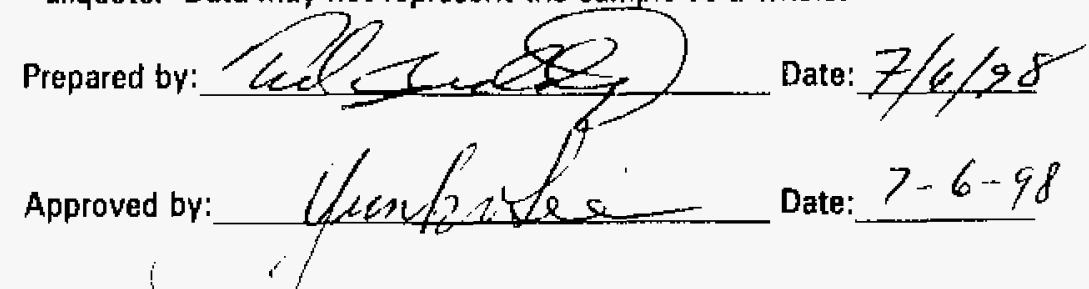

Qualification Flags:

Note: \% Error is the 2.0 Sigma Error

$E=$ Estimated Quantity

$H=$ High Recovery for Sample

$J=$ Result is less than the RDL.

$L=$ Low Recovery for Sample

$P=$ Preliminary Results

$0=$ Bad Instrument Quality Control, Result is OK

$\mathrm{A}=$ Results are Unusable, Resampling is Necessary

$\mathrm{U}=$ Result is less than Minimum Detectable Activity 


\section{Bechtel Nevada Corporation}

\section{ANALYTICAL SERVICES LABORATORY}

P.O.Box 3936, N. Las Vegas, NV 89036

Reported to: Area 6 Decon Pond Closure

Rernediation Projects

J. L. Smith, M/S NTS306

Report Date: 6 -JUL-98

Sample Delivery Group: B435

Batch: M559

Program: 888

Report No. :

\begin{tabular}{|c|c|c|c|c|c|c|c|c|c|c|c|c|c|c|c|c|}
\hline Sampla & Isotope & Analysis & Pessult & $\begin{array}{c}\text { Error } \\
\%\end{array}$ & $\begin{array}{l}\text { Oual } \\
\text { Flag }\end{array}$ & MDA & $\begin{array}{l}\text { Ressu!t } \\
\text { Units }\end{array}$ & $\begin{array}{c}\text { Analysis } \\
\text { Date }\end{array}$ & $\begin{array}{r}\text { Sample } \\
\text { Coll Date }\end{array}$ & Sizo & \begin{tabular}{|r|} 
Size \\
Units
\end{tabular} & $\begin{array}{l}\text { Type } \\
\text { Matrix }\end{array}$ & $\begin{array}{l}\text { Tracer } \\
\text { Yield } \%\end{array}$ & \begin{tabular}{|l|} 
Spikn \\
Recr $\%$ \\
\end{tabular} & $\begin{array}{l}\text { SYstern } \\
\text { Detector }\end{array}$ & $\begin{array}{l}\text { Packat-Item } \\
\text { Sample }\end{array}$ \\
\hline OA REA & Gross Beta & Gross B & $-2.67 \mathrm{E}-01$ & $2.1 E+02$ & u & 9. BE-01 & $\mathrm{pCi}$ & $07.02-98$ & $06-02-98$ & $1.00 E+\infty \mid$ & $\mathrm{sm}$ & OUAL & & & $14-01$ & G9100-0-06776 \\
\hline QA SP:GAOSSAB & Gross Bata & Gross B & $4.65 E+00$ & $1.5 \mathrm{E}+01$ & & 9.8E-01 & $\mathrm{pCi}$ & 07-02-98 & $01-06-98$ & $1.00 E+00$ & $\mathrm{sm}$ & QUAL & & 89.0 & $14-01$ & 69100-1-06777 \\
\hline
\end{tabular}

\section{Comment:}

Data generated from analyses of samples submitted on 6/3/98.

Gross alpha/beta determinations are performed on small sample

aliquots. Data may not represent the sample as a whole.

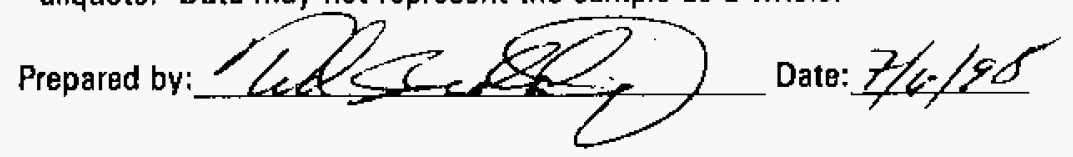

Approved by: Date: $7-6-98$
Oualification Flags:

Note: \% Error is the 2.0 Sigma Error

$E=$ Estimated Quantity

$\mathrm{H}=$ High Recovery for Sample

$\mathrm{J}=$ Result is less than the RDL

$L=$ Low Recovery for Sample

$P=$ Prelirninary Results

$\mathrm{O}=$ Bad Instrument Quality Control, Result is OK

$R=$ Results are Unusable, Resampling is Necessary

$\mathrm{U}=$ Result is less than Minimum Detectable Activity 


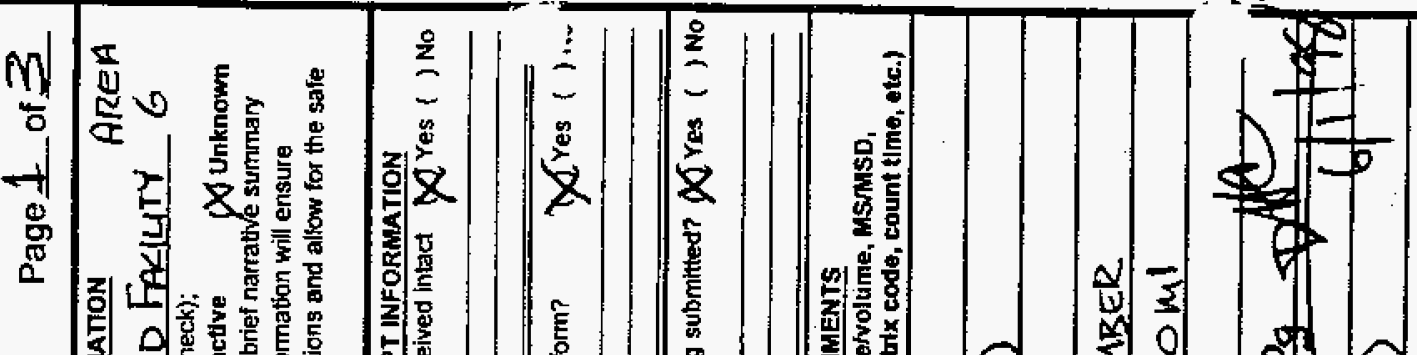




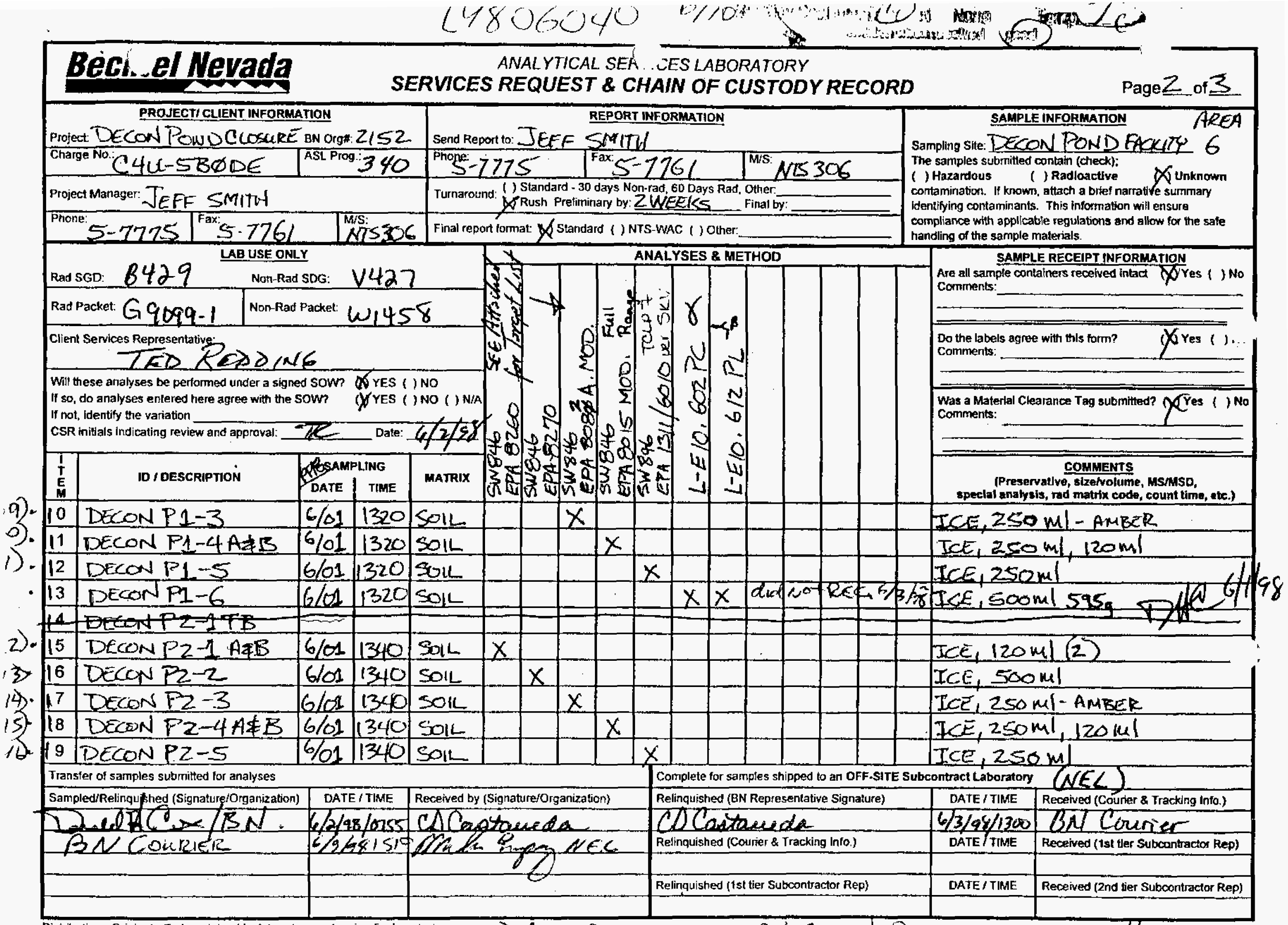




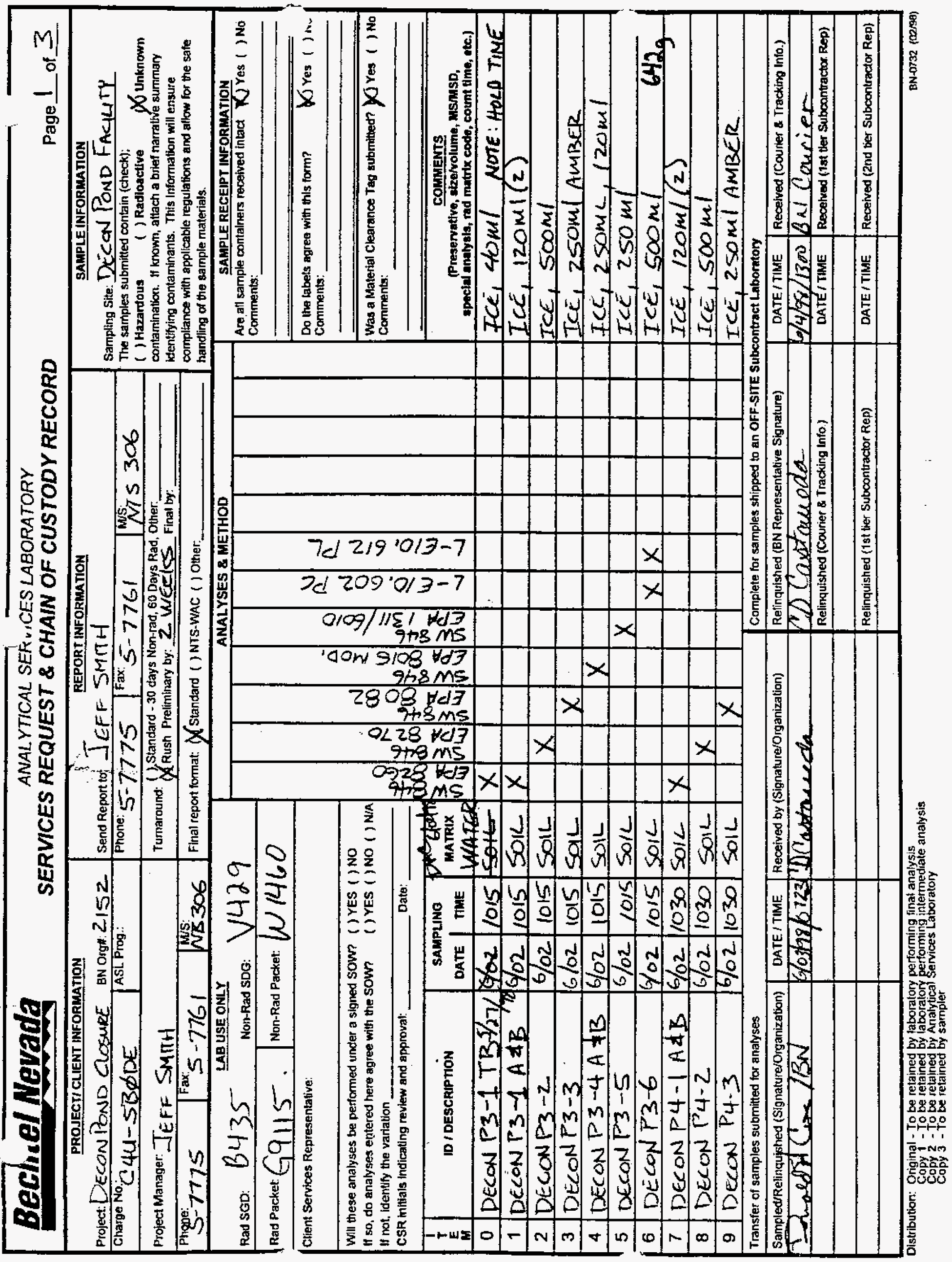




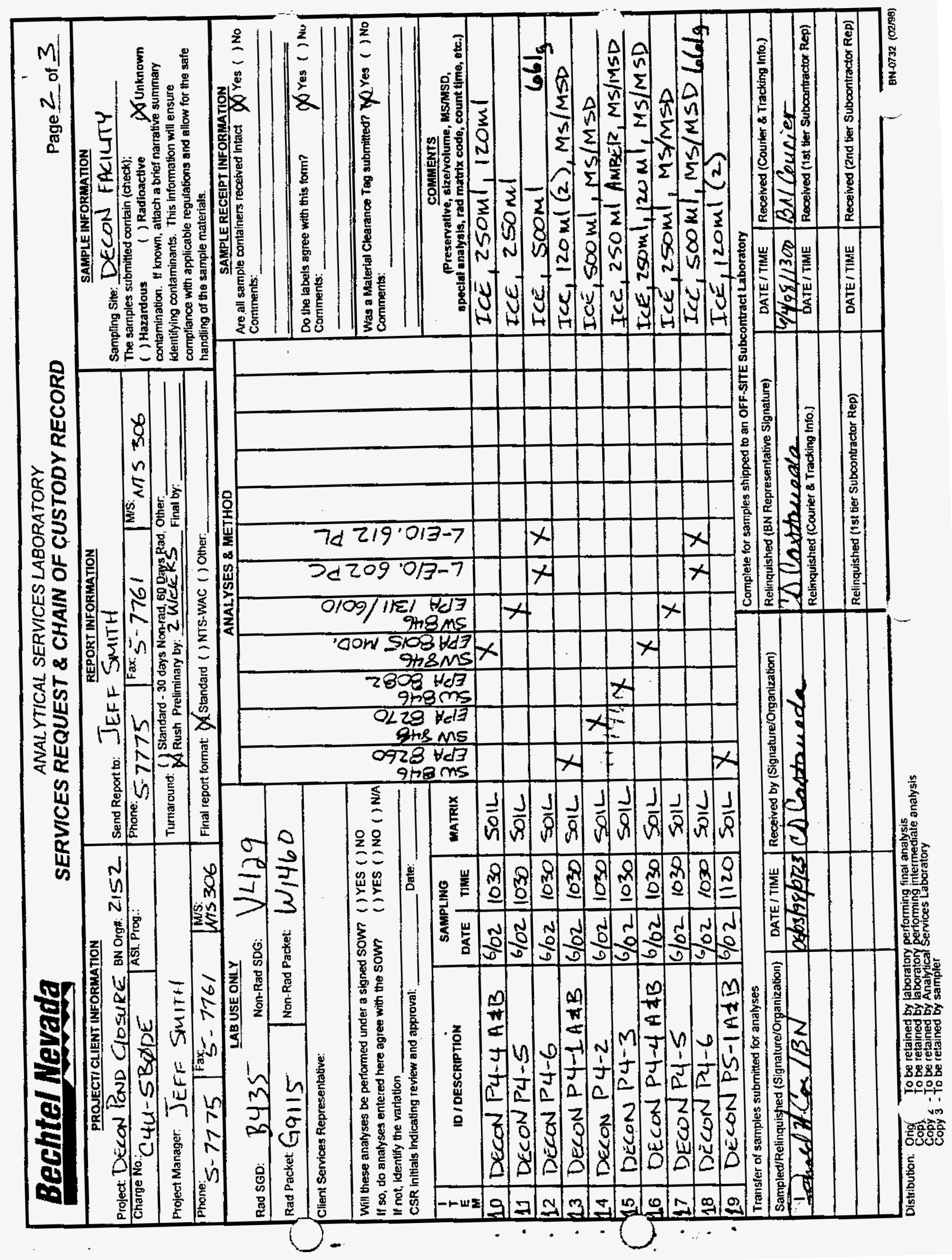


Project Decon PoND COOSRE BN Org: Z152 Send Report to: JEFF SMITH

Charge No $\mathrm{C} 4 \mathrm{U}-5 \mathrm{~B} \Phi \mathrm{DC}$

Project Manager: JEFF SMITH

\begin{tabular}{|c|c|}
\hline Phone: & Fax: \\
\hline
\end{tabular}

Phone: $5 \cdot 7775{ }^{\text {Fax }} 5.7761$

${ }^{\text {MS: }}$ TS 306

Turnaround: () Standard - 30 days Nontrad, 60 Days Rad, Other.

Q Rush Prelininary by: $Z$ WEEKS Final by: \begin{tabular}{|l|l}
\hline NIS 306 & Final report fomat $\phi$ standard ( ) NTS-WAC () Other.
\end{tabular}

Samping Site: DECON FACKCITY The samples submitted contain (check);
() Hazardous ( Radionctlve () Hazardous () Radloactlve OUnknown contamination. If krown, altach a brief nartelive sumurnery identifying contaminants. This Information will ensure complance with applicable regulations and allow for the safe handting of the sample materials.
Rad SGD: B435 $\frac{\text { LAB USE OMLY }}{\text { Non-Rad SDG: }} \sqrt{429}$

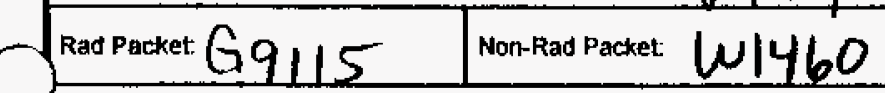

Clienl Services Representative:

Will these analyses be performed under a signed SOW? () YES () NO If so, do analyses entered here agree with the SOW7 () YES () NO () N/A If not, identify the variation CSR miltals indicating review and approval:

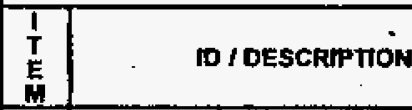

- 20 DECON P5-2

- 21 DECON P5-3

- 22 DECON P5-4 AEB

- 23 DeCON PS-S

24 Decon P5-6

$-16$

7

8

.9

Transfer of samples submitted for analyses

\begin{tabular}{|l|l|l|}
\hline Sampled/Relinquished (Signature/Organization) & DATE / TIME & Received by (Signature'Organization)
\end{tabular} Itraed $2 C_{A D N}$ Date:

1

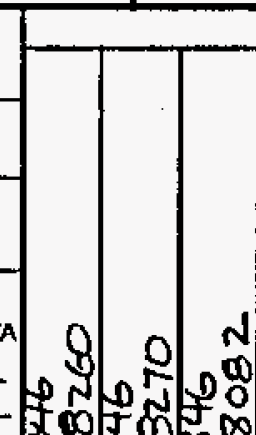

ANALYSES \& METHOD

\begin{tabular}{|c|c|c|c|}
\hline \multicolumn{2}{|c|}{ SAMPLiNo } & matRiX \\
DATE & TIME & \\
\hline
\end{tabular}

3

$6 / 021120$ $6 / 1120$ 6021120 $\% / 1120$ Sol $6 / 02,1120$

\begin{tabular}{|l|l}
$0 / 02$ & 1120 \\
& \\
& \\
&
\end{tabular}
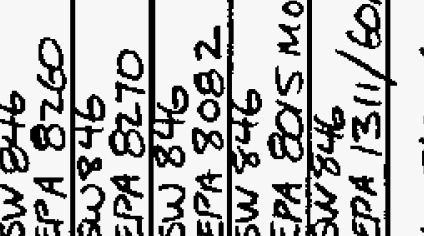

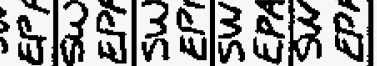

Soll
Soll
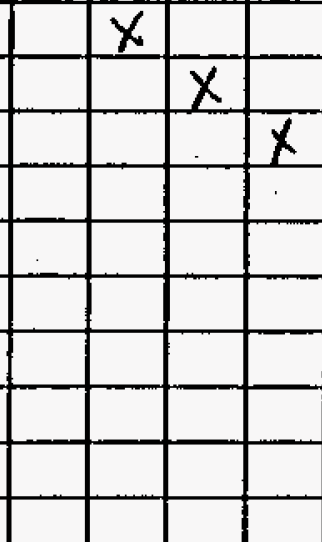

\begin{tabular}{l|l}
$x$ & \\
& $x$
\end{tabular} soll

SAMPLE RECEIPT INFORMATIOH Ars afl sample containers received intact 7 Yes ( ) No Comments:

C.

Do the labels agree with this form? (X) Yes ( ) Mu Comments:

Was a Material Clearance Tag submittedt $($ ) Yes ( ) No Comments:

COMMEMTS

(Preservative stzervolume, MrinsD,

spectal analysis, red matrix code, count time, wte.)

ICE, s00 ml

ICÉ, $250 \mathrm{ml}$ AMBER

ICE, $250 \mathrm{ml}, 120 \mathrm{ml}$

ICE, $250 \mathrm{ml}$

$I C E_{1} 500 \mathrm{ml}$

6739

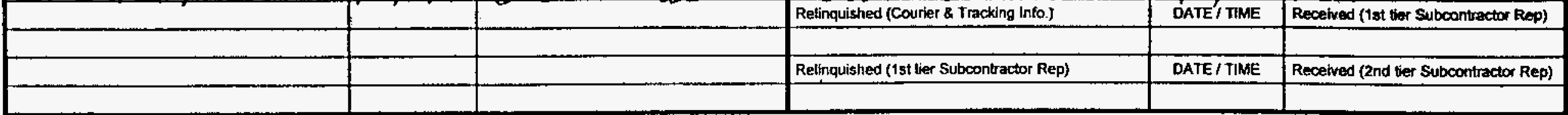

Complete for samples shipped to an OFF-SITE Subcontract Laboratory

\begin{tabular}{|l|l|l}
\hline Relinquished (BN Representative Signatture) & DATE / TIME & Received (Courier \& Tracking Info.)
\end{tabular}

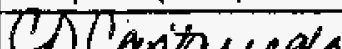

CDCaxtounde

Relfinquished (1st lier Subcontractor Rep) 


\section{VOLATILE \\ ORGANICS}

DATA 
NEL LABORATORIES
CLIENT: Bechtel Nevada
OJECT NAME: V431
CLIENT ID:
Decon W2-1 A\&B
DATE SAMPLED: $6 / 3 / 98$
YXOIECT NUMBER: 17777
NEL SAMPLE ID: L9806093-01

TEST: Volatile Organic Compounds by EPA SW846 Method 8260A, Sept. 1994

$\begin{array}{lrl}\text { MATRIX: Solid } & \text { EXTRACTED: } 6 / 11 / 98 & \text { ANALYST: Suzanne } \\ \text { DILUTION: } 1 & \text { ANALYZED: } 6 / 11 / 98 & \end{array}$

\begin{tabular}{|c|c|c|c|c|c|c|}
\hline PARAMETER & $\begin{array}{l}\text { Result } \\
\mu \mathrm{g} / \mathrm{kg}\end{array}$ & & $\begin{array}{l}\text { Reporting } \\
\text { Limit }\end{array}$ & PARAMETER & $\begin{array}{l}\text { Result } \\
\mu \mathrm{g} / \mathrm{kg}\end{array}$ & $\begin{array}{c}\text { Reporting } \\
\text { Limit }\end{array}$ \\
\hline Acetone & $\mathrm{ND}$ & & $25 . \mu \mathrm{g} / \mathrm{kg}$ & & & \\
\hline Benzene & $\mathrm{ND}$ & is & 5. $\mu \mathrm{g} / \mathrm{kg}$ & & & \\
\hline Bromodichloromethane & ND & Js & 5. $\mu \mathrm{g} / \mathrm{kg}$ & & & \\
\hline Btomoform & $\mathrm{ND}$ & Js & 5. $\mu \mathrm{g} / \mathrm{kg}$ & & & \\
\hline Bromomethane & $\mathrm{ND}$ & & 5. $11 \mathrm{~g} / \mathrm{kg}$ & & & \\
\hline 2-Butanone & ND & & 25. $\mu \mathrm{g} / \mathrm{kg}$ & & & \\
\hline Carbon disulfide & $\mathrm{ND}$ & & 5. $\mu \mathrm{g} / \mathrm{kg}$ & & & \\
\hline Carbon tetrachloride & ND & & 5. $\mu g / \mathrm{kg}$ & & & \\
\hline ChIorobenzene & 5 & Js & 5. $\mu \mathrm{g} / \mathrm{kg}$ & & & \\
\hline Chloroethane & ND & & 5. $\mu \mathrm{g} / \mathrm{kg}$ & & & \\
\hline Chloroform & $\mathrm{ND}$ & & 5. $\mu \mathrm{g} / \mathrm{kg}$ & & & \\
\hline Chloromethane & $\mathrm{ND}$ & & 5. $\mu g / k g$ & & & \\
\hline Dibromochloromethane & $\mathrm{ND}$ & is & 5. $\mu g / \mathrm{kg}$ & & & \\
\hline I.I-Dichloroethane (1.1-DCA) & $\mathrm{ND}$ & & 5. $\mu \mathrm{g} / \mathrm{kg}$ & & & \\
\hline 1.2-Dichloroethane (1,2-DCA) & ND & js & 5. $\mu \mathrm{g} / \mathrm{kg}$ & & & \\
\hline 1.1-Dichloroethene (1, I-DCE) & ND & & 5. $\mu \mathrm{g} / \mathrm{kg}$ & & & \\
\hline sis-1.2-Dichloroethene & ND & & 5. $\mu \mathrm{g} / \mathrm{kg}$ & & & \\
\hline s-1,2-Dichloroethene & ND & & 5. $\mu \mathrm{g} / \mathrm{kg}$ & & & \\
\hline 1.2-Dichloropropane & $\mathrm{ND}$ & Js & 5. $\mu \mathrm{g} / \mathrm{kg}$ & & & \\
\hline cis-1.3-Dichloropropene & ND & $\mathrm{Js}$ & 5. $\mu \mathrm{g} / \mathrm{kg}$ & & & \\
\hline Irans-1,3-Dichloropropene & $\mathrm{ND}$ & Js & 5. $\mu \mathrm{g} / \mathrm{kg}$ & & & \\
\hline Ethylbenzene & ND & Js & 5. $12 \mathrm{~g} / \mathrm{kg}$ & & & \\
\hline 2-Hexanone & ND & Js & 25. $\mu \mathrm{g} / \mathrm{kg}$ & & & \\
\hline Methylene chloride (Dichloromethane) & $\mathrm{ND}$ & $\mathbf{B}$ & 5. $\mu \mathrm{g} / \mathrm{kg}$ & & & \\
\hline 4-Nethyl-2-pentanone & ND & Js & 25. $\mu \mathrm{g} / \mathrm{kg}$ & & & \\
\hline Styrene- & ND & Js & 5. $\mu \mathrm{g} / \mathrm{kg}$ & & & \\
\hline 1.1.2.2-Tutrachlorocthane & $\mathrm{ND}$ & $\sqrt{5}$ & 5. $\mu \mathrm{g} / \mathrm{kg}$ & & & \\
\hline Tetrachlorothene (PCE) & $\mathrm{ND}$ & $\mathrm{J} 5$ & 5. $\mu \mathrm{g} / \mathrm{kg}$ & & & \\
\hline Toluene & ND & Js & 5. $\mu \mathrm{g} / \mathrm{kg}$ & & & \\
\hline 1,1, I-Trichloroethane (1,1,1-TCA) & ND & & 5. $\mu \mathrm{g} / \mathrm{kg}$ & & & \\
\hline 1,1,2-Trichloroethane (1,1,2-TCA) & ND & J5 & 5. $\mu \mathrm{g} / \mathrm{kg}$ & & & \\
\hline Trichloroethene (TCE) & ND & J5 & 5. $\mu g / \mathrm{kg}$ & & & \\
\hline Yinyl chloride & ND & & 6. $\mu \mathrm{g} / \mathrm{kg}$ & & & \\
\hline it Xylene & $\mathrm{ND}$ & j5 & 5. $\mu \mathrm{g} / \mathrm{kg}$ & & & \\
\hline m.p-Xylene & $\mathrm{ND}$ & $f_{s}$ & 5. $\mu \mathrm{g} / \mathrm{kg}$ & & & \\
\hline
\end{tabular}

ND - Not Detected

OUALITY CONTROL DATA:

\section{Surrogate}

4-Bromofluorobenzene

Dibromofluoromethane tene-d8

\section{\% Recovery}

50

107

78
Acceptable Range

$74-121$

$80-120$

$81-117$

This report shall not be reproduced except in full, without the written approval of the laboratory. 
NEL LABORATORIES

CLIENT: Bechtel Nevada

PROIECT NAME: V431

PROJECT NUMBER: 17777
CLIENT ID: Decon P6-1 TB

DATE SAMPLED: $6 / 3 / 98$

NEL SAMPLE ID: L9806093-06

TEST: Volatile Organic Compounds by EPA SW846 Method 8260B, Dec. 1996

MATRIX: Aqueous

DILUTION: 1
EXTRACTED: $6 / 9 / 98$

ANALYZED: $6 / 9 / 98$

ANALYST: Suzanne

\begin{tabular}{|c|c|c|c|c|c|}
\hline PARAMETER & $\begin{array}{l}\text { Result } \\
\mu \mathrm{g} / \mathrm{L}\end{array}$ & $\begin{array}{c}\text { Reporting } \\
\text { Limit }\end{array}$ & PARAMETER & $\begin{array}{l}\text { Result } \\
\mu \mathrm{g} / \mathrm{L}\end{array}$ & $\begin{array}{c}\text { Reporting } \\
\text { Limit }\end{array}$ \\
\hline Acetone & $\overline{\mathrm{ND}}$ & 25. $\mu \mathrm{g} L$ & & & \\
\hline Benzene & $\mathrm{ND}$ & 5. $\mu \mathrm{g} / \mathrm{L}$ & & & \\
\hline Bromodichloromethane & ND & 5. $\mu \mathrm{g} / \mathrm{L}$ & & & \\
\hline Bromoform & $\mathrm{ND}$ & 5. $\quad \mathrm{Hg} / \mathrm{L}$ & & & \\
\hline Bromomethane & $\mathrm{ND}$ & 5. $\mu \mathrm{g} / \mathrm{L}$ & & & \\
\hline 2-Butanone & ND & 25. $\mathrm{Hg} \mathrm{L}$ & & & \\
\hline Carbon disultide & $\mathrm{ND}$ & 5. $\mu \mathrm{g} / \mathrm{L}$ & & & \\
\hline Carbon tetrachloride & $\mathrm{ND}$ & 5. $\mu \mathrm{g} \mathrm{L}$ & & & \\
\hline ChLorobenzene & ND & 5. $\lg \mathrm{L}$ & & & \\
\hline Chioroethane & ND & 5. $\mu \mathrm{g} / \mathrm{L}$ & & & \\
\hline Chloroform & ND & 5. $\mu \mathrm{gL}$ & & & \\
\hline Chloromethane & $\mathrm{ND}$ & 5. $11 \mathrm{~g} / \mathrm{L}$ & & & \\
\hline Dibromochloromethane & ND & 5. $\mu g / L$ & & & \\
\hline 1.1-Dichlorperhane (1,1-DCA) & $N D$ & 5. $\mu \mathrm{g} i \mathrm{~L}$ & & & \\
\hline 1.2-Dichloroethane (1,2-DCA) & $\mathrm{ND}$ & 5. $1 \mathrm{~g}: \mathrm{L}$ & & & \\
\hline 1.1-Dichloroethene (1.1-DCE) & $\mathrm{ND}$ & 5. $\mu \mathrm{g}$ & & & \\
\hline cis-1,2-Dichloroethene & $\mathrm{ND}$ & 5. $\mu \mathrm{g} / \mathrm{L}$ & & & \\
\hline trans-1.2-Dichloroethene & $\mathrm{ND}$ & 5. $1 \mathrm{~g} / \mathrm{L}$ & & & \\
\hline 1.2-Dichloropropane & $\mathrm{ND}$ & 5. $\mu \mathrm{gL}$ & & & \\
\hline cis-1.3-Dichloropropene & $\mathrm{ND}$ & 5. $\mu \mathrm{g} \mathrm{L}$ & & & \\
\hline trans-1.3. Dichloropropere & $\mathrm{ND}$ & 5. $\mathrm{Hg} / \mathrm{L}$ & & & \\
\hline Ethylbenzene & $\mathrm{ND}$ & 5. $\mu \mathrm{g} / \mathrm{L}$ & & & \\
\hline 2-Hexanone & $\mathrm{ND}$ & 25. $\mu \mathrm{g} / \mathrm{L}$ & & & \\
\hline Mettrylene chloride (Dichloromethane) & $N D$ & 5. $\mathrm{Hg} \mathrm{L}$ & & & \\
\hline 4-Vern 1-2-pentanone & $\mathrm{ND}$ & 25. $H \mathrm{gLL}$ & & & \\
\hline Styrene & ND & 5. $\mu g / L$ & & & \\
\hline 1.1.2,2-I etrachloroethane & $\mathrm{ND}$ & 5. $\mu \mathrm{gl}$ l. & & & \\
\hline Tetrachlorouthene (PCE) & $\mathrm{ND}$ & 5. $\mu g i L$ & & & \\
\hline Toluene & ND & 5. $\mu g / L$ & & & \\
\hline 1.1.1-Trichloroethane (1.1.1-TCA) & ND & 5. $\mu \mathrm{gL}$ & & & \\
\hline 1.1,2-Trichloroethane (1,1,2-TCA) & $\mathrm{ND}$ & 5. $\mu \mathrm{g} / \mathrm{L}$ & & & \\
\hline rrichloroethene (TCE) & ND & 5. $\mu \mathrm{gL}$ & & & \\
\hline Yinyl chloride & $N D$ & 5. $\mu \mathrm{g} / \mathrm{L}$ & & & \\
\hline o-Xylene & $\mathrm{ND}$ & 5. $\mu g / L$ & & & \\
\hline m,p-Xylent & $\mathrm{ND}$ & 5. $\mu g / L$ & & & \\
\hline
\end{tabular}

ND - Not Detected

QUALITY CONTROL DATA:

\section{Surrogate}

4-Bromofiuoroberizene

Dibromotluoromethane

Ioluene-d8
$\%$ Recovery

104

\section{Acceptable Range}

$70-130$

$70-130$

$70-130$ 
NEL LABORATORIES

$\begin{array}{lll}\text { CLIENT: Bechtel Nevada } & \text { CLIENT ID: } & \text { Decon P6-1 A\&B } \\ \text { ROJECT NAME: V431 } & \text { DATE SAMPLED: } 6 / 3 / 98 \\ \text { PROJECT NUMBER: } 17777 & \text { NEL SAMPLE ID: } \text { L9806093-07 }\end{array}$

TEST: Volatile Organic Compounds by EPA SW846 Method 8260A, Sept. 1994

MATRIX: Solid

DILUTION: 1
EXTRACTED: $6 / 11 / 98$

ANALYZED: $6 / 11 / 98$

ANALYST: Suzanne

\begin{tabular}{|c|c|c|c|c|c|}
\hline PARAMETER & $\begin{array}{l}\text { Result } \\
\mu \mathrm{g} / \mathrm{kg}\end{array}$ & $\begin{array}{l}\text { Reporting } \\
\text { Limit } \\
\end{array}$ & PARAMETER & $\begin{array}{l}\text { Result } \\
\mu \mathrm{g} / \mathrm{kg}\end{array}$ & $\begin{array}{l}\text { Reporting } \\
\text { Limit }\end{array}$ \\
\hline Acetone & $\overline{N D}$ & $25 . \mu \mathrm{g} / \mathrm{kg}$ & & & \\
\hline Benzene & $\mathrm{ND}$ & 5. $\mu \mathrm{g} / \mathrm{kg}$ & & & \\
\hline Bromodichloromethane & $\mathrm{ND}$ & 5. $\mu \mathrm{g} / \mathrm{kg}$ & & & \\
\hline Bromofom & $\mathrm{ND}$ & 5. $\mathrm{\mu g} / \mathrm{kg}$ & & & \\
\hline Bromomethane & $\mathrm{ND}$ & 5. $\mu \mathrm{g} / \mathrm{kg}$ & & & \\
\hline 2-Butanone & $\mathrm{ND}$ & 25. $\mu \mathrm{g} / \mathrm{kg}$ & & & \\
\hline Carbon disulfide & $\mathrm{ND}$ & 5. $\mu \mathrm{g} / \mathrm{kg}$ & & & \\
\hline Carbon tetrachloride & $\mathrm{ND}$ & 5. $\mu \mathrm{g} / \mathrm{kg}$ & & & \\
\hline Chlorobenzene & $\mathrm{ND}$ & 5. $\mu \mathrm{g} / \mathrm{kg}$ & & & \\
\hline Chloroethane & $\mathrm{ND}$ & 5. $\mu \mathrm{g} / \mathrm{kg}$ & & & \\
\hline Chloroform & $\mathrm{ND}$ & 5. $\mu g / \mathrm{kg}$ & & & \\
\hline Chloromethane & ND & 5. $\mu g / \mathrm{kg}$ & & & \\
\hline Dibromochloromethane & $\mathrm{ND}$ & 5. $\mu \mathrm{g} / \mathrm{kg}$ & & & \\
\hline 1.1-Dichloroethane (1,1-DCA) & $\mathrm{ND}$ & 5. $\mu \mathrm{g} / \mathrm{kg}$ & & & \\
\hline 1.2-Dichlorothane (1,2-DCA) & $N D$ & 5. $\mu \mathrm{g} / \mathrm{kg}$ & & & \\
\hline 1.1-Dichloroethene (1, i-DCE) & $\mathrm{ND}$ & 5. $\mu \mathrm{g} / \mathrm{kg}$ & & & \\
\hline is-1.2-Dichloroethene & ND & 5. $\mu \mathrm{g} / \mathrm{kg}$ & & & \\
\hline - rans-1,2-Dichforoethene & $\mathrm{ND}$ & 5. $\mu g / k g$ & & & \\
\hline 1.2-Dichloropropane & $N D$ & 5. $\mu \mathrm{g} / \mathrm{kg}$ & & & \\
\hline cis-1.3-Dichloropropene & ND & 5. $\mu \mathrm{g} / \mathrm{kg}$ & & & \\
\hline trans-1,3-Dichloropropene & $\mathrm{ND}$ & 5. $\mu \mathrm{g} / \mathrm{kg}$ & & & \\
\hline Ethylbenzene & $N D$ & 5. $119 / \mathrm{kg}$ & & & \\
\hline 2-Hexanone & ND & 25. $\mu \mathrm{gg} / \mathrm{kg}$ & & & \\
\hline Methylene chloride (Dichloromethane) & $\mathrm{ND}$ & 5. $\mu \mathrm{g} / \mathrm{kg}$ & & & \\
\hline 4-Methyl-2-pentanone & $N D$ & 25. $11 \mathrm{~g} / \mathrm{kg}$ & & & \\
\hline Styrene & $\mathrm{ND}$ & 5. $\mathrm{Hg} / \mathrm{kg}$ & & & \\
\hline 1.1.2.2-Tetrachioroethane & $\mathrm{ND}$ & 5. $\mu \mathrm{g} / \mathrm{kg}$ & & & \\
\hline Tetrachlorocthene (PCE) & $\mathrm{ND}$ & 5. $\mu \mathrm{g} / \mathrm{kg}$ & & & \\
\hline Toivene & $\mathrm{ND}$ & 5. $\mu \mathrm{g} / \mathrm{kg}$ & & & \\
\hline 1.1,1-Trichloroethane (1,1,1-TCA) & $\mathrm{ND}$ & 5. $\mu \mathrm{g} / \mathrm{kg}$ & & & \\
\hline 1.1.2-Trichlornethane $(1,1,2-\mathrm{TCA})$ & ND & 5. $\mu \mathrm{g} / \mathrm{kg}$ & & & \\
\hline Trichioroethene (TCE) & $\mathrm{ND}$ & 5. $\mu \mathrm{g} / \mathrm{kg}$ & & & \\
\hline Vinyl chloride & $\mathrm{ND}$ & 6. $\mu \mathrm{g} / \mathrm{kg}$ & & & \\
\hline 0 -xylene & ND & 5. $\mu \mathrm{g} / \mathrm{kg}$ & & & \\
\hline m.p-Xylene & $\mathrm{ND}$ & 5. $\mu \mathrm{g} / \mathrm{kg}$ & & & \\
\hline
\end{tabular}

$\mathrm{ND}$ - Not Detected

QUALITY CONTROL DATA:

\section{Surrogate}

4-Bromotluorobenzene

Dibromofluoromethane

ioluene-d8
\% Recovery

96
Acceptable Range

$74-121$

$80-120$

$81-117$ 
NEL LABORATORIES

CLIENT: Bechtel Nevada

PROJECT NAME: $\quad$ V431

PROJECT NUMBER: 17777
CLIENT ID: Decon P7-1 A\&B

DATE SAMPLED: $6 / 3 / 98$

NEL SAMPLE ID: L9806093-12

TEST: Volatile Organic Compounds by EPA SW846 Method 8260A, Sept. 1994

MATRIX: Solid $\quad \begin{aligned} & \text { EXTRACTED: } 6 / 11 / 98 \\ & \text { DILUTION: I }\end{aligned}$
ANALYZED: $6 / 11 / 98$

\begin{tabular}{|c|c|c|c|c|c|}
\hline PARAMETER & $\begin{array}{l}\text { Result } \\
\mu \mathrm{g} / \mathrm{kg}\end{array}$ & $\begin{array}{c}\text { Reporting } \\
\text { Limit }\end{array}$ & PARAMETER & $\begin{array}{l}\text { Result } \\
\mu \mathrm{g} / \mathrm{kg}\end{array}$ & $\begin{array}{c}\text { Reporting } \\
\text { Limit }\end{array}$ \\
\hline Acetone & $\sqrt{\mathrm{ND}}$ & 25. $\mu \mathrm{g} / \mathrm{kg}$ & & & \\
\hline Benzene & $\mathrm{ND}$ & 5. $\mu \mathrm{g} / \mathrm{kg}$ & & & \\
\hline Bromodichloromethane & ND & 5. $\mu \mathrm{g} / \mathrm{kg}$ & & & \\
\hline Bromotorm & ND & 5. $\mu \mathrm{g} / \mathrm{kg}$ & & & \\
\hline Bromomethane & ND & 5. $\mu \mathrm{g}: \mathrm{kg}$ & & & \\
\hline 2-Butanone & $\mathrm{ND}$ & 25. $\mu \mathrm{g} / \mathrm{kg}$ & & & \\
\hline Carbon disulfide & ND & 5. $\mu \mathrm{g} / \mathrm{kg}$ & & & \\
\hline Carbon tetrachloride & ND & 5. $\mu \mathrm{g} / \mathrm{kg}$ & & & \\
\hline Chlorobenzene & ND & 5. $\mu g / \mathrm{kg}$ & & & \\
\hline Chloroethane & $\mathrm{ND}$ & 5. $\mu \mathrm{g} / \mathrm{kg}$ & & & \\
\hline Chloroform & $\mathrm{ND}$ & 5. $\mu g / \mathrm{kg}$ & & & \\
\hline Chloromethane & ND & 5. $\mu \mathrm{g} / \mathrm{kg}$ & & & \\
\hline Dibromochloromethane & $N D$ & 5. $\mu \mathrm{gkg}$ & & & \\
\hline 1.1-Dichtoroethane (1.1-DCA) & $\mathrm{ND}$ & 5. $\mu \mathrm{g} / \mathrm{kg}$ & & & \\
\hline 1.2-Dichloroethane (1.2-DCA) & $\mathrm{ND}$ & 5. $\mu g / k g$ & & & \\
\hline [.1-Dichloroethene (1,1-DCE) & ND & 5. $\mu g / k g$ & & & \\
\hline cis-1.2-Dichloroethene & $N D$ & 5. $\mu \mathrm{g} / \mathrm{kg}$ & & & \\
\hline trans-1.2-Dichloroethene & $\mathrm{ND}$ & 5. $\mu \mathrm{g} / \mathrm{kg}$ & & & \\
\hline \$.2-Dichloropropant & ND & 5. $\mathrm{Hg} \mathrm{kg}$ & & & \\
\hline cis-1.3-Dichloropronene & ND & 5. $\mu g \mathrm{~kg}$ & & & \\
\hline uans-1.3-Dichloropropene & ND & 5. $\mu \mathrm{g} / \mathrm{kg}$ & & & \\
\hline Ethylbenzene & $\mathrm{ND}$ & 5. $\mu \mathrm{gg} / \mathrm{kg}$ & & & \\
\hline 2-Hexanone & $\mathrm{ND}$ & 25. $\mu g / k g$ & & & \\
\hline Wethylene chloride (Dichloromethane) & $\mathrm{ND}$ & 5. $\mu \mathrm{g} / \mathrm{kg}$ & & & \\
\hline 4-Methy-2-pentanone & $\mathrm{ND}$ & 25. $\mu \mathrm{gg} / \mathrm{kg}$ & & & \\
\hline Sţrene & $\mathrm{ND}$ & 5. $\mu \mathrm{g} / \mathrm{kg}$ & & & \\
\hline 1, 1,2.2-Tetrachlorouthanc & $\mathrm{ND}$ & 5. $\mu \mathrm{g} / \mathrm{kg}$ & & & \\
\hline Tetrachioroethene ( $\mathrm{PCE}$ ) & $\mathrm{ND}$ & 5. $\mu \mathrm{g} / \mathrm{kg}$ & & & \\
\hline Toluene & $\mathrm{ND}$ & 5. $\mu g i k g$ & & & \\
\hline 1.1.1-Trichloroethane (1,1,1-TCA) & ND & 5. $\mu g \mathrm{~kg}$ & & & \\
\hline 1,1,2-Trichloroethane (1,1.2-TCA) & $\mathrm{ND}$ & 5. $4 \mathrm{~g} / \mathrm{kg}$ & & & \\
\hline Trichloroethene (TCE) & $\mathrm{ND}$ & 5. $\quad H \mathrm{~g} \mathrm{~kg}$ & & & \\
\hline Vinyl chloride & $\mathrm{ND}$ & 6. $\mu \mathrm{g} / \mathrm{kg}$ & & & \\
\hline (1)-Xylene & $\mathrm{ND}$ & 5. $\mu \mathrm{gg} / \mathrm{kg}$ & & & \\
\hline$n, p-X y l e n e$ & ND & 5. $\mu g / \mathrm{kg}$ & & & \\
\hline
\end{tabular}

ND - Not Detected

QUALITY CONTROL DATA:

\section{Surrogate}

4-13romohluorobenzene

Dibromofluoromethane

Toluene-dis
\% Recovery

87

92

98
Acceptable Range

$74-121$

$80-120$

$81-117$ 
NEL LABORATORIES

CLIENT: Bechtel Nevada

ROJECT NAME: V431

PROJECT NUMBER: 17777
CLIENTID: Decon P8-1 A\&B

DATE SAMPLED: $63: 98$

NEL SAMPLE ID: L9806093-17

TEST: Volatile Organic Compounds by EPA SW846 Method 8260A, Sept. 1994

MATRIX: Solid

DILUTION: 1
EXTRACTED: $6 / 11 / 98$

ANALYZED: 6/11/98
ANALYST: Suzanne

\begin{tabular}{|c|c|c|c|c|c|c|}
\hline PARAMETEK & $\begin{array}{l}\text { Restrit } \\
\mu \mathrm{g} / \mathrm{kg}\end{array}$ & & $\begin{array}{c}\text { Reporting } \\
\text { Limit } \\
\end{array}$ & PARAMETER & $\begin{array}{l}\text { Result } \\
\mu \mathrm{g} / \mathrm{kg}\end{array}$ & $\begin{array}{c}\text { Reporting } \\
\text { Limit } \\
\end{array}$ \\
\hline Acerone & $\widetilde{N D}$ & & $25 . \mu \mathrm{g} / \mathrm{kg}$ & & & \\
\hline Benzene & ND & & 5. $\mu \mathrm{g} / \mathrm{kg}$ & & & \\
\hline Bromodichloromethane & $N D$ & & 5. $\mu g / \mathrm{kg}$ & & & \\
\hline Bromofom & $N D$ & & 5. $\mu g \mathrm{~kg}$ & & & \\
\hline Btomomethane & $\mathrm{ND}$ & & 5. $\mu g / k g$ & & & \\
\hline 2-Butanone & ND & & 25. $\mu \mathrm{gkg}$ & & & \\
\hline Carbon disuifide & $N D$ & & 5. $\mu \mathrm{g} \mathrm{kg}$ & & & \\
\hline Carbon tetrachloride & $\mathrm{ND}$ & & 5. $\mu \mathrm{g} / \mathrm{kg}$ & & & \\
\hline Chlorobenzene & $\mathrm{ND}$ & & 5. $\mu \mathrm{g} / \mathrm{kg}$ & & & \\
\hline Chloroethane & $\mathrm{ND}$ & & 5. $\mathrm{Hg} / \mathrm{kg}$ & & & \\
\hline Chloroform & ND & & 5. $\mathrm{Hg} / \mathrm{kg}$ & & & \\
\hline Chloromethane & ND & & 5. $11 \mathrm{~g} / \mathrm{kg}$ & & & \\
\hline Dibromochloromethane & $\mathrm{ND}$ & & 5. $\mu \mathrm{gg} / \mathrm{kg}$ & & & \\
\hline :.1-Dichlorothane $(1,1-D C A\}$ & $\mathrm{ND}$ & & 5. $\mu \mathrm{g} / \mathrm{kg}$ & & & \\
\hline :2-Dichlorothane (1.2-DCA) & $\mathrm{ND}$ & & 5. $\mu g \mathrm{~kg}$ & & & \\
\hline (. 1-Dicislorothene (1,1-DCE) & ND & $\mathrm{m}$ & 5. $\mu g \mathrm{~kg}$ & & & \\
\hline is-1.2-Dienbroethene & ND & & 5. $\mu \mathrm{g} / \mathrm{kg}$ & & & \\
\hline rans-1.2-Dichloroethene & $N D$ & & 5. $\mu \mathrm{g} / \mathrm{kg}$ & & & \\
\hline 1.2-Dichloropropane & ND & & 5. $\mu \mathrm{g} / \mathrm{kg}$ & & & \\
\hline ais-1.3-Dichloropropene & ND & & 5. $\mu \mathrm{g} / \mathrm{kg}$ & & & \\
\hline trans-1,3-Dichloropropene & $\mathrm{ND}$ & & 5., $4 \mathrm{~g} / \mathrm{kg}$ & & & \\
\hline Ethy!benzene & ND & & 5. $\mathrm{Hg} / \mathrm{kg}$ & & & \\
\hline 2-Hexanone & $\mathrm{ND}$ & & 25. $\mu \mathrm{gkg}$ & & & \\
\hline Wethyene chloride (Dichloromethane) & ND & B & 5. $\mu g / k g$ & & & \\
\hline 4-Methy $[-2$-pentanone & ND & & 25. $\mu g / k g$ & & & \\
\hline Styrene & $\mathrm{ND}$ & & 5. $\mu \mathrm{g} / \mathrm{kg}$ & & & \\
\hline 1. I.2,2-Tetrachlorocthane & $\mathrm{ND}$ & & 5. $4 \mathrm{gg} / \mathrm{kg}$ & & & \\
\hline Tetrachloroethene (PCE) & $\mathrm{ND}$ & & 5. $\mu \mathrm{g} / \mathrm{kg}$ & & & \\
\hline Toluene & $N D$ & & 5. $\mu \mathrm{g} / \mathrm{kg}$ & & & \\
\hline 1.1.1-Trichloroethane (1.1.1-TCA) & $\mathrm{ND}$ & & 5. $4 \mathrm{~g} / \mathrm{kg}$ & & & \\
\hline 1,1.2-Trichlorocthane $(1,1,2$-TCA $)$ & $\mathrm{ND}$ & & 5. $\mu \mathrm{g} / \mathrm{kg}$ & & & \\
\hline [richloroethene (TCE) & $\mathrm{ND}$ & & 5. $\mu \mathrm{g} / \mathrm{kg}$ & & & \\
\hline Vinyl chloride & $\mathrm{ND}$ & & 6. $\mu \mathrm{g} / \mathrm{kg}$ & & & \\
\hline 1)-Xyiene & $\mathrm{KD}$ & & 5. $\mu \mathrm{g} / \mathrm{kg}$ & & & \\
\hline m.p-Xylane & $\mathrm{ND}$ & & 5. $1 \mathrm{gg} \mathrm{kg}$ & & & \\
\hline
\end{tabular}

VD - Not Detected

QUALITY CONTROL DATA:

\section{Surrogate}

4-Bromofluorobenzene

Dibromofluoromethane

5oluene-d8
$\%$ Recovery

87

92

97
Acceptable Range

$74-121$

$80-120$

$81-117$ 
NEL LABORATORIES

CLIENT: Bechtel Nevada

CLIENT ID:

Matrix Spike

PROJECT ID: V431/17777

DATE SAMPLED: NA

PROJECT \#: $\quad 17777$

NEL SAMPLE ID: L9806093-17-MS

TEST: $\quad$ Volatile Organic Compounds by EPA SW846 Method 8260A, Sept. 1994

MATRIX: Solid

EXTRACTED: $6 / 11 / 98$

ANALYZED: $6 / 11 / 98$

ANALYST: Suzanne

\section{PARAMETER}

Benzene

Chlorobenzene

1,1-Dichloroethene (1,1-DCE)

Toluene

Trichloroethene (TCE)

OUALITY CONTROL DATA:

Surrogate

4-Bromofluorobenzene

Dibromofluoromethane

Toluene-d8

\section{Percent Recovery}

94

100

142

96

98

\section{Acceptable Range}

$66-142$

$60-133$

$59-172$

$59-139$

$62-137$
Percent Recovery

85

93

97

\section{Acceptable Range}

$74-121$

$80-120$

$81-117$ 
NEL LABORATORIES

$\begin{array}{llll}\text { CLIENT: } & \text { Bechtel Nevade } & \text { CLIENT ID: } & \text { Matrix Spike Duplicate } \\ \text { 'ROJECT ID: } & \text { V431/17777 } & \text { DATE SAMPLED: } & \text { NA } \\ \text { ROJECT } \#: & 17777 & \text { NEL SAMPLE ID: L9806093-17-MSD }\end{array}$

TEST: $\quad$ Volatile Organic Compounds by EPA SW846 Method 8260A, Sept. 1994

MATRIX: Solid EXTRACTED: $6 / 11 / 98$ ANALYZED: $6 / 11 / 98$

ANALYST: Suzanne

\section{PARAMETER}

Benzene

Percent Recovery

Acceptable Range

$\underline{\text { RPD }}$

Chlorobenzene

96

1,1-Dichloroethene (1,1-DCE)

98

Toluene

156

Trichloroethene (TCE)

94

98

$66-142$
$60-133$
$59-172$
$59-139$
$62-137$

2.1

2.

9.4

2.1

OUALITY CONTROL DATA:

Surrogate

4-Bromofluorobenzene

Dibromofluoromethane

Toluene-d8

\section{Percent Recovery}

86

93

97

\section{Acceptable Range}

$74-12$ I

$80-120$

$81-117$ 
NEL LABORATORIES

CLIENT: Bechtel Nevada

PROJECT NAME: V431

PROIECT NUMBER: 17777
CLIENT ID: Method Blank

DATE SAMPLED: NA

NEL SAMPLE ID: 9806092 W60B-BLK

TEST: $\quad$ Volatile Organic Compounds by EPA SW846 Method 8260B, Dec. 1996

MATRIX: Aqueous

EXTRACTED: $\quad 6 / 9 / 98$

ANALYZED: $\quad 6 / 9 / 98$

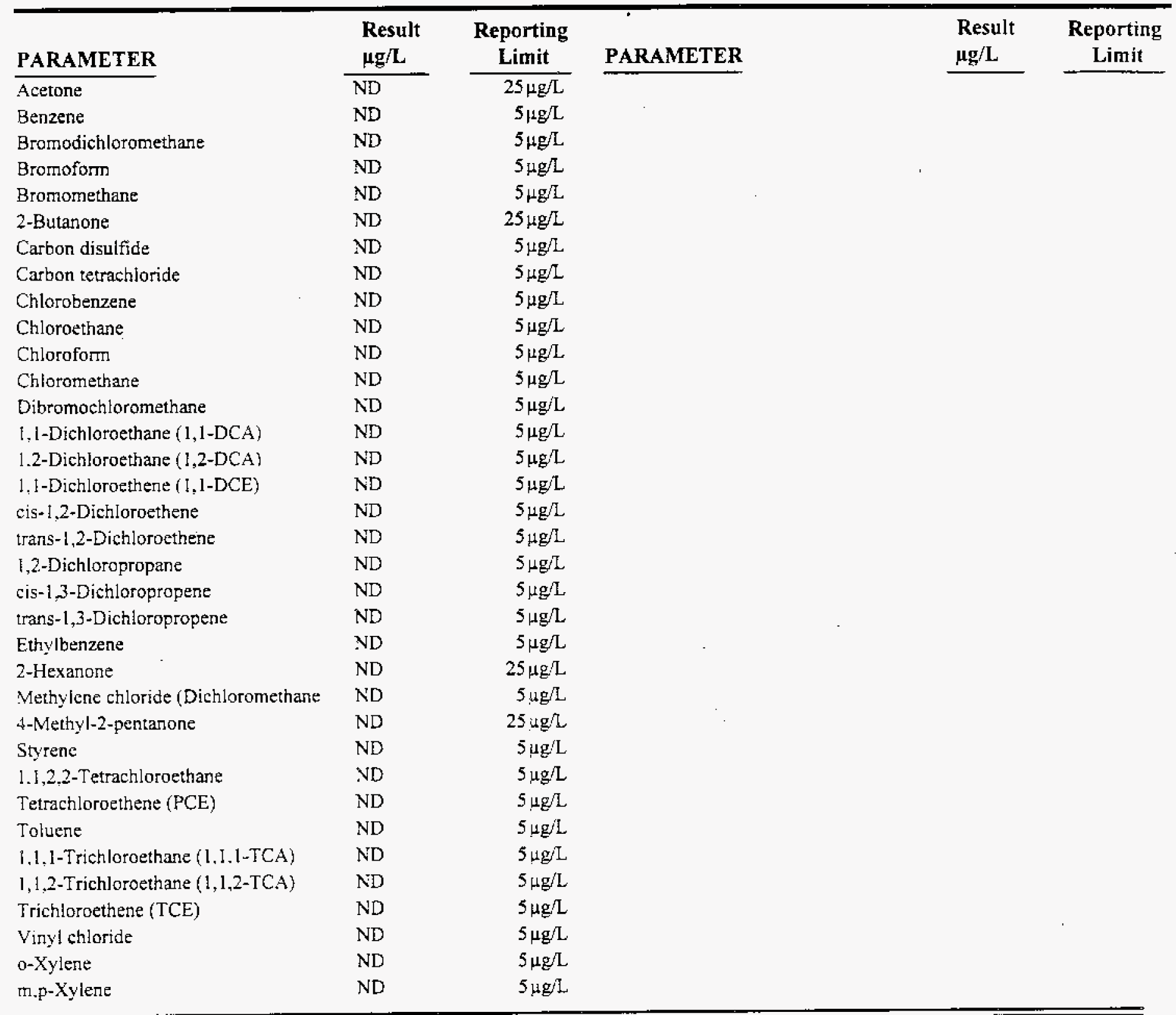

ND - Not Detected

QUALITY CONTROL DATA:

Surrogate

4-Bromofluorobenzene

Dibromofluoromethane

Toluene-d8
\% Recovery
Acceptable Range

$70 \cdot 130$

$70-130$

$70 \cdot 130$

This report shall not be reproduced except in full, without the written approval of the laboratory. 
NEL LABORATORIES

$\begin{array}{llll}\text { CLIENT: } & \text { Bechtel Nevada } & \text { CLIENT ID: } & \text { Laboratory Control Spike } \\ \text {-OJECT ID: } & \text { V431/17777 } & \text { DATE SAMPLED: } & \text { NA } \\ \text {.OJECT \#: } & 17777 & \text { NEL SAMPLE ID: } 9806092 \text { W60B-LCS }\end{array}$

TEST: Volatile Organic Compounds by EPA SW846 Method 8260B, Dec. 1996

MATRIX: Aqueous

EXTRACTED: 6/9:98

ANALYZED: $6 / 9 / 98$

ANALYST: Suzanne

\section{PARAMETER}

Benzene

Chlorobenzene

1,1-Dichloroethene (1,1-DCE)

Toluene

Trichloroethene (TCE)

\section{Percent Recovery}

97

93

93

96

92

OUALITY CONTROL DATA:

\section{Surrogate}

-Bromofluorobenzene

Dibromofiuoromethane

Toluene- $d 8$

\section{Percent Recoverv}

109

103

113

\section{Acceptable Range}

$70-130$

$70-130$

$70-130$

$70-130$

$70-130$

\section{Acceptable Range}

$70-130$

$70-130$

$70 \cdot 130$ 
NEL LABORATORIES

CLIENT: Bechtel Nevada

PROJECT NAME: V431

PROJECT NUMBER: 17777
CLIENT ID: Method Blank

DATE SAMPLED: NA

NEL SAMPLE ID: $9806111260 \mathrm{~B} 2-\mathrm{BLK}$

$\begin{array}{lll}\text { TEST: } & \text { Volatile Organic Compounds by EPA SW846 Method 8260A, Sept. } 1994 \\ \text { MATRIX: } & \text { Solid } & \text { EXTRACTED: }\end{array}$

ANALYZED: $\quad 6 / 11 / 98$

\begin{tabular}{|c|c|c|c|c|c|}
\hline PARAMETER & $\begin{array}{l}\text { Result } \\
\mu \mathrm{g} / \mathrm{kg}\end{array}$ & $\begin{array}{l}\text { Reporting } \\
\text { Limit } \\
\end{array}$ & PARAMETER & $\begin{array}{l}\text { Resuit } \\
\mu \mathrm{g} / \mathrm{kg}\end{array}$ & $\begin{array}{c}\text { Reporting } \\
\text { Limit }\end{array}$ \\
\hline$\overline{\text { Acetone }}$ & ND & $25 \mu \mathrm{g} / \mathrm{kg}$ & & & \\
\hline Benzene & ND & $5 \mu \mathrm{g} / \mathrm{kg}$ & & & \\
\hline Bromodichloromethane & ND & $5 \mu \mathrm{g} / \mathrm{kg}$ & & & \\
\hline Bromoform & ND & $5 \mu \mathrm{kg}$ & & & \\
\hline Bromomethane & ND & $5 \mathrm{Hg} / \mathrm{kg}$ & & & \\
\hline 2-Butarone & ND & $25 \mathrm{ug} / \mathrm{kg}$ & & & \\
\hline Carbon disulfide & $\mathrm{ND}$ & $5 \mu \mathrm{g} / \mathrm{kg}$ & & & \\
\hline Carbon tetrachloride & $\mathrm{ND}$ & $5 \mathrm{ug} / \mathrm{kg}$ & & & \\
\hline Chlorobenzene & ND & $5 \mu \mathrm{g} / \mathrm{kg}$ & & & \\
\hline Chloroethane & ND & $5.1 \mathrm{gg} / \mathrm{kg}$ & & & \\
\hline Chlorofom & ND & $5 \cdot 1 \mathrm{~g} / \mathrm{kg}$ & & & \\
\hline Chloromethane & ND & $5 \mu \mathrm{g} / \mathrm{kg}$ & & & \\
\hline Dibromochloromethane & ND & $5 \mu \mathrm{g} / \mathrm{kg}$ & & & \\
\hline 1.]-Dichlorothane (1.1-DCA) & $\mathrm{ND}$ & $5 \mu \mathrm{g} / \mathrm{kg}$ & & & \\
\hline 1.2-Dichforoethane (1,2-DCA) & ND & $5 \mu \mathrm{g} / \mathrm{g}$ & & & \\
\hline 1.1-Dichloroethene (1.1-DCE) & ND & $5 \mu \mathrm{g} / \mathrm{kg}$ & & & \\
\hline cis-1,2-Dichloroethene & ND & $5 \mu \mathrm{g} / \mathrm{kg}$ & & & \\
\hline trans-1.2-Dichlorocthene & $N D$ & $5 \mu g / \mathrm{kg}$ & & & \\
\hline [.2-Dichioropropane & ND & $5 \mu \mathrm{g} / \mathrm{kg}$ & & & \\
\hline cis-1,3-Dichloropropene & ND & $5 \mu \mathrm{g} / \mathrm{kg}$ & & & \\
\hline tans-1.3-Dichloropropene & ND & $5 \mu \mathrm{g} / \mathrm{kg}$ & & & \\
\hline Ethylbenzene & $\mathrm{ND}$ & $5 \mu g / \mathrm{kg}$ & & & \\
\hline 2-Hexanone & $N D$ & $25 \mu \mathrm{gkg}$ & & & \\
\hline Methylene chloride (Dichloromethane & 7 & $5 \mu g \mathrm{~kg}$ & & & \\
\hline -1-Methyi-2-pentanone & ND & $25 \mu \mathrm{g} / \mathrm{kg}$ & & & \\
\hline Styrene & ND & $5 \mu \mathrm{g} / \mathrm{kg}$ & & & \\
\hline 1.1.2.2-Tetrachlorocthane & ND & $5 \mathrm{~kg} / \mathrm{kg}$ & & & \\
\hline 'Tutrachloroethene $\left(\mathrm{P}^{3} \mathrm{CE}\right)$ & ND & $5 \mu \mathrm{g} / \mathrm{kg}$ & & & \\
\hline Joluene & $\mathrm{ND}$ & $5 \mathrm{Hg} / \mathrm{kg}$ & & & \\
\hline 1.1,1-Trichlorothane (1.1.1-TCA) & $N D$ & $5 \mathrm{Hg} \mathrm{kg}$ & & & \\
\hline 1, 1.2-Trichloroethane (1,1,2-TCA) & $\mathrm{ND}$ & $51 \mathrm{gg} / \mathrm{kg}$ & & & \\
\hline Trichloroethene (TCE) & ND & $5 \mu \mathrm{g} / \mathrm{kg}$ & & & \\
\hline Vinyi chloride & ND & $6 \mu \mathrm{g} / \mathrm{kg}$ & & & \\
\hline axyiene & ND & $5 \mu g / \mathrm{kg}$ & & & \\
\hline m.p-Xylene & ND & $5 \mu g / k g$ & & & \\
\hline
\end{tabular}

ND - Not Detected

QUALTY CONTROL DATA:

\section{Surrogate}

4-Bromofluorobenzene

Dibromofluoromethane

Toluene-d8

\section{$\%$ Recoverv}

92

93

98

\section{Acceptable Range}

$74-121$

$80-120$

$81-117$

This report shall not be reproduced except in full, without the written approval of the laboraron. 
NEL LABORATORIES

$\begin{array}{llll}\text { CLIENT: } & \text { Bechtel Nevada } & \text { CLIENT ID: } & \text { Laboratory Control Spike } \\ \text {-ROJECT ID: } & \text { V431/17777 } & \text { DATE SAMPLED: NA } \\ \text { ROJECT \#: } & 17777 & \text { NEL SAMPLE ID: } 9806111260 B 2-L C S\end{array}$

TEST: $\quad$ Volatile Organic Compounds by EPA SW846 Method 8260A, Sept. 1994

MATRIX: Solid

EXTRACTED: $6 / 11 / 98$

ANALYZED: $6 / 11 / 98$

ANALYST: Suzanne

\section{PARAMETER}

Benzene

Chlorobenzene

1,1-Dichloroethene (1,1-DCE)

Toluene

Trichloroethene (TCE)

OUALITY CONTROL DATA:

Surrogate

4-Bromofluorobenzene

Dibromofluoromethane

Toluene-d8
Percent Recovery

99

102

89

104

100

\section{Acceptable Range}
$80-120$
$80-120$
$80-120$
$80-120$
$80-120$

Percent Recovery

91

91

99

\section{Acceptable Range}

$74-121$

$80-120$

8]- 117 
SEMI-VOLATILE

ORGANICS

DATA 
CLIENT: Bechtel Nevada

OJECT NAME: V431

PROJECT NUMBER: 17777
CLIENT ID: Decon W2-2

DATE SAMPLED: $6 / 398$

NEL SAMPLE ID: L9806093-02

TEST: Semi-Volatile Organic Compounds by EPA 8270C, Dec. 1996

MATRIX: Solid

DILUTION: 10
EXTRACTED: $6 / 11 / 98$

ANALYZED: $6 / 12 / 98$
ANALYST: CCJ

\begin{tabular}{|c|c|c|c|c|c|}
\hline PARAMETER & $\begin{array}{c}\text { Result } \\
\mu \mathrm{g} / \mathrm{Kg}\end{array}$ & $\begin{array}{c}\text { Reporting } \\
\text { Limit }\end{array}$ & PARAMETER & $\begin{array}{l}\text { Result } \\
\mu \mathrm{g} / \mathrm{Kg}\end{array}$ & $\begin{array}{c}\text { Reporting } \\
\text { Limit }\end{array}$ \\
\hline Acenaphthene & $\overline{\mathrm{ND}}$ & $5000 . \mu \mathrm{g} / \mathrm{Kg}$ & $\overline{\text { Di-n-octyl phthalate }}$ & $\mathrm{ND}$ & $\overline{5000 . \mu \mathrm{g}} \mathrm{K}$ \\
\hline Acenaphthylene & ND & 5000. $\mu \mathrm{g} / \mathrm{Kg}$ & Fhoranthene & $\mathrm{ND}$ & $5000.1 \mathrm{gKg}$ \\
\hline Anthracene & ND & $5000 . \mu \mathrm{g} / \mathrm{Kg}$ & Fhorene & $\mathrm{ND}$ & 5000. $\mu \mathrm{gkg}$ \\
\hline Benzo (a) anthracene & $\mathrm{ND}$ & $5000 . \mu \mathrm{g} / \mathrm{Kg}$ & Hexachlorobenzene & $\mathrm{ND}$ & 5000. $\mu \mathrm{g} \mathrm{Kg}$ \\
\hline Benzo (b\&k) fluoranthene & $\mathrm{ND}$ & 5000. $\mu \mathrm{g} / \mathrm{Kg}$ & Hexachlorobutadiene & $\mathrm{ND}$ & $5000 . \mu \mathrm{gKg}$ \\
\hline Benzo (g,h.j) pery.lene & $\mathrm{ND}$ & $5000 . \mu \mathrm{g} / \mathrm{Kg}$ & Hexachlorocyclopentadiene & ND & 5000. $\mu \mathrm{ggg}$ \\
\hline Benzo (a) pyrene & ND & $5000 . \mu \mathrm{g} / \mathrm{Kg}$ & Hexachloroethane & ND & 5000. $\mu \mathrm{g} \mathrm{Ko}$ \\
\hline bis (2-Chlorocthy $]$ ) ether & ND & $5000 . \mu \mathrm{g} / \mathrm{Kg}$ & Indeno $(1,2.3-c . d)$ pyrene & $\mathrm{ND}$ & 5000. $H \mathrm{~g} \mathrm{Kg}$ \\
\hline bis (2-Cnloroethoxy) methane & $\mathrm{ND}$ & $5000 . \mu g / \mathrm{Kg}$ & 1sophorone & $\mathrm{ND}$ & 5000. LgKg \\
\hline bis (2-chloroisopropyl) ether & $\mathrm{ND}$ & $5000 . \mu \mathrm{g} / \mathrm{Kg}$ & 2-Methy'tnaphthalene & $\mathrm{ND}$ & $5000 . \mu \mathrm{g} \mathrm{Kg}$ \\
\hline bis (2-Ethylhexyl)phthalate & ND & 5000. $\mu \mathrm{g} / \mathrm{Kg}$ & 2-Methyiphenol & $\mathrm{ND}$ & $5000 . \mu \mathrm{g} \mathrm{K}$ \\
\hline Butylbenty tphtinalate & ND & 5000. $\mu \mathrm{g} / \mathrm{Kg}$ & 4-Methylphenol & $N D$ & $5000.4 \mathrm{Kg}$ \\
\hline$\downarrow$-Bromophenyl phenyl ether & ND & $5000 . \mu \mathrm{g} \mathrm{Kg}$ & Naphthalene & $\mathrm{ND}$ & $5000 . \mathrm{Lg} \mathrm{Kg}$ \\
\hline Carbazole & $\mathrm{ND}$ & $5000 . \mu \mathrm{g} / \mathrm{Kg}$ & 2-Nitroaniline & ND & $25000 \mathrm{Hg} \cdot \mathrm{Ka}$ \\
\hline 4-Chloroanaine & $\mathrm{ND}$ & $10000.4 \mathrm{~g} \mathrm{Kg}$ & 3.Nitroariline & ND & 25000 . \\
\hline 4-Cojloro-3-methy] phenol & $\mathrm{ND}$ & $10000 . \mu \mathrm{g} / \mathrm{Kg}$ & 4-Nitroaniline & $\mathrm{ND}$ & $10000 . \mu \mathrm{g} K$ \\
\hline Criloronanhthaiene & $\mathrm{ND}$ & $5000 . \mu \mathrm{g} / \mathrm{Kg}$ & Nitrobenzene & $\mathrm{ND}$ & 5000 . ug $\mathrm{Kg}$ \\
\hline -Chtorophenol & $\mathrm{ND}$ & $5000 . \mu \mathrm{g} / \mathrm{Kg}$ & 2-Nitropinenol & $\mathrm{ND}$ & $5000 \mathrm{Hg} \cdot \mathrm{Kg}$ \\
\hline 4-Cnlorophenyl phenyl ether & $\mathrm{ND}$ & $5000 . \mu \mathrm{g} / \mathrm{Kg}$ & 4-Nitropheno: & $N D$ & $25000 . \mathrm{ug} \cdot \mathrm{kg}$ \\
\hline Chrysens & $\mathrm{ND}$ & 5000. $\mu \mathrm{g} / \mathrm{Kg}$ & N-Nitrosodi-n-propylamine & ND & $5000.4 \mathrm{~g} \mathrm{Kg}$ \\
\hline Dibenzo (uh histhracene & $\mathrm{ND}$ & 5000. $\mu \mathrm{g} / \mathrm{Kg}$ & N-Nitrosodiphenyiamine & $\mathrm{ND}$ & $5000 \mathrm{ag} K \mathrm{O}$ \\
\hline Dibenzofuran & $\mathrm{ND}$ & $5000 . \mu \mathrm{g} / \mathrm{Kg}$ & Pentachlorophenol & $N D$ & $25000 . \mathrm{Hg} \mathrm{Kg}$ \\
\hline Di-r-butyl phthajate & $\mathrm{ND}$ & 5000. $\mu \mathrm{g} / \mathrm{Kg}$ & Phenol & $N D$ & $5000.19 k \mathrm{~kg}$ \\
\hline 1.2-Dichlorotenzent (0-DCB) & ND & 5000. $\mu \mathrm{g} / \mathrm{Kg}$ & Phenanthrene & ND & $5000 . \mu \mathrm{g} \cdot \mathrm{K}$ \\
\hline i.3-Dichlorobenzene $(\mathrm{m}$-DCB $)$ & $N D$ & 5000. $\mu \mathrm{g} / \mathrm{Kg}$ & Pyrene & ND & 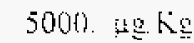 \\
\hline 1.4-Dichlorobenzene (p-DCB) & $\mathrm{ND}$ & $5000 . \mu \mathrm{g} / \mathrm{Kg}$ & 1.2.4-Trichlorobenzene & ND & $5000 . \mu g k$ \\
\hline 2.4-Dichlorophenol & $\mathrm{ND}$ & 5000. $\mu \mathrm{g} / \mathrm{Kg}$ & 2,4,5-Trichloropienol & $\mathrm{ND}$ & 5000 ug $k$ \\
\hline 3.3'-Dichlorobenzidine & $\mathrm{ND}$ & $10000 \mu \mathrm{\mu g} / \mathrm{Kg}$ & $2.4,6$-Trichiorophenol & ND & 5000 HIK $K$ \\
\hline Disthylphthalate & $N D$ & 5000. $\mu \mathrm{g} / \mathrm{Kg}$ & & & \\
\hline 2.4-Dimethy iphenol & $\mathrm{ND}$ & $5000 . \mu \mathrm{g} / \mathrm{Kg}$ & & & \\
\hline Simethyiphthala:e & $\mathrm{ND}$ & 5000. $\mathrm{Mg} \mathrm{Kg}$ & & & \\
\hline 4.6-Dinitro-2-methy] phenol & $\mathrm{ND}$ & 25000. $\mathrm{Hg} \mathrm{Kg}$ & & & \\
\hline 2.4-Dinitrotoluene (DNT) & $\mathrm{ND}$ & $5000 . \mu \mathrm{g} / \mathrm{Kg}$ & & & \\
\hline ב.6-Dinitrotoluene (DNT) & $\mathrm{ND}$ & $5000 . \mu \mathrm{g} \mathrm{Kg}$ & & & \\
\hline 2.4-Dinitrophenol & $\mathrm{ND}$ & $25000 . \mu \mathrm{g} / \mathrm{Kg}$ & & & \\
\hline
\end{tabular}

ND - Nor Detected

QUALITY CONTROL DATA:

\section{Surrogate}

7.4,6-Tribromophenol

-Fluorotipheny'

Fluorophenol

Vitrobenzetle-d5

p-Terpheny $\mathrm{i}-\mathrm{u} 14$

Phenol-ds

\section{\% Recover}

78

64

D

\section{Acceptable Range}

$19-122$

$30-115$

$25-121$

$23-120$

$18-137$

$24-113$ 
CLIENT: Bechtel Nevada

PROJECT NAME: V431

PROJECT NUMBER: 17777
CLIENT ID: $\quad$ Decon P6-2

DATE SAMPLED: $6 / 3 / 98$

NEL SAMPLE ID: L9806093-08

TEST: Semi-Volatile Organic Compounds by EPA 8270C, Dec. 1996

MATRLX: Solid

DILUTTON: 1
EXTRACTED: $6 / 11 / 98$

ANALYZED: $6 / 12 / 98$

ANALYST: CCJ

\begin{tabular}{|c|c|c|c|c|c|}
\hline PARAMETER & $\begin{array}{l}\text { Result } \\
\mu g / \mathrm{Kg}\end{array}$ & $\begin{array}{l}\text { Reporting } \\
\text { Limit }\end{array}$ & PARAMETER & $\begin{array}{l}\text { Result } \\
\mu \mathrm{g} / \mathrm{Kg}\end{array}$ & $\begin{array}{c}\text { Reporting } \\
\text { Limit }\end{array}$ \\
\hline Acenaphthene & $\overline{N D}$ & $500 . \mu \mathrm{g} / \mathrm{Kg}$ & $\overline{\text { Di-n-octyl phthaiate }}$ & $\mathrm{ND}^{-}$ & $500 . \mu \mathrm{g} K \mathrm{~g}$ \\
\hline Acenaphthylene & $\mathrm{ND}$ & $500 . \mu g / \mathrm{Kg}$ & Filuoranthene & ND & 500. $\mu \mathrm{g}: \mathrm{Kg}$ \\
\hline Anthracene & ND & $500 . \mu \mathrm{g} / \mathrm{Kg}$ & Fluorene & ND & $500 . \mu \mathrm{g} / \mathrm{Kg}$ \\
\hline Велzo (a) anthracene & $\mathrm{ND}$ & $500 . \mu \mathrm{g} / \mathrm{Kg}$ & Hexachlorobenzene & ND & 500. $\mu \mathrm{g} / \mathrm{Kg}$ \\
\hline Benzo (b\&k) fluoranthene & $\mathrm{ND}$ & $500 . \mu \mathrm{g} / \mathrm{Kg}$ & Hexachlorobutadiene & ND & 500. $\mathrm{\mu g} \mathrm{Kg}$ \\
\hline Benzo $(\mathrm{g}, \mathrm{h}, \mathrm{i})$ perylene & ND & $500 . \mu \mathrm{g} / \mathrm{Kg}$ & Hexachlorocyclopentadiene & ND & $500.12 \mathrm{~g} K$ \\
\hline Benzo (a) pyrene & $\mathrm{ND}$ & $500 . \mu \mathrm{g} / \mathrm{Kg}$ & Hexachloroethane & ND & 500. $\mu g \mathrm{Kg}$ \\
\hline bis (2-Chloroethyl) ether. & ND & 500. $\mu \mathrm{g} / \mathrm{Kg}$ & Indeno $(1,2,3-c, d)$ pyrene & ND & $500 . \mu \mathrm{g} K$ \\
\hline bis (2-Chloroethoxy) methane & $\mathrm{ND}$ & 500. $\mu g / K g$ & Isophorone & ND & $500 \mu \mathrm{g} / \mathrm{Kg}$ \\
\hline bis (2-chloroisopropyl) ether & $N D$ & 500. $\mu \mathrm{g} / \mathrm{Kg}$ & 2-Methylnaphthalene & ND & $500 . \mu \mathrm{K} \mathrm{K}$ \\
\hline bis (2-Ethylhexyl)phthalate & $\mathrm{ND}$ & 500. $\mu \mathrm{g} / \mathrm{Kg}$ & 2-Methylphenol & ND & 500. $\mu \mathrm{g} \mathrm{Kg}$ \\
\hline Butylbenzylphthalate & $\mathrm{ND}$ & 500. $\mathrm{Hg} / \mathrm{Kg}$ & 4-Methylphenol & ND & 500. $\mu \mathrm{g} \mathrm{Kg}$ \\
\hline 4-Bromophenyl phenyl ether & $\mathrm{ND}$ & 500. $\mathrm{Hg} / \mathrm{Kg}$ & Naphthalene & ND & 500. $\mu \mathrm{g} \mathrm{K}$ \\
\hline Carbazole & $\mathrm{ND}$ & $500 . \mu \mathrm{g} / \mathrm{Kg}$ & 2-Nitroaniline & ND & 2500. $\mu \mathrm{g} \mathrm{Kg}$ \\
\hline 4-Chloroanaline & $\mathrm{ND}$ & $1000 . \mu \mathrm{g} / \mathrm{Kg}$ & 3-Nitroaniline & ND & 2500. $\mu \mathrm{g} \mathrm{K} \mathrm{g}$ \\
\hline 4-Chloro-3-methyl phenol & ND & 1000. $\mu \mathrm{g} / \mathrm{Kg}$ & 4-Nitroaniline & $\mathrm{ND}$ & 1000. $\mu \mathrm{gikg}$ \\
\hline 2-Chloronaphthalene & $\mathrm{ND}$ & $500 . \mu \mathrm{g} / \mathrm{Kg}$ & Nitrobenzene & $N D$ & 500. $\mathrm{Lg} \mathrm{Ko}$ \\
\hline 2-Chlorophenol & $\mathrm{ND}$ & $500 . \mu \mathrm{g} / \mathrm{Kg}$ & 2-Nitrophenol & $\mathrm{ND}$ & 500. $\mu \mathrm{g}: \mathrm{h}$ \\
\hline 4-Chlorophenyl phenyl ether & ND & 500. $\mathrm{Hg} / \mathrm{Kg}$ & 4-Nitrophenol & $\mathrm{ND}$ & $2500 . \mu \mathrm{gKg}$ \\
\hline Chrysene & ND & 500. $\mathrm{Hg} / \mathrm{Kg}$ & N-Nitrosodi-n-propylamine & ND & 500. $\mu \mathrm{g} \mathrm{Kg}$ \\
\hline Dibenzo (ah) anthracene & $\mathrm{ND}$ & 500. $\mu \mathrm{g} / \mathrm{Kg}$ & N-Nitrosodiphenylamine & ND & $500 . \mu \mathrm{g} / \mathrm{Kg}$ \\
\hline Dibenzofuran & $\mathrm{ND}$ & 500. $\mathrm{Hg} / \mathrm{Kg}$ & Pentachlorophenol & ND & $2500 . \mu g \mathrm{Kg}$ \\
\hline Di-n-bucyl phthalate & $\mathrm{ND}$ & 500. $\mathrm{Hg} / \mathrm{Kg}$ & Phenol & ND & 500. $\mu \mathrm{g}: \mathrm{K}$ \\
\hline 1,2-Dichlorobenzenc (0-DCB) & ND & 500. $\mu \mathrm{g} / \mathrm{Kg}$ & Phenanthrene & $\mathrm{ND}$ & 500. แg Kg \\
\hline I.3-Dichlorohenzene (m-DCB) & $N D$ & 500. $\mathrm{Hg} / \mathrm{Kg}$ & Pyrene & $\mathrm{ND}$ & 500. $\mu \mathrm{g}: \mathrm{Kg}$ \\
\hline 1.4-Dichlorobenzene ( $\mathrm{p}$-DCB) & ND & 500. $\mathrm{Hg} / \mathrm{Kg}$ & 1.2,4-Trichlorobenzene & $\mathrm{ND}$ & $500.4 \mathrm{giKg}$ \\
\hline 2,4-Dichlorophenol & ND & 500. $\mu \mathrm{g} / \mathrm{Kg}$ & 2,4,5-Trichlorophenol & ND & $500.4 \mathrm{~g} K \mathrm{Kg}$ \\
\hline 3.3'-Dichlorobenzidine & $\mathrm{ND}$ & 1000. $\mu \mathrm{g} / \mathrm{Kg}$ & $2,4,6$-Trichlorophenoi & ND & 500. kigkg \\
\hline Diethylphthalate & $\mathrm{ND}$ & 500. $\mu \mathrm{g} / \mathrm{Kg}$ & & & \\
\hline 2,4-Dimethylphenol & ND & $500 . \mu \mathrm{g} / \mathrm{Kg}$ & & & \\
\hline Dimethylphthalate & ND & 500. $\mu \mathrm{g} / \mathrm{Kg}$ & & & \\
\hline 46-Dinitro-2-methyl phenol & $\mathrm{ND}$ & 2500. $\mu \mathrm{g} / \mathrm{Kg}$ & & & \\
\hline 2.4-Dinitrotoluene (DNT) & $\mathrm{ND}$ & $500 . \mu \mathrm{g} / \mathrm{Kg}$ & & & \\
\hline 2.6-Dinitrotoluene (DNT) & $\mathrm{ND}$ & $500 . \mu \mathrm{g} / \mathrm{Kg}$ & & & \\
\hline 2.4-Dinitrophenol & $\mathrm{ND}$ & 2500. $11 \mathrm{~g} / \mathrm{Kg}$ & & & \\
\hline
\end{tabular}

ND - Not Detected

QUALITY CONTROL DATA:

\section{Surrogate}

2.4.6-Tribromophenol

2-Fluorobipheny!

2-Fluorophenol

Nitrobenzene-d5

p-Terphenyl-d 14

Phenol-d5

\section{\% Recovery}

67

64

55

63

70

66
Acceptable Range

19. 122

$30-115$

$25-121$

$23-120$

$18-137$

$24-113$ 
CLIENT: Bechtel Nevada

-ROJECT NAME: V431

ROJECT NUMBER: 17777
CLIENT ID: Decon P7-2

DATE SAMPLED: 6399

NEL SAMPLE ID: L9806093-13

TEST: Semi-Volatile Organic Compounds by EPA 8270C, Dec. 1996

MATRIX: Solid

DILUTION: 1
EXTRACTED: $6 / 11 / 98$

ANALYZED: 6/12/98
ANALYST: CCJ

\begin{tabular}{|c|c|c|c|c|c|}
\hline PARAMETER & $\begin{array}{l}\text { Result } \\
\mu \mathrm{g} / \mathrm{Kg}\end{array}$ & $\begin{array}{c}\text { Reporting } \\
\text { Limit } \\
\end{array}$ & PARAMETER & $\begin{array}{l}\text { Result } \\
\mu \mathrm{g} / \mathrm{Kg}\end{array}$ & $\begin{array}{l}\text { Reporting } \\
\text { Limit }\end{array}$ \\
\hline Acenapnthene & $\overline{\mathrm{ND}}$ & $500 . \mu \mathrm{g} / \mathrm{Kg}$ & Di-ri-octyl phthaiate & $\mathrm{ND}$ & $500 . \mu \mathrm{gKg}$ \\
\hline Acenaphthylene & $\mathrm{ND}$ & $500 . \mu \mathrm{g} / \mathrm{Kg}$ & Fluoranthene & $N D$ & 500. $\mu \mathrm{gKg}$ \\
\hline Anthracene & $\mathrm{ND}$ & $500 . \mu \mathrm{g} / \mathrm{Kg}$ & Fluorene & $\mathrm{ND}$ & 500. $\mu \mathrm{g} / \mathrm{Kg}$ \\
\hline Benzo (a) anthracene & $\mathrm{ND}$ & 500. $\mu \mathrm{g} / \mathrm{Kg}$ & Hexachlorobenzene & $\mathrm{ND}$ & $500 . \mu \mathrm{g} / \mathrm{Kg}$ \\
\hline Benzo (b\&k) fluoranthene & $\mathrm{ND}$ & $500 . \mu \mathrm{g} / \mathrm{Kg}$ & Hexachlorobutadiene & $\mathrm{ND}$ & 500. $\mathrm{kg} / \mathrm{Kg}$ \\
\hline Benzo (g.h.i) perylene & ND & 500. $\mu \mathrm{g} / \mathrm{Kg}$ & Hexachforocyciopentadiene & $N D$ & 500. $\mathrm{Hg} \mathrm{Kg}$ \\
\hline Benzo al pyrene & ND & 500. $\mu \mathrm{g} / \mathrm{Kg}$ & Hexachloroethane & $N D$ & 500. $4 \mathrm{~g}: \mathrm{Kg}$ \\
\hline bis $\{2$-Chloroethyl) ther & $\mathrm{ND}$ & 500. $\mu \mathrm{g} / \mathrm{kg}$ & Indeno $(1,2.3-c . d)$ pyrene & $\mathrm{ND}$ & 500. $. \mathrm{g}: \mathrm{Kg}$ \\
\hline his (2-Chloroethoxy) methane & $\mathrm{ND}$ & $500 . \mu g / \mathrm{Kg}$ & Isophorone & ND & 500. $\mathrm{t} \mathrm{g} / \mathrm{Kg}$ \\
\hline his (2-chioroisopropy 1) ether & $\mathrm{ND}$ & 500. $\mu \mathrm{g} / \mathrm{Kg}$ & 2-Methylnaphthalene & $\mathrm{ND}$ & 500. $\mu \mathrm{g} i \mathrm{Kg}$ \\
\hline bis (a-Fthy lhexyliphthaiate & $N D$ & 500. $\mu \mathrm{g} / \mathrm{Kg}$ & 2-Methylphenol & $\mathrm{ND}$ & 500. $\mathrm{kg} \mathrm{hg}$ \\
\hline Buty Ebenzy tpinthalate & $M D$ & 500. $\mu \mathrm{g} / \mathrm{Kg}$ & 4-Methylphenol & $\mathrm{ND}$ & $500 . \mu \mathrm{gKg}$ \\
\hline 4-Bromopheny phenyl ether & $\mathrm{ND}$ & $500 . \mathrm{Hg} / \mathrm{Kg}$ & Naphthalene & $\mathrm{ND}$ & 500. $4 \mathrm{EKg}$ \\
\hline Carbazoie & $\mathrm{ND}$ & 500. $\mu \mathrm{g} / \mathrm{Kg}$ & 2-Nitroanitine & ND & 2500. $\mu \mathrm{gKg}$ \\
\hline$\downarrow$-Chioroanaline & $N D$ & 1000. $\mu \mathrm{g} / \mathrm{Kg}$ & 3-Nitroaniline & $\mathrm{ND}$ & 2500. ugin \\
\hline ¿-Chloro-j-metinyl phenol &.$V D$ & 1000. $\mu \mathrm{g} \mathrm{Kg}$ & 4-Nitroaniline & $\mathrm{NI}$ & 1000. $\mu \mathrm{g}$ \\
\hline Z-Chloronaphthalene & $\mathrm{ND}$ & 500. $\mu \mathrm{g} / \mathrm{Kg}$ & Nitrobenzene & $\mathrm{ND}$ & $500 . \mu \mathrm{g}: \mathrm{Kg}$ \\
\hline hiorophenol & $N D$ & $500 . \mu \mathrm{g} / \mathrm{Kg}$ & 2-Nitrophenol & $\mathrm{ND}$ & $500.4 g i K g$ \\
\hline +Chloropieny] pheny ethar & $\mathrm{YD}$ & 500. $\mu \mathrm{g} / \mathrm{Kg}$ & 4-Nitrophenol & $\mathrm{ND}$ & $2500 . \mu \mathrm{K} \mathrm{Kg}$ \\
\hline Ghруеле & $\mathrm{ND}$ & $500 . \mu \mathrm{g} / \mathrm{Kg}$ & N-Nitrosodi-n-propylamine & $\mathrm{VD}$ & $500 . \mu \mathrm{gKg}$ \\
\hline Dibenzó lahi anthracene & $N D$ & 500. $\mu \mathrm{g} / \mathrm{Kg}$ & N-Nitrosodiphenylamine & ND & $500.4 \mathrm{~g} \mathrm{Kg}$ \\
\hline Dibenzoîtan & $N D$ & 500. $\mathrm{Hg} / \mathrm{Kg}$ & Pentachiorophenol & $\mathrm{YD}$ & 2500. $\mu \mathrm{g} \mathrm{Kg}$ \\
\hline Ji-n-hury phthalate & $N D$ & 500. $4 \mathrm{~g} / \mathrm{Kg}$ & Phenol & $N D$ & 500. แg K \\
\hline 1.2-Dictiorobenzene (o-DCB) & $\mathrm{ND}$ & 500. $\mu \mathrm{g} / \mathrm{Kg}$ & Phenanthrene & $A D$ & $500 . \mu g \mathrm{~K}$ \\
\hline 1.j-Dichlorobenzere $(m-D C B)$ & $\mathrm{ND}$ & $500 . \mu \mathrm{g} / \mathrm{Kg}$ & Pyrene & $N D$ & 500. $4 \mathrm{gikg}$ \\
\hline i.4-Dichlorobenzene (p-DCB) & ND & $500 . \mu \mathrm{g} \mathrm{Kg}$ & 1,2.4-Trichlorobenzene & $\mathrm{NiS}$ & $500.4 \mathrm{Kg}$ \\
\hline 4-Dichiorophenol & $K D$ & $500 . \mu \mathrm{g} / \mathrm{Kg}$ & 2.4,5-Trichlorophenol & $N D$ & $500 . \mu g \mathrm{~K}$ \\
\hline 3.3'-Dichlorobenzidine & ND & [000. $\mu \mathrm{g} / \mathrm{Kg}$ & 2,4,6-TrichIorophenol & $N D$ & 500. higkg \\
\hline Diethylphthalate & $\mathrm{ND}$ & $500 . \mu \mathrm{g} K \mathrm{Kg}$ & & & \\
\hline 2.4-Dimenylphenoi & $\mathrm{ND}$ & 500. $\mu \mathrm{g} / \mathrm{Kg}$ & & & \\
\hline Dimethylpinthaiate & $\mathrm{ND}$ & 500. $\mu \mathrm{g} / \mathrm{Kg}$ & & & \\
\hline 4.6-Dinitro-2-methyl phenol & ND & 2500. $\mu \mathrm{gKg}$ & & & \\
\hline 2.4-Dinitrotoiuene (DNT) & $\mathrm{KD}$ & 500. $\mu \mathrm{g} / \mathrm{Kg}$ & & & \\
\hline 2.6-Dinitrotoiuene (DNT) & $N D$ & 500. $\mu \mathrm{g} / \mathrm{Kg}$ & & & \\
\hline 2.4-Dinitropienol & $\mathrm{ND}$ & 2500. $\mathrm{Hg} / \mathrm{Kg}$ & & & \\
\hline
\end{tabular}

ND- Vot Detected

QLALITY CONTROL DATA:

\section{Surrogate}

2.4.6-Tribromophenol

2-Fluorobiprery-l lorophenol

Virooenzene-d5

n-Terohengy $|+1| 4$

phenoi-its
\% Recovery

71

62

52

$6 !$

73

61
Acceptable Range

$19-122$

$30-115$

$25-121$

$23-120$

$18-137$

$24-113$

Fhis report shall not be reproduced except in full. without the written approval of the laborator: 
CLIENT: Bechtel Nevada

PROJECT NAME: V431

PROJECT NUMBER: 17777
CLIENT ID: Decon P8-2

DATE SAMPLED: $6 / 3 / 98$

NEL SAMPLE ID: L9806093-18

TEST: Semi-Volatile Organic Compounds by EPA 8270C, Dec. 1996

MATRIX: Solid

DILUTION: 1
EXTRACTED: $6 / 11 / 98$

ANALYZED: $6 / 12 / 98$

ANALYST: CCJ

\begin{tabular}{|c|c|c|c|c|c|}
\hline PARAMETER & $\begin{array}{l}\text { Result } \\
\mu \mathrm{g} / \mathrm{Kg}\end{array}$ & $\begin{array}{l}\text { Reporting } \\
\text { Limit }\end{array}$ & PARAMETER & $\begin{array}{l}\text { Result } \\
\mu \mathrm{g} / \mathrm{Kg}\end{array}$ & $\begin{array}{c}\text { Reporting } \\
\text { Limit }\end{array}$ \\
\hline Acenaphthene & $\overline{\mathrm{ND}}$ & $500 . \mu \mathrm{g} / \mathrm{Kg}$ & $\overline{\text { Di-n-octyl phthalate }}$ & $N D$ & $500 . \mu \mathrm{g} / \mathrm{Kg}$ \\
\hline Acenaphthylene & ND & $500 . \mu \mathrm{g} / \mathrm{Kg}$ & Fluoranthene & ND & 500. $\mu \mathrm{g} / \mathrm{Kg}$ \\
\hline Anthracene & ND & 500. $\mu \mathrm{g} / \mathrm{Kg}$ & Fluorene & ND & 500. $\mu \mathrm{g} / \mathrm{Kg}$ \\
\hline Benzo (a) arthracene & $\mathrm{ND}$ & 500. $\mu \mathrm{g} / \mathrm{Kg}$ & Hexachlorobenzene & ND & 500. $\mu \mathrm{g} / \mathrm{Kg}$ \\
\hline Benzo (b\&k) fluoranthene & ND & 500. $\mu \mathrm{g} / \mathrm{Kg}$ & Hexachlorobutadiene & ND & 500. $\mu \mathrm{g} / \mathrm{Kg}$ \\
\hline Benzo $(\mathrm{g}, \mathrm{h}, \mathrm{i})$ perylene & $\mathrm{ND}$ & 500. $\mu g / \mathrm{Kg}$ & Hexachlorocyclopentadiene & ND & $500.4 \mathrm{~g} / \mathrm{Kg}$ \\
\hline Benzo (a) pyrene & ND & 500. $\mu \mathrm{g} / \mathrm{Kg}$ & Hexachloroethane & $\mathrm{ND}$ & 500. $\mathrm{Lg} \mathrm{Kg}$ \\
\hline bis (2-Chioroethyl) ether & $N D$ & $500 . \mu \mathrm{g} / \mathrm{Kg}$ & Indeno $(1,2, \hat{\jmath}-\mathrm{c}, \mathrm{d})$ pyrene & ND & 500. $1 \mathrm{Eg} \mathrm{Kg}$ \\
\hline bis (2-Chloroethoxy) methane & $\mathrm{ND}$ & 500. $\mu \mathrm{g} / \mathrm{Kg}$ & Isophorone & ND & $500 . \mu \mathrm{g}: \mathrm{Kg}$ \\
\hline bis (2-chloroisopropyt) ether & $N D$ & $500 . \mu \mathrm{g} / \mathrm{Kg}$ & 2-Methylnaphthaiene & $\mathrm{ND}$ & $500.4 \mathrm{~g} / \mathrm{Kg}$ \\
\hline bis (2-Ethylhexyl)phthalate & $N D$ & 500. $\mu \mathrm{g} / \mathrm{Kg}$ & 2-Methylphenol & ND & 500. $\mathrm{LEKT}$ \\
\hline Butylbenzetphthalate & $\mathrm{ND}$ & 500. $\mu \mathrm{g} / \mathrm{Kg}$ & 4-Methylphenol & ND & $500 . \mu \mathrm{g} / \mathrm{Kg}$ \\
\hline 4-Bromophenyl phenyl ether & ND & 500. $1 \mathrm{~g} \mathrm{Kg}$ & Naphthalene & $\mathrm{ND}$ & 500. $\mu \mathrm{g}^{\prime} \mathrm{K} \mathrm{g}$ \\
\hline Carbazole & ND & 500. $\mu \mathrm{g} / \mathrm{Kg}$ & 2-Nitroaniline & $N D$ & 2500. $\mu \mathrm{g} \mathrm{K}$ \\
\hline 4-Chloroanaline & $\mathrm{ND}$ & $1000.1 \mathrm{~g} / \mathrm{Kg}$ & 3-Nitroaniline & $\mathrm{ND}$ & 2500. $\mathrm{HgKg}$ \\
\hline 4-Chloro-3-methyl phenoi & ND & 1000. $\mu \mathrm{g} / \mathrm{Kg}$ & 4-Nitroaniline & $\mathrm{ND}$ & 1000. $\mu \mathrm{g} \mathrm{Kg}$ \\
\hline 2-Chloronaphthaiene & ND & 500. $\mathrm{Hg} / \mathrm{Kg}$ & Nitrobenzene & $\mathrm{ND}$ & 500. Hgki \\
\hline 2-Chiorophenol & $N D$ & 500. $4 \mathrm{~g} \mathrm{Kg}$ & 2-Nitrophenol & ND & $500.4 \mathrm{gih}$ \\
\hline 4-Chlorophenyl phenyl ether & $\mathrm{ND}$ & 500. $\mu \mathrm{g} / \mathrm{Kg}$ & 4 -Nitrophenol & $\mathrm{MD}$ & 2500. HEX \\
\hline Chrysene & VD & $500 . \mu \mathrm{g} / \mathrm{Kg}$ & N-iNitrosodi-n-propylamine & $\mathrm{ND}$ & 500. $\mu \mathrm{gKg}$ \\
\hline Dibenzo $(a, h)$ anthracene & ND & 500. $\mu \mathrm{g} / \mathrm{Kg}$ & N-Nitrosodiphenylamitle & $\mathrm{ND}$ & 500. 山g: $k_{\underline{1 T}}$ \\
\hline Dibenzofuran & $Y D$ & 500. $\mu \mathrm{g} / \mathrm{Kg}$ & Pentachlorophenol & $\mathrm{ND}$ & 2500. $\mu \mathrm{g} \mathrm{Kg}$ \\
\hline Di-n-butyl phthalate & ND & 500. $\mu \mathrm{g} / \mathrm{Kg}$ & Pheno] & $\mathrm{ND}$ & 500. $1 \mathrm{ggKg}$ \\
\hline 1.2-Dichlorobenzene (O-DCB) & ND & $500 . \mu \mathrm{g} / \mathrm{Kg}$ & Phenasthrene & $\mathrm{ND}$ & 500. $\mathrm{ug}: \mathrm{Kg}$ \\
\hline 1,3-Dichlorobenzene (m-DCB) & ND & 500. $\mathrm{Hg} / \mathrm{Kg}$ & Pyrene & ND & $500 . \mu \mathrm{g} K \underline{\underline{g}}$ \\
\hline 1.4-Dichiorubenzene (p-DCB) & $\mathrm{ND}$ & 500. $\mu \mathrm{g} / \mathrm{Kg}$ & 1.2.4-Trichlorobenzene & $\mathrm{MD}$ & $500 \mu \mathrm{Hg}$ \\
\hline 2.4-Dichlorophenol & $\mathrm{ND}$ & 500. $\mu \mathrm{g} / \mathrm{Kg}$ & 2.4,5-Trichlorophenol & $\mathrm{ND}$ & $500 . \mu \mathrm{g} i \mathrm{~K} \stackrel{\mathrm{g}}{=}$ \\
\hline 3.3'-Dichlorobenzidine & $\mathrm{ND}$ & 1000. $\mu \mathrm{gg}$ & 3.4,6-Trichlorophenol & ND & $500, \mathrm{~kg} \mathrm{~kg}$ \\
\hline Dicthyphthalat: & $\mathrm{ND}$ & 500. $4 \mathrm{~g} / \mathrm{Kg}$ & & & \\
\hline 2.4-Dimethylphenol & ND & 500. $\mu \mathrm{g} \mathrm{Kg}$ & & & \\
\hline Dimethylphthalate & $\mathrm{ND}$ & $500.1 \mathrm{~g} / \mathrm{Kg}$ & & & \\
\hline 4.6-Dinitro-2-methyl phenoi & $\mathrm{ND}$ & 2500. $\mathrm{Hg} \mathrm{Kg}$ & & & \\
\hline 2.4-Dinitrotoluene (DXT) & ND & $500.4 \mathrm{~g} / \mathrm{Kg}$ & & & \\
\hline 2.6-Dinitrotoluene (DNT) & $\mathrm{ND}$ & 500. $\mu \mathrm{g} / \mathrm{Kg}$ & & & \\
\hline 2.4-Dinitrophenol & ND & 2500. $4 \mathrm{~g} / \mathrm{Kg}$ & & & \\
\hline
\end{tabular}

ND- Not Detected

QUALITY CONTROL DATA:

\section{Surrogate}

2.4.6-Tribromophenol

2-Fluotobiphenv?

2-Fluorophenol

virrobenzene-ds

p-Terpheny -d 14

Phenol-d5
$\%$ Recovery

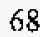

59

52

60

71

59
Acceptable Range

$19-122$

$30-115$

$25+121$

$23-120$

18. 137

$24-113$

This report shall not be reproduced except in full, without the written approval of the laboratory. 


\section{NEL LABORATORIES}

CLIENT: Bechtel Nevada

- ZOJECT ID: V431/17777

.OFECT \#: 17777
CLIENT ID: Matrix Spike

DATE SAMPLED: NA

NEL SAMPLE ID: L9806093-18-MS

TEST: $\quad$ Semi-Volatile Organic Compounds by EPA 8270C, Dec. 1996

MATRLX: Solid

EXTRACTED: $6 / 11 / 98$

ANALYZED: $6 / 12 / 98$

ANALYST: CCJ

\section{PARAMETER}

Acenaphthene

4-Chloro-3-methyl phenol

2.Chlorophenol

1,4-Dichlorobenzene ( $\mathrm{p}$-DCB)

2,4-Dinjtrotoluene (DNT)

4-Nitrophenol

N-Nitrosodi-n-propylamine

Pentachlorophenol

Phenol

1,2,4-Trichlorobenzene

\section{Percent Recovery}

50

54

56

49

54

53

65

49

50

53

\section{Acceptable Range}

$31-137$

$26-103$

$25-102$

$28-104$

$28-89$

$11-114$

$41-126$

$17-109$

$26-90$

$28-104$

QUALITY CONTROL DATA:

\section{Surrogate}

2,4,6-Tribromophenol

2-Fluorobiphenyl

2-Fluorophenol

Nitrobenzene-d5

Terphenyl-d 14

...2nol-d5

\section{Percent Recovery}

56

54

50

56

63

60

\section{Acceptable Range}

$19 \cdot 122$

$30-115$

$25-121$

$23 \cdot 120$

I8 - 137

$24-113$ 
NEL LABORATORIES

CLIENT: Bechtel Nevada

PROJECT ID: V431/17777

PROJECT H: 17777

\section{CLIENT ID: Matrix Spike Duplicate}

DATE SAMPLED: NA

NEL SAMPLE ID: L9806093-18-MSD

TEST:

Semi-Volatile Organic Compounds by EPA 8270C, Dec. 1996

MATRIX: Solid

EXTRACTED: $6 / 11 / 98$

ANALYZED: $6 / 12 / 98$

ANALYST: CCJ

PARAMETER

Acenaphthene

4-Chloro-3-methyl phenol

2-Chloropheno]

1,4-Dichlorobenzene ( 0 -DCB)

2,4-Dinitrotoluene (DNT)

4-Nitrophenol

$\mathrm{N}-\mathrm{Nitrosodi}-\mathrm{n}$-propylamine

Pentachlorophenol

Phenol

1,2,4-Trichlorobenzene

QLALITY CONTROL DATA:

\section{Surrogate}

2,4,6-Tribromophenol

2-Fluorobiphenyl

2-Fluorophenol

Nitrobenzene-d5

p-Terphenyl-dl4

Phenol-d5

\section{Percent Recovery}

55

62

54

49

62

64

63

58

49

54
Acceptable Range

$31-137$

$26-103$

$25-102$

$28-104$

$28-89$

$11-114$

$41-126$

$17-109$

$26-90$

$38-107$
RPD

2.8

14.7

1.6

9.3

13.9

17.8

2.7

16.6

0.3

2.4

\section{Acceptable Range}

61

78

63

76

85

80
$19-122$

$30-115$

25- 121

23. 120

$18-137$

$24-113$ 
CLIENT: Bechtel Nevada

NDOJECT NAME: V431

OJECT NUMBER: 17777
CLIENT ID: Method Blank

DATE SAMPLED: NA

NEL SAMPLE ID: 061098-E1-BLK

TEST:

Semi-Volatile Organic Compounds by EPA 8270C, Dec. 1996

MATRIX:

Solid

EXTRACTED:

$6 / 11 / 98$

ANALYZED:

$6 / 12 / 98$

\begin{tabular}{|c|c|c|c|c|c|}
\hline PARAMETER & $\begin{array}{c}\text { Result } \\
\mu \mathrm{g} / \mathrm{Kg}\end{array}$ & $\begin{array}{c}\text { Reporting } \\
\text { Limit }\end{array}$ & PARAMETER & $\begin{array}{r}\text { Result } \\
\mu \mathrm{g} / \mathrm{Kg}\end{array}$ & $\begin{array}{c}\text { Reporting } \\
\text { Limit }\end{array}$ \\
\hline Acenaphthene & ND & $\overline{500 \mu \mathrm{KKg}}$ & Di-n-octyl phthalate & $\widehat{N D}$ & $500 \mu \mathrm{g} / \mathrm{Kg}$ \\
\hline Acenaphthylene & ND & $500 \mathrm{\mu g} / \mathrm{Kg}$ & Fluorarthene & ND & $500 \mu \mathrm{g} / \mathrm{Kg}$ \\
\hline Anthracene & ND & $500 \mu \mathrm{g} / \mathrm{Kg}$ & Fluorene & ND & $500 \mu \mathrm{g} / \mathrm{Kg}$ \\
\hline Benzo (a) anthracene & ND & $500 \mathrm{Hg} / \mathrm{Kg}$ & Hexachiorobenzene & ND & $500 \mu \mathrm{g} K \mathrm{~K}$ \\
\hline Benzo (b\&k) fluoranthene & ND & $500 \mu g / \mathrm{Kg}_{\mathrm{g}}$ & Hexachlorobutadiene & ND & $500 \mu \mathrm{g} / \mathrm{Kg}$ \\
\hline Benzo (g,h.i) perylene & ND & $500 \mu \mathrm{g} / \mathrm{Kg}$ & HexachJorocyclopentadiene & $\mathrm{ND}$ & $500 \mathrm{\mu g} \mathrm{Kg}$ \\
\hline Benzo (a) pyrene & ND & $500 \mu \mathrm{Hg} / \mathrm{Kg}$ & Hexachloroethane & ND & $500 \mu \mathrm{g} / \mathrm{Kg}$ \\
\hline bis (2-Chloroethyi) ether & $\mathrm{ND}$ & $500 \mu \mathrm{g} / \mathrm{Kg}$ & Indeno $(1,2,3-c, d)$ pyrene & $\mathrm{ND}$ & $500 \mathrm{~kg} \mathrm{Kg}$ \\
\hline bis (2-Chlorocthoxy) methane & $\mathrm{ND}$ & $500 \mu \mathrm{g} / \mathrm{Kg}$ & Isophorone & ND & $500 \mu \mathrm{g} \mathrm{Kg}$ \\
\hline bis (2-chloroisopropyl) ether & ND & $500 \mu \mathrm{g} / \mathrm{Kg}$ & 2-Methylnaphthalene & $\mathrm{ND}$ & $500 \mu \mathrm{Kg}$ \\
\hline bis (2-Ethy fhexyl)phtbalate & ND & $500 \mu g / \mathrm{Kg}$ & 2-Methylphenol & $\mathrm{ND}$ & $500 \mu \mathrm{g} \mathrm{Kg}$ \\
\hline Butylbenfylphthalate & $\mathrm{ND}$ & $500 \mu \mathrm{g} / \mathrm{Kg}$ & 4-Methylphenol & $\mathrm{ND}$ & $500 \mu \mathrm{g} / \mathrm{Kg}$ \\
\hline 4-Bromophenyl phenyl ether & ND & $500 \mu \mathrm{g} / \mathrm{Kg}$ & Naphthaiene & ND & $500 \mu \mathrm{g} / \mathrm{Kg}$ \\
\hline Catbazole & ND & $50 \mu_{\mu g} / \mathrm{Kg}$ & 2-Nitroaniline & ND & $2500 \mu \mathrm{g} \mathrm{Kg}$ \\
\hline 4-Chloroanaline & $\mathrm{ND}$ & $1000 \mu \mathrm{g} / \mathrm{Kg}$ & 3-Nitroaniline & $\mathrm{ND}$ & $2500 \mu \mathrm{g} K \mathrm{Kg}$ \\
\hline 4-Chloro-3-methyl phenol & ND & $1000 \mu \mathrm{g} / \mathrm{Kg}$ & 4-Nitroaniline & ND & $1000 \mu \mathrm{g} / \mathrm{Kg}$ \\
\hline 2-Chloronaphthaiene & ND & $500 \mu \mathrm{g} / \mathrm{Kg}$ & Nitrobenzene & ND & $500 \mu \mathrm{g}$ \\
\hline - hlorophenol & $N D$ & $500 \mu g / K g$ & 2-Nitrophenol & ND & $500 \mu \mathrm{g} \mathrm{Kg}$ \\
\hline -nlorophenyl phenyl ether & ND & $500 \mathrm{\mu g} / \mathrm{Kg}$ & 4-Nitrophenol & $\mathrm{ND}$ & $2500 \mu \mathrm{g} \mathrm{Kg}$ \\
\hline Chrysene & ND & $50011 \mathrm{~g} / \mathrm{Kg}$ & N-Nitrosodi-n-propylamine & ND & $500 \mu \mathrm{g} K \mathrm{Kg}$ \\
\hline Dibenzo $(\mathrm{a} h$ ) anthracene & ND & $500 \mu \mathrm{g} / \mathrm{Kg}$ & N-Nitrosodiphenylamine & ND & $500 \mu \mathrm{Kg}$ \\
\hline Dibenzofuran & ND & $500 \mu \mathrm{g} / \mathrm{Kg}$ & Pentachlorophenol & $\mathrm{ND}$ & $2500+\mathrm{g} / \mathrm{Kg}$ \\
\hline Di-n-butyl phthalate & $\wedge \mathrm{D}$ & $500 \mu g / \mathrm{Kg}$ & Phenol & ND & $500 \mu g i \mathrm{Kg}$ \\
\hline 1.2-Dichlorobenzene (o-DCB) & ND & $500 \mathrm{\mu g} / \mathrm{Kg}$ & Phenanthrene & $\mathrm{ND}$ & 500 ug $\mathrm{Kg}$ \\
\hline 1.3-Dichlorobenzene (m-DCB) & $\mathrm{ND}$ & $500 \mu \mathrm{g} / \mathrm{Kg}$ & Рутеле & $\mathrm{ND}$ & $500 \mu \mathrm{g} K \mathrm{Kg}$ \\
\hline I,4-Dichlorobenzene ( $\mathrm{p}$-DCB) & ND & $500 \mu g / K g$ & 1,2,4-Trichlorobenzene & ND & $500 \mu \mathrm{g} / \mathrm{Kg}$ \\
\hline 2,4-Dichlorophenol & $\mathrm{ND}$ & $500 \mu \mathrm{g} / \mathrm{Kg}$ & 2,4,5-Trichlorophenol & $\mathrm{ND}$ & $500 \mu \mathrm{g} \mathrm{Kg}$ \\
\hline 3.3'-Dichlorobenzidine & $\mathrm{ND}$ & $1000 \mu \mathrm{g} / \mathrm{Kg}$ & 2,4,6-Trichlorophenol & $\mathrm{ND}$ & $500+\mathrm{g} / \mathrm{K}$ \\
\hline Diethylphthalate & ND & $500 \mu \mathrm{g} / \mathrm{Kg}$ & & & \\
\hline 2.4-Dimethylphenol & $\mathrm{ND}$ & $500 \mu g / \mathrm{Kg}$ & & & \\
\hline Dimethviphthalate & ND & $500 \mu \mathrm{g} / \mathrm{Kg}$ & & & \\
\hline 4.6-Dinitro-2-methyl phenol & $\mathrm{ND}$ & $2500 \mu \mathrm{g} / \mathrm{Kg}$ & & & \\
\hline 2.4-Dinitrotoluene (DNT) & ND & $500 \mu \mathrm{g} / \mathrm{Kg}$ & & & \\
\hline 2.6-Dinitrololuene (DNT) & $\mathrm{ND}$ & $500 \mu \mathrm{g} / \mathrm{Kg}$ & & & \\
\hline 2.4-Dinitrophenoi & $N D$ & $2500 \mu \mathrm{g} / \mathrm{Kg}$ & & & \\
\hline
\end{tabular}

ND - Nor Detected

QUALITY CONTROL DATA.

\section{Surrogate}

2,4,6-Tribromophenol

2-Fluorobiphenyl

2-Fluorophenol

$:$ benzene-d5

$p$-Terphenyi-dl4

Phenol-d5

\section{\% Recovery}

88

78

66

75

83

77
Acceptable Range

$19-122$

$30-115$

$25-121$

$23-120$

$18-137$

$24-113$

This report shall not be reproduced except in full, without the written approval of the laboratory. 


\section{NEL LABORATORIES}

$\begin{array}{ll}\text { CLIENT: } & \text { BechteI Nevada } \\ \text { PROJECT ID: } & \text { V431/17777 } \\ \text { PROJECT } \sharp: & 17777\end{array}$

CLIENT ID:

DATE SAMPLED: NA

NEL SAMPLE ID: 061098-E1-LCS

TEST: $\quad$ Semi-Volatile Organic Compounds by EPA 8270C, Dec. 1996

MATRIX: Solid

EXTRACTED: 6/11/98

ANALYZED: $6 / 12 / 98$

ANALYST: CCI

\section{PARAMETER}

Acenaphthene

4-Chloro-3-metbyl phenol

2-Chlorophenol

1,4-Dichlorobenzene ( $\mathrm{p}-\mathrm{DCB}$ )

2,4-Dinitrotoluene (DNT)

4-Nitrophenol

N-Nitrosodi-n-propylamine

Pentachlorophenol

Phenol

1.2,4-Trichlorobenzene
QLALITY CONTROL DATA:

\section{Surrogate}

2.4.6-Tribromophenol

2-Fiuorobipheny]

2-Fluorophenol

Nitrobenzene-ds

p-Terphenyl-di 4

Phenol-d5

\section{Percent Recovery}

79

85

83

80

90

89

108

89

79

87

\section{Acceptable Range}

$31-137$

$26-103$

$25-102$

$28-104$

$28-89$

$11-114$

$41-126$

$17-109$

$26-90$

$38-107$

\section{Percent Recovery}

69

91

73

90

105

94

\section{Acceptable Range}

$19-122$

$30-115$

$25-121$

$23 \cdot 120$

$18-137$

$24-113$ 
NEL LABORATORIES

$\begin{array}{llll}\text { CLIENT: } & \text { Bechtel Nevada } & \text { CLIENT ID: } & \text { Laboratory Control Spike Duplicate } \\ \text { 'ROJECT ID: } & \text { V431/17777 } & \text { DATE SAMPLED: NA } \\ \text { ROJECT \#: } & 17777 & \text { NEL SAMPLE ID: } 061098-E 1-L C S D\end{array}$

TEST: $\quad$ Semi-Volatile Organic Compounds by EPA 8270C, Dec. 1996

MATRIX: Solid

EXTRACTED: $6 / 11 / 98$

ANALYZED: $6 / 12 / 98$

ANALYST: CCJ

\section{PARAMETER}

Acenaphthene

4-Chioro-3-methyl phenol

2-Chlorophenol

1.4-Dichlorobenzene (p-DCB)

2.4-Dinitrotoluene (DNT)

4-Nitrophenol

N-Nitrosodi-n-propylamine

Pentachlorophenol

Phenol

1,2.4-Trichlorobenzene

QLALITY CONTROL DATA.

\section{Surrogate}

2.4,6-Tribromophenol

2-Fluotobiphenyl

2-FIuorophenol

Vitrobenzene-d5

Terphenyl-dit

senol-d5
Percent Recovery

69

75

71

66

75

74

89

73

66

73

\section{Percent Recovery}

61

78

63

76

85

80

\section{Acceptable Range $\quad \underline{\text { RPD }}$}

$31-137$

$26-103$

$25-102$

$28-104$

$28-89$

$11-114$

$41-126$

$17-109$

$26-90$

$38 \cdot 107$

14.2

12.3

15.4

19.9

17.9

18.7

18.8

19.8

18.5

17.3

Acceptable Range

$19-122$

$30-115$

$25-121$

$23-120$

$18-137$

$24-113$ 
POLYCHLORINATED

\section{BIPHENYLS}

DATA 
NEL LABORATORIES

CLIENT: Bechte! Nevada

POJECT NAME: V431

ROJECT NUMBER: 17777

CLIENT ID: Decon W2-3

DATE SAMPLED: 6/3.98

NEL SAMPLE ID: L9806093-03

TEST: Polychlorinated Biphenyl's (PCB's) by EPA 8082, Dec. 1996

MATRIX: Solid

DILUTION: 1

\begin{tabular}{l} 
PARAMETER \\
Arochlor-1016 \\
Arochlor-1221 \\
Arochlor-1232 \\
Arochlor-1242 \\
Arochlor-1248 \\
Arochlor-1254 \\
Arochlor-1260 \\
\hline \hline ND- Not Detected \\
QUALITY CONTROL DATA:
\end{tabular}

\section{Surrogate}

Decachlorobipheny]

]etraciloro-[m-xy]ene
EXTRACTED: $6 / 11 / 98$

ANALYZED: $6 / 13 / 98$

ANALYST: John

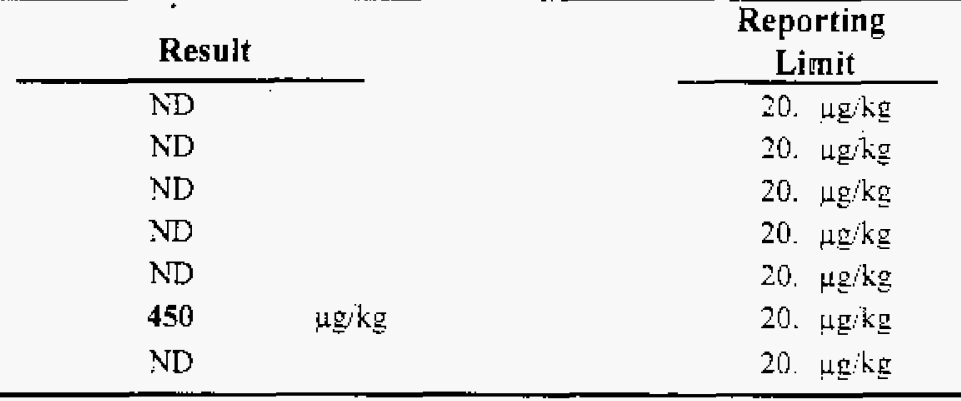

$\%$ Recovery

103

95
Acceptable Range

$70-130$

$70-130$ 
NEL LABORATORIES

CLIENT: Bechtel Nevada

PROJECT NAME: V431

PROJECT NUMBER: 17777

CLIENT ID: Decon P6-3

DATE SAMPLED: $6 / 3 / 98$

NEL SAMPLE ID: L9806093-09

TEST: Polychlorinated Biphenyl's (PCB's) by EPA 8082, Dec. 1996

MATRIX: Solid

EXTRACTED: $6 / 11 / 98$

DILUTION: 1

ANALYZED: $6 / 1398$

ANALYST: John

PARAMETER

Arochlor-1016

Arochlor-1221

Arochlor 1232

Arochlor- 1242

Arochlor- 1248

Arochlor-1254

Arochlor-1260

\begin{tabular}{l} 
Result \\
\hline $\mathrm{ND}$ \\
$\mathrm{ND}$ \\
$\mathrm{ND}$ \\
$\mathrm{ND}$ \\
$\mathrm{ND}$ \\
$\mathrm{ND}$ \\
$\mathrm{ND}$
\end{tabular}

Reporting

Limit

20. $\mu \mathrm{g} / \mathrm{kg}$

20. $\mu \mathrm{g} / \mathrm{kg}$

20. $\mu \mathrm{g} / \mathrm{kg}$

20. $\mu \mathrm{g} / \mathrm{kg}$

20. $\mu \mathrm{g} / \mathrm{kg}$

20. $\mathrm{Hg} / \mathrm{kg}$

20. $1 \mathrm{ggkg}$

$\mathrm{VD}$ - Not Detected

OUALITY CONTROL DATA:

\section{Surrogate}

Decachlorobiphenyi

Tetrachloro-m-xylene
$\%$ Recovery

102

98
Acceptable Range

$70-130$

$70-130$ 
NEL LABORATORIES

CLIENT: Bechtel Nevada

CLIENT ID: Decon P7-3

DROJECT NAME: V431

.OJECT NUMBER: 17777

DATE SAMPLED: $6 / 3 / 98$

NEL SAMPLE ID; L9806093-14

TEST: Polychlorinated Biphenyl's (PCB's) by EPA 8082, Dec. 1996

MATRIX: Solid

EXTRACTED: $6 / 11 / 98$

DILUTION: 1

ANALYZED: $6 / 12 / 98$

ANALYST: John

\begin{tabular}{lll}
\hline PARAMETER & Result & \multicolumn{1}{c}{$\begin{array}{c}\text { Reporting } \\
\text { Limit }\end{array}$} \\
\cline { 2 - 3 } Arochlor-1016 & $\mathrm{ND}$ & $20 . \mu \mathrm{g} / \mathrm{kg}$ \\
Arochlor-1221 & $\mathrm{ND}$ & $20 . \mu \mathrm{g} / \mathrm{kg}$ \\
Arochlor-1232 & $\mathrm{ND}$ & $20 . \mu \mathrm{g} / \mathrm{kg}$ \\
Arochlor-1242 & $\mathrm{ND}$ & $20 . \mu \mathrm{g} / \mathrm{kg}$ \\
Arochlor-1248 & $\mathrm{ND}$ & $20 . \mu \mathrm{g} / \mathrm{kg}$ \\
Arochlor-1254 & $\mathrm{ND}$ & $20 . \mu \mathrm{kg} / \mathrm{kg}$ \\
Arochlor-1260 & $\mathrm{ND}$ & $20 . \mu \mathrm{kg} g$ \\
\hline
\end{tabular}

ND - Not Detected

QUALITY CONTROL DATA

\section{Surrogate}

Decachlorobiphenyl

Tetrachloro-m-xylene
$\%$ Recovery

92

83
Acceptable Range

$70-130$

$70-130$ 
NEL LABORATORIES

CLIENT: Bechtel Nevada

PROJECT NAME: V431

CLIENT ID: Decon P8-3

PROJECT NUMBER: 17777

DATE SAMPLED: $6 / 3 / 98$

NEL SAMPLE ID: L9806093-19

TEST: Polychlorinated Biphenyl's (PCB's) by EPA 8082, Dec. 1996

MATRIX: Solid

EXTRACTED: $6 / 11 / 98$

DILLTION: 1

ANALYZED: $6 / 13 / 98$

ANALYST: John

PARAMETER

Arochlor-1016

Arochlor-1221

Arochlor-1232

Arochlor 1242

\begin{tabular}{ll} 
Result \\
\hline ND \\
ND \\
ND \\
ND \\
ND \\
ND \\
ND
\end{tabular}

Reporting

Limit

20.. $\mathrm{gg} \mathrm{kg}$

20. $\mu \mathrm{gg} / \mathrm{kg}$

20. $\mu \mathrm{g} / \mathrm{kg}$

20. $\mu \mathrm{g} / \mathrm{kg}$

Arochlor-1248

20. $\mu \mathrm{g} / \mathrm{kg}$

Arochlor-1254

Arochlor -1260

20. $\mu \mathrm{g} / \mathrm{kg}$

20. $\mu \mathrm{g} / \mathrm{kg}$

ND - Not Detected

QUALITY CONTROL DATA:

Surrogate

Decachlorobipheryl

Tetrachloro-m-xyiene
\% Recovery

87

83
Acceptable Range

$70 \cdot 130$

$70-130$ 
NEL LABORATORIES

$\begin{array}{clll}\text { CLIENT: } & \text { Bechtel Nevada } & \text { CLIENT ID: } & \text { Matrix Spike } \\ \text { DROJECT ID: } & \text { V431/17777 } & \text { DATE SAMPLED: } & \text { NA } \\ \text { OJECT H: } & 17777 & \text { NEL SAMPLE ID: } & \text { L9806093-19-MS }\end{array}$

TEST: $\quad$ Polychlorinated Biphenyl's (PCB's) by EPA 8082, Dec. 1996

MATRIX: Solid EXTRACTED: 6/11/98

ANALYZED: $6 / 12 / 98 \quad$ ANALYST: John

PARAMETER $\quad$ Percent Recovery Acceptable Range

$\begin{array}{llr}\text { Arochlor-1260 } & 85 & 48-129\end{array}$

OUALITY CONTROL DATA:

Surrogate

Decachlorobiphenyl

Tetrachioro-m-xylene
Percent Recovery

97

94
Acceptable Range

$70-130$

$70-130$ 
NEL LABORATORIES

CLIENT: Bechtel Nevada

PROJECT ID: V431/17777

PROJECT \#: 1777 ;

\section{CLIENT ID: Matrix Spike Duplicate}

DATE SAMPLED: NA

NEL SAMPLE ID: L9806093-19-MSD

TEST: $\quad$ Polychlorinated Biphenyl's (PCB's) by EPA 8082, Dec. 1996

MATRIX: Solid

EXTRACTED: 6/11/98

ANALYZED: $6 / 12 / 98$

ANALYST: John

\begin{tabular}{l} 
PARAMETER \\
Arochlor- 1260 \\
\hline QUALITY CONTROL DATA:
\end{tabular}

\section{Surrogate}

Decachlorobiphenyl

Tetrachioro-m1-xylene
Percent Recovery

69

Percent Recovery

86

90

\section{Acceptable Range}

$48-129$

$\underline{\text { RPD }}$

20.7

\section{Acceptable Range}

$70-130$

$70-130$ 
NEL LABORATORIES

CLIENT: Bechtel Nevada

"ROJECT NAME:

V431

ROJECT NUMBER: 17777
CLIENT ID: Method Blank

DATE SAMPLED: NA

NEL SAMPLE ID: $98061158082-B K$

TEST: Polychlorinated Biphenyl's (PCB's) by EPA 8082, Dec. 1996

MATRIX: Solid

EXTRACTED: 6/11/98

ANALYZED: $6 / 12 / 98$
ANALYST: John

\begin{tabular}{c} 
Result \\
\hline ND \\
ND \\
ND \\
ND \\
ND \\
ND \\
ND
\end{tabular}

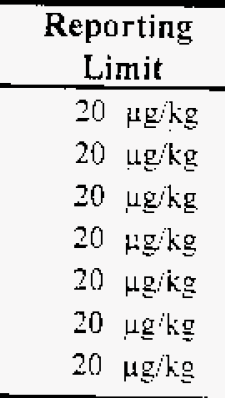

ND - Not Detected

QUALITY CONTROL DATA:

Surrogate

Decachlorobiphenyl

Tetrachloro-m-xylene
$\%$ Recovery

84

68
Acceptable Range

$70-130$

$70-130$ 


\section{NEL LABORATORIES}

CLIENT: Bechtel Nevada

PROJECT ID: V431/17777

CLIENT ID:

DATE SAMPLED

Laboratory Control Spike

PROJECT \#: $\quad 17777$

NEL SAMPLE ID: 980611 s8082-LCS

TEST: $\quad$ Polychlorinated Biphenyl's (PCB's) by EPA 8082, Dec. 1996

MATRIX: Solid

EXTRACTED: $6 / 11 / 98$

ANALYZED: $6 / 12 / 98$

ANALYST: John

\section{PARAMETER}

Arochlor-1016

Arochlor- 1260

Percent Recovery

86

82

Acceptable Range

QUALITY CONTROL DATA:

\section{Surrogate}

Decachiorobiphenyl

Tetrachloro-m-xylene
Percent Recovery

101

100
$80-120$

$80-120$

\section{Acceptable Range}

$70-130$

$70-130$ 


\section{TOTAL \\ PETROLEUM \\ HYDROCARBON \\ DATA}


CLIENT: Bechtel Nevada

TROJECT NAME: V431

OJECT NUMBER: 17777

TEST: $\quad$ Total Extractable Petroleum Hydrocarbons by EPA Method 8015M, July 1992

METHOD: $\quad$ EPA 8015M

MATRIX: Solid

ANALYST: John

\begin{tabular}{|c|c|c|c|c|c|c|c|c|}
\hline $\begin{array}{l}\text { CLIENT } \\
\text { SAMPLE ID } \\
\end{array}$ & $\begin{array}{c}\text { SAMPLE } \\
\text { DATE }\end{array}$ & $\begin{array}{c}\text { NEL } \\
\text { SAMPLE ID }\end{array}$ & $\begin{array}{r}\text { RESULT } \\
\mathrm{mg} / \mathrm{kg} \\
\end{array}$ & C.R. & $\begin{array}{c}\text { Reporting } \\
\text { Limit } \\
\end{array}$ & $\begin{array}{l}\text { Surrogate } \\
\text { Recovery }\end{array}$ & EXTRACTED & ANALYZED \\
\hline Decon W2-4 A\&B & $6 / 3 / 98$ & L9806093-04 & 5500 & GO & $100 . \mathrm{mg} / \mathrm{kg}$ & $70 \%$ & $6 / 10 / 98$ & $6 / 10 / 98$ \\
\hline Decon P6 4 A\&B & $6 / 3 / 98$ & L9806093.10 & 94 & 0 & $10 . \mathrm{mg} / \mathrm{kg}$ & $101 \%$ & $6 / 10 / 98$ & $6: 10: 98$ \\
\hline Decon P7-4 A\&B & $6 / 3 / 98$ & L9806093-15 & $\mathrm{ND}$ & & 10. $\mathrm{mg} / \mathrm{kg}$ & $116 \%$ & $6 / 10 / 98$ & $6 / 1098$ \\
\hline Decon P8-4 A\&B & $6 / 3 / 98$ & L9806093-20 & ND & & $10 . \mathrm{mg} / \mathrm{kg}$ & $103 \%$ & $6 / 10 / 98$ & $6: 10: 98$ \\
\hline
\end{tabular}

\section{CR: Carbon Range}

GO Gas Range Organics (C8 to $\mathrm{C} 12$ ) and Oil Range Organics (C12 to $\mathrm{C} 38$ ).

$\mathrm{O}$ Oil Range Otganics ( $\mathrm{C} 12$ to $\mathrm{C} 38$ ).

Note: The reporting limit for Oil Range Organics in soil is $50 \mathrm{mg} / \mathrm{kg}$.

QUALITY CONTROL DATA (Total for Gas and Diesel Range):

Sample ID

Blank, 980610TPH-BLK

I $980610 \mathrm{TP}$ - LCS

Ms; $980610 \mathrm{TP}$ - MS

MSD, 980610TP - MSD

ND - Not Detected

* Surrogate used was Octacosane. acceptance limits $54-130 \%$ for solids, $60-121 \%$ for aqueous samples

This report shall not be reproduced except in full, without the written approval of the laboraton. 
TCLP

\section{METALS}

DATA 
NEL LABORATORIES

CLIENT: Bechtel Nevada

ROJECT NAME: V431

$\checkmark$ ROJECT NUMBER: 17777
CLIENT ID: Decon W2-5

DATE SAMPLED: $6 / 3 / 98$

NEL SAMPLE ID; L9806093-05

TEST:

TCLP-8 Metals

MATRIX: Solid

\begin{tabular}{|c|c|c|c|c|c|c|c|}
\hline PARAMETER & $\begin{array}{c}\text { RESULT } \\
\text { mg/L } \\
\end{array}$ & $\begin{array}{c}\text { REPORTING } \\
\text { LIMIT } \\
\end{array}$ & D. F. & METHOD & $\begin{array}{c}\text { TCLP/STLC } \\
\text { EXTRACTION } \\
\text { DATE } \\
\end{array}$ & DIGESTED & ANALYZED \\
\hline Arsenic & $\mathrm{ND}$ & $0.1 \mathrm{mg} / \mathrm{L}$ & 1 & EPA $6010 A$ & $6 / 11 / 98$ & $6 / 12 / 98$ & $6 / 14 / 98$ \\
\hline Barium & ND & $1 . \mathrm{mg} / \mathrm{L}$ & 1 & EPA $6010 \mathrm{~A}$ & $6 / 11 / 98$ & $6 / 12 / 98$ & $6 / 14 / 98$ \\
\hline Cadmium & 0.011 & $0.01 \mathrm{mg} / \mathrm{L}$ & 1 & EPA $6010 \mathrm{~A}$ & $6 / 11 / 98$ & $6 / 12 / 98$ & $6 / 14 / 98$ \\
\hline Chromium & $\mathrm{ND}$ & $0.01 \mathrm{mg} / \mathrm{L}$ & 1 & EPA $6010 A$ & $6 / 11 / 98$ & $6 / 12 / 98$ & $6 / 14 / 98$ \\
\hline Lead & $\mathrm{ND}$ & $0.05 \mathrm{mg} / \mathrm{L}$ & 1 & EPA $6010 A$ & $6 / 11 / 98$ & $6 / 12 / 98$ & $6 / 14 / 98$ \\
\hline Mercury & $\mathrm{ND}$ & $0.002 \mathrm{mg} / \mathrm{L}$ & 10 & EPA $7470 \mathrm{~A}$ & $6 / 11 / 98$ & $6 / 12 / 98$ & $6 / 14 / 98$ \\
\hline Selenium & ND & $0.1 \mathrm{mg} / \mathrm{L}$ & 1 & EPA $6010 \mathrm{~A}$ & $6 / 11 / 98$ & $6 / 12 / 98$ & $6 / 14 / 98$ \\
\hline Silver & ND & $0.02 \mathrm{mg} / \mathrm{L}$ & 1 & EPA $6010 A$ & $6 / 11 / 98$ & $6 / 12 / 98$ & $6 / 14 / 98$ \\
\hline
\end{tabular}

D.F. - Dilution Factor

ND - Not Detected

This report shall not be reproduced except in full, without the written approval of the laboratory. 
NEL LABORATORIES

CLIENT: Bechtel Nevada

PROJECT NAME: V431

PROJECT NUMBER: 17777
CLIENT ID: $\quad$ Decon P6-5

DATE SAMPLED: $6 / 3 / 98$

NEL SAMPLE ID: L9806093-11

TEST: $\quad$ TCLP-8 Metals

MATRIX: Solid

PARAMETER

Arsenic

Barium

Cadmium

Chromium

Lead

Mercun:

Selenium

Silver
RESULT REPORTING

$\mathrm{mg} / \mathrm{h}$

$\mathrm{ND}$

$\mathrm{ND}$

ND

ND

ND

ND

ND

ND
LIMIT

$0.1 \mathrm{mg} / \mathrm{L}$

1. $\mathrm{mg} / \mathrm{L}$

$0.01 \mathrm{mg} /$

$0.01 \mathrm{mg} / \mathrm{L}$

$0.05 \mathrm{mg} / \mathrm{L}$

$0.002 \mathrm{mg} / \mathrm{L}$

$0.1 \mathrm{mg} / \mathrm{L}$

$0.02 \mathrm{mg} / \mathrm{L}$

TCLP/STLC EXTRACTION

D.F. METHOD

I EPA 6010A

EPA 6010A

EPA 6010A

EPA 6010A

EPA 6010A

EPA 7470A

EPA 6010A

EPA 6010A
DATE

$6 / 11 / 98$

$6 / 11 / 98$

$6 / 11 / 98$

$6 / 11 / 98$

$6 / 11 / 98$

$6 / 11 / 98$

$6 / 11 / 98$

$6 / 11 / 98$
DIGESTED ANALYZED

$6 / 12 / 98 \quad 6 / 14 / 98$

$6 / 12 / 98 \quad 6 / 14 / 98$

$6 / 12 / 98 \quad 6 / 14 / 98$

$6 / 12 / 98 \quad 6 / 14 / 98$

$6 / 12 / 98 \quad 6 / 14 / 98$

$6 / 12 / 98 \quad 6 / 14 / 98$

$6 / 12 / 98 \quad 6 / 14 / 98$

$6 / 12 / 98 \quad 6 / 14 / 98$

D.F - Dilution Factor

ND - Not Detected

This report shall not be reproduced except in full, without the wrinen approval of the laboratory. 
NEL LABORATORIES

CLIENT: Bechtel Nevada

?OJECT NAME: V431

$\ldots$ - $\angle$ OJECT NUMBER: 17777
CLIENT ID: Decon P7-5

DATE SAMPLED: $6 / 3 / 98$

NEL SAMPLE ID: L9806093-16

TEST:

TCLP-8 Metals

MATRIX: Solid

PARAMETER

Arsenic

Barium

Cadmium

Chromium

Lead

Mercur:

Selenium

Silver

\section{RESULT REPORTING} $\mathrm{mg} / \mathrm{L} \quad$ LIMIT

ND

ND

$\mathrm{ND}$

$\mathrm{ND}$

ND

ND

$\mathrm{ND}$

$\mathrm{ND}$
$0.1 \mathrm{mg} / \mathrm{L}$

$$
\text { 1. } \mathrm{mg} / \mathrm{L}
$$

$0.01 \mathrm{mg} / \mathrm{L}$

$0.01 \mathrm{mg} / \mathrm{L}$

$0.05 \mathrm{mg} / \mathrm{L}$

$0.002 \mathrm{mg} L$

$0.1 \mathrm{mg} / \mathrm{L}$

$0.02 \mathrm{mg} / \mathrm{L}$
TCLP/STLC EXTRACTION

D. F. METHOD DATE

$6 / 11 / 98$

$6 / 11 / 98$

$6 / 11 / 98$

$6 / 11 / 98$

$6 / 11 / 98$

$6 / 11 / 98$

$6 / 11.98$

$6 /[1 / 98$
DIGESTED ANALYZED

$6 / 12 / 98 \quad 6 / 14 / 98$

$6 / 12 / 98 \quad 6 / 14 / 98$

$6 / 12 / 98 \quad 6 / 14 / 98$

$6 / 12 / 98 \quad 6 / 14 / 98$

$6 / 12 / 98 \quad 6 / 14 / 98$

$6 / 12 / 98 \quad 6 / 14 / 98$

$6 / 12 / 98 \quad 6 / 14 / 98$

$6 / 12 / 98 \quad 6 / 14 / 98$

D.F. - Dilution Factor

ND - Not Detected

This report shall not be reproduced except in full, without the written approval of the laborator. 
NEL LABORATORIES

CLIENT: Bechtel Nevada

PROJECT NAME: V431

PROJECT NUMBER: 17777
CLIENT ID:

DATE SAMPLED: $6 / 3 / 98$

NEL SAMPLE ID: L9806093-21

\section{TEST: $\quad$ TCLP-8 Metals}

MATRIX: Solid

\begin{tabular}{|c|c|c|c|c|c|c|c|}
\hline PARAMETER & $\begin{array}{c}\text { RESULT } \\
\mathrm{mg} / \mathrm{L} \\
\end{array}$ & $\begin{array}{c}\text { REPORTING } \\
\text { LIMIT } \\
\end{array}$ & D. F. & METHOD & $\begin{array}{c}\text { TCLP/STLC } \\
\text { EXTRACTION } \\
\text { DATE } \\
\end{array}$ & DIGESTED & ANALYZED \\
\hline Arsenic & ND & $0.1 \mathrm{mg} / \mathrm{L}$ & 1 & EPA $6010 \mathrm{~A}$ & $6 / 11 / 98$ & $6 / 12 / 98$ & $6 / 14 / 98$ \\
\hline Barium & ND & $1 . \mathrm{mg} / \mathrm{L}$ & 1 & EPA 6010A & $6 / 11 / 98$ & $6 / 12 / 98$ & $6 / 14 / 98$ \\
\hline Cadmium & ND & $0.01 \mathrm{mg} / \mathrm{L}$ & 1 & EPA 6010A & $6 / 11 / 98$ & $6 / 12 / 98$ & $6 / 14 / 98$ \\
\hline Chromitum & ND & $0.01 \mathrm{mg} / \mathrm{L}$ & 1 & EPA $6010 \mathrm{~A}$ & $6 / 11 / 98$ & $6 / 12 / 98$ & $6 / 14 / 98$ \\
\hline Lead & ND & $0.05 \mathrm{mg} / \mathrm{L}$ & 1 & EPA $6010 \mathrm{~A}$ & $6 / 11 / 98$ & $6 / 12 / 98$ & $6 / 14 / 98$ \\
\hline Mercury & ND & $0.002 \mathrm{mg} / \mathrm{L}$ & 10 & EPA $7470 A$ & $6 / 11 / 98$ & $6 / 12 / 98$ & $6 / 14 / 98$ \\
\hline Selenium & $\mathrm{ND}$ & $0.1 \mathrm{mg} / \mathrm{L}$ & 1 & EPA $6010 A$ & $6 / 11 / 98$ & $6 / 12 / 98$ & $6 / 14 / 98$ \\
\hline Silver & $\mathrm{ND}$ & $0.02 \mathrm{mg} / \mathrm{h}$ & 1 & EPA $6010 A$ & $6 / 11 / 98$ & $6 / 12 / 98$ & $6 / 14 / 98$ \\
\hline
\end{tabular}

D.F. - Dilution Factor

ND - Not Detected

This report shall not be reproduced except in full, without the written approval of the laboratory. 
NEL LABORATORIES

$\begin{array}{llll}\text { CLIENT: } & \text { Bechtel Nevada } & \text { CLIENT ID: } & \text { Matrix Spike } \\ \text { nOIECT ID: } & \text { V431/17777 } & \text { DATE SAMPLED: } & \text { NA } \\ \ldots . \text { OJECT \#: } & 17777 & \text { NEL SAMPLE ID: } & \text { L9806093-21-MS }\end{array}$

TEST:

TCLP by EPA 1311, July 1992 \& 7 Metals by EPA 6010A, July 1992

MATRIX: Solid

EXTRACTED: $6 / 12 / 98$

ANALYZED: $6 / 14 / 98$

ANALYST: EMF

\section{PARAMETER}

Arsenic

Silver

Barium

Cadmium

Chromium

Lead

Selenium
Percent Recovery

106

100

91

105

98

101

111

\section{Acceptable Range}

$85-115$

$85-115$

$85-115$

$85-115$

$85-115$

$85-115$

$85-115$ 
NEL LABORATORIES

$\begin{array}{llll}\text { CLIENT: } & \text { Bechtel Nevada } & \text { CLIENT ID: } & \text { Matrix Spike Duplicate } \\ \text { PROJECT ID: } & \text { V431/17777 } & \text { DATE SAMPLED: } & \text { NA } \\ \text { PROJECT \#: } & 17777 & \text { NEL SAMPLE ID: } & \text { L9806093-21-MSD }\end{array}$

TEST: $\quad$ TCLP by EPA 1311, July 1992 \& 7 Metals by EPA 6010A, July 1992

MATRIX: Solid

EXTRACTED: $6 / 12 / 98$

ANALYZED: $6 / 14 / 98$

ANALYST: EMF

\section{PARAMETER}

Silver

Arsenic

Barium

Cadmium

Chromium

Lead

Selenium

\section{Percent Recovery}

100

105

90

104

97

101

111

\section{Acceptable Range $\quad \underline{\text { RPD }}$}

$85-115$

$85-115$

$85-115$

$85-115$

$85-115$

$85-115$

$85-115$
0.4

0.2

I1 1.1

3.9

3. 


\begin{tabular}{llll}
\multicolumn{1}{c}{ NEL LABORATORIES } & \\
\hline CLIENT: & Bechtel Nevada & CLIENT ID: & Matrix Spike Duplicate \\
MOJECT ID: & V431/17777 & DATE SAMPLED: NA \\
. OJECT \#: & 17777 & NEL SAMPLE ID: L9806093-21-MSD
\end{tabular}

TEST: $\quad$ TCLP by EPA 1311, July 1992 \& Mercury by EPA 7470A, July 1992

MATRIX: Solid

EXTRACTED: $6 / 12 / 98$

ANALYZED: $6 / 14 / 98$

ANALYST: EMF

PARAMETER

Percent Recovery

Acceptable Range

RPD

Mercury

99

$85-115$ 
NEL LABORATORIES

CLIENT: Bechtel Nevada

PROJECT NAME: V43I

CLIENT ID:

Method Blank

PROJECT NUMBER: 17777

DATE SAMPLED: NA

NEL SAMPLE ID: L06093I-BLK

TEST: $\quad$ TCLP by EPA 1311, July 1992 \& 7 Metals by EPA 6010A, July 1992

MATRIX: TCLP Extract

\begin{tabular}{|c|c|c|c|c|c|c|c|}
\hline \multirow[b]{2}{*}{ PARAMETER } & \multirow{2}{*}{\multicolumn{2}{|c|}{$\begin{array}{l}\text { REPORTING } \\
\text { LIMIT }\end{array}$}} & \multicolumn{4}{|c|}{$\begin{array}{c}\text { TCLP/STLC } \\
\text { EXTRACTION }\end{array}$} & \multirow[b]{2}{*}{ ANALYZED } \\
\hline & & & D. F. & METHOD & DATE & DIGESTED & \\
\hline Arsenic & ND & $0.1 \mathrm{mg} / \mathrm{L}$ & 1 & EPA $6010 \mathrm{~A}$ & $6 / 11 / 98$ & $6 / 12 / 98$ & $6 / 14 / 98$ \\
\hline Barium & ND & $1 \mathrm{mg} / \mathrm{L}$ & 1 & EPA $6010 \mathrm{~A}$ & $6 / 11 / 98$ & $6 / 12 / 98$ & $6 / 14 / 98$ \\
\hline Cadmium & ND & $0.01 \mathrm{mg} / \mathrm{L}$ & 1 & EPA $6010 \mathrm{~A}$ & $6 / 11 / 98$ & $6 / 12 / 98$ & $6 / 14 / 98$ \\
\hline Chromium & ND & $0.01 \mathrm{mg} /$ & 1 & EPA $6010 \mathrm{~A}$ & $6 / 11 / 98$ & $6 / 12 / 98$ & $6 / 14 / 98$ \\
\hline Lead & ND & $0.05 \mathrm{mg} / \mathrm{L}$ & 1 & EPA 6010A & $6 / 11 / 98$ & $6 / 12 / 98$ & $6 / 14 / 98$ \\
\hline Selenium & $N D$ & $0.1 \mathrm{mg} / \mathrm{L}$ & 1 & EPA $6010 \mathrm{~A}$ & $6 / 11 / 98$ & $6 / 12 / 98$ & $6 / 14 / 98$ \\
\hline Silver & ND & $0.02 \mathrm{mg} / \mathrm{L}$ & 1 & EPA 6010A & $6 / 1 / 98$ & $6 / 12 / 98$ & $6 / 14 / 98$ \\
\hline
\end{tabular}

D.F. - Dilution Factor

ND - Not Detected

This report shall not be reproduced except in full, without the written approval of the laboratory. 
NEL LABORATORIES

CLIENT: Bechtel Nevada

ROJECT NAME:

V431

.OJECT NUMBER: 17777

CLIENT ID:

Method Blank

DATE SAMPLED: NA

NEL SAMPLE ID: L06093HG-BLK

TEST:

TCLP by EPA 1311 , July 1992 \& Mercury by EPA 7470A, July 1992

MATRIX:

TCLP Extract

\begin{tabular}{|c|c|c|c|c|c|c|c|}
\hline \multirow[b]{2}{*}{ PARAMETER } & \multirow{2}{*}{\multicolumn{2}{|c|}{ REPORTING }} & \multicolumn{4}{|c|}{$\begin{array}{c}\text { TCLP/STLC } \\
\text { EXTRACTION }\end{array}$} & \\
\hline & & & D. F & METHOD & DATE & DIGESTED & ANALYZED \\
\hline Mercury & ND & $0.002 \mathrm{mg} / \mathrm{L}$ & 10 & EPA $7470 \mathrm{~A}$ & $6 / 11 / 98$ & $6 / 12 / 98$ & $6 / 14 / 98$ \\
\hline
\end{tabular}

D.F. - Dilution Factor

ND - Not Detected

$T^{\text {thi }}$ repori shall not be reproduced except in full, without the written approval of the laboratory 
NEL LABORATORIES

$\begin{array}{llll}\text { CLIENT: } & \text { Bechtel Nevada } & \text { CLIENT ID: } & \text { Laboratory Control Spike } \\ \text { PROJECT ID: } & \text { V431/17777 } & \text { DATE SAMPLED: NA } \\ \text { PROJECT \#: } & 17777 & \text { NEL SAMPLE ID: L06093I-LCS }\end{array}$

TEST: $\quad$ TCLP by EPA 1311, July 1992 \& 7 Metals by EPA 6010A, July 1992

MATRIX: Solid

EXTRACTED: $6 / 12 / 98$

ANALYZED: $6 / 14 / 98$

ANALYST: EMF

\section{PARAMETER}

Silver

Arsenic

Percent Recovery

99

Barium

104

Cadmium

95

Chromium

105

Lead

102

104

Selenium

107

\section{Acceptable Range}

$85-115$

$88-118.5$

$86-128.2$

$85-115$

$85-115$

$85-115$

$85-115$

This report shall not be reproduced except in full, without the written approval of the laboratory. 


\section{NEL LABORATORIES}

CLIENT: Bechtel Nevada

'ROJECT ID: V431/17777

ROJECT H: $\quad 17777$
CLIENT ID: DATE SAMPLED: NA

NEL SAMPLE ID: L06093HG-LCS

TEST:

TCLP by EPA 1311, July 1992 \& Mercury by EPA 7470A, July 1992

MATRIX: Solid

EXTRACTED: $6 / 12 / 98$

ANALYZED: $6 / 14 / 98$

Percent Recovery

97
ANALYST: JML

\section{Acceptable Range}

$85-115$

Mercury 


\section{SDG B437 \\ RADIOANALYTICAL RESULTS}




\section{Bechtel Nevada Corporation}

\section{ANALYTICAL SERVICES LABORATORY}

P.O.Box 3936, N. Las Vegas, NV 89036

Reported to: Area 6 Decon Pond Closure

Rernediation Projects

Report Date: 6-JUL-98

J. L. Smith, M/S NTS306

Sample Delivery Group: B437

Batch: 0820

Program: 720

Report No.:

\begin{tabular}{|c|c|c|c|c|c|c|c|c|c|c|c|c|c|c|c|c|}
\hline $\begin{array}{c}\text { Sample } \\
* * * * * \text { Identification * *** }\end{array}$ & Isotope & Analysis & Hesult & $\begin{array}{c}\text { Error } \\
\%\end{array}$ & $\begin{array}{l}\text { Oual } \\
\text { Flag }\end{array}$ & $M D A$ & $\begin{array}{l}\text { Result } \\
\text { Urits }\end{array}$ & $\begin{array}{c}\text { Analysis } \\
\text { Date }\end{array}$ & $\begin{array}{l}\text { Sample } \\
\text { Coll Date }\end{array}$ & Size & $\begin{array}{r}\text { Size } \\
\text { Units }\end{array}$ & $\begin{array}{c}\text { Type } \\
\text { Matrix }\end{array}$ & $\begin{array}{l}\text { Tracer } \\
\text { Yield \% }\end{array}$ & \begin{tabular}{|l|} 
Spike \\
Recv $\%$
\end{tabular} & $\begin{array}{l}\text { Systert } \\
\text { Detector }\end{array}$ & $\begin{array}{l}\text { Packet-lten } \\
\text { Sample }\end{array}$ \\
\hline DECON P6-6 & $K 40$ & GAM20 & $2.00 E+01$ & $2.1 \mathrm{E}+01$ & & $8.6 E-01$ & pCilg. & $06-04-98$ & $06-03-98$ & $5.86 \mathrm{E}+02$ & gm & SOIL G & & & $05-01$ & $69118-1 \cdot 75087$ \\
\hline DECON P6-6 & RA226 & GAM20 & $1.08 \mathrm{E}+00$ & $3.1 E+01$ & & 1.7E-01 & $\mathrm{pCi} / \mathrm{g}$ & $06-04-98$ & $06-03-98$ & $5.86 E+02$ & gm & SOIL G & & & 05.01 & G9118-1-75087 \\
\hline DECON P6-6 & $\mathrm{TH} 228$ & GAM20 & $2.36 E+00$ & $1.9 E+01$ & & $1.5 \mathrm{E}-01$ & $\mathrm{pCi} / \mathrm{g}$ & $06-04-98$ & $06-03-98$ & $5.86 \mathrm{E}+02$ & $\mathrm{gm}$ & SOIL G & & & $05-01$ & $69118-1-76087$ \\
\hline DECON P6-6 & TH232 & GAM20 & $1.81 E+00$ & $3.4 E+01$ & & $4.0 \mathrm{E}-01$ & pCilg & $06-04-98$ & $06-03.96$ & $5.86 E+02 \mid$ & $g m$ & SOIL G & & & $05-01$ & G91†8-1-75087 \\
\hline DECON P7.6 & K 40 & GAM20 & $2.41 E+01$ & $1.9 E+01$ & & $8.4 E-01$ & $\mathrm{pCi} / \mathrm{g}$ & 06-04-98 & $06-03-98$ & $6.26 \mathrm{E}+02$ & $\mathrm{gm}$ & SOIL G & & & $05-01$ & 69118-2-75089 \\
\hline DECON P7.6 & RA226 & GAM20 & $1.30 E+00$ & $2.7 E+01$ & & 1.7E-01 & $\rho \mathrm{Ci} / \mathrm{\theta}$ & $06-04-98$ & 06-03-98 & $6.26 E+02$ & gm & SOIL G & & & $05-01$ & 69118-2-75089 \\
\hline DECON P7-6 & $\mathrm{TH} 228$ & GAM20 & $2.58 \mathrm{E}+00$ & $1.8 \mathrm{E}+01$ & & $1.5 \mathrm{E}-01$ & pCi/g & $06-04-98$ & $06-03-98$ & $6.26 E+02$ & gan & SOL $\mathrm{G}$ & & & $05-01$ & 69118-2-750B9 \\
\hline DECON P7.6 & TH232 & GAM20 & $1.78 E+00$ & $3.5 E+01$ & & 3.9E-01 & $\rho \mathrm{Ci} / \mathrm{g}$ & $06-04-98$ & $06-03-98$ & $6.26 \mathrm{E}+02$ & $\mathrm{gm}$ & soll $\mathrm{G}$ & & & $05-01$ & G9118-2-75089 \\
\hline DECON P8-6 & K 40 & GAM20 & $2.14 \mathrm{E}+01$ & $2.1 E+01$ & & B.8E-01 & pCilg & $06-04-98$ & $06-03-9 B$ & $5.41 E+02$ & $\mathrm{gm}$ & SOLL G & & & $05-01$ & $69118-3-75091$ \\
\hline DECON PB-6 & मA226 & GAM20 & $1.22 \mathrm{E}+00$ & $3.1 E+01$ & & 1.7E-01 & pCilg & $06-04.98$ & 06-03-98 & $5.41 E+02$ & $\mathrm{gm}$ & SOlL G & & & $05-01$ & G9118-3-75091 \\
\hline
\end{tabular}

\section{Comment:}

Data generated from analyses of samples submitted on 6/4/98.

Gross alpha/beta determinations are performed on small sample

aliquots. Data may.not represent the sample as a whole.

Prepared by:

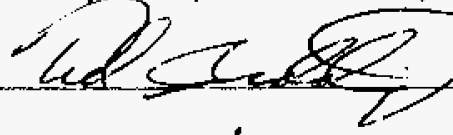

Date: $7 / 4 / 98$

Approved by: Somy fatenos Date: $7 / 4 / 98$
Qualification Flags:

$E=$ Estimated Quantity

$\mathrm{H}=$ High Recovery for Sample

$J=$ Result is less than the RDL

$\mathrm{L}=$ Low Recovery for Sample

$\mathrm{P}=$ Preliminary Results

$\mathrm{O}=$ Bad Instrument Quality Control, Result is $O K$

$R=$ Results are Unusable, Resampling is Necessary

$\mathrm{U}=$ Result is Iess than Minimum Detectable Activity
Note: \% Error is the 2.0 Sigma Error 


\section{Bechtel Nevada Corporation}

ANALYTICAL SERVICES LABORATORY

P.O.Box 3936, N. Las Vegas, NV 89036

Reported to: Area 6 Decon Pond Closure

Rernediation Projects

Feport Date: 6-JUL-98

J. L. Smith, M/S NTS306

Sample Delivery Group: B437

Batch: 0820

Program: 720

Report No. :

\begin{tabular}{|c|c|c|c|c|c|c|c|c|c|c|c|c|c|c|c|c|}
\hline $\begin{array}{c}\text { Sample } \\
\ldots * * \text { Identífication } \\
* * * * *\end{array}$ & Isotope & Analysis & Result & $\begin{array}{c}\text { Error } \\
\%\end{array}$ & $\begin{array}{l}\text { Qual } \\
\text { Flag }\end{array}$ & $M D A$ & $\begin{array}{l}\text { Result } \\
\text { Units }\end{array}$ & $\begin{array}{c}\text { Analysis } \\
\text { Date }\end{array}$ & $\begin{array}{r}\text { Sample } \\
\text { Coll Date }\end{array}$ & Size & $\begin{array}{r}\text { Sizo } \\
\text { Units }\end{array}$ & $\begin{array}{l}\text { Type } \\
\text { Matrix }\end{array}$ & $\left|\begin{array}{l}\text { Tracer } \\
\text { Yield \% }\end{array}\right|$ & $\begin{array}{l}\text { Spike } \\
\text { Recv \% }\end{array}$ & $\begin{array}{l}\text { Systern } \\
\text { Detector }\end{array}$ & $\begin{array}{l}\text { Packet-Item } \\
\text { Sample }\end{array}$ \\
\hline DECON PQ.6 & TH228 & GAM20 & $2.44 E+00$ & $1.9 E+01$ & & $1.6 \mathrm{E}-01$ & pCilg & $06-04-98$ & $06-03-98$ & $5.41 E+02$ & $\mathrm{gm}$ & SOIL $\mathrm{G}$ & & & $05-01$ & $69118-3-75091$ \\
\hline DECON PQ-6 & TH232 & GAM2O & $2.02 \mathrm{E}+00$ & $3.3 E+01$ & & $3.6 \mathrm{E}-01$ & $\mathrm{pCi} / \mathrm{g}$ & $06-04-98$ & $06-03-98$ & $5.41 E+02$ & $g m$ & SOIL G & & & 05-01 & G911日-3-75091 \\
\hline DECON P8-6 MS\&MSD & $K 40$ & GAM20 & $2.32 \mathrm{E}+01$ & $2.0 E+01$ & & $8.5 E-01$ & $\mathrm{pCi} / \mathrm{g}$ & $06-04-98$ & $06-03-98$ & $6.06 \mathrm{E}+02$ & gm & SOll G & & & 05-01 & $69118-4-75093$ \\
\hline DECON PQ.6 MS\&MSD & RA226 & GAM20 & $1.27 \mathrm{E}+00$ & $2.7 E+01$ & & $1.7 \mathrm{E}-01$ & pCilg & $06-04-98$ & $06-03-98$ & $6.06 \mathrm{E}+02$ & $\mathrm{gm}$ & SOlL G & & & $05-01$ & $69118-4-75093$ \\
\hline DECON Pg.6 MSAMSD & TH228 & GAM2O & $2.67 \mathrm{E}+00$ & $1.8 E+01$ & & $1.5 E-01$ & $\mathrm{pCil/9}$ & $06-04-98$ & $08-03-98$ & $6.06 E+02$ & $g m$ & SOlL G & & & 05-01 & G9118-4-75093 \\
\hline DECON P8.6 MS\&MSD & TH232 & GAM20 & $2.16 \mathrm{E}+00$ & $3.2 E+01$ & & 3.9E-01 & pCi/g & $06-04-98$ & 06.03 .98 & $6.06 \mathrm{E}+02$ & $g m$ & SOIL G & & & $05-01$ & G9118-4-75093 \\
\hline DECON W2-6 & $\mathrm{CO60}$ & GAM20 & $7.95 \mathrm{E}+01$ & $9.1 E+00$ & & 8.0E-02 & pCi/g & $06-04-98$ & $06-03-9 B$ & $6.03 E+02$ & $g m$ & SOIL G & & & $05-01$ & G9118-0-750B5 \\
\hline DECON W2-6 & $\operatorname{cs} 137$ & GAM20 & $0.80 E+01$ & $8.7 E+00$ & & $9.1 \mathrm{E}-02$ & $\mathrm{pCi} / \mathrm{g}$ & $06-04-98$ & $06-03-98$ & $6.03 E+02$ & $g m$ & SOIL, G & & & $05-01$ & 69118-0-75085 \\
\hline DECON W2-6 & EU152 & GAM20 & $2.92 \mathrm{E}+00$ & $4.0 \mathrm{E}+01$ & & 5.9E-01 & pCi/g & $06-04-98$ & $06-03-98$ & $6.03 E+02$ & $9 m$ & SOll, G & & & $05-01$ & 69118-0-75085 \\
\hline DECON W2-6 & EU154 & GAM20 & $4.08 E+00$ & $B .1 E+01$ & & 5.5E-01 & pCi/g & $06-04-98$ & $06-03-98$ & $6.03 E+02$ & gm & SOlL G & & & $05-01$ & $69118-0-75085$ \\
\hline
\end{tabular}

Comment:

Data generated from analyses of samples submitted on 6/4/9B.

Gross alpha/beta determinations are performed on small sampla

aliquots. Data may not represent the sample as a whole.

Prepared by

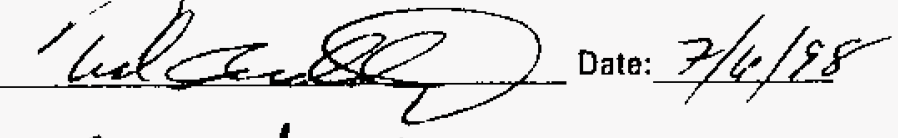

Approved by: Shwarffetente Date: $7 / 6 / 98$
Qualification Flags:

$\mathbf{E}=$ Estimated Quantity

$H=$ High Recovery for Sample

$J=$ Result is less than the RDL

$L=$ Low Pecovery for Sample

$P=$ Preliminary Results

$\mathbf{Q}=$ Bad Instrument Quality Control, Result is OK

$\mathbf{R}=$ Results are Unusable, Resampling is Necessary

$\mathbf{U}=$ Result is less than Minimum Detectable Activity

Note: \% Error is the 2.0 Sigma Error 


\section{Bechtel Nevada Corporation}

\section{ANALYTICAL SERVICES LABORATORY}

P.O.Box 3936, N. Las Vegas, NV 89036

Reported to: Area 6 Decon Pond Closure

Remediation Projects

J. L. Smith, M/S NTS306

Report Date: 6-JUL-98

Sample Delivery Group: B437

Batch: 0820

Program: 720

Report No.:

\begin{tabular}{|c|c|c|c|c|c|c|c|c|c|c|c|c|c|c|c|c|}
\hline $\begin{array}{c}\text { Sample } \\
\text {....." Identification ..... }\end{array}$ & Isotope & Analysis & Hesult & $\begin{array}{c}\text { Error } \\
\%\end{array}$ & $\begin{array}{l}\text { Oual } \\
\text { Flag }\end{array}$ & MDA & $\begin{array}{l}\text { Result } \\
\text { Units }\end{array}$ & $\begin{array}{c}\text { Analysis } \\
\text { Date }\end{array}$ & \begin{tabular}{|} 
Sample \\
Coll Date
\end{tabular} & Size & \begin{tabular}{|r|} 
Size \\
Units
\end{tabular} & \begin{tabular}{|c|} 
Typв \\
Matrix
\end{tabular} & $\begin{array}{l}\text { Tracer } \\
\text { Yield } \%\end{array}$ & \begin{tabular}{|l|} 
Spike \\
fecv $\%$
\end{tabular} & $\begin{array}{l}\text { System } \\
\text { Detector }\end{array}$ & $\begin{array}{l}\text { Packet-Item } \\
\text { Sample }\end{array}$ \\
\hline DECON W2-6 & EUt55 & GAM20 & $2.62 \mathrm{E}+00$ & $5.6 E+01$ & & 3.5E-01 & pCilg & $06.04-98$ & $06-03-98$ & $6.03 E+02$ & $g m$ & soll G & & & $05-01$ & $69118-0-75085$ \\
\hline DECON W2-6 & $k 40$ & GAM20 & $2.07 E+01$ & $2.2 \mathrm{E}+01$ & & 0.5E-01 & pCilg & $06-04-98$ & $06-03-9 B$ & $6.03 E+02$ & $\mathrm{gm}$ & SOIL G & & & $05-01$ & $69118 \cdot 0-75085$ \\
\hline DECON W2-6 & NB94 & GAM20 & $2.12 \mathrm{E}+00$ & $2.6 \mathrm{E}+01$ & & $6.5 \mathrm{E}-02$ & pCile & $06.04-98$ & 06-03-98 & $6.03 E+02$ & $\mathrm{gm}$ & SOIL G & & & 05.01 & G9118-0-75085 \\
\hline DECON W2-6 & RA226 & GAM20 & $1.79 E+00$ & $5.3 E+01$ & & $2.1 \mathrm{E}-01$ & pCilg & $06-04-98$ & $06-03-98$ & $6.03 E+02$ & $\mathrm{gm}$ & SOIL. G & & & 05.01 & 69118-0-75085 \\
\hline DECON W2-6 & $T H 228$ & GAM2O & $1.45 E+00$ & $5.5 E+01$ & & 1.3E-01 & $\mathrm{pClig}$ & 06.04 .98 & 06-03-98 & $6.03 E+02$ & $\mathrm{gm}$ & soll. G & & & $05-01$ & G9118-0-75085 \\
\hline DECON W2-6 & TH232 & GAM2O & $2.50 E+00$ & $9.3 E+01$ & & 3.4E-01 & $\mathrm{pCli} / \mathrm{g}$ & 06-04-98 & 06-03-98 & $6.03 E+02$ & grm & SOlt G & & & $05-01$ & $69118-0-75085$ \\
\hline OA BKG EMPTY BTL U05 & No Nucl Det & GAM20 & $0.00 E+00$ & $0.0 E+00$ & $v$ & $0.0 \mathrm{E}+00$ & NA & $06-04-98$ & 07.01 .95 & $1.00 E+\infty 0$ & $\begin{array}{l}5, \mu \\
\frac{6 m}{7}\end{array}$ & QUAL & & & $05-01$ & $00539-0-0020 B$ \\
\hline DA BKG EMPTY BTL U05 & AM241 & GAM20 & $0.00 E+00$ & $0.0 \mathrm{E}+00$ & u & $2.6 \mathrm{E}+01$ & $\mathrm{pCi}$ & 06-04-98 & $07-01-95$ & $1.00 E+00$ & $\mathrm{sm}$ & QUAL & & & $05-01$ & $00539-0-00208$ \\
\hline QA BKG EMPTY BTL UD5 & $\mathrm{CO60}$ & GAM 20 & $0.00 \mathrm{E}+00$ & $0.0 \mathrm{E}+00$ & U & $7.9 E+\infty 0$ & $\mathrm{pCi}$ & $06-04-98$ & $07-01-95$ & $1.00 E+\infty \mid$ & $\mathrm{sm}$ & OUAL & & & $05-01$ & $00539-0.00208$ \\
\hline QA BKG EMPTY BTL U05 & $\operatorname{cs} 137$ & GAM 20 & $0.00 E+00$ & $0.0 \mathrm{E}+\infty 0$ & U & $9.4 E+\infty 0$ & $\mathrm{pCi}$ & $06-04-98$ & 07-01-95 & $1.00 E+00$ & $\mathrm{sm}$ & QUAL & & & $05-01$ & $00539-0-00208$ \\
\hline
\end{tabular}

\section{Comment:}

Data generated from analyses of samples submitted on 6/4/98.

Gross alpha/beta determinations are performed on small sample

aliquots. Data may not represent the sample as a whole.

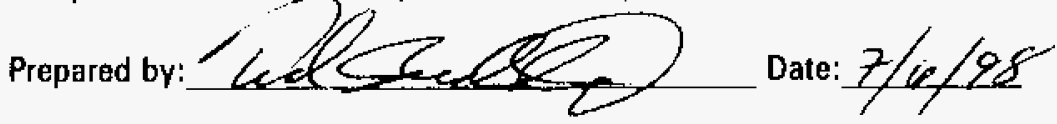

Approved by: Sarsy f(atuber

\section{Qualification Flags:}

$E=$ Estimated Quantity

$H=$ High Recovery for Sample

$J=$ Result is less than the RDL

$\mathrm{L}=$ Low Recovery for Sample

$P=$ Preliminary Results

$0=$ Bad Instrument Quality Contral, Result is OK

$\mathrm{R}=$ Results are Unusable, Resampling is Necessary

$\mathrm{U}=$ Result is less than Minimum Detectable Activity
Note: \% Error is the 2.0 Sigma Error 


\section{Bechtel Nevada Corporation}

\section{ANALYTICAL SERVICES LABORATORY}

P.O.Box 3936, N. Las Vegas, NV 89036

Reported to: Area 6 Decon Pond Closure

Remediation Projects

Report Date: 6-JUL-98

J. L. Smith, M/S NTS306

Sample Delivery Group: $B 437$

Batch: 0820

Program: 117

Report No.:

\begin{tabular}{|c|c|c|c|c|c|c|c|c|c|c|c|c|c|c|c|c|}
\hline 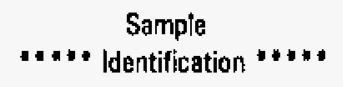 & Isotope & Analysis & Result & $\begin{array}{c}\text { Error } \\
\%\end{array}$ & $\begin{array}{l}\text { Qual } \\
\text { Flag }\end{array}$ & MDA & $\begin{array}{l}\text { Result } \\
\text { Units }\end{array}$ & $\begin{array}{c}\text { Analysis } \\
\text { Date }\end{array}$ & \begin{tabular}{|l} 
Sample \\
Coll Date
\end{tabular} & Siza & \begin{tabular}{|l|} 
Size \\
Units
\end{tabular} & \begin{tabular}{|l|} 
Type \\
Matrix
\end{tabular} & \begin{tabular}{|l|} 
Tracer \\
Yield \%
\end{tabular} & \begin{tabular}{|l|} 
Spikg \\
Recv \%
\end{tabular} & $\begin{array}{l}\text { System } \\
\text { Detector }\end{array}$ & $\begin{array}{l}\text { Packet-Item } \\
\text { Sample }\end{array}$ \\
\hline QA SP:NAS-A0271 & AM241 & GAM20 & 1.81E +05 & B. $B E+\infty$ & & $3.3 E+02$ & $\mathrm{pCi}$ & 06-04-98 & $04-01-92$ & $1.00 E+\infty 0 \mid$ & $8 \mathrm{~m}$ & QUAL & & 111.8 & 05.01 & 00534-3-14463 \\
\hline OA SP:NAS-A0271 & C060 & GAM20 & $3.00 E+05$ & $8.5 E+00$ & & $1.5 \mathrm{E}+02$ & $\mathrm{pCi}$ & 06.04-98 & $04-01-92$ & $1.00 E+\infty$ & $\mathrm{sm}$ & QUAL & & 100.7 & $05-01$ & $00534-3-14463$ \\
\hline OA SP:NAS-A0271 & CS137 & GAM20 & $2.0 B E+05$ & $8.1 E+00$ & & $9.2 E+01$ & $\mathrm{pCi}$ & 06-04-98 & 04-01-92 & $1.00 E+\infty)$ & $\mathrm{sm}$ & QUAL & & 102.5 & $05-01$ & $00534-3-14463$ \\
\hline
\end{tabular}

Comment:

Data generated from analyses of samples submitted on 6/4/98.

Gross alpha/beta determinations are performed on small sample

aliquots. Data may not represent the sample as a whole.

Prepared by:

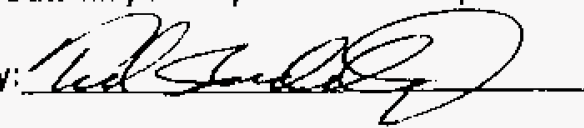

Date: $z / 6 / 28$

Approved by: Sanuyffats tow Date: $7 / 6 / 98$
Qualification Flags:

$\mathrm{E}=$ Estimated Quantity

$H=$ High Recovery for Sample

$\mathrm{J}=$ Result is less than the RDL.

$\mathbf{L}=$ Low Recovery for Sample

$P=$ Preliminary Results

Q = Bad Instrument Quality Control, Result is OK

$\mathbf{R}=$ Results are Unusable, Resampling is Necessary

$\mathbf{U}=$ Result is less than Minimum Detectable Activity
Note: \% Error is the 2.0 Sigma Error 


\section{Bechtel Nevada Corporation}

ANALYTICAL SERVICES LABORATORY

P.O.Box 3936, N. Las Vegas, NV 89036

Page 5

Reported to: Area 6 Decon Pond Closure

Remediation Projects

J. L. Smith, M/S NTS306

Report Date: 6-JUL-98

Sample Delivery Group: 8437

Batch: M559

Program: 720

Report No. :

\begin{tabular}{|c|c|c|c|c|c|c|c|c|c|c|c|c|c|c|c|c|}
\hline $\begin{array}{c}\text { Sample } \\
\ldots \ldots . . \text { Identification ..... }\end{array}$ & Isotope & Analysis & Result & $\begin{array}{l}\text { Error } \\
\%\end{array}$ & $\begin{array}{l}\text { Dual } \\
\text { Flag }\end{array}$ & MDA & $\begin{array}{l}\text { Result } \\
\text { Units }\end{array}$ & $\begin{array}{c}\text { Analysis } \\
\text { Date }\end{array}$ & $\begin{array}{l}\text { Sample } \\
\text { Coll Date }\end{array}$ & Sizo & \begin{tabular}{|c|} 
Size \\
Units
\end{tabular} & \begin{tabular}{|l|} 
Type \\
Miatrix \\
\end{tabular} & \begin{tabular}{|l|} 
Tracer \\
Yield $\%$ \\
\end{tabular} & $\begin{array}{l}\text { Spike } \\
\text { Hecr } \%\end{array}$ & $\begin{array}{l}\text { Systam } \\
\text { Detector }\end{array}$ & $\begin{array}{l}\text { Packet-Item } \\
\text { Sample }\end{array}$ \\
\hline DECON P6-6 & Gross Alpha & Gross A & $2.00 E+01$ & $1.6 E+01$ & & $2.7 \mathrm{E}+00$ & $\mathrm{pCi} / \mathrm{g}$ & $07-03-98$ & $06.03-98$ & $5.00 \mathrm{E}-01$ & $\mathrm{gmm}$ & solt G & & & 14-01 & G9118-1.75086 \\
\hline DECON P7-6 & Gross Alpho & Gross A & $1.83 E+01$ & $1.7 E+01$ & & $2.6 \mathrm{E}+00$ & pCi/g & 07-03-9B & $08-03-98$ & $5.00 \mathrm{E}-01$ & $g m$ & soll g & & & $14-01$ & G9118-2-75088 \\
\hline DECON PQ-6 & Gross Alpha & Gross A & $2.24 E+01$ & $1.6 \mathrm{E}+01$ & & $2.9 \mathrm{E}+00$ & $\mathrm{pCi} / \mathrm{g}$ & 07-03-98 & $06.03-98$ & $5.00 E-01$ & grn & soll G & & & $14-01$ & G9118-3-75090 \\
\hline DECON PA-6 MS\&MSD & Gross Alpha & Gross A & $2.11 E+01$ & $1.6 E+01$ & & $2.8 E+00$ & $\mathrm{pCi} / \mathrm{g}$ & 07-03-98 & $06-03-98$ & $5.00 \mathrm{E}-01$ & $\mathrm{gm}$ & SOIL G & & & $14-01$ & G9118-4-75092 \\
\hline DECON W2-6 & Gross Alpha & Gross A & $6.42 E+01$ & $6.4 E+\infty$ & & $1.6 \mathrm{E}+00$ & pCilg & 07.03 .98 & $06.03-98$ & $5.00 \mathrm{E}-01$ & $\mathrm{gm}$ & solL G & & & 14.01 & G9118-0-75084 \\
\hline QA REA & Gross Alpha & Gross A & $1.65 \mathrm{E}-02$ & $1.4 E+03$ & u & $4.2 \mathrm{E}-01$ & $\mathrm{pCi}$ & $07.02-98$ & $06-02-98$ & $1.00 E+00$ & $\mathrm{sm}$ & OUAL & & & $14-01$ & G9100-0-06776 \\
\hline QA SP:GROSSAB $\quad 05$ & Gross Alpha & Gross A & $3.38 E+\infty$ & $1.6 \mathrm{E}+01$ & & $4.2 \mathrm{E}-01$ & $\mathrm{pCi}$ & 07.02 .98 & $01-06-98$ & $1.00 E+00$ & $\mathrm{sm}$ & QUAL & & 98.6 & 14.01 & G9100-1-06777 \\
\hline DECON P6-6 & Gross Beta & Gross B & $1.80 E+01$ & $9.7 E+00$ & & $2.2 \mathrm{E}+00$ & pCi/g & 07-03-98 & $06.03-98$ & $5.00 \mathrm{E}-01$ & gin & SOIL G & & & $14-01$ & G911B-1-750B6 \\
\hline DECON P7-6 & Gross Bata & Gross B & $1.46 \mathrm{E}+01$ & $1.1 \mathrm{E}+01$ & & $2.2 E+00$ & pCi/g & $07-03-98$ & $06-03-98$ & $5.00 \mathrm{E}-01$ & $\mathrm{gm}$ & soll G & & & $14-01$ & G9118-2-7508B \\
\hline DECON PB.6 & Gross Beta & Gross B & $1.99 E+01$ & B. $9 E+00$ & & $2.2 \mathrm{E}+00$ & pCitg & $07-03-98$ & $06-03-98$ & 5.00E-01 & $\mathrm{gm}$ & SOL G & & & $14-01$ & G9118-3-75090 \\
\hline
\end{tabular}

\section{Comment:}

Data generated from analyses of samples submitted on $6 / 4 / 98$.

Gross alpha/beta determinations are performed on small sample

aliquots. Data may not represent the sample as a whole.

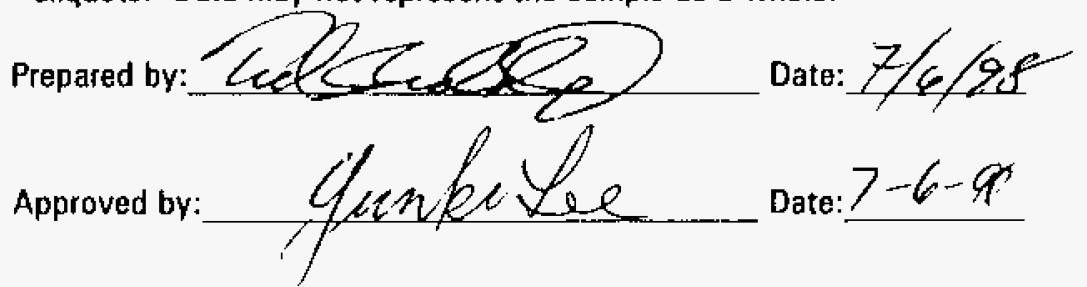

\section{Qualification Flags:}

$E=$ Estimated Quantity

$H=$ High Recovery for Sample

$J=$ Result is less than the RDI.

$L=$ Low Recovery for Sample

$\mathbf{P}=$ Prelirninary Results

$\mathrm{O}=$ Bad Instrument Quality Control, Result is OK

$\mathrm{R}=$ Results are Unusable, Resampling is Necessary

$\mathrm{U}=$ Result is less than Minimum Detectable Activity

Note: * Error is the 2.0 Sigma Error 


\section{Bechtel Nevada Corporation}

\section{ANALYTICAL SERVICES LABORATORY}

P.O.Box 3936, N. Las Vegas, NV 89036

Reported to: Area 6 Decon Pond Closure

Remediation Projects

Report Date: 6-JUL-98

J. L. Smith, M/S NTS306

Sample Delivery Group: B437

Batch: M559

Program: 720

Report No.:

\begin{tabular}{|c|c|c|c|c|c|c|c|c|c|c|c|c|c|c|c|c|}
\hline $\begin{array}{c}\text { Sample } \\
\ldots \ldots . . \\
\text { Identilication }\end{array}$..... & Isotope & Analysis & Resutt & $\begin{array}{c}\text { Error } \\
\%\end{array}$ & $\begin{array}{l}\text { Qual } \\
\text { Flag }\end{array}$ & MDA & $\begin{array}{l}\text { Result } \\
\text { Units }\end{array}$ & $\begin{array}{l}\text { Analysis } \\
\text { Date }\end{array}$ & $\begin{array}{c}\text { Somple } \\
\text { Coll Data }\end{array}$ & Size & $\begin{array}{l}\text { Size } \\
\text { Units }\end{array}$ & $\begin{array}{c}\text { Type } \\
\text { Matrix }\end{array}$ & $\begin{array}{l}\text { Tracear } \\
\text { Yreeld } \%\end{array}$ & $\begin{array}{l}\text { Spike } \\
\text { Recr } \%\end{array}$ & $\begin{array}{l}\text { System } \\
\text { Detector }\end{array}$ & $\begin{array}{l}\text { Packot-ttem } \\
\text { Sample }\end{array}$ \\
\hline DECON PB-6 MS\&MSD & Gross Beta & Gross. B & $1.67 \mathrm{E}+01$ & $1.0 E+01$ & & $2.2 \mathrm{E}+\infty 0$ & $\mathrm{pCli} / \mathrm{g}$. & 07-03-98 & 06-03-98 & $5.00 E-01$ & $\mathrm{gm}$ & SOIL G & & & 14.01 & G9118-4-75092 \\
\hline DECON W2.6 & Gross Beta & Gross B & $4.82 E+01$ & $4.7 E+\infty 0$ & & $2.2 E+\infty$ & $\mathrm{pCi} / \mathrm{g}$ & 07.03-98 & 06-03-98 & $5.00 \mathrm{E}-01$ & $g m$ & soll. G & & & $14-01$ & $69118-0.75084$ \\
\hline OA REA & Gross Bota & Gross B & $-2,67 \mathrm{E}-01$ & $2.1 E+02$ & U & 9.8E-01 & $\mathrm{pCi}$ & $07.02-98$ & $06-02-98$ & $1.00 E+\infty 0$ & $\mathrm{sm}$ & OUAL & & & $14-01$ & $69100-0-06776$ \\
\hline OA SP:GROSSAB & Gross Bata & Gross B & $4.65 E+00$ & $1.5 E+01$ & & $9.8 \mathrm{E}-01$ & $\mathrm{pCi}$ & 07.02 .98 & 01-06-98 & $1.00 E+\infty 0$ & $8 m$ & QUAL & & 89.0 & $14-01$ & G9100-1.06777 \\
\hline
\end{tabular}

Comment:

Data generated from analyses of samples submitted on 6/4/98.

Gross alpha/beta determinations are performed on small sample aliquots. Data may not represent the sample as a whole.

Prepared by:

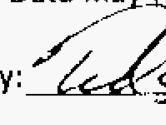

Approved by: Date: $2 / 6 / 98$ Date: $7-6-9 f$
Qualification Flags:

Note: \% Error is the 2.0 Sigma Error

$E=$ Estimated Quantity

$H=$ High Recovery for Sample

$J=$ Result is less than the RDL

$L=$ Low Recovery for Sample

$\mathrm{P}=$ Preliminary Results

$\mathrm{O}=$ Bad instrument Quality Control, Result is OK

$R=$ Results are Unusable, Resampling is Necessary

$\mathrm{U}=$ Result is less than Minimum Detectable Activity 


\section{Bechrel Nevada}

corotedes

SERVICES REQUEST \& CHAIN OF CUSTODY RECORD

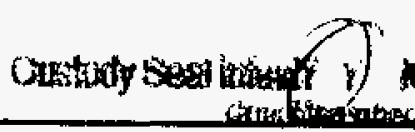

$C$

PROJECT/ CLIENT INFORMATION

TT REPORIINFORMATION

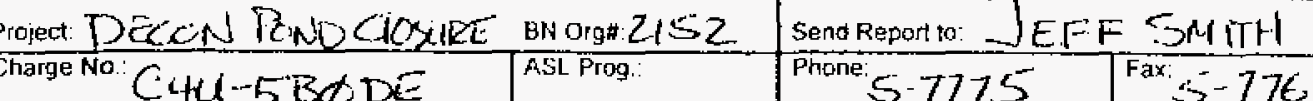

Project Manager: JEFF SUTTH

\begin{tabular}{l|l|}
\hline $5-777=5$ & Fax: $5-7761$ \\
\hline
\end{tabular}

NS $3 \subset 6$ Final report format: $\mathcal{X}$ standard () NTS-WMC () Othe

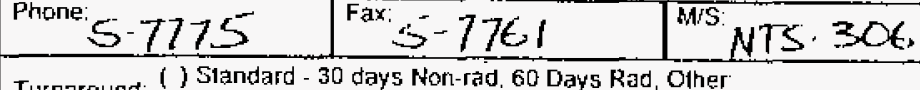
Turnaround: WRash Prelininary by: 2 WeCKS Final by

\begin{tabular}{|c|c|c|}
\hline & LAB USE ONLY & \\
\hline$d S G D$ & Non-Rad SDG: & 131 \\
\hline Packet $G_{9}$ & Non-Rad Packet: & 62 \\
\hline
\end{tabular}

Client Services Representative

Will these analyses be performed under a signed SOW? ( ) YES (I NO

If so, do analyses entered here agree with the SOW? () YES () NO () N/A
If not, identify the vartation

If not, identify the vatiation

CSR initials indicating review and approval:"

I

ID I DESCRIPJION

SAMPLING

DECON P8-2.

1 DECON P8-3

2 DETSN PB - 4 AAB

DECON PB-S

4 DECONPE-6

5 DECONPE- $1 A$ A B

6 DiCON FE-Z

7 DEON PQ - -3
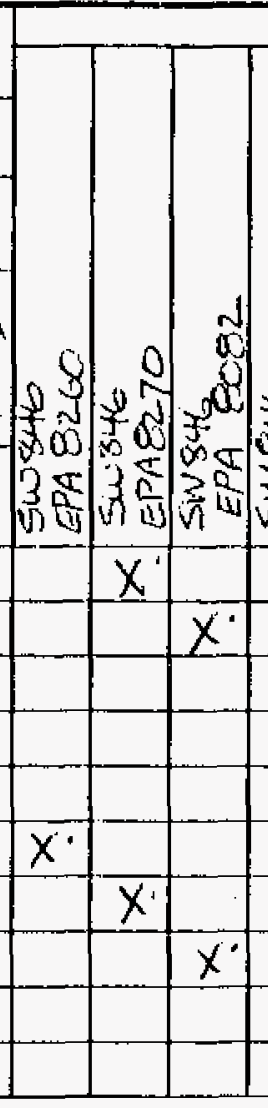

ANALYSES \& METHOD

by.

SAMPLE INFORMATION

Sampling site: DECON, FACA $\leq T T$

The samples submitted contain (cteck);

f ) Hazardous ( ) Radioactive (Unknown

contamination. If known, attach a brief narrative summary

identilying contaminanls. This information will ensure

compliance with applicable regulations and allow for the safe handling of the sample materiats.

\section{SAMPLE RECEIPT INFORMATION}

Are all sample containers received intact ( IY Yes ( ) No

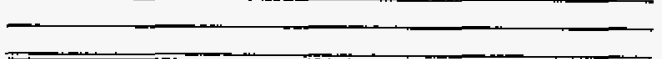

Do the labels agree with this form?

( ) Yes \{ ) No

Comments:

Was a Material Clearance Tag submitted? ( Y Yes ( ) No Corminents:

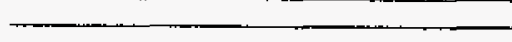

\section{COMMENTS}

(Preservative, size/volume, MS/MSD

spechal analysls, rad matrix code, count time, etc.

$\mathrm{ICF}_{1}$ scoml

ICE, ZSO MI-AMPER

ICE, $250 \mathrm{ml}, 120 \mathrm{ml}$

ILE, $250 \mathrm{MI}$

$I C E_{1} 500 \mathrm{ml} \quad 54 \mathrm{~g}_{\mathrm{g}}$

$6 / 031330$ sol

$6 / 1330$ sols

$6 / 031330$ SOL

$6 / 031330$ SOL

$6 / 031330$ SOL

$6 / 031330 \mid 5012$

9 DECSN FE -S

Transter of samples subritted for analyses

\begin{tabular}{l|l|l|l}
\hline Sampled/Relinquyshed (Signature/Organization) & DATE / TMME & Received by (Signature/Organization)
\end{tabular}

Draren C Cox/BN 1 i4

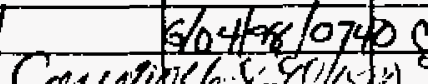

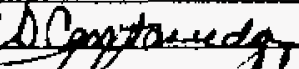

trex

$I \angle E, 120 M \mid, M S / M S D$

ICE, SOOMI, MSIMSD

$I C C, 250 \mathrm{~mL}-A M B E R, M S / M S D$ ICE, $25 \mathrm{cml}, 120 \mathrm{Ml}, M S / M S D$

ICE, $2 S O M I, M S / M S D$ DATE / TME Received (Courier \& Tracking Info.)

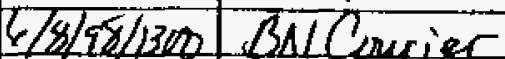
DATE TIME Received (1st lier Subcontractor Rep)

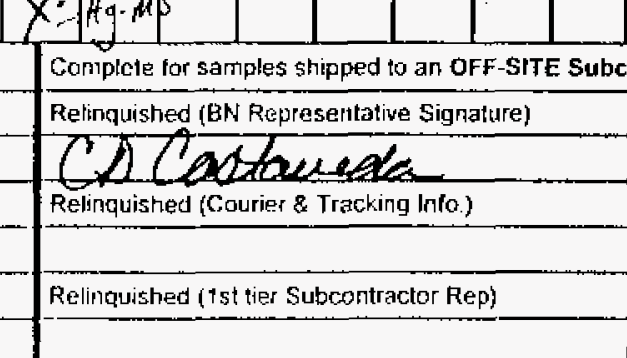

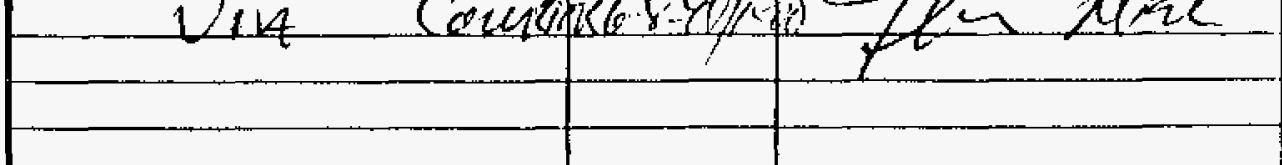

Distribution: Original - To be retained by laboratory performing final analysis Copy 2 : To be retained by Analytical services Lathoratory DATE / TIME Received (2nd tier Subcontractor Rep) 
REPORT INFORMATION

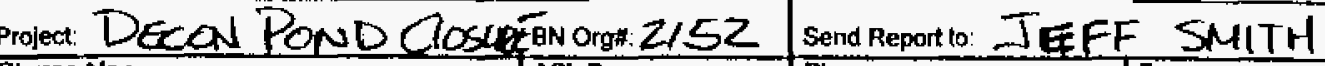

Charge No C4t $-5 B \Phi D E$ ASL Prog.

Profect Manager: TE FF SMiTH

\begin{tabular}{|r|r|}
\hline Phone: 57775 & Fax: $5-7761$ \\
\hline
\end{tabular}

\begin{tabular}{|c|c|c|}
\hline & USE ONLY & \\
\hline $045 t$ & Non-Rad SDG: & \\
\hline ad Packet 69118 & Non-Rad Packet: & W1462 \\
\hline
\end{tabular}

Client Services Representative:

Will these analyses be performed under a signed SOW? () YES ( ) NO

If so, do analyses entered here agree with the SOW7 () YES (I NO () N/A

If not, identify the vartation

CSR initials indicating review and approval: ___ Date: _____________

\begin{tabular}{|c|c|c|c|}
\hline $\mathbf{I}$ & IO /DESCRIPTION & SAMPLING & MATRIX \\
\hline
\end{tabular}

. DUECON WZ-1 A EB

1 DECONWZ-Z

2 DECON W2-3

3 DECONWZ-4 A\&B

- 4 DeCoN W2-5

5 DECON W2-6

\begin{tabular}{|c|c|c|} 
DATE & TME & MATRIX \\
\hline $6 / 03$ & 1115 & SLIDSE \\
\hline
\end{tabular}

6103 1115 SLADE $6 / 03$ 1115 SUDE

$6 / 03$ 1115 SLUOSE $6 / 03$ 1115 SUUDE 6103 1115 SLApEE

- 6 Decon PG-1 TB T27 $_{\text {Tos }} 1130$

- 7 DECON PG-1 A\&B

8 DECON PG-2

9 Decon PG-3

Transier of samples submitted for analyses

\begin{tabular}{|l|l|l|l}
\hline Sampled/Relinquighed (Signature/Organization) & DATE I TIME & Received by (Signature/Organization)
\end{tabular}

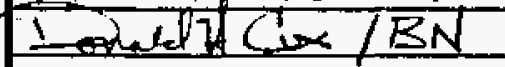

$6 / 031130$ 4031130 $6 / 231130$

WaicR SOlL $x$ SOIL Soll

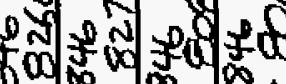
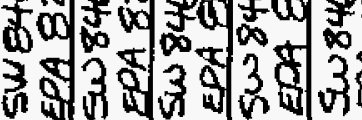

$\mathbf{X}$

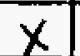

$x$

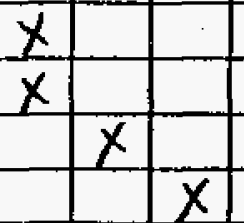
$06 / 04$ tre lokito 20 Casta

Complete for samples shipped to an OFF-SIte S Relinquished (BN Representative Signature)

Tlastaueda.

Relinquished (Courier \& Tracking Info.)

Reltrquished (1st tier Subcontractor Rep)

\begin{abstract}
SAMPLE INFORMATION Sampling Site: DECON. FACILITY

The samples submitted contain (check): W Uukno () Hazandous $\varnothing$ Radioactive $($ Unknown contamination. If knowh, attach a brief narrative summary identifying contaminants. This information will enstre

compliance with applicable regulations and allow for the safe handling of the sample materials.
\end{abstract}

\begin{tabular}{|c|c|c|c|c|c|}
\hline & & & & & \\
\hline Sampled/Reilinguig hed (Signature/Organization) & DATE / TIME & Received by (Signature/Organization) & Relinquished (BN Representative Signature) & DATE / TIME & Recelved (Courier \& Tracking Into.) \\
\hline$I \operatorname{col} d] \mathrm{C}$ ix $/ B N$ & $06 / 04 / 786$ & k100 Castamed & Inlastaueda & $6 / 8 / 98 / 1300$ & Bal Courier \\
\hline & & & Relinquished (Courier \& Tracking Info.) & DATE TIME & Received (1st Her Subrontractor Rep) \\
\hline & & & Rellthquished (1st tier Subcontractor Rep) & DATE / TIME & Recoived (2nd ther Subcontractor Rep) \\
\hline & & & & & • \\
\hline
\end{tabular}


Project DECON.POND CIOSARE BN Org\#:Z152 Charge No. C4U-SBQDE ASL Prog.: Send Report to: JEFF SMITH Phone: $5-7775 \quad$ Fax: $5-7761$ MS: NTS 306 Turnaround: () Standard - 30 days Non-rad, 60 Days Rad, Other Project Manager JEFF SMTH

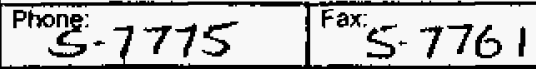
Mis: $3 \propto 6$ Final teport format: $\mathrm{O}$ Standard () NTS-WAC ( ) Other.

her:

ANALYSES \& METHOD

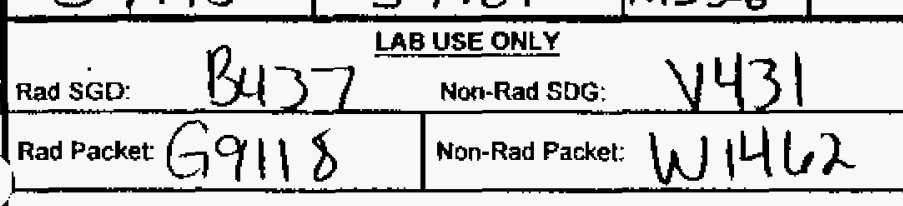

Client Services Representative:

Wil these analyses be performed under a signed SOW7 ( ) YES () NO

If so, do analyses entered here agree with the SOW (I YES (I NO () N/A If not, identity the variation

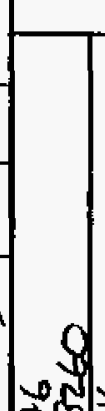

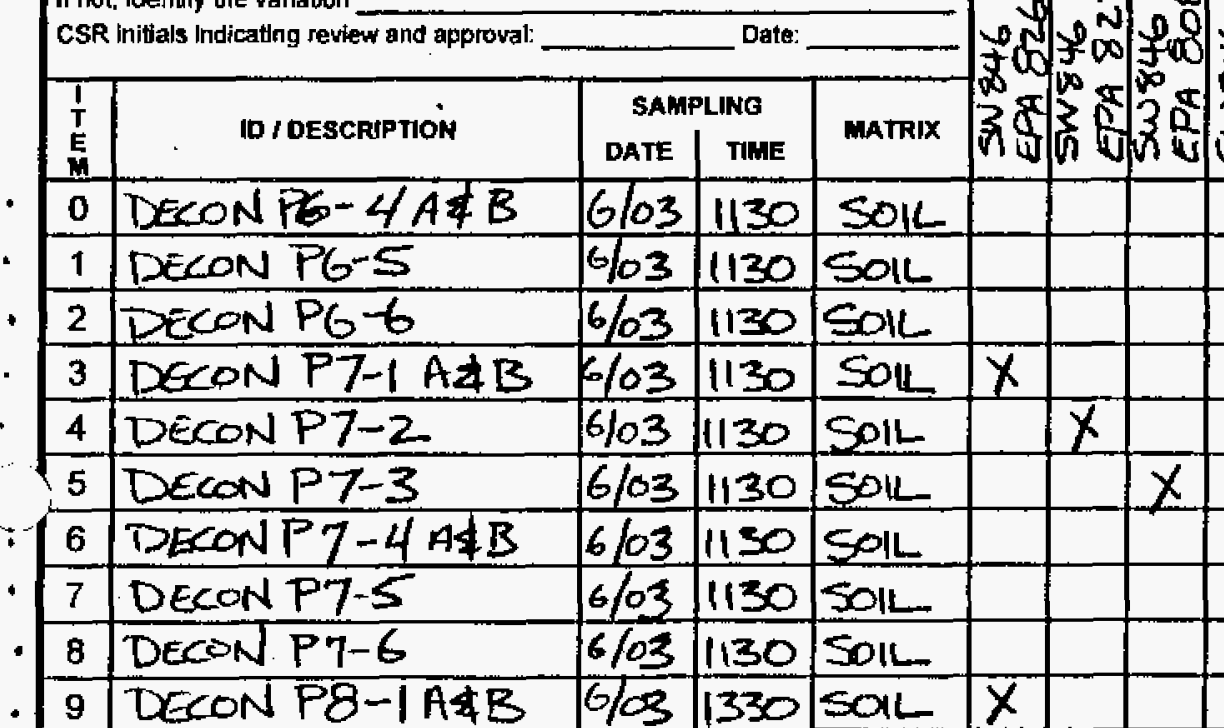

Transfer of samples submitted for analyses

\begin{tabular}{|l|l|l|}
\hline Sampled/Relinquished (Signature/Organization) & DATE / TME & Received by (Signature/Organization) \\
\hline
\end{tabular} Thalit. Cos JBN

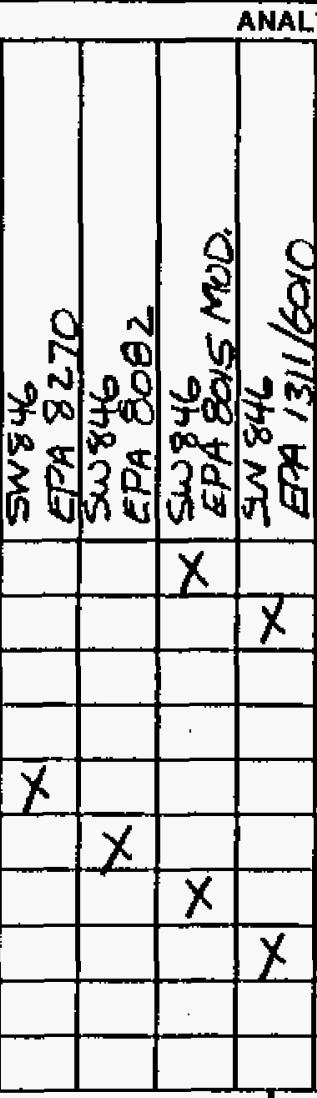

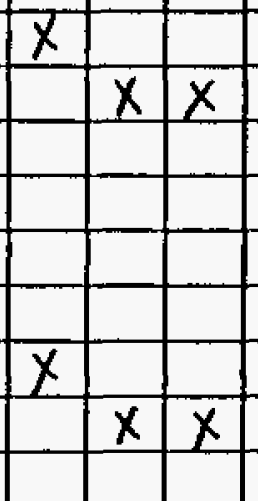

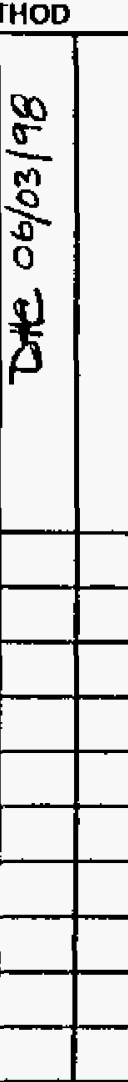

Complete for samples shipped to an OFF-SITE Subcontract Laboratory \begin{tabular}{l|l|l|l|l}
\hline Relinquished (BN Representative Signature) & DATE / TME & Recelved (Counder a Tracking Info.)
\end{tabular} TD Tastaueda Relinquished (Courier T Tracking info) Relinquished (1st tier Subontractor Rep)
SAMPLE INFORMATION Sampling ste: DECON, FACILITY The samples submitted contain (check); () Hazandous () Radioactlve $\bigotimes$ Unknown contamination. If known, attach a brief narrative summary identifying contaminants. This information will ensure compliance with applicable regulations and allow for the safe handling of the sample materials.

SAMPLE RECEIPT INFORMATION Are all sample containers received intact (i) Yes ( ) No Comments:

- ב........ Do the labels agree with this form? () Yes () No

Comment: agre wis this form? was a Materia clearance Tog outhe Comments:

COMHENTS

Preseruative stzolvolume, MSIMSD. speclal analysis, rad matrix code, count time, otc.J IEE, $250 \mathrm{ml}, 120 \mathrm{Ml}$ Ice, $250 \mathrm{~m} \mid$ ICE, $500 \mathrm{~m}$ ICE, $120 \mathrm{~m} /(2)$ ICE, $5 \mathrm{com} 1$ ICE, $250 \mathrm{ml}$-AMRER ICC, $250 \mathrm{~m} /, 120 \mathrm{Ml}$ ICE, $250 \mathrm{Ml}$. ICE $500 M$ TCE $120 \mathrm{Ml}(\mathrm{z})$ 6269

\begin{tabular}{|c|c|}
\hline $58 / 98 / 1300$ & BAT Courier \\
\hline DATE I TMEE & Reocived (1st the Subcontractor Rep) \\
\hline DATE $/ 1$ & Received (2nd tier Subcontractor Rep) \\
\hline
\end{tabular}

Distribution: Original - To be retained by laboratory pertorining final analysis 
Project DECON PNDCIOSURE EN Orgn:ZISZ

Charge No: $C 4 U-5 B \phi D E$

Project Manager: JEFF SMITH \begin{tabular}{|c|c|}
\hline Phone: 57775 & Fax: $5-7761$ \\
\hline
\end{tabular} LAB USE ONLY

\begin{tabular}{|c|c|c|}
\hline \multicolumn{3}{|c|}{ LAB USE ONLY } \\
\hline Rat SGO: $\quad B 437$ & Non-Rad SDG: & \\
\hline Rad Packet $G 9118$ & | Non-Rad Packet: & W|4 \\
\hline
\end{tabular}

Clien Services Representative:

Witl these analyses be performed under a skgned SOW? () YES () NO

If so, do analyses entered here agree with the SOW? (I YES () NO () N/A

If not. Identify the vartation

CSR Initials Indicating review and approval:

\begin{tabular}{c|c}
\hline $\mathbf{I}$ & ID / DESCRIPTION \\
\hline E &
\end{tabular}

- DECON P8-2

1 DECON P8-3

2 DECON PB-4ABB

3 DECON PB-S

4 DECON P8-6

5 DECON PB-1 AAB

6 DECON P8-2

7 DECON P8-3

8 DECON PB-4 AAB

9 DECON PB-5

Transfer of samples submitted tor analyses

\begin{tabular}{|l|l|l|}
\hline Sampled/Relinqu/shed (Signature/Organization) & DATE / TIME & Received by (Signature/Organization)
\end{tabular} Dmald CX/BN

\section{martector} Goskrozed colantouda \begin{tabular}{c|c|c|c|} 
DATE & TME & \\
\hline $6 / 03$ & 1330 & SOIL \\
\hline
\end{tabular} $6103 \quad 1330$ SOL $6 / 13330$ SOIL $6 / 031330 \mathrm{Sal}$ $6 / 631330$ SOIL $6 / 631330$ sol $x$ $6 / 1031330 \mathrm{SOL}$ $6 \% 31330$ SOL $6 / 031330$ SaL $6 / 03$ 1330 SOL
T REPORT INFORMATON

Send Report to: JEFF $S M \pi T H$

Phone: $5-7775$ Fax: $5-7761$

M Rush Preliminary by: 2 WeCK $K$ Rad, Other.

al report format: $\not$ Standard ( ) NTS-WAC ( ) Other: Final by:

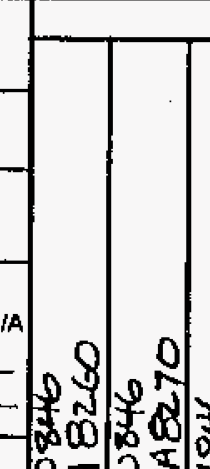

SAMPLE INFORMATION

Sampling site: DECON, FACILITY

The samples submitted contain (check):

() Hazardous () Radioactive (Jnknown

contamination. If known, attach a brlef narretive summery

identifying contaminants. This Information will ensure

compliance with appllcable regulations and allow for the sofe

handling of the sample materials.
SAMPLE RECEIPT IMFORMATION
Aro all sample containers recelved Intact ( ) Yes ( ) No
Comments:

Dom

Do the labels agree with this form?

( ) Yes ( ) N

Distribution: Orif To be re!ained by kboratory performing final analysis

Cop To be retained by taboralony performing intermediate analysis

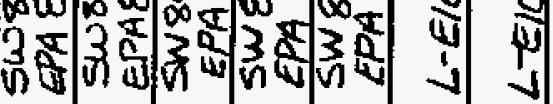
COMHENTS

Was a Materlal Clearance Tag submitted? ( ) Yes ( ) No Comments:

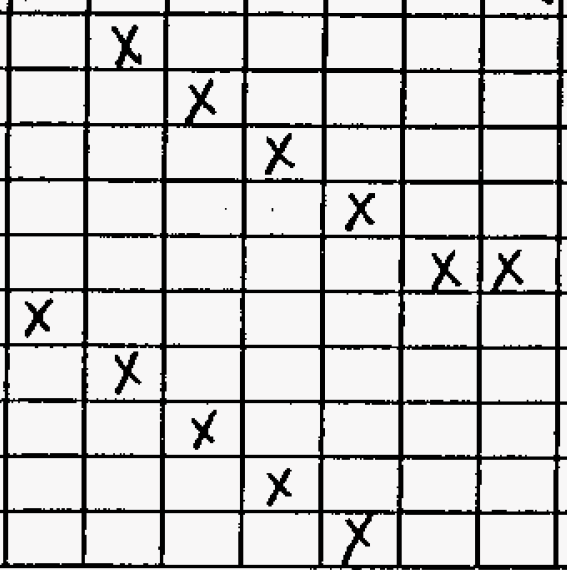

Preservative, size'volume, MSMSD, spocial analysis, rad matrix code, count time, etc.)

$\mathrm{ICE}_{1} 500 \mathrm{ml}$

ICE, $250 \mathrm{~m} /$-AMBER

ICE, $250 \mathrm{ml}, 120 \mathrm{ml}$

ICE, $250 \mathrm{ml}$

ICE, $500 \mathrm{ml}$

ICE, $|2 O M|, M S / M S D$

IEe, $500 \mathrm{ml}, M S / M S D$

ICE, $250 \mathrm{ML}$-AMBER, MS/MSD

ICE, $250 \mathrm{ml}$, $120 \mathrm{Ml}$, MS/MSD

ICE, $25 O M I, M S / M S D$

Complete for samples shipped to an OFF-SITE Subeontract Laboratory

Relinquished (BN Representative Signalure)

TJ Tasbueda

\begin{tabular}{|c|c|c|}
\hline DATE I TIME & Recelved (Courler \& Tracking Into.) \\
\hline
\end{tabular}

Relinquished (Courier \& Tracking info.)

6/3/58/3300 BAIConcier DATE TIME Recelved (1st ter Subcontractor Rep) 


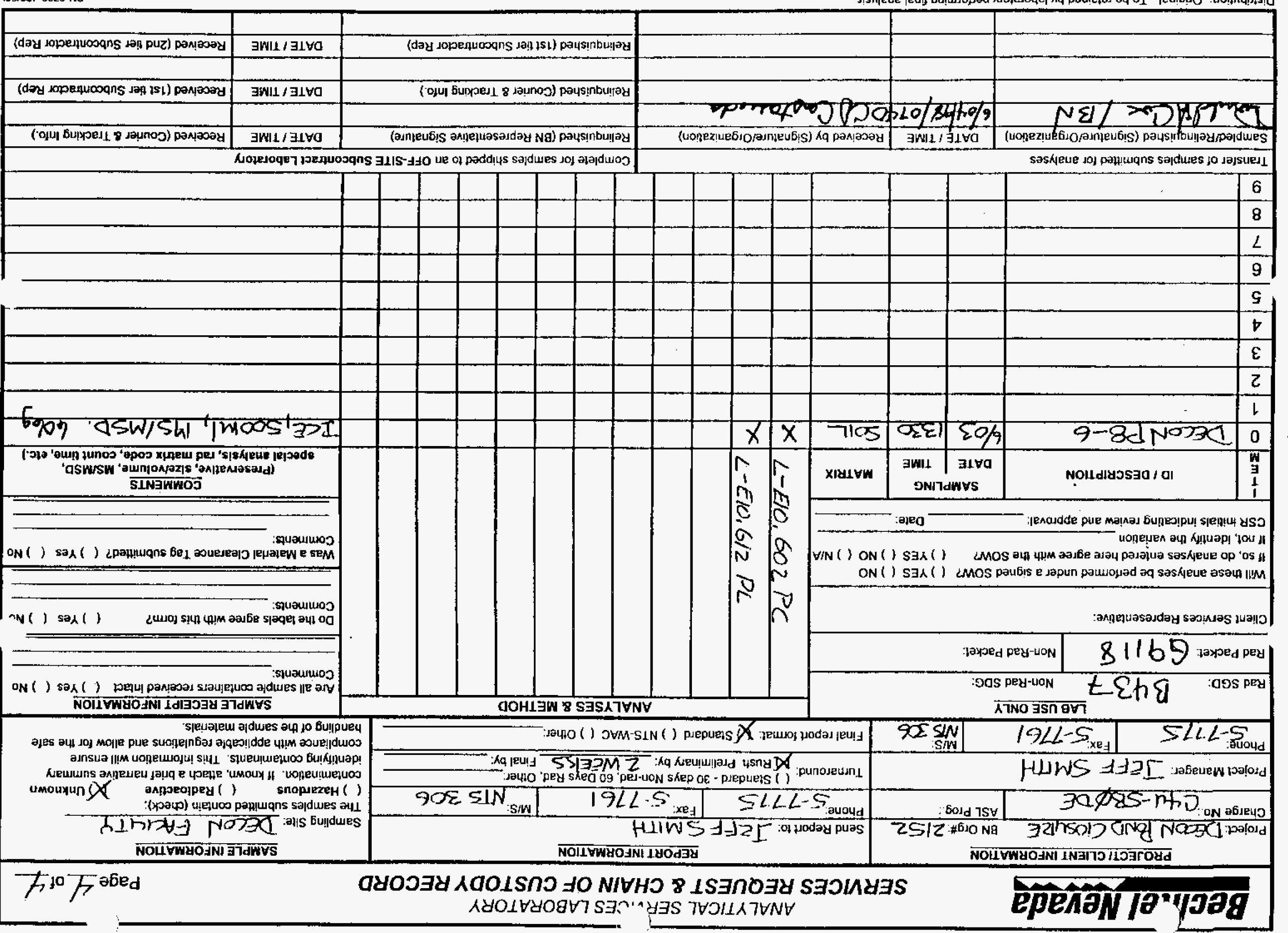




\section{VOLATILE}

ORGANICS

DATA 
CLIENT: Bechtel Nevada

OJECT NAME: V432

OSECT NUMBER: 17777
CLIENT ID: Decon P9-1 TB

DATE SAMPLED: $5 / 27 / 98$

NEL SAMPLE ID: L9806094-01

TEST: Volatile Organic Compounds by EPA \$W846 Method 8260B, Dec. 1996

MATRIX: Aqueous

DILUTION: 1
EXTRACTED: $6 / 9 / 98$

ANALYZED: $6 / 9 / 98$
ANALYST: Suzanne

\begin{tabular}{|c|c|c|c|c|c|}
\hline PARAMETER & $\begin{array}{l}\text { Result } \\
\mu g / L\end{array}$ & $\begin{array}{c}\text { Reporting } \\
\text { Limit } \\
\end{array}$ & PARAMETER & $\begin{array}{l}\text { Result } \\
\mu \mathrm{g} / \mathrm{L} \\
\end{array}$ & $\begin{array}{c}\text { Reporting } \\
\text { Limit } \\
\end{array}$ \\
\hline Acetone & $\overline{\mathrm{ND}}$ & $25 . \quad \mu g / L$ & & & \\
\hline Benzene & $\mathrm{ND}$ & 5. $\mu \mathrm{g} / \mathrm{L}$ & & & \\
\hline Bromodichloromethane & $\mathrm{ND}$ & 5. $\mu \mathrm{g} / \mathrm{L}$ & & & \\
\hline Bromoform & ND & 5. $\mu g / L$ & & & \\
\hline Bromomethane & ND & 5. $\mu \mathrm{g} / \mathrm{L}$ & & & \\
\hline 2-Butanone & ND & 25. $\mu \mathrm{g} / \mathrm{L}$ & & & \\
\hline Carbon disulfide & ND & 5. $\mu \mathrm{g} / \mathrm{L}$ & & & \\
\hline Carbon tetrachloride & ND & 5. $\mu \mathrm{g} / \mathrm{L}$ & & & \\
\hline Chlorobenzene & ND & 5. $\mu \mathrm{g} / \mathrm{L}$ & & & \\
\hline Chloroethane & ND & 5. $\mu \mathrm{g} / \mathrm{L}$ & & & \\
\hline Chloroform & $\mathrm{ND}$ & 5. $\mu \mathrm{g} / \mathrm{L}$ & & & \\
\hline Chloromethane & $\mathrm{ND}$ & 5. $11 \mathrm{~g} / \mathrm{L}$ & & & \\
\hline Dibromochloromethane & ND & 5. $\mu \mathrm{g} /$ & & & \\
\hline 1.1-Dichloroethane (1.1-DCA) & ND & 5. $\mu g / L$ & & & \\
\hline 1,2-Dichloroethane (1,2-DCA) & ND & 5. $\mu \mathrm{g} / \mathrm{L}$ & & & \\
\hline 1.1-Dichloroethene (1,I-DCE) & ND & 5. $\mu \mathrm{g} / \mathrm{L}$ & & & \\
\hline cis-1.2-Dichloroethene & ND & 5. $\mu g / \mathrm{L}$ & & & \\
\hline i-1,2-Dichloroethene & ND & 5. $\mu \mathrm{g} / \mathrm{L}$ & & & \\
\hline 1.2-Dichloropropane & ND & 5. $\mu \mathrm{g} / \mathrm{L}$ & & & \\
\hline cis-1,3-Dichloropropene & $\mathrm{ND}$ & 5. $\mu \mathrm{g} / \mathrm{L}$ & & & \\
\hline trans-1,3-Dichloropropene & $\mathrm{ND}$ & 5. $\mu g / L$ & & & \\
\hline Ethylbenzene & $\mathrm{ND}$ & 5. $\mu g / L$ & & & \\
\hline 2-Hexanone & ND & 25. $\mu g / L$ & & & \\
\hline Methylene chloride (Dichloromethane) & ND & 5. $\mu g / L$ & & & \\
\hline 4-Methyl-2-pentanone & $\mathrm{ND}$ & 25. $\mu \mathrm{g} / \mathrm{L}$ & & & \\
\hline Styrene & ND & 5. $\mu g / L$ & & & \\
\hline 1.1,2.2-Tetrachloroethane & $\mathrm{ND}$ & 5. $H g / L$ & & & \\
\hline Tetrachloroethene (PCE) & $\mathrm{ND}$ & 5. $\mu g / L$ & & & \\
\hline Toluene & ND & 5. $\mu \mathrm{g} / \mathrm{L}$ & & & \\
\hline $1.1,1$-Trichloroethane $(1,1,1-T C A)$ & ND & 5. $\mu g / L$ & & & \\
\hline 1.1.2-Trichloroethane (1,1.2-TCA) & ND & 5. $\mu \mathrm{g} / \mathrm{L}$ & & & \\
\hline Trichloroethene (TCE) & $\mathrm{ND}$ & 5. $\mu \mathrm{g} / \mathrm{L}$ & & & \\
\hline Vinyl chloride & $\mathrm{ND}$ & 5. $\mu g / L$ & & & \\
\hline $0-X y l e n e$ & ND & 5. $\mu \mathrm{g} / \mathrm{L}$ & & & \\
\hline mi,p-Xylene & ND & 5. $\mu \mathrm{g} / \mathrm{L}$ & & & \\
\hline
\end{tabular}

ND - Not Detected

QUALITY CONTROL DATA:

\section{Surrogate}

4-Bromofluorobenzene

Dibromofluoromethane

- sne-dg
\% Recovery

103

103

108
Acceptable Range

$70-130$
$70-130$
$70-130$ 
NEL LABORATORIES

CLIENT: Bechtel Nevada

PROJECT NAME: $\quad \mathrm{V} 432$

PROJECT NUMBER: 17777
CLIENT ID: Decon P9-1 A\&B

DATE SAMPLED: $6 / 4 / 98$

NEL SAMPLE ID: L.9806094-02

TEST: Volatile Organic Compounds by EPA SW846 Method 8260A, Sept. 1994

MATRIX: Solid DILUTION: 1
EXTRACTED: 6/11/98

ANALYZED: $6 / 11 / 98$

ANALYST: Suzanne

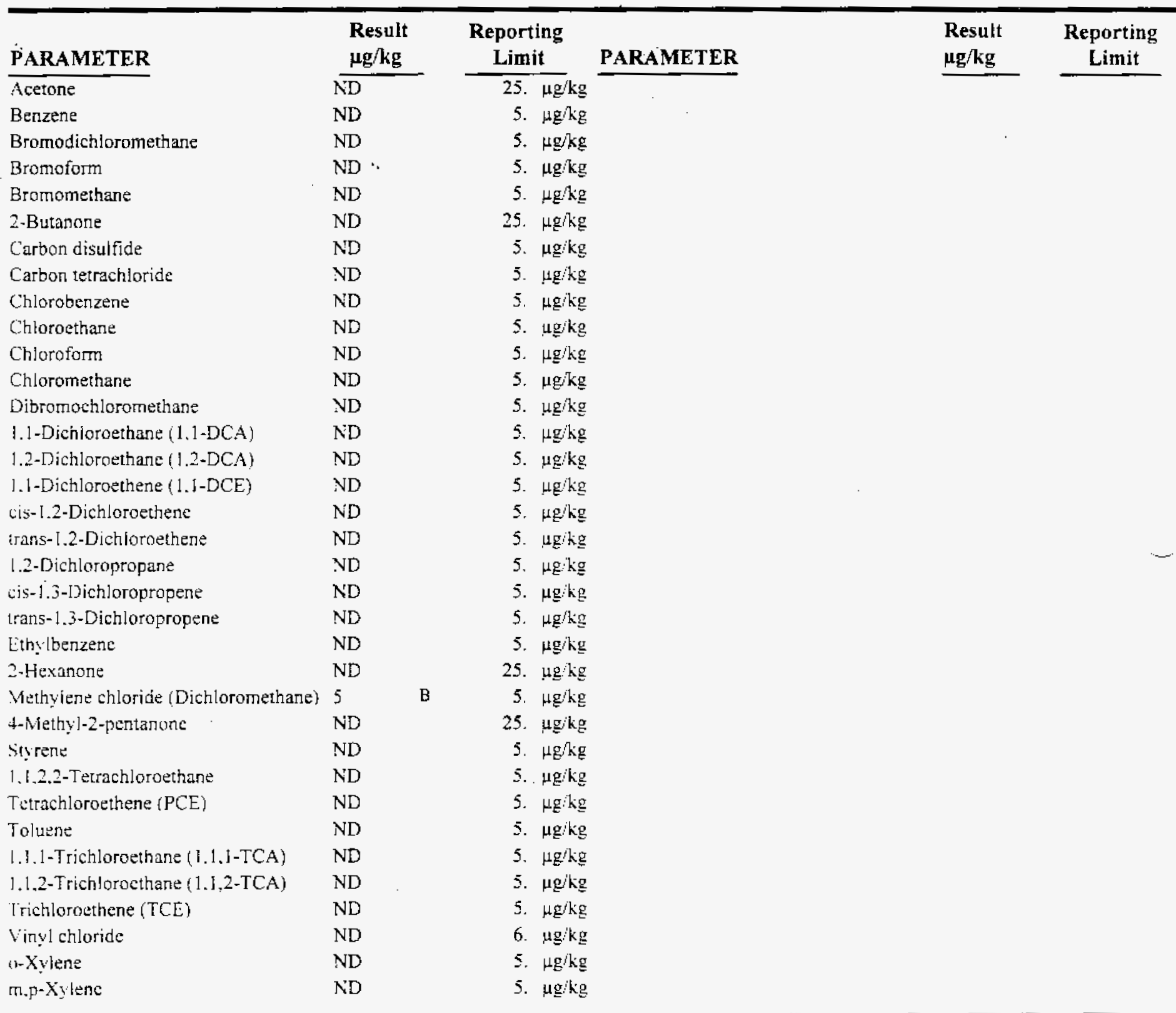

ND - Not Detected

QUALITY CONTROL DATA:

\section{Surrogate}

4-Bromoituorobenzene

Dibromofluoromethane

roluene-d8

\section{\% Recovery}

89

93

98
Acceptable Range

$74-121$

$80-120$

$81-117$ 
NEL LABORATORIES

LIENT: Bechtel Nevada

- ROJECT NAME: V432

PROJECT NUMBER: 17777
CLIENT ID: Decon P10-1 A\&B

DATE SAMPLED: $6 / 4: 98$

NEL SAMPLE ID: L9806094-07

TEST: Volatile Organic Compounds by EPA SW846 Method 8260A, Sept. 1994

MATRIX: Solid

DILUTION: 1
EXTRACTED: $6 / 11 / 98$

ANALYZED: $6 / 11 / 98$

ANALYST: Suzanne

\begin{tabular}{|c|c|c|c|c|}
\hline PARAMETER & $\begin{array}{l}\text { Result } \\
\mu \mathrm{g} / \mathrm{kg}\end{array}$ & $\begin{array}{c}\text { Reporting } \\
\text { Limit } \\
\end{array}$ & $\begin{array}{l}\text { Result } \\
\mu \mathrm{g} / \mathrm{kg}\end{array}$ & $\begin{array}{c}\text { Reporting } \\
\text { Limit } \\
\end{array}$ \\
\hline Acetone & $\overline{\mathrm{ND}}$ & $25 . \mu \mathrm{g} / \mathrm{kg}$ & & \\
\hline Benzene & $\mathrm{ND}$ & 5. $12 \mathrm{~g} / \mathrm{kg}$ & & \\
\hline Bromodichloromethane & ND & 5. $\mu \mathrm{g} / \mathrm{kg}$ & & \\
\hline Bromoform & $\mathrm{ND}$ & 5. $\mu \mathrm{g} / \mathrm{kg}$ & & \\
\hline Bromomethane & ND & 5. $\mu \mathrm{g} / \mathrm{kg}$ & & \\
\hline 2-Butanone & $\mathrm{ND}$ & 25. $\mu \mathrm{g} / \mathrm{kg}$ & & \\
\hline Carbon disulfide & ND & 5. $\mu \mathrm{g} / \mathrm{kg}$ & & \\
\hline Carbon tetrachloride & ND & 5. $\mu \mathrm{g} / \mathrm{kg}$ & & \\
\hline Chlorobenzene & $\mathrm{VD}$ & 5. $\mu \mathrm{g} / \mathrm{kg}$ & & \\
\hline Chloroethane & ND & 5. $\mu \mathrm{g} / \mathrm{kg}$ & & \\
\hline Chloroform & $\mathrm{ND}$ & 5. $\mu \mathrm{gkg}$ & & \\
\hline Chtoromethane & ND & 5. $\mu \mathrm{g} / \mathrm{kg}$ & & \\
\hline Dibromochloromethane & $N D$ & 5. $\mu \mathrm{g} / \mathrm{kg}$ & & \\
\hline 1.1-Dichlorothane (1,1-DCA) & $\mathrm{ND}$ & 5. $\mu g \mathrm{~kg}$ & & \\
\hline 1.2-Dichloroethane (1.2-DCA) & ND & 5. $\mu g \mathrm{~kg}$ & & \\
\hline 1, I-Dichlowethene (1,1-DCE) & $N D$ & 5. $4 \mathrm{~g} / \mathrm{kg}$ & & \\
\hline is-1.2-Dichlorothene & $\mathrm{ND}$ & 5. $\mu \mathrm{g} / \mathrm{kg}$ & & \\
\hline irass-1.2-Dichloroethene & $\mathrm{ND}$ & 5. $\mu \mathrm{g} / \mathrm{kg}$ & & \\
\hline 1,2-Dichioropropane & $N D$ & 5. $\mu \mathrm{g} k \mathrm{~g}$ & & \\
\hline cis-1.3-Dichloropropene & ND & 5. $\mu \mathrm{g} / \mathrm{kg}$ & & \\
\hline trans-1.3-Dichloropropene & $\mathrm{ND}$ & 5. $\mu \mathrm{g} / \mathrm{kg}$ & & \\
\hline Sithylbenzene & $\mathrm{ND}$ & 5. $\mu g / k g$ & & \\
\hline 2-Hexanone & $\mathrm{ND}$ & 25. $11 \mathrm{~g} / \mathrm{kg}$ & & \\
\hline Methylene chloride (Dichloromethane) & $\gamma D$ & 5. $\mu \mathrm{g} / \mathrm{kg}$ & & \\
\hline 4-Methy:-2-pentanone & $\mathrm{ND}$ & 25. $\mu g \mathrm{~kg}$ & & \\
\hline Stytene & ND & 5. $\mu g \mathrm{~kg}$ & & \\
\hline 1.1,2.2-Tetrachlorsethane & $N D$ & 5. $\mu \mathrm{g} / \mathrm{kg}$ & & \\
\hline Tetrachlorothene (PCE) & $\mathrm{ND}$ & 5. $\mu \mathrm{gg} / \mathrm{kg}$ & & \\
\hline Toluene & ND & 5. $\mu g / k g$ & & \\
\hline 1.1.1-Trichioroethane $(1.1,1-\mathrm{TCA})$ & ND & 5. $4 \mathrm{~g} / \mathrm{kg}$ & & \\
\hline 1.1,2-Irichloroethane (1,1,2-TCA) & ND & 5. $\mu \mathrm{g} / \mathrm{kg}$ & & \\
\hline Trichloruethene (TCE) & $\mathrm{ND}$ & 5. $\mu \mathrm{g} / \mathrm{kg}$ & & \\
\hline Vinyl chloride & ND & 6. $\mu \mathrm{g} / \mathrm{kg}$ & & \\
\hline 0 -Xylone & ND & 5. $11 \mathrm{~g} / \mathrm{kg}$ & & \\
\hline $17 . \mathrm{p}-\mathrm{X}$ ylene & ND & 5. $\mu \mathrm{g} / \mathrm{kg}$ & & \\
\hline
\end{tabular}

$\overline{\text { ND - Not Detected }}$

QUALITY CONTROL DATA.

\section{Surrogate}

4-Bromotiugrobenzene

Dibromofluoromethane

- Toluene-ds

\section{$\%$ Recovery}

87

92

98
Acceptable Range

$74-121$

$80-120$

$81-117$ 
NEL LABORATORIES

CLIENT: Bechtel Nevada

PROJECT NAME: V432

PROJECT NUMBER: 17777
CLIENT ID: Method Blank

DATE SAMPLED: NA

NEL SAMPLE ID: 9806092 W60B-BLK

TEST: $\quad$ Volatile Organic Compounds by EPA SW846 Method 8260B, Dec. 1996

MATRIX: Aqueous

EXTRACTED: $\quad 6 / 9 / 98$

ANALYZED: $\quad 6 / 9 / 98$

\begin{tabular}{|c|c|c|c|c|c|}
\hline PARAMETER & $\begin{array}{l}\text { Result } \\
\mu \mathrm{g} / \mathrm{L}\end{array}$ & $\begin{array}{c}\text { Reporting } \\
\text { Limit }\end{array}$ & PARAMETER & $\begin{array}{l}\text { Result } \\
\mu \mathrm{g} / \mathrm{L}\end{array}$ & $\begin{array}{c}\text { Reporting } \\
\text { Limit }\end{array}$ \\
\hline Acetone & ND & $\overline{25 \mu g / L}$ & & & \\
\hline Benzene & ND & $5 \mu \mathrm{g} / \mathrm{L}$ & & & \\
\hline Bromodichloromethane & ND & $5 \mu \mathrm{g} / \mathrm{L}$ & & & \\
\hline Bromoform & ND & $5 \mu \mathrm{g} / \mathrm{L}$ & & & \\
\hline Bromomethane & ND & $5 \mu \mathrm{g} / \mathrm{L}$ & & & \\
\hline 2-Butanone & ND & $25 \mu \mathrm{g} / \mathrm{L}$ & & & \\
\hline Carbon disulfide & ND & $5 \mu \mathrm{g} / \mathrm{L}$ & & & \\
\hline Carbon tetrachloride & ND & $5 \mu g / L$ & & & \\
\hline Chlorobenzene & ND & $5 \mu \mathrm{g} / \mathrm{L}$ & & & \\
\hline Chloroethane & NO & $5 \mu \mathrm{g} / \mathrm{L}$ & & & \\
\hline Chloroform & ND & $5 \mu \mathrm{g} / \mathrm{L}$ & & & \\
\hline Chloromethane & ND & $5 \mu \mathrm{g} /$ & & & \\
\hline Dibromochloromethane & ND & $5 \mu \mathrm{g} / \mathrm{L}$ & & & \\
\hline 1.1-Dichloroethane (1,1-DCA) & $N D$ & $5 \mu \mathrm{g} / \mathrm{L}$ & & & \\
\hline 1.2-Dichloroethane (1,2-DCA) & ND & $5 \mu \mathrm{g} / \mathrm{L}$ & & & \\
\hline 1,1-Dichloroethene (1,1-DCE) & ND & $5 \mu \mathrm{g} / \mathrm{L}$ & & & \\
\hline cis-1.2-Dichloroethene & ND & $5 \mu \mathrm{g} / \mathrm{L}$ & & & \\
\hline trans-1.2-Dichloroethene & ND & $5 \mu \mathrm{g} / \mathrm{L}$ & & & \\
\hline 1.2-Dichloropropane & ND & $5 \mu \mathrm{g} / \mathrm{L}$ & & & \\
\hline cis-1,3-Dichloropropene & ND & $5 \mu \mathrm{g} / \mathrm{L}$ & & & \\
\hline trans-1,3-Dichloropropene & $\mathrm{ND}$ & $5 \mu \mathrm{g} / \mathrm{L}$ & & & \\
\hline Ethylbenzene & ND & $5 \mu \mathrm{g} / \mathrm{L}$ & & & \\
\hline 2-Hexanone & $\mathrm{ND}$ & $25 \mu \mathrm{g} / \mathrm{L}$ & & & \\
\hline Methylene chloride (Dichloromethane. & ND & $5,1 \mathrm{~g} / \mathrm{L}$ & & & \\
\hline 4-Methyl-2-pentanone & ND & $25 \mu \mathrm{g} / \mathrm{L}$ & & & \\
\hline Styrene & $\mathrm{ND}$ & $5 \mu \mathrm{g} / \mathrm{L}$ & & & \\
\hline 1,1,2,2-Tetrachloroethane & ND & $5 \mu \mathrm{g} / \mathrm{L}$ & & & \\
\hline Tetrachloroethene (PCE) & $\mathrm{ND}$ & $5 \mu \mathrm{g} / \mathrm{L}$ & & & \\
\hline Toluene & ND & $5 \mu g / L$ & & & \\
\hline 1,1,1-Trichloroethane $(1,1,1-\mathrm{TCA})$ & $\mathrm{ND}$ & $5 \mu \mathrm{g} / \mathrm{L}$ & & & \\
\hline 1.1,2-Trichforoethane (1.1,2-TCA) & ND & $5 \mu \mathrm{g} / \mathrm{L}$ & & & \\
\hline Trichloroethene (TCE) & ND & $5 \mu \mathrm{g} / \mathrm{L}$ & & & \\
\hline Vinyl chloride & ND & $5 \mu \mathrm{g} / \mathrm{L}$ & & & \\
\hline o-Xylene & ND & $5 \mu \mathrm{g} / \mathrm{L}$ & & & \\
\hline $\mathrm{m}, \mathrm{p}-\mathrm{Xyj}$ Iene & ND & $5_{\mu \mathrm{g} / \mathrm{L}}$ & & & \\
\hline
\end{tabular}

ND - Not Detected

QUALITY CONTROL DATA:

Surrogate

4-Bromofluorobenzene

Dibromofluoromethane

Toluene-d8
$\%$ Recovery

93

103

105
Acceptable Range

$70-130$

$70-130$

$70-130$

This report shall not be reproduced except in full, without the written approval of the laboratory. 
NEL LABORATORIES

CLIENT: Bechtel Nevada

'ROJECT ID: V432/17777

ROJECT \#: 17777
CLIENT ID: Matrix Spike Duplicate

DATE SAMPLED: NA

NEL SAMPLE ID: L9806093-17-MSD

TEST: Volatile Organic Compounds by EPA SW846 Method 8260A, Sept. 1994

MATRIX: Solid

EXTRACTED: 6/1 1/98

ANALYZED: $6 / 11 / 98$

ANALYST: Suzanne

\section{PARAMETER}

Benzene

Chiorobenzene

1,1-Dichloroethene (1,1-DCE)

Toluene

Trichloroethene (TCE)

Percent Recovery

96

98

156

94

98

\section{Percent Recovery}

86

93

97
Acceptable Range $\quad \underline{\text { RPD }}$

\begin{tabular}{cc}
$66-142$ & $2 . \mathrm{I}$ \\
$60-133$ & 2. \\
$59-172$ & 9.4 \\
$59-139$ & 2.1 \\
$62-137$ & 0. \\
\hline
\end{tabular}

$66-142$

2. I

2.

9.4

0 .

OLALITY CONTROL DATA:

\section{Surrogate}

Toluene-d8

\section{Acceptable Range}

$74 \cdot 121$

$80-120$

$81-117$ 
NEL LABORATORIES

CLIENT: Bechtel Nevada

PROJECT ID: V432/17777

PROJECT \#: $\quad 17777$
CLIENT ID: Laboratory Control Spike

DATE SAMPLED: NA

NEL SAMPLE ID: 9806092 W60B-LCS

TEST: $\quad$ Volatile. Organic Compounds by EPA SW846 Method 8260B, Dec. 1996

MATRIX: Aqueous

EXTRACTED: $6 / 9 / 98$

ANALYZED: $6 / 9 / 98$

ANALYST: Suzanne

\section{PARAMETER}

Benzene

Chlorobenzene

1,1-Dichloroethene (1,1-DCE)

Toluene

Trichloroethene (TCE)

Percent Recovery

97

93

93

96

92

OUALITY CONTROL DATA:

Surrogate

4-Bromofluorobenzene

Dibromofluoromethane

Toluene-d8
Percent Recovery

109

103

113
Acceptable Range

$70-130$

$70-130$

$70-130$

$70-130$

$70-130$

\section{Acceptabie Range}

$70-130$

$70-130$

$70-130$ 
NEL LABORATORIES

CLIENT: Bechtel Nevada

ROJECT NAME; V432

PROJECT NUMBER: 17777
CLIENT ID: Method Blank

DATE SAMPLED: NA

NEL SAMPLE ID: 9806 I I I260B2-BLK
TEST:

MATRIX:
Volatile
Solid

\section{PARAMETER}

Acetone

Benzene

Bromodichloromethane

Bromoform

Bromomethane

3-Butanone

Carbon disulifide

Carbon tetrachloride

Chjorobenzene

Chloroethane

Chloroform.

Chloromethane

Dibromochloromethane

i. 1-Dichloroethane (1.1-DCA)

1.2-Dichloroethane (1.2-DCA)

:. 1-Dichloroethene (1.1-DCE)

i5-1.2-Dichloroethene

anis-1.2-Dichlorocthene

1.2-Dichloropropane

Lis-1.3-Dichloropropene

:rans-1.3-Dichloropropene

Ethylbenzene

-Hexanone

Wethylene coloride (Dichloromethane

4-Methyl-2-pentanone

sty rene

5.1.2.2-Tatractoroethane

jetrachlorostbere (PCE)

Toluene

1.1.1-Trichlorosthane (1.1.1-TCA)

1.1.2-Trichloroethane (1.1.2-TCA)

rrichlorothene (TCE)

vinyi ethloride

(1) Xylene

m. $\mathrm{x}$ Xylene

\begin{tabular}{|c|c|}
\hline $\begin{array}{l}\text { Result } \\
\mu \mathrm{g} / \mathrm{kg}\end{array}$ & $\begin{array}{l}\text { Reporting } \\
\text { Limit }\end{array}$ \\
\hline$\overline{\mathrm{ND}}$ & $\overline{25} \mu \mathrm{g} / \mathrm{kg}$ \\
\hline ND & $5 \mu \mathrm{g} / \mathrm{kg}$ \\
\hline ND & $5 \mu \mathrm{g} / \mathrm{kg}$ \\
\hline ND & $5 \mu \mathrm{g} / \mathrm{kg}$ \\
\hline ND & $5 \mu \mathrm{g} / \mathrm{kg}$ \\
\hline ND & $25 \mu \mathrm{g} / \mathrm{kg}$ \\
\hline ND & $5 \mu \mathrm{g} / \mathrm{kg}$ \\
\hline ND & $5 \mathrm{\mu g} / \mathrm{kg}$ \\
\hline ND & $5 \mu \mathrm{gkg}$ \\
\hline $\mathrm{ND}$ & $5 \mu \mathrm{g} / \mathrm{kg}$ \\
\hline ND & $5 \mu \mathrm{g} / \mathrm{kg}$ \\
\hline $\mathrm{ND}$ & $5 \mu \mathrm{g} / \mathrm{kg}$ \\
\hline ND & $5 \mu \mathrm{g} / \mathrm{kg}$ \\
\hline ND & $5 \mu \mathrm{g} / \mathrm{kg}$ \\
\hline ND & $5 \mu \mathrm{gkg}$ \\
\hline ND & $5 \mu \mathrm{g} / \mathrm{kg}$ \\
\hline $\mathrm{ND}$ & $5 \mu \mathrm{g} / \mathrm{kg}$ \\
\hline ND & 5 ugikg \\
\hline ND & $5 \mu \mathrm{g} / \mathrm{kg}$ \\
\hline ND & $5 \mu \mathrm{g} k$ \\
\hline $\mathrm{ND}$ & $5 \mu \mathrm{g} / \mathrm{kg}$ \\
\hline $\mathrm{ND}$ & $5 \mathrm{~kg} k \mathrm{~g}$ \\
\hline $\mathrm{ND}$ & $25 \mu \mathrm{g} / \mathrm{kg}$ \\
\hline 7 & 5 ugkg \\
\hline $\mathrm{ND}$ & $25 \mu \mathrm{g} / \mathrm{kg}$ \\
\hline$N D$ & $5 \mu \mathrm{g} / \mathrm{kg}$ \\
\hline $\mathrm{ND}$ & $5 \mu \mathrm{g} k \mathrm{~kg}$ \\
\hline $\mathrm{ND}$ & $5 \mu \mathrm{g} / \mathrm{kg}$ \\
\hline ND & $5 \mu \mathrm{gkg}$ \\
\hline $\mathrm{ND}$ & $5 \mathrm{Hg} / \mathrm{kg}$ \\
\hline $\mathrm{ND}$ & $5 \mu \mathrm{g} / \mathrm{kg}$ \\
\hline ND & $5 \mu \mathrm{g} \mathrm{kg}$ \\
\hline $\mathrm{ND}$ & $6 \mu \mathrm{g} / \mathrm{kg}$ \\
\hline $\mathrm{ND}$ & $5,1 \mathrm{~g} / \mathrm{kg}$ \\
\hline ND & $5 \mu \mathrm{g} k g$ \\
\hline
\end{tabular}

\section{Result Reporting}

PARAMFTER

$\mu \mathrm{g} / \mathrm{kg}$

$6 / 11 / 98$

$6 / 11 / 98$

\section{ND - Vor Detected \\ OUALITY CONTROL DATA.}

\section{Surrogate}

4-Bromofluorobenzene

Dibromofluoromethane

olluene-d8
$\%$ Recovery

92

93

98

\section{Acceptable Range}

$74-121$

$80-120$

$81 \cdot 117$

This report shall not be reproduced except in full, without the written approval of the laboratorv. 
NEL LABORATORIES

CLIENT: Bechtel Nevada

mOJECT ID: V432/17777

OJECT \#: $\quad 17777$
CLIENT ID: Laboratory Control Spike

DATE SAMPLED: NA

NEL SAMPLE ID: 9806111260 B2-LCS

TEST:

Volatile Organic Compounds by EPA SW846 Method 8260A, Sept. 1994

MATRIX: Solid

EXTRACTED: $6 / 11 / 98$

ANALYZED: $6 / 11 / 98$

ANALYST: Suzanne

\section{PARAMETER}

Benzene

Chlorobenzene

1,1-Dichloroethere (1,1-DCE)

Toluene

Trichloroethene (TCE)

QUALITY CONTROL DATA:

Surrogate

4-Bromoftuorobenzene

Dibromofluotomethane

Toluene-d $d 8$

\section{Percent Recovery}

99

102

89

104

100

\section{Percent Recovery}

91

91

99

\section{Acceptable Range}

$80-120$

$80-120$

$80-120$

$80-120$

$80-120$

\section{Acceptable Range}

$74-121$

$80-120$

$81-117$ 
NEL LABORATORIES

CLIENT: $\quad$ Bechtel Nevada

PROJECT ID: V432/17777

CLIENT ID: Matrix Spike

PROJECT \#: $\quad 17777$

DATE SAMPLED: NA

NEL SAMPLE ID: L9806093-17-MS

TEST: Volatile Organic Compounds by EPA SW846 Method 8260A, Sept. 1994

MATRIX: Solid

EXTRACTED: $6 / 11 / 98$

ANALYZED: $6 / 11 / 98$

ANALYST: Suzanne

\section{PARAMETER}

Benzene

Chlorobenzene

1,1-Dichloroethene (1,1-DCE)

Toluene

Trichloroethene (TCE)

OUALITY CONTROL DATA:

Surrogate

4-Bromofluorobenzene

Dibromofluoromethane

Toluene-d8

\section{Percent Recovery}

94

100

142

96

98

\section{Acceptable Range}

$66-142$

$60-133$

$59-172$

$59-139$

$62-137$
Percent Recovery

85

93

97

\section{Acceptable Range}

$74-121$

$80-120$

$81-117$ 
SEMI-VOLATILE

ORGANICS

DATA 
TIENT: Bechtel Nevada

.OJECT NAME: V432

PROJECT NUMBER: 17777

\section{CLIENT ID: \\ Decon P9-2}

DATE SAMPLED: $6 / 4 / 98$

NEL SAMPLE ID: L9806094-03

TEST: Semi-Volatile Organic Compounds by EPA 8270C, Dec. 1996

MATRIX: Solid

DILUTION: 1
EXTRACTED: $6 / 11 / 98$

ANALYZED: $6 / 15 / 98$
ANALYST: CCJ

\section{PARAMETER}

Acenaphthene

Acenaphthylene

Anthracene

Benzo (a) anthracene

Benzo (b\&k) fluoranthene

Benzo (g,h,i) perylene

Benzo (a) pyrene

bis (2-Chloroethyi) ether

bis (2-Chloroethoxy) methane

bis (2-chloroisopropyl) ether

bis (2-Ethylhexyl)phthalate

Butylbenzylphthalate

4-Bromophenyl phenyl ether

Carbazole

4-Chloroanaline

4-Chioro-3-methyl phenol

Shloronaphthalene

$\checkmark$-Chiorophenol

4-Chlorophenyl phenyl ether

Chrysene

Dibenzo $\left(a_{1} h\right)$ anthracene

Dibenzofuran

Di-n-buty: phthalate

1,2-Dichlorobenzene (o-DCB)

1.3-Dichtorobenzene (m-DCB)

1,4-Dichjorobenzene (p-DCB)

2,4-Dichlorophenol

3.3'-Dichlorobenzidine

Diethylphthalate

2.4-Dimethylphenol

Dimethylphthalate

4.6-Dinitro-2-methyl phenol

2.4-Dinitrotoluene (DNT)

2,6-Dinitrotoluene (DNT)

2,4-Dinitrophenol

\begin{tabular}{l}
$\begin{array}{c}\text { Result } \\
\mu g / K g\end{array}$ \\
\hline ND \\
ND \\
ND \\
ND \\
ND \\
ND \\
ND \\
ND \\
ND \\
ND \\
ND \\
ND \\
ND \\
ND \\
ND \\
ND \\
ND \\
ND \\
ND \\
ND \\
ND \\
ND \\
ND \\
ND \\
ND \\
ND \\
ND \\
ND \\
ND \\
ND \\
ND \\
ND \\
ND \\
ND \\
ND \\
\hline
\end{tabular}

\section{Reporting}

Limit

$500 . \mu \mathrm{g} / \mathrm{K} \overline{\text { Di-n-0ctyl phthalate }}$

$500, \mu \mathrm{g} / \mathrm{Kg}$ Fluoranthene

500. $\mu \mathrm{g} / \mathrm{Kg}$ Fluorene

$500 . \mu \mathrm{g} / \mathrm{Kg}$ Hexachjorobenzene

500. $\mu \mathrm{g} / \mathrm{Kg}$ Hexachlorobutadiene

500. $\mu \mathrm{g} / \mathrm{Kg}$ Hexachlorocyclopentadiene

500. $\mu \mathrm{g} / \mathrm{Kg}$ Hexachloroethane

500. $\mu \mathrm{g} / \mathrm{Kg}$ Indeno $(1,2,3-\mathrm{c}, \mathrm{d})$ pyrene

500. $\mu \mathrm{g} / \mathrm{Kg}$ Isophorone

500. $\mu \mathrm{g} / \mathrm{Kg}$ 2-Methyinaphthalene

500. $\mathrm{Hg} / \mathrm{Kg}$ 2-Methylphenol

500. $\mu \mathrm{g} / \mathrm{Kg}$ 4-Methylphenol

500. $\mu \mathrm{g} / \mathrm{Kg}$ Naphthalene

500. $\mu \mathrm{g} / \mathrm{Kg}$ 2-Nitroaniline

1000. $\mu g / \mathrm{Kg} 3-\mathrm{Nitroaniline}$

1000. $\mu \mathrm{g} / \mathrm{Kg}$ 4-Nitoantline

500. $\mu \mathrm{g} / \mathrm{Kg}$ Nitrobenzene

500. $\mu \mathrm{g} / \mathrm{Kg}$ 2-Nitrophenol

500. $\mathrm{Hg} / \mathrm{Kg}$ 4-Nitrophenol

500. $\mu \mathrm{g} / \mathrm{Kg}$ N-Nitrosodi-n-propylamine

500. $\mu \mathrm{g} / \mathrm{Kg}$ N-Nitrosodiphenylamine

500. $\mu \mathrm{g} / \mathrm{Kg}$ Pentachlorophenol

500. $\mu \mathrm{g} / \mathrm{Kg}$ Phenol

500. $\mu \mathrm{g} / \mathrm{Kg}$ Phenanthrene

500. $\mathrm{tg} / \mathrm{Kg}$ Pyrene

500. $\mu \mathrm{g} / \mathrm{Kg} \mathrm{1,2,4-Trichlorobenzene}$

500. $\mu \mathrm{g} / \mathrm{Kg}$ 2,4,5-Trichtorophenol

1000. $\mu \mathrm{g} / \mathrm{Kg} \quad 2,4,6$-Trichlorophenol

500. $\mu \mathrm{g} / \mathrm{Kg}$

500. $\mu \mathrm{g} / \mathrm{Kg}$

500. $\mu \mathrm{g} / \mathrm{Kg}$

2500. $\mu \mathrm{g} / \mathrm{Kg}$

500. $\mu \mathrm{g} / \mathrm{Kg}$

500. $\mu \mathrm{g} / \mathrm{Kg}$

2500. $\mu \mathrm{g} / \mathrm{Kg}$

ND - Not Detected

\section{QUALITY CONTROL DATA:}

\section{Surrogate}

2,4,6-Tribromophenol

-Fluorobiphenyl

-Fluorophenol

Nitrobenzere-dS

p-Terphenyl-d 14

Phenol-d5
$\%$ Recovery

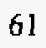

51

43

50

65

49

\begin{tabular}{|c|c|}
\hline $\begin{array}{l}\text { Result } \\
\mu \mathrm{g} / \mathrm{Kg}\end{array}$ & $\begin{array}{c}\text { Reporting } \\
\text { Limit }\end{array}$ \\
\hline $\mathrm{ND}$ & $\overline{500 . \mu \mathrm{g} / \mathrm{Kg}}$ \\
\hline ND & 500. $\mu \mathrm{g} / \mathrm{Kg}$ \\
\hline $\mathrm{ND}$ & $500 . \mu \mathrm{g} / \mathrm{Kg}$ \\
\hline $\mathrm{ND}$ & $500 . \mu \mathrm{g} / \mathrm{Kg}$ \\
\hline ND & 500. $\mu \mathrm{g} \mathrm{Kg}$ \\
\hline $\mathrm{ND}$ & 500. $\mu \mathrm{g} / \mathrm{Kg}$ \\
\hline $\mathrm{ND}$ & 500. $\mu \mathrm{g} / \mathrm{K}_{\mathrm{E}}$ \\
\hline $\mathrm{ND}$ & $500 . \mu \mathrm{g} K \mathrm{Kg}$ \\
\hline $\mathrm{ND}$ & $500, \mu \mathrm{g} / \mathrm{Kg}$ \\
\hline $\mathrm{ND}$ & 500. $\mu \mathrm{g} / \mathrm{Kg}$ \\
\hline ND & $500 . \mu \mathrm{K} / \mathrm{Kg}$ \\
\hline $\mathrm{ND}$ & $500 . \mu \mathrm{K} \mathrm{Kg}$ \\
\hline $\mathrm{ND}$ & $500 . \mu g / K \underline{L}$ \\
\hline ND & $2500 \mu \mathrm{g} K g$ \\
\hline $\mathrm{ND}$ & 2500. $\mu \mathrm{g} \mathrm{Kg}$ \\
\hline ND & $1000.4 \mathrm{~g} / \mathrm{KE}$ \\
\hline ND & $500 . \mu \mathrm{g} / \mathrm{Kg}$ \\
\hline $\mathrm{ND}$ & 500. 过 $\mathrm{Kg}$ \\
\hline $\mathrm{ND}$ & 2500. $\mu \mathrm{g} \mathrm{KE}$ \\
\hline ND & $500 . \mu \mathrm{g} / \mathrm{Kg}$ \\
\hline$N D$ & 500. \\
\hline $\mathrm{ND}$ & 2500. $\mu \mathrm{g} / \mathrm{Kg}$ \\
\hline $\mathrm{ND}$ & 500. $1 \mathrm{~g} / \mathrm{Kg}$ \\
\hline$N D$ & 500. $\mu \mathrm{g} K g$ \\
\hline $\mathrm{ND}$ & $500.4 \mathrm{~g} K \mathrm{Kg}$ \\
\hline $\mathrm{ND}$ & 500. $\mu g \mathrm{Kg}$ \\
\hline $\mathrm{ND}$ & 500. $\mu \mathrm{g} K g$ \\
\hline $\mathrm{ND}$ & 500. $\mu \mathrm{g} / \mathrm{Kg}$ \\
\hline
\end{tabular}

Acceptable Range

19.122

$30-115$

$25-121$

$23-120$

$18-137$

$24-113$ 
CLIENT: Bechtel Nevada

PROJECT NAME: $\quad$ V432

PROJECT NUMBER: 17777
CLIENT ID: Decon P10-2

DATE SAMPLED: $6 / 4 / 98$

NEL SAMPLE ID: L9806094-08

TEST: Semi-Volatile Organic Compounds by EPA 8270C, Dec. 1996

MATRIX: Solid

DILUTION: 1
EXTRACTED: 6/11/98

ANALYZED: $6 / 15 / 98$

ANALYST: CCJ

\begin{tabular}{|c|c|c|c|c|c|}
\hline PARAMETER & $\begin{array}{l}\text { Result } \\
\mu \mathrm{g} / \mathrm{Kg}\end{array}$ & $\begin{array}{c}\text { Reporting } \\
\text { Limit }\end{array}$ & PARAMETER & $\begin{array}{l}\text { Result } \\
\mu \mathrm{g} / \mathrm{Kg}\end{array}$ & $\begin{array}{c}\text { Reporting } \\
\text { Limit }\end{array}$ \\
\hline Acenaphthene & $\overline{\mathrm{ND}}$ & $\overline{500.11 \mathrm{~g} / \mathrm{Kg}}$ & Di-n-octyl phthalate & $\mathrm{ND}$ & $500 . \mu \mathrm{g} / \mathrm{Kg}$ \\
\hline Acenaphthylene & $\mathrm{ND}$ & 500. $\mu g \mathrm{Kg}$ & Fluoranthene & $\mathrm{ND}$ & 500. $\mu \mathrm{g} / \mathrm{Kg}$ \\
\hline Anthracene & $\mathrm{ND}$ & 500. $\mu \mathrm{g} / \mathrm{Kg}$ & Fluorene & $\mathrm{ND}$ & 500. $4 \mathrm{~g} \mathrm{Kg}$ \\
\hline Benzo (a) anthracene & ND & $500 . \mu \mathrm{g} / \mathrm{Kg}$ & Hexachlorobenzene & ND & 500. $\mu \mathrm{g} / \mathrm{Kg}$ \\
\hline Benzo (b\&k) fluoranthene & VD & $500 . \mu \mathrm{g} / \mathrm{Kg}$ & Hexachlorobutadiene & $\mathrm{ND}$ & $500.4 \mathrm{~g} \mathrm{Kg}$ \\
\hline Benzo (g.h.i) perylene & $\mathrm{ND}$ & 500. $11 \mathrm{~g} / \mathrm{Kg}$ & Hexachlorocyclopentadiene & $\mathrm{ND}$ & 500. $\mu \mathrm{g} / \mathrm{Kg}$ \\
\hline Benzo (a) pyrene & $\mathrm{KD}$ & 500. $\mu \mathrm{g} / \mathrm{Kg}$ & Hexachloroethane & $\mathrm{ND}$ & $500 . \mu \mathrm{g} \mathrm{Kg}$ \\
\hline bis (2-Chloroethyl) ether & $\mathrm{ND}$ & $500 . \mu \mathrm{g} / \mathrm{Kg}$ & Indeno $(1,2,3-c, d)$ pyrene & $\mathrm{ND}$ & 500. $1 \mathrm{gg} \mathrm{Kg}$ \\
\hline bis (2-Chloroethoxy) methane & $\mathrm{ND}$ & 500. $\mu \mathrm{g} / \mathrm{Kg}$ & Isophorone & $\mathrm{ND}$ & 500. $\mathrm{Hg} / \mathrm{K} \underline{\mathrm{g}}$ \\
\hline bis (2-chloroisopropy]) ether & $\mathrm{ND}$ & 500. $\mu g / \mathrm{Kg}$ & 2-Methylnaphthalene & $\mathrm{ND}$ & 500. $\mu g / K \underline{\underline{g}}$ \\
\hline bis (2-Ethylhexyl)phthalate & $\mathrm{ND}$ & 500. $\mathrm{Hg} / \mathrm{Kg}$ & 2-Methylphenol & ND & 500. $11 \mathrm{~g} / \mathrm{Kg}$ \\
\hline Butylbenzylphthalate & ND & 500. $\mu \mathrm{g} / \mathrm{Kg}$ & 4-Methylphenol & $\mathrm{ND}$ & $500 . \mu \mathrm{g} / \mathrm{Kg}$ \\
\hline 4-Bromophenyi phenyl ether & $\mathrm{ND}$ & 500. $\mu \mathrm{g} / \mathrm{Kg}$ & Naphthalene & $\mathrm{ND}$ & 500. $\mathrm{Hg} \mathrm{Kg}$ \\
\hline Carbazole & $\mathrm{ND}$ & 500. $\mu \mathrm{g} / \mathrm{Kg}$ & 2-Nitroaniline & $\mathrm{ND}$ & 2500. $\mu \mathrm{g} / \mathrm{Kg}$ \\
\hline 4-Chloroanaline & $\mathrm{ND}$ & 1000. $\mu \mathrm{g} / \mathrm{Kg}$ & 3-Nitroaniline & $\mathrm{ND}$ & 2500. $\mu \mathrm{gKO}$ \\
\hline 4-Chloro-3-methyl phenol & $\mathrm{ND}$ & $1000.1 \mathrm{~g} / \mathrm{Kg}$ & 4-Nitroaniline & ND & $1000 . \mu g R g$ \\
\hline 2-Chloronaphthalene & ND & 500. $\mu \mathrm{g} / \mathrm{Kg}$ & Nitrobenzene & $\mathrm{MD}$ & 500. $\mu \mathrm{g} \mathrm{Ko}$ \\
\hline 2-Chlorophenol & ND & 500. $\mu \mathrm{g} / \mathrm{Kg}$ & 2-Nitropinenol & $\mathrm{ND}$ & $500 . \mu \Omega$ \\
\hline 4-Chlorophenyl phenyl ether & $\mathrm{ND}$ & 500. $\mu \mathrm{g} / \mathrm{Kg}$ & 4-Nitrophenol & $\mathrm{ND}$ & 2500. $\lfloor\underline{G} \mathrm{~K}$ \\
\hline Chrysene & ND & 500. $\mathrm{Hg} / \mathrm{Kg}$ & N-Nitrosodi-n-propylanine & $\mathrm{ND}$ & $500 . \mu \mathrm{g} K \mathrm{Kg}$ \\
\hline Dibenzo (ah) anthracene & $\mathrm{ND}$ & 500. $\mu \mathrm{g} \mathrm{Kg}$ & N-Nitrosodiphenylamine & $\mathrm{ND}$ & $500.4 \mathrm{Kg}$ \\
\hline Dibenzoturan & $\mathrm{ND}$ & 500. $\mu \mathrm{g} / \mathrm{Kg}$ & Pentachiorophenol & $\mathrm{ND}$ & 2500. $\mu \mathrm{g} / \mathrm{Kg}$ \\
\hline Di-n-butyl phthalate & ND & 500. $\mu g / \mathrm{Kg}$ & Phenol & $N D$ & $500.11 \mathrm{~g} / \mathrm{Kg}$ \\
\hline 1.2-Dichlorobenzene (O-DCB) & $\mathrm{ND}$ & 500. $\mu \mathrm{g} / \mathrm{Kg}$ & Phenanthrene & $\mathrm{ND}$ & 500. $\mu \mathrm{g} / \mathrm{Kg}$ \\
\hline 1.3-Dichlorobenzene (m-DCB) & $\mathrm{ND}$ & 500. $1 \mathrm{~g} / \mathrm{Kg}$ & Pyrene & $\mathrm{ND}$ & 500. $H_{\mathrm{E}} / \mathrm{Kg}$ \\
\hline 1.4-Dichlorobenzene (p-DCB) & ND & 500. $\mu \mathrm{g} / \mathrm{Kg}$ & 1.2,4-Trichlorobenzene & $\mathrm{ND}$ & 500. $\mu \mathrm{g} \mathrm{Kg}$ \\
\hline 2.4-Dichlorophenol & $N D$ & 500. $\mu \mathrm{g} / \mathrm{Kg}$ & 2,4,5-Trichlorophenoi & $\mathrm{ND}$ & $500 . \mu \mathrm{E} \mathrm{K}_{\mathrm{S}}$ \\
\hline 3.3'-Dichlorobenzidine & ND & 1000. $\mu \mathrm{g} / \mathrm{Kg}$ & 2.4,6-Trichlorophenol & $\mathrm{ND}$ & 500. $\mu \mathrm{g} \mathrm{Kg}$ \\
\hline Diethylphthaiate & ND & 500. $\mu \mathrm{g} \mathrm{Kg}$ & & & \\
\hline 2,4-Dimethylphenol & ND & 500. $\mu \mathrm{g} / \mathrm{Kg}$ & & & \\
\hline Dimethylphthalate & ND & 500. $11 \mathrm{~g} / \mathrm{Kg}$ & & & \\
\hline 4.6-Dinitro-2-methy' phenol & ND & $2500.11 \mathrm{~g} \mathrm{Kg}$ & & & \\
\hline 2.4-Dinitrotoluene (DNT) & ND & 500. $\mu \mathrm{g} / \mathrm{Kg}$ & & & \\
\hline 2.6-Dinitrotoluene (DNT) & $\mathrm{ND}$ & 500. $11 \mathrm{~g} \mathrm{Kg}$ & & & \\
\hline 2.4-Dinitrophenol & $N D$ & 2500. $\mu \mathrm{g} \mathrm{Kg}$ & & & \\
\hline
\end{tabular}

ND - Not Detected

QUALITY CONTROL DATA:

\section{Surrogate}

2.4.6-Tribromophenol

2-Ftuorobipheny?

2-Fluoropheno]

Nitrobenzene-d5

p-Terphenyl-d 14

Phenol-d5
\% Recovery
Acceptable Range

$19-122$

$30-115$

$25-121$

$23-120$

$18-137$

$24-113$

This report shall not be reproduced except in full, without the written approval of the laboratory 
NEL LABORATORIES

CLIENT: Bechtel Nevada

-ROJECT ID: V432/17777

....OJECT H: $\quad 17777$

\section{CLIENT ID: Matrix Spike}

DATE SAMPLED: NA

NEL SAMPLE ID: L9806093-18-MS

TEST: $\quad$ Semi-Volatile Organic Compounds by EPA 8270C, Dec. 1996

MATRIX: Solid

EXTRACTED: $6 / 11 / 98$

ANALYZED: $6 / 12 / 98$

ANALYST: CCJ

PARAMETER

Acenaphthene

4-Chloro-3-methyl phenol

2-Chlorophenol .

1,4-Dichlorobenzene ( $\mathrm{p}$-DCB)

2.4-Dinitrotoluene (DNT)

4-Nitrophenol

N-Nitrosodi-n-propylamine

Pentachlorophenol

Phenol

1,2,4-Trichlorobenzene

QUALITY CONTROL DATA:

\section{Surrogate}

2,4,6-Tribromophenol

2-Fluorobiphenyl

2-Fluorophenol

Nitrobenzene-d5

Terphenyl-d 14

....nol-d5

\section{Percent Recovery}

50

54

56

49

54

53

65

49

50

53

\section{Acceptable Range}

$31-137$

$26-103$

$25-102$

$28-104$

$28-89$

$11-114$

$41-126$

$17-109$

$26-90$

$28-104$

\section{Percent Recovery}

56

54

50

56

63

60

\section{Acceptable Range}

$19-122$
$30-115$
$25-121$
$23-120$
$18-137$
$24-113$ 
NEL LABORATORIES

$\begin{array}{llll}\text { CLIENT: } & \text { Bechtel Nevada } & \text { CLIENT ID: } & \text { Matrix Spike Duplicate } \\ \text { PROJECT ID: } & \text { V432/17777 } & \text { DATE SAMPLED: } & \text { NA } \\ \text { PROJECT \#: } & 17777 & \text { NEL SAMPLE ID: } & \text { L9806093-18-MSD }\end{array}$

TEST: $\quad$ Semi-Volatile Organic Compounds by EPA 8270C, Dec. 1996

MATRIX: Solid EXTRACTED: 6/1 1/98

ANALYZED: $6 / 12 / 98$

ANALYST: CCJ

\section{PARAMETER}

Percent Recovery

\section{Acceptable Range}

$\underline{\text { RPD }}$

Acenaphthene

4-Chloro-3-methyl phenol

2-Chlorophenol.

1,4-Dichlorobenzene (p-DCB)

2,4-Dinitrotoluene (DNT)

4-Nitrophenol

$\mathrm{N}-\mathrm{Nitrosodi-n-propylamine}$

Pentachlorophenol

Phenol

1, 2,4-Trichlorobenzene

QUALITY CONTROL DATA:

\section{Surrogate}

2,4,6-Tribromophenol

2-Fluorobiphenyl

2-Fluorophenol

Nitrobenzene-d5

p-Terphenyl-d 14

Phenol-ds
55

62

$31-137$

$26-103$

$25-102$

$28-104$

$28-89$

$11-114$

$41-126$

$17-109$

$26=90$

$38-107$

54

Percent Recovery

61

78

63

76

85

80
Acceptable Range

$19-122$

$30-115$

$25-121$

$23-120$

$18-137$

$24-113$ 
NEL LABORATORIES

LIENT: Bechtel Nevada

ROJECT NAME: V432

PROJECT NUMBER: 17777
CLIENT ID: Method Blank

DATE SAMPLED: NA

NEL SAMPLE ID: 061098-E1-BLK

TEST: $\quad$ Semi-Volatile Organic Compounds by EPA 8270C, Dec. 1996

MATRIX: Solid EXTRACTED:

\begin{tabular}{|c|c|c|c|c|c|}
\hline PARAMETER & $\begin{array}{c}\text { Result } \\
\mu \mathrm{g} / \mathrm{Kg}\end{array}$ & $\begin{array}{c}\text { Reporting } \\
\text { Limit }\end{array}$ & PARAMETER & $\begin{array}{c}\text { Result } \\
\mu \mathrm{g} / \mathrm{Kg}\end{array}$ & $\begin{array}{c}\text { Reporting } \\
\text { Limit }\end{array}$ \\
\hline Acenaphthene & $\overline{\mathrm{ND}}$ & $\overline{500 \mu \mathrm{g} / \mathrm{Kg}}$ & $\overline{\text { Di-n-octyl phthalate }}$ & ND & $500 \mu \mathrm{g} / \mathrm{Kg}$ \\
\hline Acenaphthylene & ND & $500 \mu \mathrm{g} / \mathrm{Kg}$ & Fluorarthene & $\mathrm{ND}$ & 500 ug $/ \mathrm{Kg}$ \\
\hline Anthracene & ND & $500 \mu \mathrm{g} / \mathrm{Kg}$ & Fluorene & $\mathrm{ND}$ & $300 \mu \mathrm{g} / \mathrm{Kg}$ \\
\hline Benzo (a) anthracene & ND & $500 \mu \mathrm{g} / \mathrm{Kg}$ & Hexachlorobenzene & ND & $500 \mu \mathrm{g} / \mathrm{Kg}$ \\
\hline Benzo (b\&k) fluoranthene & $\mathrm{ND}$ & $500 \mu \mathrm{g} / \mathrm{Kg}$ & Hexachlorobutadiene & $N D$ & $5001 \mathrm{Kg}$ \\
\hline Benzo $(\mathrm{g}, \mathrm{h} . \mathrm{i})$ peryiene & $\mathrm{ND}$ & $50049 \mathrm{Kg}$ & Hexachlorocyclopentadiene & ND & $500 \mu \mathrm{g} \mathrm{Kg}$ \\
\hline Benzo (a) pyrene & ND & $500 \mu \mathrm{g} / \mathrm{Kg}$ & Hexachloroethane & $\mathrm{ND}$ & $500 \mu \mathrm{Kg}$ \\
\hline bis $\{2$-Chloroethyl $\}$ ether & ND & $500 \mu \mathrm{g} / \mathrm{Kg}$ & Indeno $(1,2,3-c, d) p y$ rene & $\mathrm{ND}$ & $500 \mu \mathrm{g} / \mathrm{R} \underline{\mathrm{g}}$ \\
\hline bis (2-Chloroethoxy) methane & ND & $50011 \mathrm{~g} / \mathrm{Kg}$ & Isophorone & ND & $500 \mu \mathrm{g}: \mathrm{Kg}$ \\
\hline bis (2-chloroisopropyl) ether & ND & $500 \mu \mathrm{g} \mathrm{Kg}$ & 2-Methylnaphthalene & $\mathrm{ND}$ & $500 \mu \mathrm{g} \cdot \mathrm{Kg}$ \\
\hline bis (2-Ethylibexyl)phthalate & $\mathrm{ND}$ & $500 \mu \mathrm{g} / \mathrm{Kg}$ & 2-Methylphenol & ND & $500 \mu \mathrm{g} \mathrm{Kg}$ \\
\hline Butylbenzy/phthalate & $\mathrm{ND}$ & $500 \mathrm{ug} / \mathrm{Kg}$ & 4-Methylphenol & ND & $500 \mathrm{ug}: \mathrm{Kg}$ \\
\hline 4-Bromophenyl pheny i ether & $\mathrm{ND}$ & $500 \mu \mathrm{g} / \mathrm{Kg}$ & Naphthalene & $\mathrm{XD}$ & $500 \mu \mathrm{g} / \mathrm{Kg}$ \\
\hline Carbazole & ND & $500 \mu \mathrm{g} \mathrm{Kg}$ & 2-Nitroaniline & ND & $2500 \mu \mathrm{g} \mathrm{Kg}$ \\
\hline 4-Chloroanaline & ND & $1000 \mu \mathrm{g} / \mathrm{Kg}$ & 3-Nitroaniline & $\mathrm{ND}$ & $2500 \mu \mathrm{gr} \mathrm{Kg}$ \\
\hline -Chloro-3-methyl phenol & $\mathrm{ND}$ & $1000 \mu \mathrm{g} \mathrm{Kg}$ & 4-Nitroaniline & $\mathrm{NO}$ & $1000 \mu \mathrm{kg}$ \\
\hline -Chloronaphthalene & ND & $500 \mu \mathrm{gKE}$ & Nitrobenzene & $\mathrm{ND}$ & $500 \mu \mathrm{g} \mathrm{Kg}$ \\
\hline -Chlorophenol & ND & $500 \mathrm{ug} \mathrm{Kg}$ & 2-Nitrophenol & $\mathrm{ND}$ & $500 \mu \mathrm{g} \mathrm{Kg}$ \\
\hline 4-Chlorophenyi pheny's cther & ND & $500 \mu g \mathrm{Kg}$ & 4-Nitrophenol & $N D$ & $2500 \mu \mathrm{g} / \mathrm{K} \underline{\mathrm{g}}$ \\
\hline Cnpisene & ND & $500 \mu g / \mathrm{Kg}$ & N-Nitrosodi-n-propylaminz & ND & $500 \mu \mathrm{g} K \mathrm{~K}$ \\
\hline Dibenzo (ah) anthracene & ND & $500 \mu \mathrm{g} / \mathrm{Kg}$ & N-Nitrosodipheryiamine & $\mathrm{ND}$ & $500 \mu \mathrm{g} \cdot \mathrm{Kg}$ \\
\hline Dibenzofuran & ND & $500 \mathrm{\mu g} / \mathrm{Kg}$ & Pentachlorophenol & ND & $2500 \mu \mathrm{g} \mathrm{Ko}$ \\
\hline Di-n-butyl phthalate & ND & $500 \mu \mathrm{g} / \mathrm{Kg}$ & Phenol & ND & 500 Lekg \\
\hline 1.2-Dichlurobenzene (o-DCB) & $\mathrm{ND}$ & $500 \mu \mathrm{gkg}$ & Phenanthrene & $N D$ & $500 \mu \mathrm{g}: \mathrm{Kg}$ \\
\hline 1.3-Dichlorobenzene (m-DCB) & $N D$ & $\$ 00 \mu \mathrm{g} / \mathrm{Kg}$ & Pугеле & ND & $500 \mu \mathrm{g} K \mathrm{Kg}$ \\
\hline 1,7-Dichlorobenzene (p-DCB) & $\mathrm{ND}$ & $500 \mu \mathrm{g} K \mathrm{Kg}$ & 1.2.4-Trichlorobenzene & $\mathrm{ND}$ & 500 ug: Kg \\
\hline I.4-Dichlorophenol & ND & $5001 \mathrm{gg} / \mathrm{Kg}$ & 2.4.5-Trichlorophenol & $\mathrm{ND}$ & $500 \mathrm{ug} \mathrm{Kg}$ \\
\hline 3.3'-Dichlorobenzidine & $N D$ & $1000 \mu \mathrm{g} / \mathrm{Kg}$ & 2.4,6-Trichlorophenol & ND & $500 \mu \mathrm{g} \mathrm{K}$ \\
\hline Diethylphthalate & $\mathrm{ND}$ & $500 \mu \mathrm{g} / \mathrm{Kg}$ & & & \\
\hline 2.4-Dimethylphenol & ND & $500 \mu \mathrm{g} / \mathrm{Kg}$ & & & \\
\hline Dimethy'lphthalate & ND & $500 \mu \mathrm{gg}$ & & & \\
\hline 4,6.Dinitro-2-methyl phenol & $\mathrm{ND}$ & $2500 \mu \mathrm{g} K \mathrm{Kg}$ & & & \\
\hline 2.4-Dinitrotoluene (DNT) & $\mathrm{ND}$ & $500 \mu \mathrm{g} / \mathrm{Kg}$ & & & \\
\hline 2,6-Dinitrotoluene (DNT) & $\mathrm{ND}$ & $500 \mu \mathrm{g} / \mathrm{Kg}$ & & & \\
\hline 2.4-Dinitrophenol & ND & $2500 \mu \mathrm{giKg}$ & & & \\
\hline
\end{tabular}

VD - Not Detected

QUALITY CONTROL DATA:

Surrogate

2.4,6-Tribromophenol

2-Fluorobiphenyl

-Fluorophenol

Nitrobenzene-d5

p-Terphenyl-dl4

Phenol-ds

\section{$\%$ Recovery}

88

78

66

75

83

77
$6 / 11 / 98$

$6: 12 / 98$

This report shall not be reproduced except in full, without the written approval of the laboratory

\section{Accentable Range}

19. 122

$30-115$

$25-121$

$23-120$

$18-137$

$24-113$ 
NEL LABORATORIES

CLIENT: Bechtel Nevada

PROJECT ID: V432/17777

PROJECT
CLIENT ID: Laboratory Control Spike

DATE SAMPLED: NA

NEL SAMPLE ID: 061098-El-LCS

TEST: $\quad$ Semi-Volatile Organic Compounds by EPA 8270C, Dec. 1996

MATRIX: Solid

EXTRACTED: $6 / 11 / 98$

ANALYZED: $6 / 12 / 98$
ANALYST: $C C J$

\section{Acceptable Range}

$$
\begin{aligned}
& 31-137 \\
& 26-103 \\
& 25-102 \\
& 28-104 \\
& 28-89 \\
& 11-114 \\
& 41-126 \\
& 17-109 \\
& 26-90 \\
& 38-107
\end{aligned}
$$

1,2,4-Trichlorobenzene

\section{Percent Recovery}

Surrogate

2,4,6-Tribromophenol

2-Fluorobiphenyl

2-Fluorophenol

Nitrobenzene-d5

p-Terphenyl-dI 4

Phenol-ds
69

91

73

90

105

94

\section{Acceptable Range}

$19-122$
$30-115$
$25-121$
$23-120$
$18-137$
$24-113$


NEL LABORATORIES

$\begin{array}{llll}\text { CLIENT: } & \text { Bechtel Nevada } & \text { CLIENT ID: } & \text { Laboratory Control Spike Dupitcate } \\ \text { "ROJECT ID: } & \text { V432/17777 } & \text { DATE SAMPLED: } & \text { NA } \\ \text {. ROJECT \#: } & 17777 & \text { NEL SAMPLE ID: } & 061098-E 1-L C S D\end{array}$

TEST: $\quad$ Semi-Volatile Organic Compounds by EPA 8270C, Dec. 1996

MATRIX: Solid

EXTRACTED: 6/11/98

ANALYZED: $6 / 12 / 98$

ANALYST: CCJ

PARAMETER

Acenaphthene

4-Chloro-3-methyl phenol

2-Chlorophenol

1.4-Dichlorobenzene ( $\mathrm{p}-\mathrm{DCB}$ )

2.4-Dinitrotoluene (DNT)

4-Nitrophenol

N-Nitrosodi-n-propylamine

Pentachlorophenol

Phenol

1.2.4-Trichlorobenzene

OUALITY CONTROL DATA:

\section{Surrogate}

3.4.6-Tribromophenol

2-Fluorobiphenyl

2-Fluorophenol

Nitrobenzene-d5

Terphenyl-d14

-.enoi-d5
Percent Recoverv

69

75

71

66

75

74

89

73

66

73

Percent Recoyery

61

78

63

76

85

80

\section{Acceptable Range $\quad \underline{\text { RPD }}$}

$31-137$

$26-103$

14.2

$25-102$

12.3

15.4

$28-104$

19.9

$28-89$

II - 114

$41-126$

I7 - 109

$26-90$

$38-107$

17.9

18.7

18.8

19.8

18.5

17.3

\section{Acceptable Range}

$19-122$

$30-115$

$25-121$

$23-120$

$18-137$

$24-113$ 
POLYCHLORINATED

BIPHENYLS

DATA 
NEL LABORATORIES

CLIENT: Bechtel Nevada

2OJECT NAME: V432

-PROJECT NUMBER: 17777
CLIENT ID: Decon P9-3

DATE SAMPLED: $6 / 4 / 98$

NEL SAMPLE ID: L9806094-04

TEST: Polychlorinated Biphenyl's (PCB's) by EPA 8082, Dec. 1996

MATRIX: Solid

DILUTION: 1
EXTRACTED: $6 / 1 \mathrm{I} / 98$

ANALYZED: $6 / 13 / 98$
ANALYST: John

\begin{tabular}{c}
$\begin{array}{c}\text { Reporting } \\
\text { Limit }\end{array}$ \\
\hline $20 . \mu \mathrm{gg}$ \\
$20 . \mu \mathrm{kg} / \mathrm{kg}$ \\
20. $\mu \mathrm{g} / \mathrm{kg}$ \\
$20 . \mu \mathrm{g} / \mathrm{kg}$ \\
$20 . \mu \mathrm{gg}$ \\
$20 . \mu \mathrm{gg} / \mathrm{kg}$ \\
$20 . \mu \mathrm{g} / \mathrm{kg}$
\end{tabular}

PARAMETER

Arochlor-1016

Arochlor-1221

Arochlor-1232

Arochlor -1242

Arochlor-1248

Arochlor-1254

Arochlor -1260

\begin{tabular}{l} 
Result \\
\hline $\mathrm{ND}$ \\
$\mathrm{ND}$ \\
$\mathrm{ND}$ \\
$\mathrm{ND}$ \\
$\mathrm{ND}$ \\
$\mathrm{ND}$ \\
$\mathrm{ND}$
\end{tabular}

Acceptable Range

$70-130$

$70-130$
Decachlorobipheny

Tetrachloro-m-xylene
\% Recovery

74

71 
NEL LABORATORIES

CLIENT: Bechtel Nevada

PROJECT NAME: $\quad \mathrm{V} 432$

CLIENT ID: Decon P10-3

PROJECT NUMBER: 17777

DATE SAMPLED: $6 / 4 / 98$

NEL SAMPLE ID: L9806094-09

TEST: Polychlorinated Biphenyl's (PCB's) by EPA 8082, Dec. 1996

MATRIX: Solid

EXTRACTED: $6 / 11 / 98$

DILLTION: 1

ANALYZED: $6 / 12 / 98$

ANALYST: John

\section{PARAMETER}

Arochlor-1016

Arochlor-1221

Arochlor 1232

Arochlor- 1242

Arochlor-1248

Arochlor-1254

Arochlor- 1260

ND - Not Detected

OUALITY CONTROL DATA:

\section{Surrogate}

Decachlorobiphenyl

Tetrachloro-m*xylene

\section{$\%$ Recoverv}

70

64

\section{Acceptable Range}

$70-130$

$70-130$ 
NEL LABORATORIES

CLIENT: Bechtel Nevada

ROJECT ID: V432/17777

-.OJECT \#: 17777

\section{CLIENT ID: Matrix Spike}

DATE SAMPLED: NA

NEL SAMPLE ID: L9806093-19-MS

TEST:

Polychlorinated Biphenyl's (PCB's) by EPA 8082, Dec. 1996

MATRIX: Solid

EXTRACTED: 6/11/98

ANALYZED: $6 / 12 / 98$

ANALYST: John

PARAMETER

Arochlor -1260

OUALITY CONTROL DATA:

Surrogate

Decachlorobiphenyl

Tetrachloro-m-xylene
Percent Recovery

85

Percent Recovery

97

94

\section{Acceptable Range}

$48-129$

\section{Acceptable Range}

$70 \cdot 130$

$70-130$

This report shall not be reproduced except in full, without the written approval of the laboratory. 
NEL LABORATORIES

CLIENT: Bechtel Nevada

PROJECT ID: V432/17777

PROJECT \#: $\quad 17777$
CLIENT ID:

DATE SAMPLED: NA

NEL SAMPLE ID: L9806093-19-MSD

TEST: $\quad$ Polychlorinated Biphenyl's (PCB's) by EPA 8082, Dec. 1996

MATRIX: Solid

EXTRACTED: $6 / 11 / 98$

ANALYZED: $6 / 12 / 98$

ANALYST: John

\section{PARAMETER}

Percent Recovery

Arochlor -1260

69

Acceptable Range

RPD

OUALITY CONTROL DATA:

\section{Surrogate}

Decachlorobipheny]

Tetrachloro-m-xylene

\section{Percent Recovery}

86

90
$48-129$

20.7

\section{Acceptable Range}

$70-130$

$70-130$ 
NEL LABORATORIES

CLIENT: Bechtel Nevada

SOJECT NAME: V432

LOJECT NUMBER: 17777

CLIENT ID: Method Blank

DATE SAMPLED: NA

NEL SAMPLE ID: 980611 s8082-BK

TEST: Polychlorinated Biphenyl's (PCB's) by EPA 8082, Dec. 1996

MATRIX: Solid

EXTRACTED: $6 / 11 / 98$

ANALYZED: $6 / 12 / 98$

ANALYST: John

\begin{tabular}{|c|c|c|}
\hline PARAMETER & Result & $\begin{array}{c}\text { Reporting } \\
\text { Limit }\end{array}$ \\
\hline Arochlor-1016 & ND & $20 \mu g / \mathrm{kg}$ \\
\hline Arocinlor- 1221 & $\mathrm{ND}$ & $20 \mu \mathrm{g} / \mathrm{kg}$ \\
\hline Arochlor- 1232 & $\mathrm{ND}$ & $20 \mu \mathrm{g} / \mathrm{kg}$ \\
\hline Arochlor $=1242$ & $\mathrm{ND}$ & $20 \mu \mathrm{g} / \mathrm{kg}$ \\
\hline Arochlor- 1248 & ND & $20 \mu \mathrm{g} / \mathrm{kg}$ \\
\hline Arochlor- 1254 & $\mathrm{ND}$ & $20 \mu \mathrm{\mu g} / \mathrm{kg}$ \\
\hline Arochlor- 1260 & $\mathrm{ND}$ & $20 \mu \mathrm{Hg} / \mathrm{kg}$ \\
\hline
\end{tabular}

ND - Not Detected

QUALITY CONTROL DATA:

Surrogate

Decachlorobipheny]

Tetrachloro-m-xylene
\% Recovery

84

68
Acceptable Range

$70 \cdot 130$

$70-130$ 
NEL LABORATORIES

$\begin{array}{llll}\text { CLIENT: } & \text { Bechtel Nevada } & \text { CLIENT ID: } & \text { Laboratory Control Spike } \\ \text { PROJECT ID: } & \text { V432/17777 } & \text { DATE SAMPLED: } & \text { NA } \\ \text { PROJECT \#: } & 17777 & \text { NEL SAMPLE ID: } 980611 \text { s8082-LCS }\end{array}$

TEST: $\quad$ Polychlorinated Biphenyl's (PCB's) by EPA 8082, Dec. 1996

MATRIX: Solid $\quad$ EXTRACTED: $6 / 11 / 98$

ANALYZED: $6 / 12 / 98 \quad$ ANALYST: John

PARAMETER

Percent Recovery

Acceptable Range

Arochlor- 1016

86

$80-120$

Arochlor -1260

82

$80-120$

QUALITY CONTROL DATA:

Surrogate

Decachlorobiphenyl

Tetrachloro-m-xylene

\section{Percent Recovery}

101

100

\section{Acceptable Range}

$70-130$

$70-130$ 
TOTAL

PETROLEUM

HYDROCARBON

DATA 
CLIENT: Bechtel Nevada IOJECT NAME: V432

PROJECT NUMBER: 17777

TEST: $\quad$ Total Extractable Petroleum Hydrocarbons by EPA Method 8015 M, July 1992

METHOD: EPA 8015M

MATRIX: Solid ANALYST: John

\begin{tabular}{|c|c|c|c|c|c|c|c|c|}
\hline $\begin{array}{l}\text { CLIENT } \\
\text { SAMPLE 1D } \\
\end{array}$ & $\begin{array}{c}\text { SAMPLE } \\
\text { DATE } \\
\end{array}$ & $\begin{array}{c}\text { NEL } \\
\text { SAMPLE ID } \\
\end{array}$ & $\begin{array}{c}\text { RESULT } \\
\mathrm{mg} / \mathrm{kg}\end{array}$ & C.R. & $\begin{array}{l}\text { Reporting } \\
\text { Limit }\end{array}$ & $\begin{array}{l}\text { Surrogate } \\
\text { Recovery }\end{array}$ & EXTRACTED & ANALYZED \\
\hline Decon P9-4 A\&B & $6 / 4 / 98$ & L9806094-0S & $\mathrm{ND}$ & & $10 . \mathrm{mg} / \mathrm{kg}$ & $101 \%$ & $6 / 10 / 98$ & $6 / 10 / 98$ \\
\hline Decon P104 A\&B & $6 / 4 / 98$ & L9806094-10 & ND & & 10. $\mathrm{mg} / \mathrm{kg}$ & $110 \%$ & $6 / 10 / 98$ & $6 / 10 / 98$ \\
\hline
\end{tabular}

Note: The reporting limit for Oil Range Organics in soil is $50 \mathrm{mg} / \mathrm{kg}$.

QUALITY CONTROL DATA (Total for Gas and Diesel Range):

\section{Sample ID}

Tlank, 980610TPH-BLK

ØS, 980610TP - LCS

MS, 980610TP - MS

MSD. 980610TP - MSD
Result Acceptable Range Surrogate Recovery* Sample Number

\begin{tabular}{|c|c|c|c|c|c|}
\hline ND & $<<$ & 10. $\mathrm{mg} / \mathrm{kg}$ & 99 & $\%$ & NA \\
\hline $69 \%$ & 55 & $-102 \%$ & 99 & $\%$ & NA \\
\hline $67 \%$ & 38 & $-107 \%$ & 94 & $\%$ & L9806093-20 \\
\hline $57 \%$ & 38 & $-107 \%$ & 78 & $\%$ & L9806093.20 \\
\hline
\end{tabular}

ND - Not Detected

*Surrogate used was Octacosane, acceptance limits 54-130\% for solids, 60-121\% for aqueous samples

This report shall not be reproduced except in full, without the written approval of the laboratory. 
TCLP

\section{METALS}

DATA 
CLIENT: Bechtel Nevada

?OJECT NAME: $\quad \mathrm{V} 432$

$r$ ROJECT NUMBER: 17777

\section{CLIENT ID: Decon P9-5}

DATE SAMPLED: $6 / 4 / 98$

NEL SAMPLE ID: L9806094-06

TEST:

TCLP-8 Metals

MATRIX:

Solid

\begin{tabular}{|c|c|c|c|c|c|c|c|}
\hline PARAMETER & $\begin{array}{c}\text { RESULT } \\
\mathrm{mg} / \mathrm{L} \\
\end{array}$ & $\begin{array}{c}\text { REPORTING } \\
\text { LIMIT } \\
\end{array}$ & D. F. & METHOD & $\begin{array}{l}\text { TCLP/STLC } \\
\text { EXTRACTION } \\
\text { DATE } \\
\end{array}$ & DIGESTED & ANALYZED \\
\hline Atsenic & $\mathrm{ND}$ & $0.1 \mathrm{mg} / \mathrm{L}$ & 1 & EPA $6010 \mathrm{~A}$ & $6 / 11 / 98$ & $6 / 12 / 98$ & $6 / 14 / 98$ \\
\hline Barium & $\mathrm{ND}$ & $1 . \mathrm{mg} / \mathrm{L}$ & 1 & EPA 6010A & $6 / 11 / 98$ & $6 / 12 / 98$ & $6 / 14 / 98$ \\
\hline Cadmium & ND & $0.01 \mathrm{mg} / \mathrm{L}$ & 1 & EPA $6010 A$ & $6 / 11 / 98$ & $6 / 12 / 98$ & $6 / 14 / 98$ \\
\hline Chromium & $\mathrm{ND}$ & $0.01 \mathrm{mg} / \mathrm{L}$ & 1 & EPA $6010 \mathrm{~A}$ & $6 / 11 / 98$ & $6 / 12 / 98$ & $6 / 14 / 98$ \\
\hline Lead & ND & $0.05 \mathrm{mg} / \mathrm{L}$ & 1 & EPA $6010 \mathrm{~A}$ & $6 / 11 / 98$ & $6 / 12 / 98$ & $6 / 14 / 98$ \\
\hline Mercury & ND & $0.002 \mathrm{mg} / \mathrm{L}$ & 10 & EPA $7470 A$ & $6 / 11 / 98$ & $6 / 12 / 98$ & $6 / 14: 98$ \\
\hline Selenium & ND & $0.1 \mathrm{mg} / \mathrm{L}$ & 1 & EPA $6010 \mathrm{~A}$ & $6 / 11 / 98$ & $6 / 12 / 98$ & $6 / 1498$ \\
\hline Silver & $\mathrm{ND}$ & $0.02 \mathrm{mg} / \mathrm{L}$ & 1 & EPA $6010 \mathrm{~A}$ & $6 / 11 / 98$ & $6 / 12 / 98$ & $6 / 14: 98$ \\
\hline
\end{tabular}

D.F. - Dilution Factor

ND - Not Detected

This report shall not be reproduced except in full, without the written approval of the laboratory. 
NEL LABORATORIES

CLIENT: Bechtel Nevada

PROJECT NAME: V432

PROJECT NUMBER: 17777
CLIENT ID: Decon P10-5

DATE SAMPLED: $6 / 4 / 98$

NEL SAMPLE ID: L9806094-11

TEST: $\quad$ TCLP-8 Metals

MATRIX: Solid

\begin{tabular}{|c|c|c|c|c|c|c|c|}
\hline PARAMETER & $\begin{array}{c}\text { RESULT } \\
\mathrm{mg} / \mathrm{L} \\
\end{array}$ & $\begin{array}{c}\text { REPORTING } \\
\text { LIMIT } \\
\end{array}$ & D. F. & METHOD & $\begin{array}{c}\text { TCLP/STLC } \\
\text { EXTRACTION } \\
\text { DATE } \\
\end{array}$ & DIGESTED & ANALYZED \\
\hline Arsenic & $\mathrm{ND}$ & $0.1 \mathrm{mg} / \mathrm{L}$ & 1 & EPA $6010 \mathrm{~A}$ & $6 / 11 / 98$ & $6 / 12 / 98$ & $6 / 14 / 98$ \\
\hline Barium & ND & $1 . \mathrm{mg} / \mathrm{L}$ & 1 & EPA 6010A & $6 / 11 / 98$ & $6 / 12 / 98$ & $6 / 14 / 98$ \\
\hline Cadmium & $\mathrm{ND}$ & $0.01 \mathrm{mg} / \mathrm{L}$ & 1 & EPA $6010 \mathrm{~A}$ & $6 / 11 / 98$ & $6 / 12 / 98$ & $6 / 14 / 98$ \\
\hline Chromium & ND & $.0 .01 \mathrm{mg} \mathrm{L}$ & 1 & EPA $6010 A$ & $6 / 11 / 98$ & $6 / 12 / 98$ & $6 / 14 / 98$ \\
\hline Lead & ND & $0.05 \mathrm{mg} / \mathrm{L}$ & 1 & EPA $6010 \mathrm{~A}$ & $6 / 11 / 98$ & $6 / 12 / 98$ & $6 / 14 / 98$ \\
\hline Mercury & ND & $0.002 \mathrm{mg} / \mathrm{L}$ & 10 & EPA $7470 \mathrm{~A}$ & $6 / 11 / 98$ & $6 / 12 / 98$ & $6 / 14 / 98$ \\
\hline Selenium & ND & $0.1 \mathrm{mg} / \mathrm{L}$ & 1 & EPA $6010 \mathrm{~A}$ & $6 / 11 / 98$ & $6 / 12 / 98$ & $6 / 14: 98$ \\
\hline Silver & $\mathrm{ND}$ & $0.02 \mathrm{mg} / \mathrm{L}$ & 1 & EPA $6010 A$ & $6 / 11 / 98$ & $6 / 12 / 98$ & $6 / 14: 98$ \\
\hline
\end{tabular}

D.F. - Dilution Factor

ND - Not Detected

This report shall not be reproduced except in full, without the written approval of the laboratory. 


\section{NEL LABORATORIES}

CLIENT:

Bechtel Nevada

POJECT ID: V432/17777

COJECT \#: 17777
CLIENT ID: $\quad$ Matrix Spike

DATE SAMPLED: NA

NEL SAMPLE ID: L9806093-21-MS

TEST:

TCLP by EPA 13I1, July 1992 \& 7 Metals by EPA 6010A, July 1992

MATRIX: Solid

EXTRACTED: $6 / 12 / 98$

ANALYZED: $6 / 14 / 98$

ANALYST: EMF

\section{PARAMETER}

Arsenic

Silver

Barium

Cadmium

Chromium

Lead

Selenium

\section{Percent Recovery}

106

100

91

105

98

101

111

\section{Acceptable Range}

$85-115$

$85-115$

$85-115$

$85-115$

$85-115$

$85-115$

$85-115$ 
NEL LABORATORIES

$\begin{array}{llll}\text { CLIENT: } & \text { Bechtel Nevada } & \text { CLIENT ID: } & \text { Matrix Spike Duplicate } \\ \text { PROJECT ID: } & V 432 / 17777 & \text { DATE SAMPLED: NA } \\ \text { PROJECT \#: } & 17777 & \text { NEL SAMPLE ID: L9806093-21-MSD }\end{array}$

TEST: $\quad$ TCLP by EPA 1311, July 1992 \& 7 Metals by EPA 6010A, July 1992

MATRIX: Solid

EXTRACTED: 6/12/98

ANALYZED: $6 / 14 / 98$

ANALYST: EMF

\section{PARAMETER}

Percent Recovery

100

Silver

Arsenic

Barium

Cadmium

Chromium

Lead

Selenium.
105

90

104

97

101

111

\section{Acceptable Range}

85- 115

$85-115$

$85-115$

$85-115$

$85-115$

$85-115$

$85-115$

\section{$\underline{\text { RPD }}$}

0.4

0.2

11.1

3.9

3. 


\begin{tabular}{llll}
\multicolumn{2}{c}{ NEL LABORATORIES } & \\
\hline CLIENT: & Bechtel Nevada & CLIENT ID: & Matrix Spike Duplicate \\
'ROJECT ID: & V432/17777 & DATE SAMPLED: NA \\
. ROJECT \#: & 17777 & NEL SAMPLE ID: & L9806093-21-MSD
\end{tabular}

TEST: $\quad$ TCLP by EPA 1311, July 1992 \& Mercury by EPA 7470A, July 1992

MATRIX: Solid

EXTRACTED: 6/12/98

ANALYZED: $6 / 14 / 98$

ANALYST: EMF

PARAMETER

Mercury
Percent Recoverv

99
Acceptable Range

$85-115$ $\underline{\text { RPD }}$ 
NEL LABORATORIES

CLIENT: Bechtel Nevada

PROJECT ID: V432/17777

PROJECT \#: $\quad 17777$
CLIENT ID: Laboratory Control Spike

DATE SAMPLED: NA

NEL SAMPLE ID: L06093I-LCS

TEST: $\quad$ TCLP by EPA 1311, July 1992 \& 7 Metals by EPA 6010A, July 1992

MATRIX: Solid

EXTRACTED: $6 / 12 / 98$

ANALYZED: $6 / 14 / 98$

ANALYST: EMF

PARAMETER

Silver

Arsenic

Barium

Cadmium

Chromium

Lead

Selenium
Percent Recovery

99

104

95

105

102

104

107
Acceptable Range

85- 115

$88-118.5$

$86-128.2$

$85-115$

$85-115$

$85-115$

$85-115$ 
NEL LABORATORIES

CLIENT: Bechtel Nevada

ROJECT ID: V432/17777

CLIENT ID:

Laboratory Control Spike

SOJECT \#: 17777

DATE SAMPLED: NA

NEL SAMPLE ID: L06093HG-LCS

TEST:

TCLP by EPA 1311, July 1992 \& Mercury by EPA 7470A, July 1992

MATRIX: Solid

EXTRACTED: $6 / 12 / 98$

ANALYZED: $6 / 14 / 98$

ANALYST: JML

PARAMETER

Mercury
Percent Recoverv

97
Acceptable Range

$85-115$ 
NEL LABORATORIES

CLIENT: Bechtel Nevada

PROJECT NAME: V432

PROJECT NUMBER: 17777

CLIENT ID:

DATE SAMPLED:

NEL SAMPLE ID: L06093HG-BLK

TEST: $\quad$ TCLP by EPA 1311, July 1992 \& Mercury by EPA 7470A, July 1992

MATRIX: TCLP Extract

\begin{tabular}{|c|c|c|c|c|c|c|c|}
\hline \multirow[b]{2}{*}{ PARAMETER } & \multirow[b]{2}{*}{ RESULT } & \multirow{2}{*}{$\begin{array}{c}\text { REPORTING } \\
\text { LIMIT } \\
\end{array}$} & \multicolumn{4}{|c|}{$\begin{array}{l}\text { TCLP/STLC } \\
\text { EXTRACTION }\end{array}$} & \\
\hline & & & D. F. & METHOD & DATE & DIGESTED & ANALYZED \\
\hline Mercury & ND & $0.002 \mathrm{mg} / \mathrm{L}$ & 10 & EPA 7470A & $6 / 11 / 98$ & $6 / 12 / 98$ & $6: 14 / 98$ \\
\hline
\end{tabular}

D.F. - Dilution Factor

ND - Not Detected

This report shall not be reproduced except in full, withoul the written approval of the laborator: 
NEL LABORATORIES

CLIENT: Bechtel Nevada

"ROJECT NAME: V432

.OJECT NUMBER: 17777
CLIENT ID: Method Blank

DATE SAMPLED: NA

NEL SAMPLE ID: L06093I-BLK

TEST: $\quad$ TCLP by EPA 1311, July 1992 \& 7 Metais by EPA 6010A, July 1992

MATRIX: TCLP Extract

\begin{tabular}{|c|c|c|c|c|c|c|c|}
\hline \multirow[b]{2}{*}{ PARAMETER } & \multirow{2}{*}{\multicolumn{2}{|c|}{$\begin{array}{c}\text { REPORTING } \\
\text { LIMIT }\end{array}$}} & \multicolumn{4}{|c|}{$\begin{array}{l}\text { TCLP/STLC } \\
\text { EXTRACTION }\end{array}$} & \multirow[b]{2}{*}{ ANALYZEI } \\
\hline & & & $\underline{\text { D. } F}$. & METHOD & DATE & DIGESTED & \\
\hline Arsenic & ND & $0.1 \mathrm{mg} / \mathrm{L}$ & 1 & EPA $6010 \mathrm{~A}$ & $6 / 11 / 98$ & $6 / 12 / 98$ & $6 / 14 / 98$ \\
\hline Barium & $\mathrm{ND}$ & $1 \mathrm{mg} / \mathrm{L}$ & 1 & EPA $6010 A$ & $6 / 11 / 98$ & $6 / 12 / 98$ & $6 / 14 / 98$ \\
\hline Cadmium & ND & $0.01 \mathrm{mg} / \mathrm{L}$ & 1 & EPA $6010 \mathrm{~A}$ & $6 / 11 / 98$ & $6 / 12 / 98$ & $6 / 14 / 98$ \\
\hline Chromium & ND & $0.01 \mathrm{mg} / \mathrm{L}$ & 1 & EPA $6010 \mathrm{~A}$ & $6 / 11 / 98$ & $6 / 12 / 98$ & $6 / 14 / 98$ \\
\hline Lead & ND & $0.05 \mathrm{mg} / \mathrm{L}$ & 1 & EPA 6010A & $6 / 11 / 98$ & $6 / 12 / 98$ & $6 / 14 / 98$ \\
\hline Selenium & ND & $0.1 \mathrm{mg} / \mathrm{L}$ & 1 & EPA $6010 \mathrm{~A}$ & $6 / 11 / 98$ & $6 / 12 / 98$ & $6 / 14 / 98$ \\
\hline Silver & $\mathrm{ND}$ & $0.02 \mathrm{mg} / \mathrm{L}$ & 1 & EPA $6010 A$ & $6 / 11 / 98$ & $6 / 12 / 98$ & $6: 14 / 98$ \\
\hline
\end{tabular}

D.F. - Dilution Factor

ND - Not Detected

$T^{\text {sis }}$ report shall not be reproduced except in full, without the written approval of the laboratory. 


\section{SDG B438}

RADIOANALYTICAL

RESULTS 


\section{Bechtel Nevada Corporation}

\section{ANALYTICAL SERVICES LABORATORY}

P.O.Box 3936, N. Las Vegas, NV 89036

Feported to: Area 6 Decon Pond Closure

Remediation Projects

Report Date: 6-JUL-98

J. L. Smith

Sample Delivery Group: $\mathrm{B438}$

M/S NTS306

Batch: 0820

Program: 720

Report No. :

\begin{tabular}{|c|c|c|c|c|c|c|c|c|c|c|c|c|c|c|c|c|}
\hline $\begin{array}{c}\text { Sample } \\
* * * * * \text { Identífication *...** }\end{array}$ & Isotope & Analysis & Result: & $\begin{array}{c}\text { Error } \\
\%\end{array}$ & $\begin{array}{l}\text { Qual } \\
\text { Flag }\end{array}$ & MDA & $\begin{array}{l}\text { Result } \\
\text { Units }\end{array}$ & $\begin{array}{c}\text { Analysis } \\
\text { Date }\end{array}$ & $\begin{array}{l}\text { Sample } \\
\text { Coll Date }\end{array}$ & Size & $\begin{array}{c}\text { Size } \\
\text { Units }\end{array}$ & \begin{tabular}{c|} 
Typo \\
Matrix
\end{tabular} & $\begin{array}{l}\text { Tracer } \\
\text { Yield } x_{0}\end{array}$ & $\begin{array}{l}\text { Spike } \\
\text { Recr } \%\end{array}$ & $\begin{array}{l}\text { System } \\
\text { Detector }\end{array}$ & $\begin{array}{l}\text { Pecket-Item } \\
\text { Sample }\end{array}$ \\
\hline DECON P10-6 & $K 40$ & GAM20 & $2.03 E+01$ & $2.1 E+01$ & & B.4E-01 & pCi/g & $06-04-98$ & $06-04-98$ & $6.40 E+02$ & $\mathrm{gm}$ & SOLL G & & & $05-01$ & $69121-1-75097$ \\
\hline DECON P10-6 & RA226 & GAM 20 & $1.20 E+00$ & $2.7 E+01$ & & $1.7 \mathrm{E}-01$ & $\mathrm{pCl} / \mathrm{g}$ & $06-04-98$ & $06-04-98$ & $6.40 E+02 \mid$ & gmm & solt G & & & 05.01 & 69121-1.75097 \\
\hline DECON P10-6 & TH228 & GAM20 & $2.38 \mathrm{BE}+00$ & $1.9 E+01$ & & $1.5 \mathrm{E}-01$ & $\mathrm{pCi} / \mathrm{g}$ & 06-04-98 & $06-04-98$ & $6.40 E+02$ & $\mathrm{am}$ & solt G & & & 05.01 & G9121-1-75097 \\
\hline DECON P10-6 & TH232 & GAM20 & $2.13 E+00$ & $3.1 E+01$ & & $3.9 E-01$ & $\mathrm{pCl} / \mathrm{g}$ & $06-04-98$ & $06-04 \cdot 98$ & $6.40 E+02$ & $\mathrm{gm}$ & SOIL G & & & 05-01 & G9121.1.75097 \\
\hline DECON P9.6 & K 40 & GAM20 & $1.81 E+01$ & $2.2 E+01$ & & 0.5E-01 & $\mathrm{pCi} / \mathrm{g}$ & $06-04-98$ & $06-04-98$ & $6.04 \mathrm{E}+02$ & $\mathrm{gm}$ & SOIL G & & & 05.01 & G9121-0.75095 \\
\hline DECON P9.6 & RA226 & GAM20 & $1.46 E+00$ & $2.4 E+01$ & & $1.7 \mathrm{E}-01$ & $\rho \mathrm{Ci} / \mathrm{g}$ & $06-04-98$ & $06-04-9 B$ & $6.04 \mathrm{E}+02$ & $\mathrm{gm}$ & soll. G & & & $05-01$ & G9121-0-75095 \\
\hline DECON P9-6 & TH228 & GAM20 & $2.07 E+00$ & $2.0 E+01$ & & $1.5 E-01$ & $\mathrm{pCi} / \mathrm{g}$ & $06-04-98$ & $06.04 .9 \mathrm{~B}$ & $6.04 E+02$ & $g m$ & SOIL G & & & 05-01 & G9121-0-75095 \\
\hline DECON P9-6 & $\mathrm{TH} 232$ & GAM20 & $2.03 E+00$ & $3.2 E+01$ & & 3.9E-01 & $\mathrm{pCi} / \mathrm{g}$ & $06-04-98$ & 06-04-98 & $6.04 E+02$ & $\mathrm{gmm}$ & Soll G & & & 05-01 & G9121-0.75095 \\
\hline QA BKG EMPTY BTL UO5 & No Nucl Det & GAM20 & $0.00 \mathrm{E}+00$ & $0.0 E+00$ & u & $0.0 \mathrm{E}+00$ & NA & $06-04-98$ & 07-01-95 & $1.00 E+00 \mid$ & $\frac{9 m}{x p}$ & QUAL & & & 05-01 & $00539-0.00208$ \\
\hline QA BKG EMPTY BTL U05 & AM241 & GAM20 & $0.00 E+00$ & $0.0 E+00$ & U & $2.6 E+01$ & $\mathrm{pCi}$ & 06-04-98 & $07-01-95$ & $1.00 \mathrm{E}+00$ & $\mathrm{sm}$ & QUAL & & & 05-01 & $00539-0-00208$ \\
\hline
\end{tabular}

\section{Comment:}

Data generated from analyses of samples submitted on 6/4/98. Gross alpha/beta determinations are performed on small sample aliquots. Data may not represent sample activity as a whoie.

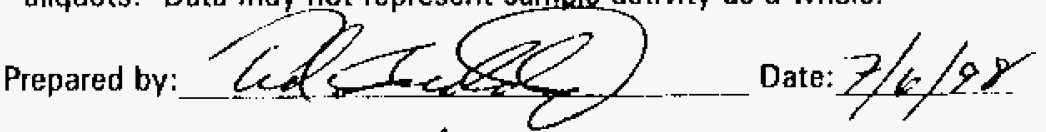
approved by: Souryftatena Date: $7 / 6 / 98$

\section{Qualification Flags:}

$\mathrm{E}=$ Estimated Quantity

$\mathrm{H}=$ High Recovery for Sample

$J=$ Result is less than the RDL

$L=$ Low Recovery for Sample

$P=$ Preliminary Results

$\mathrm{O}=$ Bad Instrument Quality Control, Result is $\mathrm{OK}$

$\mathrm{R}=$ Results are Unusable, Resampling is Necessary

$\mathrm{U}=$ Result is less than Minimum Detectable Activity

Note: \% Error is the 2.0 Sigma Error 


\section{Bechtel Nevada Corporation}

\section{ANALYTICAL SERVICES LABORATORY}

P.O.Box 3936, N. Las Vegas, NV 89036

Reported to: Area 6 Decon Pond Closure

Remediation Projects

Report Date: 6-JUL-98

J. L. Smith

Sample Delivery Group: B438

M/S NTS306

Batch: 0820

Program: 500

Report No. :

\begin{tabular}{|c|c|c|c|c|c|c|c|c|c|c|c|c|c|c|c|c|}
\hline $\begin{array}{c}\text { Sample } \\
\ldots \ldots * * \text { Identification *...* }\end{array}$ & Isotope & Analysis & Ressult & $\begin{array}{l}\text { Error } \\
\%\end{array}$ & \begin{tabular}{|l} 
Qual \\
Flas
\end{tabular} & MDA & \begin{tabular}{|l} 
Result \\
Units
\end{tabular} & $\begin{array}{c}\text { Analysis } \\
\text { Dato }\end{array}$ & $\begin{array}{l}\text { Sample } \\
\text { Coll Date }\end{array}$ & Size & \begin{tabular}{|l|} 
Size \\
Units
\end{tabular} & \begin{tabular}{|c} 
Typa \\
Matrix
\end{tabular} & $\begin{array}{l}\text { Tracer } \\
\text { Yield \% }\end{array}$ & $\begin{array}{l}\text { Spike } \\
\text { Recv \% }\end{array}$ & $\begin{array}{l}\text { System } \\
\text { Detecto }\end{array}$ & $\begin{array}{l}\text { Packet-ltem } \\
\text { Samplo }\end{array}$ \\
\hline OA BKG EMPTY BTL UOS & $\mathrm{CO60}$ & AM20 & $0.00 E+\infty$ & $0.0 \mathrm{E}+0 \mathrm{OS}$ & u & $7.9 \mathrm{E}+00$ & $\mathrm{pCi}$ & $06-04-98$ & $0701-95$ & $1.00 E+00$ & $\mathrm{sm}$ & QUAL & & & 05-01 & $00539.0-00208$ \\
\hline QA BKG EMPTY BTL UO5 & $\operatorname{cs} 137$ & GAM20 & $0.00 E+00$ & $0.0 E+00$ & $u$ & $9.4 E+\infty 0$ & $\mathrm{pCi}$ & 06.04 .98 & $07-01-95$ & $1.00 E+00$ & $\mathrm{sm}$ & QUAL & & & 05.01 & 00539-0.0020B \\
\hline QA SP:NAS-A0271 & AM241 & GAM20 & $1.81 E+05$ & $8.8 E+00$ & & $3.3 E+02$ & $\mathrm{pCi}$ & $06-04-98$ & 04-01-92 & $1.00 \mathrm{E}+00$ & $\sin$ & QUAL & & 111,8 & $05-01$ & 00534 \\
\hline QA SP:NAS-A0271 & C060 & GAM20 & $3.00 E+05$ & $8.5 \mathrm{E}+00$ & & $1.5 E+02$ & pCi & 06-04-98 & $04-01-92$ & $1.00 E+00$ & $\mathrm{sm}$ & QUAL & & 100.7 & $05-01$ & $00534-3 \cdot 14463$ \\
\hline QA SP:NAS-A0271 & $\operatorname{cs} 137$ & GAM20 & $2.08 E+05$ & $8.1 E+\infty$ & & $9.2 E+01$ & $\mathrm{pCi}$ & 06-04-98 & $04-01-92$ & $1.00 E+00$ & $\mathrm{sm}$ & OUAL & & 102.5 & $05-01$ & $00534-3-14463$ \\
\hline
\end{tabular}

\section{Comment:}

Data generated from analyses of samples submitted on 6/4/98. Gross alpha/beta determinations are performed on small sample aliquots. Data may not represent sample activity as a whole.

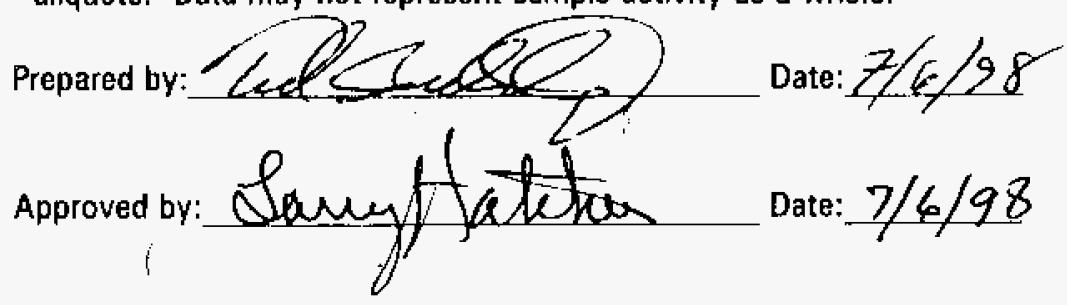

Qualification Flags:

$\mathrm{E}=$ Estimated Quantity

Note: $\%$ Error is the 2.0 Sigma Error

$\mathrm{H}=$ High Recovery for Sample

$\mathrm{J}=$ Result is less than the RDL

$L=$ Low Recovery for Sample

$P=$ Preliminary Results

$\mathrm{Q}=$ Bad Instrument Quality Control, Result is OK

$R=$ Results are Unusable, Resampling is Necessary

$\mathrm{U}=$ Result is less than Minimum Detectable Activity 


\section{Bechtel Nevada Corporation}

\section{ANALYTICAL SERVICES LABORATORY}

P.O.Box 3936, N. Las Vegas, NV 89036

Reported to: Area 6 Decon Pond Closure

Remediation Projects

Report Date: 6-JUL-98

J. L. Smith

Sample Delivery Group: B438

M/S NTS306

Batch: M559

Program: 720

Report No.

\begin{tabular}{|c|c|c|c|c|c|c|c|c|c|c|c|c|c|c|c|c|}
\hline $\begin{array}{c}\text { Sample } \\
* * * \text { Identification }\end{array}$ & Isotope & Analysis & Hesult & $\begin{array}{c}\text { Error } \\
\%\end{array}$ & $\begin{array}{l}\text { Outal } \\
\text { Flag }\end{array}$ & MDA & $\begin{array}{l}\text { Pesult } \\
\text { Units }\end{array}$ & $\begin{array}{c}\text { Analysis } \\
\text { Date }\end{array}$ & $\begin{array}{l}\text { Sample } \\
\text { Coll Date }\end{array}$ & Size & $\begin{array}{r}\text { Size } \\
\text { Units }\end{array}$ & $\begin{array}{c}\text { Type } \\
\text { Matrix }\end{array}$ & $\mid \begin{array}{l}\text { Tracer } \\
\text { Yield \% }\end{array}$ & $\begin{array}{l}\text { Spike } \\
\text { Recu } \%\end{array}$ & $\begin{array}{l}\text { System } \\
\text { Detector }\end{array}$ & $\begin{array}{l}\text { Packet-Item } \\
\text { Sample }\end{array}$ \\
\hline DECON P10-6 & Gross Alpha & Gross A & $1.96 \mathrm{~F}+01$ & $1.7 E+01$ & & $2.9 E+00$ & $\mathrm{pCi} / \mathrm{g}$ & $07-03-98$ & $06 \cdot 04-98$ & $5.00 \mathrm{E}-01$ & $g m$ & SOL G & & & 14.01 & G9121- $\uparrow-75096$ \\
\hline DECON P9-6 & Gross Alpha & Gross A & $1.80 E+01$ & $1.8 E+01$ & & $2.9 E+00$ & $\mathrm{pCi} / \mathrm{\theta}$ & $07.03-98$ & $06-04-98$ & $5.00 \mathrm{E}-01$ & $\mathrm{gm}$ & SOL G & & & $14-01$ & 69121-0-75094 \\
\hline QA REA & Gross Alpha & Gross A & $1.65 \mathrm{E}-02$ & $1.4 \mathrm{E}+03$ & U & $4.2 \mathrm{E}-01$ & $\mathrm{pCi}$ & $07-02-98$ & $06-02-98$ & $1.00 E+\infty$ & $\mathrm{sm}$ & OUAL. & & & $14-01$ & G9100-0.06776 \\
\hline QA SP:GROSSAB & Gross Alphs & Gross A & $3.39 \mathrm{gE}+00$ & $1.6 E+01$ & & $4.2 \mathrm{E}-01$ & $\mathrm{pCi}$ & $07-02-98$ & $01-06-98$ & $1.00 E+00$ & $\mathrm{sm}$ & QUAL & & 98.6 & 1401 & $69100-1.06777$ \\
\hline DECON P10-6 & Gross Bets & Gross B & $1.43 \mathrm{E}+01$ & $1.2 E+01$ & & $2.2 \mathrm{E}+00$ & $\mathrm{pCi} / \mathrm{g}$ & 07-03-98 & $06-04-98$ & $5.00 \mathrm{E}-01$ & $\mathrm{gm}$ & SOIL G & & & 1401 & $69121-1-75096$ \\
\hline DECON P9-6 & Gross Beta & Gross B & $1.41 E+01$ & $1.2 \mathrm{E}+01$ & & $2.2 \mathrm{E}+00$ & pCilg & 07-03-98 & $06-04-98$ & $5.00 E-01$ & $\mathrm{gm}$ & Soll. G & & & 14.01 & G9121-0-75094 \\
\hline QA AEA & Gross Beta & Gross B & $-2.67 \mathrm{E}-01$ & $2.1 \mathrm{E}+02$ & U & $9.8 E-01$ & $\mathrm{pCi}$ & $07-02-98$ & $06-02-98$ & $1.00 \mathrm{E}+00$ & $\mathrm{sm}$ & OUAL & & & $14-01$ & G9100-0-06776 \\
\hline OA SP:GHOSSAB & Gross Beta & Gross & $4.65 E+00$ & $1.5 \mathrm{E}+01$ & & $9.8 \mathrm{E}-01$ & $\mathrm{pCi}$ & 07-02-98 & $01-06-98$ & $1.00 E+00$ & $\sin$ & QUAL & & 89.0 & 14.01 & G9100-1-06777 \\
\hline
\end{tabular}

\section{Comment:}

Data generated from analyses of samples submitted on 6/4/98.

Gross alpha/beta determinations are performed on small sample aliquots. Data may not represent sample activity as a whole.

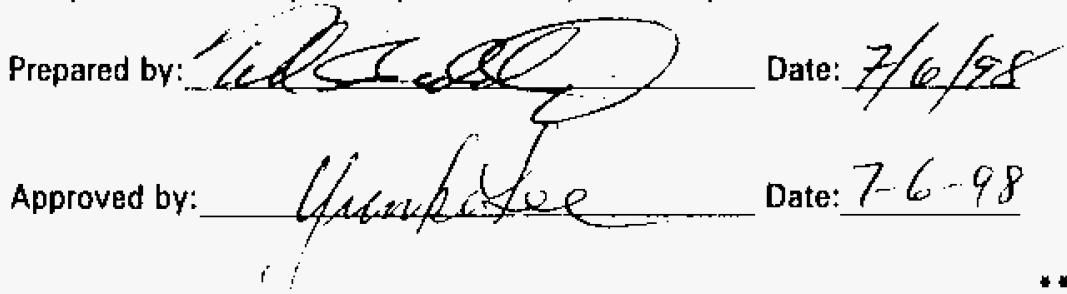

Qualification Flags:

Note: \% Error is the 2.0 Sigma Error

E $=$ Estimated Quantity

$\mathrm{H}=$ High Recovery for Sample

$\mathrm{J}=$ Result is less than the RDL

$L=$ Low Recovery for Sample

$P=$ Preliminary Results

$0=$ Bad Instrument Quality Control, Result is OK

$\mathbf{R}=$ Results are Unusable, Hesampling is Necessary

$\mathrm{U}=$ Result is less than Minimum Detectable Activity 


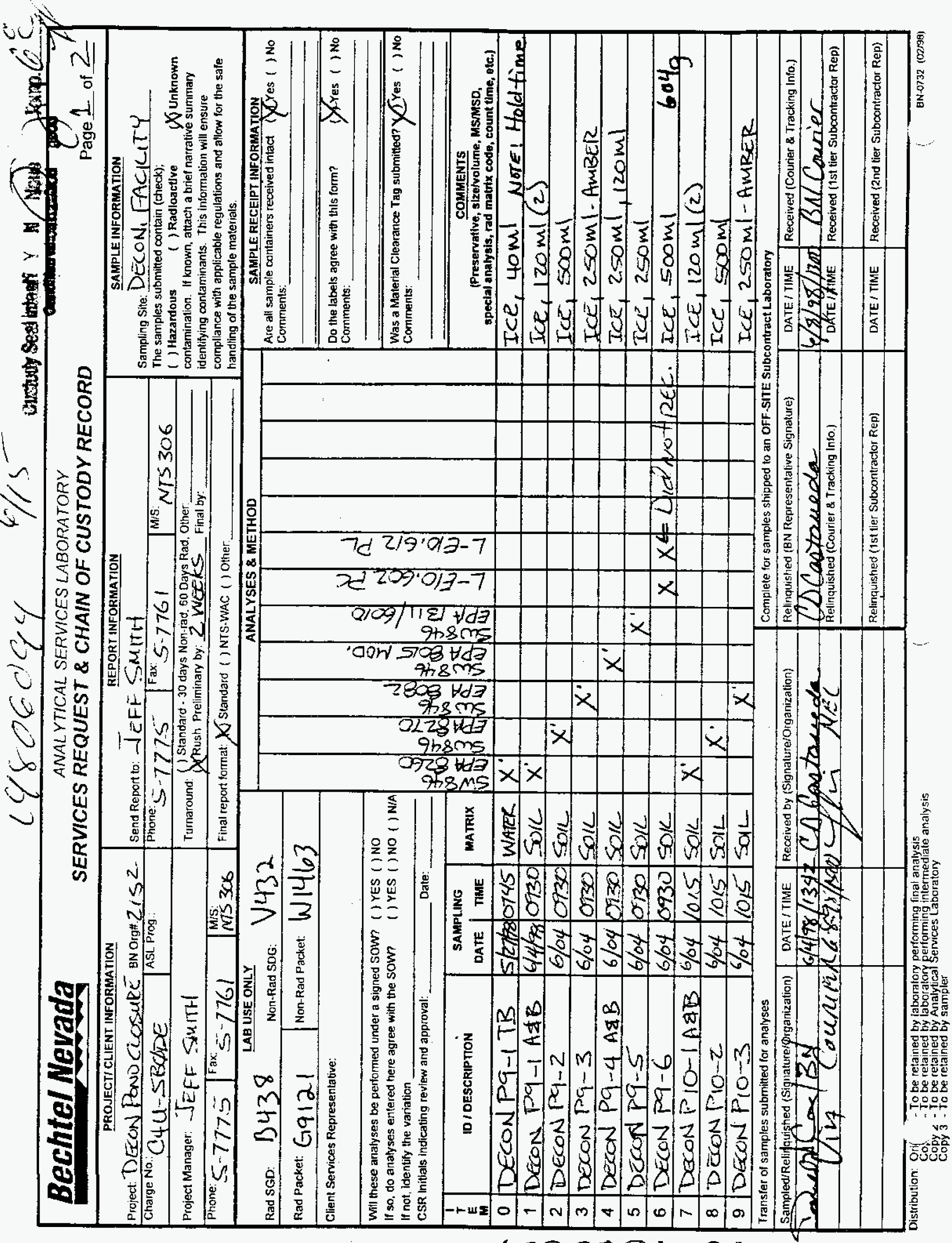


PROJECTICLIENT INFORMATION

Project DECON PENu CLOSURE EN Org* $2 / 52$

Project Manager. TEFF SM ASL Prog: Phone: $5-7775$ Fax: $5-7761$ Mis: NTS 306

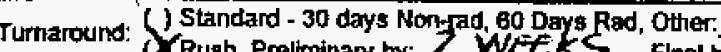
\begin{tabular}{l|l|l|l|l}
\hline Phome: $S-7775$ & Fax & $5-776)$ & MSS 306 & Final report format $X$ Standard () NTS-WAC () Other.
\end{tabular} Final by:

Rad SGD: B438 $\frac{\text { LAB USE ONLY }}{\text { Non-Rad SDG: }}$ V432

\begin{tabular}{l|l|l} 
Rad Packet & -19121 & Non-Rad Packet: 61463 \\
\hline
\end{tabular}

\section{Client Services Representative:}

Wilt thene analyses be performed under a signed SOW? () YES () NO If so, do anatyses entered hore agree with the SOWn (I) YES () NO () N/A If not, identify the variation

ANALYSES \& METHOD

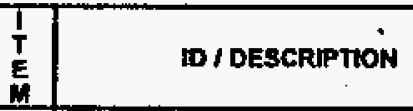

- 0 TECON PG-1 TB

1 Decon Pq-1 AEB

- 2 DECON PQ-2

- 3 DECON PG-3

- 4 DECON PQ-4 AхB

5 Decort pq-5

6 DECon Pq-6

7 Deron PIO-IAAB

- 8 Decon Pio-z

- 9 Decon P1O-3 Transfer of samples submitted for analyses

\begin{tabular}{|l|l|l|l|l}
\hline Sampled/Relin quighed (Signature/Crgankation) & DATE / TIME & Received by (Signature/Organizatton)
\end{tabular}

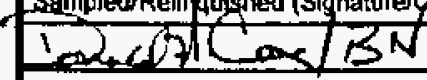

\begin{tabular}{|c|c|c|}
\multicolumn{2}{c|}{ SAMPLMG } & MATRIX \\
DATE & TME & \\
\hline
\end{tabular}

$5 k 7$ OOT45 WATCR

$447 \%$ OTBO

6/04 0930

6\%4

$6 / 040130$

6.040930

6640930

6,641015

Goy

404105 SOL

\begin{tabular}{l|l|l|}
$\%$ & 10,5 & SOL \\
\hline
\end{tabular}

$5012 x$

SOL

Solk.

sok

Sok

SOK

X 00 dof

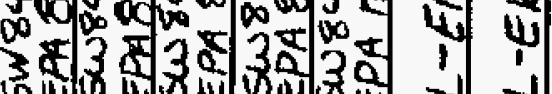

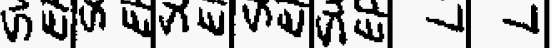

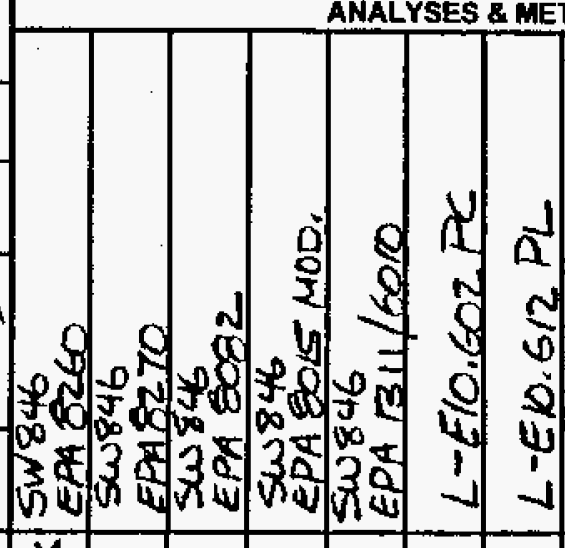

SAMPLE ANFORMATIOM Samping Site: DECON, FACKLITY

The samples subritted contain (check):

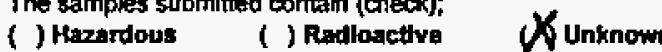
contamination. If known, altach a brlef narrative summary identifying contamisants. This inlormation will ensuse compliance with applicable regulations and allow for the sate handling of the sample materials.

$$
\text { SAMPLE RECEIPT IAFORMATIOM }
$$
Are, an samplo containers recetived intact (YCYes ( ) No Comments:

- . - .

Do the labels agree with this form? S/Yes ( ) No

Comments:

(2)

Was a Matertal Clearance Teg submitted? Y G Yes ( I No

Comrments:

COMMENTS

Preservutlve, sizoivolǘme, msnsD epecial anabysts, red mutrix code, cotsmt time, etc.)

ICE, $40 \mathrm{ml}$ NoTe: Hold time ICE, $120 \mathrm{ml}(\mathrm{z})$

$I C C_{1}$ Sooml

ICE, ZSOMl. AmiBER

ICE, $250 \mathrm{ml}$, $20 \mathrm{ml}$

ICL, 250ml

$I C E, 500 \mathrm{ml}$

$I C E, 120 \mathrm{ml}(2)$

$\mathrm{ICC}_{1} 50 \mathrm{ml}$

ICE, $250 \mathrm{MI}$ - AMBER

Complete for samples shipped to an OFF-STE Subcontract Laboratory \begin{tabular}{l|l|l} 
Rellinquished (BN Representative Signatiru) & DATE / TIMEF & Recelved (Courier a Tracking Info.)
\end{tabular}

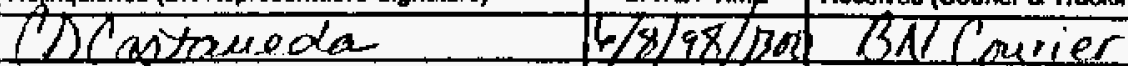
Retinguishued CCourier \& Tracking Info I

QATE/TRE Recelved (1st ter Subourtractor Rep) 
PROJECT/CLIENT INFORMATION

Project DecoN BNDClosuRE BN Orgat: 2152 send Report to: JEFF SMMH

\begin{tabular}{|l|l|l}
\hline Protect Manager: JEFF SMITH \\
\hline Phone: $5-7775$ & Fax $S-7761$ \\
\hline
\end{tabular}

\section{SL Prog.: $\quad$ Phone: 5.7775 Fax 5.7761}

\begin{tabular}{|l|l|l|} 
Phone: 5.7775 & Fax $5.7 \% 1$ & Mis: NTS.306
\end{tabular}

Turnaround: ()Standard - 30 days Non-rad 60 Days Rad, Other.

WFS 306 Final report format (X) (standard () NTS-WAC () Other.

\section{by:}

ANALYSES \& METHOD

Rad SGD: $\quad 3438$ Non-Rad SDG: $V 432$

Rad Packet $(9121)$ Nor-Rad Packet: W/4 63

Client Services Representative:

Wil these analyses be performed under a signed SOW7 () YES () NO it so, do analyses entered here ggree with the sown (YYES () NO () N/A If not, bentify the variation

CSR Initals Indicating review and epproval: ___ Data:____________

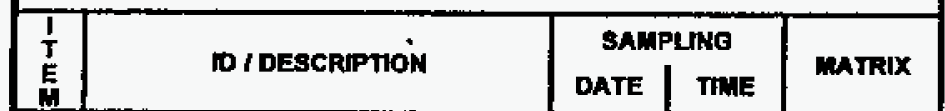

- 10 DECON PDO-4 A $4 B$

- 11 DECON PIO-5

- 12 DECON P10-6

\begin{tabular}{|l|l|}
\hline 3 & \\
\hline 4 & \\
\hline 5 & \\
\hline 6 & \\
\hline 7 & \\
\hline 8 & \\
\hline 9 & \\
\hline
\end{tabular}

Transfer of samples submitted for analyses

G.4 1015 SOIL

$6 \% 4$ 1015 sal

\begin{tabular}{ll|l}
$6 / 04$ & 1015 & Soll \\
\hline
\end{tabular}

Sempled/Relin huished (Signature/Organization)

$$
\text { (1) }
$$
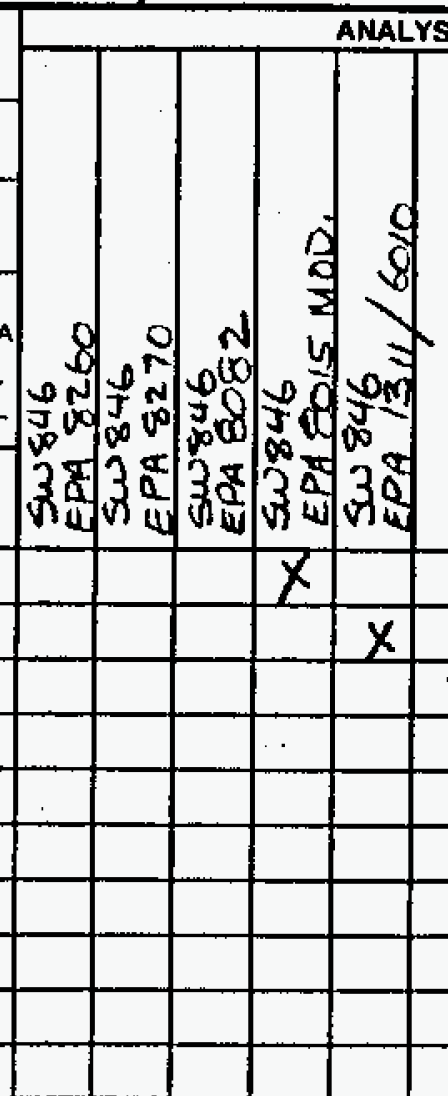

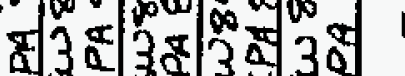

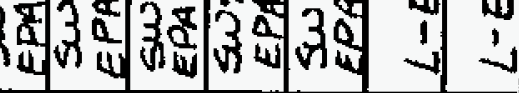

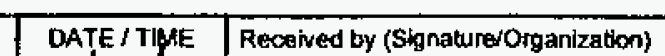
$6 / 3 / 5 / 342$ CD C astoreda

\section{SAMPLE ANFORLATON Sampling site: DECON FACILITY

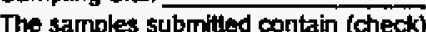 () Hazardous () Radloactive "No Unknown contamination. If known, attach a brief narrattive sumatrary dientifyin contaminants. This intormation will tonsure compliance with applicable regulations and allow for the safo handling of the sample materials.}

SAMPLE RECEPT INFORMATION
Are all sample containers recelved mitict S(Yes ( ) No
Comments:

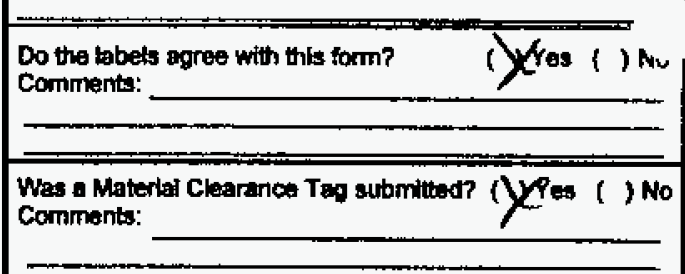

COMMENTS

(Preservattve, tizeholum msisp.

epecial anabyth, red matrix code, count time, ote.)

$x+12 \quad|I C E, 250 \mathrm{~m}|, 120 \mathrm{ml}$

ICE, $250 \mathrm{~m} \mid$

Ice, $500 \mathrm{ml}$

$640 \mathrm{~g}$

Complete for samples shipped to an OFF-SITE Subcontract Laborttory

\begin{tabular}{|l|l|l|}
\hline Relingulghed (BN Representative Signature) & DATE / TME & Received (Courher 4 Tracking Into.)
\end{tabular} CDCostaneda Relinquished (Courier a Tracking info.) 6/1/48/3on BAl Cavier dATE/T THAE Rocehed (19t ter Subcontractor Rep) 
VOLATILE

ORGANICS

DATA 
NEL LABORATORIES

CLIENT: Bechtel Nevada

DROJECT NAME: V435

OJECT NUMBER: 17777
CLIENT ID: $\quad$ Decon P11-1 TB

DATE SAMPLED: $6 / 4 / 98$

NEL SAMPLE ID: L9806156-06

TEST: Volatile Organic Compounds by EPA SW846 Method 8260B, Dec 1996

$\begin{array}{lll}\text { MATRIX: Aqueous } & \text { EXTRACTED: 6/15/98 } & \text { ANALYST: Scott } \\ \text { DILUTION: } 1 & \text { ANALYZED: } 6 / 15 / 98 & \end{array}$

\begin{tabular}{|c|c|c|c|c|c|}
\hline PARAMETER & $\begin{array}{l}\text { Result } \\
\mu \mathrm{g} / \mathrm{L}\end{array}$ & $\begin{array}{c}\text { Reporting } \\
\text { Limit } \\
\end{array}$ & PARAMETER & $\begin{array}{l}\text { ResuIt } \\
\mu \mathrm{g} / \mathrm{L} \\
\end{array}$ & $\begin{array}{c}\text { Reporting } \\
\text { Limit } \\
\end{array}$ \\
\hline Acetone & $\overline{\mathrm{ND}}$ & 25. $\mu g / L$ & & & \\
\hline Benzene & $\mathrm{ND}$ & 5. $\mu \mathrm{g} / \mathrm{L}$ & & & \\
\hline Bromodichloromethane & ND & 5. $\mu g / L$ & & & \\
\hline Bromoform & ND & 5. $\mu g / L$ & & & \\
\hline Bromomethane & $\mathrm{ND}$ & 5. $\mu \mathrm{g} / \mathrm{L}$ & & & \\
\hline 2-Butanone & ND & 25. $\mu \mathrm{g} / \mathrm{L}$ & & & \\
\hline Carbon disulfide & ND & 5. $\mu g / L$ & & & \\
\hline Carbon tetrachioride & ND & 5. $\mu \mathrm{g} / \mathrm{L}$ & & & \\
\hline Chlorobenzene & ND & 5. $\mu \mathrm{g} / \mathrm{L}$ & & & \\
\hline Chloroethane & ND & 5. $\mu \mathrm{g} / \mathrm{L}$ & & & \\
\hline Chloroform & ND & 5. $\mu \mathrm{g} / \mathrm{L}$ & & & \\
\hline Chloromethane & ND & 5. $\mathrm{Hg} / \mathrm{L}$ & & & \\
\hline Dibromochloromethane & ND & 5. $\mu g / \mathrm{L}$ & & & \\
\hline 1,1-Dichloroethane (1,1-DCA) & ND & 5. $\mu g / \mathrm{L}$ & & & \\
\hline 1,2-Dichloroethane (1,2-DCA) & $\mathrm{ND}$ & 5. $\mu \mathrm{g} / \mathrm{L}$ & & & \\
\hline 1,1-Dichloroethene (1,1-DCE) & $\mathrm{ND}$ & 5. $11 \mathrm{~g} / \mathrm{L}$ & & & \\
\hline cis-1,2-Dichloroethene & ND & 5. $\mu \mathrm{g} / \mathrm{L}$ & & & \\
\hline 5-1,2-Dichloroethene & $\mathrm{ND}$ & 5. $\mu g / L$ & & & \\
\hline$r \approx$-Dichloropropane & ND & 5. $\mu \mathrm{g} / \mathrm{L}$ & & & \\
\hline cis-1,3-Dichloropropene & ND & 5. $\mu \mathrm{g} L$ & & & \\
\hline trans-1,3-Dichloropropene & ND & 5. $\mu \mathrm{g} / \mathrm{L}$ & & & \\
\hline Ethylbenzene & ND & 5. $\mu g / L$ & & & \\
\hline 2-Hexanone & ND & 25. $\mu \mathrm{g} / \mathrm{L}$ & & & \\
\hline Methylene chloride (Dichloromethane) & ND & 5. $\mu \mathrm{g} / \mathrm{L}$ & & & \\
\hline 4-Methyl-2-pentanone & ND & 25. $\mu \mathrm{g} / \mathrm{L}$ & & & \\
\hline Styrene & $\mathrm{ND}$ & 5. $\mu \mathrm{g} / \mathrm{L}$ & & & \\
\hline 1,1,2,2-Tetrachloroethane & ND & 5. $\mu \mathrm{g} / \mathrm{L}$ & & & \\
\hline Tetrachloroethene (PCE) & ND & 5. $\mu g / L$ & & & \\
\hline Toluene & ND & 5. $\mu \mathrm{g} / \mathrm{L}$ & & & \\
\hline 1, I, 1-Trichloroethane (1,1,1-TCA) & ND & 5. $\mathrm{Hg} / \mathrm{L}$ & & & \\
\hline 1,1,2-Trichloroethane (1,1,2-TCA) & ND & 5. $\mu \mathrm{g} / \mathrm{L}$ & & & \\
\hline Trichloroethene (TCE) & ND & 5. $\mu \mathrm{g} / \mathrm{L}$ & & & \\
\hline Vinyl chloride & ND & 5. $\mu \mathrm{g} / \mathrm{L}$ & & & \\
\hline o-Xylene & ND & 5. $\mu \mathrm{g} / \mathrm{L}$ & & & \\
\hline$m, p$-Xylene & ND & 5. $\mu g / L$ & & & \\
\hline
\end{tabular}

ND - Not Detected

QUALITY CONTROL DATA:

\section{Surrogate}

4-Bromofluorobenzene

Dibromofluoromethane

$T$ re $-\mathrm{d} 8$
\% Recovery

\section{4}

93

99
Acceptable Range

$86-115$

$86 \cdot 118$

$88-110$ 
CLIENT: Bechtel Nevada

PROJECT NAME: V435

PROJECT NUMBER: 17777
CLIENT ID: Decon W3-1 A\&B

DATE SAMPLED: $6 / 8 / 98$

NEL SAMPLE ID： L9806156-01

TEST: Volatile Organic Compounds by EPA SW846 Method 8260A, Sept. 1994

MATRIX: Solid

DILUTION: 1
EXTRACTED: $6 / 11 / 98$

ANALYZED: $6 / 11 / 98$

ANALYST: Suzanne

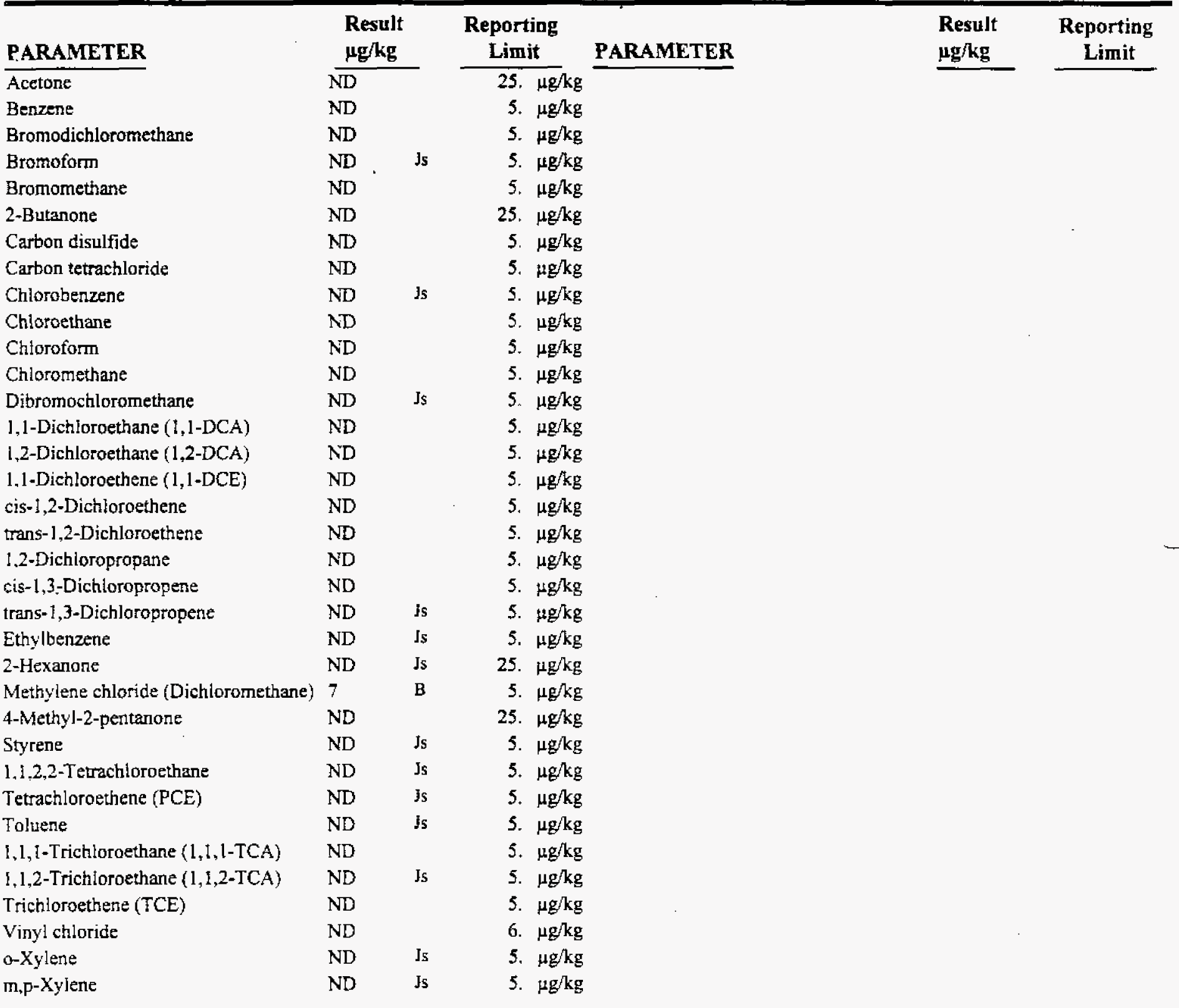

ND - Not Detected

QUALITY CONTROL DATA:

Surrogate

4-Bromafluorobenzene

Dibromofluoromethane

Toluene-d8

\section{$\%$ Recoverv}

57

94

84

\section{Acceptable Range}

$74-121$

$80 \cdot-120$

$81-117$

This report shall not be reproduced except in full, without the written approval of the laboratory. 
CLIENT: Bechtel Nevada

DROJECT NAME: V435

LOJECT NUMBER: 17777
CLIENT ID: Decon P11-1 A\&B

DATE SAMPLED: $6 / 8 / 98$

NEL SAMPLE ID: L9806156-07

TEST: Volatile Organic Compounds by EPA SW846 Method 8260A, Sept. 1994

MATRIX: Solid

DILUTION: 1
EXTRACTED: 6/11/98

ANALYZED: $6 / 11 / 98$

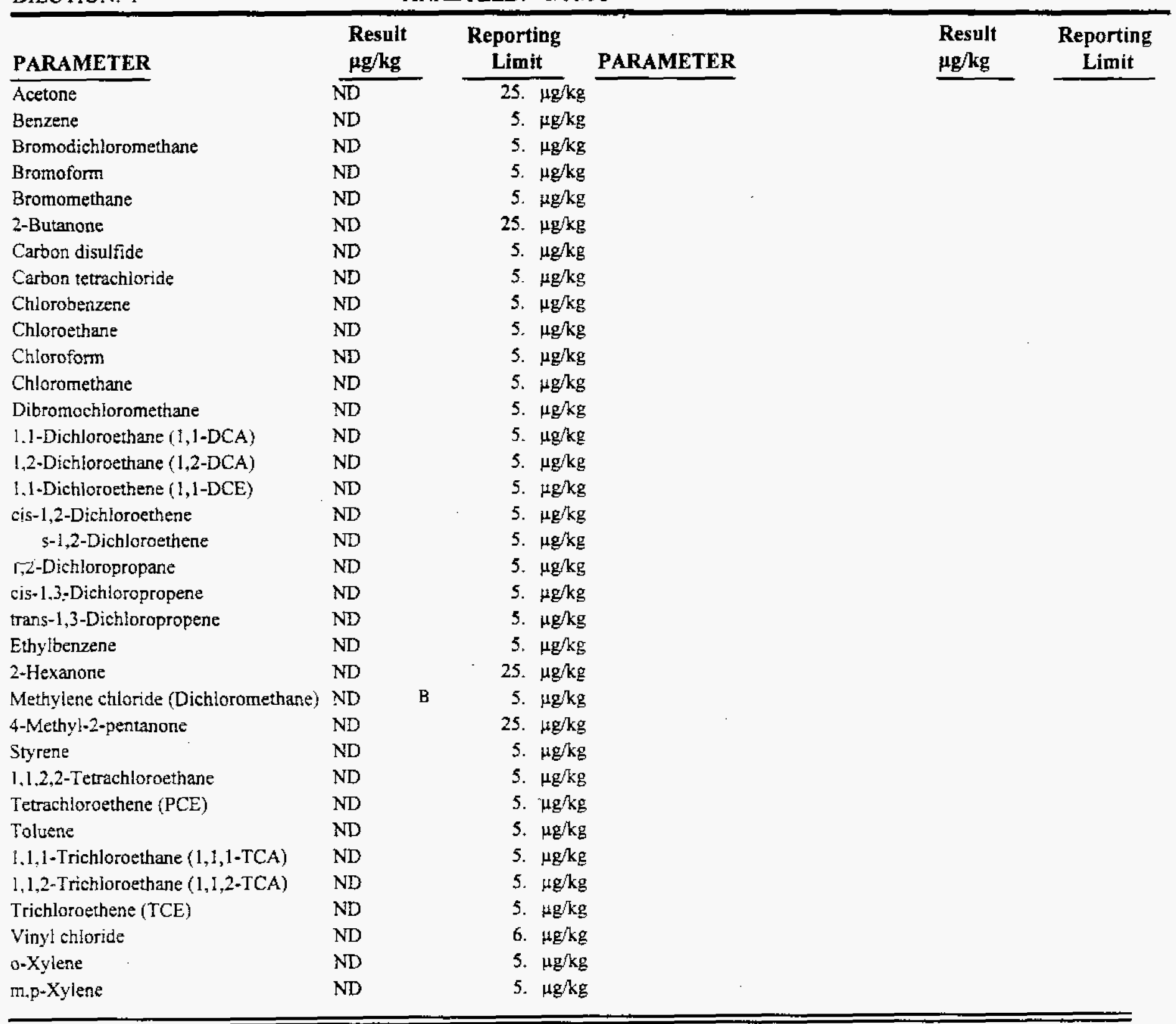

ND - Not Detected

OUALITY CONTROL DATA:

\section{Surrogate}

4-Bromofluorobenzene

Dibromofluoromethane

$T$ sne-d8
\% Recovery

84

91

96
ANALYST: Suzanne

Result

eporting

5. $11 \mathrm{~g} / \mathrm{kg}$

5. $\mu \mathrm{g} / \mathrm{kg}$

25. $\mu \mathrm{g} / \mathrm{kg}$

$\mu \mathrm{g} / \mathrm{kg}$

$5.4 \mathrm{~g} / \mathrm{kg}$

5. $\mu \mathrm{g} / \mathrm{kg}$

5. $\mu \mathrm{g} / \mathrm{kg}$

5. $\mu \mathrm{g} / \mathrm{kg}$

5. $\mu \mathrm{g} / \mathrm{kg}$

5. $\mu \mathrm{g} / \mathrm{kg}$

5. $\mu \mathrm{g} / \mathrm{kg}$

5. $\mu \mathrm{g} / \mathrm{kg}$

5. $\mu \mathrm{g} / \mathrm{kg}$

5. $\mu \mathrm{g} / \mathrm{kg}$

5. $\mu \mathrm{g} / \mathrm{kg}$

5. $\mu g / \mathrm{kg}$

5. $\mu \mathrm{g} / \mathrm{kg}$

5. $\mu \mathrm{g} / \mathrm{kg}$

5. $\mu \mathrm{g} / \mathrm{kg}$

.

This report shall not be reproduced except in full, without the written approval of the laboratory. 


\section{CLIENT: Bechtel Nevada}

PROJECT NAME: V435

PROJECT NUMBER: 17777
CLIENT ID: Decon R 1-1

DATE SAMPLED: $6 / 9 / 98$

NEL SAMPLE ID: L9806155-0I

TEST: Volatile Organic Compounds by EPA SW846 Method 8260B, Dec. 1996

MATRIX: Aqueous

DILUTION: 1
EXTRACTED: $6 / 15 / 98$

ANALYZED: $6 / 15 / 98$

ANALYST: Scott

\begin{tabular}{|c|c|c|c|c|c|}
\hline PARAMETER & $\begin{array}{c}\text { Result } \\
\mu \mathrm{g} / \mathrm{L}\end{array}$ & $\begin{array}{c}\text { Reporting } \\
\text { Limit } \\
\end{array}$ & PARAMETER & $\begin{array}{l}\text { Result } \\
\mu \mathrm{g} / \mathrm{L}\end{array}$ & $\begin{array}{c}\text { Reporting } \\
\text { Limit }\end{array}$ \\
\hline Acetone & $\mathrm{ND}$ & $25 . \mu \mathrm{g} / \mathrm{L}$ & & & \\
\hline Benzene & $\mathrm{ND}$ & 5. $\mu \mathrm{g} / \mathrm{L}$ & & & \\
\hline Bromodichloromethane & 7 & 5. $\mu g / L$ & & & \\
\hline Bromoform & $\mathrm{ND}$ & 5. $\mu g / L$ & & & \\
\hline Bromomethane & $\mathrm{ND}$ & 5. $\mu g / L$ & & & \\
\hline 2-Butanone & $\mathrm{ND}$ & 25. $\mu \mathrm{g} / \mathrm{L}$ & & & \\
\hline Carbon disulfide & $\mathrm{ND}$ & 5. $\mu \mathrm{g} / \mathrm{L}$ & & & \\
\hline Carbon tetrachloride & $\mathrm{ND}$ & 5. $\mu g / L$ & & & \\
\hline Chlorobenzene & $\mathrm{ND}$ & 5. $\mu g / L$ & & & \\
\hline Chloroethane & $\mathrm{ND}$ & 5. $\mu g / L$ & & & \\
\hline Chloroform & 8 & 5. $\mu g / L$ & & & \\
\hline Chloromethane & ND & 5. $\mu g / \mathrm{L}$ & & & \\
\hline Dibromochloromethane & ND & 5. $\mu \mathrm{g} / \mathrm{L}$ & & & \\
\hline 1,1-Dichloroethane (1,1-DCA) & ND & 5. $\mu \mathrm{g} / \mathrm{L}$ & & & \\
\hline 1,2-Dichioroethane (1,2-DCA) & $\mathrm{ND}$ & 5. $\mu g / L$ & & & \\
\hline 1,1-Dichloroethene (1,1-DCE) & $\mathrm{ND}$ & 5. $\mu g / \mathrm{L}$ & & & \\
\hline cis-1,2-Dichloroethene & ND & 5. $\mu g / L$ & & & \\
\hline trans-1,2-Dichloroethene & $\mathrm{ND}$ & 5. $\mathrm{Hg} / \mathrm{L}$ & & & \\
\hline 1,2-Dichtoropropane & $\mathrm{ND}$ & 5. $\mu \mathrm{g} / \mathrm{L}$ & & & \\
\hline cis-1,3-Dichloropropene & ND & 5. $\mu \mathrm{g} / \mathrm{L}$ & & & \\
\hline trans-1,3-Dichloropropene & ND & 5. $\mu \mathrm{g} / \mathrm{L}$ & & & \\
\hline Ethylbenzene & ND & 5. $\mu g / L$ & & & \\
\hline 2-Hexanone & ND & 25. $\mu \mathrm{g} / \mathrm{L}$ & & & \\
\hline Methylene chloride (Dichloromethane) & $\mathrm{ND}$ & 5. $\mu \mathrm{g} / \mathrm{L}$ & & & \\
\hline 4-Methyl-2-pentanone & ND & 25. $\mu \mathrm{g} / \mathrm{L}$ & & & \\
\hline Styrene & ND & 5. $\mu \mathrm{g} / \mathrm{L}$ & & & \\
\hline 1,1,2,2-Tetrachloroethane & $\mathrm{ND}$ & 5. $\mu \mathrm{g} / \mathrm{L}$ & & & \\
\hline Tetrachloroethene (PCE) & $\mathrm{ND}$ & 5. $\mu g / \mathrm{L}$ & & & \\
\hline Toluene & $\mathrm{ND}$ & 5. $\mu \mathrm{g} / \mathrm{L}$ & & & \\
\hline 1,1,1-Trichloroethane $(1,1,1-\mathrm{TCA})$ & $\mathrm{ND}$ & 5. $\mu g / \mathrm{L}$ & & & \\
\hline 1,1,2-Trichjoroethane $(1,1,2-\mathrm{TCA})$ & ND & 5. $\mu g / L$ & & & \\
\hline Trichloroethene (TCE) & $\mathrm{ND}$ & 5. $\mu g / \mathrm{L}$ & & & \\
\hline Vinyl chloride & $\mathrm{ND}$ & 5. $\mu g / L$ & & & \\
\hline o-Xylene & ND & 5. $\mu g / \mathrm{L}$ & & & \\
\hline m,p-Xylene & $\mathrm{ND}$ & 5. $\mu g / L$ & & & \\
\hline
\end{tabular}

ND - Not Detected

QUALITY CONTROL DATA:

\section{Surrogate}

4-Bromofluorobenzene

Dibromofluoromethane

Toluene-d8

\section{\% Recovery}

93

93

99
Acceptable Range

$86-115$

$86-118$

$88-110$

This report shall not be reproduced except in full, without the written approval of the laboratory. 
NEL LABORATORIES

CLIENT: Bechtel Nevada

PROJECT NAME: V435

JJECT NUMBER: 17777
CLIENT ID: Method Blank

DATE SAMPLED: NA

NEL SAMPLE ID: 9806111260B2-BLK

TEST: Volatile Organic Compounds by EPA SW846 Method 8260A, Sept. 1994

MATRIX: Solid EXTRACTED: 6/11/98

ANALYZED: $\quad 6 / 11 / 98$

\begin{tabular}{|c|c|c|c|c|c|}
\hline PARAMETER & $\begin{array}{c}\text { Result } \\
\mu \mathrm{g} / \mathrm{kg}\end{array}$ & $\begin{array}{l}\text { Reporting } \\
\text { Limit }\end{array}$ & PARAMETER & $\begin{array}{l}\text { Result } \\
\mu \mathrm{g} / \mathrm{kg}\end{array}$ & $\begin{array}{c}\text { Reporting } \\
\text { Limit }\end{array}$ \\
\hline$\overline{\text { Acetone }}$ & ND & $25 \mu \mathrm{g} / \mathrm{kg}$ & & & \\
\hline Benzene & $\mathrm{ND}$ & $5 \mu \mathrm{g} / \mathrm{kg}$ & & & \\
\hline Bromodichloromethane & ND & $5 \mu \mathrm{g} / \mathrm{kg}$ & & & \\
\hline Bromoform & ND & $5 \mu \mathrm{g} / \mathrm{kg}$ & & & \\
\hline Bromomethane & ND & $5 \mathrm{Hg} / \mathrm{kg}$ & & & \\
\hline 2-Butanone & ND & $25 \mu \mathrm{g} / \mathrm{kg}$ & & & \\
\hline Carbon disulfide & ND & $5 \mu \mathrm{g} / \mathrm{kg}$ & & & \\
\hline Carbon tetrachloride & ND & $5 \mu g / \mathrm{kg}$ & & & \\
\hline Chlorobenzene & ND & $5 \mu \mathrm{g} / \mathrm{kg}$ & & & \\
\hline Chloroethane & ND & $5 \mu \mathrm{g} / \mathrm{kg}$ & & & \\
\hline Chloroform & ND & $5 \mu \mathrm{g} / \mathrm{kg}$ & & & \\
\hline Chloromethane & ND & $5 \mu \mathrm{g} / \mathrm{kg}$ & & & \\
\hline Dibromochloromethane & ND & $5 \mu \mathrm{g} / \mathrm{kg}$ & & & \\
\hline 1,1-Dichloroethane (1, [-DCA) & ND & $5 \mu \mathrm{g} / \mathrm{kg}$ & & & \\
\hline 1,2-Dichlotoethane (1,2-DCA) & ND & $5 \mu \mathrm{g} / \mathrm{kg}$ & & & \\
\hline [,1-Dichloroethene (1,1-DCE) & ND & $5 \mu \mathrm{g} / \mathrm{kg}$ & & & \\
\hline cis-1,2-Dichloroethene & ND & $5 \mu \mathrm{g} / \mathrm{kg}$ & & & \\
\hline it - 1,2 -Dichloroethene & ND & $5 \mu \mathrm{g} / \mathrm{kg}$ & & & \\
\hline$A_{w-\ldots}$ )ichloropropane & ND & $5 \mu \mathrm{g} / \mathrm{kg}$ & & & \\
\hline cis-1,3-Dichloropropene & ND & $5 \mu \mathrm{g} / \mathrm{kg}$ & & & \\
\hline trans-1,3-Dichloropropene & ND & $5 \mu \mathrm{g} / \mathrm{kg}$ & & & \\
\hline Ethylbenzene & $\mathrm{ND}$ & $5 \mu \mathrm{g} / \mathrm{kg}$ & & & \\
\hline 2-Hexanone & ND & $25 \mu \mathrm{g} / \mathrm{kg}$ & & & \\
\hline Methylene chloride (Dichloromethane & 7 & $5 \mu \mathrm{g} / \mathrm{kg}$ & & & \\
\hline 4-Methyl-2-pentanone & ND & $25 \mu \mathrm{g} / \mathrm{kg}$ & & & \\
\hline Styrene & ND & $5 \mu \mathrm{g} / \mathrm{kg}$ & & & \\
\hline 1,i,2,2-Tetrachloroethane & ND & $5 \mu \mathrm{g} / \mathrm{kg}$ & & & \\
\hline Tetrachloroethene (PCE) & $\mathrm{ND}$ & $5 \mu \mathrm{g} / \mathrm{kg}$ & & & \\
\hline Toluene & $\mathrm{ND}$ & $5 \mu g / \mathrm{kg}$ & & & \\
\hline 1,1,1-Trichloroethane $(1,1,1$-TCA $)$ & ND & $5 \mu \mathrm{g} / \mathrm{kg}$ & & & \\
\hline 1,1,2-Trichloroethane (1,1,2-TCA) & ND & $5 \mu \mathrm{g} / \mathrm{kg}$ & & & \\
\hline Trichloroethene (TCE) & ND & $5 \mathrm{Fg} / \mathrm{kg}$ & & & \\
\hline Vinyl chioride & ND & $6 \mu \mathrm{g} / \mathrm{kg}$ & & & \\
\hline $0-X y l e n e$ & ND & $5 \mu \mathrm{g} / \mathrm{kg}$ & & & \\
\hline m,p-Xylene & ND & $5 \mu \mathrm{g} / \mathrm{kg}$ & & & \\
\hline
\end{tabular}

ND- Not Detected

QUALITY CONTROL DATA:

\section{Surrogate}

4-Bromofluorobenzene

Dibromofluoromethane

Toluene-d8
$\%$ Recovery

92

93

98
Acceptable Range

$74-121$

$80-120$

$81-117$

This report shall not be reproduced except in full, without the written approval of the laboratory. 
CLIENT: Bechtel Nevada

PROJECT NAME: V435

PROJECT NUMBER: 17777
CLIENT ID: Method Blank

DATE SAMPLED: NA

NEL SAMPLE ID: 980615I W60M-BLK

TEST: $\quad$ Volatile Organic Compounds by EPA SW846 Method 8260B, Dec. 1996

MATRIX: Aqueous

EXTRACTED: $\quad 6 / 15 / 98$

ANALYZED: $\quad 6 / 15 / 98$

\begin{tabular}{|c|c|c|c|c|c|}
\hline PARAMETER & $\begin{array}{l}\text { Result } \\
\mu g / L\end{array}$ & $\begin{array}{l}\text { Reporting } \\
\text { Limit }\end{array}$ & PARAMETER & $\begin{array}{l}\text { Result } \\
\mu \mathrm{g} / \mathrm{L}\end{array}$ & $\begin{array}{c}\text { Reporting } \\
\text { Limit }\end{array}$ \\
\hline Acetone & ND & $25 \mu g / \mathrm{L}$ & & & \\
\hline Benzene & ND & $\$ \mu g / L$ & & & \\
\hline Bromodichloromethane & ND & $5 \mu \mathrm{g} / \mathrm{L}$ & & & \\
\hline Bromoform & ND & $5 \mu g / L$ & & & \\
\hline Bromotretsane & ND & $5 \mu \mathrm{g} / \mathrm{L}$ & & & \\
\hline 2-Butanone & ND & $25 \mu g / \mathrm{L}$ & & & \\
\hline Carbon disulfide & ND & $5 \mu g / L$ & & & \\
\hline Carbon tetrachloride & ND & $5 \mu g / L$ & & & \\
\hline Chlorobenzene & $\mathrm{ND}$ & $5 \mu \mathrm{g} / \mathrm{L}$ & & & \\
\hline Chloroethane & $\mathrm{ND}$ & $5 \mu \mathrm{g} / \mathrm{L}$ & & & \\
\hline Chloroform & $\mathrm{ND}$ & $5 \mu \mathrm{g} / \mathrm{L}$ & & & \\
\hline Chloromethane & ND & $5 \mu g / L$ & & & \\
\hline Dibromochloromethane & ND & $5 \mu g / L$ & & & \\
\hline 1,1-Dichlorothane (1,1-DCA) & ND & $S_{\mu \mathrm{g} / \mathrm{L}}$ & & & \\
\hline 1,2-Dichloroethane (1,2-DCA) & ND & $5 \mu \mathrm{g} / \mathrm{L}$ & & & \\
\hline 1,1-Dichloroethene (1, I-DCE) & $\mathrm{ND}$ & $5 \mu g / L$ & & & \\
\hline cis-1,2-Dichloroethene & ND & $5 \mu \mathrm{g} / \mathrm{L}$ & & & \\
\hline trans-1,2-Dichloroethene & ND & $5 \mu \mathrm{g} / \mathrm{L}$ & & & \\
\hline 1,2-Dichloropropane & ND & $5 \mu \mathrm{g} / \mathrm{L}$ & & & \\
\hline cis-1,3-Dichloropropene & ND & $5 \mathrm{Hg} / \mathrm{L}$ & & & \\
\hline trans-1,3-Dichloropropene & ND & $5 \mu \mathrm{g} / \mathrm{L}$ & & & \\
\hline Ethylbenzene & $\mathrm{ND}$ & $S \mu g / L$ & & & \\
\hline 2-Hexanone & ND & $25 \mu \mathrm{g} / \mathrm{L}$ & & & \\
\hline Methylene chloride (Dichloromethane & ND & $5 \mu \mathrm{g} / \mathrm{L}$ & & & \\
\hline 4-Methyl-2-pentanone & $\mathrm{ND}$ & $25 \mu g / L$ & & & \\
\hline Styrene & ND & $5 \mu g / \mathrm{L}$ & & & \\
\hline 1,1,2,2-Tetrachloroethane & ND & $5 \mu \mathrm{g} / \mathrm{L}$ & & & \\
\hline Tetrachloroethene (PCE) & ND & $5 \mu g / L$ & & & \\
\hline Toluene & $\mathrm{ND}$ & $5 \mu \mathrm{g} / \mathrm{L}$ & & & \\
\hline 1,1,1-Trichloroethane $(1,1,1-\mathrm{TCA})$ & $\mathrm{ND}$ & $5 \mu \mathrm{g} / \mathrm{L}$ & & & \\
\hline 1,1,2-Trichloroethane $(1,1,2-\mathrm{TCA})$ & ND & $5 \mu \mathrm{g} / \mathrm{L}$ & & & \\
\hline Trichloroethene (TCE) & ND & $5 \mu g / L$ & & & \\
\hline Vinyl chloride & ND & $5 \mu \mathrm{g} / \mathrm{L}$ & & & \\
\hline o-Xylene & ND & $5 \mu \mathrm{g} / \mathrm{L}$ & & & \\
\hline $\mathrm{m}, \mathrm{p}-\mathrm{Xylene}$ & ND & $5 \mu \mathrm{g} / \mathrm{L}$ & & & \\
\hline
\end{tabular}

ND - Not Detected

QUALITY CONTROL DATA:

Surrogate

4-Bromofluorobenzene

Dibromofluoromethane

Toluene-d8
\% Recovery

94

100

95
Acceptable Range

$86-115$

$86-118$

$88-110$

This report shall not be reproduced except in full, without the written approval of the laboratory. 
SEMI-VOLATILE

ORGANICS

DATA 
CLIENT: Bechtel Nevada

"OJECT NAME: V435

..OJECT NUMBER: 17777
CLIENT ID: $\quad$ Decon R1-2

DATE SAMPLED: $6 / 8 / 98$

NEL SAMPLE ID: L9806156-13

TEST: Semi-Volatile Organic Compounds by EPA 8270C, Dec. 1996

MATRIX: Aqueous

DILUTION: 1
EXTRACTED: $6 / 17 / 98$

ANALYZED: $6 / 18 / 98$

ANALYST: CCJ

\begin{tabular}{|c|c|c|c|c|c|c|}
\hline \multirow{2}{*}{$\frac{\text { PARAMETER }}{\text { Acenaphthene }}$} & \multirow{2}{*}{$\begin{array}{l}\begin{array}{c}\text { Result } \\
\mu \mathrm{g} / \mathrm{L}\end{array} \\
\mathrm{ND}\end{array}$} & $\begin{array}{l}\text { Reporting } \\
\text { Limit }\end{array}$ & \multirow{2}{*}{$\frac{\text { PARAMETER }}{\text { 2,4-Dinitrophenol }}$} & \multirow{2}{*}{$\begin{array}{l}\begin{array}{l}\text { Result } \\
\mathrm{Hg} / \mathrm{L}\end{array} \\
\mathrm{ND}\end{array}$} & \multicolumn{2}{|c|}{$\begin{array}{c}\text { Reporting } \\
\text { Limit } \\
\end{array}$} \\
\hline & & $10 . \mu \mathrm{g} / \mathrm{L}$ & & & 50 . & $\overline{\mu g} / \mathrm{L}$ \\
\hline Acenaphthylene & ND & 10. $\mu \mathrm{g} / \mathrm{L}$ & Di-n-octyl phthatate & $\mathrm{ND}$ & 10. & $\mu \mathrm{g} L$ \\
\hline Anthracene & $\mathrm{ND}$ & 10. $\mu g / L$ & Fluoranthene & $\mathrm{ND}$ & 10. & $\mu g / \mathrm{L}$ \\
\hline Benzo (a) anthracene & ND & 10. $\mu \mathrm{g} / \mathrm{L}$ & Fluorene & $\mathrm{ND}$ & 10. & $\mu \mathrm{g} L$ \\
\hline Benzo (b\&k) fluoranthene & $\mathrm{ND}$ & 10. $\mathrm{Hg} / \mathrm{L}$ & Hexachlorobenzene & $\mathrm{ND}$ & 10. & $\mu \mathrm{g} / \mathrm{L}$ \\
\hline Benzoic Acid & $\mathrm{ND}$ & 50. $\mu g / L$ & Hexachlorobutadiene & ND & 10. & $\mu g / L$ \\
\hline Benzo $(g, h, i)$ perylene & $\mathrm{ND}$ & 10. $\mu \mathrm{g} / \mathrm{L}$ & Hexachlorocyciopentadiene & $\mathrm{ND}$ & 10. & $\mu g L$ \\
\hline Benzo (a) pyrene & ND & 10. $\mu \mathrm{g} / \mathrm{L}$ & Hexachloroethane & ND & 10. & $\mathrm{~kg} / \mathrm{L}$ \\
\hline Benzyl alcohol & $\mathrm{ND}$ & 20. $\mu \mathrm{g} / \mathrm{L}$ & Indeno $(1,2,3-c, d)$ pyrene & ND & 10. & $\mu g / L$ \\
\hline bis (2-Chloroethyl) ether & ND & 10. $\mu g / L$ & Isophorone & ND & 10. & $\mathrm{HEL}$ \\
\hline bis (2-Chloroethoxy) methane & $\mathrm{ND}$ & 10. $\mathrm{Hg} / \mathrm{L}$ & 2-Methylnaphthalene & ND & 10. & $\mu g: \mathrm{L}$ \\
\hline bis (2-Ethylhexyl)phthalate & $\mathrm{ND}$ & 10. $\mu \mathrm{gL}$ & 2-Methylphenol & ND & 10. & $\mu g / \mathrm{L}$ \\
\hline Butylbenzylphthalate & ND & 10. $\mu \mathrm{g} / \mathrm{L}$ & 4-Methylphenol & $\mathrm{ND}$ & 10. & $\mu \mathrm{g}^{\prime} \mathrm{t}$ \\
\hline 4-Bromophenyl phenyl ether & ND & 10. $\mu g / L$ & Naphthaiene & ND & 10. & $\mu g i L$ \\
\hline Carbazole & ND & 10. $\mu \mathrm{g} / \mathrm{L}$ & 2-Nitroaniline & ND & 50. & $\mu g / L$ \\
\hline 4-Chloroanaline & ND & 20. $\mu \mathrm{g} / \mathrm{L}$ & 3-Nitroaniline & ND & 50. & $\mu g L$ \\
\hline 4-Chloro-3-methyl phenol & ND & 20. $\mathrm{Hg} / \mathrm{L}$ & 4-Nitroaniline & $\mathrm{ND}$ & 20. & HEl \\
\hline Ioronaphthalene & ND & 10. $\mu \mathrm{g} / \mathrm{L}$ & Nitrobenzene & ND & 10. & $\mu g: L$ \\
\hline 2-Chlorophenol & ND & 10. $\mu \mathrm{g} / \mathrm{L}$ & 2-NitrophenoI & ND & 10 & $\mu \mathrm{g}: \mathrm{L}$ \\
\hline 4-Chlorophenyl phenyl ether & ND & 10. $\mu \mathrm{g} / \mathrm{L}$ & 4-Nitrophenol & ND & 50. & $\mu g r$ \\
\hline Chrysene & $\mathrm{ND}$ & 10. $\mu \mathrm{g} / \mathrm{L}$ & N-Nitrosodi-n-propylamine & ND & 10. & $\mu g: L$ \\
\hline Dibenzo $(a, h)$ anthracene & ND & 10. $\mu g / L$ & N-Nitroso-Diethylamine & ND & 10. & $\mu g^{\prime} \mathrm{L}$ \\
\hline Dibenzofuran & ND & 10. $\mu \mathrm{g} / \mathrm{L}$ & N-Nitrosodiphenylamine & $\mathrm{ND}$ & 10. & $\mu \mathrm{g}^{\prime} \mathrm{L}$ \\
\hline Di-n-butyl phthalate & ND & 10. $\mu \mathrm{g} / \mathrm{L}$ & Pentachlorophenol & $\mathrm{ND}$ & 50. & $\mu g / L$ \\
\hline 1,2-Dichlorobenzene (o-DCB) & $\mathrm{ND}$ & 10. $\mu \mathrm{g} / \mathrm{L}$ & Phenol & ND & 10. & $\mu g \mathrm{~L}$ \\
\hline 1,3-Dichlorobenzene (m-DCB) & ND & 10. $\mathrm{Hg} / \mathrm{L}$ & Phenanthrene & ND & 10. & Hg'L \\
\hline 1.4-Dichlorobenzene (p-DCB) & ND & 10. $\mu \mathrm{g} / \mathrm{L}$ & Pyrent & $\mathrm{ND}$ & 10. & $\mu \mathrm{g} \mathrm{L}$ \\
\hline 2.4-Dichlorophenol & ND & 10. $\mu \mathrm{g} / \mathrm{L}$ & 1,2,4-Trtehlorobenzene & $\mathrm{ND}$ & 10 . & $\mu \mathrm{e} L$ \\
\hline 3.3'-Dichlorobenziơine & $\mathrm{ND}$ & 20. $\mathrm{Hg} / \mathrm{L}$ & 2,4,5-Trichlorophenol & ND & 10. & $\mu g \cdot L$ \\
\hline Diethylphthafate & ND & 10. $\mu \mathrm{g} / \mathrm{L}$ & 2,4,6-Trichlorophenol & ND & 10. & $\mu \mathrm{g} \cdot \mathrm{L}$ \\
\hline 2,4-Dimethylphenol & $\mathrm{ND}$ & 10. $\mu \mathrm{g} / \mathrm{L}$ & & & & \\
\hline Dimethylphthalate & ND & 10. $\mu \mathrm{g} / \mathrm{L}$ & & & & \\
\hline 4.6-Dinitro-2-methyl phenol & ND & 50. $\mu \mathrm{g} / \mathrm{L}$ & & & & \\
\hline 2,4-Dinitrotoluene (DNT) & $\mathrm{ND}$ & 10. $\mu \mathrm{g} / \mathrm{L}$ & & & & \\
\hline 2,6-Dinitrotoluene (DNT) & $\mathrm{ND}$ & 10. $\mu \mathrm{g} / \mathrm{L}$ & & & & \\
\hline
\end{tabular}

ND - Not Detected

QUALITY CONTROL DATA:

\section{Surrogate}

2,4,6-Tribromophenol

2.Fluorobiphenyl

torophenol

Norobenzene-d5

p-Terphenyi-d 14

Phenol-d5
$\%$ Recovery
Acceptable Range

$10-123$

$43-116$

$21 \cdot 100$

$35-114$

$33-141$

$10-94$ 


$\begin{array}{ll}\text { CLIENT: Bechtel Nevada } & \text { CLIENT ID: Method Blank } \\ \text { PROJECT NAME: V435 } & \text { DATE SAMPLED: NA } \\ \text { PROJECT NUMBER: } 17777 & \text { NEL SAMPLE ID: 061798-E1-BLK }\end{array}$

TEST:

MATRIX

Semi-Volatile Organic Compounds by EPA 8270C, Dec. 1996

Aqueous

EXIRACIED:

$6 / 17 / 98$

ANALYZED:

$6 / 18 / 98$

\begin{tabular}{|c|c|c|c|c|c|}
\hline PARAMETER & $\begin{array}{l}\text { Result } \\
\mu g / L\end{array}$ & $\begin{array}{c}\text { Reporting } \\
\text { Limit } \\
\end{array}$ & PARAMETER & $\begin{array}{c}\text { Result } \\
\mu \mathrm{g} / \mathrm{L}\end{array}$ & $\begin{array}{c}\text { Reporting } \\
\text { Limit }\end{array}$ \\
\hline Acenaphthene & $\mathrm{ND}$ & $10 \mu g / L$ & $\overline{\text { 2,4-Dinitrophenol }}$ & $\overline{N D}$ & $50 \mu \mathrm{g} / \mathrm{L}$ \\
\hline Acenaphthylene & $\mathrm{ND}$ & $10 \mu g / L$ & Di-n-octyl phthatate & ND & $10 \mu \mathrm{g} / \mathrm{L}$ \\
\hline Anthracene & $\mathrm{ND}$ & $10 \mu \mathrm{g} / \mathrm{L}$ & Fluoranthene & ND & $10 \mu \mathrm{g} / \mathrm{L}$ \\
\hline Benzo (a) anthracene & ND & $10 \mu g / L$ & Fluorene & ND & $10 \mu \mathrm{g} / \mathrm{L}$ \\
\hline Benzo (b\&k) fluoranthene & ND & $10 \mu g / L$ & Hexachlorobenzene & ND & $10 \mu g / \mathrm{L}$ \\
\hline Benzoic Acid & ND & $50 \mu \mathrm{g} / \mathrm{L}$ & Hexachlorobutadiene & ND & $10 \mu \mathrm{g} / \mathrm{L}$ \\
\hline Benzo $(\mathrm{g}, \mathrm{h}, \mathrm{i})$ perylene & ND & $10 \mu \mathrm{g} / \mathrm{L}$ & Hexachlorocyclopentadiene & ND & $10 \mu \mathrm{g} / \mathrm{L}$ \\
\hline Benzo (a) pyrene & ND & $10 \mu g / L$ & Hexachloroethane & $\mathrm{ND}$ & $10 \mu g / L$ \\
\hline Benzyl alcohol & ND & $20 \mu g / L$ & Indeno $(1,2,3-c, d)$ pyrene & $\mathrm{ND}$ & $10 \mu \mathrm{g} / \mathrm{L}$ \\
\hline bis (2-Chloroethyl) ether & ND & $10 \mu \mathrm{g} / \mathrm{L}$ & Isophorone & ND & $10 \mu \mathrm{g} / \mathrm{L}$ \\
\hline bis (2-Chloroethoxy) methane & ND & $10 \mu \mathrm{g} / \mathrm{L}$ & 2-Methylnaphthalene & ND & $10 \mu \mathrm{gg} / \mathrm{L}$ \\
\hline bis (2-Ethylhexyl)phthalate & ND & $10 \mu \mathrm{g} / \mathrm{L}$ & 2-Methylphenol & ND & $10 \mu g / \mathrm{L}$ \\
\hline Butylbenzylphthalate & $\mathrm{ND}$ & $10 \mu \mathrm{g} / \mathrm{L}$ & 4-Methylphenol & ND & $10 \mu \mathrm{g} / \mathrm{L}$ \\
\hline 4-Bromophenyl phenyl ether & ND & $10 \mu \mathrm{g} / \mathrm{L}$ & Naphthalene & $\mathrm{ND}$ & $10 \mathrm{\mu g} / \mathrm{L}$ \\
\hline Carbazole & $\mathrm{ND}$ & $10 \mu \mathrm{g} / \mathrm{L}$ & 2-Nitroanitine & ND & $50 \mu \mathrm{g} / \mathrm{L}$ \\
\hline 4-Chloroanaline & ND & $20 \mu \mathrm{g} / \mathrm{L}$ & 3-Nitroaniline & $\mathrm{ND}$ & $50 \mu \mathrm{gg} / \mathrm{L}$ \\
\hline 4-Chloro-3-methyl phenol & ND & $20 \mu g / L$ & 4-Nitroaniline & ND & $20 \mu \mathrm{g} / \mathrm{L}$ \\
\hline 2-Chloronaphthalene & ND & $10 \mu \mathrm{g} / \mathrm{L}$ & Nitrobenzene & $\mathrm{ND}$ & $10 \mu \mathrm{g} / \mathrm{L}$ \\
\hline 2-Chlorophenol & ND & $10 \mu \mathrm{g} / \mathrm{L}$ & 2-Nitrophenol & ND & $10 \mu \mathrm{g} / \mathrm{L}$ \\
\hline 4-Chlorophenyl phenyl ether & $\mathrm{ND}$ & $10 \mu \mathrm{g} / \mathrm{L}$ & 4-Nitrophenol & ND & $50 \mu \mathrm{g} / \mathrm{L}$ \\
\hline Chrysene & $\mathrm{ND}$ & $10 \mu \mathrm{g} / \mathrm{L}$ & N-Nitrosodi-n-propylamine & ND & $10 \mu \mathrm{g} / \mathrm{L}$ \\
\hline Dibenzo $(a, h)$ anthracene & ND & $10 \mu \mathrm{g} / \mathrm{L}$ & N-Nitroso-Diethylamine & ND & $10 \mu \mathrm{g} / \mathrm{L}$ \\
\hline Dibenzofuran & $\mathrm{ND}$ & $10 \mu \mathrm{g} / \mathrm{L}$ & N-Nitrosodiphenylamine & ND & $10 \mu \mathrm{g} / \mathrm{L}$ \\
\hline Di-n-butyl phthalate & ND & $10 \mu g / L$ & Pentachlorophenol & ND & $50 \mu \mathrm{g} / \mathrm{L}$ \\
\hline 1,2-Dichlorobenzene (o-DCB) & ND & $10 \mu \mathrm{g} / \mathrm{L}$ & Phenol & ND & $1011 \mathrm{~g} / \mathrm{L}$ \\
\hline 1,3-Dichlorobenzene (m-DCB) & ND & $10 \mu \mathrm{g} / \mathrm{L}$ & Phenanthrene & $\mathrm{ND}$ & $10 \mu \mathrm{g} / \mathrm{L}$ \\
\hline 1,4-Dichlorobenzene (p-DCB) & ND & $10 \mu g / L$ & Pyrene & $\mathrm{ND}$ & $10 \mu \mathrm{g} / \mathrm{L}$ \\
\hline 2,4-Dichlorophenol & ND & $10 \mu \mathrm{g} / \mathrm{L}$ & 1,2,4-Trichlorobenzene & ND & $10 \mu \mathrm{g} / \mathrm{L}$ \\
\hline 3,3'-Dichlorobenzidine & ND & $20 \mu \mathrm{g} / \mathrm{L}$ & 2,4,5-Trichlorophenol & $\mathrm{ND}$ & $10 \mu \mathrm{g} / \mathrm{L}$ \\
\hline Diethylphthalate & ND & $10 \mu \mathrm{g} / \mathrm{L}$ & 2,4,6-Trichlorophenol & $\mathrm{ND}$ & $10 \mu \mathrm{g} / \mathrm{L}$ \\
\hline 2,4-Dimethylphenol & ND & $10 \mu \mathrm{g} / \mathrm{L}$ & & & \\
\hline Dimethylphthalate & $\mathrm{ND}$ & $10 \mu g / \mathrm{L}$ & & & \\
\hline 4,6-Dinitro-2-methyl phenol & ND & $50 \mu g / \mathrm{L}$ & & & \\
\hline 2,4-Dinitrotoluene (DNT) & ND & $10 \mu g / \mathrm{L}$ & & & \\
\hline 2,6-Dinitrotoluene (DNT) & ND & $10 \mu \mathrm{g} / \mathrm{L}$ & & & \\
\hline
\end{tabular}

ND - Not Detected

QUALITY CONTROL DATA:

\section{Surrogate}

2,4,6-Tribromophenol

2-Fluorobiphenyl

2-Fluorophenol

Nitrobenzene-d5

p-Terphenyl-dl4

phenol-ds
\%.Recovery

65

62

40

65

76

25
Acceptable Range

10.123

$43-116$

$21-100$

$35-114$

$33-141$

$10=94$

This report shall not be reproduced except in full, without the written approval of the laboratory. 


\section{POLYCHLORINATED}

\section{BIPHENYLS}

DATA 
NEL LABORATORIES

CLIENT: Bechtel Nevada

DROJECT NAME: V435

OJECT NUMBER: 17777
CLIENT ID: Decon W3-3

DATE SAMPLED: $6 / 8 / 98$

NEL SAMPLE ID: L9806156-03

TEST: Polychlorinated Biphenyl's (PCB's) by EPA 8082, Dec. 1996

EXTRACTED: $6 / 11 / 98$

ANALYZED: $6 / 12 / 98$
MATRIX: Solid

DILUTION: 1

\section{PARAMETER}

ATochlor-1254

Arochtor- 1232

Arochior-1221

Arochlor 1260
Arochlor -1248

Arochlor-1 242

Arochlor- 1016

ANALYST: John

\begin{tabular}{llc} 
Result & & $\begin{array}{c}\text { Reporting } \\
\text { Limit }\end{array}$ \\
\cline { 3 - 3 } $\mathbf{1 4 0}$ & $\mu \mathrm{g} / \mathrm{kg}$ & $20 . \mu \mathrm{g} / \mathrm{kg}$ \\
$\mathrm{ND}$ & & $20 . \mu \mathrm{g} / \mathrm{kg}$ \\
$\mathrm{ND}$ & & $20 . \mu \mathrm{g} / \mathrm{kg}$ \\
$\mathrm{ND}$ & & $20 . \mu \mathrm{kg}$ \\
$\mathrm{ND}$ & & $20 . \mu \mathrm{g} / \mathrm{kg}$ \\
$\mathrm{ND}$ & & $20 . \mu \mathrm{kg}$ \\
$\mathbf{7 4}$ & $\mu \mathrm{g} / \mathrm{kg}$ & $20 . \mu \mathrm{\mu g} / \mathrm{kg}$ \\
\hline
\end{tabular}

ND - Not Detected

QUALITY CONTROL DATA:

\section{Surrogate}

DecachlorobiphenyI

Tetrachloro-m-xylene

\section{\% Recovery}

85

74
Acceptable Range

$70 \cdot 130$

$70 \cdot 130$ 
NEL LABORATORIES

CLIENT: Bechtel Nevada

PROJECT NAME: V435

PROJECT NUMBER: 17777
CLIENT ID: Decon P11-3

DATE SAMPLED: $6 / 8 / 98$

NEL SAMPLE ID： L9806156.09

TEST: Polychlorinated Biphenyl's (PCB's) by EPA 8082, Dec. 1996

MATRIX: Solid

EXTRACTED: 6/11/98

DILUTION: 1

ANALYZED: $6 / 12 / 98$

ANALYST: John

PARAMETER

Arochlor-I242

Arochlor-1016

Arochlor-1232

Arochlor- 1248

Arochlor- 1254

Arochlor- 1260

Arochlor-1221

\begin{tabular}{l} 
Result \\
\hline ND \\
ND \\
ND \\
ND \\
ND \\
ND \\
ND
\end{tabular}

\section{Reporting}

Limit

20. $\mu \mathrm{g} / \mathrm{kg}$

20. $\mu \mathrm{g} / \mathrm{kg}$

20. $\mu \mathrm{g} / \mathrm{kg}$

20. $\mu \mathrm{g} / \mathrm{kg}$

20. $\mu \mathrm{g} / \mathrm{kg}$

20. $\mu \mathrm{g} / \mathrm{kg}$

20. $\mu \mathrm{g} / \mathrm{kg}$

ND - Not Detected

QUALITY CONTROL DATA:

\section{Surrogate}

Decachlorobiphenyl

Tetrachloro-m-xylene
$\%$ Recovery

95

88
Acceptable Range

$70-130$

$70-130$ 
CLIENT: Bechtel Nevada

"OJECT NAME: V435

_.OJECT NUMBER: 17777
CL $:$ NT ID: Decon RI-3

DAIE SAMPLED: $6 / 8 / 98$

NEL SAMPLE ID: L9806156-14

TEST: Polychlorinated Biphenyls (PCB's) by EPA 8082, Dec. 1996

MATRIX: Aqueous

DILUTION: 1
EXTRACTED: 6/11/98

ANALYZED: $6 / 14 / 98$
ANALYST: John

\begin{tabular}{c}
$\begin{array}{c}\text { Reporting } \\
\text { Limit }\end{array}$ \\
\hline $0.5 \mu \mathrm{g} / \mathrm{L}$ \\
$0.5 \mu \mathrm{g} / \mathrm{L}$ \\
$0.5 \mu \mathrm{g} / \mathrm{L}$ \\
$0.5 \mu \mathrm{g} / \mathrm{L}$ \\
$0.5 \mu \mathrm{g} / \mathrm{L}$ \\
$0.5 \mu \mathrm{g} / \mathrm{L}$ \\
$0.5 \mu \mathrm{g} / \mathrm{L}$
\end{tabular}

\section{PARAMETER}

Arochlor-1016

Arochlor 122 ]

Arochlor- 1232

Afochlor-1242

Arochlor-1248

Arochlor- 1254

Arocblor -1260

\begin{tabular}{l} 
Result \\
\hline $\mathrm{ND}$ \\
$\mathrm{ND}$ \\
$\mathrm{ND}$ \\
$\mathrm{ND}$ \\
$\mathrm{ND}$ \\
$\mathrm{ND}$ \\
$\mathrm{ND}$
\end{tabular}

ND - Not Detected

QUALITY CONTROL DATA:

\section{Surrogate}

Decachlorobiphenyl

Tetrachloro-m-xylene

\section{$\%$ Recovery}

95

81
Acceptable Range

$70-130$

$70-130$ 
CLIENT: Bechtel Nevada

PROJECT NAME: V435

PROJECT NUMBER: 17777
CLIENT ID: Method Blank

DATE SAMPLED: NA

NEL SAMPLE ID： 98061 1s8082-BK

TEST: Polychlorinated Biphenyl's (PCB's) by EPA 8082, Dec. 1996

MATRIX: Solid EXTRACTED: 6/11/98

ANALYZED: $6 / 12 / 98$

ANALYST: John

\section{PARAMETER}

Arochlor-1016

Arochlor-1221

Arochior-1232

Arochlor -1242

Arochlor- 1248

Arochlor- 1254

Arochlor-1260

\begin{tabular}{l} 
Result \\
\hline ND \\
ND \\
ND \\
ND \\
ND \\
ND \\
ND
\end{tabular}

\begin{tabular}{c}
$\begin{array}{c}\text { Reporting } \\
\text { Limit }\end{array}$ \\
\hline $20 \mu \mathrm{g} / \mathrm{kg}$ \\
$20 \mu \mathrm{g} / \mathrm{kg}$ \\
$20 \mu \mathrm{gg}$ \\
$20 \mu \mathrm{gg} / \mathrm{kg}$ \\
$20 \mu \mathrm{g} / \mathrm{kg}$ \\
$20 \mu \mathrm{gg} / \mathrm{kg}$ \\
$20 \mu \mathrm{g} / \mathrm{kg}$.
\end{tabular}

ND - Not Detected

QUALITY CONTROL DATA:

Surrogate

Decachlorobiphenyl'

Tetrachloro-m-xylene

\begin{tabular}{c} 
\% Recovery \\
\hline 84 \\
68
\end{tabular}

Acceptable Range

$70-130$

$70-130$
CLIENT: Bechtel Nevada

PROJECT NAME: V435

PROJECT NUMBER: 17777
CLIENT ID: Method Blank

DATE SAMPLED: NA

NEL SAMPLE ID: 980611 w8082-BK

TEST: Polychlorinated Biphenyls (PCB's) by EPA 8082, Dec. 1996

MATRIX: Aqueous $\quad$ EXTRACTED: $6 / 11 / 98$
ANALYZED: $6 / 14 / 98$

ANALYST: John

\begin{tabular}{c}
$\begin{array}{c}\text { Reporting } \\
\text { Limit }\end{array}$ \\
\hline $0.5 \mu \mathrm{g} / \mathrm{L}$ \\
$0.5 \mu \mathrm{L}$ \\
$0.5 \mu \mathrm{L} / \mathrm{L}$ \\
$0.5 \mu \mathrm{L} / \mathrm{L}$ \\
$0.5 \mu \mathrm{g} / \mathrm{L}$ \\
$0.5 \mu \mathrm{g} / \mathrm{L}$ \\
$0.5 \mu \mathrm{g} / \mathrm{L}$
\end{tabular}

\section{PARAMETER}

Arochlor-1016

Arochior-1221

Arochlor-1232

Arochlor -1242

Arochlor-1248

Arochlor-1254

Arochlor- 1260

\begin{tabular}{l} 
Result \\
\hline $\mathrm{ND}$ \\
$\mathrm{ND}$ \\
$\mathrm{ND}$ \\
$\mathrm{ND}$ \\
$\mathrm{ND}$ \\
$\mathrm{ND}$ \\
$\mathrm{ND}$
\end{tabular}

ANALYST: John

ND - Not Detected

\section{QUALITY CONTROL DATA:}

\section{Surrogate}

Decachlorobiphenyl .

Tetrachloro-m-xylene
\% Recovery

115

99
Acceptable Range

$70-130$

$70-130$ 


\section{TOTAL}

\section{PETROLEUM}

HYDROCARBON

DATA 
NEL LABORATORIES

CLIENT: Bechtel Nevada

-ROJECT NAME: V435

....OJECT NUMBER: 17777

TEST: $\quad$ Total Extractable Petroleum Hydrocarbons by EPA Method 8015M, July 1992

METHOD: $\quad$ EPA 8015M

MATRDX: Aqueous

ANALYST: Suzanne

CLIENT

SAMPLE ID

Decon Rl-4

\section{SAMPLE NEL RESULT}

DATE SAMPLE ID mg/L

6/8/98 L9806156-15

ND

C.R. $\frac{\begin{array}{c}\text { Reporting } \\ \text { Limit }\end{array}}{0.5 \mathrm{mg} / \mathrm{L}}$

Surrogate

Recovery ${ }^{*}$ EXTRACTED ANALYZED

$119 \% \quad 6 / 15 / 98$

$6 / 17 / 98$

\section{CR: Carbon Range}

GDO Gas Range Organics ( $\mathrm{C} 8$ to $\mathrm{C12}$ ), Diesel Rarge Organics (C12 to C24) and Oil Range Organics (C12 to C38).

Note: The reporting limit for Oil Range Organics in soil is $50 \mathrm{mg} / \mathrm{kg}$.

QUALITY CONTROL DATA (Total for Gas and Diesel Range):

Sample ID

Blank, 980615TPH-BLK

I 9806I5TP - LCS

LCSD 980615TP - LCSD
Result Acceptable Range Surrogate Recovery* Sample Number

$\begin{array}{lllllll}\text { ND } & & < & 0.5 \mathrm{mg} / \mathrm{L} & 117 \% & \text { NA } \\ 72 & \% & 61 & -104 \% & 115 \% & \text { NA } \\ 82 & \% & 61 & -104 \% & 111 \% & \text { NA. }\end{array}$

ND - Not Detected

*Surrogate used was Octacosane, acceptance limits $54-130 \%$ for solids, $60-121 \%$ for aqueous samples

This report shall not be reproduced except in full, without the written approval of the laboratory. 
NEL LABORATORIES

CLIENT: Bechtel Nevada

PROJECT NAME: V435

PROJECT NUMBER: 17777

TEST: $\quad$ Total Extractable Petroleum Hydrocarbons by EPA Method 8015M, July 1992

METHOD: EPA 8015M

MATRIX: Solid

ANALYST: Suzanne

\begin{tabular}{|c|c|c|c|c|c|c|c|c|}
\hline $\begin{array}{l}\text { CLIENT } \\
\text { SAMPLE ID } \\
\end{array}$ & $\begin{array}{c}\text { SAMPLE } \\
\text { DATE }\end{array}$ & $\begin{array}{c}\text { NEL } \\
\text { SAMPLE ID } \\
\end{array}$ & $\begin{array}{c}\text { RESULT } \\
\mathbf{m g} / \mathbf{k g} \\
\end{array}$ & C.R. & $\begin{array}{c}\text { Reporting } \\
\text { Limit } \\
\end{array}$ & $\begin{array}{l}\text { Surrogate } \\
\text { Recovery* }\end{array}$ & EXTRACTED & ANALYZED \\
\hline econ W3-4 A\&B & $6 / 8 / 98$ & L9806156+04 & 1100 & GDO & $10 . \mathrm{mg} / \mathrm{kg}$ & $122 \%$ & $6 / 16 / 98$ & $6 / 19 / 98$ \\
\hline Decon P11-4 A\&B & $6 / 8 / 98$ & L9806156-10 & ND & & $10 . \mathrm{mg} / \mathrm{kg}$ & $87 \%$ & $6 / 16 / 98$ & $6 / 18 / 98$ \\
\hline
\end{tabular}

\section{CR: Carbon Range}

GDO Gas Range Organics ( $\mathrm{C} 8$ to $\mathrm{C} 12$ ), Diesel Range Organics ( $\mathrm{Cl2}$ to $\mathrm{C} 24$ ) and Oil Range Organics (C12 to $\mathrm{C} 38$ ).

Note: The reporting limit for Oil Range Organies in soil is $\mathbf{5 0 ~} \mathrm{mg} / \mathrm{kg}$.

QUALITY CONTROL DATA (Total for Gas and Diesel Range):

Sample ID

Blank, 980616TPH-BLK

LCS, 980616TP - LCS
Result Acceptable Range Surrogate Recovery* Sample Number

$\begin{array}{lllllll}\text { ND } & < & 10 . \mathrm{mg} / \mathrm{kg} & 70 & \% & \text { NA } \\ 68 & \% & 55 & -102 \% & 88 & \% & \text { NA }\end{array}$

ND - Not Detected

"Surrogate used was Octacosane, acceptance limits 54-130\% for solids, 60-121\% for aqueous samples

This report shall not be reproduced except in full, without the written approval of the laboratory. 


\section{SDG B447 \\ RADIOANALYTICAL \\ RESULTS}




\section{Beutel Nevada Corporation}

\section{ANALYTICAL SERVICES LABORATORY}

P.O.Box 3936, N. Las Vegas, NV 89036

Reported to: Area 6 Decon Pond Closure

Remediation Projects

Report Date: 6-JUL-98

J. L. Smith, M/S NTS306

Sample Delivery Group: 8447

Batch: 0821

Program: 720

Report No.:

\begin{tabular}{|c|c|c|c|c|c|c|c|c|c|c|c|c|c|c|c|c|}
\hline $\begin{array}{c}\text { Sample } \\
\text {...... Identification ..... }\end{array}$ & Isotope & Analysis & Result: & $\begin{array}{c}\text { Eror } \\
\%\end{array}$ & $\begin{array}{l}\text { Oua: } \\
\text { Flag }\end{array}$ & MDA & $\begin{array}{l}\text { Result } \\
\text { Units }\end{array}$ & $\begin{array}{c}\text { Analysis } \\
\text { Date }\end{array}$ & \begin{tabular}{|c} 
Sample \\
Co:l Oate
\end{tabular} & Size & \begin{tabular}{|l} 
Size \\
Units
\end{tabular} & $\begin{array}{c}\text { Type } \\
\text { Matrix }\end{array}$ & \begin{tabular}{|l|} 
Tracer \\
Yield $\%$
\end{tabular} & $\begin{array}{l}\text { Spiks } \\
\text { hecv \% }\end{array}$ & $\begin{array}{l}\text { System } \\
\text { Detacto }\end{array}$ & $\begin{array}{l}\text { Packet-them } \\
\text { Sample }\end{array}$ \\
\hline DECON P11-6 & K 40 & GAM2O & $2.34 E+01$ & $2.0 E+01$ & & $1.1 E+\infty$ & pCilg & $06-09-98$ & 06-08-9B & $6.94 E+02$ & $\mathrm{gm}$ & soll G & & & 05-01 & G9135-1-75101 \\
\hline DECON P11-6 & RA226 & GAM20 & $1.07 E+00$ & $3.0 \mathrm{E}+01$ & & 1.5E-01 & $\mathrm{pClig}$ & 06-09-98 & 06-08-98 & $6.94 E+02$ & $\mathrm{gm}$ & soll. G| & & & 05.01 & G9135-1-75101 \\
\hline DECON P11-6 & TH228 & GAM20 & $2.55 \mathrm{E}+00$ & $1.8 \mathrm{E}+01$ & & $1.4 \mathrm{E}-01$ & pCilg & 06-09-98 & $06-08-98$ & $6.94 E+02$ & $\mathrm{gm}$ & soll, G & & & $05-01$ & $69135-1-75101$ \\
\hline DECON P11-6 & TH232 & GAM2O & $1.35 E+00$ & $4.0 E+01$ & & $3.6 E-01$ & pCi/g & $06-09-98$ & O6-OB-9B & $6.94 E+02$ & gm & soll G & & & 05.01 & G9135-1.75101 \\
\hline DECON W3-6 & AM241 & GAM20 & $1.64 E+00$ & $4.8 \mathrm{E}+01$ & & $3.1 E-01$ & $\mathrm{pCi} / \theta$ & 06-09-9B & $06-08-98$ & $8.39 E+02 \mid$ & $\operatorname{gm}$ & SOIL G & & & $05-01$ & $69135-0-75099$ \\
\hline DECON W3-6 & $\mathrm{CO} 60$ & GAM20 & $1.53 \mathrm{E}+00$ & $2.3 E+01$ & & 8.4E-02 & pCi/g & $06-09-98$ & $06-08-98$ & $8.39 E+02$ & gm & solt G & & & $05-01$ & G9135-0.75099 \\
\hline DECON W3-6 & $\operatorname{cs} 137$ & GAM20 & $9.40 E+00$ & $1.2 E+01$ & & $7.1 \mathrm{E}-02$ & $\mathrm{pCli} / \mathrm{g}$ & 06-09-98 & $06-08-98$ & $8.39 E+02 \mid$ & $\mathrm{gm}$ & SOLL $\mathrm{G}$ & & & $05-01$ & $69135-0-75099$ \\
\hline DECON W3-6 & EU152 & GAM20 & $1.35 E+00$ & $4.2 \mathrm{E}+01$ & & 2.8E-01 & oCi/g & $06-09-98$ & 06-08-9B & B. $39 \mathrm{E}+02$ & $\mathrm{gm}$ & soll. G & & & $05-01$ & G9135-0-75099 \\
\hline DECON W3-6 & EU154 & GAM20 & B.05E-01 & $5.0 E+01$ & & $3.2 \mathrm{E}-01$ & pCilg & 06-09-98 & 06-08-98 & $8.39 E+02 \mid$ & $\mathrm{gm}$ & SOIL G & & & 05-01 & G9135-0-75099 \\
\hline DECON W3-6 & EU155 & GAM2O & 7.69E-01 & $8.8 E+01$ & & $2.4 E-01$ & $\mathrm{pCi} / \mathrm{g}$ & 06-09-98 & 06-08-98 & $8.39 \mathrm{E}+02$ & $\mathrm{gm}$ & so:L G & & & $05-01$ & G9135-0-75099 \\
\hline
\end{tabular}

\section{Comment:}

Data generated from analyses of samples submitted on 6/9/98.

Gross alpha/beta determinations are performed on small sample

aliquots. Data may ngt represent sample activity as a whole.

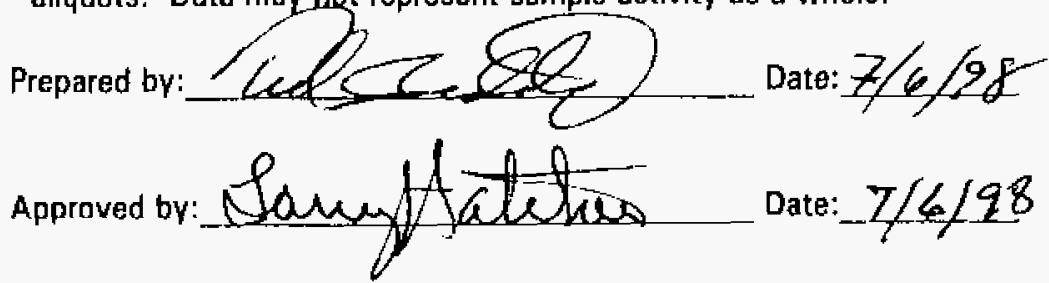

Qualification Flags:

$E$ = Estimated Quantity

$H=$ High Recovery for Sample

$\mathrm{J}=$ Result is less than the RDL

$L=$ Low Recovery for Sample

$P=$ Preliminary Resuits

$0=$ Bad Instrument Quality Control, Result is OK

$R=$ Results are Unusable, Resampling is Necessary

$\mathrm{U}=$ Result is less than Minimum Detectable Activity

Note: \% Error is the 2.0 Sigma Error 


\section{Bechtel Nevada Corporation}

ANALYTICAL SERVICES LABORATORY

P.O.Box 3936, N. Las Vegas, NV 89036

Page 2

Reported to: Area 6 Decon Pond Closure

Remediation Projects

Report Date: 6-JUL-98

J. L. Smith, M/S NTS306

Sample Delivery Group: B447

Batch: 0821

Program: 720

Report No. :

\begin{tabular}{|c|c|c|c|c|c|c|c|c|c|c|c|c|c|c|c|c|}
\hline$\underset{\text { Sampla }}{\text {...... Identification ..... }}$ & Isotope & Analysis & Result & $\begin{array}{c}\text { Error } \\
\%\end{array}$ & $\begin{array}{l}\text { Qual } \\
\text { Flag }\end{array}$ & MDA & $\begin{array}{l}\text { Alessult } \\
\text { Units }\end{array}$ & $\begin{array}{l}\text { Analysis } \\
\text { Date }\end{array}$ & $\begin{array}{l}\text { Sample } \\
\text { Coll Date }\end{array}$ & Sizo & $\begin{array}{l}\text { Size } \\
\text { Units }\end{array}$ & \begin{tabular}{|l|} 
Typo \\
Matrix
\end{tabular} & \begin{tabular}{|l|} 
Tracer \\
Yifild $\%$
\end{tabular} & $\begin{array}{l}\text { Spike } \\
\text { Hecv } x\end{array}$ & \begin{tabular}{|l|} 
System \\
Detector
\end{tabular} & $\begin{array}{l}\text { Packat-Herm } \\
\text { Sample }\end{array}$ \\
\hline DECON W3-6 & $K 40$ & GAM20 & $1.87 E+01$ & $2.1 E+01$ & & $1.1 E+00$ & pCilg & $06-09-98$ & OB-08-98 & $8.39 E+02$ & $\mathrm{gm}$ & SOIL G & & & 05-01 & G9135-0-75099 \\
\hline DECON W3-6 & RA226 & GAM20 & $1.15 E+00$ & $3.1 E+01$ & & $1.5 \mathrm{E}-01$ & $\mathrm{pCi} / \mathrm{g}$ & $06-09-98$ & 06-08-98 & $8.39 E+02$ & $\mathrm{gm}$ & soll G & & & 05.01 & G9135-0.75099 \\
\hline DECON W3-6 & SB125 & GAM20 & $2.53 E+00$ & $4.2 \mathrm{E}+01$ & & 6.3E-01 & $\mathrm{pCi} / \mathrm{g}$ & $06-09-98$ & $08-08-98$ & $8.39 E+02$ & $\mathrm{gm}$ & soll G & & & 05-01 & G9135-0-75099 \\
\hline DECON W3-6 & TH228 & GAM2O & $1.66 E+\infty$ & $2.6 \mathrm{E}+01$ & & $1.6 \mathrm{E}-01$ & $\mathrm{pCi} / \mathrm{g}$ & 08-09-98 & $06-08-98$ & $|8.39 E+02|$ & $\mathrm{gm}$ & SOHL G & & & 05-01 & G9135-0.75099 \\
\hline DECON W3-6 & TH232 & GAM20 & $1.26 \mathrm{E}+\infty 0$ & $4.3 E+01$ & & 4.0E-01 & $\mathrm{pCi} / \mathrm{g}$ & 06-09-98 & $08-08-98$ & $|8.39 E+02|$ & $\mathrm{gm}$ & SOLL G & & & 05-01 & G9135-0.75099 \\
\hline OA BKG EMPTY BTL UO5 & No Nucl Det & GAM20 & $0.00 E+00$ & $0.0 E+\infty$ & $\mathrm{u}$ & $0.0 \mathrm{E}+00$ & NA & $06-09-98$ & $07-01-95$ & $1.00 E+\infty$ & 䖪 & QUAL & & & $05-01$ & $00539-0.00213$ \\
\hline OA BKG EMPTY BTL VO5 & AM241 & GAM20 & $0.00 \mathrm{E}+00$ & $0.0 E+00$ & u & $2.2 E+01$ & $\mathrm{pCi}$ & $06-09-98$ & $07.01-95$ & $|1.00 E+00|$ & $\mathrm{sm}$ & QUAL & & & 05.01 & $00539-0.00213$ \\
\hline QA BKG EMPTY BTL U05 & CO60 & GAM20 & $0.00 E+\infty$ & $0.0 \mathrm{E}+00$ & $\mathrm{U}$ & $1.3 \mathrm{E}+01$ & $\mathrm{pCi}$ & 06-09-98 & $07-01-95$ & $1.00 E+\infty 0 \mid$ & $\operatorname{sm}$ & QUAL & & & 05-01 & $00539-0-00213$ \\
\hline OA BKG EMPTY BTL U05 & $\operatorname{cs} 137$ & GAM20 & $0.00 E+00$ & $0.0 \mathrm{E}+\infty 0$ & $\mathrm{u}$ & $8.0 \mathrm{E}+00$ & $\mathrm{pCi}$ & $06-09-98$ & $07.01-95$ & $|1.00 E+\infty 0|$ & $\mathrm{sm}$ & QUAL & & & 05.01 & $00539 \cdot 0-00213$ \\
\hline QA SP:NAS-A0271 & AM241 & GAM20 & $1.80 E+05$ & B.8E $+\infty$ & & $2.9 \mathrm{E}+02$ & $\mathrm{pCi}$ & $06-09-98$ & $04-01-92$ & $|1.00 E+00|$ & $\mathrm{sm}$ & QUAL & & 111.4 & $05-01$ & |00534-3-14536 \\
\hline
\end{tabular}

Comment:

Data generated from analyses of samples submitted on 6/9/98.

Gross alpha/beta determinations are performed on small sample.

aliquots. Data may nof represent sample activity as a whole.

Prepared by:

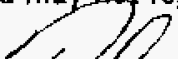

Approved by

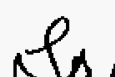

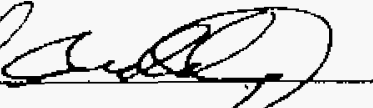

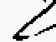
Date

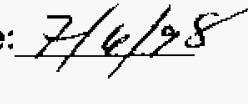
Date: $7 / 6 / 98$
Qualification Flags:

$E=$ Estimated Quantity

$H=$ High Recovery for Sample

$\mathrm{J}=$ Result is less than the RDL

$L=$ Low Recovery for Sample

$P=$ Preliminary Results

$\mathbf{Q}=$ Bad Instrument Quality Control, Result is OK

$\mathbf{R}=$ Results are Unusable, Resampling is Necessary

$\mathrm{U}=$ Result is less than Minimum Detectable Activity 


\section{Beuhtel Nevada Corporation}

ANALYTICAL SERVICES LABORATORY

P.O.Box 3936, N. Las Vegas, NV 89036

Page 3

Reported to: Area 6 Decon Pond Closure

Remediation Projects

J. L. Smith, M/S NTS306

Report Date: 6-JUL-98

Sample Delivery Group: B447

Batch: 0821

Program: 117

Report No. :

\begin{tabular}{|c|c|c|c|c|c|c|c|c|c|c|c|c|c|c|c|c|}
\hline $\begin{array}{c}\text { Sample } \\
\ldots \ldots+\cdots \\
\text { Identification } \ldots . . .\end{array}$ & Isotope & Analysis & Fesult & $\begin{array}{l}\text { Error } \\
\%\end{array}$ & \begin{tabular}{|l|} 
Qusl \\
Flag
\end{tabular} & MDA & \begin{tabular}{|l} 
Result \\
Units
\end{tabular} & $\begin{array}{c}\text { Analysis } \\
\text { Dato }\end{array}$ & \begin{tabular}{|l|} 
Sample \\
Coll Date
\end{tabular} & Siza & \begin{tabular}{|c|} 
Size \\
Units
\end{tabular} & \begin{tabular}{c|} 
Type \\
Matrix
\end{tabular} & $\begin{array}{l}\text { Tracer } \\
\text { Yiald \% }\end{array}$ & \begin{tabular}{|l|} 
Spike \\
Reev $\%$
\end{tabular} & $\begin{array}{l}\text { System } \\
\text { Detector }\end{array}$ & $\begin{array}{l}\text { Packet-ftem } \\
\text { Sample }\end{array}$ \\
\hline DA SP:NAS-A027 $\uparrow$ & $\cos 0$ & GAM20 & $3.05 E+05$ & $8.5 E+00$ & & $2.2 \mathrm{E}+02$ & $\mathrm{pCi}$ & $06-09.98$ & $04-01-92$ & $1.00 E+00 \mid$ & $\mathrm{sm}$ & QUAL & & 102.4 & 05-01 & $00534-3-14536$ \\
\hline OA SP:NAS-A0271 & $\operatorname{cs} 137$ & GAM20 & $2.06 E+05$ & $8.1 E+\infty 0$ & & $7.4 E+01$ & $\mathrm{pCi}$ & $06-09-98$ & $04-01-92$ & $|.00 E+\infty|$ & $\mathrm{sm}$ & |avAl & & 101.4 & 05-01 & $00534-3-14536$ \\
\hline
\end{tabular}

Comment:

Data generated from analyses of samples submitted on 6/9/98.

Gross alpha/beta determinations are performed on small sample

aliquots. Data may nof represent sample activity as a whole.

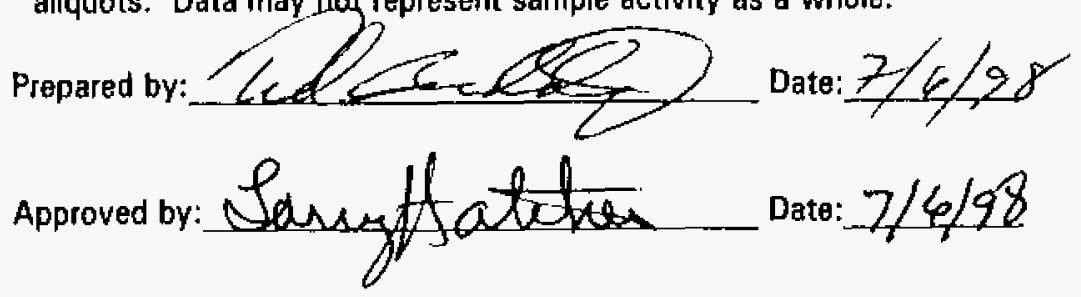

Qualification Flags:

$E$ = Estimated Quantity

$H=$ High Recovery for Sample

$J=$ Result is less than the RDL

$L=$ Low Recovery for Sample

$P=$ Preliminary Results

$\mathrm{Q}=$ Bad Instrument Quality Control, Result is OK

$\mathbf{R}=$ Results are Unusable, Resampling is Necessary

$\mathbf{U}=$ Result is less than Minimum Detectable Activity
Note: \% Error is the 2.0 Sigma Error 


\section{Bechtel Nevada Corporation}

ANALYTICAL SERVICES LABORATORY

P.O.Box 3936, N. Las Vegas, NV 89036

Reported to: Area 6 Decon Pond Closure

Remediation Projects

Report Date: 6-JUL-98

J. L. Smith, M/S NTS306

Sample Delivery Group: B447

Batch: M559

Program: 720

Report No. :

\begin{tabular}{|c|c|c|c|c|c|c|c|c|c|c|c|c|c|c|c|c|}
\hline 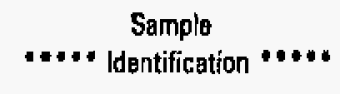 & Isotope & Analysis & Result & $\begin{array}{c}\text { Error } \\
\%\end{array}$ & $\begin{array}{l}\text { Oual } \\
\text { Flag }\end{array}$ & MDA & $\begin{array}{l}\text { Result } \\
\text { Units }\end{array}$ & $\begin{array}{c}\text { Analysis } \\
\text { Date }\end{array}$ & $\begin{array}{l}\text { Sample } \\
\text { Coll Date }\end{array}$ & Size & \begin{tabular}{|l|} 
Size \\
Units
\end{tabular} & \begin{tabular}{|c|} 
Type \\
Matrix
\end{tabular} & $\begin{array}{l}\text { Tracerr } \\
\text { Yieild \% }\end{array}$ & $\begin{array}{l}\text { Spike } \\
\text { Recr \% }\end{array}$ & \begin{tabular}{|l|} 
System \\
Detector
\end{tabular} & $\begin{array}{c}\text { Packet-Item } \\
\text { Sample }\end{array}$ \\
\hline DECON P11-6 & Gross Alpha & Gross A & $1.39 \mathrm{E}+01$ & $1.8 E+01$ & & $2.2 \mathrm{E}+00$ & $\mathrm{pClig}$ & 07-04-98 & $06-08-98$ & $5.00 \mathrm{E}-01$ & $\mathrm{gm}$ & SOlL 6 & & & 14-01 & $69136-1.75100$ \\
\hline DECON W3-6 & Gross Alpha & Gross A & $7.13 \mathrm{E}+00$ & $2.4 E+01$ & & $1.8 E+00$ & pCils & $07-03-98$ & $08-08-98$ & 5.00E-01 & $\mathrm{gm}$ & soll G & & & $14-01$ & G9135-0.75098 \\
\hline QA REA & Gross Alpha & Gross A & 1.65E-02 & $1.4 E+03$ & u & $4.2 \mathrm{E}-01$ & $\mathrm{PCi}$ & 07-02-98 & $06-02-98$ & $1.00 E+\infty \mid$ & $\mathrm{sm}$ & QUAL & & & $14-01$ & G9100-0-06776 \\
\hline OA SP:GROSSAB 05 & Gross Alphe & Gross A & $3.38 E+\infty 0$ & $1.6 \mathrm{E}+01$ & & $4.2 \mathrm{E}-01$ & $\mathrm{pCi}$ & 07-02-98 & $01.06-98$ & $|1.00 E+\infty|$ & $\mathrm{sm}$ & QUAL & & 98.6 & 14-01 & 69100-1.06777 \\
\hline DECON P11-6 & Gross Beta & Gross B & $1.20 E+01$ & $1.3 \mathrm{E}+01$ & & $2.2 \mathrm{E}+00$ & $\mathrm{pC} i / g$ & $07-04-98$ & $06-08-98$ & $5.00 \mathrm{E}-01$ & $9 m$ & solt G & & & $14-01$ & $69135-1.75100$ \\
\hline DECON W3-6 & Gross Bata & Gross B & $1.95 E+01$ & $9.0 \mathrm{E}+00$ & & $2.2 E+\infty$ & $\mathrm{pCi} / \mathrm{\theta}$ & $07-03-98$ & $06.08-98$ & $5.00 \mathrm{E}-01$ & $\mathrm{gm}$ & Solt $\mathrm{G}$ & & & 1401 & $69135-0.76098$ \\
\hline DA REA & Gross Beta & Gross B & $-2.67 E-01$ & $2.1 E+02$ & 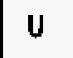 & $9.8 \mathrm{E}-01$ & $\mathrm{pCi}$ & 07-02-98 & $06-02-98$ & $1.00 \mathrm{E}+\infty 0$ & $\mathrm{sm}$ & QUAL & & & $14-01$ & G9100-0.06776 \\
\hline OA SP:GROSSAB & Gross Bela & Gross B & $4.65 E+\infty$ & $1.5 \mathrm{E}+01$ & & $9.8 \mathrm{E}-01$ & $\mathrm{pCi}$ & $07-02-98$ & $01.06-98$ & $|1.00 E+00|$ & $5 \mathrm{~m}$ & OUAL & & 89.0 & $14-01$ & G9100-1-06777 \\
\hline
\end{tabular}

\section{Comment:}

Data generated from analyses of samples submitted on 6/9/98.

Gross alpha/beta determinations are performed on small sample

aliquots. Data may not represent sample activity as a whole.

Prepared by:

Approved by:
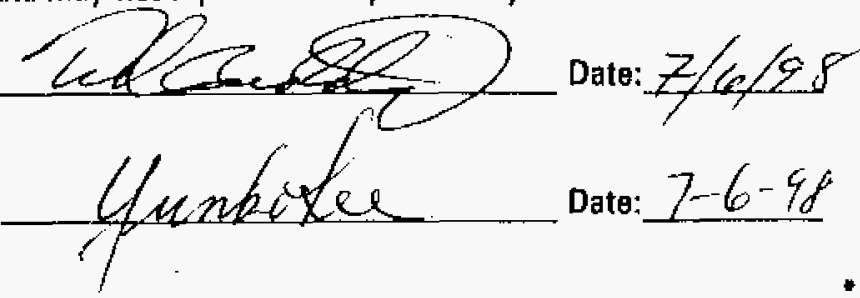

\section{Qualification Flags:}

$E=$ Estimated Quantity

$H=$ High Recovery for Sample

$J=$ Result is less than the RDL

$\mathrm{L}=$ Low Recovery for Sample

$P=$ Preliminary Results

$\mathrm{Q}=$ Bad Instrument Ouality Control, Result is OK

$\mathrm{R}=$ Results are Unusable, Resampling is Necessary

$\mathbf{U}=$ Result is less than Minimum Detectable Activity
Note: \% Error is the 2.0 Sigma Error 


\section{Bechtel Nevada Corporation}

ANALYTICAL SERVICES LABORATORY

P.O.Box 3936, N. Las Vegas, NV 89036

Page 1

Feported to: Remediation Projects

Area 6 Decon Pond Closure

J. L. Smith

M/S NTS306

Report Date: 8-SEP-98

Sample Delivery Group: B649

Batch: 0841

Program: 720

Report No. :

\begin{tabular}{|c|c|c|c|c|c|c|c|c|c|c|c|c|c|c|c|c|}
\hline $\begin{array}{c}\text { Sarmple } \\
\ldots \ldots\end{array}$ & Isotope & Analysis & Rasult & $\begin{array}{l}\text { Error } \\
\%\end{array}$ & $\begin{array}{l}\text { Oulal } \\
\text { flag }\end{array}$ & MDA & $\begin{array}{l}\text { Fesult } \\
\text { Uniits }\end{array}$ & $\begin{array}{l}\text { Analysis } \\
\text { Data }\end{array}$ & $\begin{array}{l}\text { Sample } \\
\text { Coll Date }\end{array}$ & Size & $\begin{array}{r}\text { Size } \\
\text { Units }\end{array}$ & $\begin{array}{c}\text { Type } \\
\text { Matrix } \\
\end{array}$ & 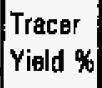 & $\begin{array}{l}\text { Spikg } \\
\text { Recv \% }\end{array}$ & $\begin{array}{l}\text { System } \\
\text { Detector }\end{array}$ & $\begin{array}{l}\text { Packet-Item } \\
\text { Sampla }\end{array}$ \\
\hline DECON P3-7 & $K 40$ & GAM20 & 2.35E +01 & $1.9 \mathrm{E}+01$ & & $1.2 E+00$ & $p C i / \theta$ & 08.04-98 & $07-28-98$ & $6.74 E+02$ & $\mathrm{gm}$ & soll G & & & 05.01 & $69511-0-75230$ \\
\hline DECON P3-7 & TH228 & GAM2O & $2.65 E+00$ & $1.8 \mathrm{E}+01$ & & $1.7 \mathrm{E}-\mathrm{D} 1$ & $\mathrm{pCi} / \mathrm{q}$ & $08-04-98$ & 07-28-98 & $6.74 E+02$ & gm & soll G & & & 05.01 & G9511-0-76230 \\
\hline QA BKG EMPTY BTL U05 & No Nucl Det & GAM20 & $0.00 E+00$ & $0.0 E+00$ & $u$ & $0.0 \mathrm{E}+\infty 0$ & NA & 08-04-98 & 07-01-95 & $1.00 E+\infty$ & gm & QUAL & & & $05-01$ & $00539-0-00264$ \\
\hline OA BKG EMPTY BTL U05 & AM241 & GAM 20 & $0.00 E+00$ & $0.0 E+\infty 0$ & u & $2.2 E+01$ & $\rho \mathrm{Ci}$ & 08-04-98 & 07.01-95 & $1.00 E+\infty 0$ & $\mathrm{sm}$ & OUAL & & & $05-01$ & $00539-0-00284$ \\
\hline OA BKG EMPTY BTL U05 & $\operatorname{co60}$ & GAM2O & $0.00 E+00$ & $0.0 E+00$ & $\mathrm{U}$ & $7,9 E+00$ & $\mathrm{pCi}$ & 08-04-98 & |07-01-95 & $1.00 E+\infty$ & $8 m$ & QUAL & & & 05.01 & $00539-0-00284$ \\
\hline QA BKG EMPTY BTL U05 & $\operatorname{CS} 137$ & GAM20 & $0.00 E+00$ & $0.0 E+00$ & u & $1.0 E+01$ & $\mathrm{pCi}$ & $08-04-98$ & $07-01-95$ & $1.00 E+\infty$ & $\mathrm{sm}$ & QUAL & & & $05-01$ & $00539 \cdot 0-00264$ \\
\hline OA SP:NAS-A0271 & AM241 & GAM20 & $1.80 E+05$ & $8.8 E+\infty 0$ & & $2.9 \mathrm{E}+02$ & $\mathrm{pCi}$ & $08-04-98$ & $04.01 \cdot 92$ & $1.00 E+\infty$ & $\operatorname{sm}$ & QUAL & & 110.8 & $05-01$ & $00534-3-15214$ \\
\hline QA SP:NAS-A0271 & $\cos 0$ & GAM20 & $3.04 \mathrm{E}+05$ & $8.5 E+00$ & & $1.3 \mathrm{E}+02$ & $\mathrm{pCi}$ & $08-04-98$ & 04-01-92 & $1.00 E+00$ & $5 m$ & OUAL & & 101.9 & $05-01$ & $00534-3 \cdot 15214$ \\
\hline OA SP:NAS-A0271 & $\operatorname{cs} 137$ & GAM20 & $2.07 E+05$ & 8.TE +00 & & 9.8E +01 & $\mathrm{pCi}$ & 08-04-98 & $04-01-92$ & $1.00 \mathrm{E}+00$ & $\mathrm{sm}$ & OUAL & & 101.9 & 05.01 & $00534-3-15214$ \\
\hline
\end{tabular}

\section{Comment:}

Data generated from analyses of a sample submitted 7/29/98.

The gross alpha/beta determination was performed on a small

aliquot. The data reflect strictly that portion analyzed.

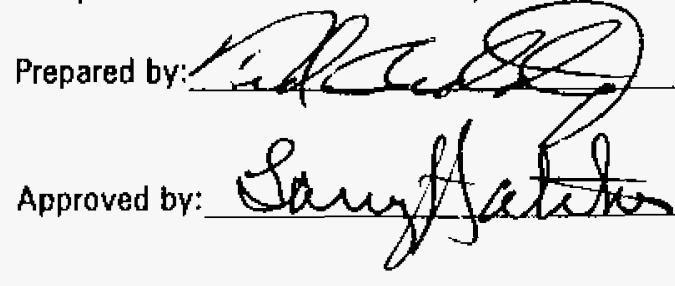

Qualification Flags:

$E=$ Estimated Quantity

$H=$ High Recovery for Sample

$J=$ Result is less than the RDL

$L=$ Low Recovery for Sample

$P=$ Preliminary Results

$\mathrm{O}=$ Bad Instrument Quality Control, Result is OK

$\mathrm{R}=$ Results are Unusable, Resampling is Necessary

$\mathrm{U}=$ Result is less than Minimum Detectable Activity 


\section{Bechtel Nevada Corporation}

ANALYTICAL SERVICES LABORATORY

P.O.Box 3936, N. Las Vegas, NV 89036

Page 2

Reported to: Remediation Projects

Area 6 Decon Pond Closure

J. L. Smith

M/S NTS306

Report Date: 8-SEP-98

Sample Delivery Group: B649

Batch: M668

Program: 720

Report No. :

\begin{tabular}{|c|c|c|c|c|c|c|c|c|c|c|c|c|c|c|c|c|}
\hline $\begin{array}{c}\text { Sample } \\
\ldots . . . \text { Identification ..... }\end{array}$ & Isotope & Analysis & Result & $\begin{array}{c}\text { Error } \\
\%\end{array}$ & $\begin{array}{l}\text { Oual } \\
\text { Flág }\end{array}$ & MDA & $\begin{array}{l}\text { Result } \\
\text { Units }\end{array}$ & $\begin{array}{c}\text { Analysis } \\
\text { Date }\end{array}$ & \begin{tabular}{|l} 
Sample \\
Coll Datg
\end{tabular} & Sizo & $\begin{array}{l}\text { Size } \\
\text { Units }\end{array}$ & \begin{tabular}{|l|} 
Type \\
Matrix
\end{tabular} & \begin{tabular}{|l|} 
Tracer \\
Yield \%:
\end{tabular} & \begin{tabular}{|l} 
Spike \\
Pecv \%
\end{tabular} & $\begin{array}{l}\text { System } \\
\text { Detector }\end{array}$ & $\begin{array}{l}\text { Packet-Item } \\
\text { Sample }\end{array}$ \\
\hline DECON P3-7 & Gross Alpha & Gross A & $1.15 E+01$ & $1.6 \mathrm{E}+01$ & & $1.5 E+00$ & $\mathrm{pCi} / \mathrm{g}$ & 09-03-98 & $07-28-98$ & $1.00 E+\infty 0$ & $\mathrm{gm}$ & soll. G & & & $14-01$ & G9511-0-75229 \\
\hline OA REA & Gross Alpha & Gross A & 1.32E-01 & $1.7 \mathrm{E}+02$ & $\mathrm{U}$ & 4.5E-01 & $\mathrm{pCi}$ & |09-02-98 & 07-31-98 & $1.00 E+\infty 0$ & $8 m$ & OUAL. & & & $14-01$ & G9512-0.06794 \\
\hline QA SP:GROSSAB 05 & Gross Aipha & Gross A & 4.D7E +00 & $1.4 \mathrm{E}+01$ & & $4.5 E-01$ & $\mathrm{pCi}$ & $09-02-98$ & 05-12-94 & $1.00 E+\infty \mid$ & $\mathrm{sm}$ & OUAL & & 118.8 & $14-01$ & G9512-1-06795 \\
\hline DECON P3-7 & Gross Beta & Gross B & $1.04 E+01$ & $8.7 \mathrm{E}+00$ & & $1.1 E+\infty$ & $\mathrm{pCi} / \mathrm{g}$ & $09-03-98$ & $07.28-98$ & $1.00 E+00$ & $\mathrm{gm}$ & Soll G & & & $14-01$ & G9511-0.75229 \\
\hline QA REA & Gross Beta & Gross B & $-1.54 E-01$ & $3.7 \mathrm{E}+02$ & $\mathrm{U}$ & 9.7E-01 & $\rho \mathrm{Ci}$ & |09-02-98 & |07.31-98 & $1.00 E+00 \mid$ & $\operatorname{sm}$ & QUAL. & & & $14-01$ & 69512-0-06794 \\
\hline QA SP:GROSSAB $\quad 05$ & Gross Bata & Gross 8 & $5.54 E+\infty 0$ & $1.4 \mathrm{E}+01$ & & $9.7 \mathrm{E}-01$ & $\mathrm{pCi}$ & |09-02-98 & 05-12-94 & $1.00 E+\infty \mid$ & $\mathbf{s m}$ & QUAL & & 106.0 & 14-01 & G9512-1.06795 \\
\hline
\end{tabular}

\section{Comment:}

Data generated from analyses of a sample submitted 7/29/98.

The gross alpha/beta determination was performed on a small

aliquot. The data reflect strictly that portion analyzed.

Prepared by:

Approved by: Hemanas Date: $9 / 9 / 98$
Qualification Flags:

$\mathrm{E}=$ Estimated Quantity

$H=$ High Recovery for Sample

$\mathrm{J}=$ Result is less than the RDL

$\mathrm{L}=$ Low Recovery for Sample

$\mathrm{P}=$ Preliminary Results

$\mathrm{Q}=$ Bad Instrument Quality Control, Result is OK

$\mathbf{R}=$ Results are Unusable, Resampling is Necessary

$\mathbf{U}=$ Result is less than Minimum Detectable Activity 


\section{$19806156-617$}

Bechrel Nevada

ANALYTICAL SERVICES LABORATORY

SERVICES REQUEST \& CHAIN OF CUSTODY RECORD

PROJECTI CLIENT INFORMATION

REPORT INFORMATION

Project: Decon Pond CLCSURE GN Org ZISZ send Report to -JEFF SMTTH

Charge No: $C 44-5 B \phi D E$

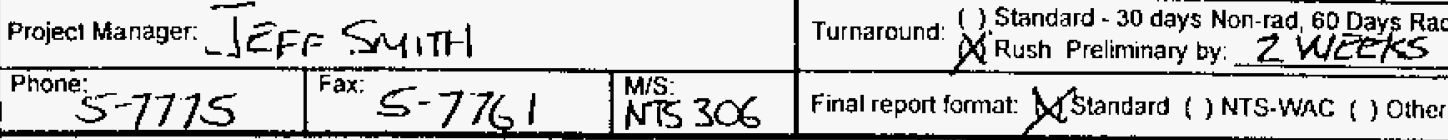

\begin{tabular}{|c|c|c|}
\hline Rat SGD: & $\frac{\text { USE ONLY }}{\text { Non-Rad SDG: }}$ & \\
\hline Rad Packel: & Non-Ract Packet: & Wi4to6 \\
\hline
\end{tabular}

Client Services Representative

Will these analyses be performed under a signed SOW? () YES () NO

If so, do analyses entered here agree with the SOW? () YES (\}) NO ( ) N/A

If not, identify the variation

CSR initials indicating review and approval:

Date:

\begin{tabular}{l|l}
\hline & ID / DESCRIPTION \\
\hline
\end{tabular}

O) DECON W3-1A4B

Q 1 DECON W3-2

Q 2 DECON W $3-3$

G 3 Decon $W / 3-4 A \pm B$

Q -4 DeCONW $3-5$

eft 5 DeCON W3-6

CO DECONP11-1TB

02 Decon P $11-1 A \neq B$

of 8 DECONP11-2

Of 9 DECON PII-3

Transfer of samples submitted for analyses

Sampled/Relinquisfed (Signature/Organization) DATE / TIME Received by (Signature/Organization) Land H. Cor $18 N$

\begin{tabular}{|c|c|c|}
\hline \multicolumn{2}{|c|}{ SAMPLING } & \multirow{2}{*}{ MATRIX } \\
DATE & TIME & \\
\hline
\end{tabular}

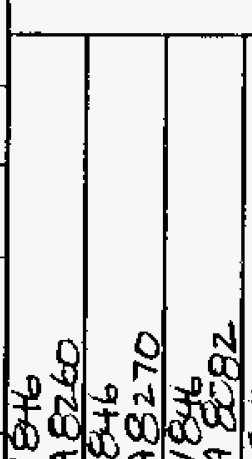

ANALYSES \& METHOD NTS 306

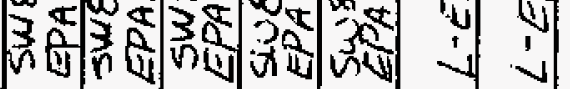

Finat by:

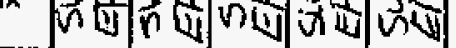

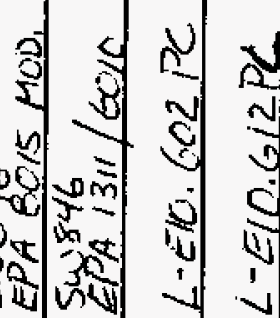

\section{SAMPLE INFORMATIO DECON FACKITY \\ De \\ The samples submitted contan (check), \\ () Hazardous $\$$ Radioactive Xunknown \\ contamination. If known, attach a brief narrative summary \\ identifying contaminants. This information will ensure \\ compliance with applicable regulations and allow for the safe handling of the sample materials.}

Page 1 of 2

618601115

$6 / 8-1115$

$6 / 1115$ 6. 115 SWTOGE $6 / 81115$ SUDGE $6 / 8$ ill5 SLuDG $6 / 8 G 40900$ WATR $X$ $6 / 841153012$ $68 \quad 145 \mathrm{SOI}$ $6 \longdiv { 8 } 1 4 1 5$ Sol $7+2$

SLIDGE $X^{\prime}$ SUUPGE $x$

\begin{tabular}{|l|l|l|}
\hline & & \\
\hline & & \\
\hline & & \\
\hline
\end{tabular}

$x$

\section{SAMPLE RECEIPT IMFORMATION} Are all sample containers received intact 4 Yes ( I wo Comments:

Do the labels agree with this form? MYYes ( ) No Comments:

GYes ( No

C......

Was a Material Clearance Tay subrnitted? OC $\mathrm{Pas}(\mathrm{No}$ Comments:

-

I $\quad 01$ ace $250 \mathrm{~m}$

Complete for samples shipped to an ofF-SITE Subcontract Laboratory \begin{tabular}{l|c|l}
\hline Relinquished (BN Representative Signature) & DATE / TMME & Received (Courier \& Tracking Into.)
\end{tabular} CLAatroude Relinquished (Courier \& Tracking Info.) BN COURIER Relinquished (1st tier Subcontractor Rep)
610/48/1300 BN COULLER DATE TIME Receivge (1st tigs Subsentractor Rep) 6/10/95/5a

DATE / TIME
COMMENTS

(Preservative, sizelvolume, MSMso, spectal anahysls, rad matrix code, count time, etc.)

$I \angle E+\angle 20 \mathrm{~m} /(2)$

ICE, $500 \mathrm{Ml}$

ICE, $250 M \mid-A M B C R$

ICE, $250 \mathrm{ml}, 120 \mathrm{ml}$

ICE, $250 \mathrm{~m} 1$

ICE $500 \mathrm{Mu}$

$40 \mathrm{~m}$

ICE $120 \mathrm{ml}(\mathrm{z})$

Ret scoml 


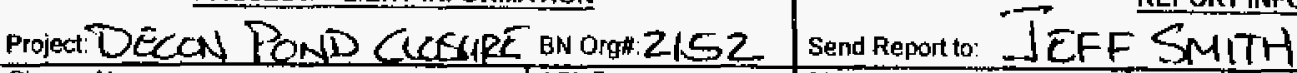

\section{Charge No C $4 U$ - SBQDE}

Project Manager: $-\widehat{J E F F} S \mathrm{~N} T H$

\section{Phone: $5-7775$ Fax: $^{\text {F }}-7761$} ASL Prog:

\section{Phone $5-7775 \mid{ }^{\text {Fax: }} 5-7761$}

$T^{M / S}$ NTS- 306 Turnaround: S Standard - 30 days Non-rad, 60 Days Rad, Other:

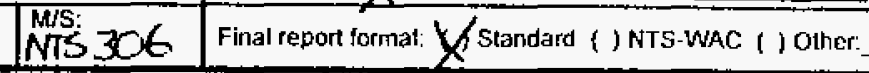
nal by:
SAMPLEINFORMATION Sampling site: DECON. FACILITY The samples subrilted contain (check) () Hazandous () Radioactive contaminalion. If knowm, attact a bref narrative summary identifying contaminants. This information will ensure compliance with applicable regulations and allow for the sate handling of the sample materiats.

\begin{tabular}{|c|c|c|}
\hline$B 447$ ․ & $\frac{\text { USE ONLY }}{\text { Non-Rad SOG: }}$ & W435 \\
\hline Rad Packet $69135-1$ & Nen-Rad Packet: & w1466 \\
\hline
\end{tabular}

Client Services Representabive:

Will these analyses be performed under a signed SOW () YES () NO II so, do anatyses entered here agree with the SOW? () YES () NO () NAA If not, identify the variation

CSR initials indicating review and approval: Date:

\begin{tabular}{|l|l}
\hline $\mathbf{T}$ & ID I DESCRIPTION \\
\hline
\end{tabular}

$+\frac{1}{10}$

.

11 DECONPI1-4A\#B

12 DECONP11-6

13 DECON R1-1

14 Decon R1-2

15 DeCoN R1-3

16 DESON R1-4

17 DECON RL -5

8

9

Transfer of samples submitted for analyses

Sampled/Reling pished (Signature/Organization) DATE / TIME Received by (Signature/Organization)

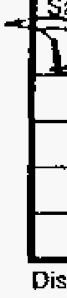

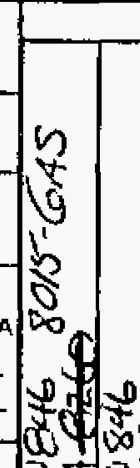

\section{6 isco watier $6 / 81500$ WATER}

ANALYSES \& METHOD

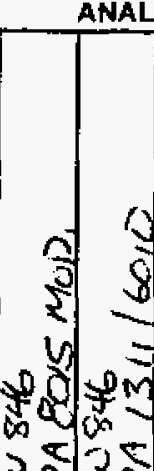

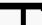

\section{SAMPLERECEIPT WFORMATION} Are all sample containers received intact $\overline{\text { CLYes }}$ ( ) No

Do the labels agree with this form? (Yyes i) No Comments:

Was a Material Clearance Tag submitted? (fyes ( ) No Comments:

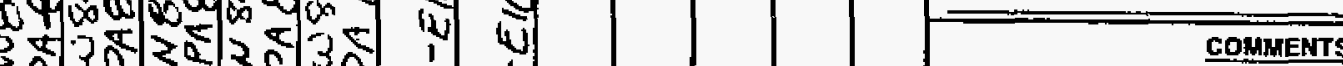

Preservative, stzejvolume, MSnsD.

10 special analysis, rad matrix code, count time, etc. ICE, $2 \mathrm{som}$, $120 \mathrm{ml}$ Ict $+250 m$ ICE, SOOM 1 LE, $694 \mathrm{~g}$ ICE, 1 LITER RCE, 1 LITER ICE, 1 LITER

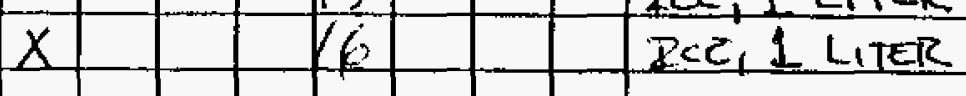

$x^{i}$ $x$ $x$ $x$
Camplete for samples shipped to an OFF-SITE Subcontract Laboratory Relinquished (BN Representative Signature) CAlantouedar Relinquished (Courier \& Tracking Into.) Dn Gorolen Relinquished (1st tier Subcontractor Rep) \begin{tabular}{|l|l}
\hline DATE / TIME & Received (Courler $\&$ Tracking Info.)
\end{tabular}

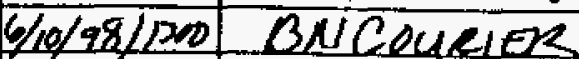

DATE TIME Received (1st ler sitgzontractor Rep) $0 / 10 / 0 ; 70$ DATE / TIME Received (2nd tie $\zeta$ Sobcontractor Rep) 
$6 \% 5 \% 6 / 5$

$6 / 17$

PROJECT/ CLLENT INFORMATION

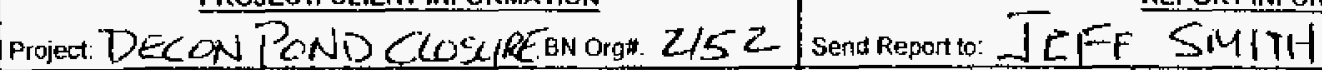

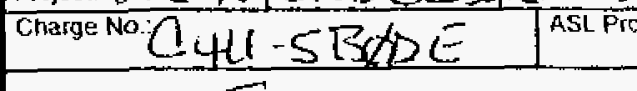
Projecl Manager: JeFF $S M \mid T H$ \begin{tabular}{|c|c|c|}
\hline Phone: $5-7775$ & $5-776.1$ & MiST \\
\hline
\end{tabular} Phone $57775 \mid$ Fax $5-7761$ $\prod^{\mathrm{M} / \mathrm{S}}$ NTS 306 Turnaround (3) Standard 30 days Non-rad, 60 Days Rad, Other Turnaround Xush Presiminary by: 2 WEeKS Final by: Final report format: (X) Standard () NTS.WAC o) Other:

by:

$$
1
$$

Paction

Client Services Representative

Will these analyses be performed under a signed sow? (I YES () NO if so, do analyses entered here agree with the SOW? (1) YES () NO () MAA

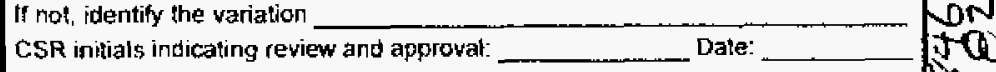
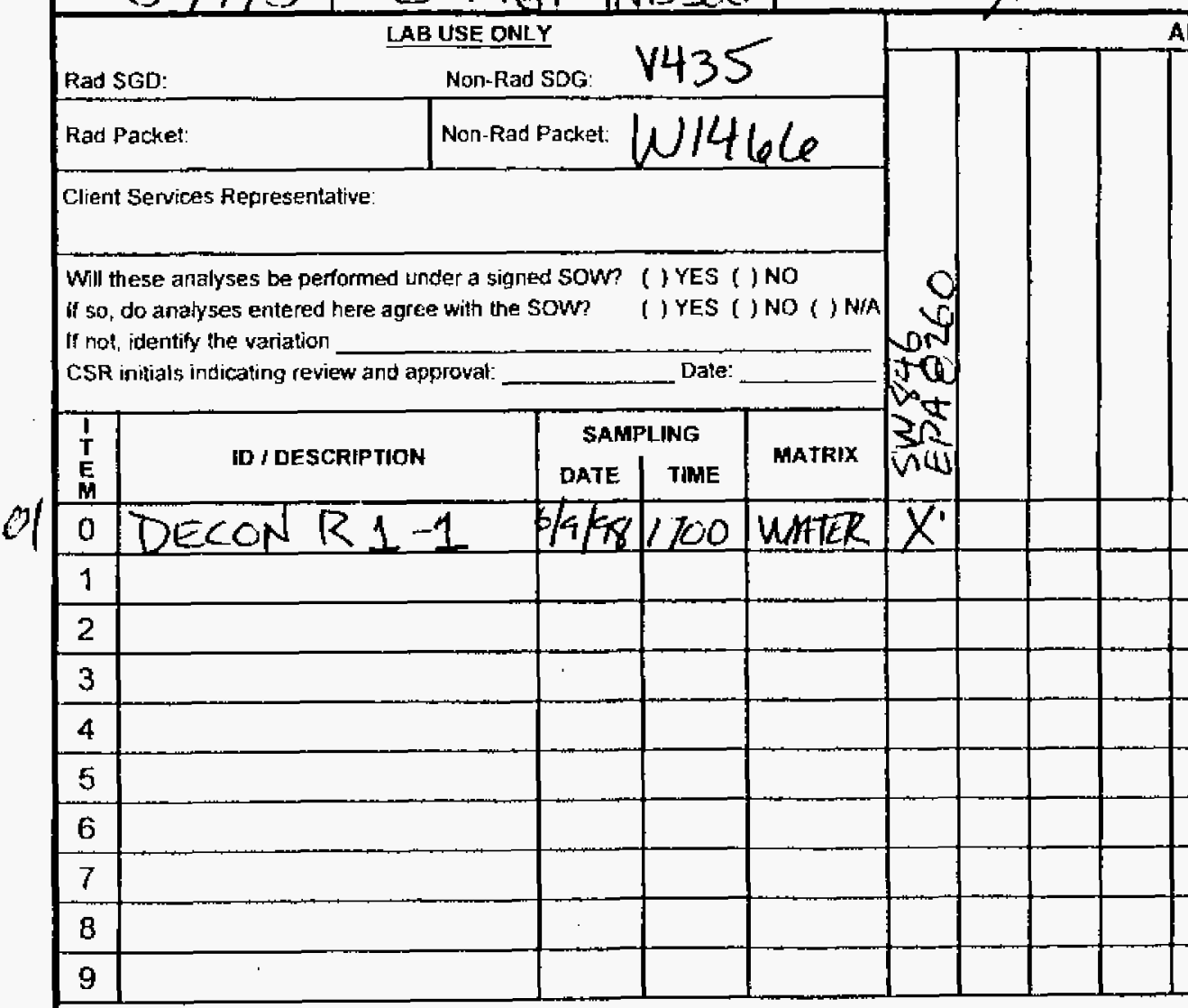

Transfer of samples submitted for anałyses

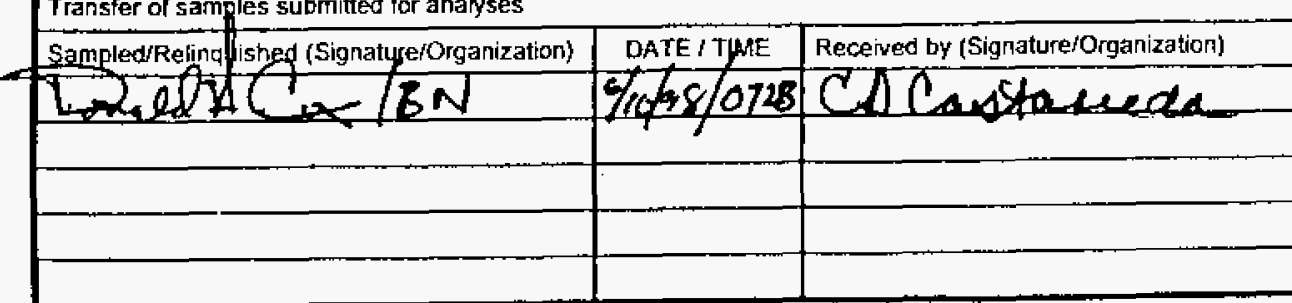
ANALYSES \& METHOD

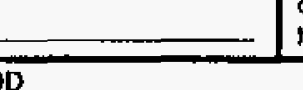

\section{SAMPLE INFORMATION Sampling site: DECON FACILTY The samples submitted contain (check): () Hazardous () Radioactive DUUnknown contamination. If krown, atlach a brief narrative summrnary identifying contarminants. This information will ensufe compliance with applicable regulations and allow tor the sale handling of the sample materials.}

SAMPLE RECEIPT INFORMATION
Are all sample containers received intact
Comments
Comments

Do the agree with this form? comments: __. _....._._._.

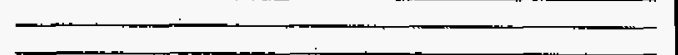
Was a Material Clearance Tag submitted? XXPes ( ) No Comments:

C.. COMHENTS (Preservative, sizelvolume, MSimsD,
pectal analysis, rad matrix code, count time, etc.)

Complete for samples shipped to an OFF-SITE Subcontract Laboratory Relinquighed (BN Representative Signature) Co Cattaneda 
Project DECON Pano CLOSURE ER Org: ZISZ Send Report to: JEFF SMITH

Charge No: $C 4 U-5 B \phi D E$

Project Manager: $\bar{I} \mathrm{CFF}_{\mathrm{FF}} \mathrm{S}$ MITH

\begin{tabular}{|c|c|}
\hline Phene: $5-7775$ & Fax: $5 \cdot 7761$ \\
\hline
\end{tabular}

\begin{tabular}{|c|c|c|}
\hline Rad SGD: $B 447$ & $\frac{\text { USE ONLY }}{\text { Non-Rad SDG: }}$ & \\
\hline Rad Packet $69135-0$ & Non-Rad Packet: & W1466 \\
\hline
\end{tabular}

Client Services Representative:

Wil these analyses be performed under a signed SOWn () YES ( ) NO

If so, do analyses entered here agree with the SOWh () YES ( ) NO () N/A

If not, identify the variation

CSR inlitals Indicating review and approval:______________ Date:

\begin{tabular}{|c|c|c|}
\hline in intorponon & SAMPLING & ATRYY \\
\hline - IDTUESCRIPIIUA & DATE $\mid$ TME & WAIRIX \\
\hline
\end{tabular}
5-7775 ||$^{\text {Fax: }} S-7761||^{\text {MS: }}$ NTS 306 Tumaround: ( Standard - 30 days Non-bad, 60 Days Rad, Other

X'Rush Prelliminary by: 2 Uiceks nal report fomat $D($ standard () NTS-WAC () Other.

ANALYSES \& METHOD

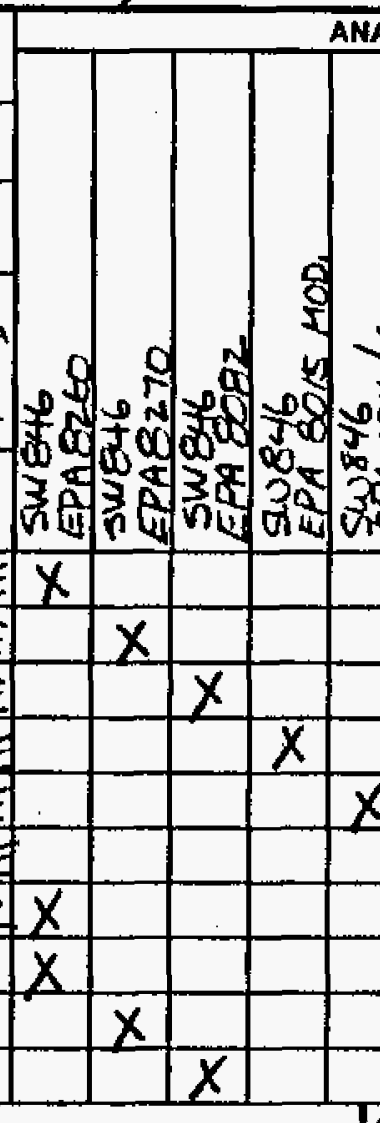
81415 SOIL $6 \longdiv { 8 } 1 4 1 5$ SOIL

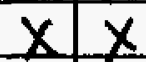

7 DECON PI1- IAAB

8 DECONPII-Z 9 DEcoNP11-3
SALPLE INFORMATON

SAMPLE INFORMATION
samping site: DECON FAC - TTY submitted onitain (check); () Hazardous OAdlaactivo OUntown contamination. If krowh, altach a brief narrative summary identifying contaminants. This information will engure complianca with applicable regutations and allow for the safe complianos with applicable regulation
handing of the stample materals.

SAMPLE RECEIPT INFORMATION Ars all sample containers recelved intact (JLYYes ( ) No Comments:

-

Do the labels agree with this form?
Comments:

Was a Materlal Clearance Tag aubmitted? GAYes ( ) No Comments:

$\longrightarrow$

COMMENTS

Preservative trelvofume, MSMisD. epecial anatyals, rad matrix code, coutnt tims, etc.) ICE, $120 \mathrm{~m} /(\mathrm{z})$

ICE, $500 \mathrm{M} 1$

ICE, ZSOMLAMBCR

$I C \bar{E}, 250 \mathrm{ml}, 120 \mathrm{ml}$

ICE, $250 \mathrm{~m} \mid$

$I C \overline{E_{1}}$ soom $40 \mathrm{ml}$.

ICE $120 \mathrm{~m} /(\mathrm{z})$

Ict, $500 \mathrm{ml}$

TIP 839

Complete for samples thipped to an OFF-sITE Subcontract Labortary

Transfer of samples submitted for analyses

DATE / TIME 1 Received by (Signature/Organization)

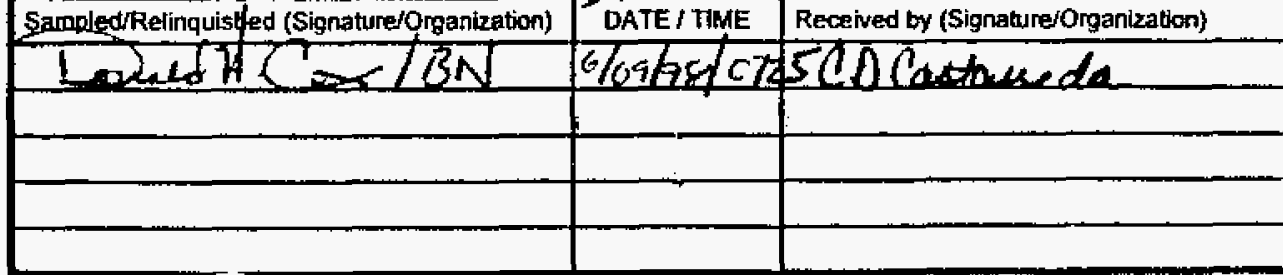

Relinquished (BN Representative Stgneture)

\begin{tabular}{|c|c|c|}
\hline Relinquished (BN Representalive Signature) & DATE / TIME & Recehved (Courler \& Tracking Info) \\
\hline Relinquished (Courier t Tracking Info.) & DATE / TIME & Riecelved (19t tior Subcouttractor Rop) \\
\hline Relinquished (1st tier Stbboontractor Rep) & DATE / TIME & Recefved (2nd ber Subcontractor Rep) \\
\hline & & . \\
\hline
\end{tabular}


PROJECT/ CLIENT INFORMATION

Propect DECON POND CLOSURE an Org*:2152 Send Report to: JEFF SMITH

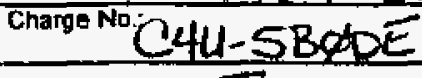

ÁsL Prog

Project Manager: JEFF $S M \pi H$

Turnaround: 2 Rush Prelminary by: 2 WMCEKS Final by:

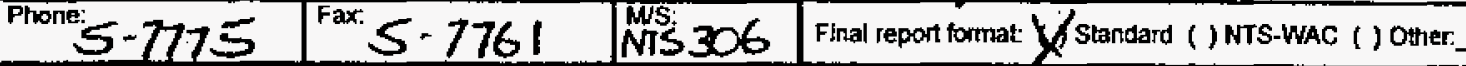

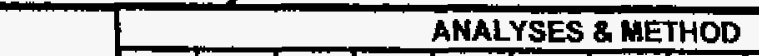

Rad SGD: $\quad 3447 \frac{\text { LAB USE ONLY }}{\text { Non-Rad SDG: }} \quad 1435$

\begin{tabular}{|l|l}
\hline Rad Packet 69135.1 & Non-Rad Packet: W1466 \\
\hline
\end{tabular}

Client Services Representative:

Will these analyses be performed under a signed SOW? () YES () NO If so, do analyses entered here agres wth the sown

If not, ldentify the variation

CSR inltiels indicating review and approval;

\begin{tabular}{|l|l|l|l|l}
\hline $\mathbf{T}$ & IO IDESCRIPTION & \multicolumn{2}{|c|}{ SAMPLINO } & MATRIX \\
\hline E & DATE & TMAE & MAT \\
\hline
\end{tabular}

10 DCCONP11-4AAB

11 DeronP11-5

12 DECONP11-6

13 DeCON R1-1

14 DECON R1-2

15 Decon R1-3

16 DECON R1-4

17 DECONRL-5

8

9

Transfer of samples submilted for analyses

\begin{tabular}{|l|l|l|}
\hline Sampled/Relingtished (Signature/Organization) & DATE / TME & Recaived by (Skgnature/Organization) \\
\hline
\end{tabular} beted h C C /BN
() YES (INO () NA Date:

6831415 Solt $6 / 8$ 14L Solt $6 / 8-1415$ SOL 46 isco WATER $6 / 8$ isOD WATER 6/8 1500 WATER 6/8 1500 WATER

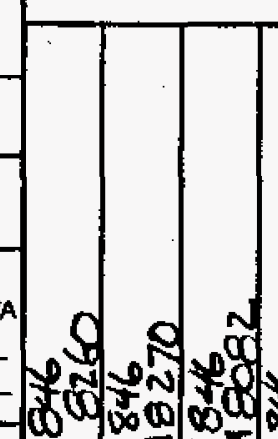
ANALYSES \& METHOD

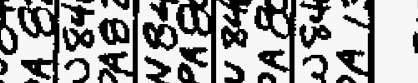

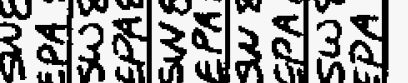

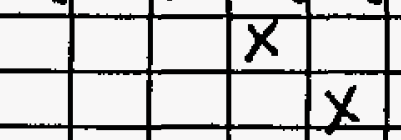

\section{$X$} $x$ $x$ $x$ $x$

$x$

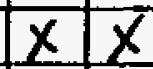
68 isQ

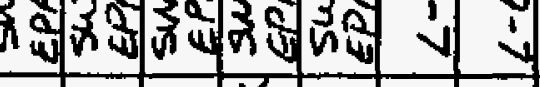

SAMPLE INFORMATIOH sampling site: DECON. FAKILITY The samples submitted contain (check): Xunk () Hazandous ( ) Radiaactive Xunknown contamination. If krown, altach a brief narrabve summany identitying contaminants. This Information will ensure compliance wth applicable regulations and allow for the safe handling of the sample materials. SAMPLE RECEIPT INAFORWATION
Are all sample containers recetved imbet XYYes ( ) No Cothiments:

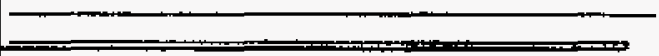

Do the labels egree with this form? Y Yes ( ) M
Comments:

Was a Materlat Clearance Tod submitted? YYYos ( ) No Comments:

COMLENTS

Preservethe comenTS mens,

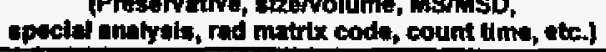

$I C \bar{c}, 250 \mathrm{~m} /, 120 \mathrm{ml}$

Ice, 2soml

ICE, 500 1

ILE, I LITER

ISC, 1 LITER

ICE, 1 LITER

ICR, 1 LATER

ICR, 1 LITER

\begin{tabular}{|c|c|c|c|c|c|}
\hline Sampled/Relinafished (Signature/Organization) & DATE / TIME & Recaived by (Skgnature/Organization) & Relinquished (BN Representalive Signature) & DATE / TIME & Recotved (Courier a Tracking Into.) \\
\hline & & & Relinqulshed (Courier \& Tracking lnio.) & DATE / TIME & Recehved (1st tler Subconttactor Rep) \\
\hline & & & & & \\
\hline & & & Relinquished (1st tier Suboontractor Rep) & DATE / TIME & Recoived (2nd tier Subcontractor Rep) \\
\hline & & & & & \\
\hline
\end{tabular}

Compiele for samples shipped to an OFF-sITE Subcontract Laboratory 


\section{- REPORT INFORMATION}

Send Report to: ICFF SMITHI

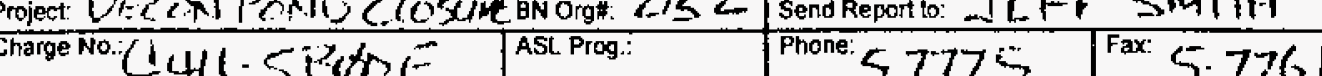

Project Manager: TEr $-5 M(17+1$

\begin{tabular}{l|l|l|}
\hline Phone: $; 7775$ & Fax: 5.7761 \\
\hline
\end{tabular}

\begin{tabular}{|c|c|}
\hline Phone: $:-7775$ & Fax: 5.7761 \\
\hline
\end{tabular}

Rad SGD: Non-Rad SOG:

Rad Packet Non-Rad Packet.

Client Services Representative

Will these analyses be performed under a signed SOW? () YES () NO It 50, do antises be perthere

If nol, tentify the vartation

CSR initials indicating revlew and approval: __ Date:

\begin{tabular}{|c|c|c|c|}
\hline 1 & ID/DESCRIPTION & SAMPLING & MATRIX \\
\hline
\end{tabular}

\begin{tabular}{|c|c|c|c|c|}
\hline 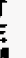 & ID / DESCRIPTION & DATE & TIME & MATRIX \\
\hline 0 & $14 C O N R 1-1$ & $/ / 1 / q$ & 170 & UMTR \\
\hline 1 & & & & \\
\hline 2 & & & & \\
\hline 3 & & & & \\
\hline 4 & & & & \\
\hline 5 & & & & \\
\hline 6 & & & & \\
\hline 7 & & & & \\
\hline 8 & & - & & \\
\hline & & & & \\
\hline
\end{tabular}

Transfer of samples submitted for analyses

Sampled/Relinquished (Signaturetorganizaton)

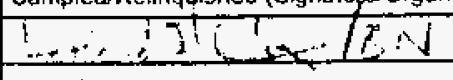

Rush Proliniays by -rad, 60 Days Rad, Other

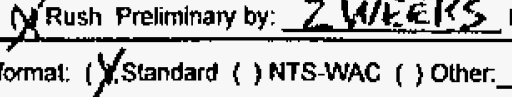

ANALYSES \& METHOD

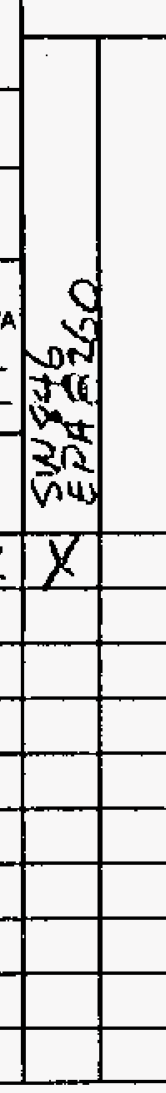

DATE
Complete for samples shipped to an OFF-SITE Subcontract Laboratory

\begin{tabular}{|l|c|l|}
\hline Retinquished (BN Representative Signature) & DATE / TIME & Received (Courler \& Tracking Info.) \\
\hline & & \\
\hline Relinquished (Courrier \& Tracking Inflo) & DATE / TIME & Recetved (1st Her Subcontbactor Rep) \\
\hline & & \\
\hline Relinquished (1st tier Subcontractor Rep) & DATE / TIME & Received (2nd tier Subcontractor Rep) \\
\hline & & \\
\hline
\end{tabular}
SAMPLE INFORMATION

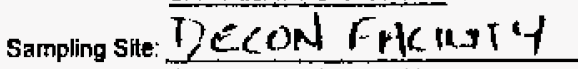
The sarnples submitted contath (cteck): () Hazardous () Radloactlve M Unknown contamination. is known, altach a brief narrative summary idenlifying contaminants. This intormatton will ensure compliance with applicable regulations and allow for the sate handling of the sample materials.

\section{SAMPLE RECEIPT INFORMATION}

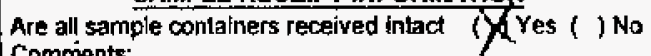
Comments

Do the labels agree with thls form? Comments: Mrres () No

Was a Material Clearance Tag submitted? M Yes ( ) Ho Comments:

COMMENTS

(Preservatlve, slzeivolume,-MSnSD,

speclal analysls, rad matrix code, count time, ete.) ICE $40 \mathrm{ml}$. 
PROJECTICLIENT INFORMATION

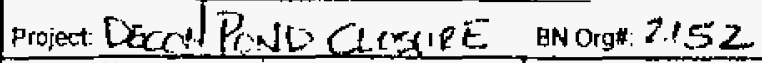
Charge No. Cif(L1-STZXTDE REPORT INFORMATION

Project Manager: TEFF SMTH

\begin{tabular}{|c|c|c|}
\hline Phone, 5.7725 & ${ }^{\mathrm{ax}} \leq-7761$ & Mis zoc \\
\hline
\end{tabular}

ANALYSES \& METHOD

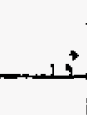

CSR initizls indicating review and approval: Date:

\begin{tabular}{|c|c|c|c|c|}
\hline \multicolumn{5}{|c|}{$R$ i } \\
\hline$i$ & \multirow{2}{*}{ ID / DESCRIP TION } & \multicolumn{2}{|c|}{ SAMPLING } & \multirow{2}{*}{ MATRIX } \\
\hline E & & DATE & TIME & \\
\hline 0 & $16 c 0$ P3 -7 & Q.7.2. & 1215 & $\mathrm{SPlL}$ \\
\hline 1 & & & & \\
\hline 2 & & & & \\
\hline 3 & & & & \\
\hline 4 & & & & \\
\hline 5 & & & & \\
\hline 6 & & & & \\
\hline 7 & & & & \\
\hline 8 & & & & \\
\hline 9 & & & & \\
\hline
\end{tabular}

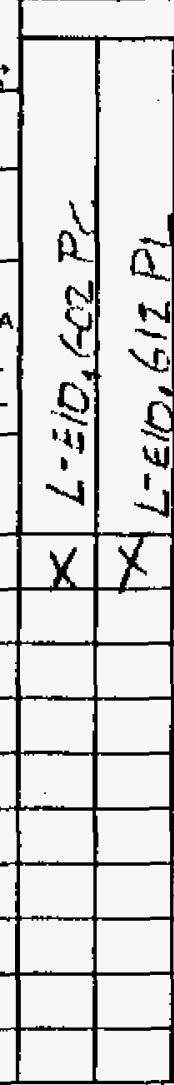

Transfer of samples submitted for analyses

Sampled/Relinctished (Signature/Organization)

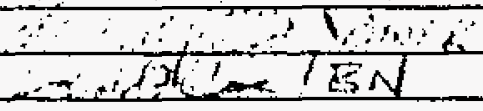

\begin{tabular}{|c|c|}
\hline DATE / TME & Received by (Sgnature/Organization) \\
\hline$\therefore \quad \therefore$ & $\operatorname{loc} / 71$ \\
\hline Hedtry $(t)$ & 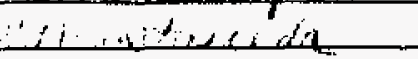 \\
\hline & \\
\hline & \\
\hline
\end{tabular}

Complete for samp/es shipped to an OFF-SITE Subtontract Laboratory

\begin{tabular}{|l|l|l|}
\hline Relinquished (BN Representative Signature) & DATE / TIME & Recelved (Courier \& Tracking Info) \\
\hline Retinquished (Courier \& Tracking Info) & & \\
\hline & DATE / TIME & Recelved (1st tler Subonttractor Rep) \\
\hline Relinquished (1s! tier Subcontractor Rep) & & \\
\hline & DATE / TIME & Received (2nd tier Subcontractor Rep) \\
\hline
\end{tabular}

The samples submitted contain (ctieck) (1) Hazandous (I) Radloaturo.

contamination OUnknown identifying contaminewts. atact a breet narralive summary compliance with applicable regulations and allow for the sale handling of the sample materials.

\section{SAMPLE RECEIPT INFORMATION} Are all sample containers received intact (XYes () Mo Comments:

Do the labels agree wth this form? (Y) Yes ( ) No

Comimenis:

Was a Materlal Clearance Tag submitted? ( $K$ Yes ( ) No

COMMENTS

(Presenutive sluetrolume, MSans special analysis, rad matrix code; count time, efc. NoNE, SOOML
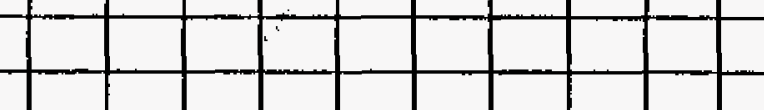
APPENDIX C

CLOSURE CERTIFICATION 


\section{CERTIFICATION OF CLOSURE BY THE DOE NEVADA OPERATIONS OFFICE}

I certify under penaity of law that the Area 6 Decontamination Pond and Oil/Water Separator, Corrective Action Unit 92, located in Area 6 at the Nevada Test site has been closed in accordance with the approved Closure Plan for CAU No. 92 Area 6 Decontamination Pond Nevada Test Site dated November 1998 and the Permit for a Hazardous Waste Management Facility Number NEV HW009. U. S. Department of Energy, Nevada Operations Office, Nevada Test Site, dated March 27, 1995. All measures required in the Closure Plan and applicable Resource Conservation and Recovery Act 42 U.S.C. 6901-6991i and Title 40 CFR 260-268 have been fully implemented and that, to the best of my knowledge, no violations exist.
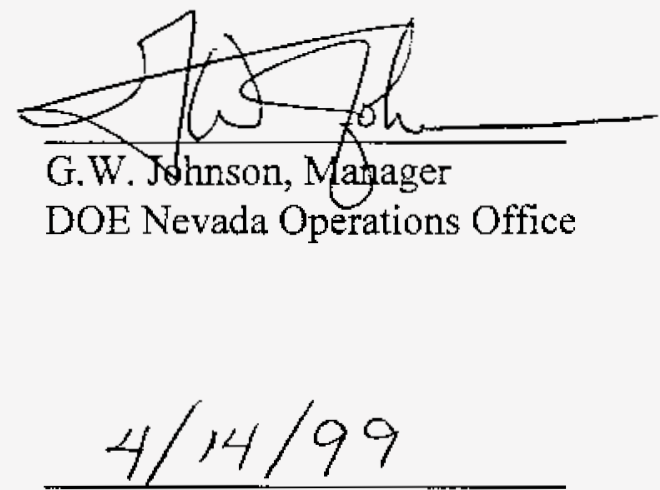

Date

DOE Nevada Operations Office

P.O. Box 98518

Las Vegas, NV 89193-8518 


\section{APPENDIX D}

\section{DESIGN CHANGE NOTICE}


15--32-1948 05:56

7022951313

BN EM

P. 62

DON NO. $99 / 12-182$

PROJ NO. 96052.106

Sheet 1 of 2

- DESIGN CHANGE NOTICE

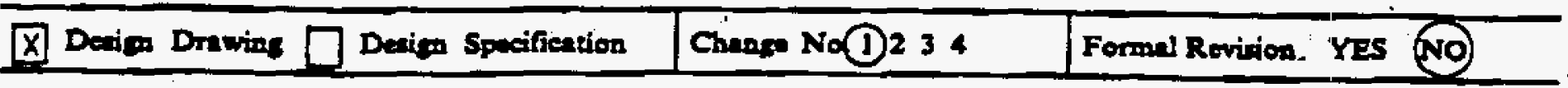

Document: No.: CAU-006-92-C3

Revision No.: 1

Title: Grading PIon

Description of Charge:

1. Revise fence corner locations per email from Jeff smith dated 12/21/98. See sheet 2 for new corner/ sign location information.

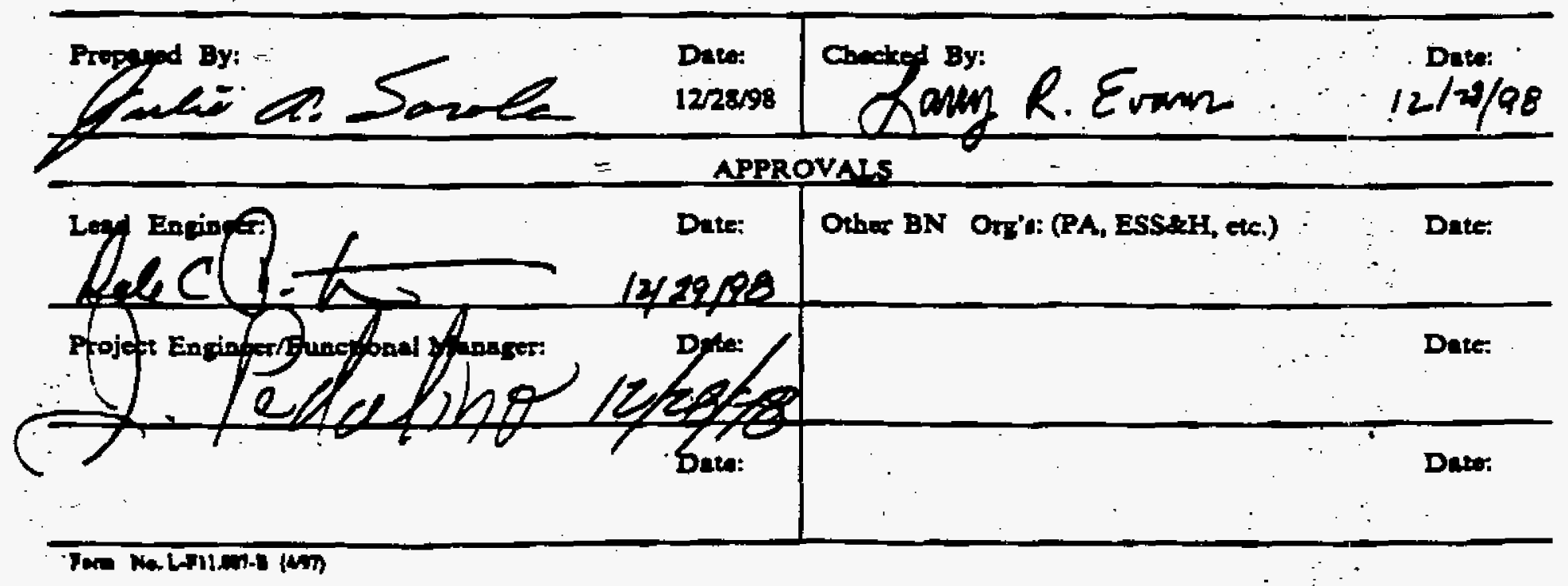


DESIGN CHANGE NOTICE

(Continuation shoet)
DCN NO. 99112.182

- PROJ NO. 95052-106

Stheet 2 of 2

\section{Ducription of Chang:}

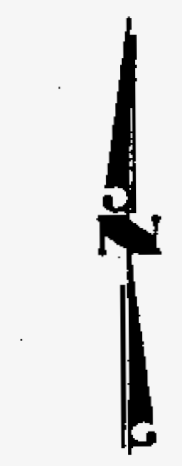

UNIT BOUNDARY

N 5242941.99
E 556106.84

MAK POINT $=2$

N 6242873.33.

E 556045.44

UNIT BOUNDRY

N 6242864,96

E 556054.99

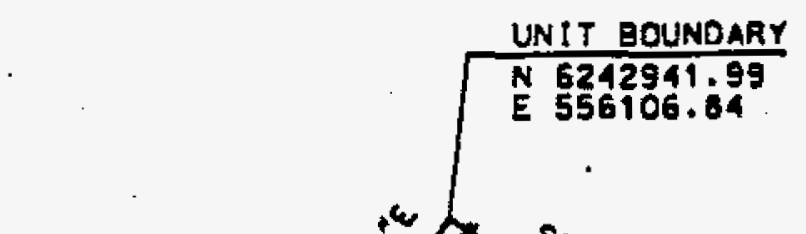

POLNT $\#$

6242911.27

1

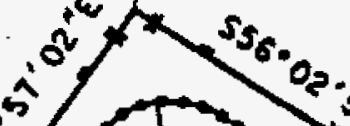

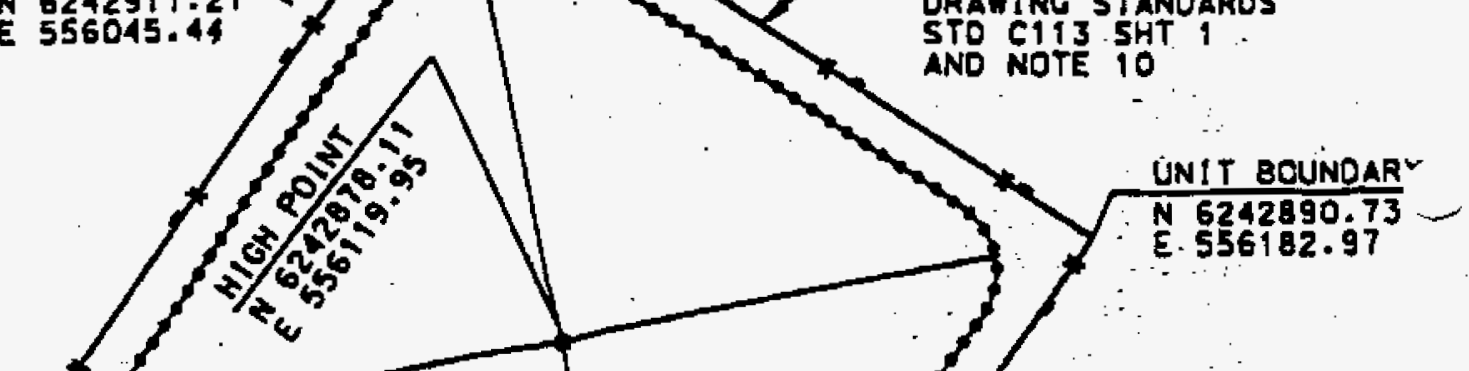

$\therefore \quad \ddots \quad$

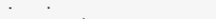


APPENDIX E AS-BUILT DRAWINGS FOR THE DECONTAMINATION POND COVER 


\section{APPENDIX E}

\section{AS-BUILT DRAWINGS FOR THE DECONTAMINATION POND}

COVER 


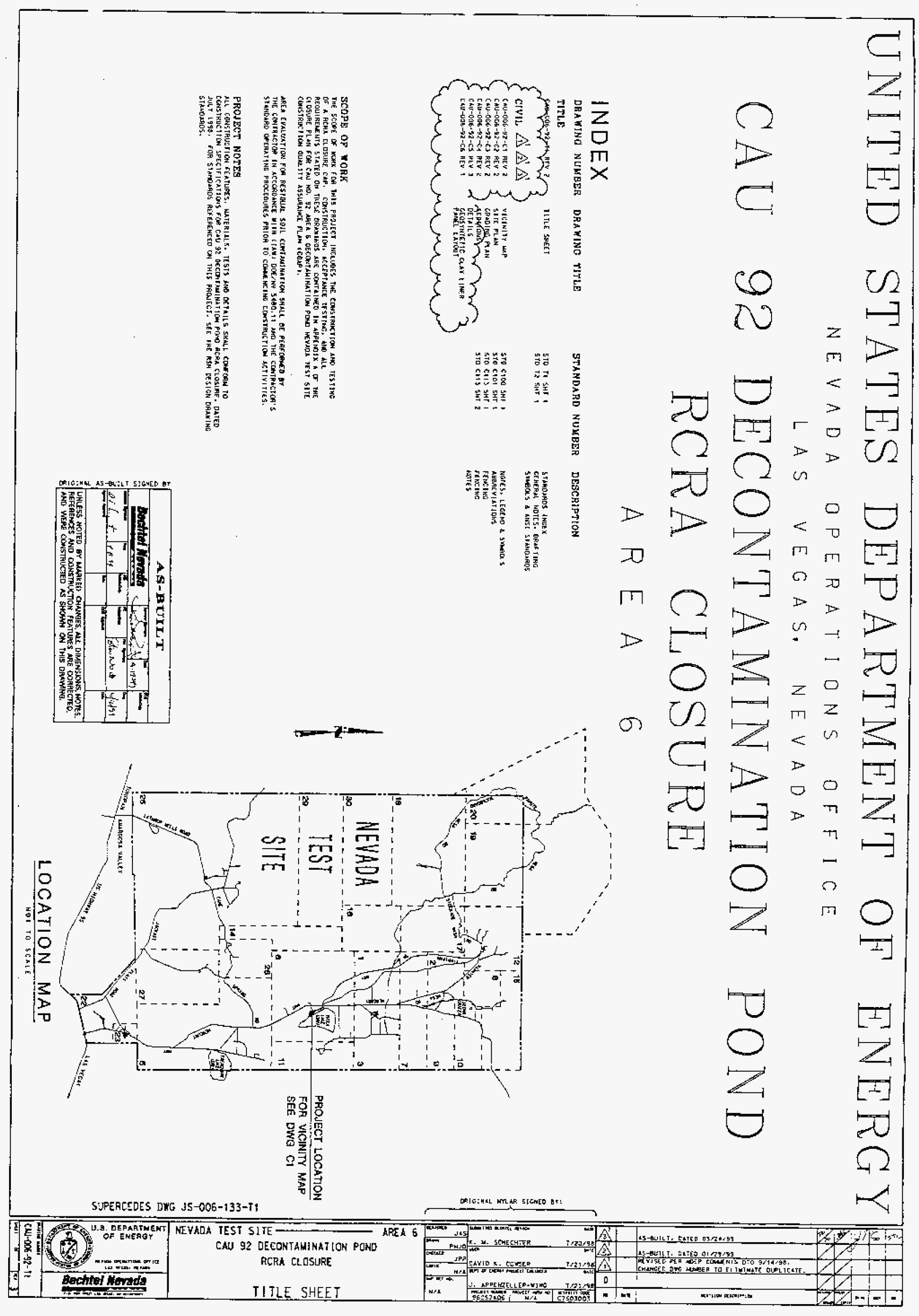




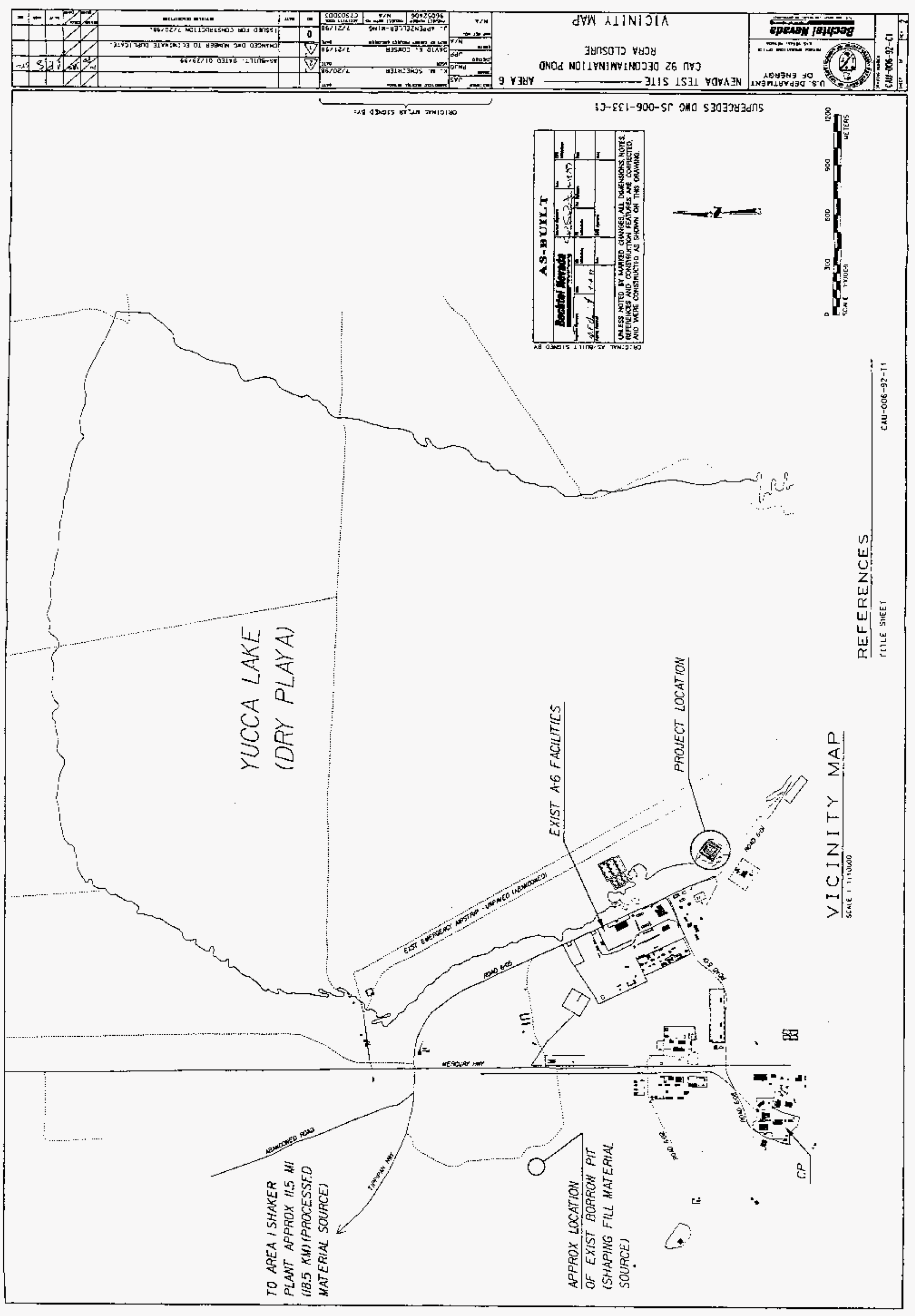




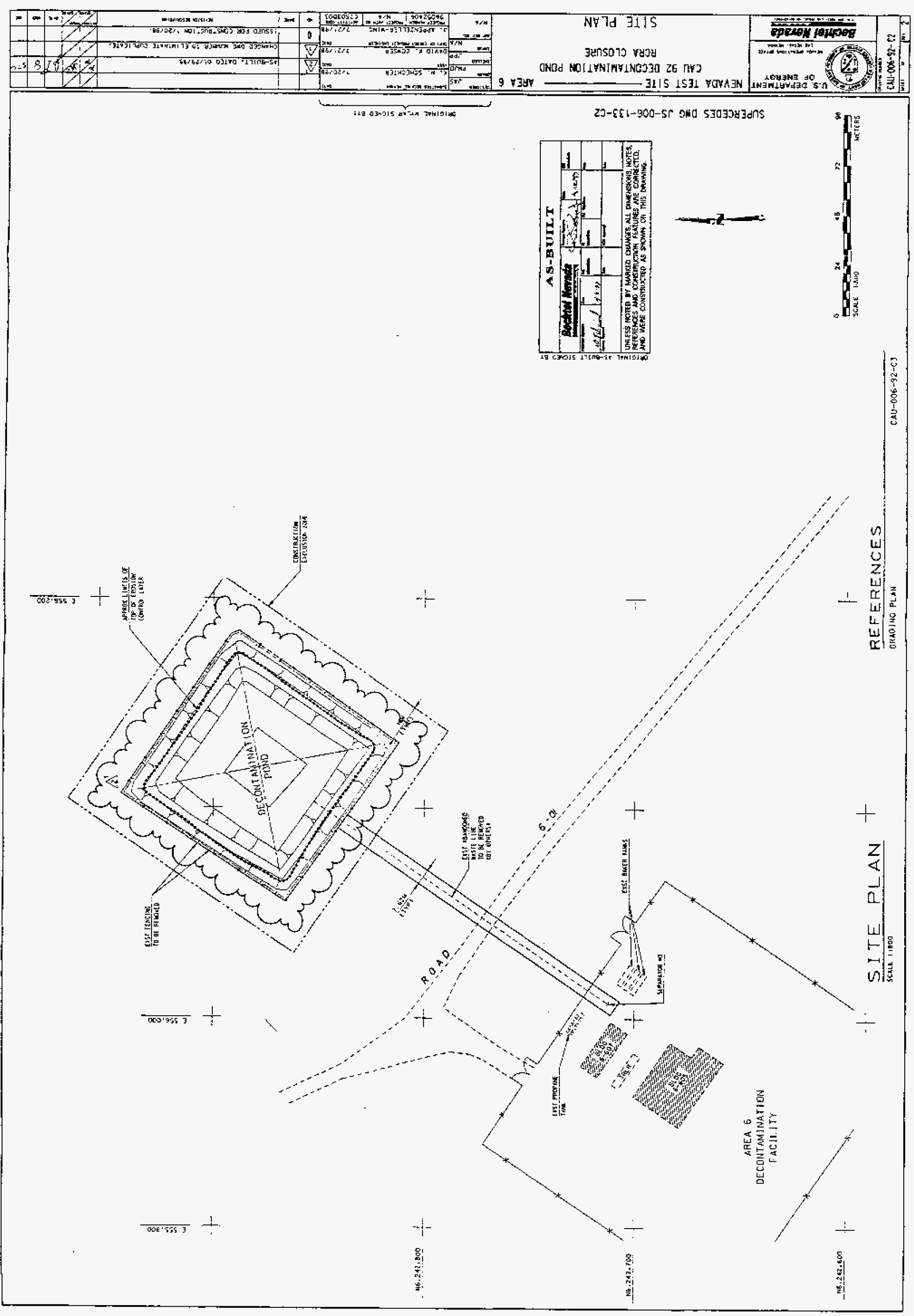




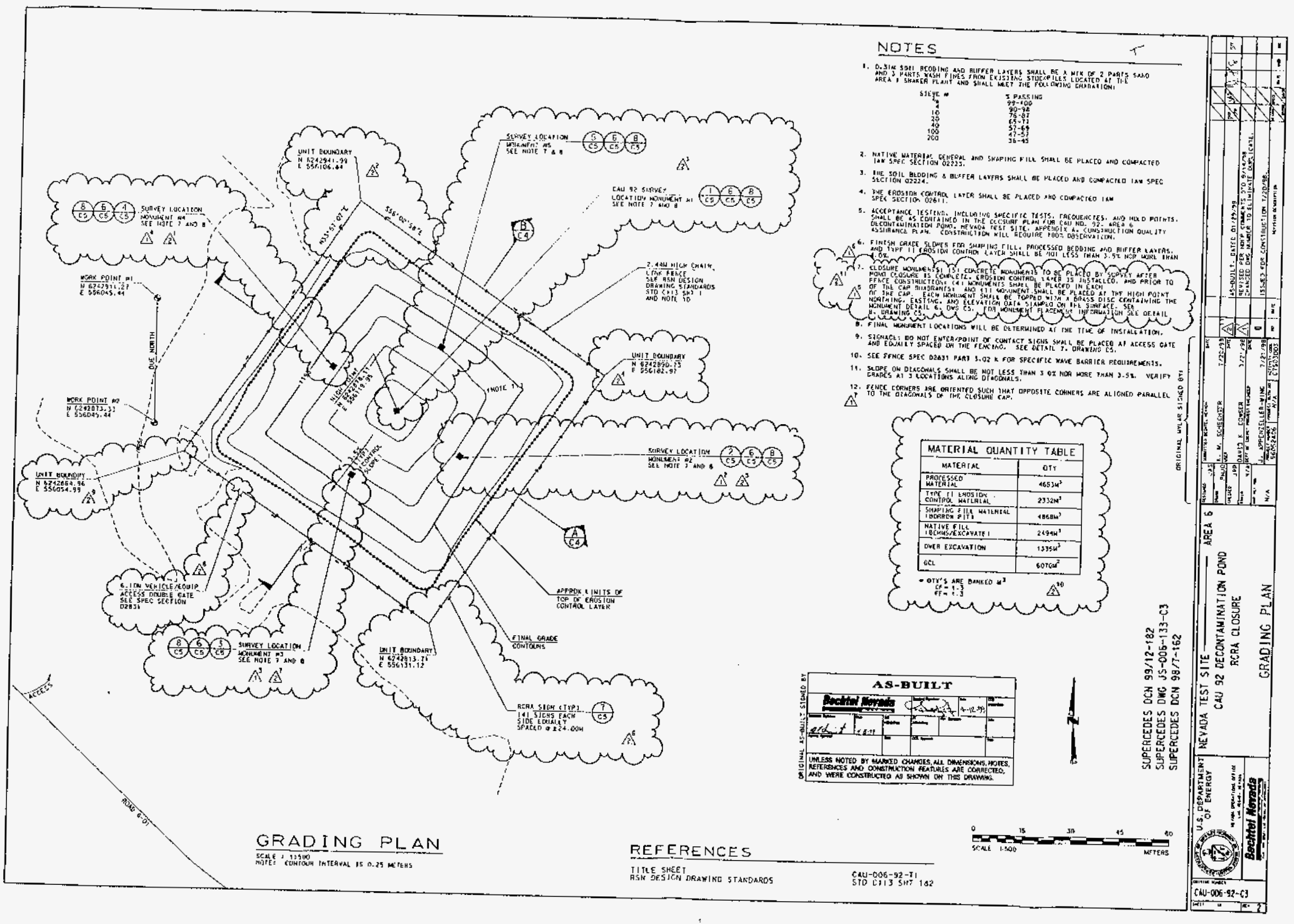




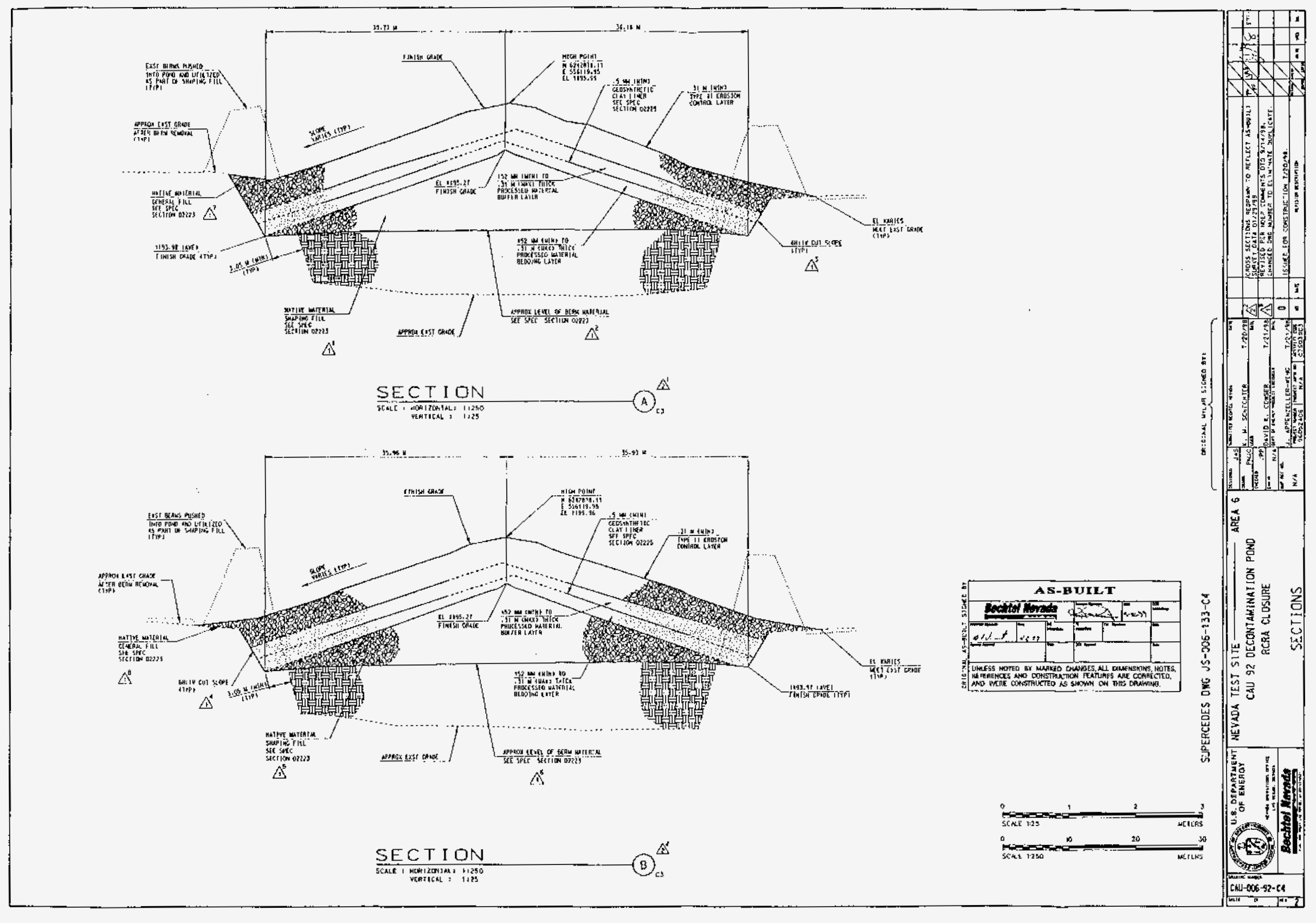



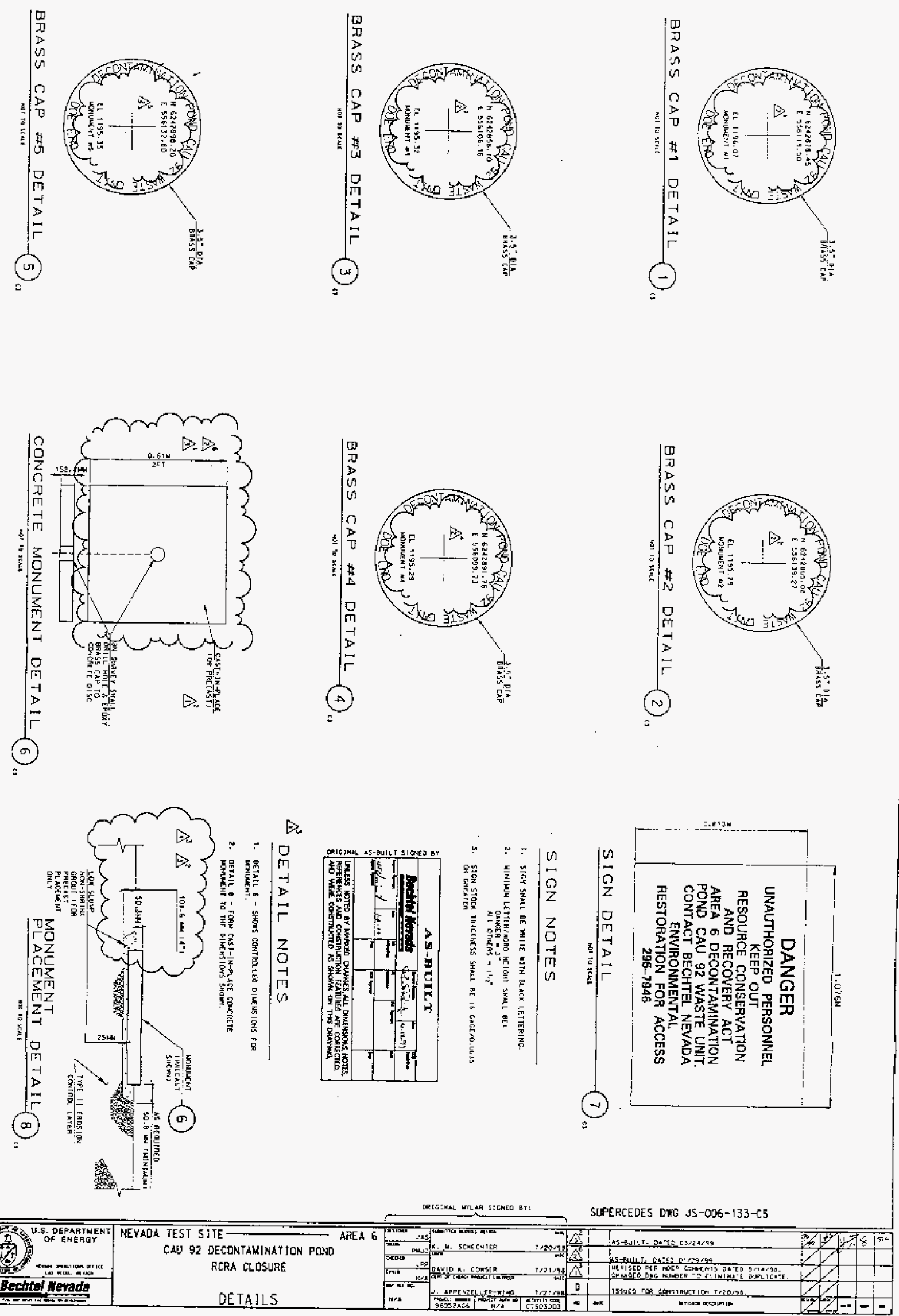

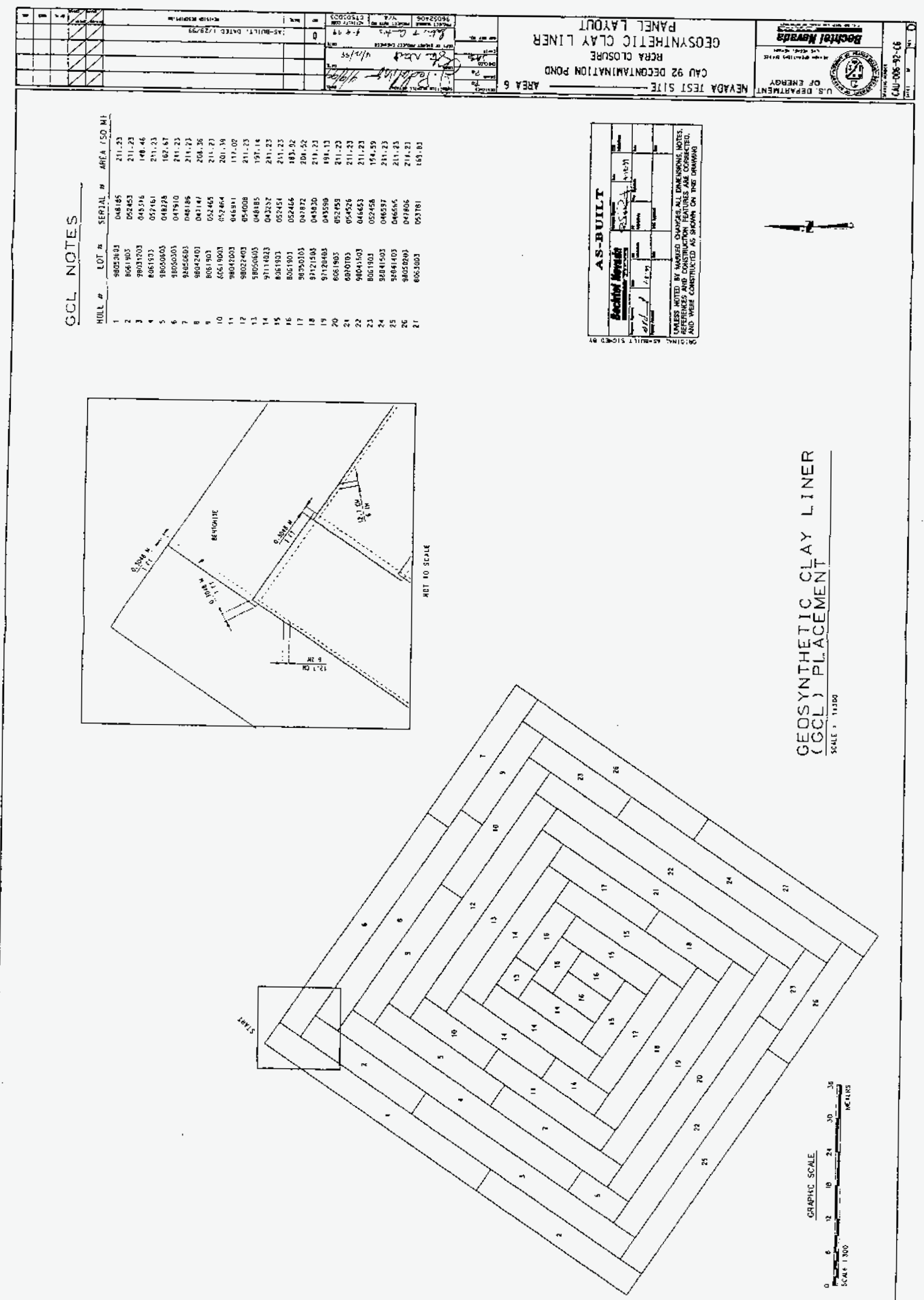


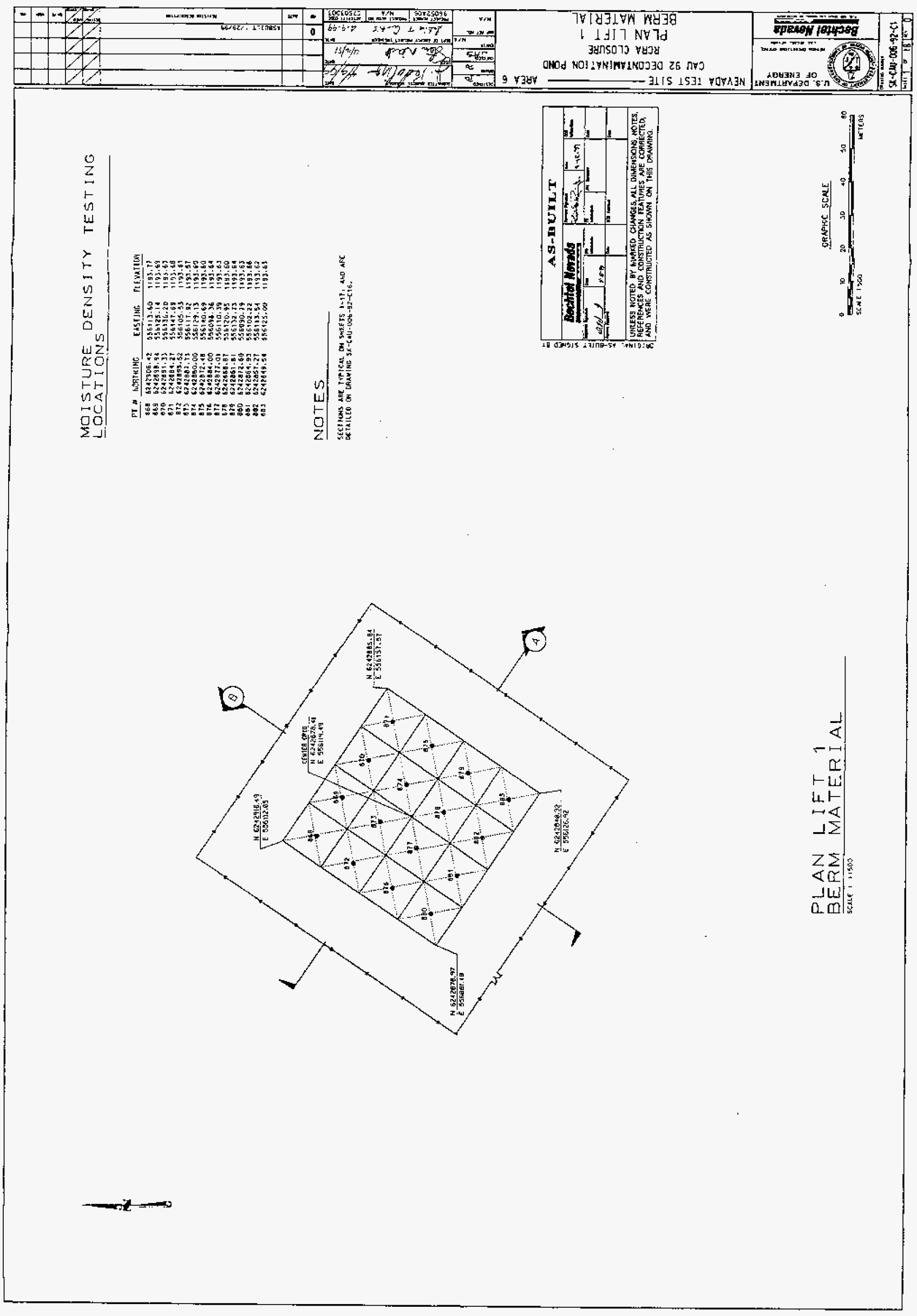




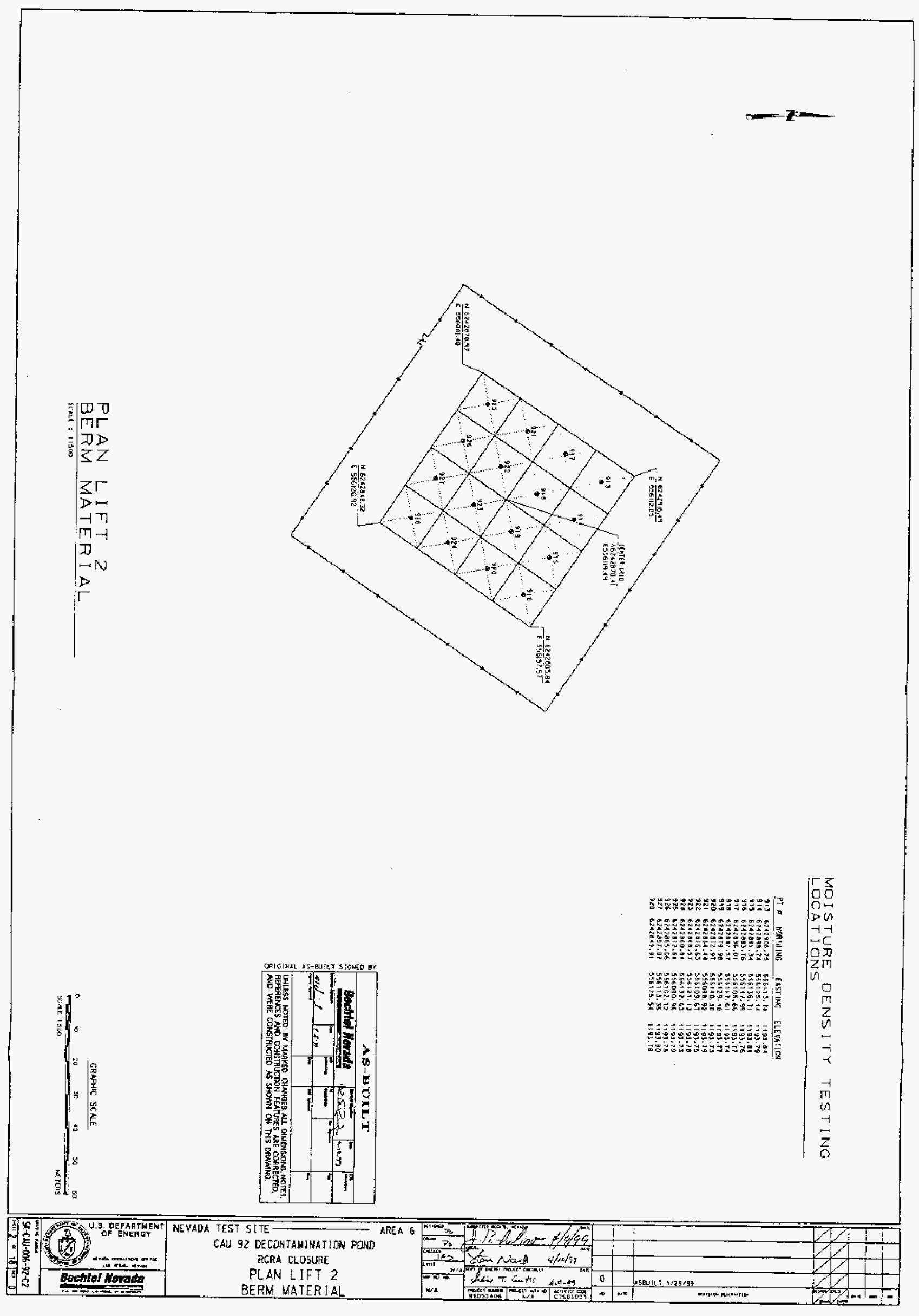




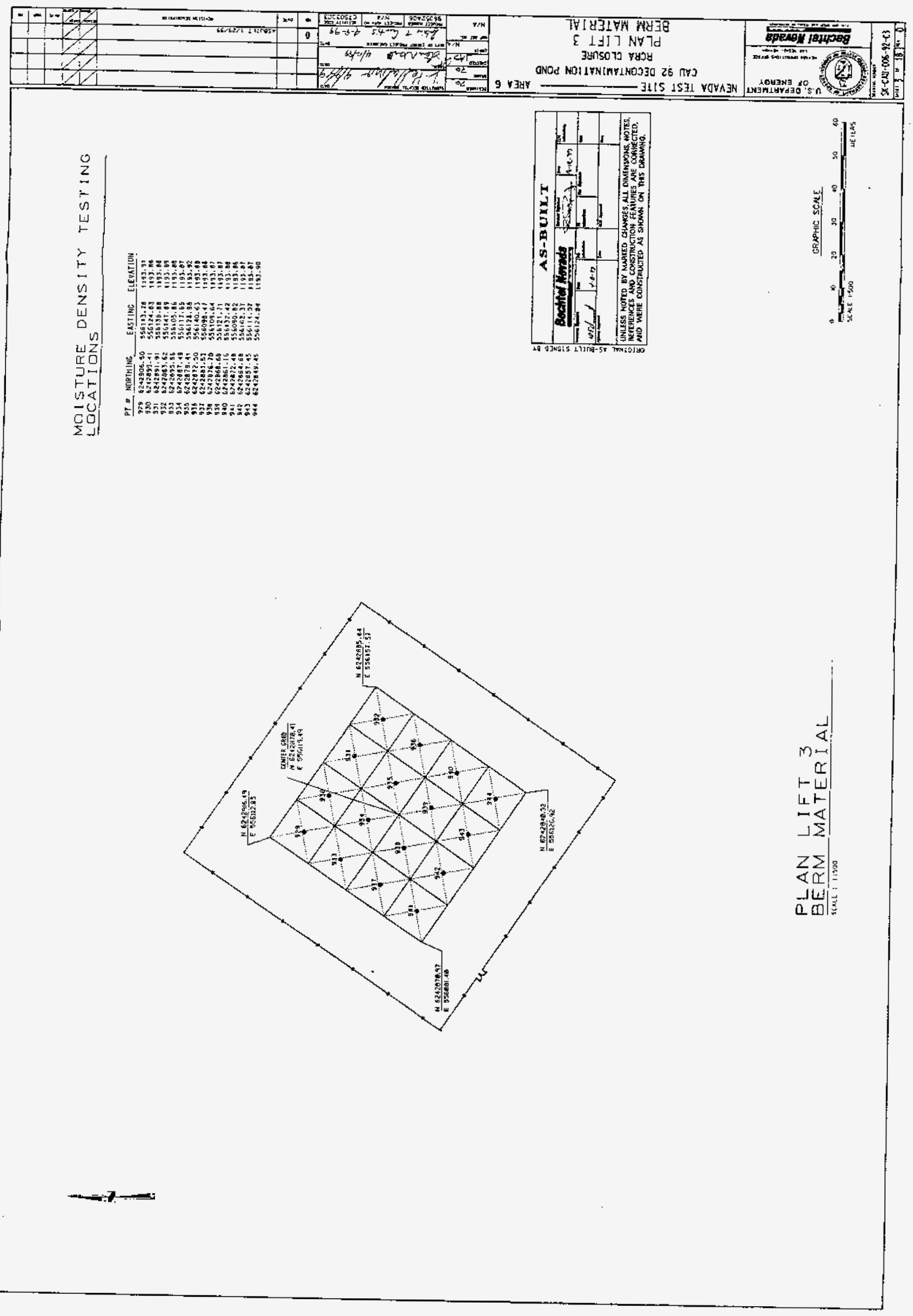




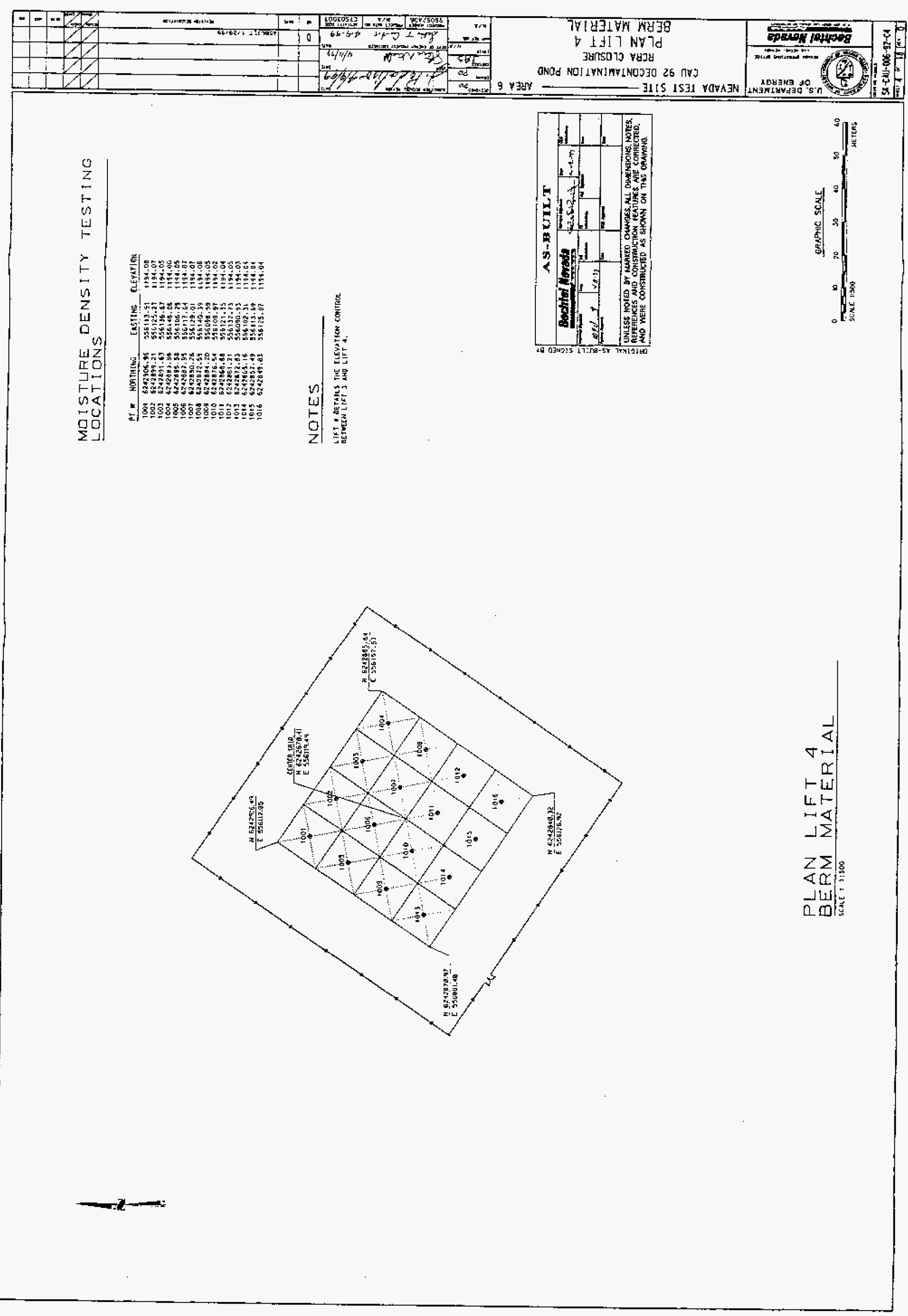




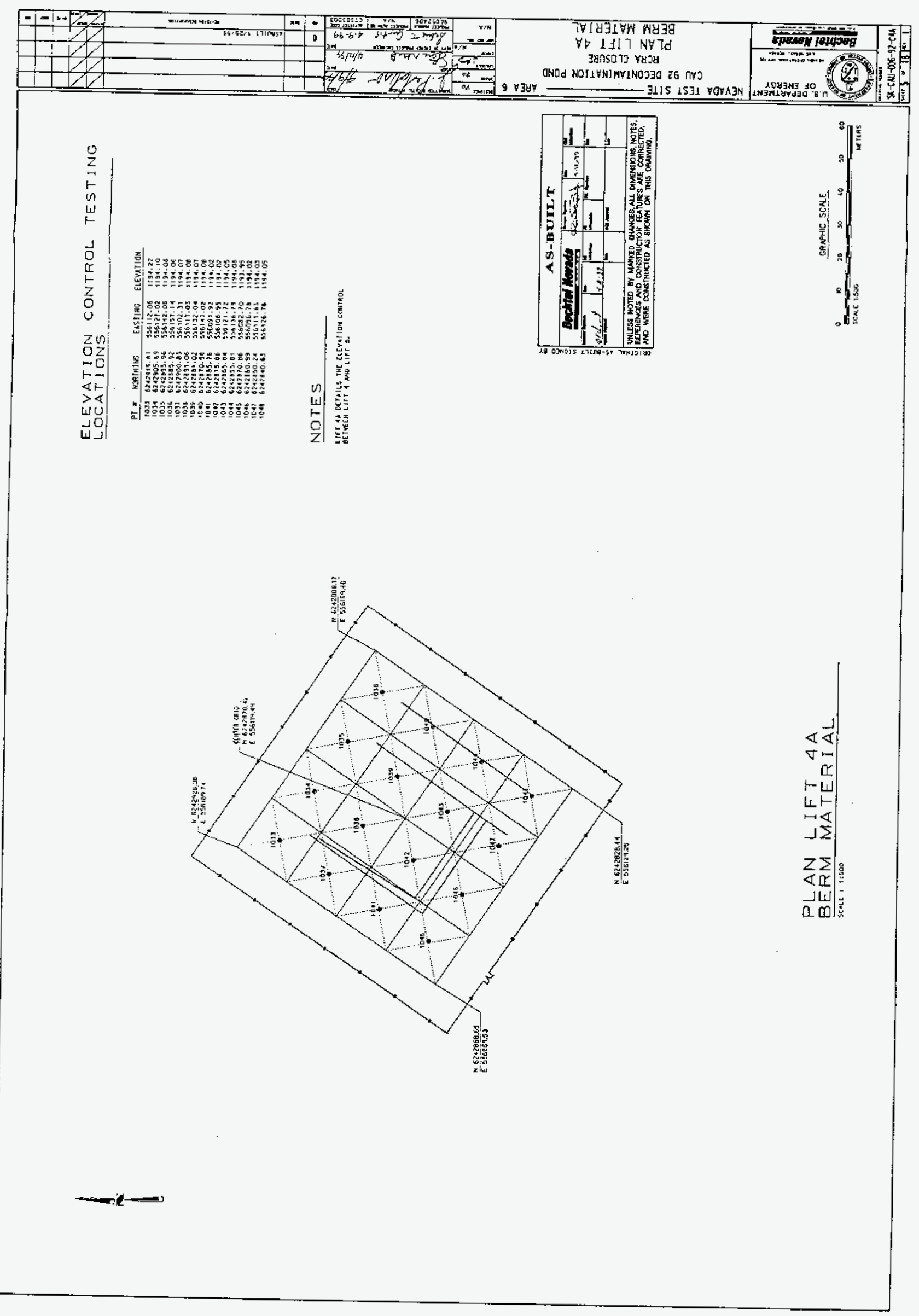




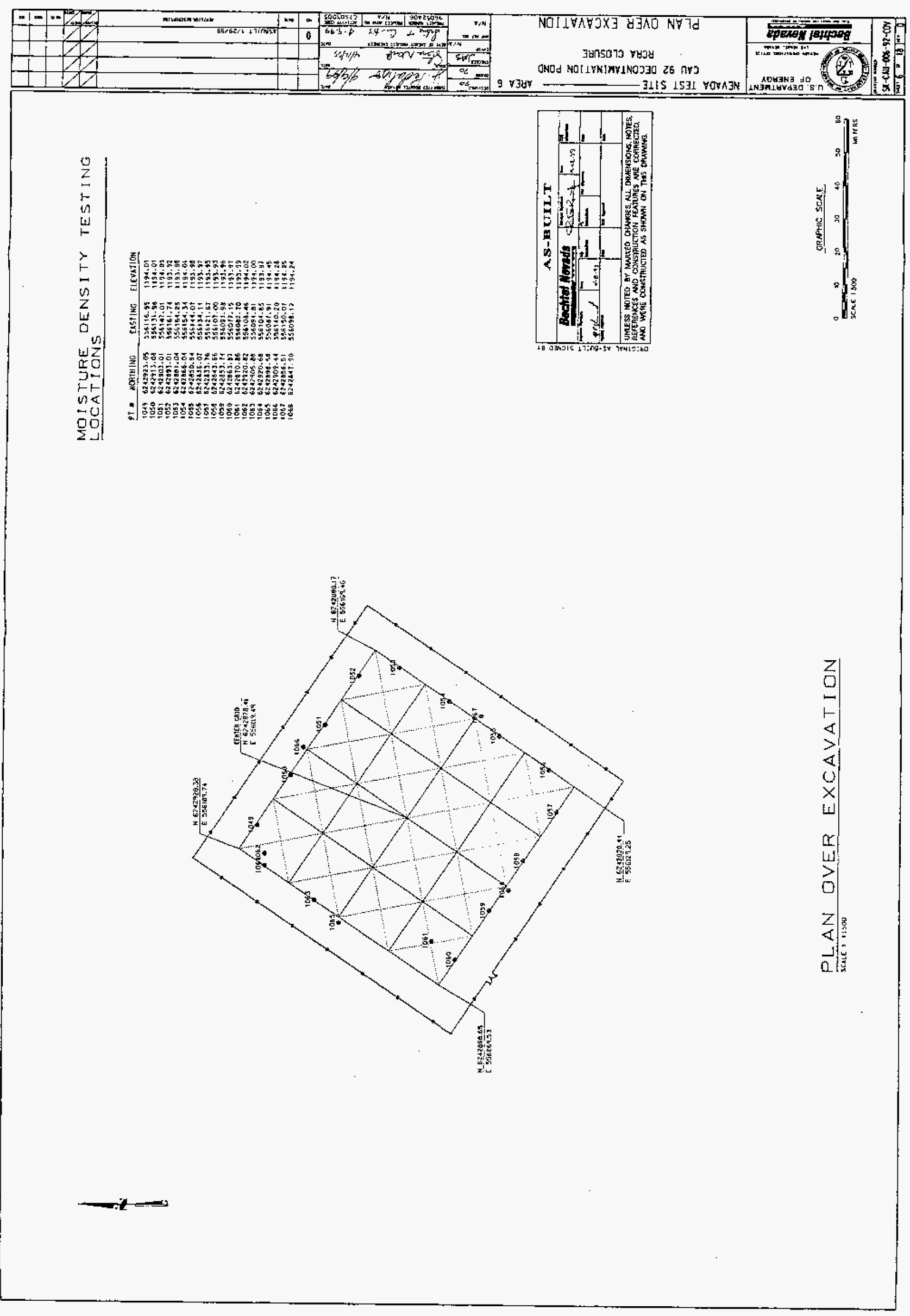




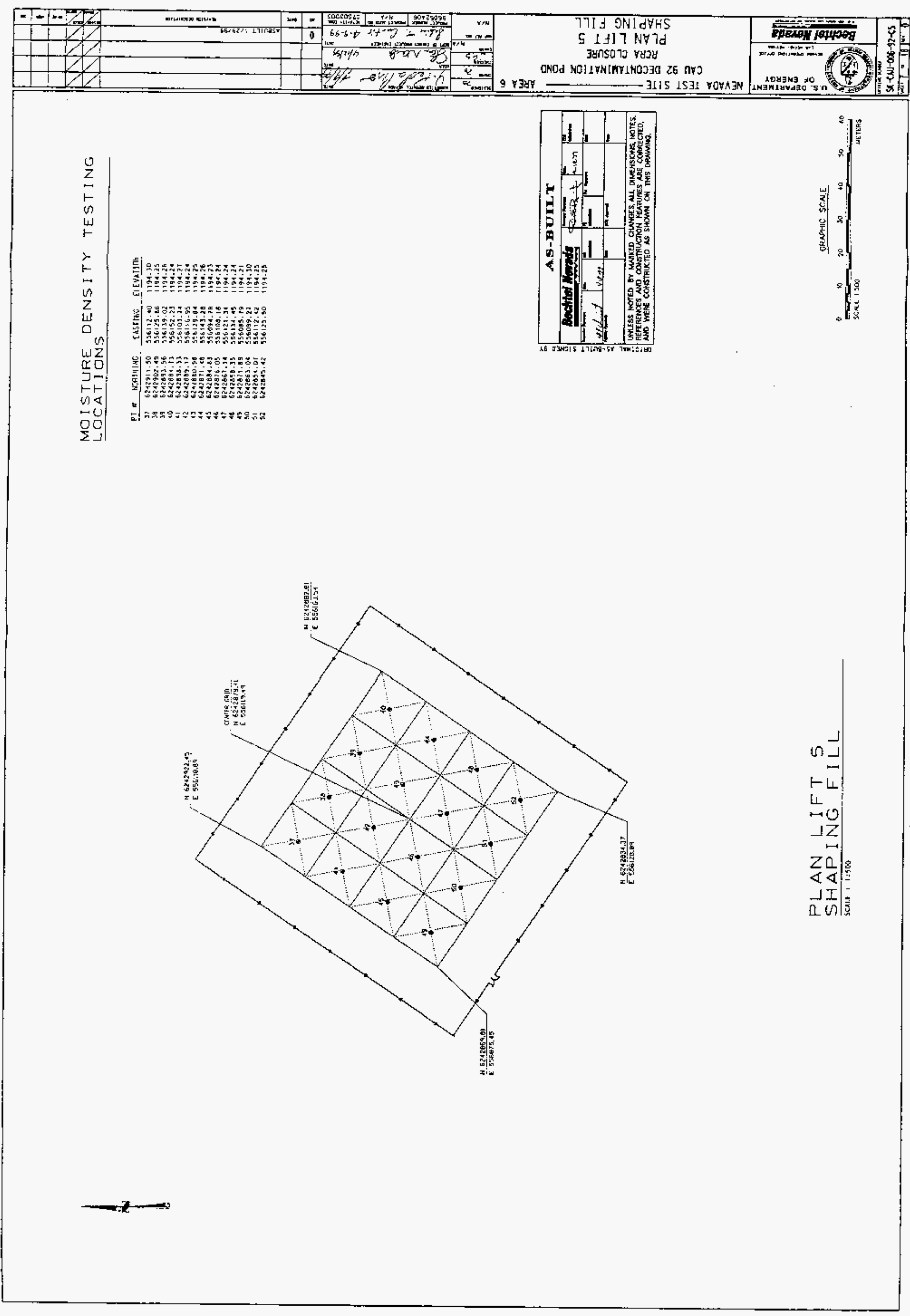




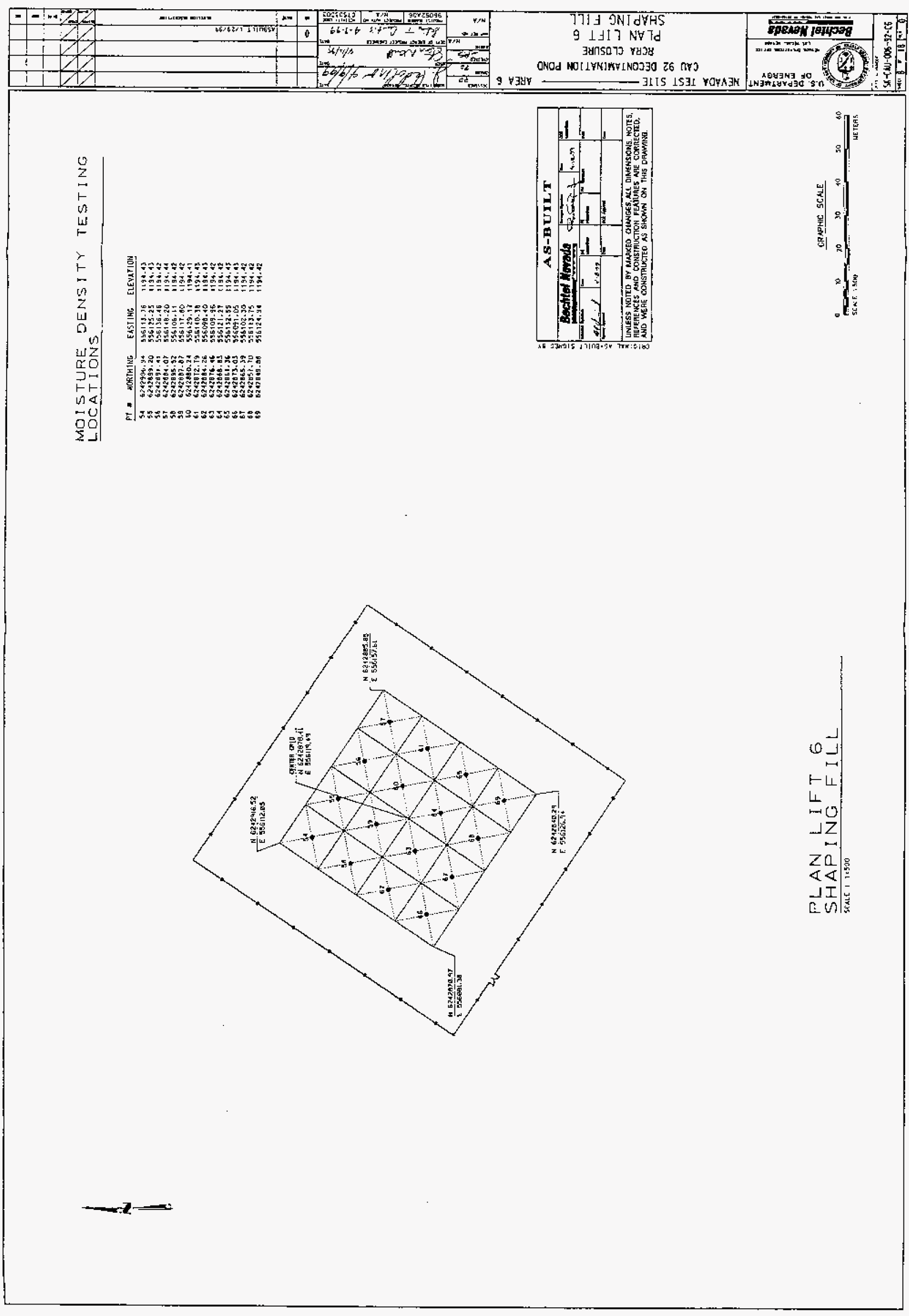




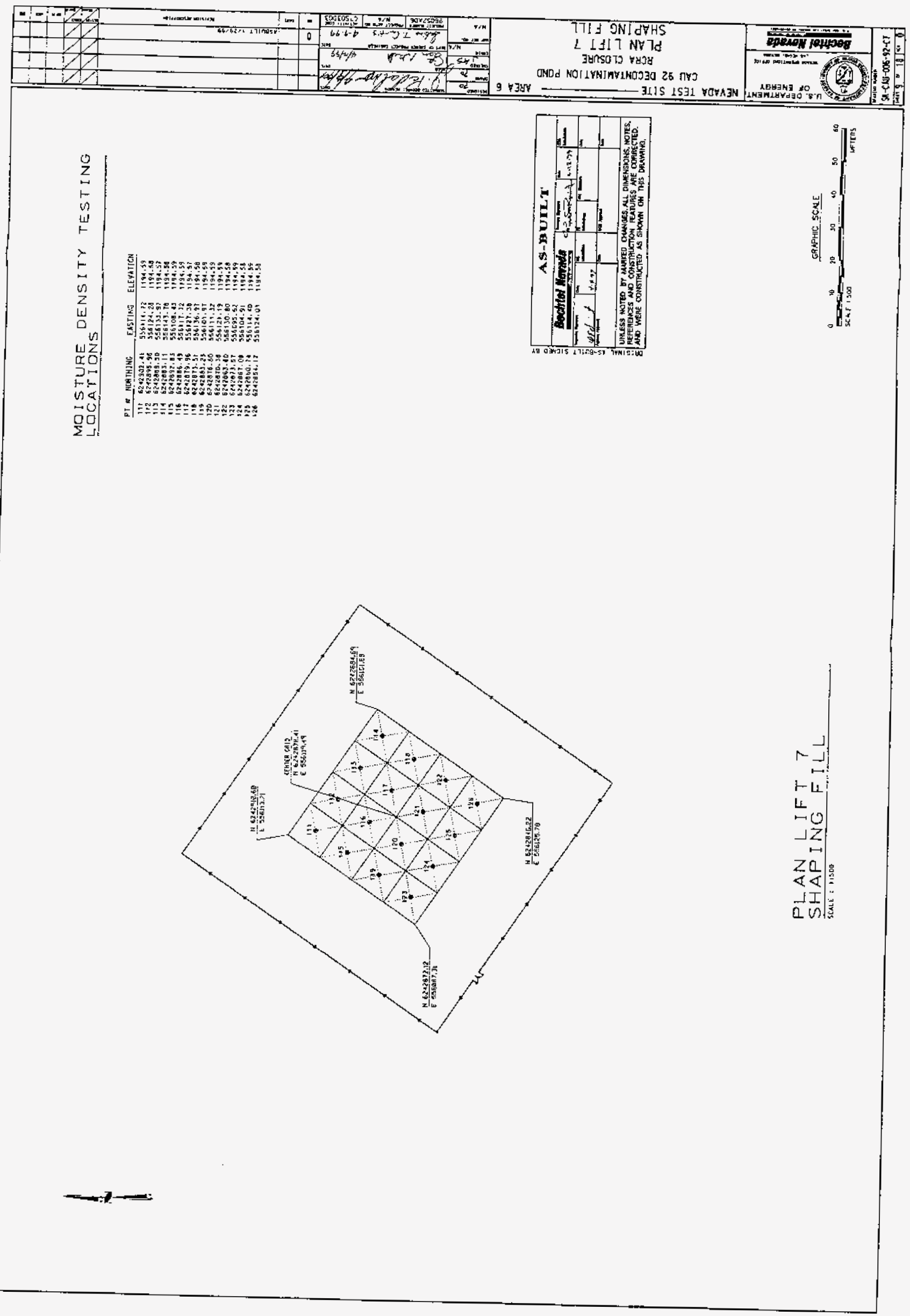




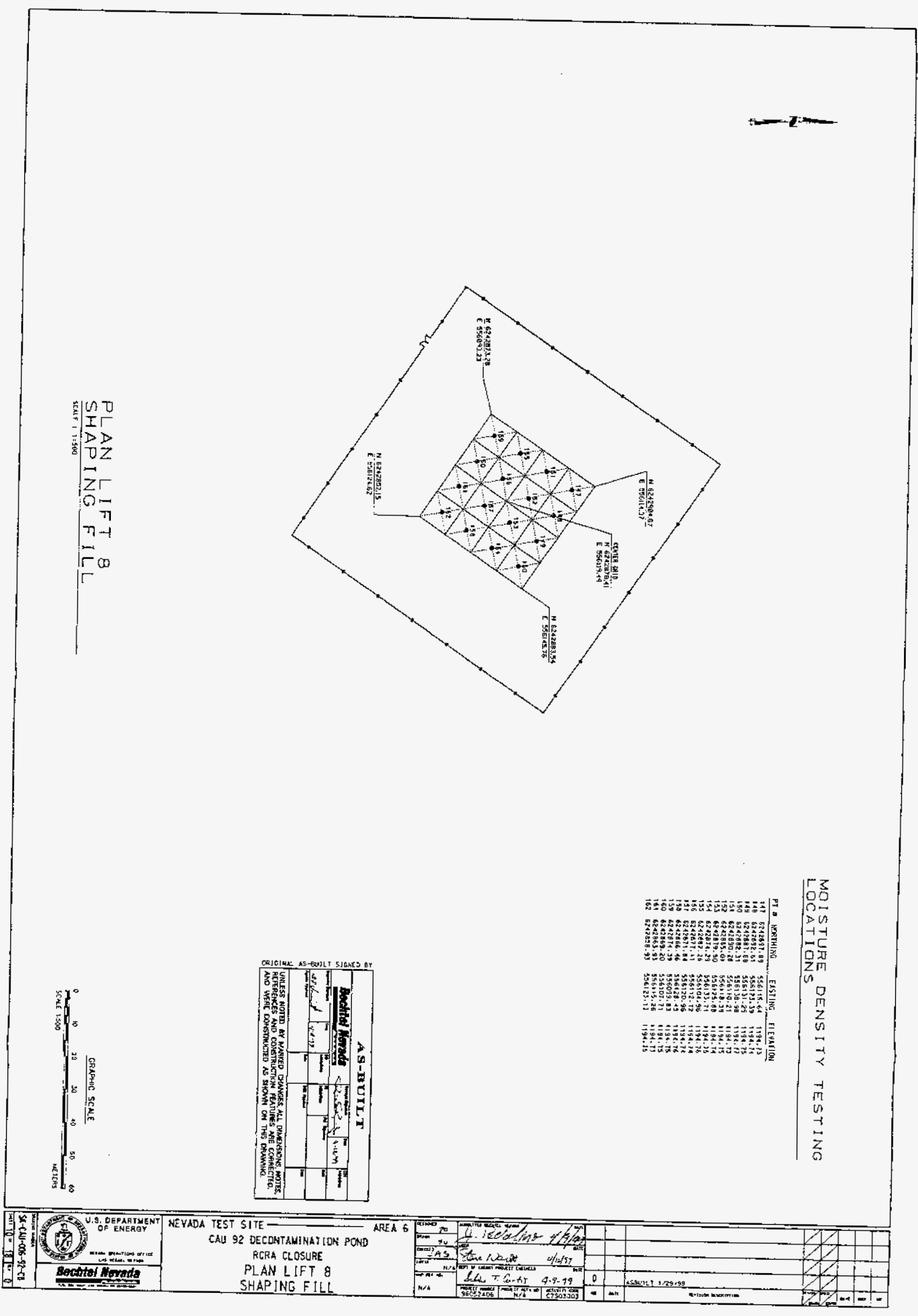




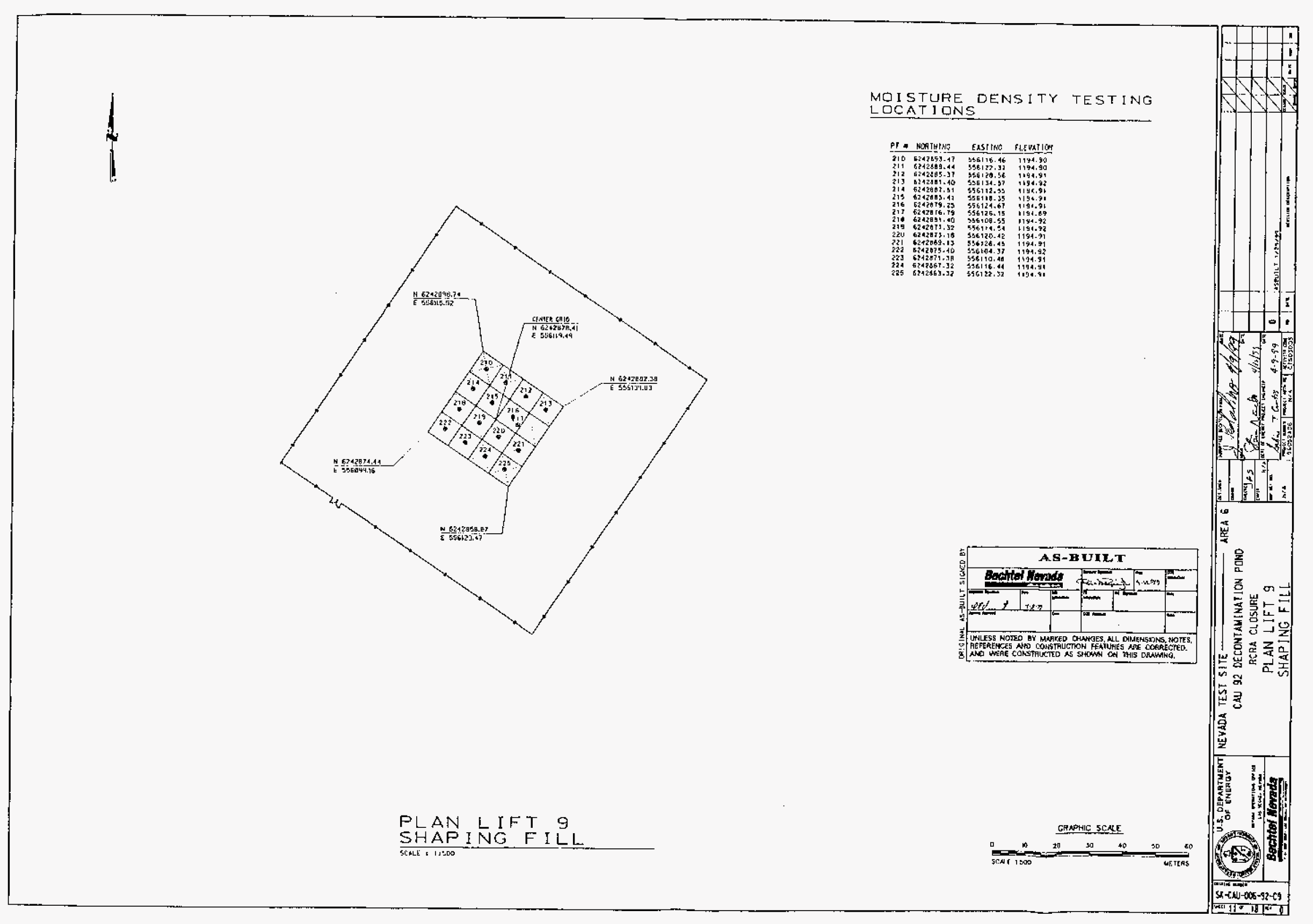




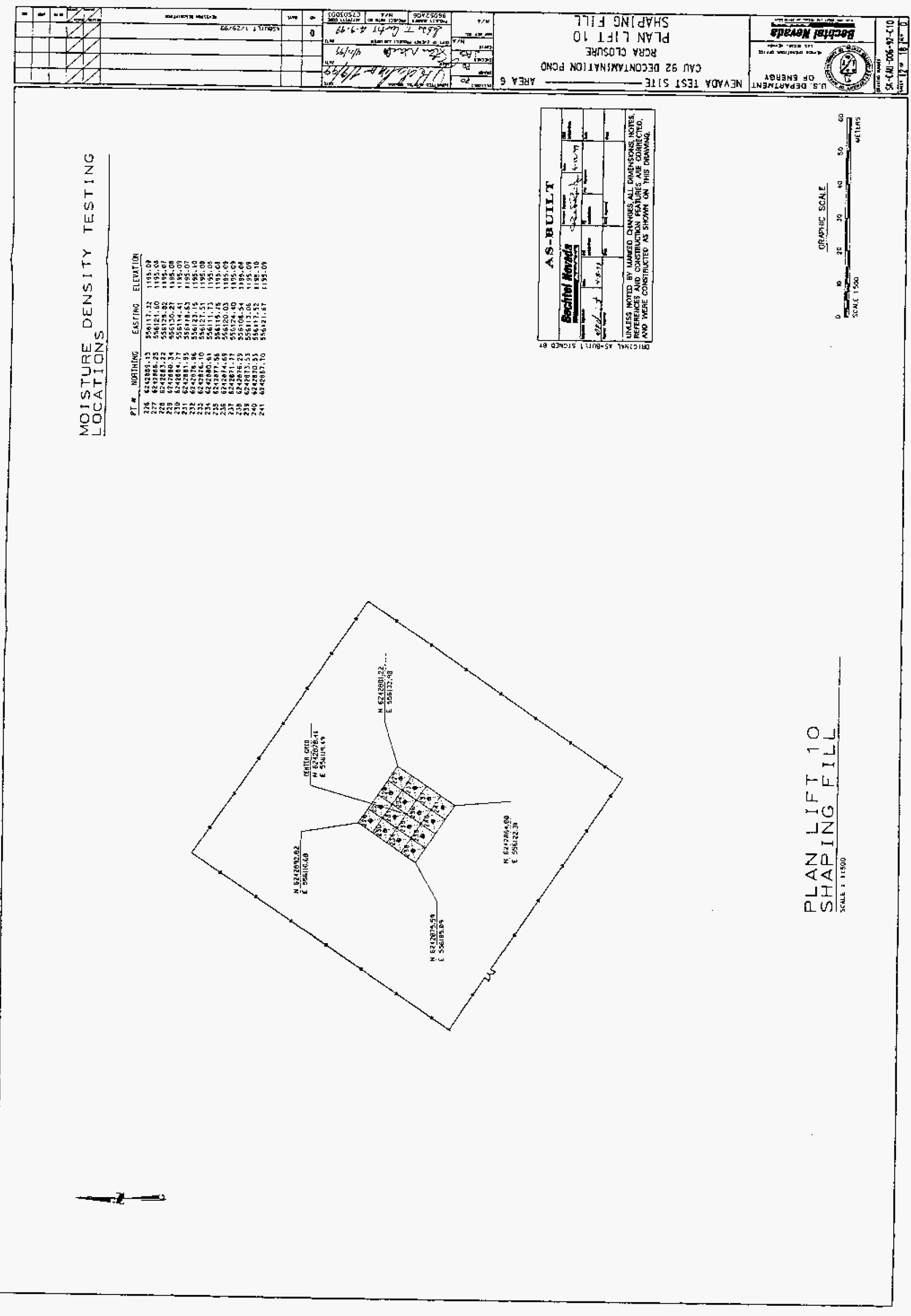




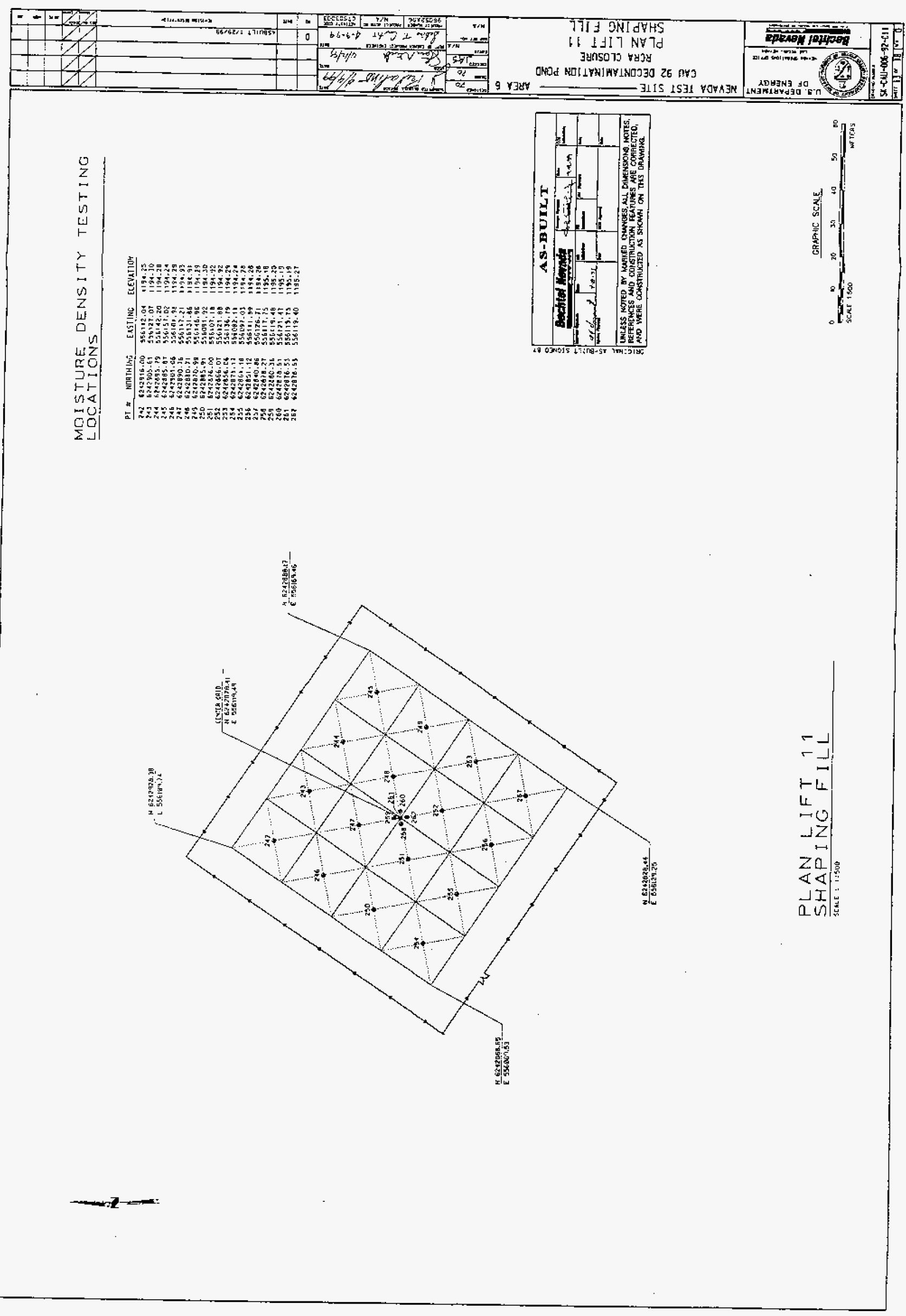




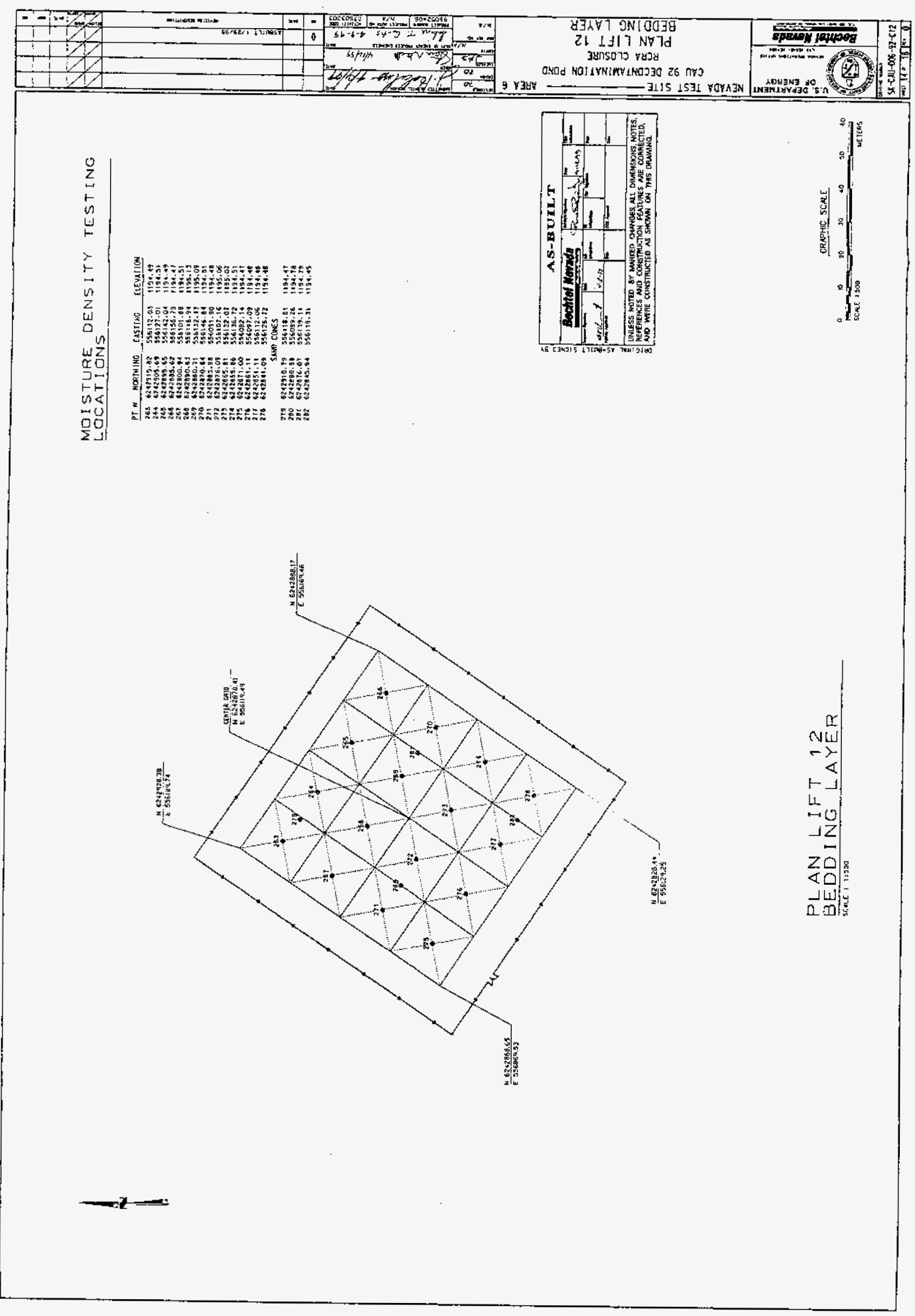




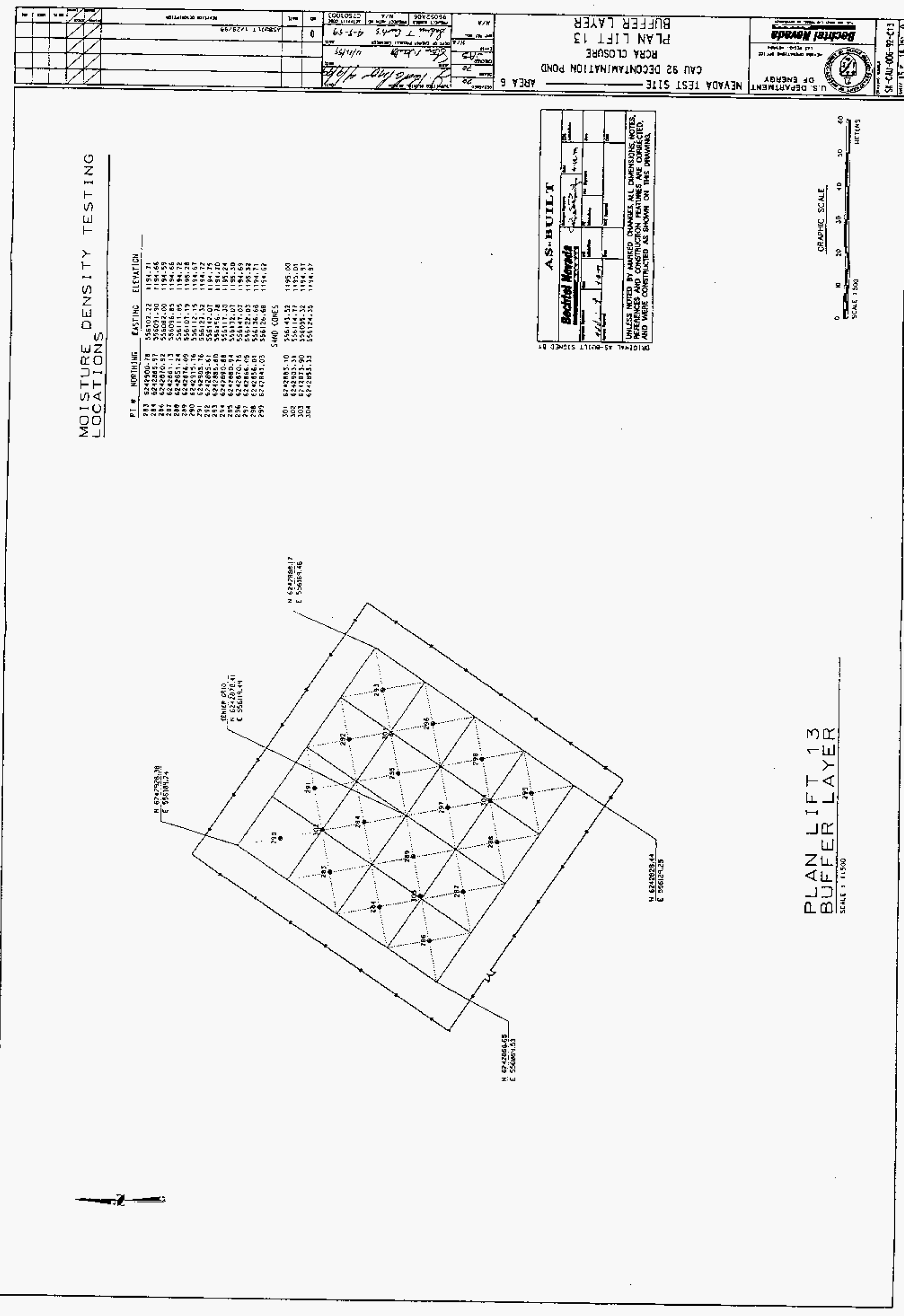




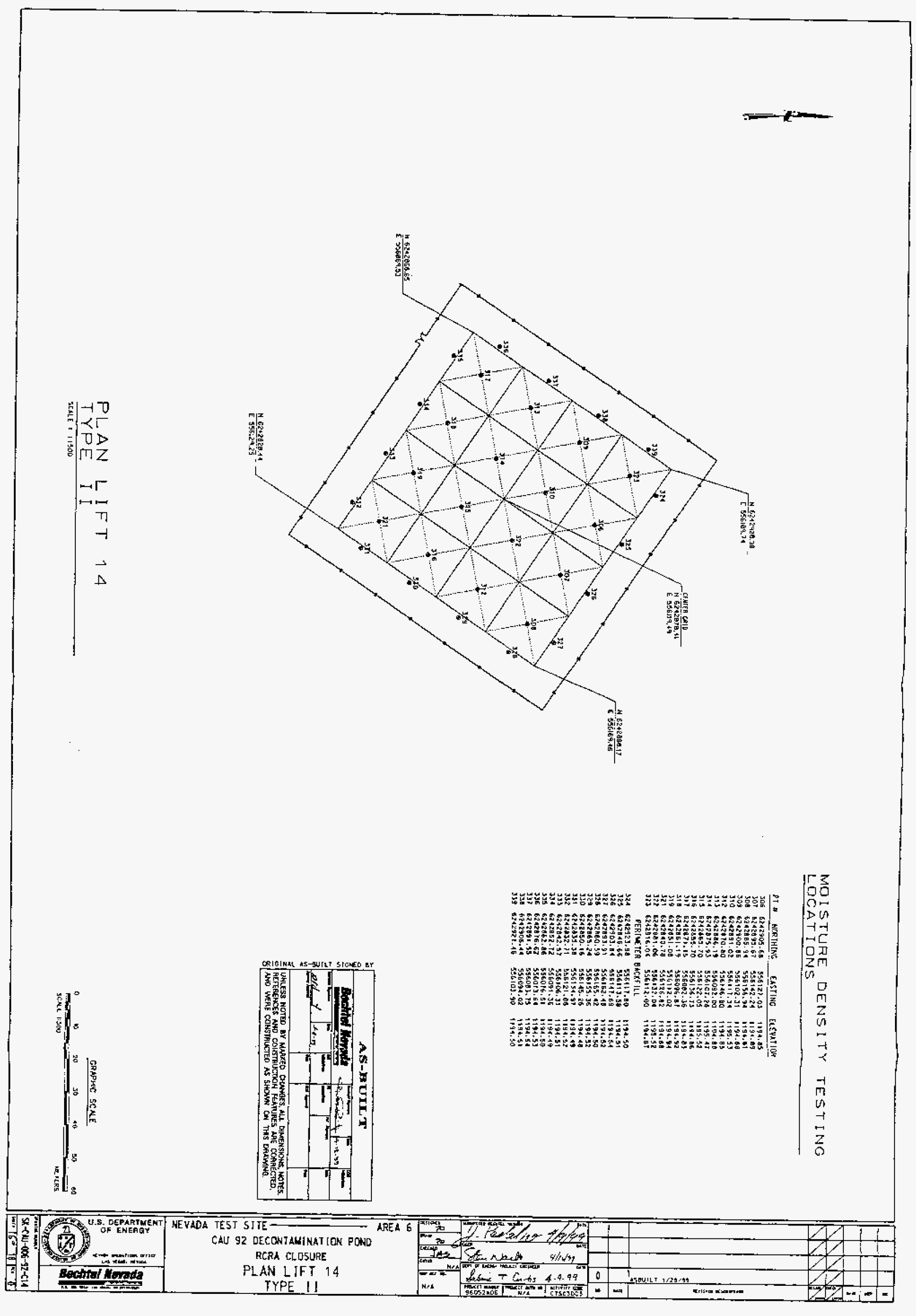




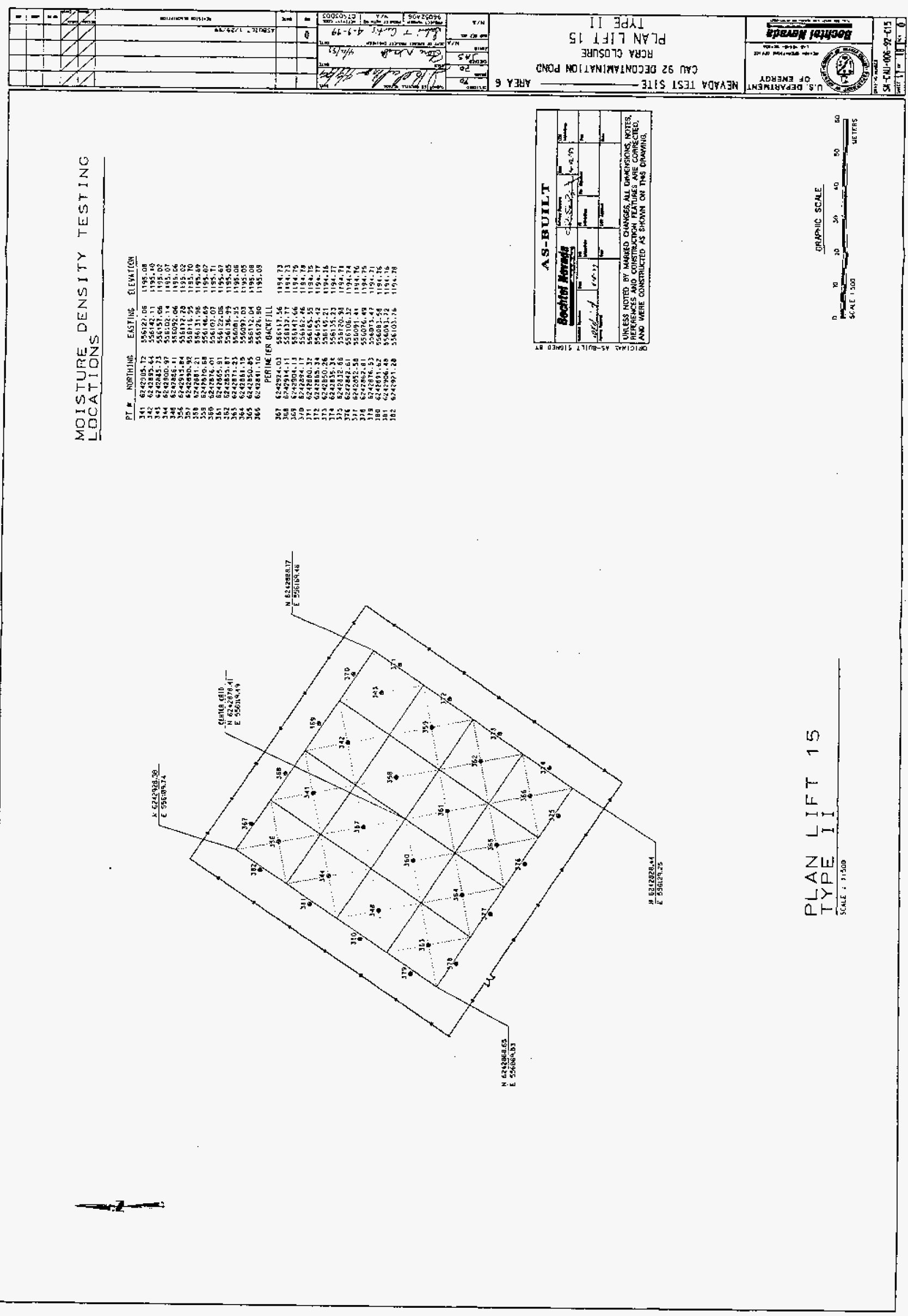




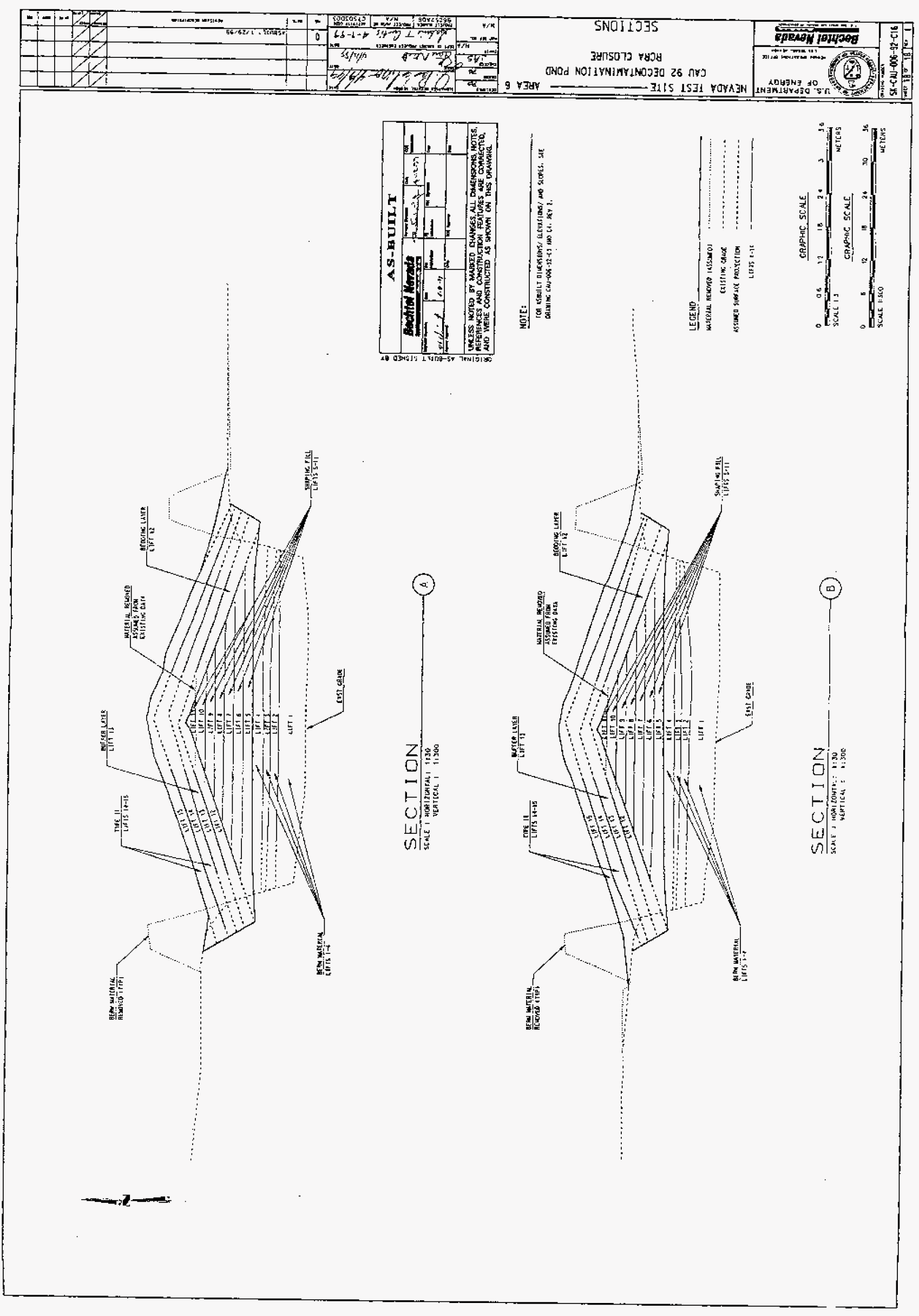




\section{APPENDIX F}

\section{APPROVAL MATRIX AND CHECKLIST}




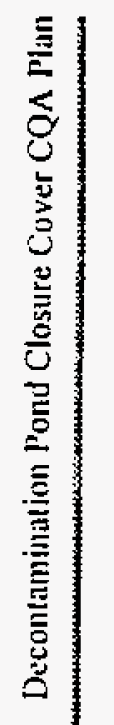

$\approx \quad$ 导 $\quad$ क

总

를

5.

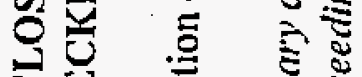

$\circlearrowleft$ 氖

워

ᄋ을

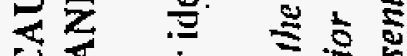

$\bigcup_{x} \stackrel{5}{5}$

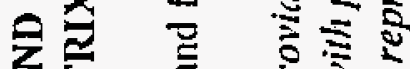

○至

z⿺

이

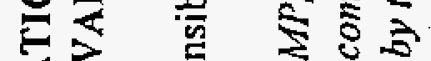

¿

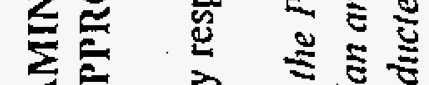

号

西

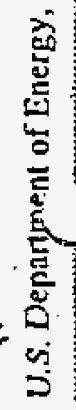

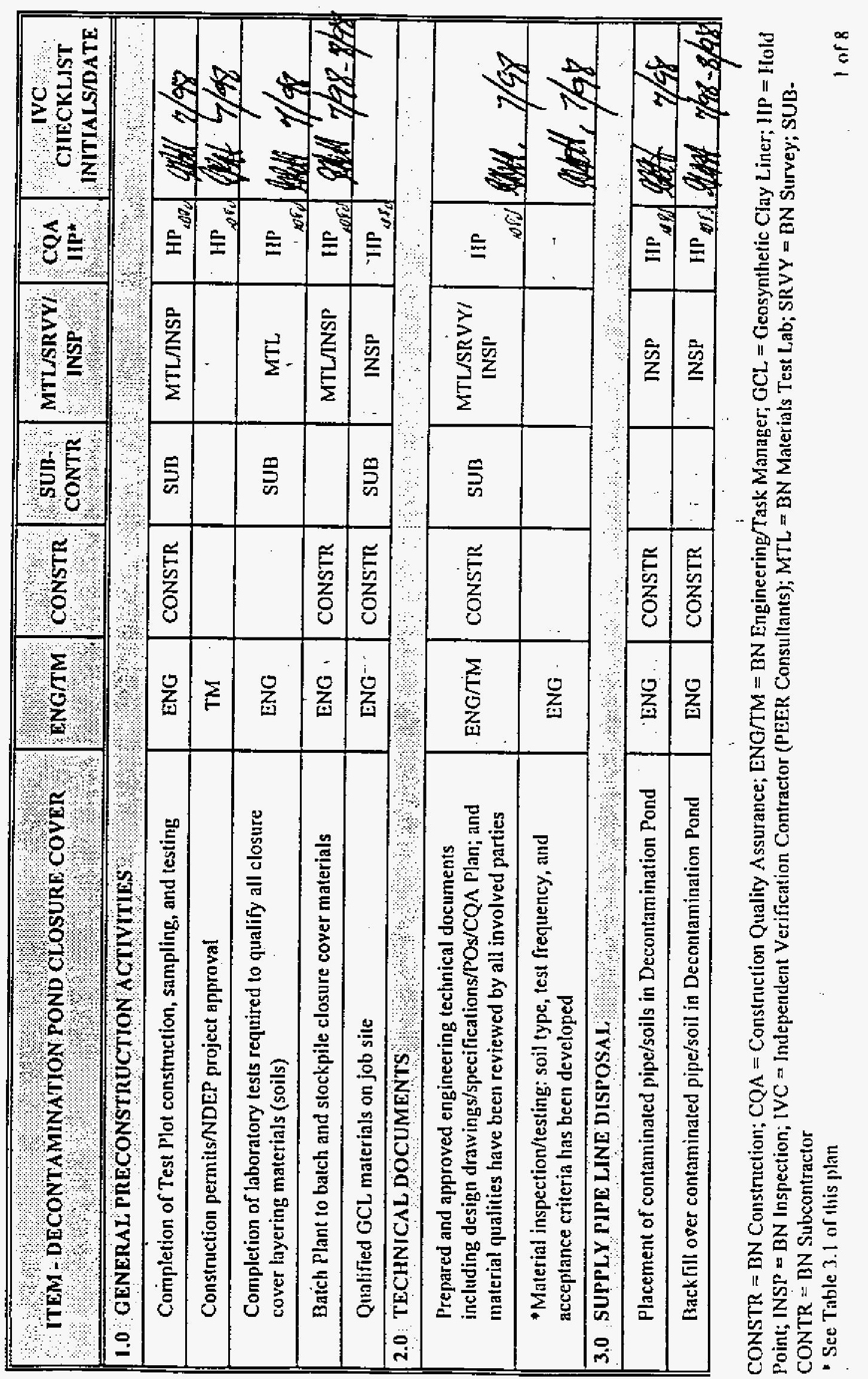




\begin{tabular}{|c|c|c|c|c|c|c|}
\hline ITEM - DECONTAMINATION POND CLOSURE COVER & ENG/TM & CONSTR & $\begin{array}{l}\text { SUB } \\
\text { CONTR }\end{array}$ & $\begin{array}{c}\text { MTL/SRVY } \\
\text { INSP } \\
\text { Pै }\end{array}$ & $\begin{array}{c}\mathrm{CQA} \\
\mathrm{HP}^{*}\end{array}$ & $\begin{array}{l}\text { IVC } \\
\text { CIIECKLIST } \\
\text { INITIALS/DATE }\end{array}$ \\
\hline \multicolumn{7}{|l|}{ 4.0 CONSTRUCTION SOLLS MANUFACTURE / ACCEPTANCE } \\
\hline \multicolumn{7}{|l|}{ 4.1 Shaping Fill Materials-Dorrow Pit/Stockpile: } \\
\hline Material stockpile segregation control (visual monitoting) & & & & INSP & god & 9616 \\
\hline -Testing: Particle size analysis of shaping fill material stockpile & ENG & & & MTL & IIPord & A- $2 / 9$ \\
\hline *esting: Modified proctor for moisture/density curve & ENG & & & MTL & HP. & ksos som \\
\hline \multicolumn{7}{|l|}{ 4.2 Processed Materials-Plant/Stockpile: Reddwed/Reffer hayer } \\
\hline Material: Processing controls/clod size (visual monitoring) & & & & INSP & 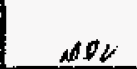 & $4 / 98$ \\
\hline *Testing: Particle size analysis of processed material stockpile & ENG & & & MTL & HP & \\
\hline *Testing: Modified proctor for moisture/density curve & ENG & & & MTL & HP & \\
\hline \multicolumn{7}{|l|}{ 4.3 Type II Materials-Plant/Stockplle: } \\
\hline Material stockpile segregation control (visual moniloring) & & & & INSP & ofo & 1411868 \\
\hline "Testing: Particle size analysis of Type II stockpile & ENG & & & MTL & HP & Kentagtang \\
\hline - Testing: Modified proctor for moisture/density curve & ENG & & & MTL & HP & \\
\hline Alt plant/stockpile testing/inspection completed & ENG & & & MTLINSP, & HP & $\lim 9 / 98$ \\
\hline \multicolumn{7}{|l|}{5.0 CONST } \\
\hline Closure cover - survey site layout established (as-built) & & & & SRVY/INSP & & 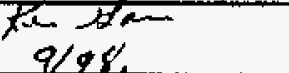 \\
\hline All material delivered/stockpiled (visual verification). & & & & INSP & & q/al \\
\hline \multicolumn{7}{|l|}{ 5.1 Berm Removal/Shaping Fill Placement: } \\
\hline Perimeter berin leveted and spread ano pond boltom & & CONSTR & & _._NSP & & \\
\hline Over excavate pend-perimeter playa malerial per Dwg C4 & & CONSTR & & ANSP & & \\
\hline
\end{tabular}

CONSTR = BN Construction; $\mathrm{CQ \Lambda}=$ Construction Quality Assurance; ENG/TM = BN Engineering/Task Manager; GCL = Geosynthetic Clay Liner; HP = Hold Point; INSP = BN Inspection; IVC = Independent Verification Contractor (PEER Consultants); MTL = BN Materials Test Lab; SRVY $=$ BN Survey; SUB-

CONTR \pm Subcontractor

- See Table 3.1 of this plan 


\section{DECONTAMINATION POND (CAU 92) CLOSURE COVER APPROVAL MATRLX AND CHECKLIST}

MATRIX: Use for further definition of quality responsibilities and for identification of relevant procedures.

NOTE: BN organizations, in accordance with the PAMP, will provide the necessary controls during construction to verify that all design-specified hold points outlined in this plan are complied with prior to proceeding with stibsequent process steps. Independent hold-point monitoring/data review will be conducted by the IVC representative.

\begin{tabular}{|c|c|c|c|c|c|c|}
\hline ITEM - DECONTAMINATION POND CLOSURE COVER & ENG/TM & CONSTR & $\begin{array}{l}\text { SUR- } \\
\text { CONTR }\end{array}$ & $\begin{array}{l}\text { MTISSRVYI } \\
\text { INSP }\end{array}$ & $\begin{array}{l}\text { CQA } \\
\text { HP* }^{*}\end{array}$ & $\begin{array}{c}\text { IVC } \\
\text { CHECKLIST } \\
\text { INITIALS/DATE }\end{array}$ \\
\hline \multicolumn{7}{|l|}{ 5.1 Berm Material Placement (Lift \#1) } \\
\hline -Lift thickness: material loose/compacted. & & & & SRVY/INSP & $\mathrm{HP}$ & Kre Garcy/g/g8 \\
\hline "Maximum clot size (visual monitoring) & & & & INSP & afo & $\operatorname{Ban} 60+9 / 15 / 91$ \\
\hline Compaction - double steel drum roller / Si was se whegh follez & & CONSTR & & INSP & $\Delta F d$ & Ramb \\
\hline "Testing: moisture content (D3017) , ? & ENG & & & MTL & Hpord & \\
\hline *Testing: in-place density (D2922) $S$ & ENG & & & MTL & HP otd & Ren \\
\hline $\begin{array}{l}\text { *Verify that all tests were taken and that applicable HPs have been } \\
\text { released by ENG, and the as-built reflects the current status }\end{array}$ & ENG & & & MTL/INSP & $(\underline{H P}$ & Ke, 6 \\
\hline \multicolumn{7}{|c|}{ Berm Matertal Placement (Lift \#2) Natue to Proceed $9 / 23 / 9$ d } \\
\hline *Lin thickness: material loose/compacted & & & & SRVYINSP & HP & Rin \\
\hline * Maximum clot size (visual monitoring) & & & & INSP & off & X. Gorro 9 \\
\hline Compaction - double steel drum roller /Gregto anthe & & CONSTR & & INSP & $\omega f_{d}$ & \\
\hline -Testing: moisture content (D3017) & ENG & & & MTL & HP & $\frac{1}{2}$ \\
\hline +Tesling: in-place density (D2922) & ENG & & & MTL & HP & Ke. \\
\hline
\end{tabular}

CONSTR = BN Construction; CQA $=$ Construction Quality A ssurance; ENG/TM = BN Engineering/Task Manager; GCl. $=$ Geosynthelic Clay Liner; HP $=$ Hold Point; INSP = BN Inspection; IVC = Independent Veriftcation Contractor (PEER Consuftants); MTL = BN Materials Test Lab; SRVY = BN Survey; SUB-CONTR

- See Table 3.1 of this plan 


\begin{tabular}{|c|c|c|c|c|c|c|}
\hline ITEM - DECONTAMINATION POND CLOSURE COVER & ENG/TM & CONSTR & $\begin{array}{l}\text { SUB- } \\
\text { CONTR }\end{array}$ & $\begin{array}{l}\text { MTLSRVY } \\
\text { INSP }\end{array}$ & $\begin{array}{l}\text { CQA } \\
\text { HPA }\end{array}$ & $\begin{array}{c}\text { IVC } \\
\text { CHECKLIST } \\
\text { INITIALS/DATE }\end{array}$ \\
\hline $\begin{array}{l}\text {-Verify that all tests were taken and that applicable HPs have been } \\
\text { reieased by ENG, and the as-built reflects the curtent status }\end{array}$ & ENG & & & MTLINSP & $\mathrm{HP}$ & $9 / 29 / 96$ \\
\hline \multicolumn{7}{|l|}{ Berm Material Placement (LIft \#3) } \\
\hline "Lin thickness: material loose/compacted & & & & SRVY/NSP & $\mathrm{HP}$ & \\
\hline -Maximum clot size (visual monitoring) & & & & INSP SFd & & \\
\hline 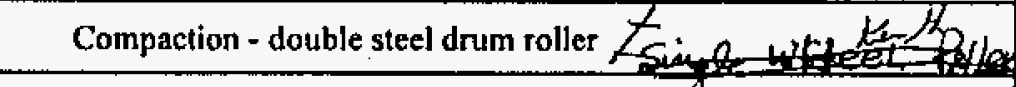 & & CONSTR & & INSP ASd & & \\
\hline Testing: moisture content (D3017) & ENG & & & MTL al. & $\mathrm{HP}$ & \\
\hline -Testing: in-place density (D2922) & ENG & & & MTL & HP & \\
\hline $\begin{array}{l}\text { "Verify that all tests were taken and that applicable HPs have been } \\
\text { released by ENG, and the as-built reflects the current status }\end{array}$ & ENG & & & \begin{tabular}{r|} 
MTLINSP \\
Afd
\end{tabular} & HP & $k$ \\
\hline Shaping fill material placement completed/verified per FCN & ENG & CONSTR & & SRVY/INSP $\omega$ & HP & Ken Gaven \\
\hline -Compaction tesis completed and accepted & ENG & CONSTR & & MTLINSP & $\mathrm{HP}$ & Pen Nane-1 \\
\hline
\end{tabular}

\section{SEE NEW CQA PIAN FOR LIFT'H}

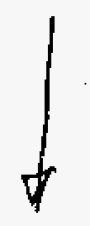

CONSTR = BN Construction; $\mathrm{CQA}=$ Construction Quality Assurance; $\mathrm{ENG} / \mathrm{TM}=\mathrm{BN}$ Engineering/Task Manager; GCL $=$ Geosynthetic Clay Liner; $H \mathrm{PP}=$ Hold Poj INSP = BN Inspection; IVC $=$ Independent Verification Contractor (PEEP ronsultants); MTL = BN Materials Test Lab; SRVY = BN Survey; SUB-CON'TR 


\begin{tabular}{|c|c|c|c|c|c|c|}
\hline ITEM - DECONTAMINATION POND CLOSURE COVER & ENG/TN & ConstrR & $\begin{array}{l}\text { SUB- } \\
\text { CONTR }\end{array}$ & $\begin{array}{c}\text { MTL/SRVY/ } \\
\text { INSP }\end{array}$ & $\begin{array}{ll}C Q A \\
I P^{*}\end{array}$ & $\begin{array}{l}\text { IVC } \\
\text { CIIECKLIST } \\
\text { INITIALSIMATE }\end{array}$ \\
\hline Over excryation completion - verified & & & & SRVY/INSP & HP & \\
\hline \multicolumn{7}{|l|}{ 5.1 Shaping Fill Placement (Lirt H1) } \\
\hline \multicolumn{7}{|l|}{-Lift thickness: materiałpoose/compacted } \\
\hline \multicolumn{7}{|l|}{-Maximum clot size (visual monitoring) } \\
\hline Compaction - double steel drum rolleh & & CONSTR & & & & \\
\hline *Testing: moisture content (D3017) & ENG & & & MTL & HP & \\
\hline *Testing: in-place density (D2922) & ENG & & & MTL & HP & \\
\hline $\begin{array}{l}\text { "Verify that all tests were taken and that ap } \\
\text { been released by ENG, and the as-built refl }\end{array}$ & ENG & & & MTL/INSP & IIP & \\
\hline \multicolumn{7}{|l|}{ 5.1 Shaping Fill Placement (Lift \#2) } \\
\hline -Lift thickness: material loose/compacted & & & & SRVY/INSP & HP & \\
\hline * Maximum clot size (visual monitoring) & & & & INSP & & \\
\hline Compaction - double steel drim roller & & CONSTR & & INSP & & \\
\hline *Testing: moisture content (D3017) & ENG & & & MTL & HP & \\
\hline "Tesling: in-place density (D2922) & ENG & & & Wa MTL & IIP & \\
\hline $\begin{array}{l}\text { *Verify that all tests were taken and that applicable HPs have } \\
\text { been released by ENG, and the as-built reflects the current status }\end{array}$ & ENG & & & MTL/INSP & $\mathrm{JIP}$ & \\
\hline \multicolumn{7}{|l|}{ 5.1 Shaping Fill gircement (Lift \#3) } \\
\hline *Lin brickness: material loose/compacted & & & & SRVY/INSP & IIP & \\
\hline * Maximum clot size (visual monitoring) & & & & INSP & & \\
\hline Compaction - double steel drum roller & & CONSTR & & INSP: & & \\
\hline
\end{tabular}

CONSTR = BN Constnction; $C Q A=$ Construction Quality Assurance; ENG/TM $=$ BN Engineering/Task Manager; GCL $=$ Geosynthetic Clay Liner; $H P=I$ Iold Point: INSP $=$ BN Inspection; IVC = Independent Verification Contractor (PEER Consultants); MTL $\Rightarrow$ BN Materials Test Lab; SRVY $=$ BN Survey; SUBCONTR $=$ BN Subcontractor

* See Table 3.1 of this plan 


\begin{tabular}{|c|c|c|c|c|c|c|}
\hline ITEM - DECONTAMINATION POND CLOSURE COVER & $\begin{array}{l}\text { ENG/TM } \\
\text { Hै। }\end{array}$ & CONSTR & $\begin{array}{l}\text { suB- } \\
\text { CONTR }\end{array}$ & $\begin{array}{l}\text { MTL/SRYYI } \\
\text { INSP }\end{array}$ & $\begin{array}{l}\mathrm{CQA} \\
\mathrm{HP}^{\mathrm{A}}\end{array}$ & $\begin{array}{l}\text { IVC } \\
\text { CIIECKLIST } \\
\text { INITIALS/DATE }\end{array}$ \\
\hline "Testing: moisture content (D3017) & ENG & & & MTL & HP & \\
\hline 'Testing: in-plice density (D2922) & ENG & & & MTL & IIP & \\
\hline $\begin{array}{l}\text { "Verify that all tests were taken and that applicable HPs have } \\
\text { been released by ENG, and the as-built reflects the current status }\end{array}$ & ENG & & & MTL/INSP & $\mathrm{IIP}$ & \\
\hline \multicolumn{7}{|l|}{ 5.1 Shaping Fill Placement (Lift $\left.H_{4}\right)$} \\
\hline${ }^{*}$ Lift thickness: material loose/compacted & & & & SRVY/NSP & $\mathrm{HP}$ & \\
\hline * Maximum clot size (visual monitoring) & & & & INSP & & \\
\hline Compaction - double steel drum roller & & CONSTR & & INSP & & \\
\hline Testing: moisture content (D3017) & ENG & & & MTL & $\mathrm{HP}$ & \\
\hline Testing: in-place density (D2922) & ÈNG & & & MTL & HP & \\
\hline $\begin{array}{l}\text { "Verify that all tests were taken and that applicable IIPs have } \\
\text { been released by ENG, and the as-built reflects the current status }\end{array}$ & ENG & & & MTL/INSP & $11 \mathrm{P}$ & \\
\hline \multicolumn{7}{|l|}{ 5.1 Shaping Fill Placement (Lift $\# \mathbf{5}$ ) } \\
\hline *Lift tlickness: material loose/compacted & & & & SRVY/INSP & IIP & \\
\hline - Maximum clot size (visual monitorifíg) & & & & $\rightarrow$ NSPP & & \\
\hline Compaction - double steel drón roller & & CONSTR & & $\mathrm{INSP} \times$ & & \\
\hline -Testing: moisture coftent (D3017) & ENG & & & MTL & Yap & \\
\hline -Testing: in-place density (D2922) & ENG & & & MTL & $\overrightarrow{I T P}$ & W \\
\hline $\begin{array}{l}\text { "Verify that all tests were taken and that applicable IJPs have } \\
\text { been released by ENG, and the as-built reflects the current status }\end{array}$ & ENG & & & MTLTNSP & $\mathrm{HP}$ & \\
\hline
\end{tabular}

CONSTR $=$ BN Construction; CQA = Construction Quality Assurance; ENG/TM = BN Engineering/Task Manager; GCL $=$ Geosynthetic Clay Liner; HP $=1$ told Point; INSP - BN Inspection; IVC = Independent Verification Contractor (PEER Consu'^nts); MTL = BN Materials Test Lab; SRVY = BN Survey; SUR-

CONTR $=($ Subcontracior

- Sce Table 3.1 of this plan 


\begin{tabular}{|c|c|c|c|c|c|c|}
\hline ITEM - DECONTAMINATION POND CLOSURE COVER & ENG/TM & CONSTR & $\begin{array}{l}\text { SUD } \\
\text { CONTR }\end{array}$ & $\begin{array}{l}\text { MTL/SRVYI } \\
\text { INSP }\end{array}$ & $\begin{array}{l}\text { CQA } \\
H P^{*}\end{array}$ & $\begin{array}{l}\text { IVC } \\
\text { CIIECKLIST } \\
\text { INITIALSIDATE } \\
\end{array}$ \\
\hline \multicolumn{7}{|l|}{5.1 Shaping Fill Placement (Lift H6) - If Required } \\
\hline -Lift thickness: material looseloompacted & & & & SRVY/NSP & HP & \\
\hline "Maximum clot size (vistual monitoring) & & & & INSP & & \\
\hline Compaction - double steel drum roller & & CONSTR & & INSP & & \\
\hline *Testing: moisture content (D3017) & ENG & & & MTL & HP & \\
\hline - Testing: in-place density (D2922) & ENG & & & MTL & HIP & \\
\hline $\begin{array}{l}\text { *Verify that alt tests were taken and that applicable HPs have } \\
\text { been released by ENG, and the as-built reflects the current status }\end{array}$ & ENG & & & MTL/INSP & IIIP & \\
\hline $\begin{array}{l}\text { Shaping fill material placement completed/verified per design } \\
\text { drawings }\end{array}$ & & CONSTR & & SRVY/INSP & HP & \\
\hline "Compaction tests completed and accepted & ENG & CONSTR & & MTL/INSP & IIP & \\
\hline \multicolumn{7}{|l|}{5.2 Processed Materials } \\
\hline "Lift thickness: material loose/compacted & & & $\checkmark$ & SRVY/INSP & IIP & \\
\hline *Maximum clod size (visual monitoring) & & & & INSP & & \\
\hline Compaction - double steel drum roller & & CONSTR & & INSP & & \\
\hline -Testing: mojstire content (D2216 and D3017) & ENG & & & MTL & HP & \\
\hline Testing: in-place density (D1556 and D2922) & ENG & & & MTL & ISP & \\
\hline $\begin{array}{l}\text { *Verify that all tests were taken and that applicable Hl's have } \\
\text { been released by ENG and the as-built reflects current status }\end{array}$ & ENG & & & MTL/INSP & & \\
\hline \multicolumn{7}{|l|}{ 5.2 Processed Material - Bedding Layer (Lift \#2) } \\
\hline "Lift thickness: material loose/compactẹd & & & & SRVYIINSP & $\mathrm{HP}$ & \\
\hline
\end{tabular}

CONSTR = BN Construction; $\mathrm{CQ \Lambda}=$ Construction Quality Assurance; ENG/TM = BN Engineering/Task Manager; GCL $=$ Geosynthetic Clay Liner; HP $=1$ lold - Point; INSP = BN Inspection; IVC = Independent Verificalion Contractor (PEER Consultants); M7L = BN Materials Test Lab; SRVY = BN Survey; SUB-

CONTR = BN Subcontractor

- See Table 3.1 of this plian 


\begin{tabular}{|c|c|c|c|c|c|c|}
\hline ITEM-DECONTAMINATION POND CLOSURE COVER & ENG TMP & CoNstr & $\begin{array}{l}\text { SÜD- } \\
\text { CONTR }\end{array}$ & $\begin{array}{l}\text { MTL/SRVY } \\
\text { INSP }\end{array}$ & $\begin{array}{c}\mathbf{C Q A} \\
\mathrm{HP}^{\mathbf{*}}\end{array}$ & $\begin{array}{l}\text { IVC } \\
\text { CHECKLIST } \\
\text { INITIALS/DATE }\end{array}$ \\
\hline -Maximum clod size (visual monitoring) & & & & INSP & & \\
\hline Compaction - double steel drum roller & & CONSTR & & INSP & & \\
\hline -Testing: motsture content (D2216 and D3017) & ENG & & & MTL & HP & \\
\hline "Testing: in-place density.(D1556 and D2922) & ENG & & & MTL & HP & \\
\hline $\begin{array}{l}\text { "Verify that all tests were taken and that applicable MPs have } \\
\text { been released by ENG and the as-built reflects current status }\end{array}$ & ENG & & & MTL/INSP' & IIP & \\
\hline \multicolumn{7}{|l|}{5.2 GCL Layer (one layer) } \\
\hline $\begin{array}{l}\text { Inspect and verify the bedding layer surface is smooth and free of } \\
\text { voids/debris }\end{array}$ & ENG & & SUB & INSP & IIP & \\
\hline $\begin{array}{l}\text { Verify the ready-to-place GCL is in good condition and is in a } \\
\text { dry state }\end{array}$ & & & SUB & INSP & HIP & \\
\hline \multicolumn{7}{|l|}{ No material sampling/testing required during placement } \\
\hline $\begin{array}{l}\text { Place liner per manufacturer's specifications/representative's } \\
\text { instnictions }\end{array}$ & & & SUB & INSP & & \\
\hline $\begin{array}{l}\text { Continuous visual inspection of liner for surface damage, } \\
\text { smootliness, and joint overlaps }\end{array}$ & & & SUB & Ni & & \\
\hline $\begin{array}{l}\text { Seal liner seams per manufacturer's specifications / } \\
\text { representative's tnstructions - if required }\end{array}$ & & & SUB & INSP & & \\
\hline Final approval of GCL completed installation & ENG & & sun & INSP & HP & \\
\hline \multicolumn{7}{|l|}{ 5.2 Processed Material - Buffer Layer (Lift 1) } \\
\hline "Lift jhičKness: material loose/compacled & & & & SRVY/INSP & HP & \\
\hline Maximum clod size (visual monitoring) & & & & INSP & & \\
\hline
\end{tabular}

CONSTR $=\mathrm{BN}$ Construction; $\mathrm{CQA}=$ Construction Quality Assurance; ENG/TM $=\mathrm{BN}$ Engineering/Task Manager; GCL $=$ Geosynthetic Clay Liner; $1 \mathrm{IP}=\mathrm{Hold}$ Point; INSP = BN Inspection; IVC = Independent Verification Contractor (PEER Consultants); MTL = BN Materials Test Lab; SRVY = BN Survey; SUB-

CONTR $=$ Subcontractor

- See Table 3.1 of this plan 


\begin{tabular}{|c|c|c|c|c|c|c|}
\hline ITEM - DECONTAMINATION POND CLOSURE COVER & ENG/TM & CONSTR & $\begin{array}{l}\text { SUB-? } \\
\text { CONTR }\end{array}$ & $\begin{array}{l}\text { MTL/SRVY } \\
\text { INSP }\end{array}$ & CQA & $\begin{array}{l}\text { IVC } \\
\text { CHECKLIST } \\
\text { INITIAIS/DATE }\end{array}$ \\
\hline Compaction - double steel drum roller & & CONSTR & & INSP & & \\
\hline *Testing: moisture content (D2216 and D3017) & ENG & & & MTL & HIP & \\
\hline Testing: in-place density (D1556 and D2922) & ENG & & & MTL & $\mathrm{HP}$ & \\
\hline $\begin{array}{l}\text {-Verify that all tests were taken and that applicable HPs have } \\
\text { been released by ENG and the as-buitt reflects current status }\end{array}$ & ENG & & & MTLJMSP & $\mathrm{HP}$ & \\
\hline \multicolumn{7}{|l|}{ 5.2 Processed material - Buffer Layer (Lift \#2) } \\
\hline -Lif lhickness: material loose/compacted & & & & SRVY/INSP & HP & \\
\hline *Maximum clod size (visual monitoring) & & تبرم & & INSP & & \\
\hline Compaction - double steel dnum roller & & CONSTR & & INSP & & \\
\hline - Tesling: moisture content (D2216 and D3017) & $\mathrm{ENG}^{2}$ & 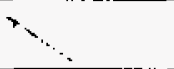 & & MTL & IIP & \\
\hline *Testing: in place density (D1556 and D2922) & ENG & $\cdots$ & & MTL & HP & \\
\hline $\begin{array}{l}\text { *Verify that all tests were taken and that applicable HPs haye } \\
\text { been released by ENG and the as-built reflects current status }\end{array}$ & ENG & & & MTL/INSP & HIP & \\
\hline \multicolumn{7}{|l|}{ 5.6 Type II Erosion Control Layer (Lift \#1) } \\
\hline Place Type Il material & & CONSTR & & INSP & & \\
\hline Lif thickness verification -- loose/compacted & & & & SRVY/INSP & XHP & \\
\hline Compaction - double steel drum roller & & CONSTR & & INSP & & . \\
\hline *Testing: in-plyee density (D2922) & ENG & & & MTL & IIP & \\
\hline -Testing. Moisture content (D30I7) & ENG & & & MTL & HIP & \\
\hline Engineering acceptance (initials) for lift thickness and testing & ENG & & & & $\mathrm{HP}$ & \\
\hline
\end{tabular}

CONSTR = BN Construction; CQA $=$ Constnction Quality Assurance; ENG/TM = BN Engineering/Task Manager; GCL $=$ Geosynthelic Clay Liner; HP $=$ Hold . Point; INSP = BN Inspection; IVC = Independent Verification Contractor (PEER Consultants); MTL = BN Materials Test Lab; SRVY $=$ BN Survey; SUB.

CONTR $=$ BN Subcontracior

- See Table 3.1 of this plan 


\begin{tabular}{|c|c|c|c|c|c|c|}
\hline ITEM - DECONTAMINATION POND CLOSURE COVER & $\begin{array}{l}\text { ENC/TM } \\
\text { Aें। }\end{array}$ & CONSTR & $\begin{array}{l}\text { SUB- } \\
\text { CONTR }\end{array}$ & MTLISRYYI & $\begin{array}{l}\text { CQA } \\
\mathrm{IP}^{*}\end{array}$ & $\begin{array}{l}\text { IVC } \\
\text { CIECKLIST } \\
\text { INITIALS/DATE }\end{array}$ \\
\hline \multicolumn{7}{|l|}{ 5.6 Type II Erosion Control Layer (Lift $\# 2)^{\circ}$} \\
\hline Place Type ll material & & CONSTR & & INSP & & \\
\hline Lift thickness verification -- loose/compacted & & & & SRVY/INSP, & $\mathrm{HP}$ & \\
\hline Compaction - double steetdrum roller & & CONSTR & & INSP' & & \\
\hline *Testing: in-place density (D2922). & ENG & & & MTL & $\mathrm{HP}$ & \\
\hline -Testing: moisture content (D3017) & ENG & & & MTL & HP & \\
\hline Engineering acceptance (initials) for lif thickness and testing & ENG & rata & & & HP & \\
\hline $\begin{array}{l}\text { FINAL ENG/IVC/INSP "WALK AROUND" COMPLÉTED } \\
\text { CLOSURE COVER ACCEPTANCE AND INITIALS }\end{array}$ & ENG & & & INSP & IIP & \\
\hline \multicolumn{7}{|l|}{ 5.7 Survey Monuments } \\
\hline $\begin{array}{l}\text { Survey monuments installed per design drawing (C3) and as } \\
\text { shown on survey as-built }\end{array}$ & ENG & & & SRVY/INSP & lip & \\
\hline \multicolumn{7}{|l|}{5.8 Site Fencing / Gates / Signage } \\
\hline Fence and access gates installed per design drawing (C3) & & CONSTR & & INSP & HP & \\
\hline Signage installed and secured per design frawing (C3) & & CONSTR & & INSP & HP & \\
\hline \multicolumn{7}{|l|}{ 6.0 POST CONSTRUCTION } \\
\hline $\begin{array}{l}\text { All test/inspection documents (test/inspection records, as-buitt } \\
\text { sketches, survey data, etc) shall be submitted to the engineering } \\
\text { team for review/evâluation and subsequent submittal to the BN } \\
\text { Task Manager }\end{array}$ & ENG & & SUB & $\begin{array}{l}\text { MTLLSRVYI } \\
\text { INSP }\end{array}$ & & \\
\hline $\begin{array}{l}\text { Other: to be determined from final design review of project } \\
\text { test/inspection results }\end{array}$ & ENG/TM & & & & & \\
\hline
\end{tabular}

CONSTR $=\mathrm{BN}$ Construction; $\mathrm{CQA}=$ Construction Quality Assurance; $\mathrm{ENG} / \mathrm{TM}=\mathrm{BN}$ Engineering/Task Manager; GCL $=$ Geosynthetic Clay Liner; $\mathrm{JIP}=\mathrm{Hold}$ Point; INSP TN Inspection; IVC = Independent Verification Contractor (PEER Consul'ants); MTL = BN Materials Test Lab; SRVY = BN Survey; SUB- 


\section{DECONTAMINATION POND (CAU 92) CLOSURE COVER APPROVAL MATRIX AND CHECKLIST}

MATRIX: Use for further definition of quality responsibilities and for identification of relevant procedures.

NOTE: BN okganizations, in accordance with the PAMP, will provide the necessary controls during construction to verify that all design-specified hold points outlined in this plan are complied with prior to proceeding with subsequent process steps. Independent hold-point monitorting/data review will be conducted by the IVC representative.

\begin{tabular}{|c|c|c|c|c|c|c|}
\hline ITEM - DECONTAMINATION POND CLOSURE COVER & ENG/TM & CONSTR & $\begin{array}{l}\text { SUR- } \\
\text { CONTR }\end{array}$ & $\begin{array}{l}\text { MTL/SRVY } / \\
\text { INSP }\end{array}$ & CQA & $\begin{array}{c}\text { IVC } \\
\text { CIECKLIST } \\
\text { INITIALS/DATE }\end{array}$ \\
\hline \multicolumn{7}{|l|}{ 5.1 Berm Material Placement (Lift \#1) } \\
\hline -Lift thickness: material loose/compacted & & & & & HP & \\
\hline *Maximum clot size (visual monitoring) & & & & INSP & & \\
\hline 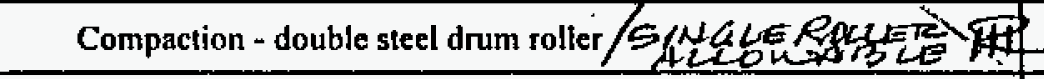 & & CONSTR & & INSP & & \\
\hline *Testing: moisture content (D3017) & ENG & & & MTL & HP & \\
\hline -Testing: in-place density (D2922) & ENG & & & MTL & $H P$ & \\
\hline $\begin{array}{l}\text { "Verify that all tests were taken and that applicable HPs have been } \\
\text { released by ENG, and the as-built reflects the current status }\end{array}$ & ENG & & & MTL/INSP' & $11 \mathrm{P}$ & \\
\hline \multicolumn{7}{|l|}{ 5.I Berm Material Placement (Lift :\#2) } \\
\hline -Lift thickness: material loose/compacted & & & & SRVY/INSP & HP & \\
\hline "Maximumelot size (visual monitoring) & & & & INSP & & \\
\hline Compaction - double steel drum roller SEE LJET & & CONSTR & & INsP & & \\
\hline "Testing: moisture content (D30I7) & ENG & & & MTL & HP & \\
\hline *Testing: in-place density (D2922) & ENG & & & MTL & Hि & \\
\hline
\end{tabular}

CONSTR = BN Construction; CQA = Construction Quality Assurance; ENG/TM = BN Engineering/Task Manager; GCL = Geosynthetic Clay Liner; IIP = Hold Point; INSP = DN Inspection; IVC = Independent Verification Contractor (PEER Consultants); MTL = BN Materials Test Lab; SRVY = BN SUrvey; SUB-CONTR = BN Subcontractor

- See Table 3.1 of this phan 


\begin{tabular}{|c|c|c|c|c|c|c|}
\hline ITEM - DEÇONTAMINATION POND CLOSURE COVER & ENG/TM & CONSTR & $\begin{array}{l}\text { SUR- } \\
\text { CONTR }\end{array}$ & $\begin{array}{l}\text { MTL/SRVY } / \\
\text { INSP }\end{array}$ & CQA & $\begin{array}{l}\text { WVC } \\
\text { INITLALSIDATE }\end{array}$ \\
\hline $\begin{array}{l}\text { Verify that all tests were taken and that applicable HPs have been } \\
\text { released by ENG, and the as-built reflects the current status }\end{array}$ & ENG & & & MTL/NSP & HP & \\
\hline \multicolumn{7}{|l|}{5.1} \\
\hline "Lift thickness: material loose/compacted & & & & SRVY/INSP & HP & \\
\hline -Maximum clot size (visual monitoring) & 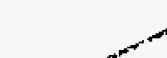 & & & INSP & & \\
\hline Compaction - double steel drum roller SRE LIFT $\mid$ ITी & & CONSTR & & INSP & & \\
\hline *Testing: moisture content (D3017) & $\mathrm{ENO}^{2}$ & $m$ & & MTL & HP & \\
\hline *Testing: in-place density (D2922) & ENG & & & MTL & HP & \\
\hline $\begin{array}{l}\text {-Verify that all tests were taken and that applicable HPs have been } \\
\text { released by ENG, and the as-builf reflects the current status }\end{array}$ & ENG & & & MTLANSP & HP & \\
\hline Shaping fill material placement completed/verified per FCN & ENG & CONSTR & & SRVYYINSP & HP & \\
\hline *Compactión tesis completed and accepted & ENG & CONSTR & & MTL/INSP & $\mathrm{HP}_{\mathrm{P}}$ & \\
\hline
\end{tabular}

CONSTR = BN Construction; CQA $=$ Construction Quality Assurance; ENG/TM $=\mathrm{BN}$ Engineering $/$ Task Manager; GCL $=$ Geosynthetic Clay Liner; $\mathrm{HP}=\mathrm{Hold}$ Point: INSP = BN Inspection; IVC = Independent Verification Contractor (PEER Consultants); MTL = BN Materials Test Lab; SRVY = BN Survey; SUD-CONTR $=\mathrm{F}$ abcontractor.

- See Table 3.1 of this plan 


\section{DECONTAMINATION POND (CAU 92) CLOSURE COVER APPROVAL MATRIX AND CHECKLIST}

PROJECT RECORD

MATRIX: Use for further definition of quality responsibilities and for identification of relevant procedures.

NOTE: BN organizations, in accordance with the PAMP, will provide the necessary controls during construction to verify that all :design-specified hold points outlined in this plan are complied with prior to procceding with subsequent process steps. Independent hold-point monitoring/data review will be conducted by the IVC representative.

\begin{tabular}{|c|c|c|c|c|c|c|}
\hline ITEM_DECONTAMINATION POND CLOSURE COVER & ENG/TM & $\begin{array}{l}\text { CoNSTR, } \\
\text { Con? }\end{array}$ & $\begin{array}{l}\text { Sun-? } \\
\text { CoNTR } \\
\end{array}$ & MrE/SRVYI & $\begin{array}{ll}\text { CQA } \\
\mathrm{HP}^{2}\end{array}$ & $\begin{array}{l}\text { OIVC } \\
\text { CIIECKLIST } \\
\text { INITIALS/DATE }\end{array}$ \\
\hline \multicolumn{7}{|l|}{ 1.0 GENERAL PRECONSTRUCTION ACTIVITIES } \\
\hline Completion of Test Plot Construction, sampling, and testing & ENG & CONSTR & SUB & MTL/NSSP & HP & \\
\hline Constnuction permits/NDEP project approva! & $\mathrm{TM}$ & & & & HP & \\
\hline $\begin{array}{l}\text { Completion of laboratory tests required to qualify all closure } \\
\text { cover layering materials (soils) }\end{array}$ & ENG & & SUB & MTL & HP & \\
\hline Batch Plant to batch and stockpile closure covet materials & ENG & CONSTR & & MTL/TNSP & IIP & \\
\hline Certified GCL materials on job silc & ENG & CONSTR & SUB & INSP & HP & \\
\hline \multicolumn{7}{|l|}{ 2.0 TECHNICAL DOCUMENTS } \\
\hline $\begin{array}{l}\text { Prepared and approved engineeting technical document including } \\
\text { design drawings/specifications/POs/CQA Plan; and material } \\
\text { qualities have been reviewed by all involved parties. }\end{array}$ & ENG/TM & CONSTR & SUB & $\begin{array}{l}\text { MTL/SRVY/ } \\
\text { INSP }\end{array}$ & IIP & \\
\hline $\begin{array}{l}\text { "Material inspection/testing: soil lypes, test frequencies, and } \\
\text { acceptance criteria has been developed perTable } 3 \text { t. }\end{array}$ & ENG & & & & HP. & \\
\hline
\end{tabular}

$+$

CONSTR = BN Construction; $\mathrm{CQA}=$ Construction Quality Assurance; ENG/TM = BN Engineering/Task Manager; GCL = Geosynthetic Clay Liner; HP = Hold Point; INSP = BN Inspection; IVCO = Independent Verification Contractor Officer (PEER Consultants); MTL - BN Materials Test Lab; SRVY * BN Survey; SUB-CONTR $=$ BN Subcontractor

- See Table 3.1 of this plan 


\begin{tabular}{|c|c|c|c|c|c|c|}
\hline ITEM - DECONTAMINATION POND CLOSURE COVER & ENG/TM & ConsTR & $\begin{array}{l}\text { SUB- } \\
\text { CONTE }\end{array}$ & $\begin{array}{l}\text { MTL/SRVYI } \\
\text { INSP }\end{array}$ & $\begin{array}{l}\text { CQA } \\
\text { I]1* }\end{array}$ & $\begin{array}{c}\text { IVC } \\
\text { CIECKLIST } \\
\text { INITIALS/DATE }\end{array}$ \\
\hline \multicolumn{7}{|l|}{ 3.0 SUPPLY PIPE LINE DISPOSAL } \\
\hline $\begin{array}{l}\text { Backfill botrow material (soil) over contaminated pipe/soit } \\
\text { placement area in Decontamination Pond }\end{array}$ & & CONSTR & & INSP & & \\
\hline Placement of contaminated pipe/soils in Deconiamination Pond & 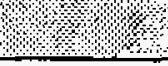 & CONSTR & & INSP & & \\
\hline \multicolumn{7}{|l|}{ 4.0 CONSTRUCTION SOILS MANUFACTURE/ACCEPTANCE } \\
\hline \multicolumn{7}{|l|}{ 4.1 Shaping Fitl Materials -- Dorrow Pit/Stockpile: } \\
\hline Material: Slockpile segregation control (visunl monitoring) & & & & INSP & & \\
\hline -Testing: Particle size analysis of shaping fill material stockpile & ENG & & & MTL & HP & \\
\hline *Tesling: Modified proctor for moisture/density curve & ENG & & & MTL & HF & \\
\hline \multicolumn{7}{|l|}{ 4.2 Processed Materials -- Plnnt/Stockpile: } \\
\hline Material: Processing controls/clod size (visual monitoring & & & & INSP & & \\
\hline *Testing: Particle size analysis of processed materials stockpile & ENG & & & MTL & It!P & \\
\hline -Testing: Modified proctor for moisture/density curve & ENG & & & MTL & IIP & \\
\hline \multicolumn{7}{|l|}{4.3 Type II Materials -- Plant/Stockpile: } \\
\hline Material: Stockpile segregation control (visual monitoring) & & & & INSP & & \\
\hline Testing: Particle size analysis of Type II materials stockpile & ENG & & & MTL & IIP & \\
\hline *Testing: Modified proctor for moisture/density curve & ENG & & & MTL & IIP & \\
\hline All plant/stockpile testing/inspection completed & ENG & & & MTL/INSP & IIP & \\
\hline \multicolumn{7}{|l|}{5.0 CONSTRUCTION -- CLOSURE COVER } \\
\hline Closure cover -- survey site layout establislied (as-built) & & & & SR VY/INSP & & \\
\hline
\end{tabular}

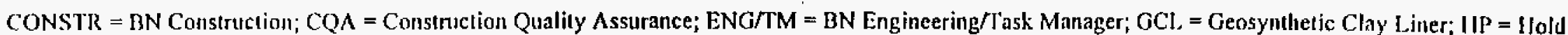
Point; INSP $=$ BN Inspection; IVCO = Independent Verification Contractor Officer (PEER Consuliants); MTL = BN Materials Test L.ab; SRVY = BN Survey; SUB-CONTR = BN Subcontractor

* Sq able 3.1 of this plan 


\begin{tabular}{|c|c|c|c|c|c|c|c|}
\hline ITEM - DECONTAMINATION POND CLOSURE COVER & ENG/TM & Constr & $\begin{array}{l}\text { SUB- } \\
\text { CONTR }\end{array}$ & $\begin{array}{r}\text { MTL/SR VYI } \\
\text { INSP }\end{array}$ & $\begin{array}{l}\text { CQA } \\
\text { IIP }^{3}\end{array}$ & $\begin{array}{l}\text { IVC } \\
\text { CIIECKLIST } \\
\text { INITIALSIDATE }\end{array}$ & $\begin{array}{l}S F E \\
O R / G / N / K L\end{array}$ \\
\hline All inaterial delivered/stockpiled (visual verification) & & & & INSP & & & \\
\hline \multicolumn{7}{|l|}{ 5.1 Berm Material Layer Placement (LIf: \#1) } & \\
\hline Perimeter berm leveled and spread onto the pond boitom & & CONSTR & & INSP & & & \\
\hline 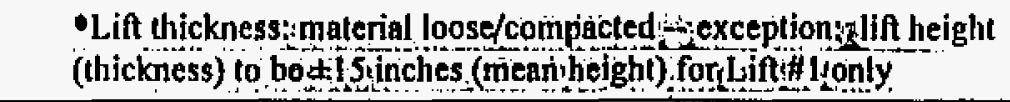 & & & & SRVY/INSP & HP & & \\
\hline * Maximum clod size (visual monitoring) & & & & INSP & & & \\
\hline Compaction - double steel drum roller & & CONSTR & & NSP & & & \\
\hline *Testling: moisturo content (D3017) & ENG & & & MTl. & & & \\
\hline *Testing: in-place density.(D2922) & ENG & & & MTL & & & \\
\hline $\begin{array}{l}\text { *erify that all tests were completed with accepteble results; that } \\
\text { applicable HPs have been released by Engineeringt;and the as- } \\
\text { buift reflects theccurrent status }\end{array}$ & ENO & & & MTL/INSP & $\mathrm{HP}$ & & \\
\hline \multicolumn{7}{|l|}{5.1 Derm Materlal Layer Placement (Lift:H2) } & \\
\hline "Lif thicknessi material:Joose/compacted per Table 3:] & & & & SRVY/INSP & $\mathrm{HP}$ & & \\
\hline Maximum clod size (visual monitoring) & & & & INSP & & & \\
\hline Compaction - double steel drum roller & & CONSTR & & INSP & & & \\
\hline Testing: moisture content (D3017) & ENG & & & MTL & & & \\
\hline Testing: in-place density (D2922) & ENG & & & MTL: & & & \\
\hline $\begin{array}{l}\text { *Verify that all tests were completed with acceptable results; ithat } \\
\text { applicable HPs have been released by ENG; and the astbuilt } \\
\text { reflects the current status }\end{array}$ & ENG & & & $\begin{array}{c}\text { MTL/INSP } \\
. \\
\end{array}$ & $H \mathrm{P}$ & & \\
\hline
\end{tabular}

CONSTR $=\mathrm{BN}$ Constnuction; $\mathrm{CQA}=$ Constnction Quality Assurance; ENG/TM $=\mathrm{BN}$ Engineering/Task Manager; GCL $=$ Geosyntlietic Clay Liner; $\mathrm{HP}=\mathrm{Hold}$ Point; INSP = AN Inspection; IVCO = Independent Verification Contractor Officer (PEER Consultants); MTL = BN Materials Test Lab; SRVY = BN Survey; SUB-CONTR = BN Subcontractor 


\begin{tabular}{|c|c|c|c|c|c|c|}
\hline ITEM - DECONTAMINATION POND CLOSURE COVER & ENG/TM & CONSTR & $\begin{array}{c}\text { SUB- } \\
\text { CONTR }\end{array}$ & $\underbrace{\text { MTLSRVYI }}_{\text {INSP }}$ & $\begin{array}{l}\text { CQA } \\
H P^{*}\end{array}$ & $\begin{array}{c}\text { IVC } \\
\text { CIHECKLIST } \\
\text { INITIALSIDATE }\end{array}$ \\
\hline \multicolumn{7}{|l|}{ 5.1 Berm Matertal Layer Placement (Lift H3) } \\
\hline Lift thicknessingaterial-loose/compacted perTable 3: & & & & SRVY/INSP & HP & \\
\hline "Moximum clod size (visual monitoring) & & & & INSP & & \\
\hline Compaction double stcel drum roller & & CONSTR & & INSP & & \\
\hline Presting: molsturécönitent (D3017) & ENG & & & MTL" & & \\
\hline *Testing: in-place density (D2922) & ENG & & & MTL & & \\
\hline $\begin{array}{l}\text { *Verify that all tesis were completed wilh accentable results; that } \\
\text { applicable HPs baye been released bydENG } \\
\text { reflects the current status }\end{array}$ & ENG & & & MTL/INSP & HP & \\
\hline \multicolumn{7}{|l|}{ 5.1 Derm MaterialLayerPlacement (Lirt \#4) } \\
\hline Lift thickness material;loose/compacted peraTable $3_{t, l}$ & & & & SRVY/INSP & HP & Fin Bancy \\
\hline *Maximum clodisizo (visualmonlloting) & & & & INSP & & tin pacer \\
\hline Compaction doublo steel drum rolles & & CONSTR & & INSP, & & th Sang \\
\hline PTesting: moisture content (D3017) & ENG & & & MTL & & Finger \\
\hline Pestingtin-place density (D2922) & ENG & & & MTL: & & $10<12<8$ \\
\hline 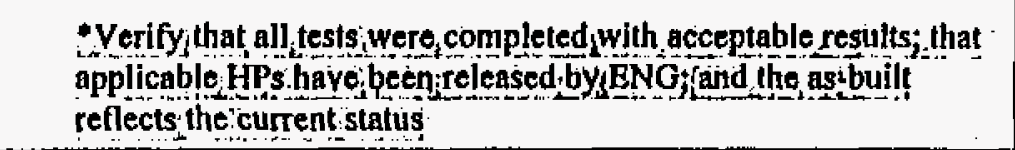 & ENG & & & MTL/INSP & HP & $10 / 12 / 96$ \\
\hline 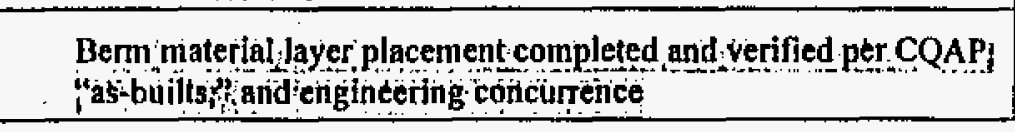 & ENG & CONSTR & & SRVY/INSP: & $\underline{\mathrm{HP}}$ & K1: Hown \\
\hline
\end{tabular}

CONSTR = BN Construction; CQA = Construction Quality Assurance; ENG/TM $=$ BN Engineering/Task Manager; GCL = Geosynthetic Clay Liner; HP = Hold Point; INSP $=$ BN Inspection; IVCO $=$ Independent Verification Contractor Officer (PEER Consultants); MTL $=$ BN Malerials Test Lab; SRVY $=$ BN Survey; SUIB-CONTR = BN Subcontractor

- Se( ble 3.1 of this plan 


\begin{tabular}{|c|c|c|c|c|c|c|c|}
\hline NoTE: & ITEM - DECONTAMTNATION POND CLOSURE COVER & $\begin{array}{c}\text { ENG/TM } \\
\text { ENG } \\
\end{array}$ & CONSTR & $\begin{array}{l}\text { SUD } \\
\text { CONTR }\end{array}$ & $\begin{array}{l}\text { MTLSRVY } \\
\text { INSP }\end{array}$ & $\begin{array}{l}\mathrm{CQA} \\
\mathrm{HH}\end{array}$ & $\begin{array}{l}\text { IVC } \\
\text { CHECKLIST } \\
\text { INITIALS/DATE }\end{array}$ \\
\hline $\begin{array}{l}\text { is DocumeF } \\
\text { EC D } 10 / 14-9\end{array}$ & \multicolumn{7}{|c|}{ Pond Perimeter Over Excavationifactivlties $/ \% / 14 / 98$ sTART } \\
\hline \multirow{14}{*}{$\begin{array}{l}\text { SPART OF } \\
\text { SUSER } \\
\text { SPFCIFARAT }\end{array}$} & Over excavate pond perimetersplaya material per.Drawing $\mathrm{C4}$ & & CONSTR & & $I_{1} P_{a} f$ & & \\
\hline & Verify pond perimeterfover excavation elevation controlpoints & ENO & & & SRVY/INSPfo & $H P$ & \\
\hline & Compaclion doublestéel druiniroller & & CONSTR & & INSP nef & & \\
\hline & Testing moistlire content (D3017) & ENG & & & MTL & & \\
\hline & Testing: in-placedensity $(\mathrm{D} 2922)$ & ENO & . & & MTLi & & \\
\hline & 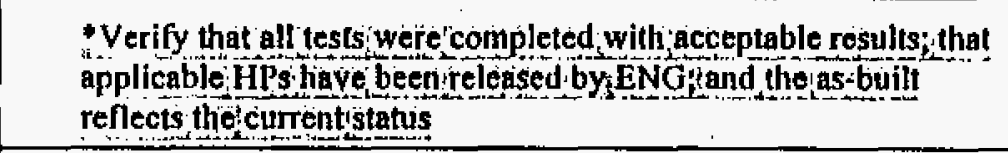 & ENG & & & $\begin{array}{r}\text { MTL/INSP } \\
\text { ant }\end{array}$ & HP & $\not$ \\
\hline & $\begin{array}{l}\text { Perimeteriexavationtconpleted and verified per.CQAPsisass } \\
\text { builts, and enginering concurtence }\end{array}$ & ENG & CONSTR & & SRVY & $\underline{11} \mathbf{P}$ & Aast \\
\hline & 5.1 Slaping Fill Layer Placement (Lift H1) & \multicolumn{6}{|l|}{$10 / 20 / 98$} \\
\hline & *Lift thickness: material loose/compacted per Table 3:1 & & & & SRVY/INSP: & IIP & \\
\hline & - Maximum clod size (visual monitoring) & & & & INSP $a n$ & & \\
\hline & Compaction - double sleel drum roller & . & CONSTR & & INSP afd & & \\
\hline & Testing: moisture content (D3017) & ENG & 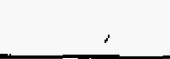 & & MTL & & \\
\hline & Testing: in-place density (D2922) & ENG & & & MTL & & 10158 \\
\hline & $\begin{array}{l}\text { * Verify that all tests were completed with acceptable; resilts that } \\
\text { applicable l-IPs have been released by ENG; and the as-built } \\
\text { reflects the current status }\end{array}$ & ENG & & & $\begin{array}{l}\text { MTLINSP: } \\
\text { ofd }\end{array}$ & HIP & $\begin{array}{l}15+10 \\
10 / 20 / 4\end{array}$ \\
\hline
\end{tabular}

CONSTR $=\mathrm{BN}$ Construction; $\mathrm{CQA}=$ Construclion Quality Assurance; ENG/TM $\approx \mathrm{BN}$ Engineering/Task Manager; GCL $=$ Geosynthetic Clay Liner; HP $=$ Hold Point; INSP = BN Inspection; IVCO = Independent Verification Contractor Officer (PEER Consultants); MTL = BN Materials Tesi Lab; $S R$ VY = BN Survey;

SUB-CONTR = BN Subcontractor

- See Table 3.1 of this plan 


\begin{tabular}{|c|c|c|c|c|c|c|}
\hline ITEM - DECONTAMINATION POND CLOSURE COVER & ENGITM & $\begin{array}{l}\text { Constr } \\
\text { const }\end{array}$ & $\begin{array}{l}\text { SUn- } \\
\text { CONTR }\end{array}$ & $\begin{array}{l}\text { MTLUSRVY/ } \\
\therefore \text { INSP }\end{array}$ & $\begin{array}{l}\text { CQA } \\
\mathrm{IP}^{4}\end{array}$ & $\begin{array}{l}\text { IVC } \\
\text { CIECKLIST } \\
\text { INITIALSTITATE }\end{array}$ \\
\hline \multicolumn{7}{|l|}{ 5.1 Slaaping Fill Layer Placement (LIft H2) } \\
\hline "Lift Ihickness: material loose/compacted per Table 3.1 & & & & SRVY/INSP & IIP & \\
\hline \multicolumn{7}{|l|}{ *Moximum clod size (visual moniloring) } \\
\hline Compaction - double steel drum roller & & CONSTR & & INSP WAS & & \\
\hline -Testing: moisture content (D3017) & ENG & & & MTI. & 8.4 & \\
\hline *Testing: in-place densily (D2922) & ENG & & & MTL & & \\
\hline $\begin{array}{l}\text { "Verify Iltat all tests were completed, with acceptable results; that } \\
\text { applicable HIS have been released by ENG; and the as-built } \\
\text {. reflects the current status }\end{array}$ & ENG & & & $\begin{array}{r}\text { MTL/INST: } \\
\text { off } \\
\end{array}$ & HP & 48 \\
\hline \multicolumn{7}{|l|}{5.1 Shaping Fill Layer Ptacement (Lift \#3) } \\
\hline "Lif thickness: material loose/compacted per Table 3.1 & & & & SRVYIINSGid & $\mathrm{IP}$ & \\
\hline *Maximum clod size (visual monitoring) & & & & INSP ard & & 198 \\
\hline Compaction - double steel drum roller & & CONSTR & & INSP a de & & Ke, Sow \\
\hline *Testing: moisture content (D3017) & ENG & & & MTL & & tarey \\
\hline *Testing: in-place density (D2922) & ENG & & & MTL & 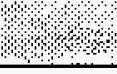 & $\lim 124,198$ \\
\hline $\begin{array}{l}\text { "Verify that all tesis were completed with acceptable results; that } \\
\text { applicable HPs have been released by ENG; and the as-built } \\
\text { reflects the current status }\end{array}$ & ENG & & & MTL/INSP' & IIP & $\begin{array}{l}\text { Kaforeq } \\
10 / 28 / 96\end{array}$ \\
\hline \multicolumn{7}{|l|}{ 5.1 Slaping Fill Layer Placentent (LIft H4) } \\
\hline "I.ifl thickness: material loose/compacted per Table 3.1 & & & & SRVY/INSLerk & IIP & Ko/ga/gis \\
\hline -Maximum clod size (visual monitoring) & & & & INSP \& ${ }^{\prime}$ & & ripolan \\
\hline
\end{tabular}

CONSTR $=$ IN Construction; $\mathrm{CQA} \Rightarrow$ Constnction Quality Assurance; ENG $/ \mathrm{TM}=\mathrm{BN}$ Engineering/Task Manager; GCL, $=$ Geosynthetic Clay Liner; HP $\Rightarrow$ Hold Point; INSP $=$ BN Inspection; IVCO = Independent Verification Contractor Officer (PLEER Consultants); MTL = BN Materials Test Lab; SRVY = BN Survey: SUB- $\Gamma$ ONTR = BN Subcontractor

- $S$ Q ible 3.1 of this plan 


\begin{tabular}{|c|c|c|c|c|c|c|}
\hline ITEM - DECONTAMINATION POND CLOSURE COVER & ENC/TM & CONSTR & $\begin{array}{l}\text { SUB- } \\
\text { CONTR }\end{array}$ & $\begin{array}{c}\text { MTLJSRVYI } \\
\text { INSP }\end{array}$ & $\begin{array}{l}\text { CQA } \\
\mathrm{HI}^{1 *}\end{array}$ & $\begin{array}{l}\text { IVC : } \\
\text { CHECKLIST } \\
\text { INITIALS/DATE }\end{array}$ \\
\hline Compaction - double steel drum roller & & CONSTR & & INSP ard & & 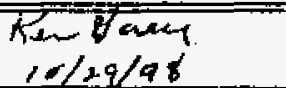 \\
\hline - Tusting: moisture content (D3017) & ENG & & & MTL & & \\
\hline "Testing: in-place density (D2922) & ENG & & & MTL & & \\
\hline $\begin{array}{l}\text { Verify lhat all tests were completed with acceplable results; that } \\
\text { applicable HPs have been released by ENG; and the as-built } \\
\text { reflects the current status }\end{array}$ & ENG & & & $\begin{array}{r}\text { MTL/INSP } \\
\Delta \mathscr{N}^{\prime} \\
\end{array}$ & $H P$ & $\begin{array}{l}\text { Facy } \\
11 / x_{1} / 9\end{array}$ \\
\hline \multicolumn{7}{|l|}{ 5.1 Sluaping Fill Layer Placement (Lifl H5) } \\
\hline "Lift thickness: material loose/compacled per Table 3.1 & & & & SRVYINSPJ & IIP & 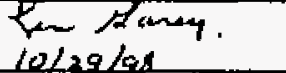 \\
\hline * Maximum clod size (visual monitoring) & & & & INSP & & Keen Fan \\
\hline Compaction - double steel drum roller & & CONSTR & & JNSP . on & & Kie tomy \\
\hline -Testing: moisture content (D3017) & ENG & & & MTL & & tiol-4/48 \\
\hline Testing: in-place density (D2922) & ENG & & & MTL & 18. & 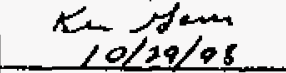 \\
\hline $\begin{array}{l}\text { "Verify that all tests were completed with acceptable results; that } \\
\text { applicable IIPs have been released by ENG; and the as-built } \\
\text { rellects the current status }\end{array}$ & ENG & & & $\begin{array}{r}\text { MTL/INSP' } \\
\text { off } \\
\end{array}$ & IIP & 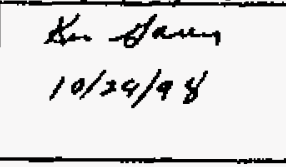 \\
\hline \multicolumn{7}{|l|}{ 5.I Slaping Fill Layer Placement (Lift H6) \ Required } \\
\hline "Lift thickness: material loose/compacted per Table 3:1 & & & & SRVY/INSPd & $H \Gamma$ & $\frac{x+1}{11}$ \\
\hline "Maximum clod size (visual monitoring) & & & & INSP ard & & Ko/2/98 \\
\hline Compaction - double steel drum roller & & CONSTR & & INSP.. if & & $k-1 / 2 / 98$ \\
\hline "Testing: moisture content (D3017) & ENG & & & MTL & & Kin dax \\
\hline "Testing: in-place densily (D2922) & ENG & & & MTL & & 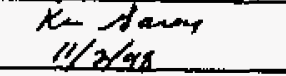 \\
\hline
\end{tabular}

CONSTR = IN Construclion; CQA $=$ ConsInction Quality Assurance; ENG/TM $=$ BN Engineering/Task Manager; GCL = Geosynthelic Clay Liner; $H$ HP $=H$ Hold Point; INSP = BN Inspection; IVCO = Independent Verification Contractor Officer (PEER Consultants); MTL = BN Materials Test Lab; SRVY = BN Survey; SUB-CONTR = BN Subcontractor

- See Table 3.1 of this plan 


\begin{tabular}{|c|c|c|c|c|c|c|}
\hline ITEM - DECONTAMINATION POND CLOSURE COVER & ENG/TM & Constr & $\begin{array}{l}\text { SUB } \\
\text { CONTR }\end{array}$ & $\begin{array}{l}\text { MTI/SRVYI } \\
\text { INSP }\end{array}$ & $\begin{array}{l}\text { CQA } \\
\text { HP* }\end{array}$ & $\begin{array}{l}\text { CIIECKLIST } \\
\text { INITIAISTIDATE }\end{array}$ \\
\hline $\begin{array}{l}\text { * Verify that all tests were completed,with acceptable results; that } \\
\text { applicable HPs have been released by ENG; and the as-buili } \\
\text { reflects the current status }\end{array}$ & ENG & & & $\begin{array}{r}\text { MTL/INSP } \\
. / f \prime\end{array}$ & HP & $\begin{array}{l}\text { Ken } \operatorname{san} t \\
11 / 9 / 98 \ldots\end{array}$ \\
\hline \multicolumn{7}{|l|}{ 5.6 Slope Control Pass (Shaping Fill Placemintnt Completed) } \\
\hline Cut/Slope surface to required gradd & & CONSTR & & INSP, ors & & \\
\hline Re-compaction - double steel drum rôller & & CONSTR & & INSP, & & \\
\hline Verify, grade slope conforms to the CQAP & & CONSTR & & SRVYITNSB & HP & \\
\hline Testing: moisture content (D3017) & ENG & & & MTL & & \\
\hline Testing: in-place density (D2922) & ENO & & & MTL & & $\tan 4$ \\
\hline 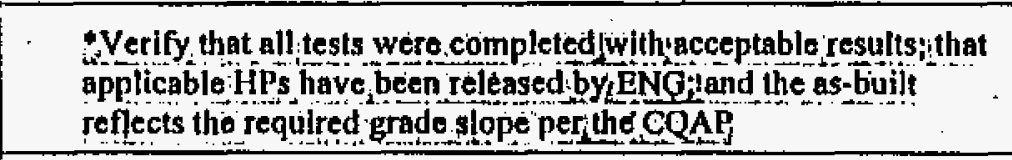 & ENG & & & $\begin{array}{r}\text { MTL/INSP } \\
\text { ofd }\end{array}$ & HP. & $11 / 4 / 8 x$. \\
\hline $\begin{array}{l}\text { Shaping fill material layer placement completed/verified per } \\
\text { CQAP, "ag-builts, }\end{array}$ & ENG & CONSTR & & $\begin{array}{r}\text { SRVY/INSP } \\
\text { AfJ }\end{array}$ & HIP & $\begin{array}{l}\text { Kony } \\
1 / 9 / 95\end{array}$ \\
\hline \multicolumn{7}{|l|}{ 5.2 Processed Materials - Dedding Layer (Lift H1) } \\
\hline -Lift thickness: material loose/compacted per Table 3:1 & & & & SRVY/INSSCE & IJP & \\
\hline *Maximum clod size (visual moniloring) & & & & INSP ifd & & \\
\hline Compaction - double steel drum roller & & CONSTR & & INSP erd & & K- Saven \\
\hline *Testing: moisture content (D2216 and D3017) & ENG & & & MTL & & Ken: \\
\hline *Testing: in-place density (D1556 and D2922) & ENG & & & MTL & & \\
\hline
\end{tabular}

CONSTR = BN Consınuction; $\mathrm{CQA}=$ Constnuction Quality Assurance; ENG/TM = BN Engineering/Task Manager; GCL $=$ Geosynthetic Clay Liner; HP $=$ Hold Point; INSP $=$ BN Inspection; IVCO = Independent Verification Conlractor Officer (PEER Consultants); MTL. = BN Materials Test Lab; SRVY = BN Survey; SUB-CONTR = DN Subcontractor

- See ${ }^{\top}$ rble 3.1 of this plan 


\begin{tabular}{|c|c|c|c|c|c|c|}
\hline ITEM - DECONTAMINATION POND CLOSURE COVER & ENG/TM & Constht & SUB & $\begin{array}{l}\text { MTL/STVY } / \\
\text { INSP }\end{array}$ & CQA & $\begin{array}{l}\text { औIVC } \\
\text { CHECKLIST } \\
\text { INITIALS/DATE }\end{array}$ \\
\hline $\begin{array}{l}\text { *Vetify ihat all tests were completed with acceptable results; that } \\
\text { applicable IIPs have been released by ENG; and the as-built } \\
\text { reflects the current status }\end{array}$ & ENO & & & MTL/INSP & HP & $\begin{array}{l}11-16-98 \\
1120 y\end{array}$ \\
\hline \multicolumn{7}{|c|}{ NoT $A P P L A B<A$} \\
\hline -Lif thickness: material loose/compacted per.Table 3:1 & & & & SRVY/INSP & HP & \\
\hline *Maximum clod size (visual monitoring) & & & & INSP & & \\
\hline Compaction - double steel drum roller & ؛ & CONSTR & & INSP & & \\
\hline Testing: moisture content (D22!6 and D3017) & ENG & & & MTL & 行 & \\
\hline *Testing: in-place density (D1556 and D2922) & ENG & & & MTL. & & \\
\hline 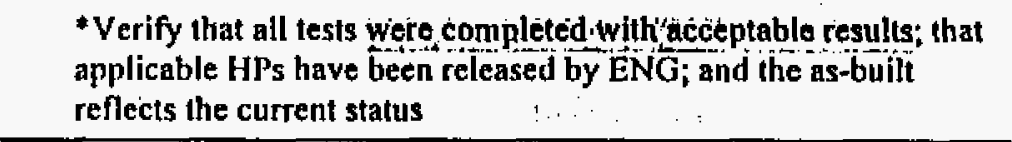 & ENG & & & MTL/INSP & IIP & \\
\hline $\begin{array}{l}\text { Bedding layer plácement completed arid verified per CQAPJïlas } \\
\text { builts "and eng Ineeringiconcurrence }\end{array}$ & ENG & CONSTR & . & SRVY/INSP & $\mathrm{HP}$ & \\
\hline 5.2 GCL Layer (one layer) & & & & & & \\
\hline $\begin{array}{l}\text { Inspect and vetify the bedding layer surface is smooth and free of } \\
\text { voids/debris }\end{array}$ & ENG & & SUB & INSP & IIP & $\begin{array}{l}\text { Sem } \\
11-23-98\end{array}$ \\
\hline $\begin{array}{l}\text { Verify that the } \mathrm{GCL} \text { is ready to place, in good condition, and is in } \\
\text { a dry state }\end{array}$ & ENG & & sun & $\begin{array}{lll} & \text { INSP } & \\
& & \text { sord } \\
\end{array}$ & IIP & 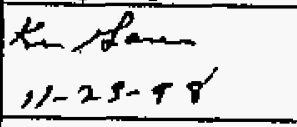 \\
\hline No material sampling/testing required during placement & & & & & & - \\
\hline $\begin{array}{l}\text { Place liner per manufacturer's specifications/representative's } \\
\text { instructions }\end{array}$ & ENQ & & SUB & INSP & & Hen Hang \\
\hline
\end{tabular}

CONSTR $=\mathrm{BN}$ Construction; $\mathrm{CQA}=$ Coustnuction Quality Assurance; $\mathrm{ENG} / \mathrm{TM}=\mathrm{BN}$ Engineering/Task Manager; $\mathrm{GCL}=$ Geosynthetic Clay Liner; HP $=\|$ lold Point; INSP = BN Inspection; IVCO = Independent Verification Contractor Officer (PEER Consultants); MTL = BN Malerials Test Lab; SRVY = BN Survey; SUB-CONTR = BN Subcontractor

- See Table 3.1 of this plan 


\begin{tabular}{|c|c|c|c|c|c|c|}
\hline ITEM - DECONTAMINATION POND CLOSURE COVER & ENG/TM & CONSTR & $\begin{array}{l}\text { SUB- } \\
\text { CONTIR }\end{array}$ & $\begin{array}{l}\text { MTL/SIRVYI } \\
\text { INSP }\end{array}$ & $\begin{array}{l}\text { CQA } \\
\text { III"* }^{*}\end{array}$ & $\begin{array}{l}\text { IVC } \\
\text { CHECKLIST } \\
\text { INITIALS/LATE }\end{array}$ \\
\hline $\begin{array}{l}\text { Continuous visual inspection of liner for surface damage, } \\
\text { smoothness, and joint overlaps }\end{array}$ & ENG & & SUI & INSP & & $\operatorname{Kem}$ \\
\hline $\begin{array}{l}\text { Seal liner searns per manufacturer's specifications/representatives } \\
\text { instructions (Bentonite) }\end{array}$ & ENG & & SUB & 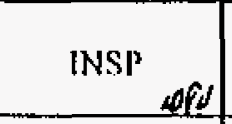 & & tang at \\
\hline $\begin{array}{l}\text { Final approval of GCL completed installation and acceplance by } \\
\text { Engineering and manufacturer's representative }\end{array}$ & ENG & & SUB & INSP & HP & \\
\hline \multicolumn{7}{|l|}{ 5.2 Processed Materials -- Buffer Layer (Lift \#I) } \\
\hline "Lift thickness: material loose/compacted per Table 3.1. & & & & SRVYIINSBR & HP & \\
\hline *Maximum clod size (vistul monitoring) & & & & INSP wN & & \\
\hline Compaction - double steel drum roller & & CONSTR & & INSP ard & & \\
\hline 'Testing: moisture content (D2216 and D1017) & ENG & & & MTL & & \\
\hline -Testing: in-place densily (D1556 and D2922) & ENG & & & MTL & & \\
\hline $\begin{array}{l}\text { "Verify that all tests were completed with acceptable results; that } \\
\text { applicable HPs have been released by ENG; and the as-built } \\
\text { reflects the current status }\end{array}$ & ENG & & & $\begin{array}{r}\text { MTL/INSP } \\
\text { ofed }\end{array}$ & $H \mathrm{P}$ & \\
\hline \multicolumn{7}{|l|}{5.21} \\
\hline "Lift thickness: naterial loose/compacted per Table 3.1 & & & & SRVYIINSP & $1 \mathrm{P}$ & \\
\hline *Maximum clod size (visual monitoring) & & & & INSP & & \\
\hline Compaction - double steel drum roller & . & CONSTR & & INSR: & & \\
\hline -Testing: moisture content (D2216 and D3017) & ENG & & & MTL & & \\
\hline -Testing: in-place density (D 1556 and D2922) & ENG & & & MTL. & & \\
\hline
\end{tabular}

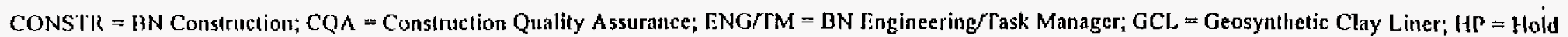
Point; INSP = BN Inspection; IVCO = Independent Verification Contractor Officer (PEER Consultants); $M T L=B N$ Materials Test Lab; SRVY $=$ BN Survey; SUR-CONT'R = BN SubeonIraclor

- Se "zble 3.1 of this plan 


\begin{tabular}{|c|c|c|c|c|c|c|}
\hline ITEM - DECONTAMINATION POND CLOSURE COVER & ENG/TM & CoNstr & $\begin{array}{l}\text { SUI- } \\
\text { CONTR }\end{array}$ & $\begin{array}{l}\text { MTU/SR VY } \\
\text { INSP }\end{array}$ & $\begin{array}{c}\mathrm{CQA} \\
\mathrm{HP} \\
\mathrm{PA}\end{array}$ & $\begin{array}{l}\text { IVC } \\
\text { CIECKLIST } \\
\text { INITIALS/DATE }\end{array}$ \\
\hline $\begin{array}{l}\text { *Verify that all tests were completed witlyaceptable results; that } \\
\text { applicable HPs have been released by ENG; and the as-built } \\
\text { reflects the current status. }\end{array}$ & ENG & & & MTL/INSP & IIP & \\
\hline $\begin{array}{l}\text { Buffer layer placement completed and verified per } C Q A P \text { isis: } \\
\text { builis," and engineering concurrence }\end{array}$ & ENG & CONSTR & & SRVY/INSP & HP. & \\
\hline \multicolumn{7}{|l|}{ 5.1 General Fill Liyerir Perimeter Placement'(Lift H1) } \\
\hline Place pond perimetergenieral filt per, Drawing $C 4$ & & CONSTR & & INSP ofd & & \\
\hline 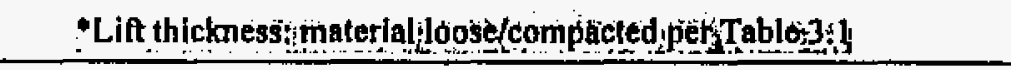 & & & & SRVY/INSP & $\mathrm{HP}$ & \\
\hline Compaction dóduble steel dnum rolter & & CONSTR & & INSP, & & \\
\hline *Testing: moisture content'(D3017) & ENG & & & MTL & & \\
\hline Testingt in-place density (D2922) & ENG & & & MTL & & \\
\hline 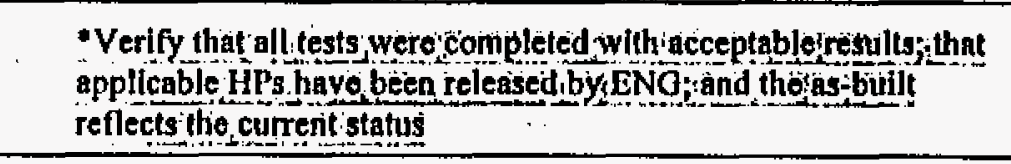 & ENO & & & MTLINSP & HP: & \\
\hline \multicolumn{7}{|l|}{5.1 General Full Layer PerImeter Placement (Lift H2) } \\
\hline Place pond perimeterigeneral frll per Drawtng C4 & & CONSTR & & 1NSP. & & \\
\hline *Lin thleknessitmaterial loose/compacted per Table 3 is & & & & SRYY/INSP & $\prod_{1}$ & \\
\hline Compaction: double'steel drum roller & & CONSTR & & İNSP. & & \\
\hline Testingt moisture content (D3017) & ENG & & & MTL & & \\
\hline -Testing: in place density (D2922) & ENG & & & MTL & & \\
\hline
\end{tabular}

CONSTR = BN Construction; CQA = Construction Quality Assurance; ENG/TM = BN Engineering/Task Manager; GCL $=$ Geosynthetic Clay Liner; HP $=$ Hold Point; INSP = BN Inspection; IVCO = Independent Verification Contractor Officer (PEER Consultants); MTL $=$ BN Materials Test Lab; SRVY = BN Survey;

SUB-CONTR $=$ BN Subcontractor

- See Table 3.1 of this plan 


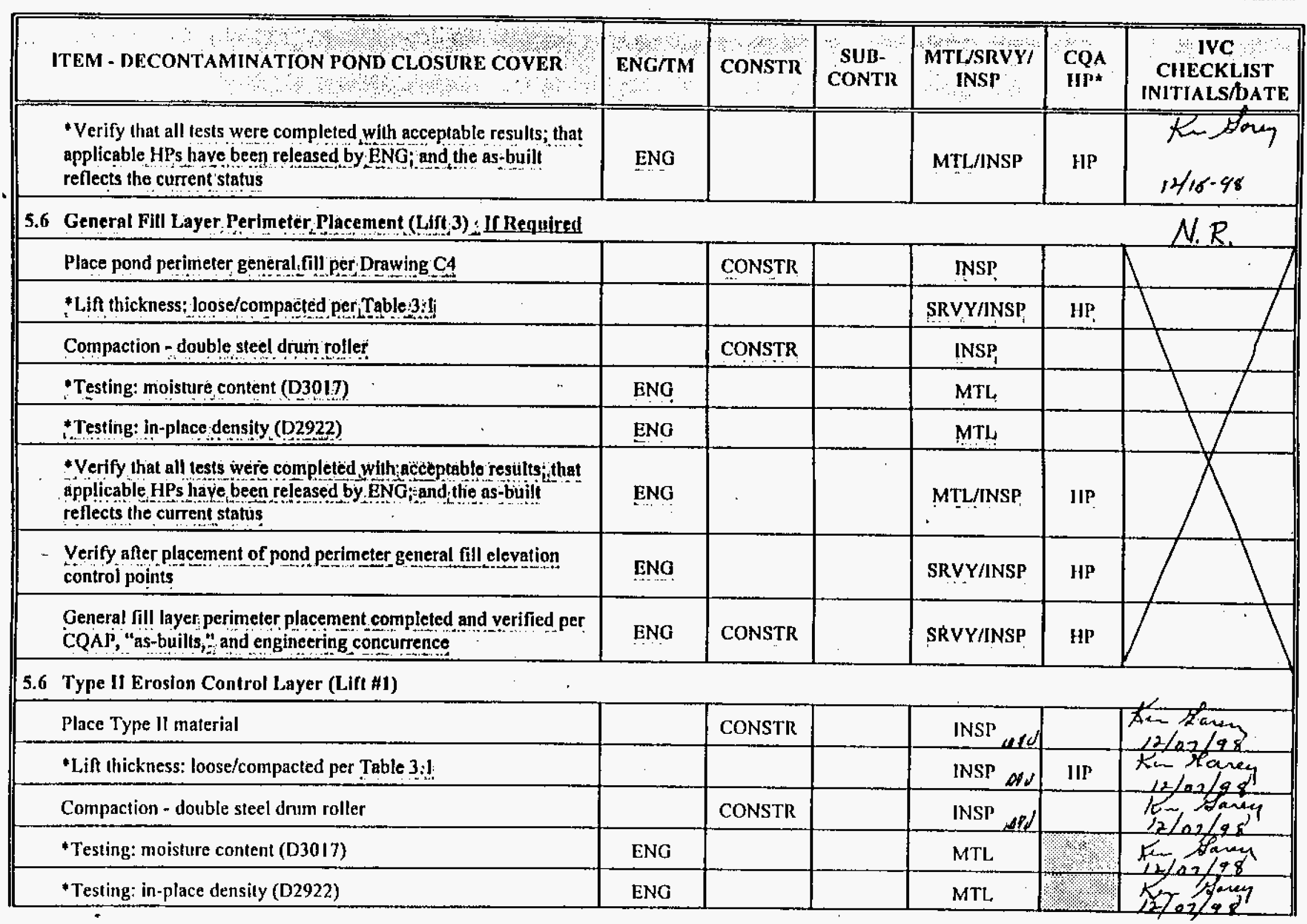

CONSTR = BN Construction; $\mathrm{CQA}=$ Construclion Quality Assurance; ENG/TM $=$ BN Engineering/Task Manager; GCL $=$ Geosynthetic Clay Liner; HP $=1$ Iold Point; INSP = BN Inspection; IVCO = Independent Verification Contractor Officer (PEER Consultants); MTL = BN Materiajs Test Lab; SRVY = BN Survey; 


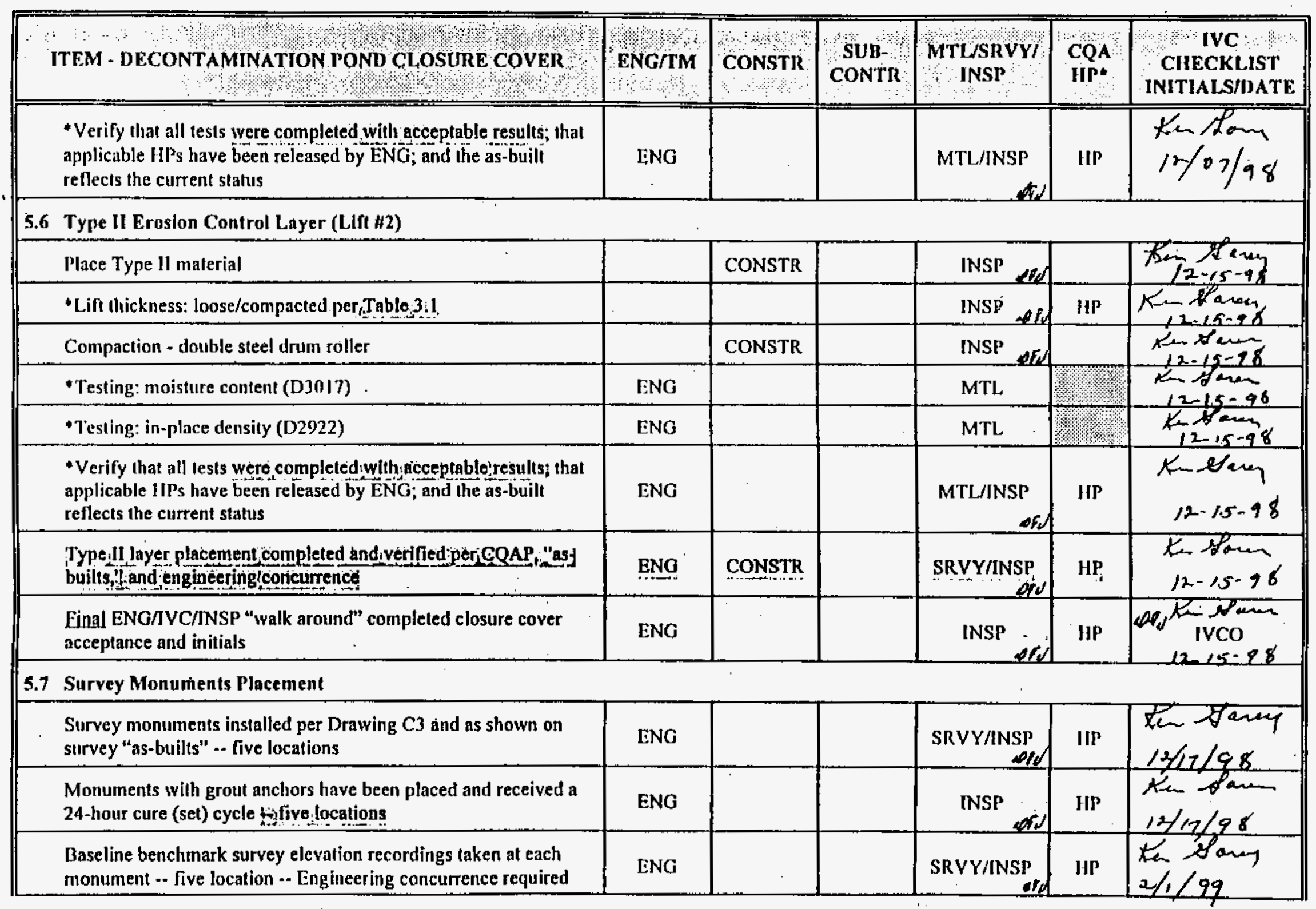

CONSTR = BN Construction; $\mathrm{CQA}=$ Construction Quality Assurance; $\mathrm{ENG} / \mathrm{TM}=\mathrm{BN}$ Engineering/Task Manager; GCL = Geosynthetic Clay Liner; $/ \mathrm{HP}=\mathrm{flold}$ Point; INSP = BN Inspection; IVCO = Independent Verification Contractor Officer (PEER Consultants); MTL = BN Malerials Test Lab; SRVY = BN Survey; SUB-CONTR $=$ BN Subcontractor 


\begin{tabular}{|c|c|c|c|c|c|c|}
\hline ITEM - DECONTAMINATION POND CLOSURE COVER & सिG/TM & $\begin{array}{l}\text { Cिएक } \\
\text { CoNsTr }\end{array}$ & $\begin{array}{l}\text { SUB- } \\
\text { CONTR }\end{array}$ & MTL/SRVY/ & $\begin{array}{cl}\text { CQA } \\
\text { HPA }^{*}\end{array}$ & $\begin{array}{l}\text { IVC } \\
\text { CIECKLIST } \\
\text { INITIALSIDATE }\end{array}$ \\
\hline \multicolumn{7}{|l|}{5.8 Sile Fencing/Gates/Signage } \\
\hline Fence and access gates installed per Drawing $\mathrm{C3}$ & ENG & CONSTR & & SRVY/INSSP & HP & the gang \\
\hline Signage inslalled and secured per Drawing C3 & ENG & CONSTR & & SRYY/INSD & HP & \\
\hline \multicolumn{7}{|l|}{ 6.0 POST CONSTRUCTION } \\
\hline 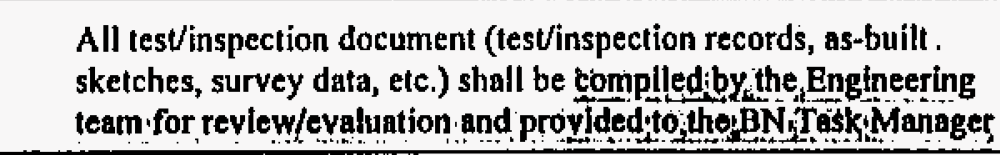 & ENG/TM & & SUB & $\begin{array}{l}\text { MTL/SRVYI } \\
\text { INSP } \\
\text { ind }\end{array}$ & HP. & $\begin{array}{l}R=96 a y \\
2-1-99\end{array}$ \\
\hline 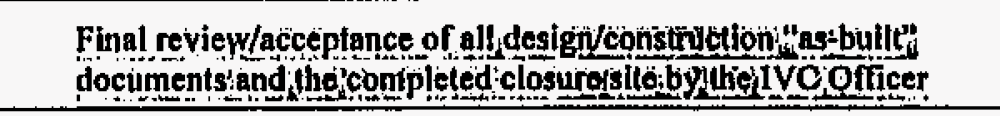 & ENG/TM & & & & $\mathrm{HP}$. & $\begin{array}{l}\text { Hin } \operatorname{Ran} 1 \\
2=-1-g G\end{array}$ \\
\hline 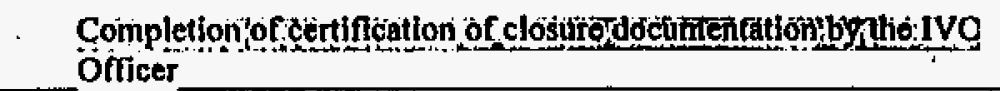 & & & & & $\mathbf{H P}$. & co \\
\hline
\end{tabular}

CONSTR = DN Construction; CQA = Construction Qualily Assurance; ENG/TM $=$ BN Engineering/Task Manager; GCL = Geosynthetic Clay Liner; HP = Hold Point; INSP = BN Inspection; IVCO = Independent Verification Contractor Officer (PEER Consultants); MTL = BN Materials Test Lab; SRVY = BN Survey; SUB.CONTR $=$ BN Subcontractor

$\mathrm{S}$ ( . ble 3.1 of this plan 


\section{APPENDIX G}

\section{COMMENT RESPONSE DOCUMENTATION}




\section{DOCUMENT REVIEW SHEET}

Document Title/Number: Closure Report for Corrective Action Unit 92: Area 6 Decontamination Pond Nevada Test Site, Nevada. DOE/NV/11718--306

Document Date: February 1999

Revision Number: 0

Originator/Organization: Thomas Fitzmaurice, Bechtel Nevada Environmental Restoration

Date Comments Duc: March, 1999

Reviewer/Organization: Matthew A. DeBurle, Staff Engineer II, Nevada Division of Environmental Protection

\begin{tabular}{||c|c|c|c||}
\hline $\begin{array}{c}\text { Comment } \\
\text { Number/ } \\
\text { Location }\end{array}$ & Type & Comment & Comment Response \\
\hline \hline $\begin{array}{c}\text { 1. Executive } \\
\text { Summary } \\
\text { fifth } \\
\text { paragraph }\end{array}$ & $\mathrm{M}$ & $\begin{array}{l}\text { Change Nevada Department of } \\
\text { Environmental Protection to Nevada } \\
\text { Division of Environmental Protection }\end{array}$ & None \\
\hline $\begin{array}{c}2 . \text { Section } \\
\text { paragraph }\end{array}$ & $\mathrm{M}$ & $\begin{array}{l}\text { Specify the date that the final closure was } \\
\text { completed. }\end{array}$ & $\begin{array}{l}\text { Because fence signage, and a few other } \\
\text { minor details were not complete when } \\
\text { the draft closure report was submitted the } \\
\text { final closure date was left blank. } \\
\text { However all the elements of the CAU 92 } \\
\text { closure have been completed and the date } \\
\text { has been included in the document. }\end{array}$ \\
\hline
\end{tabular}

a. Comment Types: M=Mandatory 'S=Suggested $\quad$ Page 1 of 8 


\section{DOCUMENT REVIEW SHEET}

\begin{tabular}{||c|c|c|l|l||}
\hline $\begin{array}{c}\text { Comment } \\
\text { Number/ } \\
\text { Location }\end{array}$ & Type & Comment & Comment Response & Accept \\
\hline \hline $\begin{array}{c}\text { 3. Section } \\
\text { 2.1.1.3 First } \\
\text { paragraph, } \\
\text { last sentence }\end{array}$ & $\mathrm{M}$ & $\begin{array}{l}\text { The meaning of this sentence is not clear to } \\
\text { the NDEP staff. The DOE/NV should } \\
\text { review and clarify this sentence. }\end{array}$ & $\begin{array}{l}\text { The last sentence describes the method } \\
\text { used to grout the remaining pipe closed. } \\
\text { The words "of the pipe" have been added } \\
\text { to the previous sentence, to help clear up } \\
\text { the confusion. A smaller pipe was } \\
\text { inserted into the historic pipeline until it } \\
\text { reached sump 3. Grout was injected } \\
\text { through the smaller (tremmie pipe) pipe. } \\
\text { As the pipeline filled with grout the } \\
\text { smaller pipe was pulled out. }\end{array}$ & Yes \\
\hline $\begin{array}{c}\text { 4. Section } \\
2.1 .2\end{array}$ & $\mathrm{M}$ & $\begin{array}{l}\text { NDEP staff infers that the DOE/NV means } \\
\text { to write "Following cover construction, } \\
\text { monuments were placed..." }\end{array}$ & $\begin{array}{l}\text { The word construct has been replaced } \\
\text { with construction. }\end{array}$ & Yes \\
\hline $\begin{array}{c}\text { 5. Section } \\
2.1 .2 .3\end{array}$ & $\mathrm{M}$ & $\begin{array}{l}\text { NDEP staff believe the contractor's name is } \\
\text { Knight Piesold. }\end{array}$ & The letter "d" was added to Piesol. & Yes \\
\hline
\end{tabular}

a. Cornment Types: M=Mandatory $S=$ Suggested

Page 2 of 8 


\section{DOCUMEN'T REVIEW SHEET}

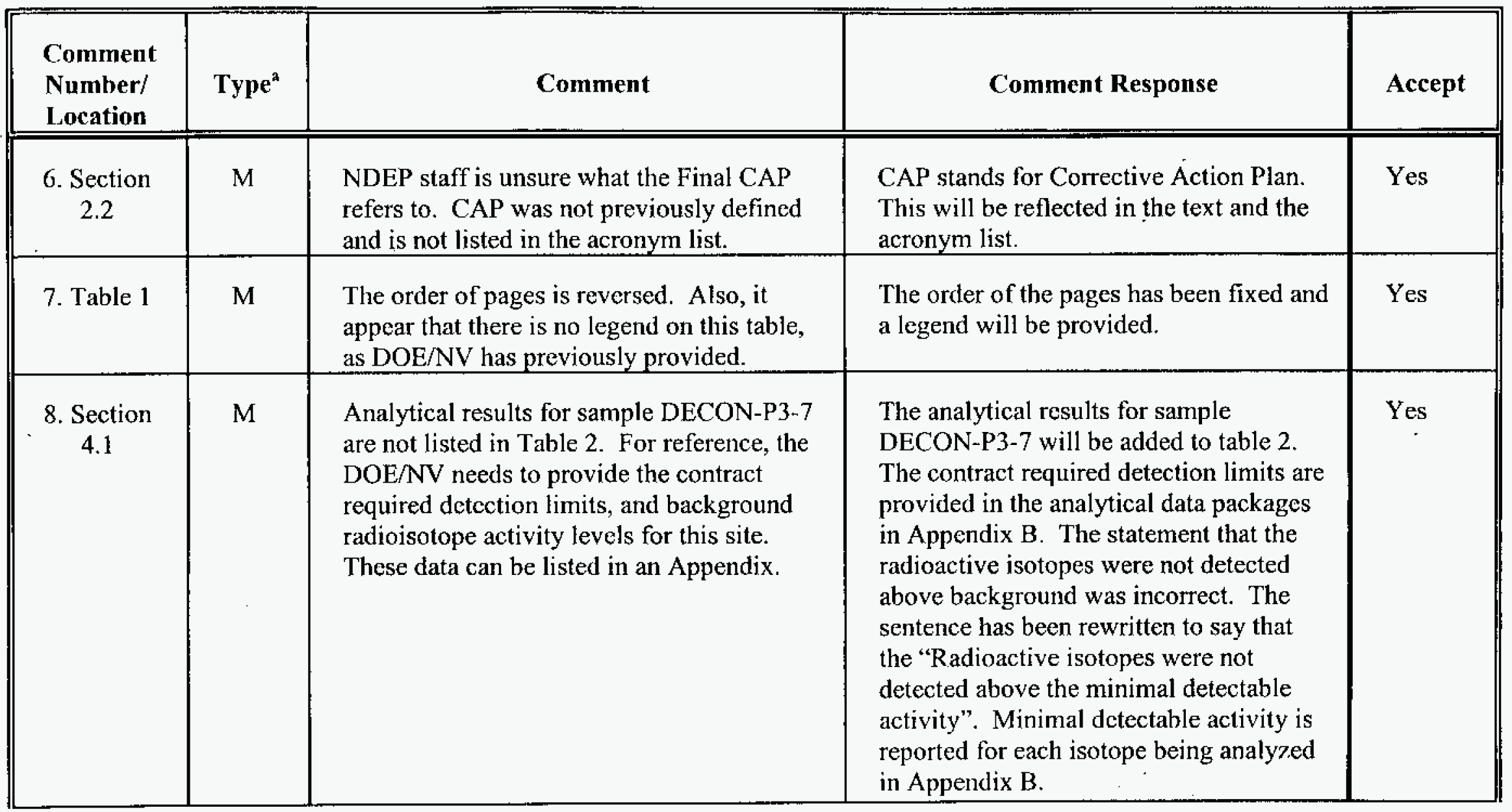

a, Comment 'Types: M-Mandatory' S'Suggested 
DOCUMENT REVIEW SHEET

\begin{tabular}{||c|c|c|c|c||}
\hline $\begin{array}{c}\text { Comment } \\
\text { Number/ } \\
\text { Location }\end{array}$ & Type & Comment & Comment Response \\
\hline \hline $\begin{array}{c}\text { 9. Section 4. } \\
\text { Last } \\
\text { paragraph }\end{array}$ & $\mathrm{M}$ & $\begin{array}{l}\text { Table 1 is referenced for analytical results } \\
\text { for the sludge samples. These data are listed } \\
\text { in Table 2 }\end{array}$ & The reference will be changed to Table 2. \\
\hline $\begin{array}{c}\text { 10. Section } \\
\text { 4.2- Table 2 }\end{array}$ & $\mathrm{M}$ & $\begin{array}{c}\text { Table 2 is referenced for the nuclear density } \\
\text { test results. These data are listed in Table 2. }\end{array}$ & The reference will be changed to Table 3. & Yes \\
\hline
\end{tabular}




\section{DOCUMENT REVIEW SHEET}

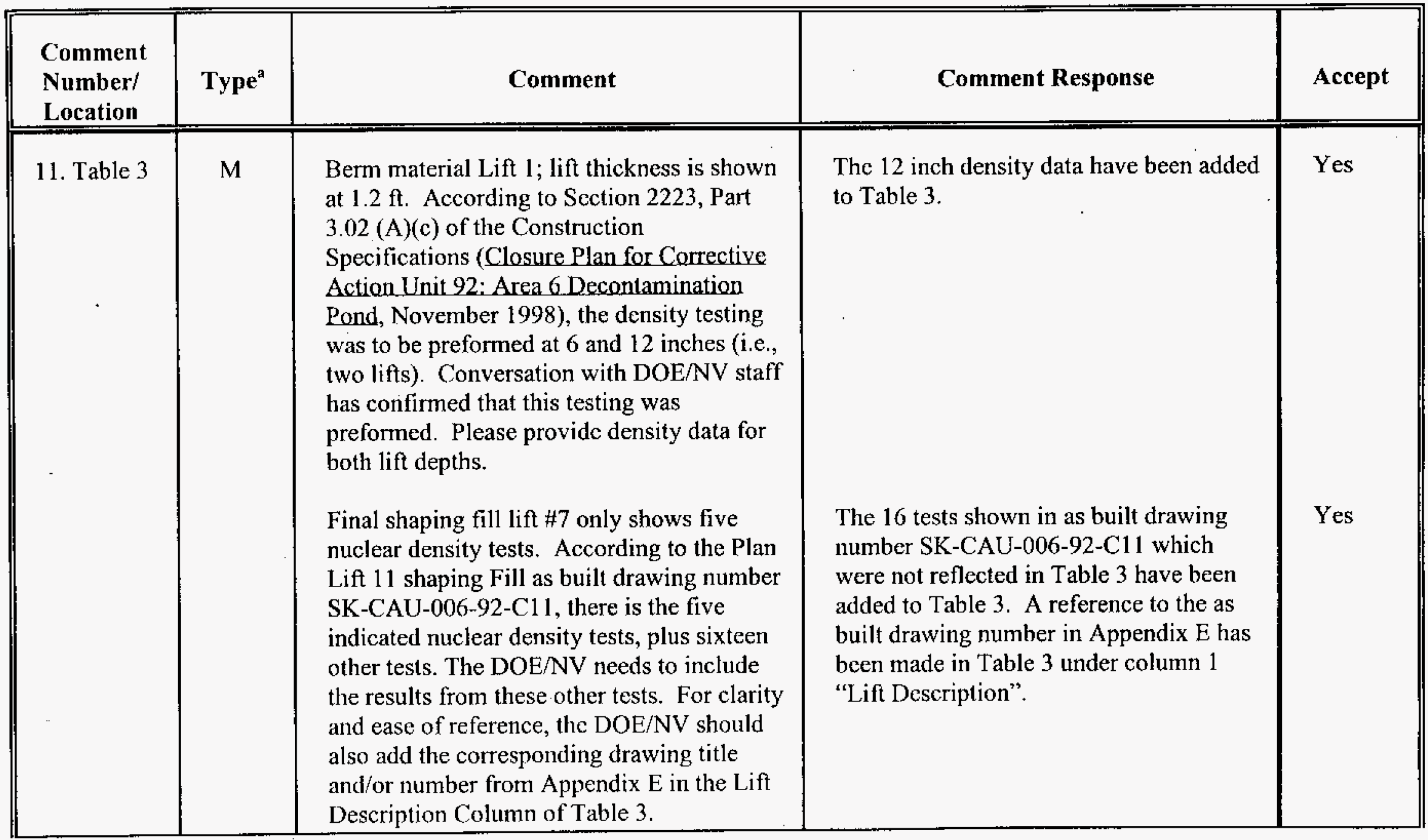

\footnotetext{
a. Comment Types: M=Mandatory $S=$ Suggested $\quad$ Page 5 of 8
} 
DOCUMENT REVIEW SHEET

\begin{tabular}{|c|c|c|c|c|}
\hline $\begin{array}{c}\text { Comment } \\
\text { Number/ } \\
\text { Location }\end{array}$ & Type & Comment & Comment Response & Accept \\
\hline \begin{tabular}{c|c|c|c|c|} 
11. Table 3 \\
(continued)
\end{tabular} & $\mathrm{M}$ & $\begin{array}{l}\text { Appendix E has drawings for a Plan Lift 4A. } \\
\text { Which nuclear density test data is reported } \\
\text { under Berm Material Lift 4 in Table 3? The } \\
\text { DOE/NV need to provide the nuclear density } \\
\text { data from both activities (Lift 4 and lift 4A). } \\
\text { Also, density test results for the Plan Over } \\
\text { Excavation are not reported. The DOE/NV } \\
\text { needs to provide these data. }\end{array}$ & $\begin{array}{l}\text { Lift 4A is the same as lift 4. It was re- } \\
\text { surveyed for elevation control only. The } \\
\text { As Built will be changed to clarify this. } \\
\text { The density results for the Plan Over } \\
\text { Excavation have been added to Table 3. }\end{array}$ & Yes \\
12. & $\mathrm{M}$ & $\begin{array}{l}\text { The photos of the Area 6 Decontamination } \\
\text { Pond Stamped Monument and Finished } \\
\text { Cover with Fencing are missing. }\end{array}$ & $\begin{array}{l}\text { Yes. At the time this draft was submitted } \\
\text { for review these activities had not been } \\
\text { completed. Now that they are photos } \\
\text { will be included. }\end{array}$ & Yes \\
\hline Appendix A & & & & \\
\hline
\end{tabular}

a. Comment Types: M Mandatory $S=$ Suggested

Page 6 of 8 


\section{DOCUMENT REVIEW SHEET}

\begin{tabular}{|c|c|c|c|c|}
\hline $\begin{array}{l}\text { Comment } \\
\text { Number/ } \\
\text { Location }\end{array}$ & Type $^{\text {a }}$ & Comment & Comment Response & Accept \\
\hline $\begin{array}{c}13 . \\
\text { Appendix E }\end{array}$ & M & $\begin{array}{l}\text { Details Drawing \# CAU-006-92-C5; Concrete } \\
\text { Monument Detail (Item 6), verify dimensions of } \\
\text { plan and horizontal views. As indicated, the } \\
\text { concrete monument height is larger than the } \\
\text { plan view length. } \\
\text { Geosynthetic Clay Liner Panel Layout Drawing } \\
\text { \# CAU-006-92-C6; There is no indication of a } \\
\text { Panel 6, however there is a large area covered } \\
\text { by Panel 5. Also, Pancl } 22 \text { seems to cover a } \\
\text { very large area (two sites), whereas, Panel } 23 \\
\text { covers a relatively small area (two sites). Is this } \\
\text { correct, or have some of the labels been } \\
\text { switched? For clarification, in addition to a lot } \\
\text { and serial number, the area of each GCL panel } \\
\text { should also be reported. }\end{array}$ & $\begin{array}{l}\text { Drawing \# CAU-006-92-C5 has been } \\
\text { corrected. } \\
\text { Some mistakes were made in the drawing } \\
\text { regarding the placement of panel numbers. } \\
\text { Drawing \# CAU-006-92-C6 has been } \\
\text { corrected. The panel areas will be addressed } \\
\text { in the Clay Liner Panel Layout Drawing. }\end{array}$ & Yes \\
\hline
\end{tabular}




\section{DOCUMENT REVIEW SHEET}

\begin{tabular}{|c|c|c|c|c|}
\hline $\begin{array}{l}\text { Comment } \\
\text { Number/ } \\
\text { Location } \\
\end{array}$ & Type $^{a}$ & Comment & Comment Response & Accept \\
\hline $\begin{array}{c}13 . \\
\text { Appendix E } \\
\text { (continued) }\end{array}$ & $M$ & $\begin{array}{l}\text { Plan Lift } 9 \text { Shaping fill Drawing \# CAU-006-92- } \\
\text { C9; The DOE/NV needs to provide the rationale } \\
\text { for taking two density tests in one quadrant, and } \\
\text { not taking any density test in another quadrant. } \\
\text { Refer to points } 216 \text { and } 217 \text {. } \\
\text { The DOE/NV should also provide the } \\
\text { nuclear density test data from the Perimeter } \\
\text { Backfill arca in Table } 3 \text {. Plan lift } 14 \text { (\& } 15 \text { ) } \\
\text { Type II Drawing \# CAU-006-92-C14 (\& } \\
\text { C15) indicate that nuclear density testing } \\
\text { was conducted on the Perimeter Backfill. }\end{array}$ & $\begin{array}{l}\text { The density tests were not taken in the same } \\
\text { quadrant, this was a transposition error in the } \\
\text { survey record. Lach point which was tested } \\
\text { for density was verified in the field by } 5 \text { sets } \\
\text { of eyes (Site Supervisor, BN Inspector, } \\
\text { Independent Verifier, Material Testing } \\
\text { Laboratory Technician and the Surveyor). } \\
\text { However, after extensive review of the } \\
\text { survey data log books we are unable to } \\
\text { provide evidence to support this. This } \\
\text { derivation to the CQA Plan will be } \\
\text { addressed within Section } 2.2 \text { of the } \\
\text { Closure Report. } \\
\text { The data form the Perimeter Backfill for } \\
\text { Lift } 14 \text { and Lift } 15 \text { have been added to } \\
\text { Table } 3 \text {. }\end{array}$ & Yes \\
\hline
\end{tabular}

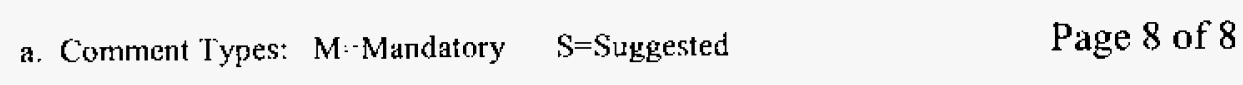




\section{DISTRIBUTION LIST}


*Provide copy of initial distribution of Revision 0; remainder of list gets Revision 0 if approved without changes. The entire list receives Revision 1, if issued.

\section{Nevada Department of Environmental Protection}

Paul Liebendorfer

Bureau of Federal Facilities

Division of Environmental Protection

333 W. Nye Lane, Room 13B

Carson City, NV 89706-0866

Michael D. McKinnon

Bureau of Federal Facilities

Division of Environmental Protection

555 E. Washington, Suite 4300

Las Vegas, NV 89101-1049
2 (Controlled)*

1 (Controlled)*

\section{U.S. Department of Energy}

Janet Appenzeller-Wing

Environmental Restoration Division

1 (Uncontrolled)*

U.S. Department of Energy, Nevada Operations Office

P.O. Box $98518 \mathrm{M} / \mathrm{S} 505$

Las Vegas, NV 89193-8518

Clayton W. Barrow

1 (Uncontrolled)*

Environmental Restoration Division

U.S. Department of Energy, Nevada Operations Office

P.O. Box $98518 \mathrm{M} / \mathrm{S} 505$

Las Vegas, NV 89193-8518

Sabrina Lawrence

1 (Controlled)*

Environmental Restoration Division

U.S. Department of Energy, Nevada Operations Office

P.O. Box $98518 \mathrm{M} / \mathrm{S} 505$

Las Vegas, NV 89193-8518

DOE Public Reading Facility

1 (Controlled)

P.O. Box $98521 \mathrm{M} / \mathrm{S}$ NLV040

Las Vegas, NV 89193-8521 
DOE/Nevada Operations Office

Technical Information Resource Center

P.O. Box $98521 \mathrm{M} / \mathrm{S} 505$

Las Vegas, NV 89193-8521

U.S. Department of Energy

Office of Scientific and Technical Information

175 Oak Ridge Turnpike

P.O. Box 62

Oak Ridge, TN 37831-0062

\section{Bechtel Nevada}

Correspondence Control

Bechtel Nevada

P.O. Box $98521 \mathrm{M} / \mathrm{S}$ NLV008

Las Vegas, NV 89193-8521

Environmental Management Correspondence Control

Bechtel Nevada

P.O. Box $98521 \mathrm{M} / \mathrm{S}$ NLV080

Las Vegas, NV 89193-8521

Jefferry Smith

Bechtel Nevada

P.O. Box 98521 M/S NTS306

Las Vegas, NV 89193-8521

Janet Cowley

Bechtel Nevada

P.O. Box 98521 M/S NTS1 10

Las Vegas, NV 89193-8521

David Cowser

Bechtel Nevada

P.O. Box $98521 \mathrm{M} / \mathrm{S}$ NLV082

Las Vegas, NV 89193-8521
1 (Uncontrolled)

2 (Uncontrolled)

1 (Uncontrolled)*

1 (Uncontrolled)*

1 (Uncontrolled)*

1 (Uncontrolled)

1 (Uncontrolled)* 


\section{DISTRIBUTION LIST (Continued)}

Thomas Fitzmaurice

Bechtel Nevada

P.O. Box 98521 M/S NTS306

Las Vegas, NV $89193-8521$

Steve Nacht

Bechtel Nevada

P.O. Box 98521 M/S NTS306

Las Vegas, NV 89193-8521
1 (Uncontrolled)*

1 (Uncontrolled)* 